\title{
Radical Carbonyl Umpolung Arylation via Dual Nickel Catalysis
}

Huan-Ming Huang‡, Peter Bellotti ${ }^{\ddagger}$, Johannes E. Erchinger, Tiffany O. Paulisch, F. Glorius*

Organisch-Chemisches Institut, Westfälische Wilhelms-Universität Münster, Corrensstraße 40, 48149 Münster, Germany

*Email: glorius@uni-muenster.de

$\neq$ These authors contributed equally. 


\section{Contents}

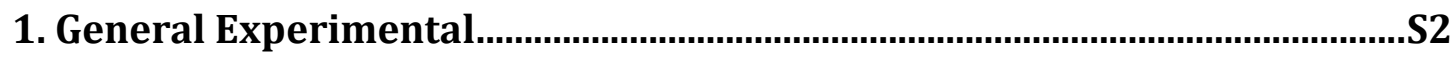

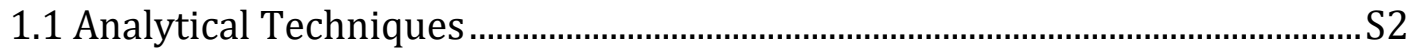

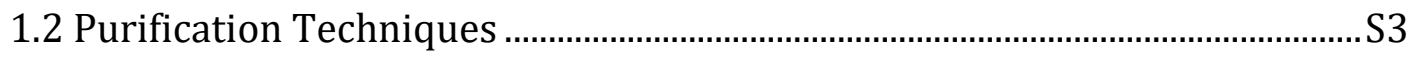

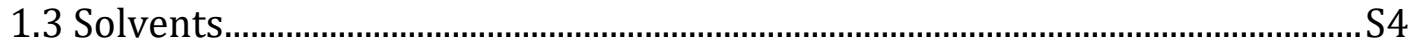

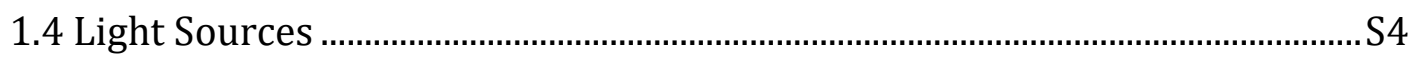

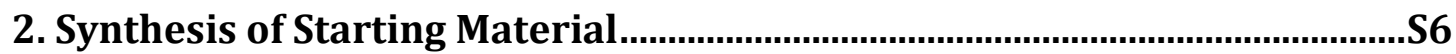

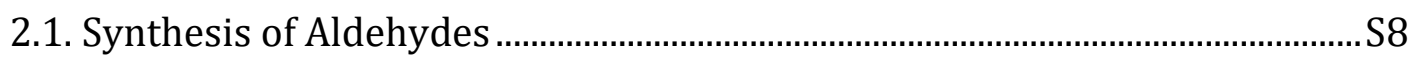

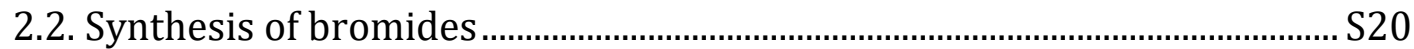

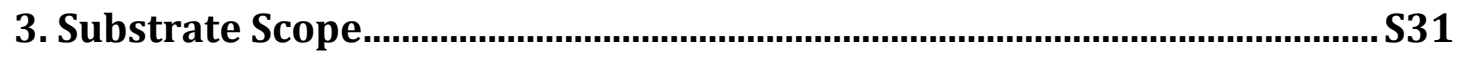

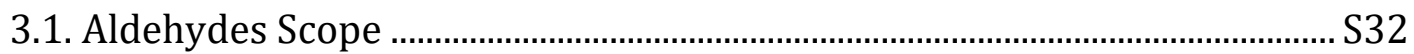

3.2. Bromides Scope ........................................................................................... S4

3.3 Evaluation of the Grignard reaction …….......................................................... 669

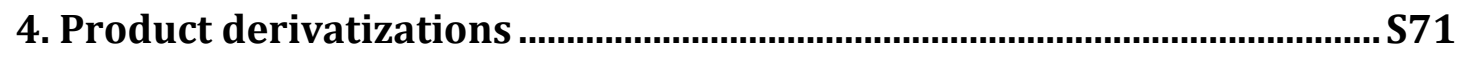

5. Troubleshooting: Frequently Asked Questions............................................S77

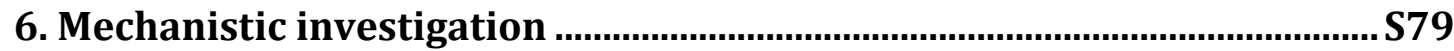

6.1. Synthesis of oxidative addition complex Ni-I ............................................... 579

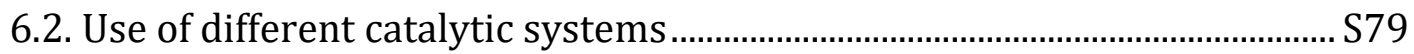

6.3. Stoichiometric reaction of $\mathrm{Ni}(\mathrm{II})$-oxidative addition complex ..................... S81

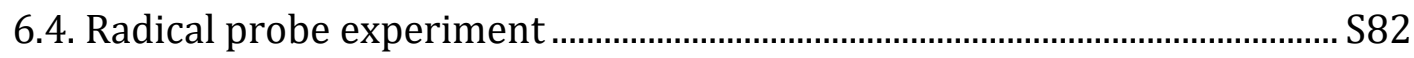

6.5. Radical cyclization experiment …………......................................................... $\mathrm{S} 86$

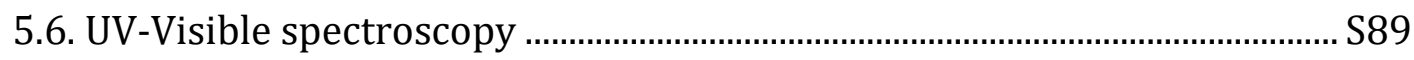

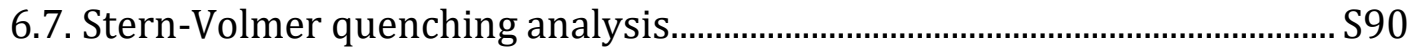

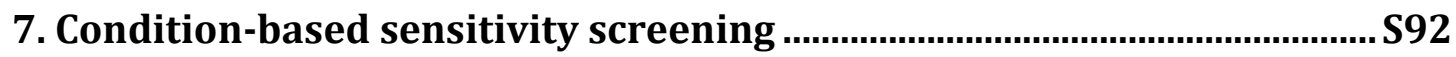

8. ${ }^{1} \mathrm{H},{ }^{13} \mathrm{C}$ and ${ }^{19} \mathrm{~F}$ NMR Spectra of Compounds ................................................ 595

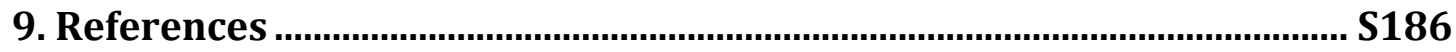




\section{General Experimental}

All reactions were performed in oven-dried glassware under argon, unless otherwise stated. Reaction temperatures are referred to the ones of the heating/cooling media (heating block, cryogenic bath), unless otherwise stated. Reagents were purchased from Sigma-Aldrich, Merck, ACROS Organics, Alfa Aesar, Fluorochem, Combiblocks, TCI and used without further purification. Reactions were stirred using PTFE-coated magnetic stirring bars at $~ 1000 \mathrm{rpm}$, unless otherwise stated. Low boiling solvents $\left(<110^{\circ} \mathrm{C}\right)$ were removed by rotary evaporation under reduced pressure, heating the solution with a water bath at $40^{\circ} \mathrm{C}$. High boiling solvents $\left(>110^{\circ} \mathrm{C}\right)$ were removed in vacuo $(<1 \mathrm{mbar})$ at room temperature or under mild heating $\left(<50^{\circ} \mathrm{C}\right)$, unless otherwise stated. The identity of literature-known compounds was assessed by comparison of ${ }^{1} \mathrm{H}$ NMR spectra and therefore reported. New compounds were characterized by means of ${ }^{1} \mathrm{H}$ NMR, ${ }^{13} \mathrm{C}$ NMR, ${ }^{19} \mathrm{~F}$ NMR (when applicable), HRMS, retention factor on thin layer chromatography, unless otherwise noted. Literature known compounds were characterized by ${ }^{1} \mathrm{H}$ NMR (and ${ }^{13} \mathrm{C}$ NMR, ${ }^{19} \mathrm{~F}$ NMR in some cases) and compared with the available analytical data to confirm their identity.

\subsection{Analytical Techniques}

Thin layer chromatography (TLC) was performed using Merck silica gel 60 F254 aluminum plates and visualization was accomplished with UV light (254 nm) and/or staining with basic $\mathrm{KMnO}_{4}$ solution $4 \mathrm{~g}$ of $\mathrm{KMnO}_{4}, 10 \mathrm{~g} \mathrm{~K}_{2} \mathrm{CO}_{3}, 1 \mathrm{~g} \mathrm{NaOH}$ in $200 \mathrm{ml}$ of distilled water). GC samples were filtered over a short plug of silica (length: $2-3 \mathrm{~cm}$, diameter: $4 \mathrm{~mm}$ ) eluting with EtOAc before analysis, if not stated otherwise. GC-MS spectra were recorded on an Agilent Technologies 7890A GCsystem (Agilent 5975C VL MSD or an Agilent 5975 MSD) or Agilent Intuvo 9000 (Agilent 5977B MSD), and a HP-5MS column $(0.25 \mathrm{~mm} \cdot 30 \mathrm{~m}$, film: $0.25 \mu \mathrm{m})$ and analyzed using MSD ChemStation E.02.02.1431. ${ }^{1} \mathrm{H},{ }^{13} \mathrm{C}$ and ${ }^{19} \mathrm{~F}$ NMR spectra were recorded at room temperature on a Bruker Avance $400\left({ }^{1} \mathrm{H}: 400.13 \mathrm{MHz} ;{ }^{13} \mathrm{C}\right.$ : 
100.62 MHz), Avance Neo 400 ( $\left.{ }^{1} \mathrm{H}: 400.23 \mathrm{MHz} ;{ }^{13} \mathrm{C}: 100.65 \mathrm{MHz}\right)$, Varian $500 \mathrm{MHz}$ INOVA (1 $\left.\mathrm{H}: 499.83 \mathrm{MHz} ;{ }^{13} \mathrm{C}: 125.70 \mathrm{MHz}\right)$ or Varian Unity plus $600\left({ }^{1} \mathrm{H}: 599.31\right.$ $\mathrm{MHz} ;{ }^{13} \mathrm{C}: 150.71 \mathrm{MHz}$ ) in deuterated solvents (> 99.5 Deuteration) purchased from Sigma-Aldrich $\left(\mathrm{CDCl}_{3}\right.$, DMSO- $d_{6} ; \mathrm{D}_{2} \mathrm{O}, \mathrm{CD}_{3} \mathrm{OD}$, THF- $\left.d_{8}, \mathrm{C}_{6} \mathrm{D}_{6}\right)$. Chemical shifts $(\delta)$ for ${ }^{1} \mathrm{H}$ and ${ }^{13} \mathrm{C}$ NMR spectra are given in parts per million (ppm) relative to tetramethylsilane (TMS) using the residual solvent signals as references for ${ }^{1} \mathrm{H}$ and ${ }^{13} \mathrm{C}$ NMR spectra $\left(\mathrm{CDCl}_{3}: \delta_{\mathrm{H}}=7.26 \mathrm{ppm}, \delta_{\mathrm{c}}=77.16 \mathrm{ppm}\right.$, DMSO- $d_{6}: \delta_{\mathrm{H}}=2.05$ ppm, $\delta_{\mathrm{C}}=29.84$ or $206.26 \mathrm{ppm}, \mathrm{CD}_{3} \mathrm{OD}: \delta_{\mathrm{H}}=3.31 \mathrm{ppm}, \delta_{\mathrm{C}}=49.00 \mathrm{ppm}, \mathrm{D}_{2} \mathrm{O}: \delta_{\mathrm{H}}=$ $4.79 \mathrm{ppm}, \delta_{c}=$ absolute referencing, acetone- $d_{6}: \delta_{H}=2.05 \mathrm{ppm}, \delta_{c}=206.26$, THF$d_{8}: \delta_{\mathrm{H}}=3.58$ or $\left.1.73 \mathrm{ppm} ; \mathrm{C}_{6} \mathrm{D}_{6}: \delta_{\mathrm{H}}=7.16 \mathrm{ppm}, \delta_{\mathrm{C}}=128.06 \mathrm{ppm}\right) .{ }^{19} \mathrm{~F}$ NMR spectra were calibrated using absolute referencing system, as suggested by IUPAC. ${ }^{1}$ NMRsignals multiplicities that can be analyzed as first order multiplets are reported using the following abbreviations (or combination thereof): $\mathrm{s}=$ singlet, $\mathrm{d}=$ doublet, $\mathrm{t}=$ triplet, $\mathrm{q}$ = quartet, $\mathrm{p}=$ quintet, $\mathrm{h}$ =sextet; hept = heptet; $\mathrm{m}=$ multiplet, br = broad signal. All spectra were processed using MestReNova 14 using standard phase and baseline correction automations. ESI accurate mass spectra (HRMS) were recorded on a MicroTof (Bruker Daltronics, Bremen) with loop injection. Mass calibration was performed using sodium formate cluster ions immediately followed by the sample in a "quasi-internal" calibration or alternatively on an LTQ Orbitap LTQ XL (Thermo-Fisher Scientific, Bremen) with nano spray.

\subsection{Purification Techniques}

Unless otherwise noted, compouns were purified by flash column chromatography, according to Still and co-workers, ${ }^{2}$ using ACROS Organics silica (0.035-0.070 mm, $60 \AA)$ and the specified solvent system under 0.3-0.5 bar overpressure. Pentane, dichloromethane and ethyl acetate used for chromatography were purchased of technical grade and preliminarly purified by atmospheric pressure distillation. 


\subsection{Solvents}

Unless otherwise stated, dry $\left(\mathrm{H}_{2} \mathrm{O}\right.$ content $\left.<50 \mathrm{ppm}\right)$ reaction solvents were used to perform reactions. The following solvents (ACROS ExtraDry solvents with ACROSeal ${ }^{\circledR}$ cap) were purchased from ACROS Organics, stored under 3 or $4 \AA$ activated molecular sieves and collected under positive argon pressure: dimethylsulfoxide (DMSO), ethyl acetate (EtOAc), 1,4-dioxane. The following solvents were purchased from ACROS Organics, Sigma-Aldrich and Fischer Scientific (HPLC grade) and purified using a custom SPS with activated aluminacolumns (built by the "Feinmechanische Werkstatt des OrganischChemischen Instituts, WWU Münster") and collected under positive argon pressure according to Grubbs procedure: ${ }^{3}$ acetonitrile $(\mathrm{MeCN})$, tetrahydrofuran (THF), diethyl ether (Et 20$), N, N$-dimethylformamide ( $N, N$-DMF), hexane, toluene, 1,2-dichloroethane (1,2-DCE) and dichloromethane (DCM).

\subsection{Light Sources}

Photochemical reactions were performed in a Hepatochem EvoluChem ${ }^{\mathrm{TM}}$ PhotoRedOx Box Duo device and irradiated with two EvoluChem ${ }^{\mathrm{TM}}$ P303-30-1 LEDs $\left(30 \mathrm{~W}, \lambda_{\max }=450 \mathrm{~nm}\right)$. The reaction temperature was measured to be between $25^{\circ} \mathrm{C}$ and $30{ }^{\circ} \mathrm{C}$ using this setup. 


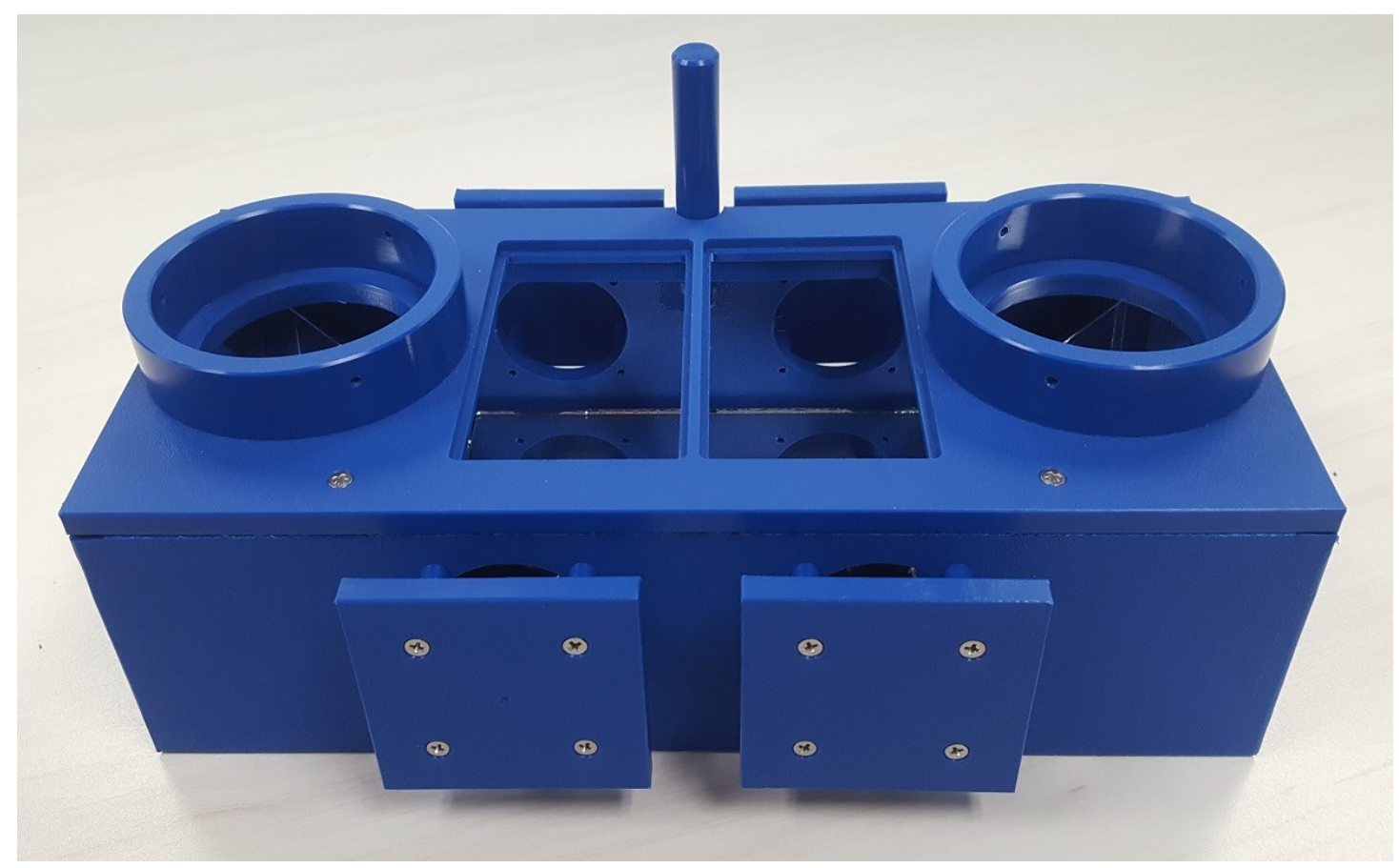

Supplementary Figure 1. Hepatochem EvoluChem ${ }^{\text {TM }}$ PhotoRedOx Box Duo (without light sources and vial holders). Courtesy of Hepatochem.

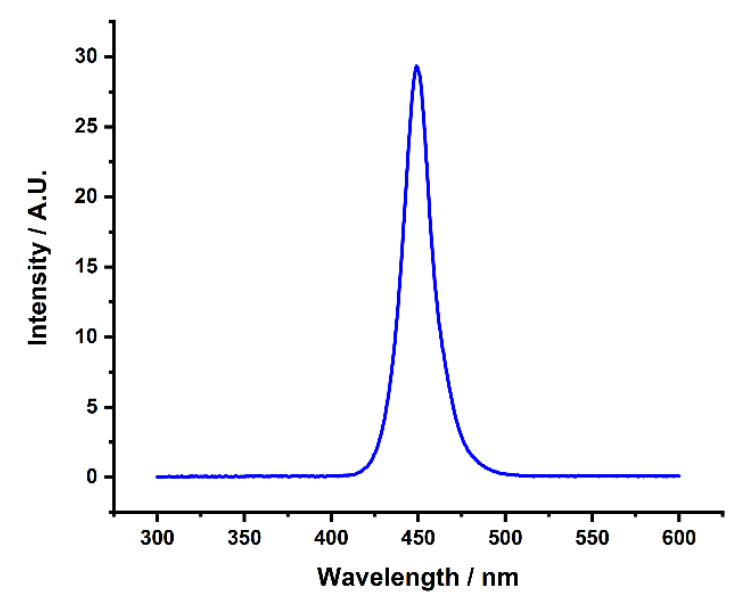

Supplementary Figure 2. Emission spectrum of the light source left - EvoluChem ${ }^{\text {TM }}$ P30330-1 LEDs. 


\section{Synthesis of Starting Material}

Aldehydes 1a-d, 1f-i, 1l were purchased from commercial sources and used as received. Aldehydes $\mathbf{1} \mathbf{m}-\mathbf{o}^{4}$ were synthesized according to the literature procedures. The other aldehydes were synthesized as detailed below.

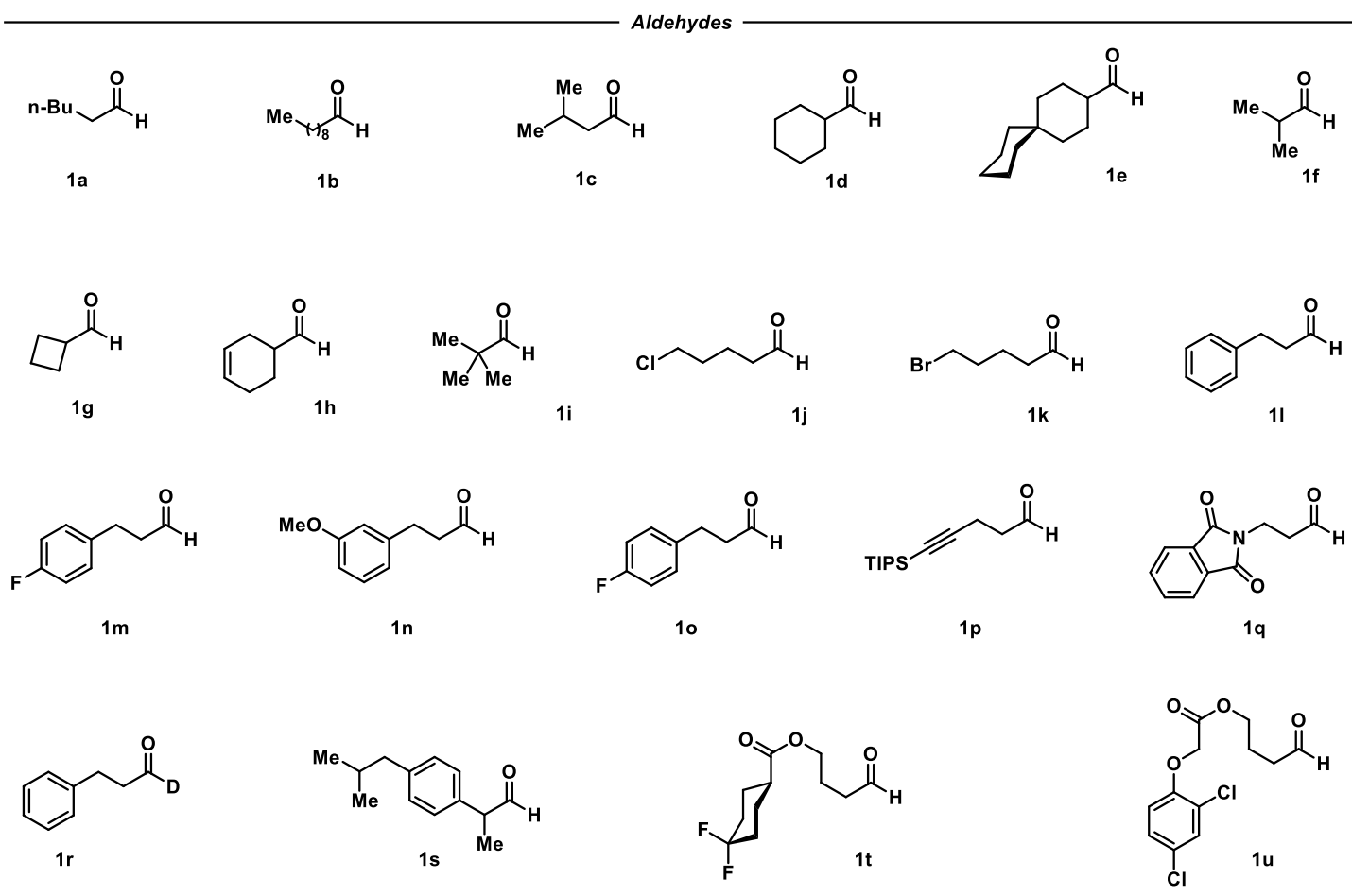

Benzoyl bromide was purchased from commercial sources and used as received, 4-chlorobenzoyl chloride was synthesized according to Palomo and co-workers. ${ }^{5}$

Bromides 2a, 2c-h, 2j-v were purchased from commercial sources and used as received. The other bromides were synthesized according to the procedures described below. 


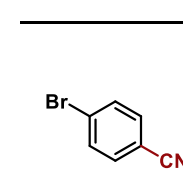

$2 a$<smiles>COC(=O)c1ccc(Br)cc1</smiles>

$2 f$<smiles>O=Cc1ccccc1Br</smiles>

2k<smiles>Clc1cccc(Br)n1</smiles>

$2 p$<smiles>FC(F)(F)c1cccc(Br)n1</smiles>

2v

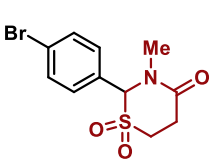

2aa

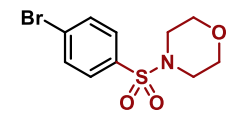

2b<smiles>O=Cc1ccc(Br)cc1</smiles>

$2 g$<smiles>O=C1OCc2cc(Br)ccc21</smiles>

2c

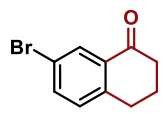

$2 h$

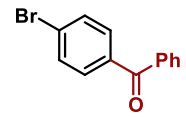

2d

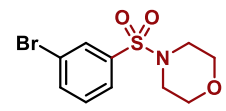

$2 i$

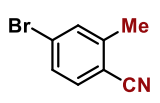

$2 m$<smiles>CC(=O)c1ccc(Br)cc1</smiles>

$2 e$

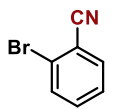

2j

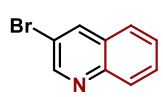

20

21<smiles>Cc1cc(Br)c(N)cc1N(C)C</smiles><smiles>[13CH3][13CH3]</smiles><smiles>[B]c1ccc(I)cn1</smiles>

$2 u$

2q

$2 s$

$2 t$

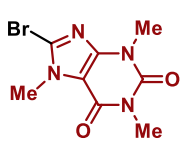

$2 w$

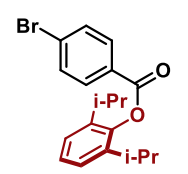

2x

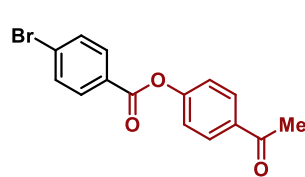

2y

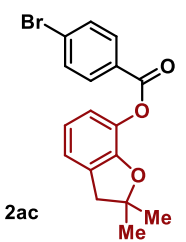

$2 z$

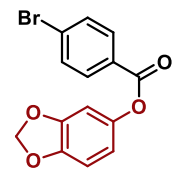

2ad
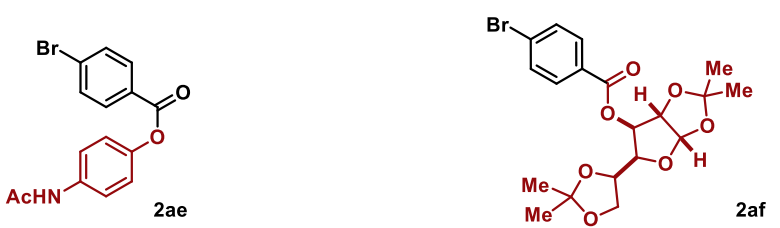

Silanes were purchased from commercial sources and used as received. (TES) ${ }_{3} \mathrm{SiH}$ could also be synthesized according to the procedure by Yamamoto and Yamsoka. ${ }^{6}$ Nickel(II)-bispyridine complexes were synthesized according to the procedure by Martin and co-workers. ${ }^{7}$

The photocatalyst $\left[\operatorname{Ir}\left\{d \mathrm{~F}\left(\mathrm{CF}_{3}\right)_{2} \mathrm{ppy}\right\}_{2}(t \mathrm{Bu}-\mathrm{bpy})\right] \mathrm{PF}_{6}$ (Ir-F) was prepared following the following literature procedure. ${ }^{8}$ 


\subsection{Synthesis of Aldehydes}

\section{General procedure for oxidation of aldehydes (GP1)}

The procedure was adapted from Shiner et al. ${ }^{9}$ An oven-dried Schlenk flask was equipped with a PTFE-coated stirring bar and pyridinium chlorochromate $(1.50$ equiv.) was charged. The vessel was evacuated and backfilled with argon, then dry $\mathrm{CH}_{2} \mathrm{Cl}_{2}(0.2 \mathrm{M})$ was added. Upon vigorous stirring, the appropriate alcohol (1.0 equiv.) was added dropwise/portionwise and the reaction mixture was stirred for three hours until all starting material was consumed (monitored by GCMS or TLC). The reaction mixture was filtered over a silica/Celite ${ }^{\circledR}$ pad and the flask was washed three times with additional $\mathrm{CH}_{2} \mathrm{Cl}_{2}(10 \mathrm{~mL}$ each time). The crude reaction mixture was concentrated in vacuo and purified by column chromatography on silica gel (pentane/Et $2 \mathrm{O}$ mixtures, as indicated in the individual entries) to afford the corresponding aldehyde.

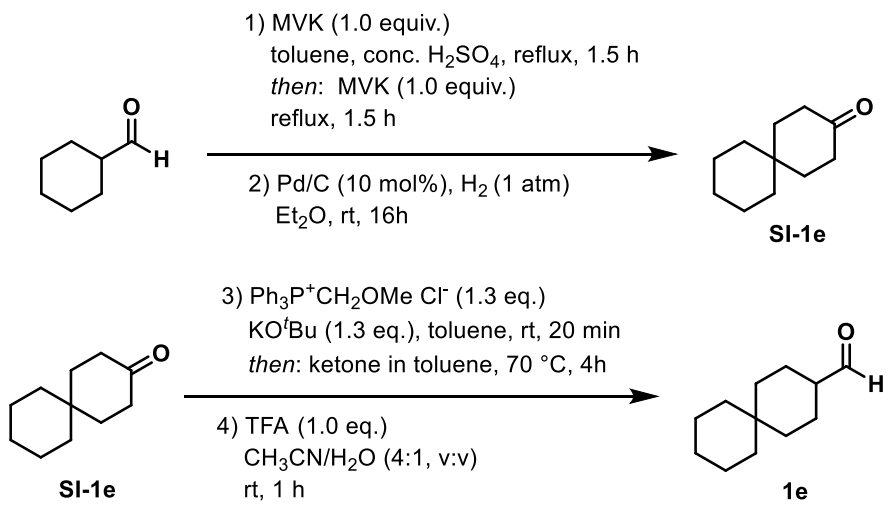

spiro[5.5] undecane-3-carbaldehyde (1e). The title compound was prepared according to the following four step procedure. in four steps.

Step 1. The procedure was adapted from Wang et al. ${ }^{10}$ Cyclohexanecarbaldehyde (2.27 mL, $20.0 \mathrm{mmol}, 1.0$ equiv.) was charged into a round-bottom flask equipped with a PTFE-coated stirring bar, followed by the addition of toluene $(20 \mathrm{~mL})$, methylvinyl ketone (1.63 mL, 20.0 mmol, 1.0 equiv.) and $\mathrm{H}_{2} \mathrm{SO}_{4} 96 \%$ (0.1 mL). The flask was equipped with a Dean-Stark apparatus with a reflux condenser and the reaction mixture was stirred at $45{ }^{\circ} \mathrm{C}$ for 90 minutes, then under reflux for additional 90 minutes. The reaction mixture was cooled to room temperature, 
then additional methylvinyl ketone (1.63 mL, $20.0 \mathrm{mmol}, 1.0$ equiv.) was added and the reaction mixture was stirred under reflux for 90 minutes. Upon cooling to room temperare, aqueous $\mathrm{NaHCO}_{3}(1 \mathrm{M}, 40 \mathrm{~mL})$ was added, the layers were separated, then the aqueous layer was extracted three times with toluene $(10 \mathrm{~mL}$ each time) and the combined organic layers were washed with brine (10 mL), then dried over $\mathrm{MgSO}_{4}$, filtered and concentrated in vacuo. Purification by column chromatography on silica gel (pentane/Et $2 \mathrm{O}=19 / 1 \rightarrow 3 / 1$ ) afforded spiro[5.5] undec-1-en-3-one (0.78 g, $4.7 \mathrm{mmol}, 24 \%$ ) as a colorless oil.

$\underline{\text { Step 2. }}$. The procedure was adapted from Snider et al. ${ }^{11}$ To a round bottom flask equipped with a PTFE-coated stirring bar, spiro[5.5] undec-1-en-3-one (0.78 g, $4.7 \mathrm{mmol}, 1.0$ equiv.) and 5\% Pd/C (300 mg, $0.47 \mathrm{mmol}, 10 \mathrm{~mol} \%$ ) were charged under argon, followed by $\mathrm{Et}_{2} \mathrm{O}(20 \mathrm{~mL})$. The atmosphere was exchanged with hydrogen and the reaction mixture was stirred at room temperature for 16 hours under 1 bar hydrogen pressure. Upon completion, the mixture was filtered over Celite $^{\circledR}$, eluting with $\mathrm{Et}_{2} \mathrm{O}$. The filtrate was concentrated in vacuo to afford spiro[5.5] undecan-3-one SI-1e (0.52 g, $3.2 \mathrm{mmol}, 67 \%$ ) as a yellow oil.

Step 3. The procedure was adapted from Matsuda et al. ${ }^{12}$ (Methoxymethyl)triphenylphosphonium chloride (1.39 g, $4.0 \mathrm{mmol}, 1.3$ equiv.), toluene $(9 \mathrm{~mL})$ and $t$-BuOK $(0.41 \mathrm{~g}, 4.1 \mathrm{mmol}, 1.3$ equiv.) were charged into a Schlenk tube equipped with a PTFE-coated stirring bar, then the reaction mixture was stirred at room temperature for 20 minutes. SI-1e $(0.52 \mathrm{~g}, 3.2 \mathrm{mmol}$, 1.0 equiv.) in toluene ( $6 \mathrm{~mL}$ ) was added dropwise and the reaction mixture stirred stirred at $70{ }^{\circ} \mathrm{C}$ for 4 hours. Upon completion, the mixture was cooled to $0{ }^{\circ} \mathrm{C}$, saturated aqueous $\mathrm{NH}_{4} \mathrm{Cl}$ solution $(5 \mathrm{~mL})$ was added, the layers were separated and the aqueous layer was extracted three times with EtOAc ( $5 \mathrm{~mL}$ each time). The combined organic layers were dried over $\mathrm{MgSO}_{4}$, filtered, concentrated in vacuo and purified by column chromatography on silica gel (pentane/Et $2 \mathrm{O}=98 / 2$ $\rightarrow 95 / 5)$ to afford 3-(methoxymethylene)spiro[5.5] undecane (0.42 g, $2.2 \mathrm{mmol}$, $69 \%$ ) as a colorless oil. 
Step 4. The procedure was adapted from Matsuda et al.12 3(methoxymethylene)spiro[5.5] undecane $\quad(0.42 \mathrm{~g}, \quad 2.2 \mathrm{mmol}, \quad 1.0$ equiv.), acetonitrile $(20 \mathrm{~mL})$, deionized $\mathrm{H}_{2} \mathrm{O}(5 \mathrm{~mL})$ and TFA $(0.17 \mathrm{~mL})$ were consecutively charged into a round-bottom flask equipped with a PTFE-coated stirring bar. The reaction mixture was stirred for 1 hour at room temperature, then cooled to $0{ }^{\circ} \mathrm{C}$ and quenched with saturated aqueous $\mathrm{NaHCO}_{3}$. The layers were separated, the aqueous layer was extracted three times with EtOAc ( $5 \mathrm{~mL}$ each time), the combined organic layers were washed with brine, filtered and concentrated in vacuo. Purification by column chromatography on silica gel (pentane/Et $2 \mathrm{O}=98 / 2 \rightarrow 96 / 4)$ afforded 1 e $(0.18 \mathrm{~g}, 1.0 \mathrm{mmol}, 45 \%)$ as a colorless oil. $\mathbf{R}_{\mathbf{f}}$ (pentane/Et $\left.2 \mathrm{O}=96 / 4\right)$ 0.40. ${ }^{1} \mathbf{H}$ NMR (400 MHz, $\left.\mathrm{CDCl}_{3}\right) \delta 9.62(\mathrm{~s}, 1 \mathrm{H}), 2.24-$ $2.12(\mathrm{~m}, 1 \mathrm{H}), 1.71(\mathrm{dq}, J=13.5,4.3 \mathrm{~Hz}, 2 \mathrm{H}), 1.62$ (dt, $J=13.7,4.3 \mathrm{~Hz}, 2 \mathrm{H}), 1.57$ $1.45(\mathrm{~m}, 2 \mathrm{H}), 1.45-1.29(\mathrm{~m}, 8 \mathrm{H}), 1.24(\mathrm{t}, J=5.6 \mathrm{~Hz}, 2 \mathrm{H}), 1.17-1.07(\mathrm{~m}, 2 \mathrm{H}) .{ }^{13} \mathrm{C}$ NMR (101 MHz, $\left.\mathrm{CDCl}_{3}\right) \delta$ 205.3, 50.4, 40.0, 35.2, 33.4, 32.2, 27.0, 21.7, 21.6, 21.0. The analyical data are in agreement with the literature report.13

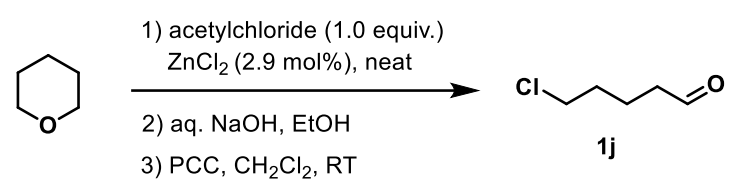

5-chloropentanal (1j). The compound was synthesized according to the following two step procedure.

Step 1. The intermediate alcohol was prepared using an adapted procedure from Cason et al. ${ }^{14}$ and Hanack et al. ${ }^{15}$ In an oven-dried Schlenk flask equipped with a PTFE-coated stirring bar, anhydrous $\mathrm{ZnCl}_{2} \quad(0.39 \mathrm{~g}, 2.9 \mathrm{mmol}, 2.9 \mathrm{~mol} \%)$, tetrahydropyran $(9.80 \mathrm{~mL}, 100 \mathrm{mmol}, 1.0$ equiv.) and acetyl chloride $(7.12 \mathrm{~mL}$, 100 mmol, 1.0 equiv.) were sequentially added. The reaction mixture was stirred under reflux for 2 hours, until the starting material was fully consumed (determined by GC-MS), then cooled to room temperature. The crude material was dissolved in EtOH $(70 \mathrm{~mL})$ and aqueous $\mathrm{NaOH}(2 \mathrm{M}, 70 \mathrm{~mL})$ and the reaction mixture was stirred for 12 hours at room temperature. Upon completion 
(determined by GC-MS), EtOH was removed in vacuo and the aqueous layer was extracted three times with EtOAc (20 mL each time). The combined organic layers were dried over $\mathrm{MgSO}_{4}$, filtered, concentrated in vacuo. The crude material was purified by Kugelrohr distillation $\left(150^{\circ} \mathrm{C}, 10 \mathrm{mbar}\right)$ to afford the intermediate alcohol compound $(4.21 \mathrm{~g}, 34.3 \mathrm{mmol}, 34 \%)$ as a colorless oil. $\mathbf{R}_{\mathbf{f}}$ (pentane/EtOAc $=4 / 1) 0.50 .{ }^{1} \mathrm{H}$ NMR $\left(400 \mathrm{MHz}, \mathrm{CDCl}_{3}\right) \delta 3.65(\mathrm{t}, J=6.4 \mathrm{~Hz}, 2 \mathrm{H})$, $3.54(\mathrm{t}, J=6.7 \mathrm{~Hz}, 2 \mathrm{H}), 3.27-3.19(\mathrm{~m}, 1 \mathrm{H}), 1.80(\mathrm{~m}, 2 \mathrm{H}), 1.56(\mathrm{~m}, 4 \mathrm{H})$. The experimental data are in agreement with the literature report. ${ }^{16}$

Step 2. The compound was synthesized following GP1 with 5-chloropentan-1-ol (1.22 g, 10.0 mmol, 1.0 equiv.), PCC (3.24 g, 15.0 mmol, 1.5 equiv.) in $\mathrm{CH}_{2} \mathrm{Cl}_{2}$ $(50 \mathrm{~mL})$ and purified by column chromatography on silica gel (pentane $\left./ \mathrm{Et}_{2} \mathrm{O}=24 / 1 \rightarrow 9 / 1\right)$, affording $\mathbf{1 j}(0.36 \mathrm{~g}, 2.96 \mathrm{mmol}, 30 \%)$ as a colorless oil. $\mathbf{R} \mathbf{f}$ (pentane $\left./ \mathrm{Et}_{2} \mathrm{O}=4 / 1\right) 0.60 .{ }^{1} \mathbf{H} \mathbf{N M R}\left(400 \mathrm{MHz}, \mathrm{CDCl}_{3}\right) \delta 9.77(\mathrm{t}, J=1.4 \mathrm{~Hz}$, 1H), $3.61-3.49(\mathrm{~m}, 2 \mathrm{H}), 2.49$ (ddt, $J=7.1,4.3,1.4 \mathrm{~Hz}, 2 \mathrm{H}), 1.88-1.71(\mathrm{~m}, 4 \mathrm{H}) .{ }^{13} \mathrm{C}$ NMR $\left(101 \mathrm{MHz}, \mathrm{CDCl}_{3}\right) \delta 202.0,44.6,43.1,31.9,19.5$ The experimental data are in agreement with the literature report. ${ }^{17}$

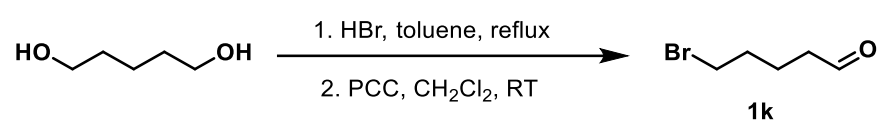

5-bromopentanal (1k). The compound was prepared according to the following two step procedure.

Step 1. The reaction was adapted from Chong et al. ${ }^{18}$ In a two-necked roundbottom flask equipped with a PTFE-coated stirring bar and an air condenser, 1,5Pentandiol (13.95 mL, $135 \mathrm{mmol}, 1.0$ equiv.), toluene ( $400 \mathrm{~mL}$ ) and aqueous $\mathrm{HBr}$ $(48 \%, 17.82 \mathrm{~mL})$ were added, then the reaction mixture was heated at reflux under vigorous stirring for 36 hours. Upon completion (determined by GC-MS and TLC), the reaction mixture was cooled to room temperature, the phases were separated and the organic layer was diluted with $\mathrm{Et}_{2} \mathrm{O}(100 \mathrm{~mL})$. The combined organic phases were consecutively washed with $1 \mathrm{M} \mathrm{NaOH}$, brine, $3 \mathrm{M}$ phosphate buffer with $\mathrm{pH}=7$ (50 mL each), dried over $\mathrm{MgSO}_{4}$, filtered and concentrated in 
vacuo. Column chromatography of the crude material on silica gel (pentane/EtOAc $=1 / 0 \rightarrow 1 / 1)$ afforded the intermediate alcohol compound $(8.75 \mathrm{~g}, 52.4 \mathrm{mmol}$, $39 \%)$ as a colorless oil. $\mathbf{R}_{\mathbf{f}}$ (pentane/EtOAc $\left.=4 / 1\right) 0.50 .{ }^{1} \mathbf{H} \mathbf{~ N M R}\left(400 \mathrm{MHz}, \mathrm{CDCl}_{3}\right)$ $\delta 3.66(\mathrm{t}, J=6.3 \mathrm{~Hz}, 2 \mathrm{H}), 3.42(\mathrm{t}, J=6.8 \mathrm{~Hz}, 2 \mathrm{H}), 1.89(\mathrm{~m}, 2 \mathrm{H}), 1.66-1.49(\mathrm{~m}, 4 \mathrm{H})$, $1.46(\mathrm{~s}, 1 \mathrm{H}) .{ }^{13} \mathrm{C}$ NMR $\left(101 \mathrm{MHz}, \mathrm{CDCl}_{3}\right) \delta 62.8,33.8,32.6,31.9$, 24.6. The experimental data are in agreement with the literature report. ${ }^{18}$

$\underline{\text { Step } 2}$. The compound was synthesized according to GP1 with 5-bromopentan-1ol (1.67 g, 10.0 mmol, 1.0 equiv.), PCC (3.24 g, 15.0 mmol, 1.5 equiv.) in $\mathrm{CH}_{2} \mathrm{Cl}_{2}$ $(50 \mathrm{~mL}$ ). Column chromatography on silica gel (pentane/Et $2 \mathrm{O}=93 / 7 \rightarrow 85 / 15$ ) afforded $\quad \mathbf{1 k} \quad(1.16 \mathrm{~g}, \quad 7.03 \mathrm{mmol}, \quad 70 \%)$ as a colorless oil. $\mathbf{R f}$ (pentane/Et $2 \mathrm{O}=9 / 1) 0.30 .{ }^{1} \mathrm{H}$ NMR $\left(400 \mathrm{MHz}, \mathrm{CDCl}_{3}\right) \delta 9.77(\mathrm{t}, J=1.5 \mathrm{~Hz}, 1 \mathrm{H})$, $3.41(\mathrm{t}, J=6.5 \mathrm{~Hz}, 2 \mathrm{H}), 2.49(\mathrm{td}, J=7.1,1.5 \mathrm{~Hz}, 2 \mathrm{H}), 1.94-1.85(\mathrm{~m}, 2 \mathrm{H}), 1.84-1.74$ (m, 2H). ${ }^{13} \mathbf{C}$ NMR $\left(101 \mathrm{MHz}, \mathrm{CDCl}_{3}\right) \delta 201.9,43.0,33.2,32.0,20.8$. The experimental data are in agreement with the literature report. ${ }^{19}$

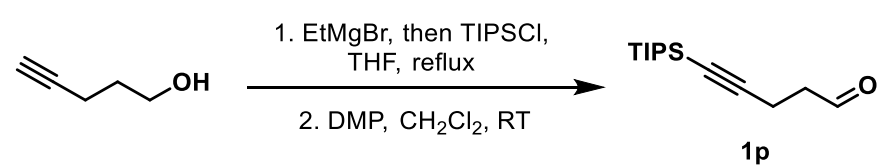

5-(triisopropylsilyl)pent-4-ynal (1p). The compound was synthesized according to the following two step procedure.

Step 1. The reaction was adapted from the procedure from Layton et al. ${ }^{20} \mathrm{~A}$ Schlenk flask equipped with a PTFE.coated stiring bar was charged with pent-4yn-1-ol (1.86 mL, $20 \mathrm{mmol}, 1.0$ equiv.), then THF ( $40 \mathrm{~mL}$ ) was added, followed by

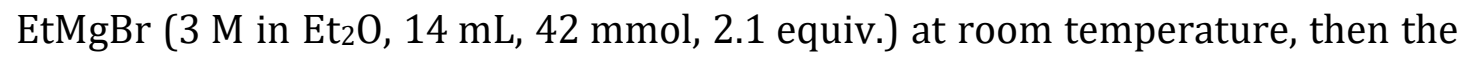
reaction mixture was stirred under reflux for 12 hours. Upon cooling to room temperature, triisopropylsilyl chloride $(4.28 \mathrm{~mL}, 20 \mathrm{mmol}, 1.0$ equiv.) in THF $(20 \mathrm{~mL})$ was added over 15 minutes and the reaction mixture was stirred under reflux for additional 6 hours. After cooling to room temperature, the reaction mixture was poured into aqueous $\mathrm{HCl}(10 \%, 50 \mathrm{~mL})$ and the mixture was extracted three times with $\mathrm{Et}_{2} \mathrm{O}$ ( $50 \mathrm{~mL}$ each time). The combined organic layers 
were dried over $\mathrm{MgSO}_{4}$, filtered and concentrated in vacuo to afford the corresponding alcohol SI-1p, which was used without further purification.

$\underline{\text { Step 2. }}$. In a round-bottom flask equipped with a PTFE-coated stirring bar, DessMartin periodane (8.89 g, $21.0 \mathrm{mmol}, 1.05$ equiv.) was dissolved in $\mathrm{CH}_{2} \mathrm{Cl}_{2}$ $(70 \mathrm{~mL})$, then the crude alcohol SI-1p in $\mathrm{CH}_{2} \mathrm{Cl}_{2}(2 \mathrm{~mL})$ and deionized $\mathrm{H}_{2} \mathrm{O}(0.37$ $\mathrm{mL}, 21.0 \mathrm{mmol}, 1.05$ equiv.) were consecutively added. The reaction mixture was stirred at room temperature for 30 minutes and then poured into a 1:1 mixture of saturated aqueous $\mathrm{Na}_{2} \mathrm{~S}_{2} \mathrm{O}_{3}$ and $\mathrm{NaHCO}_{3}$ (15 mL each), then extracted three times with $\mathrm{Et}_{2} \mathrm{O}$ (20 mL each time). The combined organic layers were washed with saturated aqueous $\mathrm{NaHCO}_{3}$ and brine (10 mL each time), dried over $\mathrm{MgSO}_{4}$, filtered and concentrated in vacuo. The crude product was purified by column chromatography on silica gel (pentane/Et $2 \mathrm{O}=24 / 1 \rightarrow 19 / 1)$ to afford $1 \mathbf{p}(0.95 \mathrm{~g}$, $3.99 \mathrm{mmol}, 20 \%$ over 2 steps) as a colorless oil. $\mathbf{R f}_{\mathbf{f}}$ (pentane $/ \mathrm{Et}_{2} \mathrm{O}=19 / 1$ ) $0.40 .{ }^{1} \mathbf{H}$ NMR (400 MHz, $\left.\mathrm{CDCl}_{3}\right) \delta 9.81(\mathrm{t}, J=1.4 \mathrm{~Hz}, 1 \mathrm{H}), 2.67(\mathrm{~m}, 2 \mathrm{H}), 2.62-2.54(\mathrm{~m}, 2 \mathrm{H})$, $1.08-1.00(\mathrm{~m}, 21 \mathrm{H}) .{ }^{13} \mathrm{C}$ NMR $\left(101 \mathrm{MHz}, \mathrm{CDCl}_{3}\right) \delta 200.8,106.5,82.0,43.0,18.7$, $13.5,11.3$. The experimental data are in agreement with the literature report. ${ }^{20}$

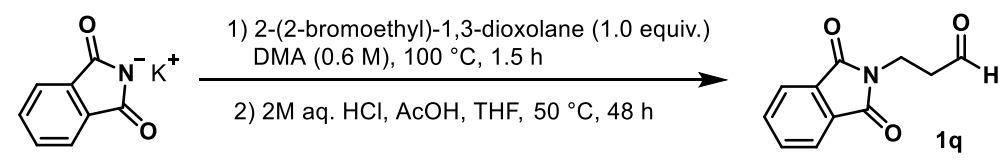

3-(1,3-dioxoisoindolin-2-yl)propanal (1q). The compound was synthesized in accordance with patent EP1640369A1 from Sumitomo. To a Schlenk flask equipped with a PTFE-coated stirring bar, potassium 1,3-dioxoisoindolin-2-ide (3.7 g, 20 mmol, 1.0 equiv.), DMA (36 mL) and 2-(2-bromoethyl)-1,3-dioxolane (90\%, 3.6 g, 20 mmol, 1.0 equiv.) were consecutively added. The reaction mixture was warmed up to $100{ }^{\circ} \mathrm{C}$ and stirred at the same temperature for 90 minutes. Upon completion (determined by TLC), brine $(10 \mathrm{~mL})$ was added, the layers were separated and the aqueous layer was extracted three times with EtOAc $(10 \mathrm{~mL}$ each time). The combined organic layers were washed with water, saturated aqueous $\mathrm{NaHCO}_{3}$ and brine, then dried over $\mathrm{MgSO}_{4}$ and concentrated in vacuo. The residue was transferred into a $200 \mathrm{~mL}$ Schlenk flask equipped with a PTFE-coated 
stirring bar and dissolved in THF (80 mL), then AcOH (27 mL) and aqueous $\mathrm{HCl}$ $(2 \mathrm{M}, 34 \mathrm{~mL})$ were added. The reaction mixture was warmed up to $50^{\circ} \mathrm{C}$ and stirred at the same temperature for 2 days. Upon completion (determined by TLC), the reaction mixture was neutralized with saturated aqueous $\mathrm{K}_{2} \mathrm{CO}_{3}$, the layers were separated, the aqueous layer was extracted three times with EtOAc $(10 \mathrm{~mL}$ each time) and the combined organic layers were dried over $\mathrm{MgSO}_{4}$, filtered and concentrated in vacuo. Column chromatography on silica gel (pentane/EtOAc = $4 / 1 \rightarrow 7 / 3$ ) of the crude material and recrystallization from boiling $\mathrm{H}_{2} \mathrm{O}$ afforded $1 q$ (1.24 g, $6.1 \mathrm{mmol}, 31 \%$ over 2 steps) as a colorless solid. Rf (pentane/EtOAc = 9/1) 0.10. ${ }^{1} \mathbf{H}$ NMR $\left(400 \mathrm{MHz}^{\mathrm{CDCl}} 3\right) \delta 9.82(\mathrm{t}, J=1.4 \mathrm{~Hz}, 1 \mathrm{H})$, $7.85(\mathrm{dd}, J=5.4,3.1 \mathrm{~Hz}, 2 \mathrm{H}), 7.77-7.67(\mathrm{~m}, 2 \mathrm{H}), 4.04(\mathrm{t}, J=7.0 \mathrm{~Hz}, 2 \mathrm{H}), 2.88(\mathrm{td}, J$ = 7.0, $1.4 \mathrm{~Hz}, 2 \mathrm{H}) .{ }^{13} \mathrm{C}$ NMR (101 MHz, $\left.\mathrm{CDCl}_{3}\right) \delta 199.6,168.2,134.3,132.1,123.5$, $42.5,31.8$. The experimental data are in agreement with the literature report. ${ }^{21}$

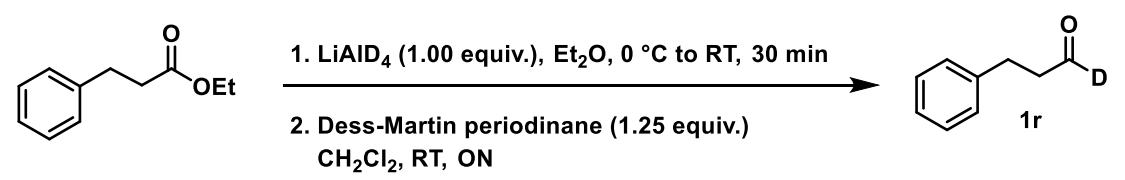

3-Phenylpropanal-1-d (1r). The compound was synthesized according to the following two-step procedure.

Step 1. In an oven-dried Schlenk tube equipped with a PTFE-coated stirring bar, ethyl 3-phenylpropanoate (1.06 ml, $6.00 \mathrm{mmol}, 1.0$ equiv.) was charged under argon and dissolved in dry $\mathrm{Et}_{2} \mathrm{O}(25 \mathrm{ml})$ at $0{ }^{\circ} \mathrm{C}$. $\mathrm{LiAlD}_{4}(252 \mathrm{mg}, 6.00 \mathrm{mmol}, 1.00$ equiv.) was added in one portion and the reaction was stirred at $0{ }^{\circ} \mathrm{C}$ for 15 minutes, the warmed at room temperature and stirred for 30 minutes. The reaction was cooled to $0{ }^{\circ} \mathrm{C}$, diluted with $\mathrm{Et}_{2} \mathrm{O}(30 \mathrm{ml})$ and consecutively quenched with water $(0.25 \mathrm{ml}), \mathrm{NaOH} 2 \mathrm{M}(0.25 \mathrm{ml})$ and water $(0.75 \mathrm{ml})$, then was warmed at room temperature and $\mathrm{MgSO}_{4}$ was added. The slurry was filtered over a short pad of Celite ${ }^{\circledR}$, rinsing thoroughly with $\mathrm{Et}_{2} \mathrm{O}$. The volatiles were removed in vacuo, affording the intermediate $\mathbf{S I - 1 r}\left(830 \mathrm{mg}, 6.00 \mathrm{mmol},>99 \%\right.$ yield, $d_{2}>95 \%$ ) as a colourless oil. ${ }^{1} \mathbf{H}$ NMR (400 MHz, $\left.\mathrm{CDCl}_{3}\right) \delta 7.32-7.26(\mathrm{~m}, 2 \mathrm{H}), 7.22-7.16(\mathrm{~m}, 3 \mathrm{H})$, 
$2.76-2.67(\mathrm{~m}, 2 \mathrm{H}), 1.89$ (t, J = 7.7 Hz, 2H). GC-MS (EI, 1.9-normal) @ $3.02 \mathrm{~min}$, $m / z=138\left(\mathrm{M}^{+}\right) \cdot{ }^{22}$

Step 2. In a round-bottom flask equipped with a PTFE-coated stirring bar, the intermediate SI-1r (830 mg, $6.00 \mathrm{mmol}, 1.00$ equiv.) dissolved in $\mathrm{CH}_{2} \mathrm{Cl}_{2}(40 \mathrm{ml})$, then Dess-Martin periodinane (3.18 g, $7.50 \mathrm{mmol}, 1.25$ equiv.) was added and the reaction was stirred at room temperature overnight, then diluted with pentane $(15 \mathrm{ml})$ and filtered over a short pad of Celite ${ }^{\circledR}$, rinsing with little $\mathrm{CH}_{2} \mathrm{Cl}_{2}$. The organic layer was washed with saturated $\mathrm{NaHCO}_{3}$, then dried over $\mathrm{MgSO}_{4}$ and the solvent was removed in vacuo. The crude residue was purified by flash column chromatography on silica (pentane/EtOAc = 20/1), affording 1r (548 mg, 4.05 mmol, $\left.68 \%, d_{1}>99 \%\right)$ as a colourless liquid. ${ }^{1} \mathbf{H}$ NMR (400 MHz, $\left.\mathrm{CDCl}_{3}\right) \delta 7.33-$ $7.27(\mathrm{~m}, 2 \mathrm{H}), 7.24-7.17(\mathrm{~m}, 3 \mathrm{H}), 2.97(\mathrm{t}, J=7.5 \mathrm{~Hz}, 2 \mathrm{H}), 2.83-2.74(\mathrm{~m}, 2 \mathrm{H}) .{ }^{13} \mathrm{C}$ NMR $\left(101 \mathrm{MHz}, \mathrm{CDCl}_{3}\right) \delta 140.5,128.8,128.4,126.5,45.2(\mathrm{t}, J=3.8 \mathrm{~Hz}), 28.2$. The experimental data are in agreement with the literature report. ${ }^{23}$
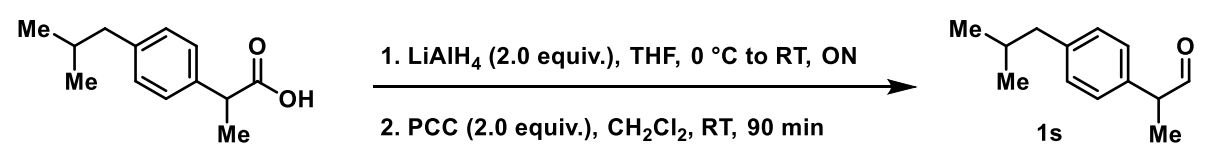

2-(4-Isobutylphenyl)propanal (1s). In an oven-dried Schlenk tube equipped with a PTFE-coated stirring bar, rac-ibuprofene (1.03 mg, $5.00 \mathrm{mmol}, 1.00$ equiv.) was dissolved in dry THF $(25 \mathrm{ml})$, then the solution was cooled to $0{ }^{\circ} \mathrm{C}$ and $\mathrm{LiAlH}_{4}$ (380 mg, $10.00 \mathrm{mmol}, 2.00$ equiv.) was added portionwise, then the reaction was warmed at room temperature and stirred overnight. The reaction was diluted with $\mathrm{Et}_{2} \mathrm{O}(30 \mathrm{ml})$, cooled to $0{ }^{\circ} \mathrm{C}$ and carefully quenched with water $(400 \mu \mathrm{l})$, $\mathrm{NaOH} 2 \mathrm{M}(400 \mu \mathrm{l})$, and water $(1.2 \mathrm{ml})$. After warming to room temperature, MgSO4 was added and the suspension was filtered over a short pad Celite ${ }^{\circledR}$, rinsing thoroughly with $\mathrm{Et}_{2} \mathrm{O}$ and the solvent was removed in vacuo. The intermediate alcohol was dissolved in $\mathrm{CH}_{2} \mathrm{Cl}_{2}(30 \mathrm{ml})$ and pyridinium chlorochromate $(2.15 \mathrm{~g}$, $10.00 \mathrm{mmol}, 2.00$ equiv.) was added portionwise. The reaction was stirred for 90 minutes, the diluted with $\mathrm{Et}_{2} \mathrm{O}(40 \mathrm{ml})$ and filtered over a pad of Celite ${ }^{\circledR}$, thoroughly rinsing with $\mathrm{Et}_{2} \mathrm{O}$. The volatiles were removed in vacuo, then the 
residue was purified by flash column chromatography on silica $($ pentane $/$ EtOAc $=$ 60/1), affording $1 \mathrm{~s}$ (327 mg, $1.72 \mathrm{mmol}, 34 \%$ over two steps) as a faint yellow oil. ${ }^{1} \mathrm{H}$ NMR (400 MHz, $\left.\mathrm{CDCl}_{3}\right) \delta 9.67(\mathrm{~d}, J=1.5 \mathrm{~Hz}, 1 \mathrm{H}), 7.17-7.09(\mathrm{~m}, 4 \mathrm{H}), 3.60$ (q, $J$ $=7.1 \mathrm{~Hz}, 1 \mathrm{H}$ ), 2.47 (d, $J=7.2 \mathrm{~Hz}, 2 \mathrm{H}), 1.86$ (hept, $J=6.7 \mathrm{~Hz}, 1 \mathrm{H}), 1.43$ (d, $J=7.1 \mathrm{~Hz}$, $3 \mathrm{H}), 0.90(\mathrm{~d}, J=6.6 \mathrm{~Hz}, 6 \mathrm{H})$. The experimental data are in agreement with the literature report. ${ }^{24}$

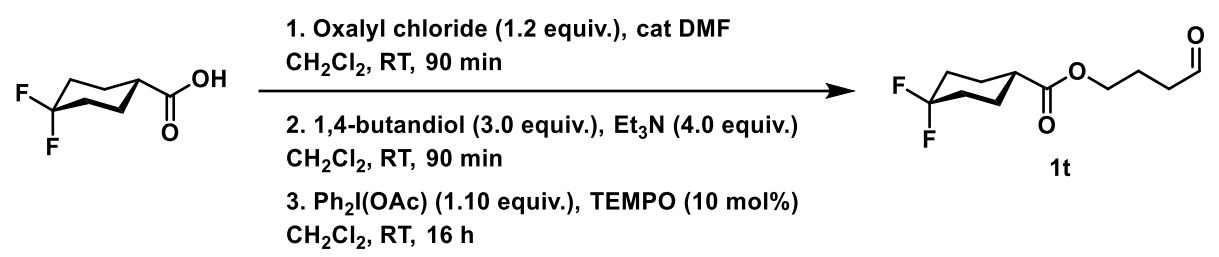

4-0xobutyl 4,4-difluorocyclohexane-1-carboxylate (1t). The title compound was synthesized using the following two-step procedure.

Step 1. In an oven-dried round-bottom flask equipped with a PTFE-coated stirring bar, 4,4-difluorocyclohexylacetic acid (821 mg, $5.00 \mathrm{mmol}, 1.00$ equiv.) was dissolved in $\mathrm{CH}_{2} \mathrm{Cl}_{2}(30 \mathrm{ml})$, then one drop of DMF followed by oxalyl chloride (515 $\mu \mathrm{l}, 6.00 \mathrm{mmol}, 1.20$ equiv.) were added. The reaction was stirred until gas evolution ceased (90 minutes), then the volatiles were removed in vacuo. The residue was dissolved in $\mathrm{CH}_{2} \mathrm{Cl}_{2}(15 \mathrm{ml})$ and added dropwise to a well-stirred solution of 1,4-butandiol (1.33 ml, $15.0 \mathrm{mmol}, 3.00$ equiv.) and $\mathrm{Et}_{3} \mathrm{~N}$ (2.70 ml, 20.0 mmol, 4.00 equiv.) in $\mathrm{CH}_{2} \mathrm{Cl}_{2}(15 \mathrm{ml})$. The reaction was stirred at room temperature for 90 minutes, then quenched with $\mathrm{HCl} 1 \mathrm{M}(40 \mathrm{ml})$. The layers were separated, then the aqueous layer was extracted twice with $\mathrm{CH}_{2} \mathrm{Cl}_{2}(30 \mathrm{ml}$ each time) and the combined organic layers were washed twice with water (30 ml each time) and once with brine $(30 \mathrm{ml})$. The organic extracts were dried over $\mathrm{MgSO}_{4}$, then the solvent was removed in vacuo and the residue purified by flash column chromatography on silica (pentane/acetone $=5 / 1 \rightarrow 4 / 1$ ), affording the intermediate SI-1t (809 mg, $3.42 \mathrm{mmol}, 68 \%$ ) as a colourless thick oil. $\mathbf{R}_{\boldsymbol{f}}$ (pentane/acetone = 4/1): 0.65. ${ }^{1} \mathbf{H}$ NMR $\left(400 \mathrm{MHz}, \mathrm{CDCl}_{3}\right) \delta 4.13(\mathrm{t}, J=6.4 \mathrm{~Hz}, 2 \mathrm{H})$, $3.68(\mathrm{t}, J=6.3 \mathrm{~Hz}, 2 \mathrm{H}), 2.45-2.35(\mathrm{~m}, 1 \mathrm{H}), 2.14-2.03(\mathrm{~m}, 2 \mathrm{H}), 2.03-1.93(\mathrm{~m}, 2 \mathrm{H})$, 
$1.90-1.79(\mathrm{~m}, 3 \mathrm{H}), 1.78-1.68(\mathrm{~m}, 3 \mathrm{H}), 1.68-1.56(\mathrm{~m}, 2 \mathrm{H}) .{ }^{13} \mathrm{C}$ NMR (101 MHz, $\left.\mathrm{CDCl}_{3}\right) \delta 174.5-174.4(\mathrm{~m}), 122.8(\mathrm{t}, J=240.9 \mathrm{~Hz}), 64.6,62.5,40.8(\mathrm{t}, J=1.0 \mathrm{~Hz})$, $32.7(\mathrm{t}, J=24.6 \mathrm{~Hz}), 29.2,25.3-25.1(\mathrm{~m}) .{ }^{19} \mathbf{F}\left\{{ }^{1} \mathbf{H}\right\} \mathbf{N M R}\left(377 \mathrm{MHz}, \mathrm{CDCl}_{3}\right) \delta-94.27$ (br d, $J=237.0 \mathrm{~Hz}$ ), -99.74 (br d, $J=237.8 \mathrm{~Hz}$ ). HRMS (ESI+): calcd. for $\mathrm{C}_{11} \mathrm{H}_{18} \mathrm{O}_{3} \mathrm{~F}_{2} \mathrm{Na}\left(\mathrm{M}+\mathrm{Na}^{+}\right): 259.1116$. Found: 259.1114 .

Step 2. In an oven-dried round-bottom flask equipped with a PTFE-coated stirring bar, the intermediate SI-1t (793 mg, $3.35 \mathrm{mmol}, 1.00$ equiv.) was dissolved in $\mathrm{CH}_{2} \mathrm{Cl}_{2}$ (5 ml), then TEMPO (53 mg, $0.34 \mathrm{mmol}, 10 \mathrm{~mol} \%$ ) and PhI(OAc) 2 (1.19 g, $3.69 \mathrm{mmol}, 1.10$ equiv.) were added. The reaction was stirred at room temperature for 16 hours, then quenched with saturated $\mathrm{Na}_{2} \mathrm{~S}_{2} \mathrm{O}_{3}(30 \mathrm{ml})$. The layers were separated and the aqueous layer was extracted twice with $\mathrm{CH}_{2} \mathrm{Cl}_{2}(20$ ml each time). The combined organic layers were washed once with saturated $\mathrm{NaHCO}_{3}(30 \mathrm{ml})$, then dried over $\mathrm{MgSO}_{4}$ and the solvent was removed in vacuo. The residue was purified by flash column chromatography on silica (pentane/EtOAc = 3/1), affording 1t $(800 \mathrm{mg}, 2.86 \mathrm{mmol}, 85 \%)$ as a colourless thick oil. $\mathbf{R}_{\boldsymbol{f}}$ (pentane/EtOAc = 9/2): 0.50. ${ }^{1} \mathbf{H} \mathbf{N M R}\left(599 \mathrm{MHz}, \mathrm{CDCl}_{3}\right) \delta 9.79(\mathrm{~s}, 1 \mathrm{H})$, $4.12(\mathrm{t}, J=6.5 \mathrm{~Hz}, 2 \mathrm{H}), 2.53(\mathrm{t}, J=7.2 \mathrm{~Hz}, 2 \mathrm{H}), 2.44-2.34(\mathrm{~m}, 1 \mathrm{H}), 2.13-2.03(\mathrm{~m}$, 2H), 2.01 - $1.94(\mathrm{~m}, 4 \mathrm{H}), 1.87$ - $1.70(\mathrm{~m}, 8 \mathrm{H}) .{ }^{13} \mathbf{C} \mathbf{~ N M R}\left(151 \mathrm{MHz}, \mathrm{CDCl}_{3}\right) \delta 201.1$, 174.2, $122.7(\mathrm{t}, J=241.0 \mathrm{~Hz}), 63.8,40.7,40.6,32.7(\mathrm{t}, J=24.5 \mathrm{~Hz}), 25.3-25.1(\mathrm{~m})$, 21.4. 19F NMR (564 MHz, $\mathrm{CDCl}_{3}$ ) $\delta-94.26$ (br d, $J=236.3 \mathrm{~Hz}$ ), -99.86 (br d, $J=235.3$ Hz). HRMS (ESI ${ }^{+}$): calcd. for $\mathrm{C}_{11} \mathrm{H}_{16} \mathrm{O}_{3} \mathrm{~F}_{2} \mathrm{Na}\left(\mathrm{M}+\mathrm{Na}^{+}\right)$: 257.0960. Found: 257.0958.

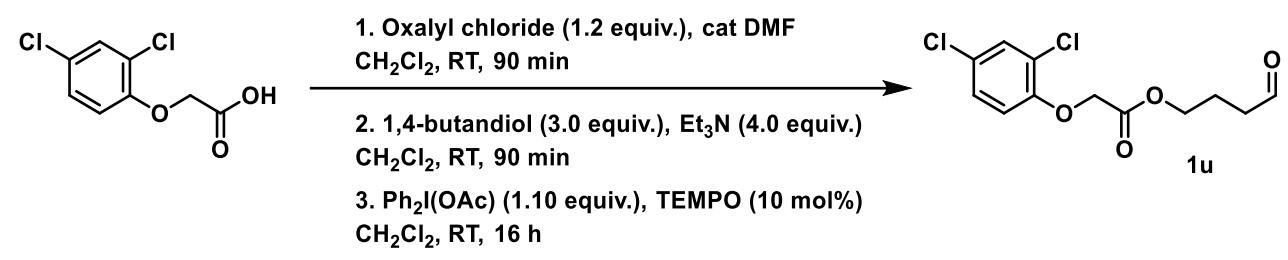

4-0xobutyl 2-(2,4-dichlorophenoxy)acetate (1u). The title compound was synthesized using the following two-step procedure.

Step 1. In an oven-dried round-bottom flask equipped with a PTFE-coated stirring bar, 2,4-D (1.11 g, $5.00 \mathrm{mmol}, 1.00$ equiv.) was dissolved in $\mathrm{CH}_{2} \mathrm{Cl}_{2}$ (30 ml), then 
one drop of DMF followed by oxalyl chloride (515 $\mu \mathrm{l}, 6.00 \mathrm{mmol}, 1.20$ equiv.) were added. The reaction was stirred until gas evolution ceased (90 minutes), then the volatiles were removed in vacuo. The residue was dissolved in $\mathrm{CH}_{2} \mathrm{Cl}_{2}(15 \mathrm{ml})$ and added dropwise to a well-stirred solution of 1,4-butandiol $(1.33 \mathrm{ml}, 15.0 \mathrm{mmol}$, 3.00 equiv.) and $\mathrm{Et}_{3} \mathrm{~N}$ (2.70 ml, $20.0 \mathrm{mmol}, 4.00$ equiv.) in $\mathrm{CH}_{2} \mathrm{Cl}_{2}(15 \mathrm{ml})$. The reaction was stirred at room temperature for 90 minutes, then quenched with $\mathrm{HCl}$ $1 \mathrm{M}(40 \mathrm{ml})$. The layers were separated, then the aqueous layer was extracted twice with $\mathrm{CH}_{2} \mathrm{Cl}_{2}$ (30 ml each time) and the combined organic layers were washed twice with water (30 ml each time) and once with brine $(30 \mathrm{ml})$. The organic extracts were dried over $\mathrm{MgSO}_{4}$, then the solvent was removed in vacuo and the residue purified by flash column chromatography on silica (pentane/acetone $=$ $4 / 1 \rightarrow 3 / 1$ ), affording the intermediate alcohol SI-1u (1.01 g, $3.44 \mathrm{mmol}, 69 \%)$ as a colourless gum. $\mathbf{R}_{\boldsymbol{f}}$ (pentane/acetone $\left.=4 / 1\right): 0.40 .{ }^{1} \mathbf{H} \mathbf{~ N M R}\left(400 \mathrm{MHz}, \mathrm{CDCl}_{3}\right) \delta$ 7.39 (d, $J=2.6 \mathrm{~Hz}, 1 \mathrm{H}$ ), $7.16(\mathrm{dd}, J=8.8,2.5 \mathrm{~Hz}, 1 \mathrm{H}$ ), 6.78 (d, $J=8.8 \mathrm{~Hz}, 1 \mathrm{H}$ ), 4.69 $(\mathrm{s}, 2 \mathrm{H}), 4.24(\mathrm{t}, J=6.5 \mathrm{~Hz}, 2 \mathrm{H}), 3.66(\mathrm{t}, J=6.3 \mathrm{~Hz}, 2 \mathrm{H}), 1.80-1.70(\mathrm{~m}, 2 \mathrm{H}), 1.63-$ $1.51(\mathrm{~m}, 3 \mathrm{H}) .{ }^{13} \mathrm{C}$ NMR $\left(101 \mathrm{MHz}, \mathrm{CDCl}_{3}\right) \delta 168.3,152.5,130.5,127.7,127.3,124.4$, 114.9, 66.6, 65.5, 62.3, 29.0, 25.1. HRMS (ESI+): calcd. for $\mathrm{C}_{12} \mathrm{H}_{14} \mathrm{O}_{4}{ }^{35} \mathrm{Cl}_{2} \mathrm{Na}$ $\left(\mathrm{M}+\mathrm{Na}^{+}\right):$315.0161. Found: 315.0161 .

Step 2. In an oven-dried round-bottom flask equipped with a PTFE-coated stirring bar, the intermediate alcohol SI-1u (992 mg, $3.38 \mathrm{mmol}, 1.00$ equiv.) was dissolved in $\mathrm{CH}_{2} \mathrm{Cl}_{2}(5 \mathrm{ml})$, then TEMPO (53 mg, $0.34 \mathrm{mmol}, 10 \mathrm{~mol} \%$ ) and $\mathrm{PhI}(\mathrm{OAc})_{2}$ (1.20 g, $3.72 \mathrm{mmol}, 1.10$ equiv.) were added. The reaction was stirred at room temperature for 16 hours, then quenched with saturated $\mathrm{Na}_{2} \mathrm{~S}_{2} \mathrm{O}_{3}(30 \mathrm{ml})$. The layers were separated and the aqueous layer was extracted twice with $\mathrm{CH}_{2} \mathrm{Cl}_{2}$ (20 ml each time). The combined organic layers were washed once with saturated $\mathrm{NaHCO}_{3}(30 \mathrm{ml})$, then dried over $\mathrm{MgSO}_{4}$ and the solvent was removed in vacuo. The residue was purified by flash column chromatography on silica (pentane/EtOAc = 9/2 $\rightarrow$ 3/1), affording 1u (852 mg, $2.92 \mathrm{mmol}, 87 \%)$ as a colourless thick oil. $\mathbf{R}_{\boldsymbol{f}}$ (pentane/EtOAc $\left.=9 / 2\right): 0.40 .{ }^{\mathbf{1}} \mathbf{H} \mathbf{~ N M R}\left(599 \mathrm{MHz}, \mathrm{CDCl}_{3}\right) \delta$ $9.77(\mathrm{~s}, 1 \mathrm{H}), 7.40(\mathrm{~s}, 1 \mathrm{H}), 7.17(\mathrm{~d}, J=8.8 \mathrm{~Hz}, 1 \mathrm{H}), 6.79$ (d, $J=8.8 \mathrm{~Hz}, 1 \mathrm{H}), 4.68(\mathrm{~s}$, 
$2 \mathrm{H}), 4.24(\mathrm{t}, J=6.5 \mathrm{~Hz}, 2 \mathrm{H}), 2.52(\mathrm{t}, J=7.1 \mathrm{~Hz}, 2 \mathrm{H}), 2.00(\mathrm{p}, J=6.3 \mathrm{~Hz}, 2 \mathrm{H}) .{ }^{13} \mathbf{C} \mathbf{~ N M R}$ (151 MHz, $\left.\mathrm{CDCl}_{3}\right) \delta 200.8,168.1,152.5,130.5,127.7,127.4,124.5,114.9,66.5$, 64.6, 40.4, 21.3. HRMS (ESI ${ }^{+}$: calcd. for $\mathrm{C}_{12} \mathrm{H}_{12} \mathrm{O}_{4}{ }^{35} \mathrm{Cl}_{2} \mathrm{Na}\left(\mathrm{M}+\mathrm{Na}^{+}\right)$: 313.0005 . Found: 313.0002 .

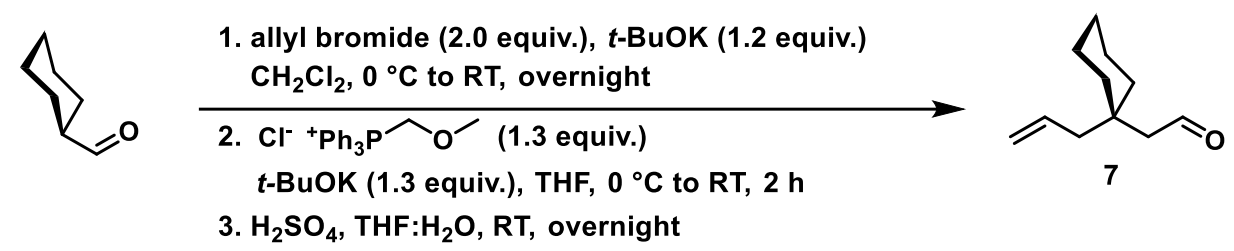

2-(1-Allylcyclohexyl)acetaldehyde (7). The title compound was synthesized according to the following two step procedure.

Step 1 was performed according to a modified procedure from Tiefenbacher and co-workers. ${ }^{25}$ In an oven-dried Schlenk tube equipped with a PTFE-coated stirring bar, cyclohexancarbaldehyde (1.21 ml, $10.0 \mathrm{mmol}, 1.00$ equiv.) was dissolved in dry $\mathrm{CH}_{2} \mathrm{Cl}_{2}(50 \mathrm{ml})$ and cooled to $0{ }^{\circ} \mathrm{C}$, then $t$-BuOK (1.35 g, $12.0 \mathrm{mmol}, 1.20$ equiv.) and allyl bromide were added in one portion. The reaction was warmed at room temperature and stirred overnight, then was carefully quenched with water (40 $\mathrm{ml}$ ), the layers were separated and the aqueous layer was extracted twice with $\mathrm{CH}_{2} \mathrm{Cl}_{2}$ (50 ml each time). The combined organic extracts were washed once with brine $(50 \mathrm{ml})$, then dried over $\mathrm{MgSO}_{4}$ and the solvent was removed in vacuo. The residue was used for the next step without further purification.

Step 2 was performed according to a modified procedure from Saicic and coworkers. ${ }^{26}$ In an oven-dried Schlenk tube equipped with a PTFE-coated stirring bar, methoxymethyl triphenylphosphonium chloride (4.46 g, $13.0 \mathrm{mmol}, 1.30$ equiv.) and dry THF $(20 \mathrm{ml})$ were charged and cooled to $0{ }^{\circ} \mathrm{C}$, then $t$-BuOK $(1.46 \mathrm{~g}$, $13.0 \mathrm{mmol}, 1.30$ equiv.) was added in one portion (the suspension turns cherry red) and the reaction was stirred at $0{ }^{\circ} \mathrm{C}$ for 60 minutes, then a solution of the intermediate aldehyde $(10.0 \mathrm{mmol})$ in dry THF (10 ml) was added dropwise. The reaction was warmed to room temperature and stirred for 2 hours, then was carefully quenched with water $(30 \mathrm{ml})$ and extracted three times with $\mathrm{CH}_{2} \mathrm{Cl}_{2}(50$ ml each time). The combined organic layers were dried over $\mathrm{MgSO}_{4}$ and the 
solvent was removed in vacuo. The residue was dissolved in THF: $\mathrm{H}_{2} \mathrm{O}(2: 1,30 \mathrm{ml})$, then $\mathrm{H}_{2} \mathrm{SO}_{4} 96 \%$ (3 ml) was added and the reaction was stirred overnight at room temperature. The reaction was neutralized with saturated $\mathrm{NaHCO}_{3}$, then the aqueous layer was extracted three times with $\mathrm{CH}_{2} \mathrm{Cl}_{2}$ (50 ml each time), the combined organic layers were washed once with brine $(50 \mathrm{ml})$, dried over $\mathrm{MgSO}_{4}$ and the solvent was removed in vacuo. The crude residue was purified by flash column chromatography on silica (pentane/EtOAc $=50 / 1)$, affording 7 (407 mg, $2.45 \mathrm{mmol}, 25 \%$ over three steps) as a faint yellow liquid. Note. The isolated yield is reduced due to the presence of co-eluted impurities. Prolonged drying under high vacuum must be avoided due to product volatility. ${ }^{\mathbf{1}} \mathbf{H} \mathbf{~ N M R}\left(400 \mathrm{MHz}, \mathrm{CDCl}_{3}\right) \delta 9.86$ $(\mathrm{t}, J=3.0 \mathrm{~Hz}, 1 \mathrm{H}), 5.82(\mathrm{ddt}, J=17.5,10.2,7.5 \mathrm{~Hz}, 1 \mathrm{H}), 5.16-5.01(\mathrm{~m}, 2 \mathrm{H}), 2.33(\mathrm{~d}$, $J=3.1 \mathrm{~Hz}, 2 \mathrm{H}), 2.21(\mathrm{~d}, J=7.5 \mathrm{~Hz}, 2 \mathrm{H}), 1.56-1.38(\mathrm{~m}, 10 \mathrm{H})$. The experimental data are in agreement with the literature report. ${ }^{26}$

\subsection{Synthesis of bromides}

General procedure for the two-step synthesis of esters (GP2)

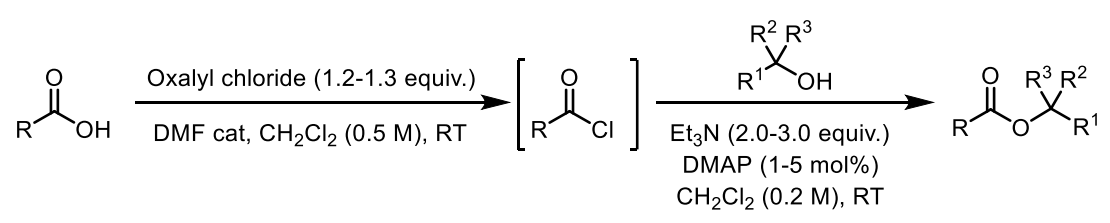

Step 1. In an oven-dried round-bottom flask equipped with a PTFE-coated stirring bar, the appropriate carboxylic acid (1.00-1.05 equiv.) and DMF (catalytic, one drop) were dissolved/suspended in dry $\mathrm{CH}_{2} \mathrm{Cl}_{2}(0.5 \mathrm{M})$, then oxalyl chloride (1.201.30 equiv.) was added dropwise (Warning: evolution of $\mathrm{HCl}$ and $\mathrm{CO}$ was observed, perform the reaction in an open-vessel under a well-ventilated fume hood). After gas evolution ceased, the volatiles were removed in vacuo, affording the acyl chloride, which was used for the next step without additional purification. (Warning: acyl chlorides are known irritating and lachrymatory agents, therefore exposure must be avoided at all times) 
Step 2. The crude acyl chloride was dissolved in dry $\mathrm{CH}_{2} \mathrm{Cl}_{2}(0.2 \mathrm{M})$, then the appropriate alcohol (1.00 equiv.), DMAP (1-5 mol\%) were added, followed by the dropwise addition of $\mathrm{Et}_{3} \mathrm{~N}$ (2.00-3.00 equiv.). Upon completion of the reaction, as monitored by TLC or GCMS, the reaction was quenched by addition of water (20 $\mathrm{ml}$ ) and stirred for 30 minutes, then the layers were separated and the aqueous layer was further extracted twice with $\mathrm{CH}_{2} \mathrm{Cl}_{2}$ (20 ml each time). The combined organic extracts were dried over $\mathrm{MgSO}_{4}$, then the solvent was removed in vacuo. The crude residue was purified by flash column chromatography on silica (pentane/EtOAc, as indicated in the individual entries), affording analytically pure esters.
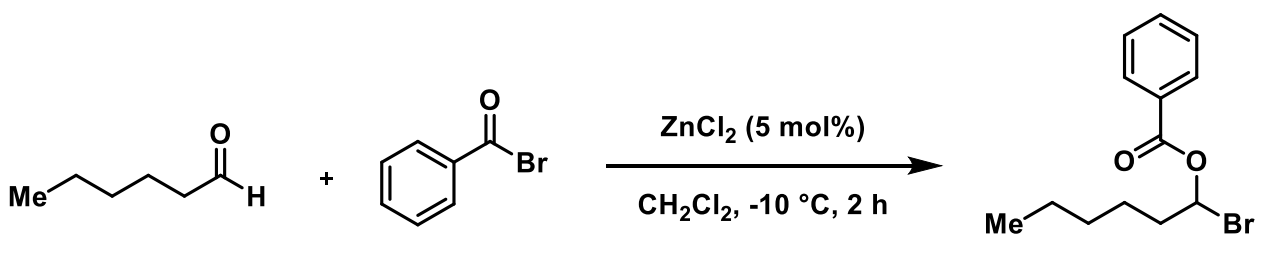

4

1-Bromohexyl benzoate (4). In an oven-dried Schlenk tube equipped with a PTFE-coated stirring bar, anhydrous $\mathrm{ZnCl}_{2}$ (272 $\mathrm{mg}, 2 \mathrm{mmol}, 5 \mathrm{~mol} \%$ ) was charged, then anhydrous $\mathrm{CH}_{2} \mathrm{Cl}_{2}(5 \mathrm{ml})$ was added and the suspension was cooled to $-10{ }^{\circ} \mathrm{C}$. Benzoyl bromide (5.7 $\mathrm{ml}, 48.0 \mathrm{mmol}, 1.2$ equiv.) was added, then after 10 minutes hexanal (4.92 ml, $40.0 \mathrm{mmol}, 1.00$ equiv.) was added dropwise over 30 minutes. The reaction was stirred at $-10{ }^{\circ} \mathrm{C}$ for 2 hours, then filtered over a short pad of basic alumina, eluting with $\mathrm{CH}_{2} \mathrm{Cl}_{2}$. The volatiles were carefully removed in vacuo and the residue was purified by vacuum distillation, affording the product 4 (7.44 g, $26.1 \mathrm{mmol}, 65 \%$ ) as a yellowish liquid. ${ }^{\mathbf{1}} \mathbf{H}$ NMR (599 MHz, $\left.\mathrm{CDCl}_{3}\right) \delta 8.07(\mathrm{~d}, J=7.9 \mathrm{~Hz}, 2 \mathrm{H}), 7.61(\mathrm{t}, J=7.7 \mathrm{~Hz}, 1 \mathrm{H}), 7.48(\mathrm{t}, J=7.3 \mathrm{~Hz}, 2 \mathrm{H}), 6.88$ $(\mathrm{t}, J=5.9 \mathrm{~Hz}, 1 \mathrm{H}), 2.34-2.22(\mathrm{~m}, 2 \mathrm{H}), 1.60-1.53(\mathrm{~m}, 2 \mathrm{H}), 1.42-1.30(\mathrm{~m}, 4 \mathrm{H}), 0.96$ - 0.87 (m, 3H). ${ }^{13}$ C NMR (151 MHz, $\left.\mathrm{CDCl}_{3}\right) \delta 164.3,134.0,130.2,129.1,128.7,77.1$, 39.7, 31.1, 25.7, 22.6, 14.1. HRMS (ESI+): calcd. for $\mathrm{C}_{13} \mathrm{H}_{17} \mathrm{O}_{2}{ }^{79} \mathrm{BrNa}\left(\mathrm{M}+\mathrm{Na}^{+}\right)$: 307.0310, Found: 307.0312. 


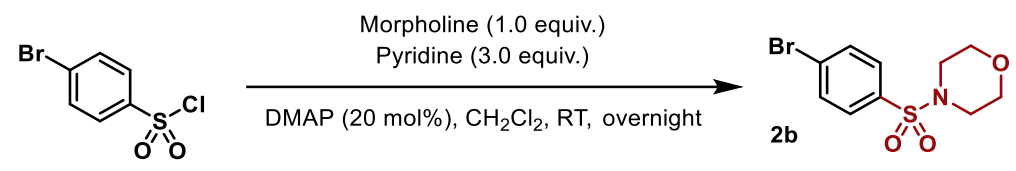

4-((4-bromophenyl)sulfonyl)morpholine (2b). The compound was synthesized according to an adapted procedure from Polites et al. ${ }^{27}$ In a Schlenk flask equipped with a PTFE-coated stirring bar, 4-bromobenzenesulfonyl chloride (6.13 g, $24.0 \mathrm{mmol}, 1.2$ equiv.) and DMAP (0.49 g, $4.8 \mathrm{mmol} 20 \mathrm{~mol} \%$ ) were dissolved in $\mathrm{CH}_{2} \mathrm{Cl}_{2}(60 \mathrm{~mL})$. Pyridine (4.83 mL, $60 \mathrm{mmol}, 3.0$ equiv.) and morpholine (1.73 mL, $20 \mathrm{mmol}, 1.0$ equiv.) were consecutively added under vigorous stirring and the reaction mixture was stirred overnight at room temperature. Upon completion, the reaction mixture was diluted with $\mathrm{CH}_{2} \mathrm{Cl}_{2}$ (25 mL) and aquoeus $\mathrm{HCl}(1 \mathrm{M}, 25 \mathrm{~mL})$ was added. The layers were separated and the aqueous layer was extracted twice with $\mathrm{CH}_{2} \mathrm{Cl}_{2}$ (30 mL each time). The combined organic layers were washed with aqueous $\mathrm{HCl}(1 \mathrm{M}, 10 \mathrm{~mL})$ and brine $(10 \mathrm{~mL})$, then dried over $\mathrm{MgSO}_{4}$, filtered and concentrated in vacuo. Recrystallization from $\mathrm{CH}_{2} \mathrm{Cl}_{2}$ at $-20{ }^{\circ} \mathrm{C}$ afforded $\mathbf{2 b}$ ( $4.58 \mathrm{~g}, 14.9 \mathrm{mmol}, 75 \%$ ) as a light orange solid. $\mathbf{R}_{\mathbf{f}}$ (pentane/EtOAc $\left.=4 / 1\right)$ 0.30. ${ }^{\mathbf{1}} \mathbf{H} \mathbf{~ N M R}\left(400 \mathrm{MHz}, \mathrm{CDCl}_{3}\right) \delta$ $7.74-7.66(\mathrm{~m}, 2 \mathrm{H}), 7.65-7.57(\mathrm{~m}, 2 \mathrm{H}), 3.78-3.71(\mathrm{~m}, 4 \mathrm{H}), 3.03-2.96(\mathrm{~m}, 4 \mathrm{H})$. ${ }^{13} \mathrm{C}$ NMR (101 $\left.\mathrm{MHz}^{\mathrm{CDCl}} 3\right) \delta 134.4,132.6,129.4,128.4,66.2,46.1$. The experimental data are in agreement with the literature report. ${ }^{27}$

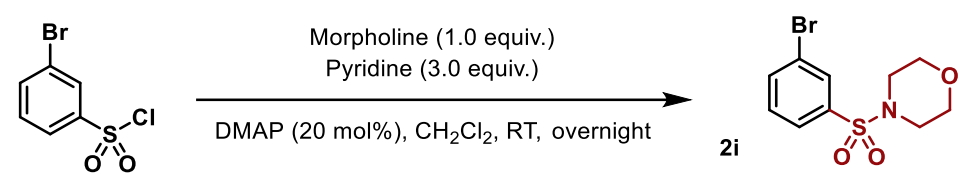

4-((3-bromophenyl)sulfonyl)morpholine (2i). The compound $2 \mathbf{i}$ (2.60 g, 8.50 mmol, 85\% yield) was obtained using the same procedure employed for the synthesis of $\mathbf{2} \mathbf{b} .{ }^{\mathbf{1}} \mathbf{H}$ NMR $\left(599 \mathrm{MHz}^{\mathrm{CDCl}} 3\right) \delta 7.91-7.88(\mathrm{~m}, 1 \mathrm{H}), 7.76(\mathrm{ddt}, J=8.0$, 1.9, $0.9 \mathrm{~Hz}, 1 \mathrm{H}), 7.69$ (ddd, $J=7.8,1.7,0.9 \mathrm{~Hz}, 1 \mathrm{H}), 7.44(\mathrm{t}, J=7.9 \mathrm{~Hz}, 1 \mathrm{H}), 3.75(\mathrm{t}, J$ $=4.7 \mathrm{~Hz}, 4 \mathrm{H}), 3.02(\mathrm{t}, J=4.8 \mathrm{~Hz}, 4 \mathrm{H}) .{ }^{13} \mathbf{C} \mathbf{~ N M R}\left(151 \mathrm{MHz}, \mathrm{CDCl}_{3}\right) \delta 137.4,136.3$, $130.8,130.7,126.5,123.4,66.2,46.1$. The experimental data are in agreement with the literature report. ${ }^{28}$ 

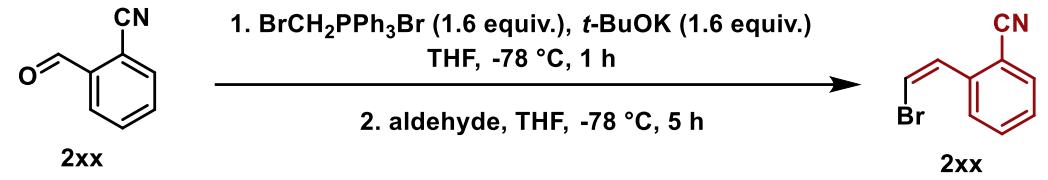

(Z)-2-(2-bromovinyl)benzonitrile (2w). The compound was prepared according to the following two-step procedure.

Step 1. The required Wittig salt was prepared according to a modified procedure from Orfanopoulos and co-workers. ${ }^{29}$ In a round-bottom flask equipped with a PTFE-coated stirring bar and a reflux condenser, triphenylphosphine $(20.0 \mathrm{~g}, 76.0$ mmol, 1.0 equiv. $)$, toluene $(100 \mathrm{~mL})$ and dibromomethane (30.0 g, $171.0 \mathrm{mmol}$, 2.3 equiv.) were charged and the reaction mixture was stirred under reflux for 24 hours. Upon cooling to $0^{\circ} \mathrm{C}$, the resulting precipitate was filtered-off, washed with hot toluene and the solid dried under vacuum to afford the corresponding phosphonium salt (18.1 g, $41.6 \mathrm{mmol}, 55 \%$ ) as a white solid. ${ }^{1} \mathbf{H}$ NMR (400 MHz, $\left.\mathrm{CDCl}_{3}\right) \delta 7.98-7.88(\mathrm{~m}, 6 \mathrm{H}), 7.79(\mathrm{ddd}, J=7.4,6.4,1.7 \mathrm{~Hz}, 3 \mathrm{H}), 7.68$ (ddd, $J$ = 9.0, 7.1, $3.7 \mathrm{~Hz}, 6 \mathrm{H}), 5.82(\mathrm{~d}, J=5.9 \mathrm{~Hz}, 2 \mathrm{H})$. The experimental data are in agreement with the literature report. ${ }^{29}$

Step 2. In an oven-dried Schlenk tube equipped with a PTFE-coated stirring bar, (bromomethyl)triphenylphosphonium bromide (1.57 g, $3.6 \mathrm{mmol}, 1.6$ equiv.) was suspended in anhydrous THF (12 ml), then the suspension was cooled to $-78{ }^{\circ} \mathrm{C}$ and $t$-BuOK (404 mg, $3.6 \mathrm{mmol}, 1.6$ equiv.) was added and the reaction was stirred for 1 hour. A solution of 2-formylbenzonitrile (288 mg, $2.2 \mathrm{mmol}, 1.0$ equiv.) in anhydrous THF ( $2 \mathrm{ml}$ ) was added dropwise, then the reaction was stirred at -78 ${ }^{\circ} \mathrm{C}$ for 5 hours. Upon completion, the reaction mixture was diluted with pentane, filtered and concentrated in vacuo. The residue was purified by flash column chromatography on silica gel (pentane/EtOAc $=98 / 2 \rightarrow 93 / 7)$, affording $2 \mathbf{w}(0.12$ g, $0.58 \mathrm{mmol}, 26 \%, Z: E>95: 5)$ as a colorless solid. $\mathbf{R}_{\mathbf{f}}($ pentane $/$ EtOAc $=95 / 5) 0.40$. 1H NMR (599 MHz, $\mathrm{CDCl}_{3}$ ) $\delta 8.05$ (ddt, $\left.J=8.0,1.2,0.6 \mathrm{~Hz}, 1 \mathrm{H}\right), 7.69$ (ddd, $J=7.7$, $1.4,0.6 \mathrm{~Hz}, 1 \mathrm{H}), 7.62(\mathrm{td}, J=7.7,1.2 \mathrm{~Hz}, 1 \mathrm{H}), 7.43(\mathrm{td}, J=7.6,1.2 \mathrm{~Hz}, 1 \mathrm{H}), 7.37(\mathrm{~d}, J$ $=8.2 \mathrm{~Hz}, 1 \mathrm{H}), 6.74(\mathrm{~d}, J=8.2 \mathrm{~Hz}, 1 \mathrm{H}) .{ }^{13} \mathrm{C} \mathbf{~ N M R}\left(151 \mathrm{MHz}, \mathrm{CDCl}_{3}\right) \delta$ 138.4, 133.0, 132.5, 129.3, 129.2, 128.6, 117.6, 112.6, 111.9. HRMS (EI+): calcd. for 
$\mathrm{C}_{9} \mathrm{H}_{6} \mathrm{~N}^{79} \mathrm{BrNa}\left(\mathrm{M}+\mathrm{Na}^{+}\right): 229.9581$, Found: 229.9577 . In addition to ${ }^{3} \mathrm{~J}$ coupling constants, the Z-geometry of the alkene was confirmed by means of NOESY experiments.

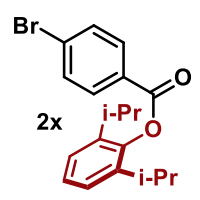

2,6-Diisopropylphenyl 4-bromobenzoate (2x). The title compound was synthesized according to GP2, starting from 4-bromobenzoic acid (603 mg, 3.00 mmol, 1.00 equiv.) and oxalyl chloride (335 $\mu$ l, $3.90 \mathrm{mmol}, 1.30$ equiv.) in dry $\mathrm{CH}_{2} \mathrm{Cl}_{2}(7 \mathrm{ml})$. The crude acyl chloride was treated with propofol $(556 \mu \mathrm{l}, 3.00$ mmol, 1.00 equiv.), DMAP (37 mg, $0.30 \mathrm{mmol}, 10 \mathrm{~mol} \%$ ) and Et $3 \mathrm{~N}$ (840 $\mu \mathrm{l}, 6.00$ mmol, 2.00 equiv.) in dry $\mathrm{CH}_{2} \mathrm{Cl}_{2}(15 \mathrm{ml})$. The crude compound was purified by flash column chromatography on silica (pentane $/ \mathrm{CH}_{2} \mathrm{Cl}_{2}=11 / 2$ ), affording $\mathbf{2 x}$ (1.02 g, $2.82 \mathrm{mmol}, 94 \%$ ) as a white solid. $\mathbf{R}_{\boldsymbol{f}}$ (pentane $/ \mathrm{CH}_{2} \mathrm{Cl}_{2}=6 / 1$ ) $=0.35 .{ }^{1} \mathbf{H}$ NMR (400 MHz, CDCl $) \delta 8.16(\mathrm{~d}, J=8.6 \mathrm{~Hz}, 2 \mathrm{H}), 7.73(\mathrm{~d}, J=8.5 \mathrm{~Hz}, 2 \mathrm{H}), 7.35-7.22$ (m, 3H), 2.97 (hept, $J=6.9 \mathrm{~Hz}, 2 \mathrm{H}), 1.24$ (d, $J=6.9 \mathrm{~Hz}, 12 \mathrm{H}) .{ }^{13} \mathbf{C}$ NMR (101 MHz, $\left.\mathrm{CDCl}_{3}\right) \delta 164.7,145.8,140.5,132.2,131.8,129.0,128.4,126.9,124.2,27.8,24.0$, 22.8. HRMS (ESI+): calcd. for $\mathrm{C}_{19} \mathrm{H}_{21} \mathrm{O}_{2} \mathrm{~S}^{79} \mathrm{BrNa}\left(\mathrm{M}+\mathrm{Na}^{+}\right)$: 383.0617, Found: 383.0616.

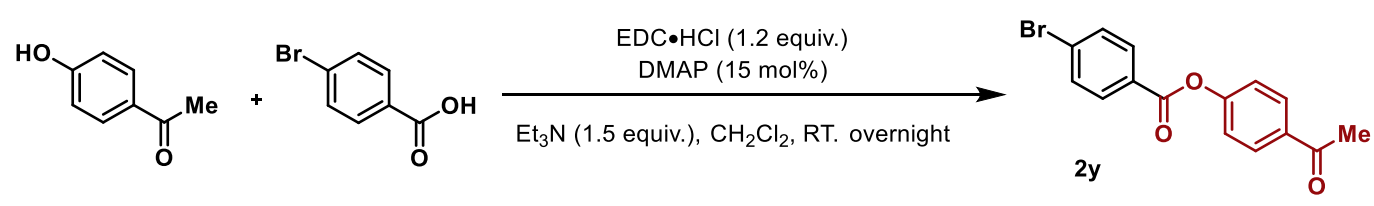

4-acetylphenyl 4-bromobenzoate (2y). In a flame-dried round bottom flask equipped with a PTFE-coated stirring bar, 4-hydroxyacetophenone (1.36 g, 10.0 mmol, 1.0 equiv.) was added under argon and dissolved in $\mathrm{CH}_{2} \mathrm{Cl}_{2}(50 \mathrm{ml})$. EDC hydrochloride (2.30 g, $12.0 \mathrm{mmol}, 1.2$ equiv.) and DMAP (183 mg, $1.5 \mathrm{mmol}$, 15 mol\%) were consecutively added, followed by 4-bromobenzoic acid (2.01 g, $10.0 \mathrm{mmol}, 1.0$ equiv.) and NEt3 (2.08 mL, $15 \mathrm{mmol}, 1.5$ equiv.). The reaction was stirred at room temperature overnight, then aqueous $\mathrm{HCl}(10 \%, 10 \mathrm{~mL})$ was 
added, the layers were separated, and the aqueous layer was extracted three times with $\mathrm{CH}_{2} \mathrm{Cl}_{2}$ (20 mL each time). The combined organic layers were washed with deionized $\mathrm{H}_{2} \mathrm{O}(20 \mathrm{~mL})$, brine $(20 \mathrm{~mL})$, dried over $\mathrm{MgSO}_{4}$, filtered, and concentrated in vacuo. Purification by column chromatography on silica gel (pentane/EtOAc $=9 / 1 \rightarrow 3 / 1)$ afforded $2 \mathbf{y}(1.06 \mathrm{~g}, 3.3 \mathrm{mmol}, 33 \%)$ as a colorless solid. Rf (pentane/EtOAc $=4 / 1)$ 0.70. ${ }^{1} \mathbf{H}$ NMR $\left(400 \mathrm{MHz}, \mathrm{CDCl}_{3}\right) \delta 8.10-8.01(\mathrm{~m}$, 4H), $7.71-7.63(\mathrm{~m}, 2 \mathrm{H}), 7.36-7.28(\mathrm{~m}, 2 \mathrm{H}), 2.62(\mathrm{~s}, 3 \mathrm{H}) .{ }^{13} \mathrm{C}$ NMR (101 MHz, $\left.\mathrm{CDCl}_{3}\right) \delta 197.0,164.1,154.6,135.1,132.2,131.8,130.2,129.4,128.1,122.0,26.8$. The experimental data are in agreement with the literature report. ${ }^{30}$

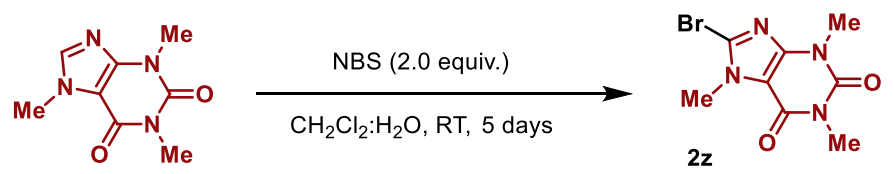

8-bromo-1,3,7-trimethyl-3,7-dihydro-1H-purine-2,6-dione

(2z). The compound was prepared by an adapted procedure from Arsenyan et al. ${ }^{31}$ Caffeine (5.0 g, $25 \mathrm{mmol}, 1.00$ equiv.) and NBS (8.8 g, $50 \mathrm{mmol}, 2.00$ equiv.) were added to $\mathrm{CH}_{2} \mathrm{Cl}_{2}(75 \mathrm{ml})$ into a round-bottom flask equipped with a PTFE-coated stirring bar. Upon dissolution of the solids, $\mathrm{H}_{2} \mathrm{O}(50 \mathrm{ml})$ was added and the reaction mixture was stirred at room temperature for 5 days. Ice-cold aqueous $\mathrm{NaOH}(2 \mathrm{M}, 30 \mathrm{ml})$ was added and the mixture was shaken until discolored. The organic layer was separated, washed twice with deionized $\mathrm{H}_{2} \mathrm{O}$ (100 ml each time), dried over $\mathrm{MgSO}_{4}$, filtered and concentrated in vacuo. Purification by column chromatography on silica gel (pentane/EtOAc $=6 / 4 \rightarrow 0 / 1)$ afforded $2 z(2.68 \mathrm{~g}$, $9.8 \mathrm{mmol}, 39 \%)$ as colorless solid. Rf (pentane/EtOAc = 4/6) 0.50. ${ }^{\mathbf{1}} \mathbf{H}$ NMR (400 $\left.\mathrm{MHz}_{\mathrm{CDCl}}\right) \delta 3.94(\mathrm{~s}, 3 \mathrm{H}), 3.53(\mathrm{~s}, 3 \mathrm{H}), 3.38(\mathrm{~s}, 3 \mathrm{H}) .{ }^{13} \mathbf{C} \mathbf{N M R}\left(101 \mathrm{MHz}, \mathrm{CDCl}_{3}\right) \delta$ $154.6,151.4,148.2,128.3,109.5,34.1,30.0,28.1$. The experimental data are in agreement with the literature report. ${ }^{31}$
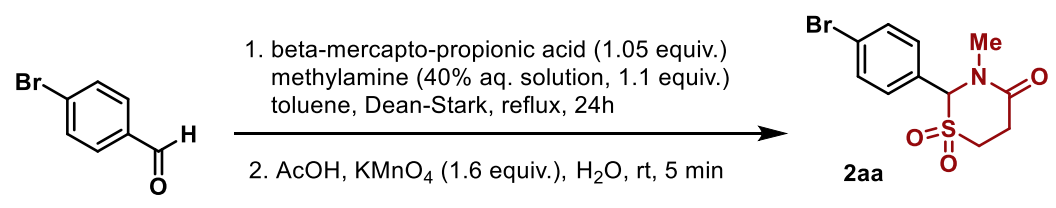
2-(4-bromophenyl)-3-methyl-1,3-thiazinan-4-one 1,1-dioxide (2aa). The compound was prepared according to the following two step procedure.

Step 1. 4-bromobenzaldehyde (1.85 g, $10.0 \mathrm{mmol}, 1.0$ equiv.) was charged into a round-bottom flask equipped with a PTFE-coated stirring bar, followed by betamercapto-propionic acid (0.92 mL, $10.5 \mathrm{mmol}, 1.05$ equiv.), methylamine (40\% solution in $\mathrm{H}_{2} \mathrm{O}, 0.85 \mathrm{~g}, 11 \mathrm{mmol}, 1.1$ equiv.) and toluene (20 mL). The flask was attached to a Dean-Stark apparatus with a reflux condenser and stirred at reflux for 24 hours. Upon completion, the solvent was removed in vacuo and the crude material was purified by column chromatography on silica gel (pentane/EtOAc $=1 / 1 \rightarrow 2 / 3$ ) to afford 2-(4-bromophenyl)-3-methyl-1,3thiazinan-4-one (2.18 g, $7.6 \mathrm{mmol}, 76 \%)$ as a colorless solid.

Step 2. In a round-bottom flask equipped with a PTFE-coated stirring bar, 2-(4bromophenyl)-3-methyl-1,3-thiazinan-4-one (1.09 g, $3.8 \mathrm{mmol}, 1.0$ equiv.) was dissolved in glacial acetic acid (9.5 mL) and $\mathrm{KMnO}_{4}(1.05 \mathrm{~g}, 6.1 \mathrm{mmol}, 1.6$ equiv.) in warm deionized water $(12.5 \mathrm{~mL})$ was added dropwise, while the temperature was kept below $30^{\circ} \mathrm{C}$ with external cooling. The reaction mixture was stirred for 5 minutes, the a saturated aqueous $\mathrm{Na}_{2} \mathrm{SO}_{3}$ solution $30 \mathrm{ml}$ ) was added and the aqueous phase was extracted thee times with EtOAc ( $10 \mathrm{~mL}$ each time). The combined organic layers were dried over $\mathrm{MgSO}_{4}$, filtered and concentrated in vacuo. Purification by column chromatography on silica gel (pentane/EtOAc $=6 / 4$ $\rightarrow 4 / 6)$ afforded 2aa $(0.64 \mathrm{~g}, 2.0 \mathrm{mmol}, 53 \%)$ as a colorless solid. Rf (pentane/EtOAc = 4/6) 0.30. ${ }^{\mathbf{1}} \mathbf{H} \mathbf{~ N M R}\left(400 \mathrm{MHz}^{\mathrm{CDCl}} 3\right) \delta 7.67-7.59(\mathrm{~m}, 2 \mathrm{H}), 7.30$ - $7.23(\mathrm{~m}, 2 \mathrm{H}), 5.25$ (d, J = 2.1 Hz, 1H), $3.39-3.04(\mathrm{~m}, 4 \mathrm{H}), 2.95$ (s, 3H). ${ }^{13}$ CNMR $\left(101 \mathrm{MHz} \mathrm{CDCl}_{3}\right) \delta 166.2,132.7,129.8,129.2,125.2,80.3,43.7,36.3,30.7$. HRMS (ESI ${ }^{+}$): calcd. for $\mathrm{C}_{11} \mathrm{H}_{12} \mathrm{NO}_{3} \mathrm{~S}^{79} \mathrm{BrNa}\left(\mathrm{M}+\mathrm{Na}^{+}\right)$: 339.9619 . Found: 339.9614 .

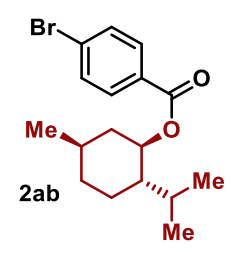


(1R,2S,5R)-2-Isopropyl-5-methylcyclohexyl 4-bromobenzoate (2ab). The title compound was synthesized according to GP2, starting from 4-bromobenzoic acid (1.01 g, $5.00 \mathrm{mmol}, 1.00$ equiv.) and oxalyl chloride (860 $\mu \mathrm{l}, 10.0 \mathrm{mmol}, 2.00$ equiv.) in dry $\mathrm{CH}_{2} \mathrm{Cl}_{2}(10 \mathrm{ml})$. The crude acyl chloride was treated with (-)-menthol (860 mg, $5.50 \mathrm{mmol}, 1.10$ equiv.), DMAP (61 mg, $0.50 \mathrm{mmol}, 10 \mathrm{~mol} \%$ ) and Et $3 \mathrm{~N}$ (1.40 ml, $10.0 \mathrm{mmol}, 2.00$ equiv.) in dry $\mathrm{CH}_{2} \mathrm{Cl}_{2}(20 \mathrm{ml})$. The crude compound was purified by flash column chromatography on silica (pentane $/ \mathrm{CH}_{2} \mathrm{Cl}_{2}=4 / 1$ ), affording 2ab (1.58 g, $4.65 \mathrm{mmol}, 93 \%$ ) as a colorless thick gum. ${ }^{\mathbf{1}} \mathbf{H}$ NMR (400 $\left.\mathrm{MHz}, \mathrm{CDCl}_{3}\right) \delta 7.93-7.86(\mathrm{~m}, 2 \mathrm{H}), 7.60-7.55(\mathrm{~m}, 2 \mathrm{H}), 4.92(\mathrm{td}, J=10.9,4.4 \mathrm{~Hz}$, 1H), $2.14-2.08(\mathrm{~m}, 1 \mathrm{H}), 1.91(\mathrm{hd}, J=7.0,2.8 \mathrm{~Hz}, 1 \mathrm{H}), 1.77-1.68(\mathrm{~m}, 2 \mathrm{H}), 1.61-$ $1.49(\mathrm{~m}, 2 \mathrm{H}), 1.19-1.04(\mathrm{~m}, 2 \mathrm{H}), 0.99-0.85(\mathrm{~m}, 7 \mathrm{H}), 0.78(\mathrm{~d}, J=7.0 \mathrm{~Hz}, 3 \mathrm{H})$. The experimental data are in agreement with the literature report. ${ }^{32}$

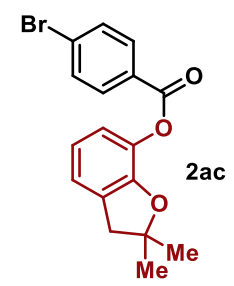

2,2-Dimethyl-2,3-dihydrobenzofuran-7-yl 4-bromobenzoate (2ac). The title compound was synthesized according to GP2, starting from 4-bromobenzoic acid (603 mg, $3.00 \mathrm{mmol}, 1.00$ equiv.) and oxalyl chloride (335 $\mu \mathrm{l}, 3.90 \mathrm{mmol}, 1.30$ equiv.) in dry $\mathrm{CH}_{2} \mathrm{Cl}_{2}(10 \mathrm{ml})$. The crude acyl chloride was treated with carbofuran (493 mg, 3.00 mmol, 1.10 equiv.), DMAP ( $37 \mathrm{mg}, 0.30 \mathrm{mmol}, 10 \mathrm{~mol} \%$ ) and $\mathrm{Et}_{3} \mathrm{~N}$ (0.84 ml, $6.00 \mathrm{mmol}, 2.00$ equiv.) in dry $\mathrm{CH}_{2} \mathrm{Cl}_{2}(15 \mathrm{ml})$. The crude compound was purified by flash column chromatography on silica (pentane $/ \mathrm{CH}_{2} \mathrm{Cl}_{2}=3 / 1$ ), affording $\mathbf{2 a c}$ (678 mg, $1.95 \mathrm{mmol}, 65 \%$ ) as a white fluffy solid. $\mathbf{R}_{\mathbf{f}}$ (pentane $/ \mathrm{CH}_{2} \mathrm{Cl}_{2}$ = 3/1): 0.20. ${ }^{1} \mathbf{H}$ NMR (400 MHz, $\left.\mathrm{CDCl}_{3}\right) \delta 8.11-8.04(\mathrm{~m}, 2 \mathrm{H}), 7.67-7.60(\mathrm{~m}, 2 \mathrm{H})$, $7.05(\mathrm{dq}, J=7.3,1.2 \mathrm{~Hz}, 1 \mathrm{H}), 6.96(\mathrm{dd}, J=8.1,1.2 \mathrm{~Hz}, 1 \mathrm{H}), 6.84(\mathrm{dd}, J=8.1,7.3 \mathrm{~Hz}$, 1H), 3.07 (s, 2H), 1.47 (s, 6H). ${ }^{13} \mathrm{C}$ NMR (101 MHz, $\left.\mathrm{CDCl}_{3}\right) \delta$ 163.8, 150.4, 134.5, 132.0, 131.9, 130.0, 128.7, 128.6, 122.9, 121.5, 120.3, 88.6, 43.2, 28.2. HRMS (ESI+): calcd. for $\mathrm{C}_{17} \mathrm{H}_{15} \mathrm{O}_{3}{ }^{79} \mathrm{BrNa}\left(\mathrm{M}+\mathrm{Na}^{+}\right)$: 369.0097. Found: 369.0097. 


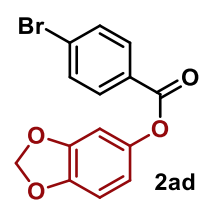

Benzo[d][1,3]dioxol-5-yl 4-bromobenzoate (2ad). The title compound was synthesized according to GP2, starting from 4-bromobenzoic acid (603 mg, 3.00 mmol, 1.00 equiv.) and oxalyl chloride (335 $\mu \mathrm{l}, 3.90 \mathrm{mmol}, 1.30$ equiv.) in dry $\mathrm{CH}_{2} \mathrm{Cl}_{2}(10 \mathrm{ml})$. The crude acyl chloride was treated with sesamol (414 mg, 3.00 mmol, 1.10 equiv.), DMAP (37 mg, $0.30 \mathrm{mmol}, 10 \mathrm{~mol} \%$ ) and Et $3 \mathrm{~N}$ (0.84 ml, 6.00 mmol, 2.00 equiv.) in dry $\mathrm{CH}_{2} \mathrm{Cl}_{2}(15 \mathrm{ml})$. The crude compound was purified by flash column chromatography on silica (pentane $/ \mathrm{CH}_{2} \mathrm{Cl}_{2} /$ EtOAc $=8 / 2 / 0.1$ ), affording 2ad (882 mg, $2.75 \mathrm{mmol}$, 92\%) as a white solid. Rf (pentane $\left./ \mathrm{CH}_{2} \mathrm{Cl}_{2} / \mathrm{EtOAc}=4 / 1 / 0.2\right): 0.65 .{ }^{1} \mathbf{H} \mathbf{~ N M R}\left(400 \mathrm{MHz}, \mathrm{CDCl}_{3}\right) \delta 8.06-8.01$ $(\mathrm{m}, 2 \mathrm{H}), 7.67-7.61(\mathrm{~m}, 2 \mathrm{H}), 6.82(\mathrm{~d}, J=8.4 \mathrm{~Hz}, 1 \mathrm{H}), 6.72(\mathrm{~d}, J=2.3 \mathrm{~Hz}, 1 \mathrm{H}), 6.64$ $(\mathrm{dd}, J=8.4,2.3 \mathrm{~Hz}, 1 \mathrm{H}), 6.01(\mathrm{~s}, 2 \mathrm{H}) .{ }^{13} \mathrm{C} \mathbf{~ N M R}\left(101 \mathrm{MHz}, \mathrm{CDCl}_{3}\right) \delta 164.9,148.2$, 145.6, 145.1, 132.0, 131.7, 129.0, 128.4, 114.1, 108.2, 103.9, 101.9. HRMS (ESI ${ }^{+}$): calcd. for $\mathrm{C}_{14} \mathrm{H}_{9} \mathrm{O}_{4}{ }^{79} \mathrm{BrNa}\left(\mathrm{M}+\mathrm{Na}^{+}\right): 342.9576$. Found: 342.9578 .

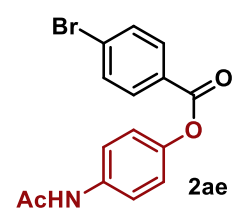

4-Acetamidophenyl 4-bromobenzoate (2ae). The title compound was synthesized according to GP2, starting from 4-bromobenzoic acid (603 mg, 3.00 mmol, 1.00 equiv.) and oxalyl chloride (335 $\mu$ l, $3.90 \mathrm{mmol}, 1.30$ equiv.) in dry $\mathrm{CH}_{2} \mathrm{Cl}_{2}(7 \mathrm{ml})$. The crude acyl chloride was treated with 4-acetamidophenol (453 mg, 3.00 mmol, 1.00 equiv.), DMAP (37 mg, $0.30 \mathrm{mmol}, 10 \mathrm{~mol} \%$ ) and $\mathrm{Et}_{3} \mathrm{~N}$ (840 $\mu \mathrm{l}, 6.00 \mathrm{mmol}, 2.00$ equiv.) in dry $\mathrm{CH}_{2} \mathrm{Cl}_{2}(15 \mathrm{ml})$. The crude compound recrystallized from boiling ethanol, affording 2ae (451 $\mathrm{mg}, 1.35 \mathrm{mmol}, 45 \%$ ) as a white solid. Note. Further product could be obtained from the mother liquors by precipitation upon water addition. $\mathbf{R}_{\boldsymbol{f}}$ (pentane $\left./ \mathrm{EtOAc}=1 / 1\right)=0.25 .{ }^{\mathbf{1}} \mathbf{H} \mathbf{N M R}(400$ MHz, DMSO-d $d_{6} \delta 10.06(\mathrm{~s}, 1 \mathrm{H}), 8.03(\mathrm{~d}, J=8.6 \mathrm{~Hz}, 2 \mathrm{H}), 7.81(\mathrm{~d}, J=8.6 \mathrm{~Hz}, 2 \mathrm{H}), 7.65$ $(\mathrm{d}, J=9.0 \mathrm{~Hz}, 2 \mathrm{H}), 7.21(\mathrm{~d}, J=9.0 \mathrm{~Hz}, 2 \mathrm{H}), 2.06$ (s, 3H). ${ }^{13}$ C NMR (101 MHz, DMSO$\left.d_{6}\right) \delta 168.3,164.1,145.6,137.3,132.1,131.7,128.2,128.1,122.0,119.8,24.0$. HRMS (ESI+): calcd. for $\mathrm{C}_{15} \mathrm{H}_{12} \mathrm{NO}_{3}{ }^{79} \mathrm{BrNa}\left(\mathrm{M}+\mathrm{Na}^{+}\right)$: 355.9893, Found: 355.9893. 


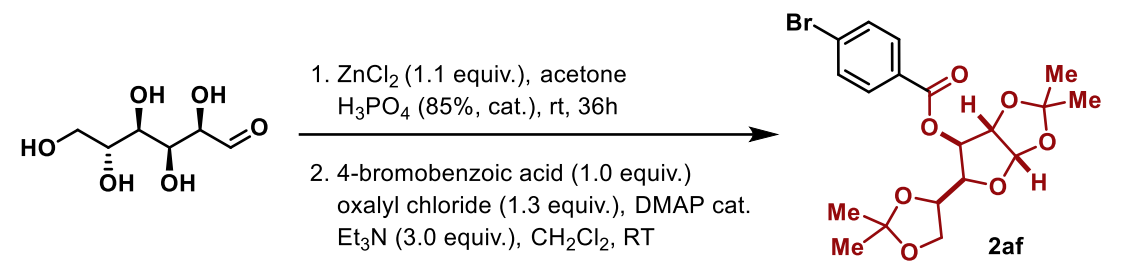

(3aR,5R,6S,6aR)-5-(2,2-dimethyl-1,3-dioxolan-4-yl)-2,2-

dimethyltetrahydrofuro[2,3-d][1,3]dioxol-6-yl 4-bromobenzoate (2af). The compound was prepared using the following two step procedure.

Step 1. Following an adapted procedure from Guo et al. ${ }^{33}$, D-Glucose (5.0 g, $27.8 \mathrm{mmol}, 1.0$ equiv.), acetone (50 mL), $\mathrm{ZnCl}_{2}(4.0 \mathrm{~g}, 29.3 \mathrm{mmol}, 1.1$ equiv.) and $85 \% \mathrm{H}_{3} \mathrm{PO}_{4}(0.15 \mathrm{~mL})$ were charged in an oven-dried round-bottom flask equipped with a PTFE-coated stirring bar. The reaction mixture was stirred at room temperature for 36 hours, filtered over Celite ${ }^{\circledR}$, neutralized by addition of $10 \% \mathrm{NaOH}$ and acetone was removed in vacuo. The aqueous layer was extracted three times with $\mathrm{CH}_{2} \mathrm{Cl}_{2}$ (50 mL each time), the combined organic layers were washed twice with deionized $\mathrm{H}_{2} \mathrm{O}$ (20 mL each time), dried over $\mathrm{MgSO}_{4}$, filtered and concentrated in vacuo. Purification by recrystallization from pentane $/ \mathrm{CH}_{2} \mathrm{Cl}_{2}$ $(1: 1, v: v)$ afforded diacetonide furanose $(0.72 \mathrm{~g}, 2.8 \mathrm{mmol}, 10 \%)$ as a colorless solid.

Step 2. The compound was synthesized following GP2 from 4-bromobenzoic acid (0.54 g, $2.7 \mathrm{mmol}, 1.0$ equiv.), $\mathrm{CH}_{2} \mathrm{Cl}_{2}(6 \mathrm{~mL})$, oxalyl chloride $(0.3 \mathrm{~mL}, 3.5 \mathrm{mmol}$, 1.3 equiv.) and then (3a $R, 5 S, 6 S, 6 \mathrm{a} R)-5$-(2,2-dimethyl-1,3-dioxolan-4-yl)-2,2dimethyltetrahydrofuro[2,3- $d][1,3]$ dioxol-6-ol $\quad(0.70 \mathrm{~g}, 2.7 \mathrm{mmol}, 1.0$ equiv.), $\mathrm{CH}_{2} \mathrm{Cl}_{2}$ (13.5 mL), DMAP (1.6 mg, $\left.0.14 \mathrm{mmol}, 5 \mathrm{~mol} \%\right)$ and $\mathrm{NEt}_{3}$ (1.12 mL, 8.1 mmol, 3.0 equiv.). The crude product was purified by flash column chromatography on silica gel (pentane/EtOAc=9/1), affording 2af $(0.92 \mathrm{~g}$, $2.1 \mathrm{mmol}, 78 \%$ ) as a colorless solid. Rf (pentane/EtOAc $=9: 1)$ 0.4. ${ }^{\mathbf{1}} \mathbf{H}$ NMR (400 $\left.\mathrm{MHz}, \mathrm{CDCl}_{3}\right) \delta 7.91-7.85(\mathrm{~m}, 2 \mathrm{H}), 7.60(\mathrm{~d}, J=8.4 \mathrm{~Hz}, 2 \mathrm{H}), 5.94(\mathrm{~d}, J=3.7 \mathrm{~Hz}, 1 \mathrm{H})$, $5.48(\mathrm{~d}, J=2.3 \mathrm{~Hz}, 1 \mathrm{H}), 4.62(\mathrm{~d}, J=3.7 \mathrm{~Hz}, 1 \mathrm{H}), 4.37-4.25(\mathrm{~m}, 2 \mathrm{H}), 4.10$ (dddd, $J=$ 12.7, 10.0, 7.9, 4.4 Hz, 2H), 2.04 (d, J = 1.0 Hz, 1H), 1.55 (s, 3H), 1.41 (s, 3H), 1.32 (s, 3H), $1.26(\mathrm{~s}, 3 \mathrm{H}) .{ }^{13} \mathrm{C}$ NMR $\left(101 \mathrm{MHz}, \mathrm{CDCl}_{3}\right) \delta 164.7,132.1,131.3,128.8,128.6$, 
112.6, 109.6, 105.3, 83.5, 80.1, 77.0, 72.7, 67.5, 27.0, 26.9, 26.4, 25.4. The experimental data are in agreement with the literature report. ${ }^{34}$ 


\section{Substrate Scope}

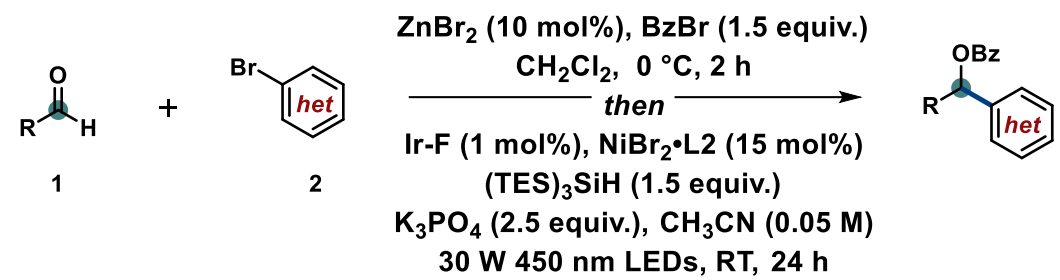

General procedure GP3. In an oven-dried Schlenk tube equipped with a PTFEcoated stirring bar, $\mathrm{ZnBr}_{2}(0.03 \mathrm{mmol}, 10 \mathrm{~mol} \%)$ and the appropriate aldehyde 1 (if solid or gum, $0.3 \mathrm{mmol}, 1.50$ equiv.) were charged under air, then the vessel was evacuated and back-filled three times with argon. Dry $\mathrm{CH}_{2} \mathrm{Cl}_{2}(0.2 \mathrm{ml})$ was added, then the solution was cooled to $0{ }^{\circ} \mathrm{C}$ and benzoyl bromide $(0.3 \mathrm{mmol}, 1.50$ equiv.) was added dropwise, followed by the appropriate aldehyde 1 (if liquid, $0.3 \mathrm{mmol}$, 1.50 equiv.). The reaction was stirred at $0{ }^{\circ} \mathrm{C}$ for two hours (usually, a change in color from colourless to yellow-orange could be observed). In another oven-dried Schlenk tube equipped with a PTFE-coated stirring bar, Ir-F ( $2 \mu \mathrm{mol}, 1 \mathrm{~mol} \%$ ), $\mathrm{NiBr}_{2} \cdot \mathrm{L} 2$ (0.03 mmol, $\left.15 \mathrm{~mol} \%\right), \mathrm{K}_{3} \mathrm{PO}_{4}(0.5 \mathrm{mmol}, 2.50$ equiv.) and the appropriate bromide 2 ( $0.2 \mathrm{mmol}, 1.00$ equiv.) were charged under air, then the vessel was evacuated and back-filled three times with argon. Dry MeCN (3 ml, $0.075 \mathrm{M}$ ) was added, then the bromide solution was transferred via syringe, rinsing with $\mathrm{MeCN}(1 \mathrm{ml}, 0.025 \mathrm{M})$, followed by (TES) $3 \mathrm{SiH}$ (0.3 mmol, 1.50 equiv.). The reaction was further degassed by freeze-pump-thaw (three times), then sealed and irradiated with 30W $450 \mathrm{~nm}$ LEDs using the standard set-up. After 24 hours, the irradiation was interrupted, then the reaction was filtered through a short pad of silica, rinsing with EtOAc. The volatiles were removed in vacuo, then the crude residue was purified by flash column chromatography on silica (pentane/EtOAc or pentane/Et ${ }_{2} \mathrm{O}$ mixtures, as detailed in the individual entries), affording the corresponding coupling product. 


\subsection{Aldehydes Scope}

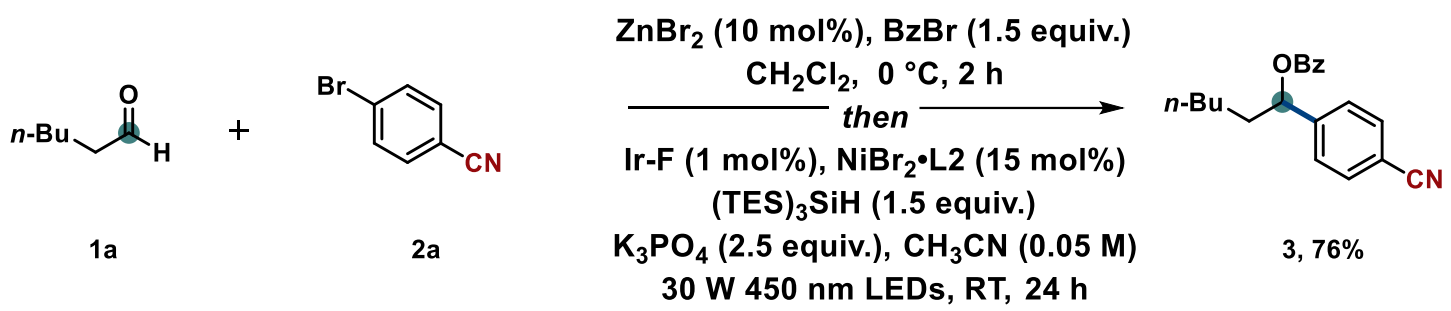

1-(4-Cyanophenyl)hexyl benzoate (3). According to the GP3, using the hexanal 1a ( $0.3 \mathrm{mmol}, 1.5$ equiv.), benzoyl bromide ( $0.3 \mathrm{mmol}, 1.5$ equiv.), aryl bromide 2a (0.2 mmol, 1.0 equiv.), Ir-F ( $2 \mu \mathrm{mol}, 1 \mathrm{~mol} \%), \mathrm{NiBr} 2 \bullet 2$ (30 $\mu \mathrm{mol}, 15 \mathrm{~mol} \%)$, (TES) ${ }_{3} \mathrm{SiH}\left(0.3 \mathrm{mmol}, 1.5\right.$ equiv.), $\mathrm{K}_{3} \mathrm{PO}_{4}(0.5 \mathrm{mmol}, 2.5$ equiv. $)$ and stirring at room temperature for $24 \mathrm{~h}$ under $30 \mathrm{~W}$ blue LEDs irradiation. The reaction mixture was filtered through a pad of silica gel and concentrated in vacuo, then purified by chromatography (pentane $/$ EtOAc $=96 / 4)$ to give $3(46.7 \mathrm{mg}, 0.152 \mathrm{mmol}, 76 \%)$ as a colorless oil. $\mathbf{R}_{\mathbf{f}}($ pentane/EtOAc $=94 / 6) 0.46 .{ }^{1} \mathbf{H} \mathbf{~ N M R}\left(599 \mathrm{MHz}, \mathrm{CDCl}_{3}\right): \delta$ ppm $8.08(\mathrm{~d}, \mathrm{~J}=7.8 \mathrm{~Hz}, 2 \mathrm{H}), 7.64(\mathrm{~d}, J=7.9 \mathrm{~Hz}, 2 \mathrm{H}), 7.59$ (t, J = 7.4 Hz, 1H), $7.51(\mathrm{~d}$, $J=8.1 \mathrm{~Hz}, 2 \mathrm{H}), 7.46(\mathrm{t}, J=7.6 \mathrm{~Hz}, 2 \mathrm{H}), 5.97$ (t, $J=7.9,5.7 \mathrm{~Hz}, 1 \mathrm{H}), 2.04(\mathrm{dt}, J=14.1$, $8.5 \mathrm{~Hz}, 1 \mathrm{H}), 1.88(\mathrm{ddt} J=14.8,10.9,5.1 \mathrm{~Hz}, 1 \mathrm{H}), 1.42(\mathrm{dt}, J=10.7,6.6 \mathrm{~Hz}, 1 \mathrm{H}), 1.36$ - $1.26(\mathrm{~m}, 5 \mathrm{H}), 0.87(\mathrm{t}, J=6.8 \mathrm{~Hz}, 3 \mathrm{H}) .{ }^{13} \mathrm{C}$ NMR (151 MHz, CDCl 3$): \delta$ ppm 165.7, 146.4, 133.3, 132.4, 129.9, 129.6, 128.5, 127.0, 118.6, 111.7, 75.8, 36.3, 31.4, 25.0, 22.4, 13.9. HRMS $\left(\mathrm{ESI}^{+}\right)$: calcd. for $\mathrm{C}_{20} \mathrm{H}_{21} \mathrm{NO}_{2} \mathrm{Na}\left(\mathrm{M}+\mathrm{Na}^{+}\right)$: 330.1465. Found: 330.1463.

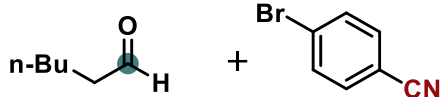

$1 \mathrm{a}$
$\mathrm{ZnBr}_{2}$ (10 mol\%), 4-Cl-BzBr (1.5 equiv.) $\mathrm{CH}_{2} \mathrm{Cl}_{2}, 0{ }^{\circ} \mathrm{C}, 2 \mathrm{~h}$

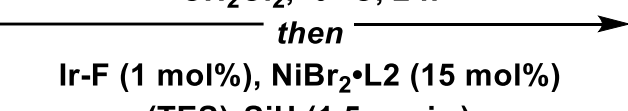

(TES) ${ }_{3} \mathrm{SiH}$ ( 1.5 equiv.)

$\mathrm{K}_{3} \mathrm{PO}_{4}$ (2.5 equiv.), $\mathrm{CH}_{3} \mathrm{CN}$ (0.05 M) $30 \mathrm{~W} 450 \mathrm{~nm}$ LEDs, RT, $24 \mathrm{~h}$

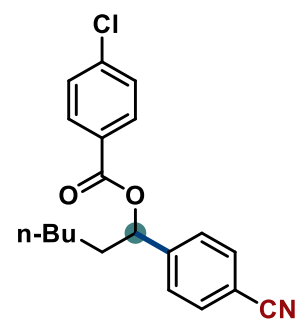

$9,49 \%$

\section{4-Acetamidophenyl 4-((benzoyloxy)(cyclohexyl)methyl)benzoate}

(9).

According to the GP3, using the aldehyde 1a (0.3 mmol, 1.5 equiv.), 4chlorobenzoyl bromide (0.3 mmol, 1.5 equiv.), aryl bromide 2a (0.2 mmol, 1.0 equiv.), Ir-F ( $2 \mu \mathrm{mol}, 1 \mathrm{~mol} \%), \mathrm{NiBr}_{2} \bullet \mathrm{L} 2$ (30 $\left.\mu \mathrm{mol}, 15 \mathrm{~mol} \%\right)$, (TES) ${ }_{3} \mathrm{SiH}(0.3 \mathrm{mmol}$, 1.5 equiv.), $\mathrm{K}_{3} \mathrm{PO}_{4}(0.5 \mathrm{mmol}, 2.5$ equiv.) and stirring at room temperature for 24 
$\mathrm{h}$ under 30W blue LEDs irradiation. The reaction mixture was filtered through a pad of silica gel and concentrated in vacuo, then purified by chromatography (pentane/EtOAc $=28 / 1 \rightarrow 24 / 1)$ to give 9 (33.5 mg, $0.098 \mathrm{mmol}, 49 \%)$ as a lightyellow gum. $\mathbf{R}_{\boldsymbol{f}}$ (pentane/EtOAc $\left.=28 / 1\right): 0.50 .{ }^{1} \mathbf{H}$ NMR $\left(400 \mathrm{MHz}, \mathrm{CDCl}_{3}\right) \delta 8.00$ (d, $J=8.5 \mathrm{~Hz}, 2 \mathrm{H}$ ), 7.65 (d, $J=8.1 \mathrm{~Hz}, 2 \mathrm{H}$ ), 7.49 (d, $J=8.1 \mathrm{~Hz}, 2 \mathrm{H}$ ), 7.43 (d, $J=8.7$ $\mathrm{Hz}, 2 \mathrm{H}), 5.94(\mathrm{dd}, J=7.9,5.8 \mathrm{~Hz}, 1 \mathrm{H}), 2.09-1.97(\mathrm{~m}, 1 \mathrm{H}), 1.92-1.81(\mathrm{~m}, 1 \mathrm{H}), 1.44$ - $1.24(\mathrm{~m}, 6 \mathrm{H}), 0.91-0.82(\mathrm{~m}, 3 \mathrm{H}) .{ }^{13} \mathrm{C}$ NMR (101 MHz, CDCl 3$) \delta$ 165.0, 146.2, 139.9, 132.6, 131.1, 129.0, 128.4, 127.1, 118.7, 111.9, 76.3, 36.4, 31.5, 25.2, 22.5, 14.1. HRMS (ESI $)$ : calcd. for $\mathrm{C}_{20} \mathrm{H}_{20} \mathrm{NO}_{2}{ }^{35} \mathrm{ClNa}\left(\mathrm{M}+\mathrm{Na}^{+}\right)$: 364.1075. Found: 364.1075.

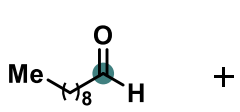

$1 \mathrm{~b}$

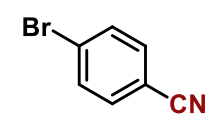

$2 a$

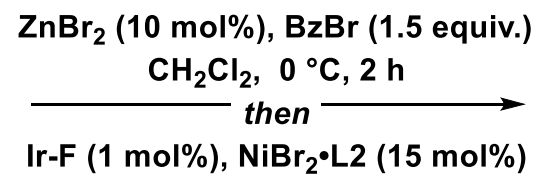

(TES) ${ }_{3} \mathrm{SiH}(1.5$ equiv.)

$\mathrm{K}_{3} \mathrm{PO}_{4}$ (2.5 equiv.), $\mathrm{CH}_{3} \mathrm{CN}$ (0.05 M) $30 \mathrm{~W} 450 \mathrm{~nm}$ LEDs, RT, $24 \mathrm{~h}$

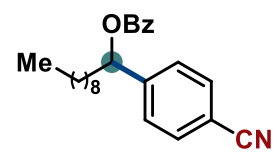

$10,53 \%$

1-(4-Cyanophenyl)propyl benzoate (10). According to the GP3, using the aldehyde $\mathbf{1 b}$ ( $0.3 \mathrm{mmol}, 1.5$ equiv.), benzoyl bromide ( $0.3 \mathrm{mmol}, 1.5$ equiv.), aryl bromide 2 a ( 0.2 mmol, 1.0 equiv.), Ir-F ( $2 \mu \mathrm{mol}, 1$ mol\%), NiBr $2 \bullet L 2$ (30 $\mu \mathrm{mol}, 15$ mol\%), (TES) ${ }_{3} \mathrm{SiH}$ (0.3 mmol, 1.5 equiv.), $\mathrm{K}_{3} \mathrm{PO}_{4}$ (0.5 mmol, 2.5 equiv.) and stirring at room temperature for $24 \mathrm{~h}$ under $30 \mathrm{~W}$ blue LEDs irradiation. The reaction mixture was filtered through a pad of silica gel and concentrated in vacuo, then purified by chromatography (pentane/EtOAc $=94 / 6)$ to give $10(35.6 \mathrm{mg}, 0.107$ mmol, 53\%) as a colorless oil. $\mathbf{R}_{\mathbf{f}}$ (pentane/EtOAc $\left.=9 / 1\right)$ 0.52. ${ }^{\mathbf{1}} \mathbf{H} \mathbf{~ N M R}(599 \mathrm{MHz}$, $\left.\mathrm{CDCl}_{3}\right): \delta$ ppm $8.13-8.04(\mathrm{~m}, 2 \mathrm{H}), 7.67-7.63(\mathrm{~m}, 2 \mathrm{H}), 7.59(\mathrm{t}, J=7.7 \mathrm{~Hz}, 1 \mathrm{H}), 7.51$ (dd, $J=7.6,2.3 \mathrm{~Hz}, 2 \mathrm{H}), 7.47(\mathrm{dt}, J=8.3,4.2 \mathrm{~Hz}, 2 \mathrm{H}), 5.97(\mathrm{q}, J=7.0,4.7 \mathrm{~Hz}, 1 \mathrm{H}$ ), $2.12-2.01(\mathrm{~m}, 1 \mathrm{H}), 1.95-1.82(\mathrm{~m}, 1 \mathrm{H}), 1.41(\mathrm{~d}, J=14.0 \mathrm{~Hz}, 1 \mathrm{H}), 1.37-1.31(\mathrm{~m}$, $3 \mathrm{H}), 1.26(\mathrm{~d}, J=16.2 \mathrm{~Hz}, 11 \mathrm{H}), 0.87(\mathrm{t}, J=8.5 \mathrm{~Hz}, 3 \mathrm{H}) .{ }^{13} \mathbf{C}$ NMR $\left(151 \mathrm{MHz}, \mathrm{CDCl}_{3}\right)$ : $\delta$ ppm 165.7, 146.4, 133.3, 132.4, 129.9, 129.6, 128.5, 127.0, 118.6, 111.7, 75.8, 36.4, 31.8, 29.43, 29.37, 29.25, 29.21, 25.3, 22.6, 14.1. HRMS (ESI+): calcd. for $\mathrm{C}_{22} \mathrm{H}_{23} \mathrm{NO}_{2} \mathrm{Na}\left(\mathrm{M}+\mathrm{Na}^{+}\right): 356.1621$. Found: 356.1626. 

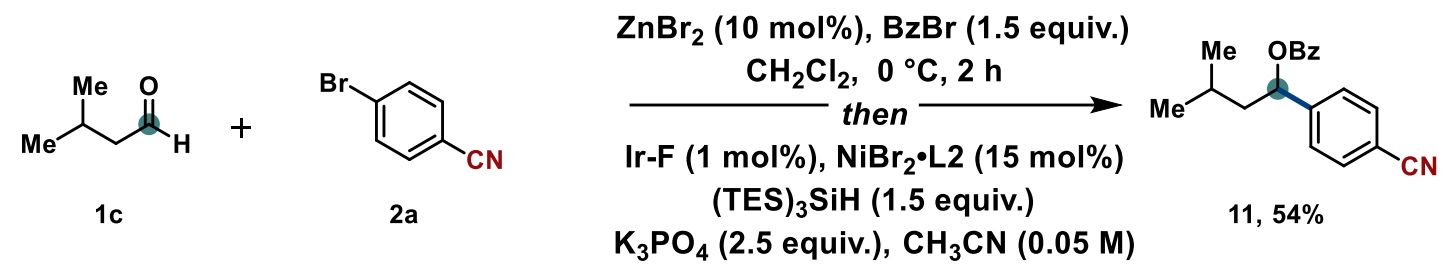
$30 \mathrm{~W} 450 \mathrm{~nm}$ LEDs, RT, $24 \mathrm{~h}$

1-(4-Cyanophenyl)-3-methylbutyl benzoate (11). According to the GP3, using the aldehyde 1c ( $0.3 \mathrm{mmol}, 1.5$ equiv.), benzoyl bromide ( $0.3 \mathrm{mmol}, 1.5$ equiv.),

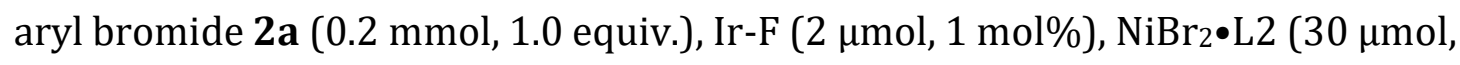
$15 \mathrm{~mol} \%$ ), (TES) $3 \mathrm{SiH}\left(0.3 \mathrm{mmol}, 1.5\right.$ equiv.), $\mathrm{K}_{3} \mathrm{PO}_{4}$ (0.5 mmol, 2.5 equiv.) and stirring at room temperature for $24 \mathrm{~h}$ under $30 \mathrm{~W}$ blue LEDs irradiation. The reaction mixture was filtered through a pad of silica gel and concentrated in vacuo, then purified by chromatography (pentane $/$ EtOAc $=94 / 6)$ to give $11(31.7 \mathrm{mg}$, $0.108 \mathrm{mmol}, 54 \%)$ as a colorless oil. $\mathbf{R}_{\mathbf{f}}$ (pentane $/$ EtOAc $\left.=94 / 6\right) 0.36 .{ }^{\mathbf{1}} \mathbf{H} \mathbf{~ N M R}$ (599 MHz, $\left.\mathrm{CDCl}_{3}\right): \delta$ ppm $8.07(\mathrm{dt}, J=8.3,1.6 \mathrm{~Hz}, 2 \mathrm{H}), 7.67-7.62(\mathrm{~m}, 2 \mathrm{H}), 7.61-$ $7.56(\mathrm{~m}, 1 \mathrm{H}), 7.54-7.49(\mathrm{~m}, 2 \mathrm{H}), 7.49-7.43(\mathrm{~m}, 2 \mathrm{H}), 6.05(\mathrm{dd}, J=9.5,4.9 \mathrm{~Hz}, 1 \mathrm{H})$, 2.02 (ddd, $J=14.8,8.3,5.5 \mathrm{~Hz}, 1 \mathrm{H}$ ), $1.77-1.70(\mathrm{~m}, 1 \mathrm{H}), 1.67$ (dd, $J=13.6,6.0 \mathrm{~Hz}$, 1H), 0.99 (dd, $J=9.3,6.6,6 \mathrm{H}) .{ }^{13} \mathrm{C}$ NMR (151 MHz, $\left.\mathrm{CDCl}_{3}\right): \delta$ ppm 165.8, 146.8, 133.4, 132.6, 130.0, 129.8, 128.6, 127.1, 118.8, 111.8, 74.5, 45.7, 25.0, 23.1, 22.3. HRMS (ESI ${ }^{+}$): calcd. for $\mathrm{C}_{19} \mathrm{H}_{19} \mathrm{NO}_{2} \mathrm{Na}\left(\mathrm{M}+\mathrm{Na}^{+}\right)$: 316.1308. Found: 316.1306 .<smiles>O=CC1CCCCC1</smiles>

$1 d$<smiles>N#Cc1ccc(Br)cc1</smiles>

2a
$\mathrm{ZnBr}_{2}$ (10 mol\%), BzBr (1.5 equiv.)

$\underset{\text { Ir-F (1 mol\%), } \mathrm{NiBr}_{2}{ }^{\circ} \mathrm{L} 2(15 \mathrm{~mol} \%)}{\mathrm{CH}_{2} \mathrm{Cl}_{2},{ }^{\circ} \mathrm{C}, 2 \mathrm{~h}}$ (TES) ${ }_{3} \mathrm{SiH}(1.5$ equiv.)

$\mathrm{K}_{3} \mathrm{PO}_{4}$ (2.5 equiv.), $\mathrm{CH}_{3} \mathrm{CN}(0.05 \mathrm{M})$ $30 \mathrm{~W} 450 \mathrm{~nm}$ LEDs, RT, $24 \mathrm{~h}$

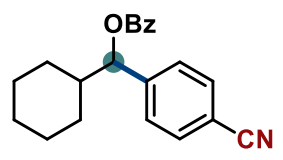

$12,58 \%$

(4-Cyanophenyl)(cyclohexyl)methyl benzoate (12). According to the GP3, using the aldehyde $\mathbf{1 d}(0.3 \mathrm{mmol}, 1.5$ equiv.), benzoyl bromide ( $0.3 \mathrm{mmol}, 1.5$ equiv.), aryl bromide 2 a ( $0.2 \mathrm{mmol}, 1.0$ equiv.), Ir-F ( $2 \mu \mathrm{mol}, 1 \mathrm{~mol} \%), \mathrm{NiBr} \bullet\llcorner 2$ (30 $\mu \mathrm{mol}, 15 \mathrm{~mol} \%$ ), (TES) $3 \mathrm{SiH}\left(0.3 \mathrm{mmol}, 1.5\right.$ equiv.), $\mathrm{K}_{3} \mathrm{PO}_{4}$ ( $0.5 \mathrm{mmol}, 2.5$ equiv.) and stirring at room temperature for $24 \mathrm{~h}$ under $30 \mathrm{~W}$ blue LEDs irradiation. The reaction mixture was filtered through a pad of silica gel and concentrated in vacuo, then purified by chromatography (pentane $/$ EtOAc $=94 / 6)$ to give $12(37.1 \mathrm{mg}$, $0.116 \mathrm{mmol}, 58 \%)$ as a colorless oil. $\mathbf{R}_{\mathbf{f}}$ (pentane $/$ EtOAc $\left.=94 / 6\right) 0.39 .{ }^{1} \mathbf{H} \mathbf{~ N M R}$ (599 MHz, $\mathrm{CDCl}_{3}$ ): $\delta$ ppm 8.13 - $8.04(\mathrm{~m}, 2 \mathrm{H}), 7.63$ (dd, $\left.J=8.2,1.8 \mathrm{~Hz}, 2 \mathrm{H}\right), 7.58$ (d, $J=7.5 \mathrm{~Hz}, 1 \mathrm{H}), 7.46(\mathrm{dd}, J=8.1,3.0 \mathrm{~Hz}, 4 \mathrm{H}), 5.74(\mathrm{~d}, J=7.0 \mathrm{~Hz}, 1 \mathrm{H}), 1.90(\mathrm{dd}, J=$ 
10.2, $6.2 \mathrm{~Hz}, 2 \mathrm{H}), 1.82-1.65(\mathrm{~m}, 3 \mathrm{H}), 1.50(\mathrm{~d}, J=12.7 \mathrm{~Hz}, 1 \mathrm{H}), 1.31-1.13(\mathrm{~m}, 4 \mathrm{H})$, $1.07-0.97(\mathrm{~m}, 1 \mathrm{H}) .{ }^{13} \mathrm{C}$ NMR $\left(151 \mathrm{MHz}, \mathrm{CDCl}_{3}\right): \delta \mathrm{ppm} 165.8,145.3,133.4,132.3$, 130.1, 129.8, 128.6, 127.7, 118.8, 111.7, 80.0, 43.3, 29.2, 28.7, 26.3, 26.0, 25.9. HRMS (ESI ${ }^{+}$): calcd. for $\mathrm{C}_{21} \mathrm{H}_{21} \mathrm{NO}_{2} \mathrm{Na}\left(\mathrm{M}+\mathrm{Na}^{+}\right)$: 342.1465. Found: 342.1462 .

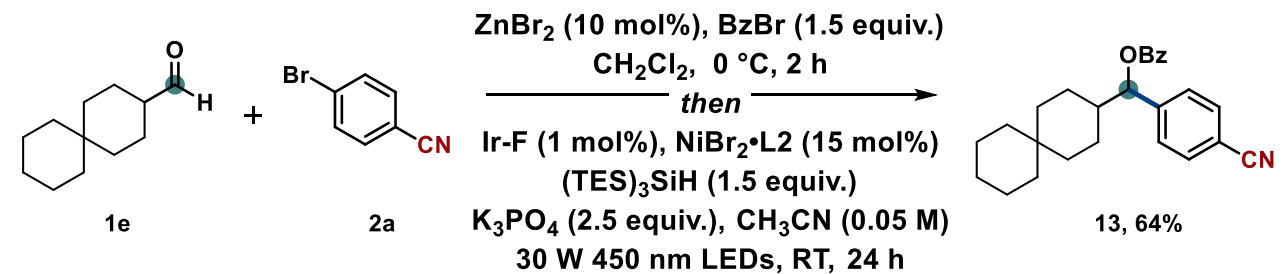

(4-cyanophenyl)(spiro[5.5] undecan-3-yl)methyl benzoate (13). According to the GP3, using the spiro[5.5] undecane-3-carbaldehyde $\mathbf{1 e}(0.3 \mathrm{mmol}, 1.5$ equiv.), benzoyl bromide ( $0.3 \mathrm{mmol}, 1.5$ equiv.), aryl bromide $2 \mathrm{a}(0.2 \mathrm{mmol}, 1.0$ equiv.), IrF (2 $\mu \mathrm{mol}, 1 \mathrm{~mol} \%), \mathrm{NiBr}_{2} \bullet \mathrm{L} 2(30 \mu \mathrm{mol}, 15 \mathrm{~mol} \%)$, (TES) $3 \mathrm{SiH}(0.3 \mathrm{mmol}, 1.5$ equiv.), $\mathrm{K}_{3} \mathrm{PO}_{4}$ (0.5 mmol, 2.5 equiv.) and stirring at room temperature for $24 \mathrm{~h}$ under 30W blue LEDs irradiation. The reaction mixture was filtered through a pad of silica gel and concentrated in vacuo, then purified by chromatography (pentane/EtOAc $=96 / 4 \rightarrow 95 / 5)$ to give $13(49.4 \mathrm{mg}, 0.127 \mathrm{mmol}, 64 \%$ ) as a colorless oil. $\mathbf{R f}($ pentane $/$ EtOAc $=96 / 4)$ 0.20. ${ }^{1} \mathbf{H}$ NMR $\left(400 \mathrm{MHz}, \mathrm{CDCl}_{3}\right) \delta(400$ $\left.\mathrm{MHz}, \mathrm{CDCl}_{3}\right) \delta 8.11-8.04(\mathrm{~m}, 2 \mathrm{H}), 7.63(\mathrm{~d}, J=8.1 \mathrm{~Hz}, 2 \mathrm{H}), 7.59(\mathrm{t}, J=7.4 \mathrm{~Hz}, 1 \mathrm{H})$, $7.47(\mathrm{dt}, J=7.5,3.5 \mathrm{~Hz}, 4 \mathrm{H}), 5.76(\mathrm{~d}, J=7.5 \mathrm{~Hz}, 1 \mathrm{H}), 1.85(\mathrm{ddt}, J=15.6,11.1,5.5 \mathrm{~Hz}$, 1H), $1.74-1.60(\mathrm{~m}, 3 \mathrm{H}), 1.45-1.30(\mathrm{~m}, 9 \mathrm{H}), 1.22$ (ddd, $J=18.2,9.9,4.1 \mathrm{~Hz}, 4 \mathrm{H}$ ), 1.00 (ddd, $J=18.0,13.3,8.5 \mathrm{~Hz}, 2 \mathrm{H}) .{ }^{13} \mathrm{C}$ NMR (101 MHz, CDCl 3$) \delta 165.8,145.4$, 133.4, 132.3, 130.1, 129.7, 128.7, 127.7, 118.8, 111.8, 80.0, 43.8, 41.6, 36.1, 32.2, 32.0, 29.8, 27.0, 24.1, 23.7, 21.8, 21.7. HRMS (ESI $\left.{ }^{+}\right)$: calcd. for $\mathrm{C}_{26} \mathrm{H}_{29} \mathrm{NO}_{2} \mathrm{Na}(\mathrm{M}+$ $\mathrm{Na}^{+}$): 410.2096. Found: 410.2091.

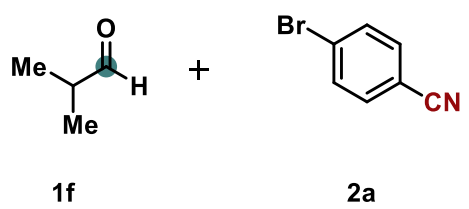

$2 a$

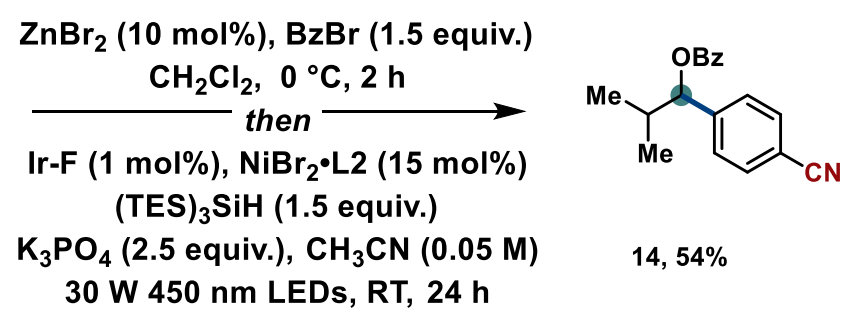


1-(4-Cyanophenyl)-2-methylpropyl benzoate (14). According to the GP3, using the aldehyde $1 \mathrm{f}$ ( $0.3 \mathrm{mmol}, 1.5$ equiv.), benzoyl bromide ( $0.3 \mathrm{mmol}, 1.5$ equiv.), aryl bromide 2 a (0.2 mmol, 1.0 equiv.), Ir-F ( $2 \mu \mathrm{mol}, 1 \mathrm{~mol} \%$ ), $\mathrm{NiBr}_{2} \bullet \mathrm{L} 2$ (30 $\mu \mathrm{mol}$, 15 mol\%), (TES) $)_{3} \mathrm{SiH}$ (0.3 mmol, 1.5 equiv.), $\mathrm{K}_{3} \mathrm{PO}_{4}$ (0.5 mmol, 2.5 equiv.) and stirring at room temperature for $24 \mathrm{~h}$ under $30 \mathrm{~W}$ blue LEDs irradiation. The reaction mixture was filtered through a pad of silica gel and concentrated in vacuo, then purified by chromatography (pentane $/$ EtOAc $=94 / 6)$ to give $14(30.4 \mathrm{mg}$, $0.109 \mathrm{mmol}, 54 \%)$ as a colorless oil. $\mathbf{R}_{\mathbf{f}}($ pentane $/$ EtOAc $=94 / 6) 0.27 .{ }^{1} \mathbf{H}$ NMR (599 MHz, $\mathrm{CDCl}_{3}$ ): $\delta$ ppm $8.09(\mathrm{~d}, J=7.6 \mathrm{~Hz}, 2 \mathrm{H}), 7.64(\mathrm{dd}, J=8.3,1.8 \mathrm{~Hz}, 2 \mathrm{H}), 7.60$ $(\mathrm{d}, J=8.2 \mathrm{~Hz}, 1 \mathrm{H}), 7.50-7.43(\mathrm{~m}, 4 \mathrm{H}), 5.74(\mathrm{~d}, J=6.9 \mathrm{~Hz}, 1 \mathrm{H}), 2.30-2.20(\mathrm{~m}, 1 \mathrm{H})$, $1.05(\mathrm{~d}, J=6.6 \mathrm{~Hz}, 3 \mathrm{H}), 0.93(\mathrm{~d}, J=6.9 \mathrm{~Hz}, 3 \mathrm{H}) .{ }^{13} \mathrm{C} \mathrm{NMR}\left(151 \mathrm{MHz}, \mathrm{CDCl}_{3}\right): \delta \mathrm{ppm}$ 165.8, 145.3, 133.4, 132.3, 130.1, 129.8, 128.7, 127.6, 118.8, 111.8, 80.6, 33.9, 18.9, 18.2. HRMS (ESI $\left.{ }^{+}\right)$: calcd. for $\mathrm{C}_{18} \mathrm{H}_{17} \mathrm{NO}_{2} \mathrm{Na}\left(\mathrm{M}+\mathrm{Na}^{+}\right): 302.1152$. Found: 302.1153 .

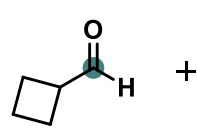

19<smiles>N#Cc1ccc(Br)cc1</smiles>

2a
$\mathrm{ZnBr}_{2}$ (10 mol\%), BzBr (1.5 equiv.)

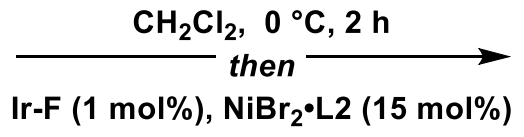

(TES) ${ }_{3} \mathrm{SiH}$ ( 1.5 equiv.)

$\mathrm{K}_{3} \mathrm{PO}_{4}$ (2.5 equiv.), $\mathrm{CH}_{3} \mathrm{CN}$ (0.05 M) $30 \mathrm{~W} 450 \mathrm{~nm}$ LEDs, RT, $24 \mathrm{~h}$

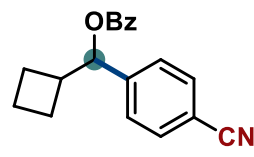

$15,56 \%$

(4-Cyanophenyl)(cyclobutyl)methyl benzoate (15). According to the GP3, using the aldehyde $1 \mathrm{~g}$ ( $0.3 \mathrm{mmol}, 1.5$ equiv.), benzoyl bromide ( $0.3 \mathrm{mmol}, 1.5$ equiv.), aryl bromide 2 a ( $0.2 \mathrm{mmol}, 1.0$ equiv.), Ir-F ( $2 \mu \mathrm{mol}, 1 \mathrm{~mol} \%), \mathrm{NiBr}_{2} \bullet \mathrm{L} 2$ (30 $\mu \mathrm{mol}, 15 \mathrm{~mol} \%$ ), (TES) ${ }_{3} \mathrm{SiH}\left(0.3 \mathrm{mmol}, 1.5\right.$ equiv.), $\mathrm{K}_{3} \mathrm{PO}_{4}$ (0.5 mmol, 2.5 equiv.) and stirring at room temperature for $24 \mathrm{~h}$ under $30 \mathrm{~W}$ blue LEDs irradiation. The reaction mixture was filtered through a pad of silica gel and concentrated in vacuo, then purified by chromatography (pentane/EtOAc $=94 / 6)$ to give $15(32.7 \mathrm{mg}$, $0.112 \mathrm{mmol}, 56 \%)$ as a colorless oil. $\mathbf{R}_{\mathbf{f}}$ (pentane $/$ EtOAc $\left.=94 / 6\right) 0.27 .{ }^{1} \mathbf{H}$ NMR (599 MHz, $\mathrm{CDCl}_{3}$ ): $\delta$ ppm $8.09(\mathrm{dd}, J=7.7,2.0 \mathrm{~Hz}, 2 \mathrm{H}), 7.65-7.55(\mathrm{~m}, 3 \mathrm{H}), 7.48(\mathrm{~d}$, $J=7.5 \mathrm{~Hz}, 4 \mathrm{H}), 5.92(\mathrm{~d}, J=7.5 \mathrm{~Hz}, 1 \mathrm{H}), 2.94-2.81(\mathrm{~m}, 1 \mathrm{H}), 2.14-2.02(\mathrm{~m}, 2 \mathrm{H})$, $1.90(\mathrm{td}, J=10.3,9.8,5.8 \mathrm{~Hz}, 4 \mathrm{H}) .{ }^{13} \mathrm{C}$ NMR (151 MHz, CDCl $): \delta \mathrm{ppm} \mathrm{166.0,} \mathrm{145.1,}$ 133.4, 132.4, 130.1, 129.8, 128.6, 127.2, 118.8, 111.8, 78.7, 40.4, 24.8, 24.5, 18.0. HRMS (ESI ${ }^{+}$): calcd. for $\mathrm{C}_{19} \mathrm{H}_{17} \mathrm{NO}_{2} \mathrm{Na}\left(\mathrm{M}+\mathrm{Na}^{+}\right)$: 314.1152. Found: 314.1151 . 


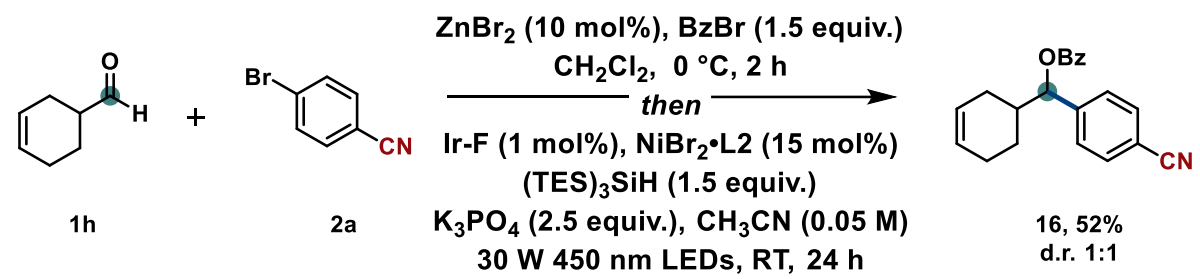

(4-cyanophenyl)(cyclohex-3-en-1-yl)methyl benzoate (16). According to the GP3, using the cyclohex-3-ene-1-carbaldehyde $\mathbf{1 h}(0.3 \mathrm{mmol}, 1.5$ equiv.), benzoyl bromide ( $0.3 \mathrm{mmol}, 1.5$ equiv.), aryl bromide $2 \mathrm{a}$ ( $0.2 \mathrm{mmol}, 1.0$ equiv.), Ir-F (2

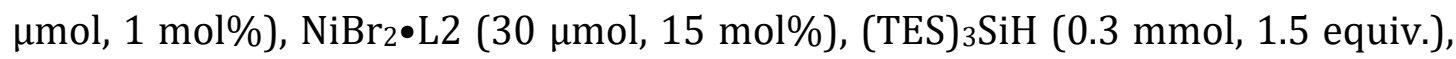
$\mathrm{K}_{3} \mathrm{PO}_{4}(0.5 \mathrm{mmol}, 2.5$ equiv.) and stirring at room temperature for $24 \mathrm{~h}$ under $30 \mathrm{~W}$ blue LEDs irradiation. The reaction mixture was filtered through a pad of silica gel and concentrated in vacuo, then purified by chromatography (pentane $/$ EtOAc $=$ $24 / 1 \rightarrow 19 / 1$ ) to give 16 (33.0 mg, $0.104 \mathrm{mmol}, 52 \%$; d.r. $1: 1$ ) as a colorless oil. $\mathbf{R}_{\mathbf{f}}$ (pentane/EtOAc $=16 / 1) 0.60 .{ }^{1} \mathrm{H}-\mathbf{N M R}\left(400 \mathrm{MHz}, \mathrm{CDCl}_{3}\right) \delta 8.11-8.04(\mathrm{~m}, 2 \mathrm{H})$, $7.65(\mathrm{dd}, J=8.3,1.8 \mathrm{~Hz}, 2 \mathrm{H}), 7.62-7.56(\mathrm{~m}, 1 \mathrm{H}), 7.53-7.43(\mathrm{~m}, 4 \mathrm{H}), 5.83(\mathrm{dd}, J=$ 11.8, 7.4 Hz, 1H), $5.74-5.57(\mathrm{~m}, 2 \mathrm{H}), 2.29-1.94(\mathrm{~m}, 3 \mathrm{H}), 1.81(\mathrm{qd}, J=5.4,2.5 \mathrm{~Hz}$, 1H), $1.67-1.56(\mathrm{~m}, 1 \mathrm{H}), 1.54-1.25(\mathrm{~m}, 1 \mathrm{H}) .{ }^{13} \mathbf{C}$ NMR (101 MHz, $\left.\mathrm{CDCl}_{3}\right) \delta 165.8$, 165.7, 145.0, 144.9, 133.4, 132.3, 129.9, 129.7, 128.6, 127.7, 127.6, 127.3, 127.0, 125.4, 125.1, 118.6, 111.8, 111.8, 79.4, 79.0, 39.5, 39.2, 27.9, 27.4, 25.0, 24.9, 24.7, 24.4. HRMS (ESI+): calcd. for $\mathrm{C}_{21} \mathrm{H}_{19} \mathrm{NO}_{2} \mathrm{Na}\left(\mathrm{M}+\mathrm{Na}^{+}\right): 340.1313$. Found: 340.1307 .

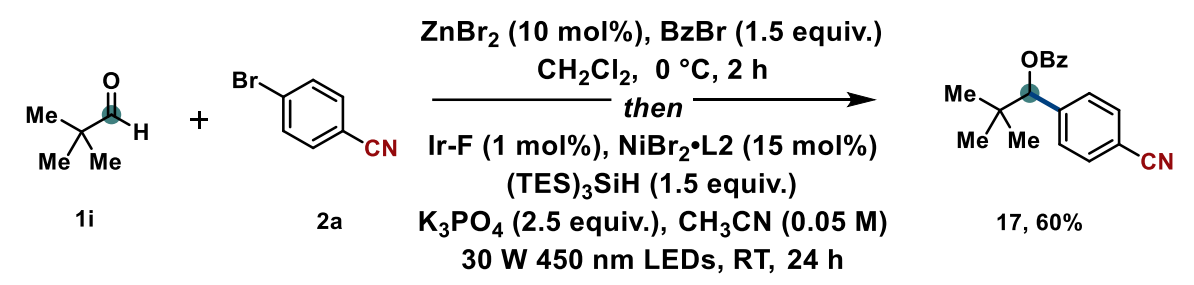

1-(4-cyanophenyl)-2,2-dimethylpropyl benzoate (17). According to the GP3, using the pivalic aldehyde $\mathbf{1 h}(0.3 \mathrm{mmol}, 1.5$ equiv.), benzoyl bromide $(0.3 \mathrm{mmol}$, 1.5 equiv.), aryl bromide $2 \mathbf{a}(0.2 \mathrm{mmol}, 1.0$ equiv.), Ir-F ( $2 \mu \mathrm{mol}, 1 \mathrm{~mol} \%), \mathrm{NiBr}_{2} \bullet \mathrm{L} 2$ (30 $\mu \mathrm{mol}, 15 \mathrm{~mol} \%$ ), (TES) 3 SiH ( $0.3 \mathrm{mmol}, 1.5$ equiv.), $\mathrm{K}_{3} \mathrm{PO}_{4}$ ( $0.5 \mathrm{mmol}, 2.5$ equiv.) and stirring at room temperature for $24 \mathrm{~h}$ under $30 \mathrm{~W}$ blue LEDs irradiation. The reaction mixture was filtered through a pad of silica gel and concentrated in vacuo, then purified by chromatography (pentane/EtOAc $=98 / 2 \rightarrow 94 / 6$ ) to give $\mathbf{1 7}$ 
(35.2 mg, $0.120 \mathrm{mmol}, 60 \%$ ) as a colorless gum. $\mathbf{R}_{\mathbf{f}}($ pentane/EtOAc $=98 / 2) 0.20$.

${ }^{1}$ H NMR (400 MHz, $\left.\mathrm{CDCl}_{3}\right) \delta 8.14-8.06(\mathrm{~m}, 2 \mathrm{H}), 7.66-7.56(\mathrm{~m}, 3 \mathrm{H}), 7.51-7.43$ (m, 4H), $5.70(\mathrm{~s}, 1 \mathrm{H}), 1.04(\mathrm{~s}, 9 \mathrm{H}) .{ }^{13} \mathrm{C}$ NMR $\left(101 \mathrm{MHz}, \mathrm{CDCl}_{3}\right) \delta 165.6,144.0,133.5$, 131.7, 130.1, 129.7, 128.7, 128.5, 118.8, 111.7, 82.9, 35.6, 26.2. HRMS (ESI+): calcd. for $\mathrm{C}_{19} \mathrm{H}_{19} \mathrm{NO}_{2} \mathrm{Na}\left(\mathrm{M}+\mathrm{Na}^{+}\right): 316.1313$. Found: 316.1308 .

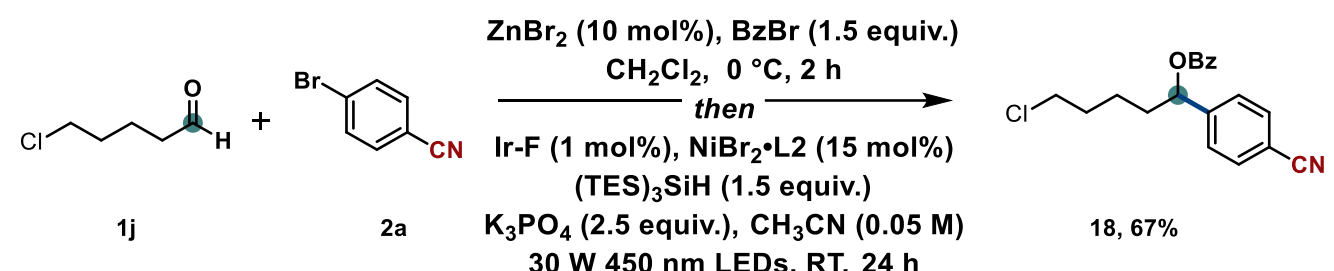

5-chloro-1-(4-cyanophenyl)pentyl benzoate (18). According to the GP3, using the chloropentanal $\mathbf{1 j}$ ( $0.3 \mathrm{mmol}, 1.5$ equiv.), benzoyl bromide ( $0.3 \mathrm{mmol}, 1.5$ equiv.), aryl bromide 2 a ( 0.2 mmol, 1.0 equiv.), Ir-F ( $2 \mu \mathrm{mol}, 1 \mathrm{~mol} \%), \mathrm{NiBr}_{2} \bullet \mathrm{L} 2$ (30 $\mu \mathrm{mol}, 15 \mathrm{~mol} \%$ ), (TES) ${ }_{3} \mathrm{SiH}\left(0.3 \mathrm{mmol}, 1.5\right.$ equiv.), $\mathrm{K}_{3} \mathrm{PO}_{4}(0.5 \mathrm{mmol}, 2.5$ equiv.) and stirring at room temperature for $24 \mathrm{~h}$ under $30 \mathrm{~W}$ blue LEDs irradiation. The reaction mixture was filtered through a pad of silica gel and concentrated in vacuo, then purified by chromatography (pentane/EtOAc $=33 / 1 \rightarrow 9 / 1$ ) to give 18 (44.0 mg, $0.13 \mathrm{mmol}, 67 \%)$ as a colorless oil. $\mathbf{R} \mathbf{f}($ pentane $/$ EtOAc $=16 / 1) 0.20 .{ }^{1} \mathbf{H}$ NMR (400 MHz, $\left.\mathrm{CDCl}_{3}\right) \delta 8.11-8.04(\mathrm{~m}, 2 \mathrm{H}), 7.65(\mathrm{~d}, J=8.1 \mathrm{~Hz}, 2 \mathrm{H}), 7.63-7.57$ (m, 1H), $7.55-7.43(\mathrm{~m}, 4 \mathrm{H}), 5.99$ (dd, $J=7.9,5.5 \mathrm{~Hz}, 1 \mathrm{H}), 3.52$ (t, $J=6.5 \mathrm{~Hz}, 2 \mathrm{H}$ ), 2.08 (dddd, $J=13.4,10.3,7.9,5.2 \mathrm{~Hz}, 1 \mathrm{H}), 1.98-1.89(\mathrm{~m}, 1 \mathrm{H}), 1.86-1.75(\mathrm{~m}, 2 \mathrm{H})$, $1.69-1.43(\mathrm{~m}, 1 \mathrm{H}), 1.05-0.48(\mathrm{~m}, 1 \mathrm{H}) .{ }^{13} \mathrm{C}$ NMR $\left(101 \mathrm{MHz}, \mathrm{CDCl}_{3}\right) \delta 165.7,146.0$, 133.5, 132.6, 129.8, 129.8, 128.7, 127.1, 118.7, 112.0, 75.5, 44.6, 35.7, 32.2, 22.8. HRMS (ESI+): calcd. for $\mathrm{C}_{19} \mathrm{H}_{18}{ }^{35} \mathrm{ClNO}_{2} \mathrm{Na}\left(\mathrm{M}+\mathrm{Na}^{+}\right)$: 350.0924. Found: 350.0920 .

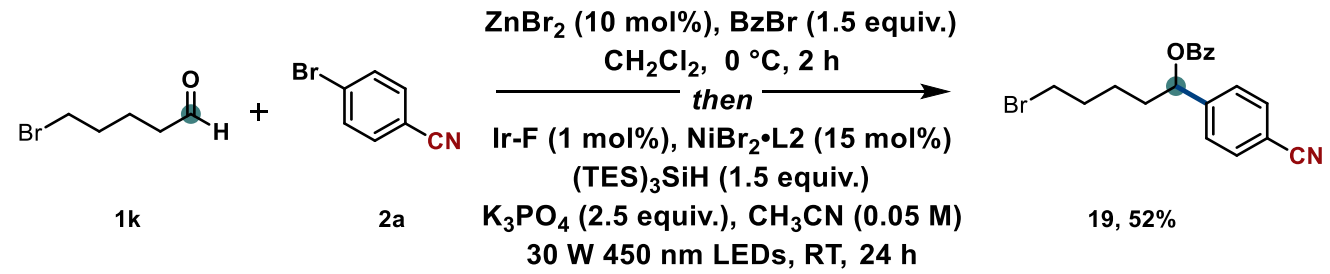


5-bromo-1-(4-cyanophenyl)pentyl benzoate (19). According to the GP3, using the bromopentanal $1 \mathbf{k}(0.3 \mathrm{mmol}, 1.5$ equiv.), benzoyl bromide (0.3 mmol, 1.5 equiv.), aryl bromide 2 a (0.2 mmol, 1.0 equiv.), Ir-F ( $2 \mu \mathrm{mol}, 1 \mathrm{~mol} \%), \mathrm{NiBr}_{2} \bullet \mathrm{L} 2$ (30 $\mu \mathrm{mol}, 15 \mathrm{~mol} \%$ ), (TES) ${ }_{3} \mathrm{SiH}\left(0.3 \mathrm{mmol}, 1.5\right.$ equiv.), $\mathrm{K}_{3} \mathrm{PO}_{4}(0.5 \mathrm{mmol}, 2.5$ equiv.) and stirring at room temperature for $24 \mathrm{~h}$ under $30 \mathrm{~W}$ blue LEDs irradiation. The reaction mixture was filtered through a pad of silica gel and concentrated in vacuo, then purified by chromatography (pentane/EtOAc $=95 / 5 \rightarrow 90 / 10$ ) to give 19 (38.7 mg, $0.104 \mathrm{mmol}, 52 \%)$ as a colorless oil. $\mathbf{R}_{\mathbf{f}}($ pentane $/$ EtOAc $=90 / 10) 0.40$. ${ }^{1} \mathbf{H}$ NMR (400 MHz, $\left.\mathrm{CDCl}_{3}\right) \delta 8.07(\mathrm{dd}, J=8.3,1.4 \mathrm{~Hz}, 2 \mathrm{H}), 7.68-7.64(\mathrm{~m}, 2 \mathrm{H}), 7.64$ - $7.55(\mathrm{~m}, 1 \mathrm{H}), 7.55-7.43(\mathrm{~m}, 4 \mathrm{H}), 5.99$ (dd, $J=7.9,5.5 \mathrm{~Hz}, 1 \mathrm{H}), 3.39$ (t, J = 6.7 Hz, 2H), 2.08 (dddd, $J=13.4,10.3,7.9,5.3 \mathrm{~Hz}, 1 \mathrm{H}), 1.99-1.84(\mathrm{~m}, 3 \mathrm{H}), 1.69-1.44$ (m, 1H), $1.09-0.65(\mathrm{~m}, 1 \mathrm{H}) .{ }^{13} \mathrm{C}$ NMR $\left(101 \mathrm{MHz}, \mathrm{CDCl}_{3}\right) \delta 165.8,146.0,133.5,132.6$, 129.8, 129.8, 128.7, 127.1, 118.7, 112.0, 75.5, 35.6, 33.2, 32.3, 24.1. HRMS (ESI+): calcd. for $\mathrm{C}_{19} \mathrm{H}_{18}{ }^{79} \mathrm{BrNO}_{2} \mathrm{Na}\left(\mathrm{M}+\mathrm{Na}^{+}\right): 394.0419$. Found: 394.0416 .
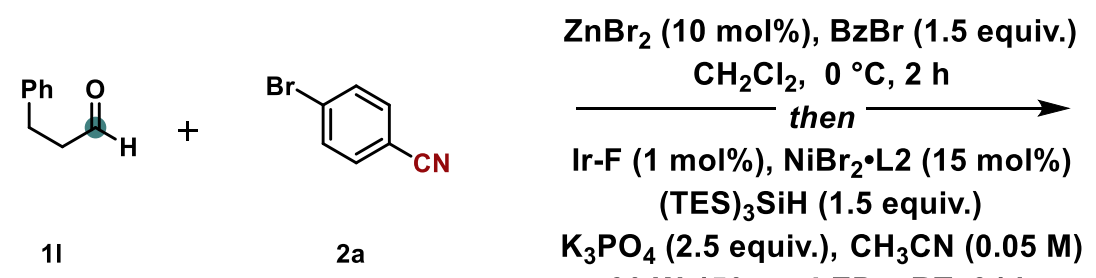
$30 \mathrm{~W} 450 \mathrm{~nm}$ LEDs, RT, $24 \mathrm{~h}$

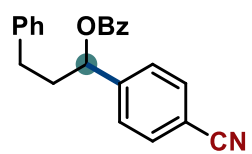

$20,63 \%$

1-(4-Cyanophenyl)-3-phenylpropyl benzoate (20). According to the GP3, using the aldehyde 11 ( $0.3 \mathrm{mmol}, 1.5$ equiv.), benzoyl bromide (0.3 mmol, 1.5 equiv.), aryl bromide 2 a ( $0.2 \mathrm{mmol}, 1.0$ equiv.), Ir-F ( $2 \mu \mathrm{mol}, 1 \mathrm{~mol} \%), \mathrm{NiBr}_{2} \bullet \mathrm{L} 2$ (30 $\mu \mathrm{mol}, 15 \mathrm{~mol} \%$ ), (TES) ${ }_{3} \mathrm{SiH}\left(0.3 \mathrm{mmol}, 1.5\right.$ equiv.), $\mathrm{K}_{3} \mathrm{PO}_{4}(0.5 \mathrm{mmol}, 2.5$ equiv.) and stirring at room temperature for $24 \mathrm{~h}$ under $30 \mathrm{~W}$ blue LEDs irradiation. The reaction mixture was filtered through a pad of silica gel and concentrated in vacuo, then purified by chromatography (pentane/EtOAc $=90 / 10)$ to give $\mathbf{2 0}(43.1 \mathrm{mg}$, $0.126 \mathrm{mmol}, 63 \%)$ as a colorless oil. $\mathbf{R}_{\mathbf{f}}$ (pentane $/$ EtOAc $\left.=80 / 20\right) 0.56 .{ }^{1} \mathbf{H}$ NMR (599 MHz, CDCl $): \delta$ ppm $8.11-8.03(\mathrm{~m}, 2 \mathrm{H}), 7.65(\mathrm{~d}, J=8.1 \mathrm{~Hz}, 2 \mathrm{H}), 7.60(\mathrm{~d}, J=$ $7.6 \mathrm{~Hz}, 1 \mathrm{H}), 7.54-7.46(\mathrm{~m}, 4 \mathrm{H}), 7.29$ (t, J=7.5 Hz, 2H), $7.21(\mathrm{~d}, J=7.4 \mathrm{~Hz}, 1 \mathrm{H}), 7.17$ (d, $J=7.5 \mathrm{~Hz}, 2 \mathrm{H}), 6.00$ (dd, $J=8.3,5.3 \mathrm{~Hz}, 1 \mathrm{H}$ ), 2.76 (ddd, $J=15.5,9.0,6.0 \mathrm{~Hz}, 2 \mathrm{H}$ ), $2.52-2.29(\mathrm{~m}, 1 \mathrm{H}), 2.26-2.00(\mathrm{~m}, 1 \mathrm{H}) .{ }^{13} \mathrm{C} \mathrm{NMR}\left(151 \mathrm{MHz}, \mathrm{CDCl}_{3}\right): \delta \mathrm{ppm} 165.6$, 
146.0, 140.5, 133.4, 132.5, 129.8, 129.7, 128.6, 128.6, 128.3, 127.0, 126.3, 118.6, 111.9, 75.2, 37.9, 31.7. HRMS (ESI $\left.{ }^{+}\right)$: calcd. for $\mathrm{C}_{23} \mathrm{H}_{19} \mathrm{NO}_{2} \mathrm{Na}\left(\mathrm{M}+\mathrm{Na}^{+}\right): 364.1308$. Found: 364.1306.

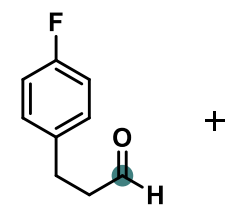

$1 \mathrm{~m}$

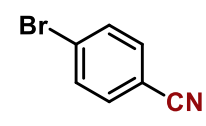

$2 a$
$\mathrm{ZnBr}_{2}$ (10 mol\%), $\mathrm{BzBr}$ (1.5 equiv.) $\mathrm{CH}_{2} \mathrm{Cl}_{2}, 0{ }^{\circ} \mathrm{C}, 2 \mathrm{~h}$

Ir-F (1 mol\%), $\mathrm{NiBr}_{2} \cdot \mathrm{L} 2$ (15 $\left.\mathrm{mol}^{2}\right)$ (TES) ${ }_{3} \mathrm{SiH}$ ( 1.5 equiv.) $\mathrm{K}_{3} \mathrm{PO}_{4}$ (2.5 equiv.), $\mathrm{CH}_{3} \mathrm{CN}(0.05 \mathrm{M})$ $30 \mathrm{~W} 450 \mathrm{~nm}$ LEDs, RT, $24 \mathrm{~h}$

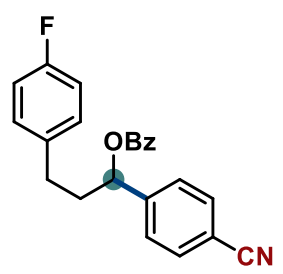

$21,56 \%$

1-(4-Cyanophenyl)-3-(4-fluorophenyl)propyl benzoate (21). According to the GP3, using the aldehyde $\mathbf{1 m}(0.3 \mathrm{mmol}, 1.5$ equiv.), benzoyl bromide $(0.3 \mathrm{mmol}$, 1.5 equiv.), aryl bromide $2 \mathrm{a}$ ( $0.2 \mathrm{mmol}, 1.0$ equiv.), Ir-F ( $2 \mu \mathrm{mol}, 1 \mathrm{~mol} \%)$, $\mathrm{NiBr}_{2} \bullet \mathrm{L} 2$ (30 $\mu \mathrm{mol}, 15 \mathrm{~mol} \%$ ), (TES) ${ }_{3} \mathrm{SiH}\left(0.3 \mathrm{mmol}, 1.5\right.$ equiv.), $\mathrm{K}_{3} \mathrm{PO}_{4}(0.5 \mathrm{mmol}, 2.5$ equiv.) and stirring at room temperature for $24 \mathrm{~h}$ under $30 \mathrm{~W}$ blue LEDs irradiation. The reaction mixture was filtered through a pad of silica gel and concentrated in vacuo, then purified by chromatography (pentane/EtOAc $=94 / 6)$ to give $21(40.5 \mathrm{mg}$, $0.113 \mathrm{mmol}, 56 \%)$ as a colorless oil. Rf (pentane $/$ EtOAc $=94 / 6) 0.16 .{ }^{1} \mathbf{H}$ NMR (599 MHz, CDCl 3 ): $\delta$ ppm $8.09-8.03(\mathrm{~m}, 2 \mathrm{H}), 7.68-7.63(\mathrm{~m}, 2 \mathrm{H}), 7.63-7.58(\mathrm{~m}$, 1H), $7.52-7.45(\mathrm{~m}, 4 \mathrm{H}), 7.13-7.09(\mathrm{~m}, 2 \mathrm{H}), 6.99-6.94(\mathrm{~m}, 2 \mathrm{H}), 5.98(\mathrm{dd}, J=8.4$, $5.1 \mathrm{~Hz}, 1 \mathrm{H}$ ), 2.72 (dddd, $J=23.2,14.1,9.2,6.2 \mathrm{~Hz}, 2 \mathrm{H}$ ), 2.38 (dddd, $J=14.2,9.1,8.4$, $5.9 \mathrm{~Hz}, 1 \mathrm{H}), 2.21-2.09$ (m, 1H). ${ }^{13} \mathrm{C}$ NMR (151 MHz, CDCl $): \delta$ ppm 165.6, 161.5 (d, $J=244.6 \mathrm{~Hz}), 145.8,136.1$ (d, $J=3.0 \mathrm{~Hz}), 133.4,132.5,129.67$ (d, $J=9.1 \mathrm{~Hz}$ ), 129.66, 129.6, 128.5, 127.0, 118.5, 115.4 (d, $J=21.1 \mathrm{~Hz}$ ), 111.9, 75.0, 38.0, 30.9. ${ }^{19}$ F NMR (564 MHz, $\mathrm{CDCl}_{3}$ ): $\delta \mathrm{ppm}-117.0$. HRMS (ESI ${ }^{+}$): calcd. for $\mathrm{C}_{23} \mathrm{H}_{18} \mathrm{NO}_{2} \mathrm{FNa}$ $\left(\mathrm{M}+\mathrm{Na}^{+}\right): 382.1214$. Found: 382.1214 .

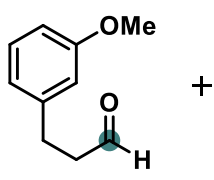

$1 \mathrm{n}$

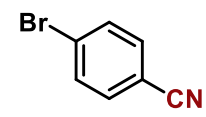

$2 \mathbf{a}$
$\mathrm{ZnBr}_{2}$ (10 mol\%), BzBr (1.5 equiv.)

$\mathrm{CH}_{2} \mathrm{Cl}_{2}, 0^{\circ} \mathrm{C}, 2 \mathrm{~h}$

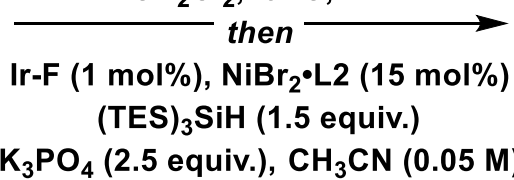

$30 \mathrm{~W} 450 \mathrm{~nm}$ LEDs, RT, $24 \mathrm{~h}$

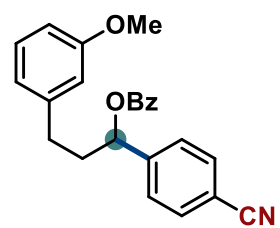

$22,47 \%$ 
1-(4-Cyanophenyl)-3-(3-methoxyphenyl)propyl benzoate (22). According to the GP3, using the aldehyde $1 \mathrm{n}(0.3 \mathrm{mmol}, 1.5$ equiv. $)$, benzoyl bromide $(0.3 \mathrm{mmol}$, 1.5 equiv.), aryl bromide 2 a ( $0.2 \mathrm{mmol}, 1.0$ equiv.), Ir-F ( $2 \mu \mathrm{mol}, 1 \mathrm{~mol} \%), \mathrm{NiBr} 2 \bullet \mathrm{L} 2$ (30 $\mu \mathrm{mol}, 15 \mathrm{~mol} \%$ ), (TES) ${ }_{3} \mathrm{SiH}\left(0.3 \mathrm{mmol}, 1.5\right.$ equiv.), $\mathrm{K}_{3} \mathrm{PO}_{4}(0.5 \mathrm{mmol}, 2.5$ equiv.) and stirring at room temperature for $24 \mathrm{~h}$ under $30 \mathrm{~W}$ blue LEDs irradiation. The reaction mixture was filtered through a pad of silica gel and concentrated in vacuo, then purified by chromatography (pentane/EtOAc $=90 / 10)$ to give $22(35.2 \mathrm{mg}$, $0.095 \mathrm{mmol}, 47 \%)$ as a colorless oil. $\mathbf{R}_{\mathbf{f}}$ (pentane/EtOAc $\left.=80 / 20\right) 0.56 .{ }^{1} \mathbf{H} \mathbf{~ N M R}$ (599 MHz, CDCl $): \delta$ ppm $8.06(\mathrm{~d}, J=7.4 \mathrm{~Hz}, 2 \mathrm{H}), 7.68-7.63(\mathrm{~m}, 2 \mathrm{H}), 7.59(\mathrm{~d}, J=$ $7.6 \mathrm{~Hz}, 1 \mathrm{H}), 7.53-7.43(\mathrm{~m}, 4 \mathrm{H}), 7.20(\mathrm{t}, J=7.6 \mathrm{~Hz}, 1 \mathrm{H}), 6.74(\mathrm{t}, J=7.1 \mathrm{~Hz}, 2 \mathrm{H}), 6.70$ $(\mathrm{s}, 1 \mathrm{H}), 5.98(\mathrm{t}, J=6.0 \mathrm{~Hz}, 1 \mathrm{H}), 3.77(\mathrm{~s}, 3 \mathrm{H}), 2.72(\mathrm{q}, J=9.7,8.2 \mathrm{~Hz}, 2 \mathrm{H}), 2.40(\mathrm{dq}, J$ $=15.4,8.1 \mathrm{~Hz}, 1 \mathrm{H}), 2.28-2.17(\mathrm{~m}, 1 \mathrm{H}) .{ }^{13} \mathrm{C}$ NMR (151 MHz, $\left.\mathrm{CDCl}_{3}\right): \delta$ ppm 165.8, 159.9, 146.1, 142.3, 133.5, 132.6, 129.9, 129.8, 129.7, 128.7, 127.1, 120.8, 118.7, 114.4, 112.0, 111.6, 75.3, 55.3, 37.9, 31.9. HRMS (ESI ${ }^{+}$): calcd. for $\mathrm{C}_{24} \mathrm{H}_{21} \mathrm{NO}_{3} \mathrm{Na}(\mathrm{M}$ $+\mathrm{Na}^{+}$): 394.1414. Found: 394.1414.

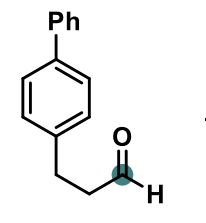

10

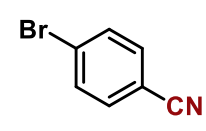

$2 a$
$\mathrm{ZnBr}_{2}$ (10 mol\%), BzBr (1.5 equiv.) $\mathrm{CH}_{2} \mathrm{Cl}_{2}, \mathrm{O}^{\circ} \mathrm{C}, 2 \mathrm{~h}$

Ir-F (1 mol\%), $\mathrm{NiBr}_{2} \cdot \mathrm{L} 2$ (15 mol\%)

(TES) ${ }_{3} \mathrm{SiH}$ ( 1.5 equiv.)

$\mathrm{K}_{3} \mathrm{PO}_{4}$ (2.5 equiv.), $\mathrm{CH}_{3} \mathrm{CN}$ (0.05 M) $30 \mathrm{~W} 450 \mathrm{~nm}$ LEDs, RT, $24 \mathrm{~h}$

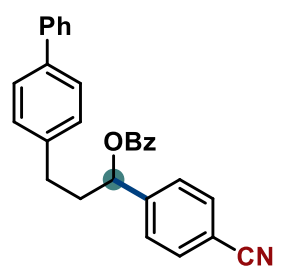

$23,54 \%$

3-([1,1'-Biphenyl]-4-yl)-1-(4-cyanophenyl)propyl benzoate (23). According to the GP3, using the aldehyde 10 ( $0.3 \mathrm{mmol}, 1.5$ equiv.), benzoyl bromide $(0.3$ mmol, 1.5 equiv.), aryl bromide 2 a ( $0.2 \mathrm{mmol}, 1.0$ equiv.), Ir-F ( $2 \mu \mathrm{mol}, 1 \mathrm{~mol} \%)$, $\mathrm{NiBr}_{2} \bullet \mathrm{L} 2$ (30 $\mu \mathrm{mol}, 15 \mathrm{~mol} \%$ ), (TES) $3 \mathrm{SiH}\left(0.3 \mathrm{mmol}, 1.5\right.$ equiv.), $\mathrm{K}_{3} \mathrm{PO}_{4}$ (0.5 mmol, 2.5 equiv.) and stirring at room temperature for $24 \mathrm{~h}$ under $30 \mathrm{~W}$ blue LEDs irradiation. The reaction mixture was filtered through a pad of silica gel and concentrated in vacuo, then purified by chromatography (pentane $/$ EtOAc $=94 / 6$ ) to give 23 (44.7 mg, $0.107 \mathrm{mmol}, 54 \%$ ) as a colorless oil. $\mathbf{R} \mathbf{f}$ (pentane/EtOAc = 94/6) 0.18. ${ }^{1} \mathbf{H}$ NMR (599 MHz, $\left.\mathrm{CDCl}_{3}\right): \delta \mathrm{ppm} 8.08(\mathrm{dd}, J=7.9,2.1 \mathrm{~Hz}, 2 \mathrm{H}), 7.70-$ $7.64(\mathrm{~m}, 2 \mathrm{H}), 7.64-7.56(\mathrm{~m}, 3 \mathrm{H}), 7.53(\mathrm{dd}, J=7.1,3.4 \mathrm{~Hz}, 4 \mathrm{H}), 7.50-7.41(\mathrm{~m}, 4 \mathrm{H})$, $7.37-7.31(\mathrm{~m}, 1 \mathrm{H}), 7.28-7.22(\mathrm{~m}, 2 \mathrm{H}), 6.04(\mathrm{dd}, J=8.5,4.9 \mathrm{~Hz}, 1 \mathrm{H}), 2.94-2.74$ (m, 2H), $2.62-2.40(\mathrm{~m}, 1 \mathrm{H}), 2.32-2.10(\mathrm{~m}, 1 \mathrm{H}) .{ }^{13} \mathbf{C} \mathbf{N M R}\left(151 \mathrm{MHz}, \mathrm{CDCl}_{3}\right): \delta$ ppm 165.7, 146.0, 140.8, 139.6, 139.3, 133.4, 132.5, 129.7, 129.7, 128.7, 128.6, 
127.3, 127.2, 127.0, 127.0, 118.6, 111.9, 75.2, 37.9, 31.4. HRMS (ESI+): calcd. for $\mathrm{C}_{29} \mathrm{H}_{23} \mathrm{NO}_{2} \mathrm{Na}\left(\mathrm{M}+\mathrm{Na}^{+}\right): 440.1621$. Found: 440.1622 .

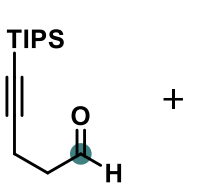

$1 p$

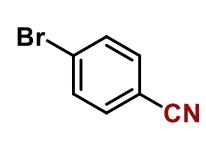

$2 a$
$\mathrm{ZnBr}_{2}(10 \mathrm{~mol} \%), \mathrm{BzBr}$ (1.5 equiv.)

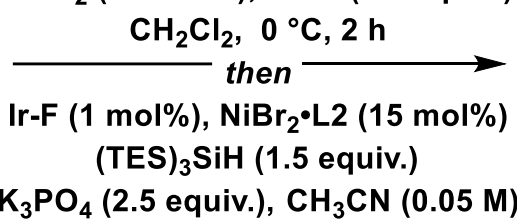

$30 \mathrm{~W} 450 \mathrm{~nm}$ LEDs, RT, $24 \mathrm{~h}$

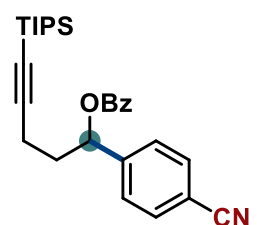

$24,43 \%$

\section{1-(4-Cyanophenyl)-5-(triisopropylsilyl)pent-4-yn-1-yl}

benzoate

According to the GP3, using the aldehyde 1p ( $0.3 \mathrm{mmol}, 1.5$ equiv.), benzoyl bromide ( $0.3 \mathrm{mmol}, 1.5$ equiv.), aryl bromide 2a ( $0.2 \mathrm{mmol}, 1.0$ equiv.), Ir-F (2

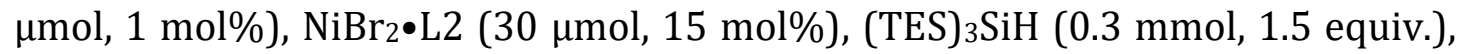
$\mathrm{K}_{3} \mathrm{PO}_{4}$ (0.5 mmol, 2.5 equiv.) and stirring at room temperature for $24 \mathrm{~h}$ under $30 \mathrm{~W}$ blue LEDs irradiation. The reaction mixture was filtered through a pad of silica gel and concentrated in vacuo, then purified by chromatography (pentane $/$ EtOAc $=$ 94/6) to give 24 (38.1 mg, $0.086 \mathrm{mmol}, 43 \%$ ) as a colorless oil. $\mathbf{R}_{\mathbf{f}}$ (pentane/EtOAc = 94/6) 0.24. ${ }^{1} \mathbf{H}$ NMR (599 MHz, $\left.\mathrm{CDCl}_{3}\right): \delta$ ppm $8.10-8.05(\mathrm{~m}, 2 \mathrm{H}), 7.69-7.64$ (m, 2H), 7.59 (t, J= 7.6 Hz, 1H), $7.55-7.52(\mathrm{~m}, 2 \mathrm{H}), 7.47$ (t, J= 7.4 Hz, 2H), $6.19-$ $6.11(\mathrm{~m}, 1 \mathrm{H}), 2.46(\mathrm{dt}, J=16.0,7.4 \mathrm{~Hz}, 1 \mathrm{H}), 2.32$ (ddd, $J=19.4,15.7,7.4 \mathrm{~Hz}, 2 \mathrm{H})$, $2.10(\mathrm{dt}, J=13.3,7.5 \mathrm{~Hz}, 1 \mathrm{H}), 1.09-1.05(\mathrm{~m}, 21 \mathrm{H}) .{ }^{13} \mathrm{C} \mathrm{NMR}\left(151 \mathrm{MHz}, \mathrm{CDCl}_{3}\right): \delta$ ppm 165.4, 145.7, 133.4, 132.6, 129.7, 129.7, 128.5, 127.0, 118.6, 112.0, 106.4, 82.1, 74.6, 35.6, 18.6, 16.5, 11.3. HRMS (ESI $\left.{ }^{+}\right)$: calcd. for $\mathrm{C}_{28} \mathrm{H}_{35} \mathrm{NO}_{2} \mathrm{SiNa}\left(\mathrm{M}+\mathrm{Na}^{+}\right)$: 468.2329. Found: 468.2327.

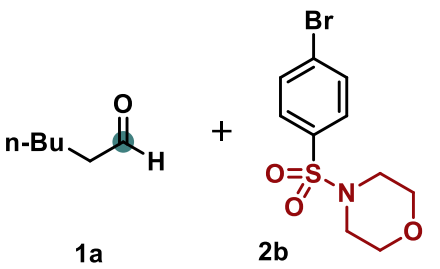

$1 \mathrm{a}$
$\mathrm{ZnBr}_{2}$ (10 mol\%), BzBr (1.5 equiv.)

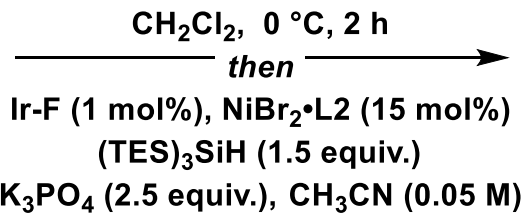

$30 \mathrm{~W} 450 \mathrm{~nm}$ LEDs, RT, $24 \mathrm{~h}$

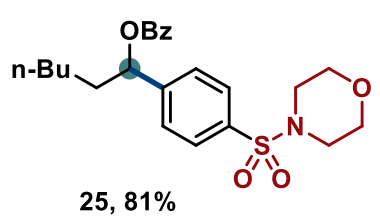

$25,81 \%$

1-(4-(Morpholinosulfonyl)phenyl)hexyl benzoate (25). According to the GP3, using the aldehyde $1 \mathrm{a}(0.3 \mathrm{mmol}, 1.5$ equiv.), benzoyl bromide (0.3 mmol, 1.5 equiv.), aryl bromide $\mathbf{2 b}$ ( $0.2 \mathrm{mmol}, 1.0$ equiv.), Ir-F ( $2 \mu \mathrm{mol}, 1 \mathrm{~mol} \%), \mathrm{NiBr}_{2} \bullet \mathrm{L} 2$ (30 $\mu \mathrm{mol}, 15 \mathrm{~mol} \%$ ), (TES) ${ }_{3} \mathrm{SiH}$ ( $0.3 \mathrm{mmol}, 1.5$ equiv.), $\mathrm{K}_{3} \mathrm{PO}_{4}$ (0.5 mmol, 2.5 equiv.) and stirring at room temperature for $24 \mathrm{~h}$ under $30 \mathrm{~W}$ blue LEDs irradiation. The reaction mixture was filtered through a pad of silica gel and concentrated in vacuo, 
then purified by chromatography (pentane/EtOAc $=80 / 20)$ to give $25(69.4 \mathrm{mg}$, $0.161 \mathrm{mmol}, 81 \%$ ) as a colorless oil. $\mathbf{R}_{\mathbf{f}}$ (pentane/EtOAc $\left.=80 / 20\right)$ 0.24. ${ }^{\mathbf{1}} \mathbf{H} \mathbf{~ N M R}$ (599 MHz, CDCl 3 ): $\delta$ ppm 8.07 (d, $J=7.7 \mathrm{~Hz}, 2 \mathrm{H}), 7.72(\mathrm{~d}, J=8.0 \mathrm{~Hz}, 2 \mathrm{H}), 7.57$ (d, $J$ $=7.8 \mathrm{~Hz}, 3 \mathrm{H}), 7.45(\mathrm{t}, J=7.6 \mathrm{~Hz}, 2 \mathrm{H}), 6.06-5.99(\mathrm{~m}, 1 \mathrm{H}), 3.71(\mathrm{t}, J=4.8 \mathrm{~Hz}, 4 \mathrm{H})$, $2.98(\mathrm{t}, J=4.8 \mathrm{~Hz}, 4 \mathrm{H}), 2.10-1.98(\mathrm{~m}, 1 \mathrm{H}), 1.88(\mathrm{ddt} J=14.8,11.0,5.2 \mathrm{~Hz}, 1 \mathrm{H})$, $1.42(\mathrm{~d}, J=6.1 \mathrm{~Hz}, 1 \mathrm{H}), 1.31(\mathrm{dd}, J=13.6,7.2 \mathrm{~Hz}, 5 \mathrm{H}), 0.85(\mathrm{t}, J=6.8 \mathrm{~Hz}, 3 \mathrm{H}) .{ }^{13} \mathrm{C}$ NMR (151 MHz, $\mathrm{CDCl}_{3}$ ): $\delta$ ppm 165.7, 146.5, 134.6, 133.2, 130.0, 129.6, 128.5, 128.1, 127.0, 75.7, 66.1, 45.9, 36.4, 31.4, 25.1, 22.4, 13.9. HRMS (ESI+): calcd. for $\mathrm{C}_{23} \mathrm{H}_{29} \mathrm{NO}_{5} \mathrm{SNa}\left(\mathrm{M}+\mathrm{Na}^{+}\right): 454.1659$. Found: 454.1655.
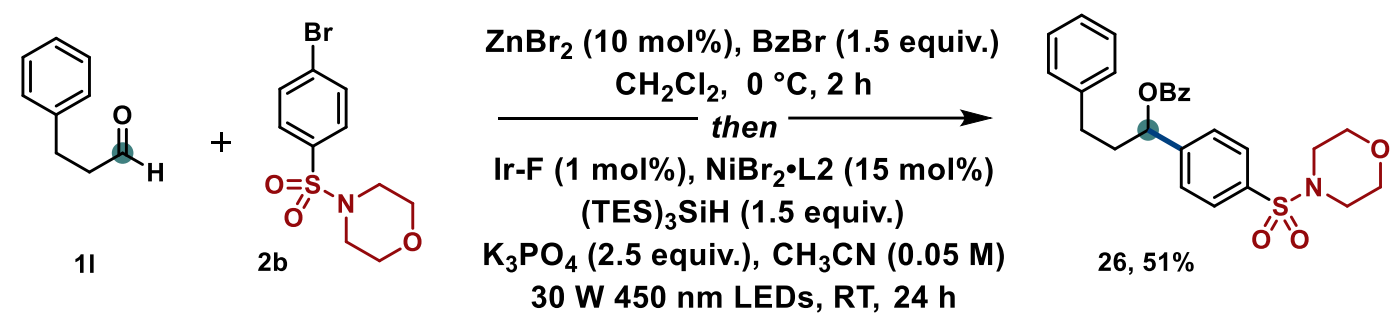

1-(4-(Morpholinosulfonyl)phenyl)-3-phenylpropyl benzoate (26). According to the GP3, using the aldehyde 11 ( $0.3 \mathrm{mmol}, 1.5$ equiv.), benzoyl bromide $(0.3$ mmol, 1.5 equiv.), aryl bromide $2 \mathbf{b}$ ( 0.2 mmol, 1.0 equiv.), Ir-F ( $2 \mu \mathrm{mol}, 1 \mathrm{~mol} \%$ ), $\mathrm{NiBr} \cdot \bullet 2(30 \mu \mathrm{mol}, 15 \mathrm{~mol} \%)$, (TES) $3 \mathrm{SiH}$ (0.3 mmol, 1.5 equiv.), $\mathrm{K}_{3} \mathrm{PO}_{4}(0.5 \mathrm{mmol}$, 2.5 equiv.) and stirring at room temperature for $24 \mathrm{~h}$ under $30 \mathrm{~W}$ blue LEDs irradiation. The reaction mixture was filtered through a pad of silica gel and concentrated in vacuo, then purified by chromatography (pentane/EtOAc = 70/30) to give 26 (47.3 $\mathrm{mg}, 0.102 \mathrm{mmol}, 51 \%$ ) as a colorless oil. $\mathbf{R}_{\mathbf{f}}$ (pentane $/$ EtOAc $=70 / 30)$ 0.21. ${ }^{1} \mathbf{H}$ NMR (599 MHz, $\left.\mathrm{CDCl}_{3}\right): \delta$ ppm $8.06(\mathrm{dd}, J=8.2$, $1.5 \mathrm{~Hz}, 2 \mathrm{H}), 7.76-7.69(\mathrm{~m}, 2 \mathrm{H}), 7.62-7.52(\mathrm{~m}, 4 \mathrm{H}), 7.46(\mathrm{t}, J=7.8 \mathrm{~Hz}, 2 \mathrm{H}), 7.26(\mathrm{t}$, $J=7.5 \mathrm{~Hz}, 2 \mathrm{H}), 7.20-7.14(\mathrm{~m}, 2 \mathrm{H}), 6.03(\mathrm{dd}, J=8.4,5.2 \mathrm{~Hz}, 1 \mathrm{H}), 3.71(\mathrm{t}, J=4.7 \mathrm{~Hz}$, $4 \mathrm{H}), 2.98(\mathrm{t}, J=4.8 \mathrm{~Hz}, 4 \mathrm{H}), 2.74(\mathrm{ddd}, J=16.2,9.0,5.9 \mathrm{~Hz}, 2 \mathrm{H}), 2.41(\mathrm{dt}, J=8.3,5.5$ $\mathrm{Hz}, 1 \mathrm{H}), 2.22(\mathrm{ddd}, J=9.2,4.7,1.5 \mathrm{~Hz}, 1 \mathrm{H}) .{ }^{13} \mathrm{C} \mathrm{NMR}\left(151 \mathrm{MHz}, \mathrm{CDCl}_{3}\right.$ ): $\delta \mathrm{ppm} 165.6$, $146.1,140.5,134.8,133.4,129.8,129.7,129.1,128.6,128.5,128.3,128.2,127.8$, 127.0, 126.2, 75.1, 66.1, 66.1, 46.0, 45.9, 38.0, 31.7. HRMS (ESI+): calcd. for $\mathrm{C}_{26} \mathrm{H}_{27} \mathrm{NO}_{5} \mathrm{Na}\left(\mathrm{M}+\mathrm{Na}^{+}\right): 488.1502$. Found: 488.1503. 

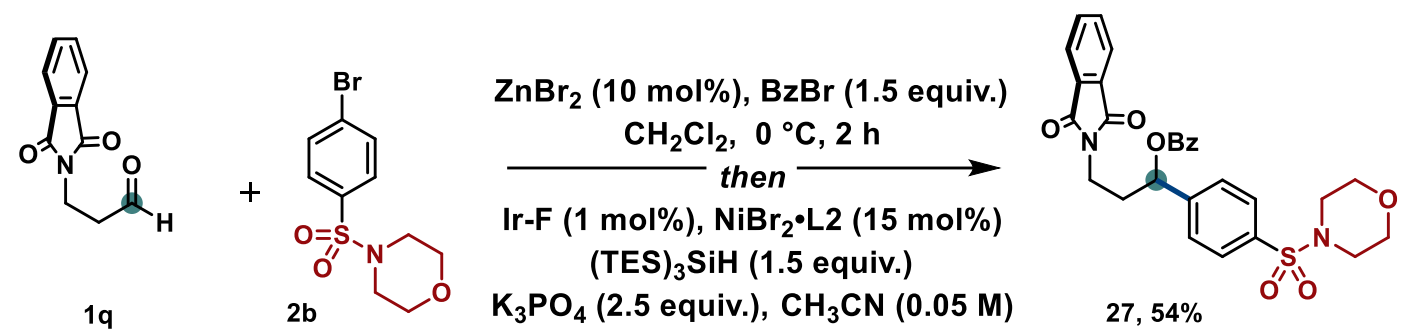

$30 \mathrm{~W} 450 \mathrm{~nm}$ LEDs, RT, $24 \mathrm{~h}$

\section{3-(1,3-Dioxoisoindolin-2-yl)-1-(4-(morpholinosulfonyl)phenyl)propyl}

benzoate (27). According to the GP3, using the aldehyde 1q ( $0.3 \mathrm{mmol}, 1.5$ equiv.), benzoyl bromide ( $0.3 \mathrm{mmol}, 1.5$ equiv.), aryl bromide $\mathbf{2 b}$ ( $0.2 \mathrm{mmol}, 1.0$ equiv.), Ir-F ( $2 \mu \mathrm{mol}, 1 \mathrm{~mol} \%)$, NiBr $2 \bullet \mathrm{L} 2$ (30 $\mu \mathrm{mol}, 15 \mathrm{~mol} \%$ ), (TES) ${ }_{3} \mathrm{SiH}$ (0.3 mmol, 1.5 equiv.), $\mathrm{K}_{3} \mathrm{PO}_{4}$ ( $0.5 \mathrm{mmol}, 2.5$ equiv.) and stirring at room temperature for 24 $\mathrm{h}$ under 30W blue LEDs irradiation. The reaction mixture was filtered through a pad of silica gel and concentrated in vacuo, then purified by chromatography (pentane/EtOAc $=50 / 50)$ to give $27(57.4 \mathrm{mg}, 0.107 \mathrm{mmol}, 54 \%)$ as a colorless oil. Rf (pentane/EtOAc $=50 / 50) 0.31 .{ }^{1} \mathbf{H}$ NMR (599 MHz, $\left.\mathrm{CDCl}_{3}\right): \delta$ ppm $8.07-$ $8.04(\mathrm{~m}, 2 \mathrm{H}), 7.75(\mathrm{dd}, J=5.4,3.0 \mathrm{~Hz}, 2 \mathrm{H}), 7.69-7.66(\mathrm{~m}, 4 \mathrm{H}), 7.60(\mathrm{~d}, J=8.4 \mathrm{~Hz}$, 2H), $7.57-7.53(\mathrm{~m}, 1 \mathrm{H}), 7.41(\mathrm{t}, J=7.8 \mathrm{~Hz}, 2 \mathrm{H}), 6.09$ (dd, $J=8.6,4.7 \mathrm{~Hz}, 1 \mathrm{H}), 3.90$ $(\mathrm{t}, J=6.8 \mathrm{~Hz}, 2 \mathrm{H}), 3.72-3.68(\mathrm{~m}, 4 \mathrm{H}), 2.95(\mathrm{t}, J=4.7 \mathrm{~Hz}, 4 \mathrm{H}), 2.56-2.48(\mathrm{~m}, 1 \mathrm{H})$, $2.36(\mathrm{ddd}, J=14.5,7.1,4.7 \mathrm{~Hz}, 1 \mathrm{H}) .{ }^{13} \mathrm{C}$ NMR (151 MHz, CDCl 3$): \delta$ ppm 168.1, 165.4, 145.3, 134.9, 134.1, 133.3, 131.8, 129.7, 129.5, 128.5, 128.2, 127.0, 123.2, 73.5, 66.0, 45.9, 34.7, 34.6. HRMS (ESI $\left.{ }^{+}\right)$: calcd. for $\mathrm{C}_{28} \mathrm{H}_{26} \mathrm{~N}_{2} \mathrm{O}_{7} \mathrm{SNa}\left(\mathrm{M}+\mathrm{Na}^{+}\right): 557.1353$. Found: 557.1355.

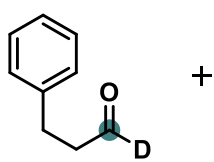

$1 \mathrm{r}$

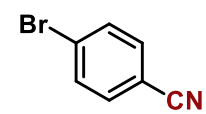

$2 a$
$\mathrm{ZnBr}_{2}$ (10 mol\%), BzBr (1.5 equiv.)

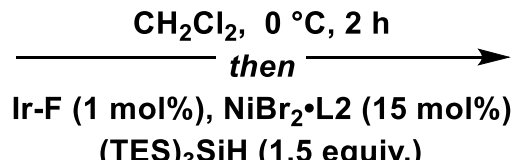

(TES) ${ }_{3} \mathrm{SiH}(1.5$ equiv.)

$\mathrm{K}_{3} \mathrm{PO}_{4}$ (2.5 equiv.), $\mathrm{CH}_{3} \mathrm{CN}(0.05 \mathrm{M})$ $30 \mathrm{~W} 450 \mathrm{~nm}$ LEDs, RT, $24 \mathrm{~h}$

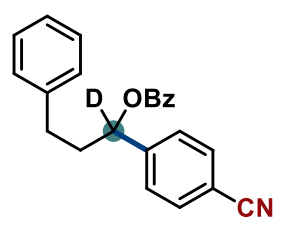

$28,72 \%$

1-(4-cyanophenyl)-3-phenylpropyl-1-d benzoate (28). According to the GP3, using the aldehyde $1 \mathbf{r}(0.3 \mathrm{mmol}, 1.5$ equiv.), benzoyl bromide ( $0.3 \mathrm{mmol}, 1.5$ equiv.), aryl bromide 2 a ( 0.2 mmol, 1.0 equiv.), Ir-F ( $2 \mu \mathrm{mol}, 1 \mathrm{~mol} \%), \mathrm{NiBr}_{2} \bullet \mathrm{L} 2$ (30 $\mu \mathrm{mol}, 15 \mathrm{~mol} \%$ ), (TES) ${ }_{3} \mathrm{SiH}\left(0.3 \mathrm{mmol}, 1.5\right.$ equiv.), $\mathrm{K}_{3} \mathrm{PO}_{4}(0.5 \mathrm{mmol}, 2.5$ equiv.) and stirring at room temperature for $24 \mathrm{~h}$ under $30 \mathrm{~W}$ blue LEDs irradiation. The reaction mixture was filtered through a pad of silica gel and concentrated in vacuo, 
then purified by chromatography (pentane/EtOAc $=93 / 7 \rightarrow 9 / 1)$ to give $28(49.1$ mg, $0.143 \mathrm{mmol}, 72 \%$ ) as a colorless oil. $\mathbf{R}_{\mathbf{f}}$ (pentane $/$ EtOAc $=9 / 1$ ) 0.60. ${ }^{1} \mathbf{H} \mathbf{~ N M R}$ $\left(400 \mathrm{MHz}^{\mathrm{CDCl}} 3\right) \delta 8.12-8.04(\mathrm{~m}, 2 \mathrm{H}), 7.66(\mathrm{~d}, J=8.4 \mathrm{~Hz}, 2 \mathrm{H}), 7.61(\mathrm{tt}, J=7.5,1.2$ Hz, 1H), $7.54-7.45$ (m, 4H), $7.33-7.25$ (m, 2H), $7.24-7.19$ (m, 1H), $7.19-7.12$ (m, 2H), $2.84-2.67(\mathrm{~m}, 2 \mathrm{H}), 2.41(\mathrm{ddd}, J=14.7,8.9,6.1 \mathrm{~Hz}, 1 \mathrm{H}), 2.28-2.18(\mathrm{~m}$, 1H). ${ }^{13}$ C NMR (101 MHz, CDCl 3 ) $\delta 165.7,146.0,140.6,133.5,132.6,129.9,129.8$, 128.7, 128.7, 128.4, 127.1, 126.4, 118.7, 112.0, 37.9, 31.8. HRMS (ESI ${ }^{+}$): calcd. for $\mathrm{C}_{23} \mathrm{H}_{28} \mathrm{NO}_{2} \mathrm{DNa}\left(\mathrm{M}+\mathrm{Na}^{+}\right)$: 365.1371. Found: 365.1366.

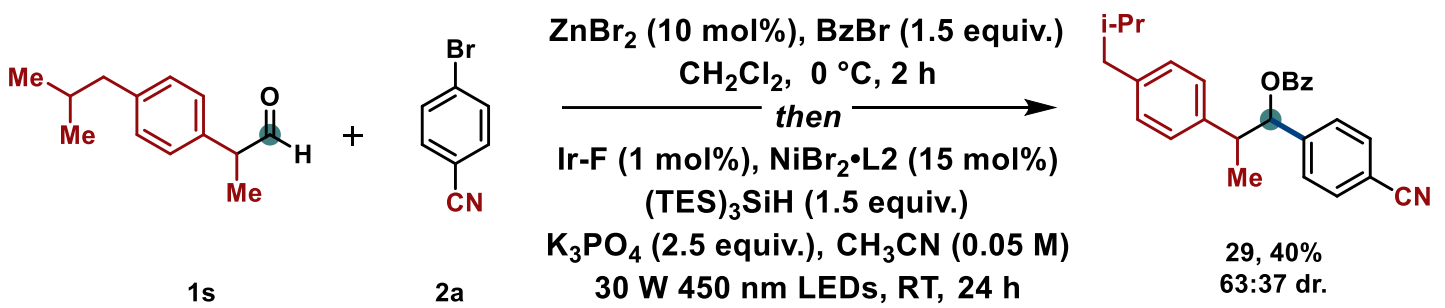

1-(4-cyanophenyl)-2-(4-isobutylphenyl)propyl benzoate (29). According to the GP3, using the aldehyde $1 \mathrm{~s}$ ( $0.3 \mathrm{mmol}, 1.5$ equiv.), benzoyl bromide $(0.3 \mathrm{mmol}$, 1.5 equiv.), aryl bromide $\mathbf{2 a}$ ( $0.2 \mathrm{mmol}, 1.0$ equiv.), $\mathrm{Ir}-\mathrm{F}(2 \mu \mathrm{mol}, 1 \mathrm{~mol} \%), \mathrm{NiBr}_{2} \bullet \mathrm{L} 2$ (30 $\mu \mathrm{mol}, 15 \mathrm{~mol} \%$ ), (TES) $33 \mathrm{SiH}\left(0.3 \mathrm{mmol}, 1.5\right.$ equiv.), $\mathrm{K}_{3} \mathrm{PO}_{4}(0.5 \mathrm{mmol}, 2.5$ equiv.) and stirring at room temperature for $24 \mathrm{~h}$ under $30 \mathrm{~W}$ blue LEDs irradiation. The reaction mixture was filtered through a pad of silica gel and concentrated in vacuo, then purified by chromatography (pentane/EtOAc $=30 / 1 \rightarrow 25 / 1 \rightarrow 20 / 1$ ) to give 29 (31.5 mg, $0.079 \mathrm{mmol}, 40 \%, 63: 37 \mathrm{dr}$.) as a colorless gum. Note. Due to overlap and limited resolution of 2D NMR methods, not all the signals could be individually assigned. $M=$ major isomer,$m=$ minor isomer. The major isomer in accordance with NMR analysis from Weissenfels and co-workers, ${ }^{35}$ can be tentatively assigned as the erythro compound. $\mathbf{R} \mathbf{f}$ (pentane/EtOAc $=40 / 1)$ 0.35. ${ }^{\mathbf{1}} \mathbf{H} \mathbf{~ N M R}\left(500 \mathrm{MHz}, \mathrm{CDCl}_{3}\right) \delta$ $8.12-8.08(\mathrm{~m}, 2 \mathrm{H}, M), 7.95-7.92(\mathrm{~m}, 2 \mathrm{H}, m), 7.61(\mathrm{tt}, J=7.5,1.3 \mathrm{~Hz}, 2 \mathrm{H}, m), 7.58$ - $7.53(\mathrm{~m}, 2 \mathrm{H}, M), 7.51-7.48(\mathrm{~m}, 2 \mathrm{H}, m), 7.46(\mathrm{~d}, J=8.6 \mathrm{~Hz}, 2 \mathrm{H}, M), 7.41(\mathrm{t}, J=8.0$ $\mathrm{Hz}, 2 \mathrm{H}, m), 7.33-7.29(\mathrm{~m}, 2 \mathrm{H}, m), 7.22-7.17(\mathrm{~m}, 2 \mathrm{H}, M), 7.08(\mathrm{~d}, J=8.2 \mathrm{~Hz}, 2 \mathrm{H}$, $m), 7.05(\mathrm{~d}, J=8.2 \mathrm{~Hz}, 2 \mathrm{H}, m), 6.98(\mathrm{~d}, J=8.1 \mathrm{~Hz}, 2 \mathrm{H}, M), 6.93(\mathrm{~d}, J=8.1 \mathrm{~Hz}, 2 \mathrm{H}, M)$, $6.07(\mathrm{~d}, J=7.0 \mathrm{~Hz}, 1 \mathrm{H}), 6.00(\mathrm{~d}, J=8.0 \mathrm{~Hz}, 1 \mathrm{H}), 3.36(\mathrm{p}, J=7.1 \mathrm{~Hz}, 1 \mathrm{H}, m), 3.30$ (p, $J$ $=7.5 \mathrm{~Hz}, 1 \mathrm{H}, M), 2.43(\mathrm{~d}, J=7.2 \mathrm{~Hz}, 1 \mathrm{H}, m), 2.41(\mathrm{~d}, J=7.3 \mathrm{~Hz}, 2 \mathrm{H}, M), 1.81$ (apt dhept $J=13.6,6.8 \mathrm{~Hz}, 1 \mathrm{H}, M+m), 1.47(\mathrm{~d}, J=7.0 \mathrm{~Hz}, 2 \mathrm{H}, M), 1.29(\mathrm{~d}, J=7.1 \mathrm{~Hz}, 2 \mathrm{H}$, $m$ ), $0.86(\mathrm{~d}, J=6.6 \mathrm{~Hz}, 6 \mathrm{H}, M), 0.85$ (apt dd, $J=6.6,3.1 \mathrm{~Hz}, 6 \mathrm{H}, m) .{ }^{13} \mathbf{C}$ NMR $(126$ 
$\left.\mathrm{MHz}, \mathrm{CDCl}_{3}\right) \delta 165.7(M), 165.6(\mathrm{~m}), 145.0(M), 144.7(\mathrm{~m}), 140.7(M), 140.6(\mathrm{~m})$, $138.5(\mathrm{~m}), 138.4(M), 133.5(M), 133.3(\mathrm{~m}), 132.1(\mathrm{~m}), 131.8(M), 129.99(M)$, $129.97(\mathrm{~m}), 129.8(M), 129.7(\mathrm{~m}), 129.21(M), 129.16(\mathrm{~m}), 128.7(M), 128.6(\mathrm{~m})$, $127.94(M), 127.92(m), 127.85(m), 127.7(M), 118.82(M), 118.80(m), 111.8(m)$, $111.6(M), 80.3(M), 80.0(m), 45.7(M), 45.09(m), 45.06(M+m), 30.30(m), 30.28$ $(M), 22.42,22.39,22.37,17.4(M), 17.2(m)$. HRMS (ESI+): calcd. for $\mathrm{C}_{27} \mathrm{H}_{27} \mathrm{NO}_{2} \mathrm{Na}$ $\left(\mathrm{M}+\mathrm{Na}^{+}\right): 420.1934$. Found: 420.1933.
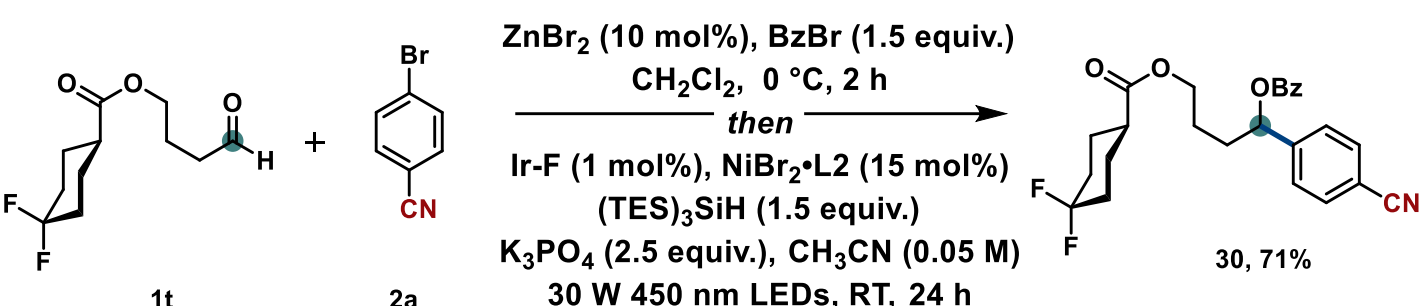

\section{1-(4-cyanophenyl)-4-((4,4-difluorocyclohexane-1-carbonyl)oxy)butyl}

benzoate (30). According to the GP3, using the aldehyde 1t ( $0.3 \mathrm{mmol}, 1.5$ equiv.), benzoyl bromide ( $0.3 \mathrm{mmol}, 1.5$ equiv.), aryl bromide 2 a ( $0.2 \mathrm{mmol}, 1.0$ equiv.), Ir-

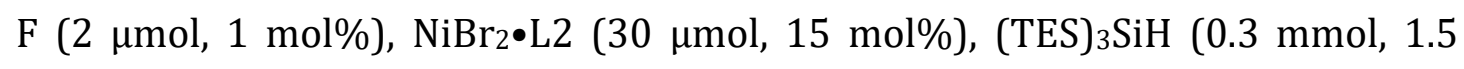
equiv.), $\mathrm{K}_{3} \mathrm{PO}_{4}$ (0.5 mmol, 2.5 equiv.) and stirring at room temperature for $24 \mathrm{~h}$ under $30 \mathrm{~W}$ blue LEDs irradiation. The reaction mixture was filtered through a pad of silica gel and concentrated in vacuo, then purified by chromatography (pentane/EtOAc = 3/1) to give $30(62.7 \mathrm{mg}, 0.142 \mathrm{mmol}, 71 \%)$ as a colorless gum. $\mathbf{R f}_{\mathbf{f}}$ (pentane/EtOAc = 3/1) 0.50. ${ }^{1} \mathbf{H}$ NMR (400 MHz, $\left.\mathrm{CDCl}_{3}\right) \delta 8.03-7.95(\mathrm{~m}, 2 \mathrm{H})$, $7.59(\mathrm{~d}, J=8.4 \mathrm{~Hz}, 2 \mathrm{H}), 7.54-7.49(\mathrm{~m}, 1 \mathrm{H}), 7.44(\mathrm{~d}, J=8.3 \mathrm{~Hz}, 2 \mathrm{H}), 7.40$ (t, $J=7.9$ $\mathrm{Hz}, 2 \mathrm{H}), 5.95(\mathrm{dd}, J=8.0,5.3 \mathrm{~Hz}, 1 \mathrm{H}), 4.10-4.02(\mathrm{~m}, 2 \mathrm{H}), 2.35-2.26(\mathrm{~m}, 1 \mathrm{H}), 2.06$ - $1.83(\mathrm{~m}, 6 \mathrm{H}), 1.79$ - $1.59(\mathrm{~m}, 6 \mathrm{H}) .{ }^{13} \mathrm{C} \mathrm{NMR}\left(101 \mathrm{MHz}, \mathrm{CDCl}_{3}\right) \delta$ 174.2, 165.7, 145.7, 133.6, 132.6, 129.7, 129.7, 128.7, 127.0, 122.7 (t, J = 241.2 Hz), 118.6, 112.1, 75.2, 63.9, $32.8(\mathrm{~d}, J=42.1 \mathrm{~Hz}), 32.6,25.2(\mathrm{~d}, J=10.2 \mathrm{~Hz}), 24.8 .{ }^{19}$ F NMR (377 MHz, $\mathrm{CDCl}_{3}$ ) $\delta-94.12$ (br d, $J=236.4 \mathrm{~Hz}$ ), -99.81 (br d, $J=237.7 \mathrm{~Hz}$ ). HRMS (ESI ${ }^{+}$): calcd. for $\mathrm{C}_{32} \mathrm{H}_{41} \mathrm{NO}_{2} \mathrm{Na}\left(\mathrm{M}+\mathrm{Na}^{+}\right)$: 494.3030. Found: 494.3027 . 


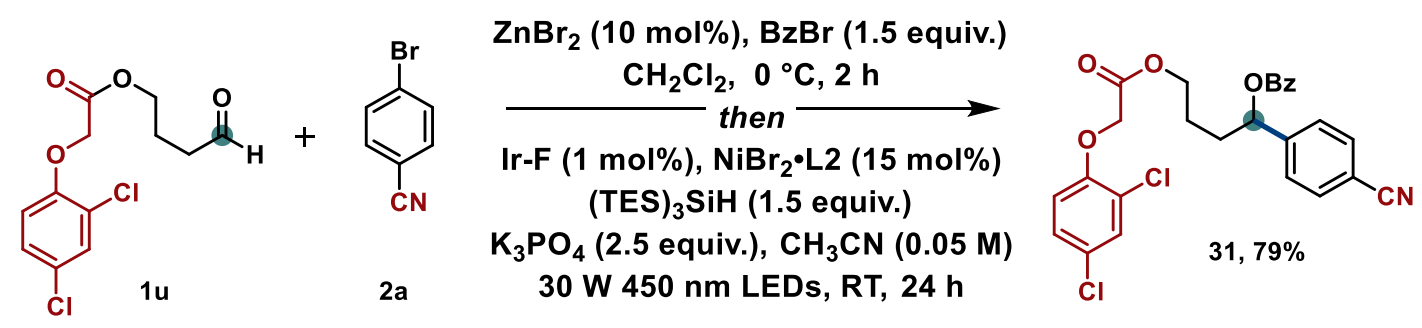

1-(4-cyanophenyl)-4-(2-(2,4-dichlorophenoxy)acetoxy)butyl benzoate

(31). According to the GP3, using the aldehyde $\mathbf{1 u}$ ( $0.3 \mathrm{mmol}, 1.5$ equiv.), benzoyl bromide ( $0.3 \mathrm{mmol}, 1.5$ equiv.), aryl bromide 2a ( $0.2 \mathrm{mmol}, 1.0$ equiv.), Ir-F (2

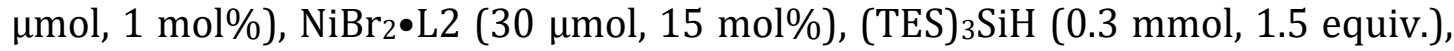
$\mathrm{K}_{3} \mathrm{PO}_{4}$ ( $0.5 \mathrm{mmol}, 2.5$ equiv.) and stirring at room temperature for $24 \mathrm{~h}$ under $30 \mathrm{~W}$ blue LEDs irradiation. The reaction mixture was filtered through a pad of silica gel and concentrated in vacuo, then purified by chromatography $($ pentane $/$ EtOAc $=$ 4/1) to give 31 (78.6 mg, $0.158 \mathrm{mmol}, 79 \%$ ) as a colorless gum. $\mathbf{R}_{\mathbf{f}}$ (pentane/EtOAc $=19 / 5)$ 0.50. ${ }^{1} \mathbf{H}$ NMR $\left(400 \mathrm{MHz}, \mathrm{CDCl}_{3}\right) \delta 8.11-8.01(\mathrm{~m}, 2 \mathrm{H}), 7.66(\mathrm{~d}, J=8.4 \mathrm{~Hz}$, 2H), $7.60(\mathrm{tt}, J=7.5,1.3 \mathrm{~Hz}, 1 \mathrm{H}), 7.50-7.42(\mathrm{~m}, 4 \mathrm{H}), 7.35(\mathrm{~d}, J=2.5 \mathrm{~Hz}, 1 \mathrm{H}), 7.12$ (dd, $J=8.8,2.6 \mathrm{~Hz}, 1 \mathrm{H}), 6.75(\mathrm{~d}, J=8.8 \mathrm{~Hz}, 1 \mathrm{H}), 5.99$ (dd, $J=8.3,5.1 \mathrm{~Hz}, 1 \mathrm{H}), 4.68$ $(\mathrm{s}, 2 \mathrm{H}), 4.24(\mathrm{dp}, J=10.6,4.9 \mathrm{~Hz}, 2 \mathrm{H}), 2.11-1.99(\mathrm{~m}, 1 \mathrm{H}), 1.98-1.86(\mathrm{~m}, 1 \mathrm{H}), 1.86$ - 1.69 (m, 2H). ${ }^{13} \mathrm{C}$ NMR (101 MHz, $\left.\mathrm{CDCl}_{3}\right) \delta$ 168.2, 165.7, 152.4, 145.7, 133.6, 132.6, 130.4, 129.7, 129.6, 128.7, 127.6, 127.2, 127.0, 124.3, 118.6, 114.6, 112.1, 75.1, 66.4, 64.8, 33.0, 24.7. HRMS (ESI $\left.{ }^{+}\right)$: calcd. for $\mathrm{C}_{26} \mathrm{H}_{21} \mathrm{NO}_{5}{ }^{35} \mathrm{Cl}_{2} \mathrm{Na}\left(\mathrm{M}+\mathrm{Na}^{+}\right)$: 520.0689. Found: 520.0689.

\subsection{Bromides Scope}

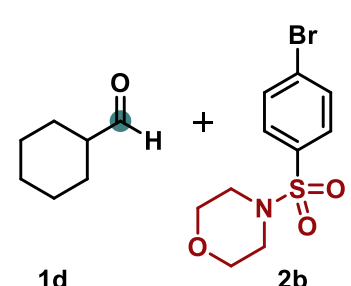

1d

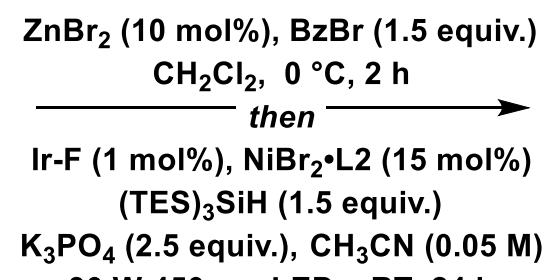

$30 \mathrm{~W} 450 \mathrm{~nm}$ LEDs, RT, $24 \mathrm{~h}$

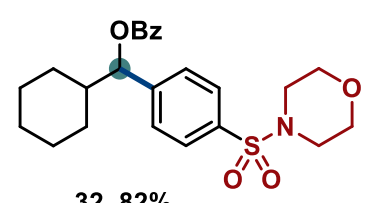

$32,82 \%$

Cyclohexyl (4-(morpholinosulfonyl)phenyl)methyl benzoate (32). According to the GP3, using the aldehyde $1 \mathbf{d}$ ( $0.3 \mathrm{mmol}, 1.5$ equiv.), benzoyl bromide $(0.3$ mmol, 1.5 equiv.), aryl bromide $2 \mathbf{b}$ ( 0.2 mmol, 1.0 equiv.), Ir-F ( $2 \mu \mathrm{mol}, 1$ mol\%), $\mathrm{NiBr}_{2} \bullet \mathrm{L} 2$ (30 $\left.\mu \mathrm{mol}, 15 \mathrm{~mol} \%\right),(\mathrm{TES})_{3} \mathrm{SiH}$ (0.3 mmol, 1.5 equiv.), $\mathrm{K}_{3} \mathrm{PO}_{4}(0.5 \mathrm{mmol}$, 2.5 equiv.) and stirring at room temperature for $24 \mathrm{~h}$ under $30 \mathrm{~W}$ blue LEDs 
irradiation. The reaction mixture was filtered through a pad of silica gel and concentrated in vacuo, then purified by chromatography (pentane/EtOAc = $80 / 20$ ) to give $32(72.9 \mathrm{mg}, 0.165 \mathrm{mmol}, 82 \%)$ as a colorless oil. $\mathbf{R f}_{\mathbf{f}}$ (pentane/EtOAc $=80 / 20)$ 0.21. ${ }^{1} \mathbf{H}$ NMR $\left(599 \mathrm{MHz}, \mathrm{CDCl}_{3}\right): \delta \mathrm{ppm} 8.09(\mathrm{~d}, J=7.7$ $\mathrm{Hz}, 2 \mathrm{H}), 7.72(\mathrm{~d}, J=8.0 \mathrm{~Hz}, 2 \mathrm{H}), 7.59$ (t, $J=7.5 \mathrm{~Hz}, 1 \mathrm{H}), 7.54(\mathrm{~d}, J=8.0 \mathrm{~Hz}, 2 \mathrm{H}), 7.47$ $(\mathrm{t}, J=7.6 \mathrm{~Hz}, 2 \mathrm{H}), 5.80(\mathrm{~d}, J=7.0 \mathrm{~Hz}, 1 \mathrm{H}), 3.72(\mathrm{t}, J=4.7 \mathrm{~Hz}, 4 \mathrm{H}), 3.00(\mathrm{t}, J=4.6 \mathrm{~Hz}$, 4H), $1.91(\mathrm{~d}, J=11.1 \mathrm{~Hz}, 2 \mathrm{H}), 1.78(\mathrm{~d}, J=12.7 \mathrm{~Hz}, 1 \mathrm{H}), 1.73(\mathrm{~d}, J=12.3 \mathrm{~Hz}, 1 \mathrm{H}), 1.67$ (d, $J=12.0 \mathrm{~Hz}, 1 \mathrm{H}), 1.49$ (d, $J=12.9 \mathrm{~Hz}, 1 \mathrm{H}), 1.25-1.11(\mathrm{~m}, 5 \mathrm{H}) .{ }^{13} \mathrm{C}$ NMR (151 $\mathrm{MHz}_{\mathrm{CDCl}}$ ): $\delta$ ppm 165.8, 145.5, 134.6, 133.4, 130.1, 129.8, 128.6, 128.0, 127.8, 79.9, 66.2, 46.1, 43.4, 29.2, 28.7, 26.3, 26.0, 25.9. HRMS (ESI+): calcd. for $\mathrm{C}_{24} \mathrm{H}_{29} \mathrm{NO}_{5} \mathrm{SNa}\left(\mathrm{M}+\mathrm{Na}^{+}\right): 466.1659$. Found: 466.1657.

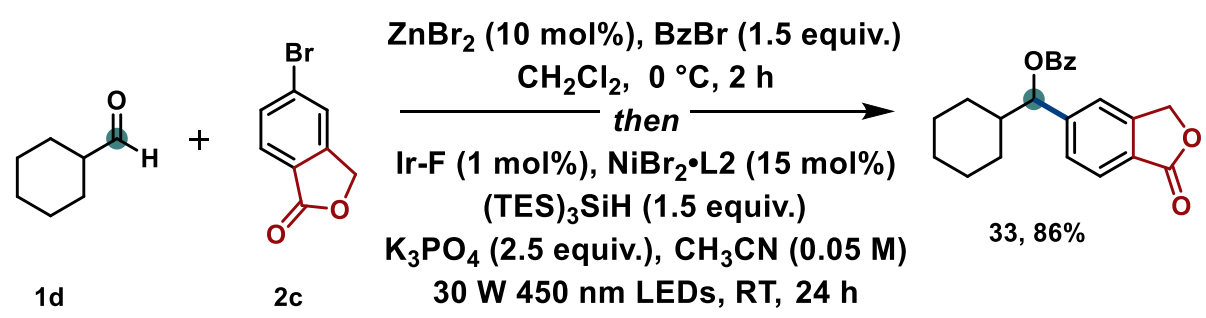

Cyclohexyl(1-oxo-1,3-dihydroisobenzofuran-5-yl)methyl benzoate (33). According to the GP3, using the aldehyde $\mathbf{1 d}(0.3 \mathrm{mmol}, 1.5$ equiv.), benzoyl bromide ( 0.3 mmol, 1.5 equiv.), aryl bromide 2 c ( 0.2 mmol, 1.0 equiv.), Ir-F (2

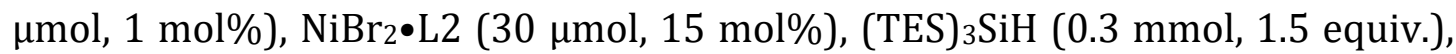
$\mathrm{K}_{3} \mathrm{PO}_{4}$ ( $0.5 \mathrm{mmol}, 2.5$ equiv.) and stirring at room temperature for $24 \mathrm{~h}$ under $30 \mathrm{~W}$ blue LEDs irradiation. The reaction mixture was filtered through a pad of silica gel and concentrated in vacuo, then purified by chromatography $($ pentane $/$ EtOAc $=$ $80 / 20)$ to give $33(60.4 \mathrm{mg}, 0.173 \mathrm{mmol}, 86 \%)$ as a colorless oil. $\mathbf{R}_{\mathbf{f}}$ (pentane/EtOAc $=80 / 20)$ 0.29. ${ }^{1} \mathbf{H}$ NMR $\left(599 \mathrm{MHz}, \mathrm{CDCl}_{3}\right): \delta \mathrm{ppm} 8.12-8.06(\mathrm{~m}$, 2H), $7.88(\mathrm{dd}, J=7.9,2.0 \mathrm{~Hz}, 1 \mathrm{H}), 7.58(\mathrm{t}, J=7.4 \mathrm{~Hz}, 1 \mathrm{H}), 7.53(\mathrm{~d}, J=8.0 \mathrm{~Hz}, 1 \mathrm{H})$, $7.50-7.42(\mathrm{~m}, 3 \mathrm{H}), 5.80(\mathrm{dd}, J=7.0,2.0 \mathrm{~Hz}, 1 \mathrm{H}), 5.34-5.24(\mathrm{~m}, 2 \mathrm{H}), 1.99-1.90$ $(\mathrm{m}, 2 \mathrm{H}), 1.78(\mathrm{~d}, J=12.9 \mathrm{~Hz}, 1 \mathrm{H}), 1.72(\mathrm{~d}, J=12.3 \mathrm{~Hz}, 1 \mathrm{H}), 1.67(\mathrm{~d}, J=12.1 \mathrm{~Hz}, 1 \mathrm{H})$, $1.50(\mathrm{~d}, J=12.8 \mathrm{~Hz}, 1 \mathrm{H}), 1.30-1.11(\mathrm{~m}, 4 \mathrm{H}), 1.11-1.04(\mathrm{~m}, 1 \mathrm{H}) .{ }^{13} \mathbf{C}$ NMR (151 $\mathrm{MHz}, \mathrm{CDCl}_{3}$ ): $\delta$ ppm 170.8, 165.9, 146.9, 146.9, 133.4, 130.1, 129.7, 128.6, 128.0, 125.8, 125.4, 120.6, 80.4, 69.7, 43.5, 29.3, 28.8, 26.3, 25.9, 25.9. HRMS (ESI+): calcd. for $\mathrm{C}_{22} \mathrm{H}_{22} \mathrm{O}_{4} \mathrm{Na}\left(\mathrm{M}+\mathrm{Na}^{+}\right): 373.1410$. Found: 373.1408 . 


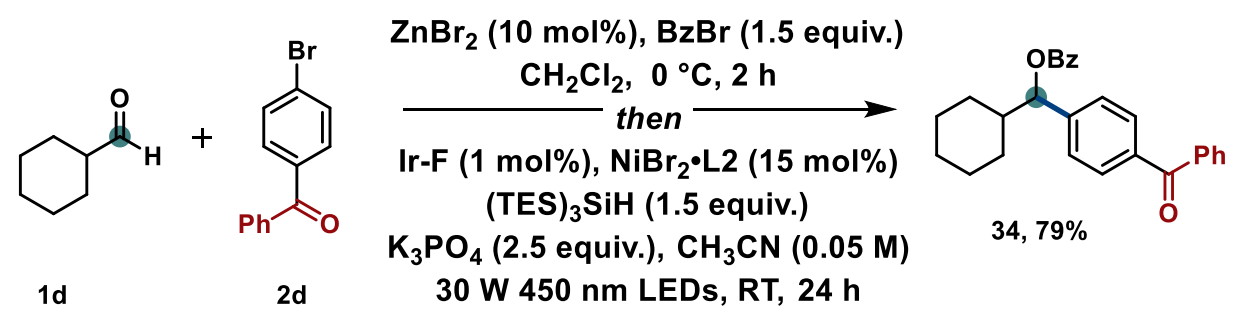

(4-Benzoylphenyl)(cyclohexyl)methyl benzoate (34). According to the GP3, using the aldehyde $\mathbf{1 d}$ ( $0.3 \mathrm{mmol}, 1.5$ equiv.), benzoyl bromide ( $0.3 \mathrm{mmol}, 1.5$ equiv.), aryl bromide $\mathbf{2 d}$ ( $0.2 \mathrm{mmol}, 1.0$ equiv.), Ir-F ( $2 \mu \mathrm{mol}, 1 \mathrm{~mol} \%)$, NiBr2 $\bullet 2$ (30 $\mu \mathrm{mol}, 15 \mathrm{~mol} \%$ ), (TES) ${ }_{3} \mathrm{SiH}\left(0.3 \mathrm{mmol}, 1.5\right.$ equiv.), $\mathrm{K}_{3} \mathrm{PO}_{4}(0.5 \mathrm{mmol}, 2.5$ equiv.) and stirring at room temperature for $24 \mathrm{~h}$ under $30 \mathrm{~W}$ blue LEDs irradiation. The reaction mixture was filtered through a pad of silica gel and concentrated in vacuo, then purified by chromatography (pentane $/$ EtOAc $=95 / 5)$ to give $34(63.0 \mathrm{mg}$, $0.158 \mathrm{mmol}, 79 \%)$ as a colorless oil. $\mathbf{R}_{\mathbf{f}}($ pentane $/$ EtOAc $=90 / 10)$ 0.48. ${ }^{\mathbf{1}} \mathbf{H}$ NMR (599 MHz, CDCl 3 ): $\delta$ ppm $8.18-8.08(\mathrm{~m}, 2 \mathrm{H}), 7.83-7.75(\mathrm{~m}, 4 \mathrm{H}), 7.57(\mathrm{~d}, J=7.7$ Hz, 2H), $7.51-7.43(\mathrm{~m}, 6 \mathrm{H}), 5.81$ (d, $J=7.1 \mathrm{~Hz}, 1 \mathrm{H}), 1.96$ (d, J = $12.0 \mathrm{~Hz}, 2 \mathrm{H}), 1.82$ - $1.76(\mathrm{~m}, 1 \mathrm{H}), 1.73$ (d, $J=12.4 \mathrm{~Hz}, 1 \mathrm{H}), 1.67$ (s, 1H), 1.55 (d, $J=13.0 \mathrm{~Hz}, 1 \mathrm{H}), 1.31$ - $1.13(\mathrm{~m}, 4 \mathrm{H}), 1.08$ (d, $J=12.1 \mathrm{~Hz}, 1 \mathrm{H}) .{ }^{13} \mathrm{C}$ NMR (151 MHz, $\left.\mathrm{CDCl}_{3}\right): \delta$ ppm 196.3, 165.8, 144.4, 137.6, 137.0, 133.1, 132.4, 130.3, 130.1, 130.0, 129.6, 128.5, 128.3, 126.8, 80.3, 43.3, 29.2, 28.7, 26.2, 25.9, 25.8. HRMS (ESI+): calcd. for $\mathrm{C}_{27} \mathrm{H}_{26} \mathrm{O}_{3} \mathrm{Na}$ $\left(\mathrm{M}+\mathrm{Na}^{+}\right):$421.1774. Found: 421.1771.

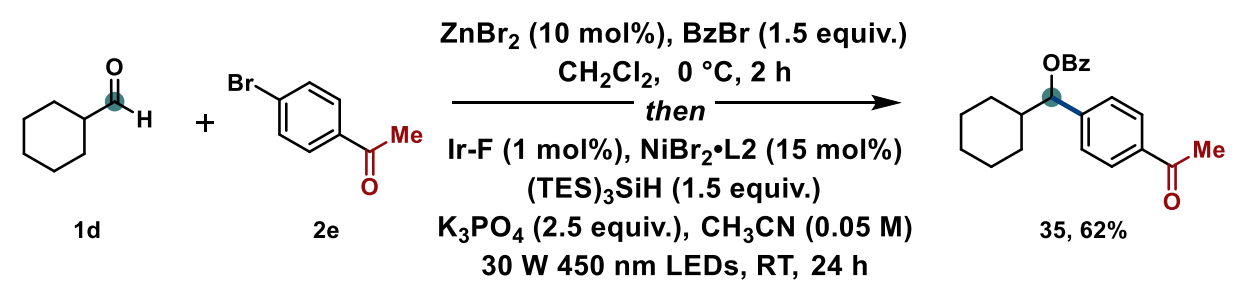

(4-acetylphenyl)(cyclohexyl)methyl benzoate (35). According to GP3, using the cyclohexanecarbaldehyde $1 \mathbf{d}(0.3 \mathrm{mmol}, 1.5$ equiv.), benzoyl bromide $[0.3$ mmol, 1.5 equiv.), 1-(4-bromophenyl)ethan-1-one 2 e ( $0.2 \mathrm{mmol}, 1.0$ equiv.), Ir-F ( $2 \mu \mathrm{mol}, 1 \mathrm{~mol} \%$ ), NiBr $2 \bullet \mathrm{L} 2$ (30 $\mu \mathrm{mol}, 15 \mathrm{~mol} \%)$, (TES) 3 SiH (0.3 mmol, 1.5 equiv.), $\mathrm{K}_{3} \mathrm{PO}_{4}$ ( $0.5 \mathrm{mmol}, 2.5$ equiv.) and stirring at room temperature for $24 \mathrm{~h}$ under $30 \mathrm{~W}$ blue LEDs irradiation. The reaction mixture was filtered through a pad of silica gel and concentrated in vacuo, then purified by chromatography $($ pentane $/$ EtOAc $=$ $93 / 7 \rightarrow 90 / 10)$ to give 35 (41.5 mg, $0.123 \mathrm{mmol}, 62 \%$ ) as a colorless solid. $\mathbf{R}_{\mathbf{f}}$ 
(pentane $/$ EtOAc $=92 / 8) 0.20 .{ }^{1} \mathbf{H}$ NMR $\left(400 \mathrm{MHz}^{\mathrm{CDCl}} 3\right) \delta 8.13-8.05(\mathrm{~m}, 2 \mathrm{H})$, $7.97-7.89$ (m, 2H), $7.62-7.53(\mathrm{~m}, 1 \mathrm{H}), 7.51-7.42(\mathrm{~m}, 4 \mathrm{H}), 5.76$ (d, J = $7.1 \mathrm{~Hz}$, $1 \mathrm{H}), 2.58(\mathrm{~s}, 3 \mathrm{H}), 1.92(\mathrm{tq}, J=10.6,3.6 \mathrm{~Hz}, 2 \mathrm{H}), 1.81-1.62(\mathrm{~m}, 3 \mathrm{H}), 1.51(\mathrm{~d}, J=12.7$ $\mathrm{Hz}, 1 \mathrm{H}), 1.30-0.99$ (m, 5H). ${ }^{13} \mathrm{C}$ NMR (101 MHz, CDCl 3 ) $\delta$ 198.0, 166.0, 145.2, 136.8, 133.3, 130.4, 129.8, 128.6, 128.5, 127.3, 80.4, 43.4, 29.3, 28.9, 26.8, 26.4, 26.0, 26.0. HRMS (ESI+): calcd. for $\mathrm{C}_{22} \mathrm{H}_{24} \mathrm{O}_{3} \mathrm{Na}\left(\mathrm{M}+\mathrm{Na}^{+}\right)$: 359.1623. Found: 359.1616.

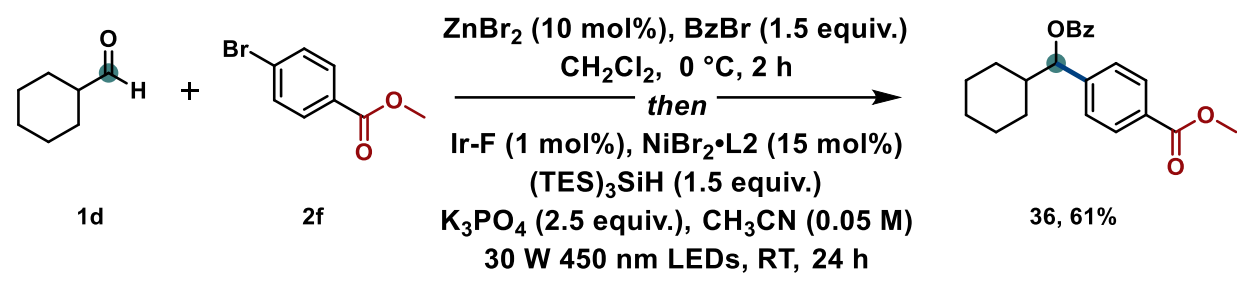

methyl 4-((benzoyloxy)(cyclohexyl)methyl)benzoate (36). According to GP3, using the cyclohexanecarbaldehyde $\mathbf{1 d}(0.3 \mathrm{mmol}, 1.5$ equiv.), benzoyl bromide ( $0.3 \mathrm{mmol}, 1.5$ equiv.), methyl 4-bromobenzoate $2 \mathrm{f}$ ( $0.2 \mathrm{mmol}, 1.0$ equiv.), Ir-F (2

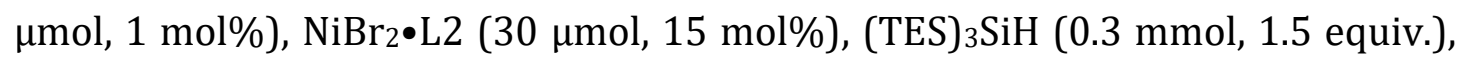
$\mathrm{K}_{3} \mathrm{PO}_{4}$ (0.5 mmol, 2.5 equiv.) and stirring at room temperature for $24 \mathrm{~h}$ under $30 \mathrm{~W}$ blue LEDs irradiation. The reaction mixture was filtered through a pad of silica gel and concentrated in vacuo, then purified by chromatography $($ pentane $/$ EtOAc $=$ $94 / 6)$ to give $36(42.8 \mathrm{mg}, 0.121 \mathrm{mmol}, 61 \%)$ as a colorless solid. $\mathbf{R}_{\mathbf{f}}$ $($ pentane $/$ EtOAc $=94 / 6)$ 0.20. ${ }^{1} \mathbf{H}$ NMR $\left(400 \mathrm{MHz}^{\mathrm{CDCl}} 3\right) \delta 8.12-8.06(\mathrm{~m}, 2 \mathrm{H})$, $8.01(\mathrm{~d}, J=8.1 \mathrm{~Hz}, 2 \mathrm{H}), 7.62-7.53(\mathrm{~m}, 1 \mathrm{H}), 7.52-7.40(\mathrm{~m}, 4 \mathrm{H}), 5.78(\mathrm{~d}, J=7.1 \mathrm{~Hz}$, $1 \mathrm{H}), 3.90(\mathrm{~d}, J=1.1 \mathrm{~Hz}, 3 \mathrm{H}), 1.92(\mathrm{qt}, J=7.6,3.5 \mathrm{~Hz}, 2 \mathrm{H}), 1.81-1.61(\mathrm{~m}, 2 \mathrm{H}), 1.51$ $(\mathrm{d}, J=12.4 \mathrm{~Hz}, 1 \mathrm{H}), 1.31-0.92(\mathrm{~m}, 6 \mathrm{H}) .{ }^{13} \mathrm{C}$ NMR (101 MHz, CDCl 3$) \delta 166.9,165.9$, 145.0, 133.2, 130.4, 129.8, 129.7, 129.7, 128.6, 127.0, 80.4, 52.2, 43.4, 29.2, 28.8, 26.3, 26.0, 26.0. HRMS (ESI+): calcd. for $\mathrm{C}_{22} \mathrm{H}_{24} \mathrm{O}_{4} \mathrm{Na}\left(\mathrm{M}+\mathrm{Na}^{+}\right)$: 375.1572. Found: 375.1567. 


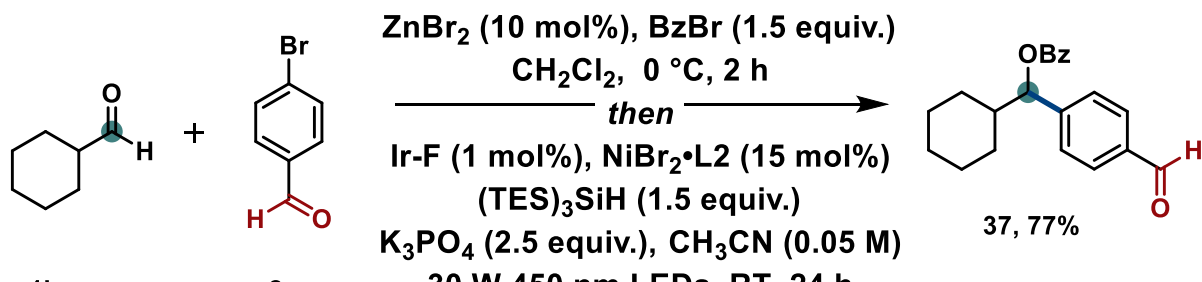

$1 b$

$2 \mathrm{~g}$

$30 \mathrm{~W} 450 \mathrm{~nm}$ LEDs, RT, $24 \mathrm{~h}$

Cyclohexyl(4-formylphenyl)methyl benzoate (37). According to the GP3, using the aldehyde $\mathbf{1 d}$ ( $0.3 \mathrm{mmol}, 1.5$ equiv.), benzoyl bromide ( $0.3 \mathrm{mmol}, 1.5$ equiv.), aryl bromide $2 \mathrm{~g}$ ( $0.2 \mathrm{mmol}, 1.0$ equiv.), Ir-F ( $2 \mu \mathrm{mol}, 1 \mathrm{~mol} \%)$, NiBr2 $\bullet 2$ ( $30 \mu \mathrm{mol}, 15 \mathrm{~mol} \%$ ), (TES) ${ }_{3} \mathrm{SiH}\left(0.3 \mathrm{mmol}, 1.5\right.$ equiv.), $\mathrm{K}_{3} \mathrm{PO}_{4}(0.5 \mathrm{mmol}, 2.5$ equiv.) and stirring at room temperature for $24 \mathrm{~h}$ under $30 \mathrm{~W}$ blue LEDs irradiation. The reaction mixture was filtered through a pad of silica gel and concentrated in vacuo, then purified by chromatography (pentane $/$ EtOAc $=95 / 5)$ to give $37(49.3 \mathrm{mg}$, $0.153 \mathrm{mmol}, 77 \%$ ) as a colorless oil. $\mathbf{R}_{\mathbf{f}}($ pentane $/$ EtOAc $=90 / 10) 0.41 .{ }^{1} \mathbf{H}$ NMR (599 MHz, CDCl 3 ): $\delta$ ppm $9.99(\mathrm{~s}, 1 \mathrm{H}), 8.16-8.07$ (m, 2H), $7.86(\mathrm{dt}, J=8.3,1.9 \mathrm{~Hz}$, $2 \mathrm{H}), 7.58(\mathrm{t}, J=7.6 \mathrm{~Hz}, 1 \mathrm{H}), 7.53(\mathrm{dd}, J=7.7,2.6 \mathrm{~Hz}, 2 \mathrm{H}), 7.47(\mathrm{td}, J=7.6,2.6 \mathrm{~Hz}$, 2H), $5.79(\mathrm{dd}, J=7.1,2.8 \mathrm{~Hz}, 1 \mathrm{H}), 1.93(\mathrm{~d}, J=11.2 \mathrm{~Hz}, 2 \mathrm{H}), 1.82-1.76(\mathrm{~m}, 1 \mathrm{H}), 1.76$ - $1.70(\mathrm{~m}, 1 \mathrm{H}), 1.70-1.64(\mathrm{~m}, 1 \mathrm{H}), 1.52$ (d, J = $12.9 \mathrm{~Hz}, 1 \mathrm{H}), 1.32-1.12(\mathrm{~m}, 4 \mathrm{H})$, 1.07 (dd, $J=12.2,3.2 \mathrm{~Hz}, 1 \mathrm{H}) .{ }^{13} \mathbf{C}$ NMR (151 MHz, CDCl 3 ): $\delta$ ppm 191.8, 165.7, 146.7, 135.9, 133.2, 130.1, 129.7, 129.6, 128.5, 127.5, 80.2, 43.3, 29.1, 28.7, 26.2, 25.9, 25.8. HRMS (ESI+): calcd. for $\mathrm{C}_{21} \mathrm{H}_{22} \mathrm{O}_{3} \mathrm{Na}\left(\mathrm{M}+\mathrm{Na}^{+}\right)$: 345.1461. Found: 345.1463.

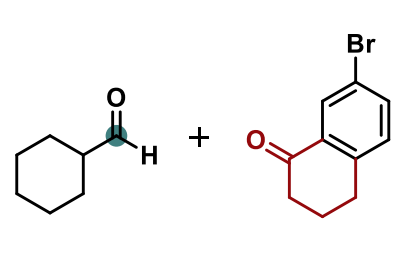

$1 d$
$\mathrm{ZnBr}_{2}$ (10 mol\%), BzBr (1.5 equiv.)

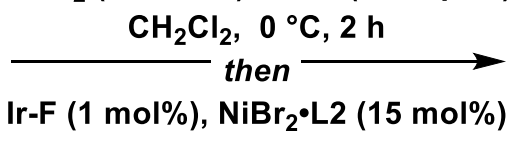

(TES) ${ }_{3} \mathrm{SiH}(1.5$ equiv.)

$\mathrm{K}_{3} \mathrm{PO}_{4}$ (2.5 equiv.), $\mathrm{CH}_{3} \mathrm{CN}(0.05 \mathrm{M})$

$30 \mathrm{~W} 450 \mathrm{~nm}$ LEDs, RT, $24 \mathrm{~h}$

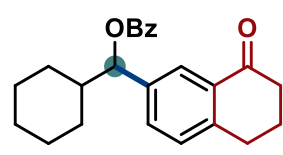

$38,30 \%$

Cyclohexyl(8-oxo-5,6,7,8-tetrahydronaphthalen-2-yl)methyl benzoate (38).

According to the GP3, using the aldehyde 1d ( $0.3 \mathrm{mmol}, 1.5$ equiv.), benzoyl bromide ( $0.3 \mathrm{mmol}, 1.5$ equiv.), aryl bromide $\mathbf{2 h}$ ( $0.2 \mathrm{mmol}, 1.0$ equiv.), Ir-F (2 $\mu \mathrm{mol}, 1 \mathrm{~mol} \%$ ), NiBr $2 \bullet \mathrm{L} 2$ (30 $\mu \mathrm{mol}, 15 \mathrm{~mol} \%$ ), (TES) ${ }_{3} \mathrm{SiH}$ (0.3 mmol, 1.5 equiv.), $\mathrm{K}_{3} \mathrm{PO}_{4}$ ( $0.5 \mathrm{mmol}, 2.5$ equiv.) and stirring at room temperature for $24 \mathrm{~h}$ under $30 \mathrm{~W}$ blue LEDs irradiation. The reaction mixture was filtered through a pad of silica gel and concentrated in vacuo, then purified by chromatography $($ pentane $/$ EtOAc $=$ 
95/5) to give 38 (21.7 mg, $0.060 \mathrm{mmol}, 30 \%$ ) as a colorless oil. $\mathbf{R}_{\mathbf{f}}$ (pentane/EtOAc = 90/10) 0.21. ${ }^{1} \mathbf{H}$ NMR (599 MHz, $\left.\mathrm{CDCl}_{3}\right): \delta$ ppm $8.12-7.94(\mathrm{~m}, 3 \mathrm{H}), 7.55(\mathrm{t}, J=$ $7.5 \mathrm{~Hz}, 1 \mathrm{H}), 7.45$ (dt, $J=15.4,7.6 \mathrm{~Hz}, 3 \mathrm{H}), 7.22$ (d, $J=7.8 \mathrm{~Hz}, 1 \mathrm{H}), 5.72$ (d, $J=7.5$ $\mathrm{Hz}, 1 \mathrm{H}), 2.94(\mathrm{t}, J=6.2 \mathrm{~Hz}, 2 \mathrm{H}), 2.64(\mathrm{t}, J=6.6 \mathrm{~Hz}, 2 \mathrm{H}), 2.15-2.08(\mathrm{~m}, 2 \mathrm{H}), 1.96(\mathrm{~d}$, $J=12.8 \mathrm{~Hz}, 2 \mathrm{H}), 1.76(\mathrm{~d}, J=13.0 \mathrm{~Hz}, 1 \mathrm{H}), 1.67(\mathrm{dd}, J=23.4,11.8 \mathrm{~Hz}, 3 \mathrm{H}), 1.48(\mathrm{~d}, J$ $=13.0 \mathrm{~Hz}, 1 \mathrm{H}), 1.27-1.09$ (m, 4H). ${ }^{13} \mathrm{C}$ NMR (151 MHz, $\left.\mathrm{CDCl}_{3}\right): \delta \mathrm{ppm} 198.2,165.9$, 143.9, 138.5, 132.9, 132.5, 132.3, 130.4, 129.6, 128.7, 128.4, 125.4, 80.4, 43.1, 39.1, 29.5, 29.1, 28.9, 26.2, 25.9, 25.8, 23.2. HRMS (ESI+): calcd. for $\mathrm{C}_{24} \mathrm{H}_{26} \mathrm{O}_{3} \mathrm{Na}(\mathrm{M}+$ $\mathrm{Na}^{+}$): 385.1774. Found: 385.1774.
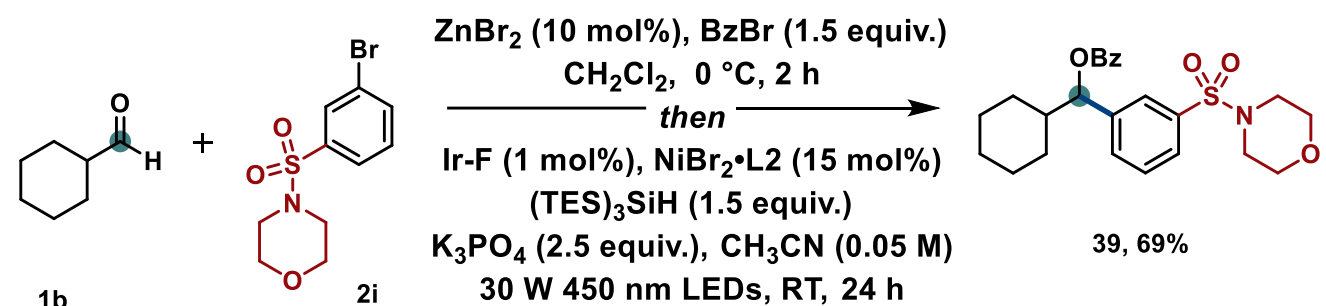

$39,69 \%$ $30 \mathrm{~W} 450 \mathrm{~nm}$ LEDs, RT, $24 \mathrm{~h}$

Cyclohexyl(3-(morpholinosulfonyl)phenyl)methyl benzoate (39). According to the GP3, using the aldehyde $\mathbf{1 d}(0.3 \mathrm{mmol}, 1.5$ equiv.), benzoyl bromide $(0.3$ mmol, 1.5 equiv.), aryl bromide $2 \mathbf{i}$ ( 0.2 mmol, 1.0 equiv.), Ir-F ( $2 \mu \mathrm{mol}, 1$ mol\%), $\mathrm{NiBr} 2 \bullet \mathrm{L} 2$ (30 $\mu \mathrm{mol}, 15 \mathrm{~mol} \%$ ), (TES) $3 \mathrm{SiH}\left(0.3 \mathrm{mmol}, 1.5\right.$ equiv.), $\mathrm{K}_{3} \mathrm{PO}_{4}$ (0.5 mmol, 2.5 equiv.) and stirring at room temperature for $24 \mathrm{~h}$ under $30 \mathrm{~W}$ blue LEDs irradiation. The reaction mixture was filtered through a pad of silica gel and concentrated in vacuo, then purified by chromatography (pentane/EtOAc = 90/10) to give 39 (61.4 $\mathrm{mg}, 0.138 \mathrm{mmol}, 69 \%)$ as a colorless oil. $\mathbf{R}_{\mathbf{f}}$ $($ pentane $/$ EtOAc $=90 / 10)$ 0.50. ${ }^{1} \mathbf{H}$ NMR (599 MHz, $\left.\mathrm{CDCl}_{3}\right): \delta$ ppm $8.16-7.96(\mathrm{~m}$, 2H), $7.75(\mathrm{~d}, J=8.2 \mathrm{~Hz}, 1 \mathrm{H}), 7.67-7.50(\mathrm{~m}, 4 \mathrm{H}), 7.45(\mathrm{dt}, J=9.4,4.9 \mathrm{~Hz}, 2 \mathrm{H}), 5.95$ - $5.64(\mathrm{~m}, 1 \mathrm{H}), 3.73(\mathrm{q}, J=4.2 \mathrm{~Hz}, 1 \mathrm{H}), 3.66(\mathrm{dd}, J=8.6,4.1 \mathrm{~Hz}, 1 \mathrm{H}), 3.61-3.57$ (m, 1H), $3.00(\mathrm{~d}, J=4.9 \mathrm{~Hz}, 1 \mathrm{H}), 2.95-2.69(\mathrm{~m}, 4 \mathrm{H}), 1.94(\mathrm{~d}, J=12.3 \mathrm{~Hz}, 2 \mathrm{H}), 1.83-$ $1.75(\mathrm{~m}, 1 \mathrm{H}), 1.72(\mathrm{~d}, J=11.0 \mathrm{~Hz}, 1 \mathrm{H}), 1.67(\mathrm{~d}, J=11.6 \mathrm{~Hz}, 1 \mathrm{H}), 1.49$ (d, $J=12.6 \mathrm{~Hz}$, $1 \mathrm{H}), 1.19(\mathrm{dq}, J=32.5,12.6,12.2 \mathrm{~Hz}, 4 \mathrm{H}), 1.05(\mathrm{q}, J=13.0,12.6 \mathrm{~Hz}, 1 \mathrm{H}) .{ }^{13} \mathbf{C} \mathbf{~ N M R}$ (151 MHz, $\left.\mathrm{CDCl}_{3}\right): \delta$ ppm 165.8, 141.7, 135.3, 133.3, 131.8, 130.1, 129.7, 129.2, 128.6, 127.9, 127.1, 126.2, 80.0, 66.2, 66.1, 46.1, 46.0, 43.3, 29.2, 28.8, 26.3, 25.9, 25.8. HRMS $\left(\mathrm{ESI}^{+}\right)$: calcd. for $\mathrm{C}_{24} \mathrm{H}_{29} \mathrm{NO}_{5} \mathrm{SNa}\left(\mathrm{M}+\mathrm{Na}^{+}\right)$: 466.1659. Found: 466.1657. 


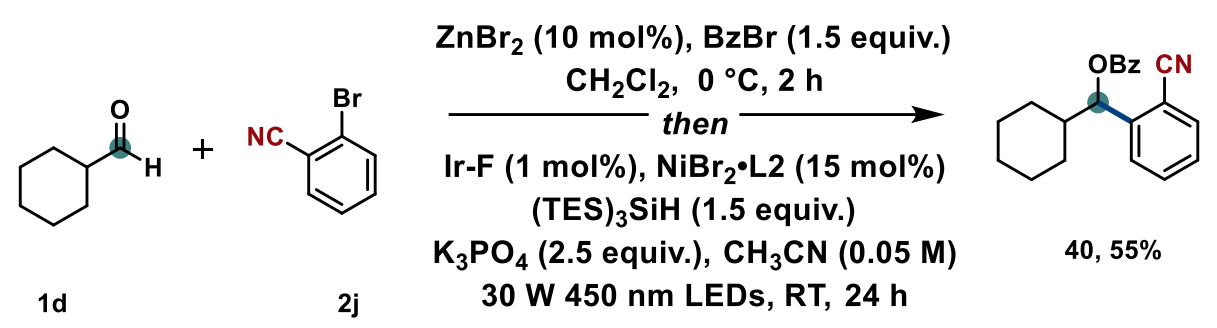

(2-Cyanophenyl)(cyclohexyl)methyl benzoate (40). According to the GP3, using the aldehyde $1 \mathbf{d}$ ( $0.3 \mathrm{mmol}, 1.5$ equiv.), benzoyl bromide ( $0.3 \mathrm{mmol}, 1.5$ equiv.), aryl bromide $2 \mathbf{j}$ ( $0.2 \mathrm{mmol}, 1.0$ equiv.), Ir-F ( $2 \mu \mathrm{mol}, 1 \mathrm{~mol} \%), \mathrm{NiBr}_{2} \bullet \mathrm{L} 2$ ( $30 \mu \mathrm{mol}, 15 \mathrm{~mol} \%$ ), (TES) ${ }_{3} \mathrm{SiH}$ ( $0.3 \mathrm{mmol}, 1.5$ equiv.), $\mathrm{K}_{3} \mathrm{PO}_{4}$ (0.5 mmol, 2.5 equiv.) and stirring at room temperature for $24 \mathrm{~h}$ under $30 \mathrm{~W}$ blue LEDs irradiation. The reaction mixture was filtered through a pad of silica gel and concentrated in vacuo, then purified by chromatography (pentane/EtOAc $=90 / 10)$ to give $\mathbf{4 0}(35.1 \mathrm{mg}$, $0.110 \mathrm{mmol}, 55 \%)$ as a colorless oil. Rf (pentane/EtOAc $=90 / 10) 0.65 .{ }^{1} \mathbf{H}$ NMR (599 MHz, $\left.\mathrm{CDCl}_{3}\right): \delta$ ppm $8.18-8.06(\mathrm{~m}, 2 \mathrm{H}), 7.70-7.62(\mathrm{~m}, 1 \mathrm{H}), 7.60-7.52(\mathrm{~m}$, 2H), $7.52-7.42(\mathrm{~m}, 3 \mathrm{H}), 7.37(\mathrm{t}, J=6.1 \mathrm{~Hz}, 1 \mathrm{H}), 5.95(\mathrm{dd}, J=8.2,2.6 \mathrm{~Hz}, 1 \mathrm{H}), 2.06$ (dd, $J=34.4,11.6 \mathrm{~Hz}, 2 \mathrm{H}), 1.79$ (d, $J=13.5 \mathrm{~Hz}, 1 \mathrm{H}), 1.73(\mathrm{~d}, J=10.2 \mathrm{~Hz}, 1 \mathrm{H}), 1.68$ (d, $J=10.9 \mathrm{~Hz}, 1 \mathrm{H}$ ), 1.43 (d, $J=11.2 \mathrm{~Hz}, 1 \mathrm{H}), 1.21(\mathrm{dt}, J=29.8,15.9 \mathrm{~Hz}, 5 \mathrm{H}) .{ }^{13} \mathrm{C}$ NMR $\left(151 \mathrm{MHz}, \mathrm{CDCl}_{3}\right)$ : $\delta$ ppm 165.6, 143.8, 133.2, 133.2, 132.7, 129.9, 129.8, 128.5, 128.1, 127.8, 117.7, 111.5, 78.9, 42.9, 29.0, 28.9, 26.1, 25.8, 25.7. HRMS (ESI+): calcd. for $\mathrm{C}_{22} \mathrm{H}_{21} \mathrm{NO}_{2} \mathrm{Na}\left(\mathrm{M}+\mathrm{Na}^{+}\right)$: 354.1465. Found: 354.1465 .

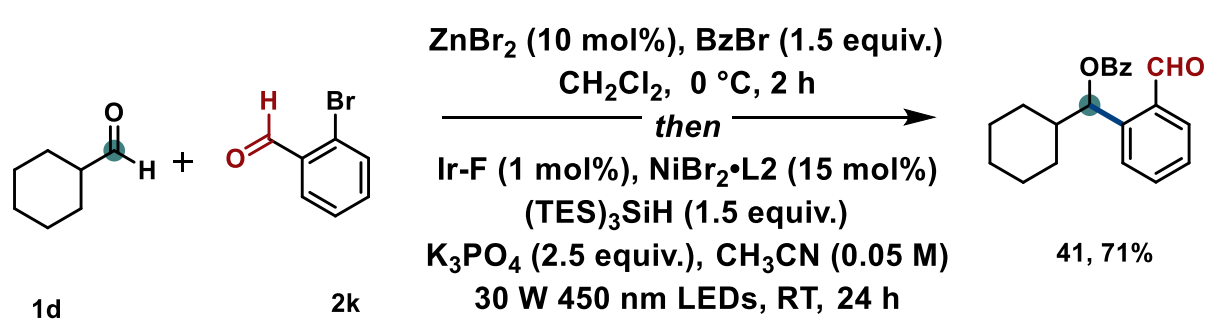

Cyclohexyl(2-formylphenyl)methyl benzoate (41). According to the GP3, using the aldehyde 1d ( $0.3 \mathrm{mmol}, 1.5$ equiv.), benzoyl bromide $(0.3 \mathrm{mmol}, 1.5$ equiv.), aryl bromide $\mathbf{2 k}$ ( $0.2 \mathrm{mmol}, 1.0$ equiv.), Ir-F ( $2 \mu \mathrm{mol}, 1 \mathrm{~mol} \%), \mathrm{NiBr}_{2} \bullet \mathrm{L} 2$ (30 $\mu \mathrm{mol}, 15 \mathrm{~mol} \%$ ), (TES) ${ }_{3} \mathrm{SiH}\left(0.3 \mathrm{mmol}, 1.5\right.$ equiv.), $\mathrm{K}_{3} \mathrm{PO}_{4}(0.5 \mathrm{mmol}, 2.5$ equiv.) and stirring at room temperature for $24 \mathrm{~h}$ under $30 \mathrm{~W}$ blue LEDs irradiation. The reaction mixture was filtered through a pad of silica gel and concentrated in vacuo, then purified by chromatography (pentane/EtOAc $=90 / 10)$ to give $\mathbf{4 1}(45.5 \mathrm{mg}$, 
$0.141 \mathrm{mmol}, 71 \%)$ as a colorless oil. $\mathbf{R}_{\mathbf{f}}($ pentane $/$ EtOAc $=90 / 10) 0.35 .{ }^{1} \mathbf{H}$ NMR (599 MHz, $\mathrm{CDCl}_{3}$ ): $\delta$ ppm $10.43(\mathrm{~s}, 1 \mathrm{H}), 8.08(\mathrm{dd}, J=7.8,2.6 \mathrm{~Hz}, 2 \mathrm{H}), 7.87(\mathrm{dd}, J=$ 7.8, 2.7 Hz, 1H), $7.62-7.52(\mathrm{~m}, 3 \mathrm{H}), 7.46(\mathrm{ddd}, J=11.4,6.3,2.9 \mathrm{~Hz}, 3 \mathrm{H}), 6.62$ (d, $J$ $=4.7 \mathrm{~Hz}, 1 \mathrm{H}), 1.98-1.88(\mathrm{~m}, 2 \mathrm{H}), 1.80-1.75(\mathrm{~m}, 1 \mathrm{H}), 1.75-1.70(\mathrm{~m}, 1 \mathrm{H}), 1.68-$ $1.64(\mathrm{~m}, 1 \mathrm{H}), 1.59-1.52(\mathrm{~m}, 1 \mathrm{H}), 1.33-1.25(\mathrm{~m}, 1 \mathrm{H}), 1.22-1.17(\mathrm{~m}, 3 \mathrm{H}), 1.04$ (ddd, $J=10.4,8.0,2.9 \mathrm{~Hz}, 1 \mathrm{H}) .{ }^{13} \mathbf{C}$ NMR (151 MHz, $\left.\mathrm{CDCl}_{3}\right): \delta$ ppm 192.2, 165.8, 142.5, 133.6, 133.6, 133.1, 132.1, 130.2, 129.6, 128.5, 127.9, 127.5, 76.4, 43.9, 29.5, 28.3, 26.2, 26.1, 26.0. HRMS (ESI ${ }^{+}$): calcd. for $\mathrm{C}_{21} \mathrm{H}_{22} \mathrm{O}_{3} \mathrm{Na}\left(\mathrm{M}+\mathrm{Na}^{+}\right): 345.1461$. Found: 345.1463.

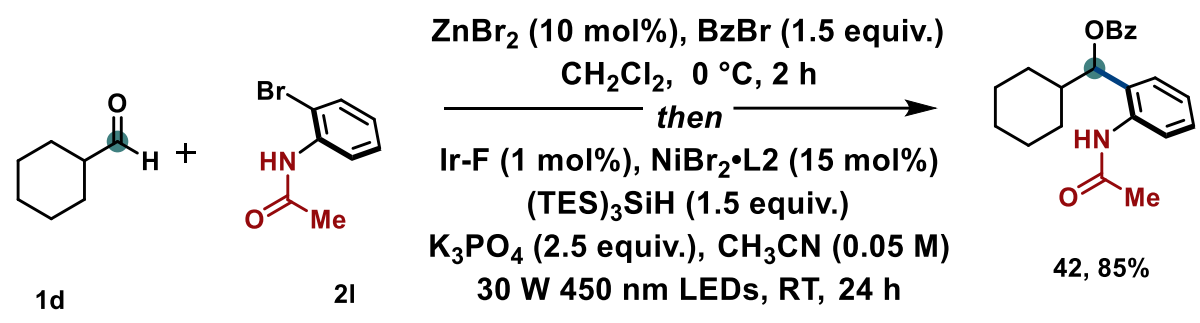

(2-Acetamidophenyl)(cyclohexyl)methyl benzoate (42). According to the GP3, using the aldehyde $1 \mathbf{d}(0.3 \mathrm{mmol}, 1.5$ equiv.), benzoyl bromide $(0.3 \mathrm{mmol}$, 1.5 equiv.), aryl bromide 21 ( $0.2 \mathrm{mmol}, 1.0$ equiv.), Ir-F ( $2 \mu \mathrm{mol}, 1 \mathrm{~mol} \%), \mathrm{NiBr}_{2} \bullet \mathrm{L} 2$ (30 $\mu \mathrm{mol}, 15 \mathrm{~mol} \%$ ), (TES) ${ }_{3} \mathrm{SiH}\left(0.3 \mathrm{mmol}, 1.5\right.$ equiv.), $\mathrm{K}_{3} \mathrm{PO}_{4}(0.5 \mathrm{mmol}, 2.5$ equiv.) and stirring at room temperature for $24 \mathrm{~h}$ under $30 \mathrm{~W}$ blue LEDs irradiation. The reaction mixture was filtered through a pad of silica gel and concentrated in vacuo, then purified by chromatography (pentane/EtOAc $=60 / 40)$ to give $42(59.6 \mathrm{mg}$, $0.170 \mathrm{mmol}, 85 \%)$ as a colorless oil. $\mathbf{R}_{\mathbf{f}}$ (pentane/EtOAc $\left.=60 / 40\right)$ 0.25. ${ }^{1} \mathbf{H} \mathbf{~ N M R}$ (599 MHz, CDCl $): \delta$ ppm $8.77(\mathrm{~s}, 1 \mathrm{H}), 8.03(\mathrm{~d}, J=7.7 \mathrm{~Hz}, 2 \mathrm{H}), 7.81(\mathrm{~d}, J=8.2 \mathrm{~Hz}$, 1H), $7.56(\mathrm{t}, J=7.5 \mathrm{~Hz}, 1 \mathrm{H}), 7.44(\mathrm{t}, J=7.3 \mathrm{~Hz}, 3 \mathrm{H}), 7.31(\mathrm{t}, J=7.8 \mathrm{~Hz}, 1 \mathrm{H}), 7.17(\mathrm{t}, J$ $=7.7 \mathrm{~Hz}, 1 \mathrm{H}), 5.65(\mathrm{~d}, J=9.9 \mathrm{~Hz}, 1 \mathrm{H}), 2.29(\mathrm{~s}, 3 \mathrm{H}), 2.17(\mathrm{t}, J=12.7 \mathrm{~Hz}, 2 \mathrm{H}), 1.80(\mathrm{~d}$, $J=13.3 \mathrm{~Hz}, 1 \mathrm{H}), 1.65(\mathrm{dd}, J=19.2,8.3 \mathrm{~Hz}, 2 \mathrm{H}), 1.31(\mathrm{~d}, J=13.1 \mathrm{~Hz}, 2 \mathrm{H}), 1.22-1.09$ (m, 3H), $0.87-0.73(\mathrm{~m}, 1 \mathrm{H}) .{ }^{13} \mathrm{C}$ NMR (151 MHz, CDCl 3$): \delta \mathrm{ppm} 169.0,167.5,136.5$, 133.5, 130.4, 129.9, 129.8, 129.1, 128.6, 127.7, 125.7, 125.2, 77.2, 41.4, 30.2, 28.9, 26.3, 25.7, 24.5. HRMS (ESI $\left.{ }^{+}\right)$: calcd. for $\mathrm{C}_{22} \mathrm{H}_{25} \mathrm{NO}_{3} \mathrm{Na}\left(\mathrm{M}+\mathrm{Na}^{+}\right): 374.1727$. Found: 374.1732 . 


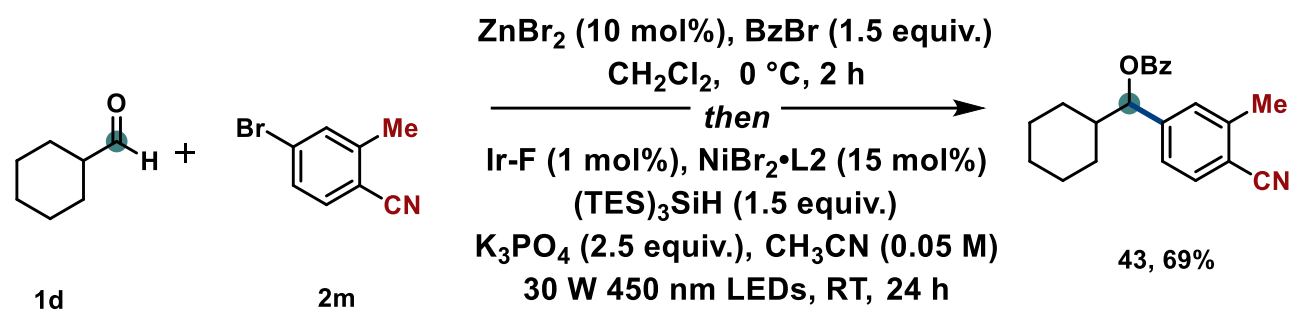

(4-Cyano-3-methylphenyl)(cyclohexyl)methyl benzoate (43). According to the GP3, using the aldehyde $1 \mathbf{d}(0.3 \mathrm{mmol}, 1.5$ equiv.), benzoyl bromide $(0.3 \mathrm{mmol}$, 1.5 equiv.), aryl bromide $2 \mathrm{~m}$ ( $0.2 \mathrm{mmol}, 1.0$ equiv.), Ir-F ( $2 \mu \mathrm{mol}, 1 \mathrm{~mol} \%)$, $\mathrm{NiBr}_{2} \bullet \mathrm{L} 2(30 \mu \mathrm{mol}, 15 \mathrm{~mol} \%)$, (TES) $3 \mathrm{SiH}\left(0.3 \mathrm{mmol}, 1.5\right.$ equiv.), $\mathrm{K}_{3} \mathrm{PO}_{4}(0.5 \mathrm{mmol}$, 2.5 equiv.) and stirring at room temperature for $24 \mathrm{~h}$ under $30 \mathrm{~W}$ blue LEDs irradiation. The reaction mixture was filtered through a pad of silica gel and concentrated in vacuo, then purified by chromatography (pentane/EtOAc $=95 / 5$ ) to give 43 (45.8 $\mathrm{mg}, 0.138 \mathrm{mmol}, 69 \%$ ) as a colorless oil. $\mathbf{R} \mathbf{f}$ (pentane $/$ EtOAc = 90/10) 0.48. ${ }^{1} \mathrm{H}$ NMR (599 MHz, $\mathrm{CDCl}_{3}$ ): $\delta$ ppm $\delta 8.12-8.00(\mathrm{~m}, 2 \mathrm{H}), 7.60-7.49$ (m, 2H), $7.44(\mathrm{td}, J=9.8,7.8,2.1 \mathrm{~Hz}, 2 \mathrm{H}), 7.30-7.18(\mathrm{~m}, 2 \mathrm{H}), 5.70-5.62(\mathrm{~m}, 1 \mathrm{H})$, $2.51(\mathrm{~s}, 3 \mathrm{H}), 1.93-1.81(\mathrm{~m}, 2 \mathrm{H}), 1.79-1.57(\mathrm{~m}, 4 \mathrm{H}), 1.46(\mathrm{~d}, J=12.7 \mathrm{~Hz}, 1 \mathrm{H}), 1.27$ - $1.08(\mathrm{~m}, 4 \mathrm{H}), 1.04-0.94(\mathrm{~m}, 1 \mathrm{H}) .{ }^{13} \mathbf{C}$ NMR $\left(151 \mathrm{MHz}, \mathrm{CDCl}_{3}\right): \delta$ ppm 165.7, 145.0, 142.0, 133.2, 132.5, 130.0, 129.6, 128.7, 128.5, 124.7, 118.0, 112.0, 80.0, 43.1, 29.1, 28.6, 26.2, 25.8, 25.8, 20.6. HRMS $\left(\mathrm{ESI}^{+}\right)$: calcd. for $\mathrm{C}_{22} \mathrm{H}_{23} \mathrm{NO}_{2} \mathrm{Na}\left(\mathrm{M}+\mathrm{Na}^{+}\right)$: 356.1621. Found: 356.1620 .<smiles>O=CC1CCCCC1</smiles>

1d<smiles>FC(F)(F)c1cc(Br)cc(C(F)(F)F)c1</smiles>

$2 n$

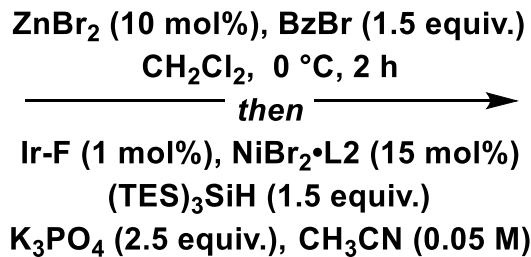

$30 \mathrm{~W} 450 \mathrm{~nm}$ LEDs, RT, $24 \mathrm{~h}$<smiles>O=C(c1cc(C(F)(F)F)cc(C(F)(F)F)c1)c1cc(C(F)(F)F)cc(C(F)(F)F)c1</smiles>

$44,87 \%$

(3,5-Bis(trifluoromethyl)phenyl)(cyclohexyl)methyl benzoate

According to the GP3, using the aldehyde 1d ( $0.3 \mathrm{mmol}, 1.5$ equiv.), benzoyl bromide ( 0.3 mmol, 1.5 equiv.), aryl bromide $2 \mathrm{n}$ ( $0.2 \mathrm{mmol}, 1.0$ equiv.), Ir-F (2

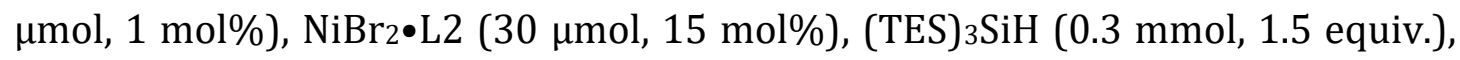
$\mathrm{K}_{3} \mathrm{PO}_{4}$ ( $0.5 \mathrm{mmol}, 2.5$ equiv.) and stirring at room temperature for $24 \mathrm{~h}$ under $30 \mathrm{~W}$ blue LEDs irradiation. The reaction mixture was filtered through a pad of silica gel and concentrated in vacuo, then purified by chromatography $($ pentane $/$ EtOAc $=$ 95/5) to give 44 (75.2 $\mathrm{mg}, 0.175 \mathrm{mmol}, 87 \%$ ) as a colorless oil. $\mathbf{R}_{\mathbf{f}}$ (pentane/EtOAc 
= 90/10) 0.18. ${ }^{1}$ H NMR (599 MHz, $\left.\mathrm{CDCl}_{3}\right): \delta$ ppm $8.18-8.04(\mathrm{~m}, 2 \mathrm{H}), 7.81(\mathrm{~d}, J=$ $3.2 \mathrm{~Hz}, 3 \mathrm{H}$ ), 7.59 (d, $J=8.0 \mathrm{~Hz}, 1 \mathrm{H}), 7.49$ (q, $J=7.6,5.7 \mathrm{~Hz}, 2 \mathrm{H}), 5.83$ (d, $J=7.2 \mathrm{~Hz}$, 1H), $1.94(\mathrm{~d}, J=12.7 \mathrm{~Hz}, 2 \mathrm{H}), 1.83-1.66(\mathrm{~m}, 3 \mathrm{H}), 1.49$ (d, $J=12.8 \mathrm{~Hz}, 1 \mathrm{H}), 1.21$ (dt, $J=45.9,13.6 \mathrm{~Hz}, 5 \mathrm{H}) \cdot{ }^{13} \mathrm{C}$ NMR $\left(151 \mathrm{MHz}, \mathrm{CDCl}_{3}\right): \delta \mathrm{ppm} 165.6,142.5,133.4,131.7$ (q, $J=32.5 \mathrm{~Hz}), 129.7,129.6,128.6,127.1$ (q, $J=3.8 \mathrm{~Hz}), 123.2$ (q, $J=266.3 \mathrm{~Hz}$ ), $121.9(\mathrm{q}, J=3.8 \mathrm{~Hz}), 79.4,43.2,29.0,28.6,26.1,25.7,25.7 .{ }^{19}$ F NMR (470 MHz, $\left.\mathrm{CDCl}_{3}\right): \delta$ ppm -62.81. HRMS $\left(\mathrm{ESI}^{+}\right)$: calcd. for $\mathrm{C}_{22} \mathrm{H}_{20} \mathrm{~F}_{6} \mathrm{O}_{2} \mathrm{Na}\left(\mathrm{M}+\mathrm{Na}^{+}\right): 453.1260$. Found: 453.1259.

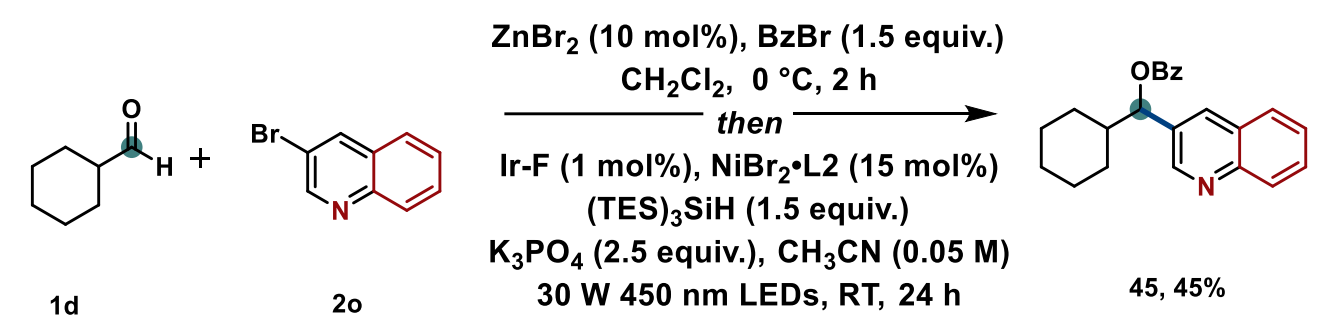

Cyclohexyl(quinolin-3-yl)methyl benzoate (45). According to the GP3, using the aldehyde $1 \mathrm{~d}$ ( $0.3 \mathrm{mmol}, 1.5$ equiv.), benzoyl bromide ( $0.3 \mathrm{mmol}, 1.5$ equiv.), aryl bromide 20 ( 0.2 mmol, 1.0 equiv.), Ir-F ( $2 \mu \mathrm{mol}, 1 \mathrm{~mol} \%$ ), NiBr $2 \bullet \mathrm{L} 2$ (30 $\mu \mathrm{mol}$, 15 mol\%), (TES) $3 \mathrm{SiH}$ (0.3 mmol, 1.5 equiv.), $\mathrm{K}_{3} \mathrm{PO}_{4}(0.5 \mathrm{mmol}, 2.5$ equiv.) and stirring at room temperature for $24 \mathrm{~h}$ under $30 \mathrm{~W}$ blue LEDs irradiation. The reaction mixture was filtered through a pad of silica gel and concentrated in vacuo, then purified by chromatography (pentane/EtOAc $=90 / 10)$ to give $45(31.2 \mathrm{mg}$, $0.090 \mathrm{mmol}, 45 \%)$ as a colorless oil. $\mathbf{R} \mathbf{f}($ pentane/EtOAc $=80 / 20) 0.48 .{ }^{1} \mathbf{H} \mathbf{~ N M R}$ (599 MHz, $\left.\mathrm{CDCl}_{3}\right): \delta$ ppm $8.98(\mathrm{~d}, J=2.4 \mathrm{~Hz}, 1 \mathrm{H}), 8.18-8.07(\mathrm{~m}, 4 \mathrm{H}), 7.82(\mathrm{~d}, J=$ $8.2 \mathrm{~Hz}, 1 \mathrm{H}), 7.70(\mathrm{ddd}, J=8.4,6.9,1.6 \mathrm{~Hz}, 1 \mathrm{H}), 7.60-7.52(\mathrm{~m}, 2 \mathrm{H}), 7.49-7.43(\mathrm{~m}$, 2H), $5.94(\mathrm{dd}, J=7.7,2.1 \mathrm{~Hz}, 1 \mathrm{H}), 2.11-1.99(\mathrm{~m}, 2 \mathrm{H}), 1.79(\mathrm{~d}, J=13.2 \mathrm{~Hz}, 1 \mathrm{H}), 1.75$ - 1.64 (m, 2H), $1.57-1.52$ (m, 1H), $1.30-1.14$ (m, 4H), 1.10 (dd, J = 13.7, $10.8 \mathrm{~Hz}$, 1H). ${ }^{13}$ C NMR (151 MHz, CDCl 3$): \delta$ ppm 165.8, 149.6, 147.7, 134.3, 133.2, 132.5, 130.1, 129.7, 129.6, 129.2, 128.5, 127.9, 127.6, 126.9, 78.9, 43.1, 29.1, 28.9, 26.2, 25.8, 25.7. HRMS (ESI+): calcd. for $\mathrm{C}_{23} \mathrm{H}_{23} \mathrm{NO}_{2} \mathrm{Na}\left(\mathrm{M}+\mathrm{Na}^{+}\right)$: 368.1621. Found: 368.1619. 


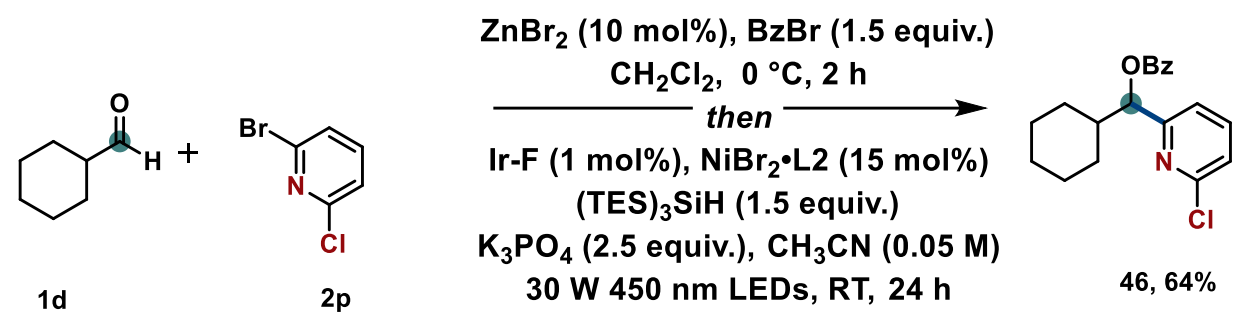

(6-Chloropyridin-2-yl)(cyclohexyl)methyl (46). According to the GP3, using the aldehyde $1 \mathbf{d}(0.3 \mathrm{mmol}, 1.5$ equiv.), benzoyl bromide ( $0.3 \mathrm{mmol}, 1.5$ equiv.), aryl bromide $2 \mathbf{p}$ ( $0.2 \mathrm{mmol}, 1.0$ equiv.), Ir-F ( $2 \mu \mathrm{mol}, 1 \mathrm{~mol} \%)$, NiBr $\bullet \bullet 2$ (30 $\mu \mathrm{mol}$, $15 \mathrm{~mol} \%$ ), (TES) ${ }_{3} \mathrm{SiH}$ (0.3 mmol, 1.5 equiv.), $\mathrm{K}_{3} \mathrm{PO}_{4}$ (0.5 mmol, 2.5 equiv.) and stirring at room temperature for $24 \mathrm{~h}$ under $30 \mathrm{~W}$ blue LEDs irradiation. The reaction mixture was filtered through a pad of silica gel and concentrated in vacuo, then purified by chromatography (pentane/EtOAc $=96 / 4)$ to give $46(42.3 \mathrm{mg}$, $0.129 \mathrm{mmol}, 64 \%)$ as a colorless oil. $\mathbf{R}_{\mathbf{f}}$ (pentane/EtOAc $\left.=94 / 6\right) 0.35 .{ }^{1} \mathbf{H} \mathbf{~ N M R}$ $\left(599 \mathrm{MHz}, \mathrm{CDCl}_{3}\right): \delta \mathrm{ppm} 8.16-8.09(\mathrm{~m}, 2 \mathrm{H}), 7.59(\mathrm{td}, J=7.6,2.7 \mathrm{~Hz}, 2 \mathrm{H}), 7.47(\mathrm{td}$, $J=7.2,4.1 \mathrm{~Hz}, 2 \mathrm{H}), 7.28-7.18(\mathrm{~m}, 2 \mathrm{H}), 5.82(\mathrm{~d}, J=6.1,2.9 \mathrm{~Hz}, 1 \mathrm{H}), 2.17(\mathrm{~d}, J=8.2$ $\mathrm{Hz}, 1 \mathrm{H}), 1.75(\mathrm{~d}, J=8.4 \mathrm{~Hz}, 3 \mathrm{H}), 1.67$ (d, $J=12.9 \mathrm{~Hz}, 1 \mathrm{H}), 1.60(\mathrm{~d}, J=13.0 \mathrm{~Hz}, 1 \mathrm{H})$, $1.30-1.19(\mathrm{~m}, 4 \mathrm{H}), 1.05-1.02(\mathrm{~m}, 1 \mathrm{H}) .{ }^{13} \mathrm{C}$ NMR (151 MHz, $\left.\mathrm{CDCl}_{3}\right): \delta$ ppm 165.9, 160.3, 151.1, 139.0, 133.3, 130.2, 129.9, 128.6, 123.2, 119.9, 80.4, 42.0, 29.7, 27.7, 26.4, 26.1, 26.0. HRMS (ESI ${ }^{+}$): calcd. for $\mathrm{C}_{19} \mathrm{H}_{20} \mathrm{NO}_{2} \mathrm{ClK}\left(\mathrm{M}+\mathrm{K}^{+}\right)$: 368.0814. Found: 368.0814 .

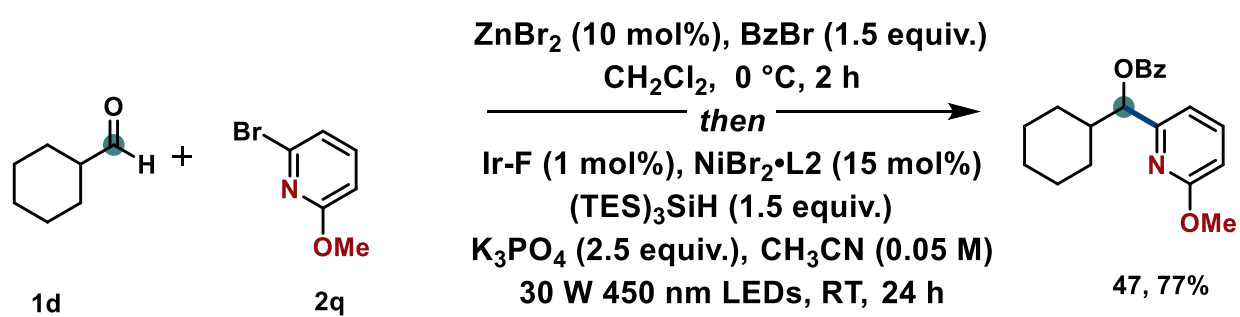

Cyclohexyl(6-methoxypyridin-2-yl)methyl benzoate (47). According to the GP3, using the aldehyde $\mathbf{1 d}(0.3 \mathrm{mmol}, 1.5$ equiv.), benzoyl bromide $(0.3 \mathrm{mmol}$, 1.5 equiv.), aryl bromide $2 q$ ( $0.2 \mathrm{mmol}, 1.0$ equiv.), Ir-F ( $2 \mu \mathrm{mol}, 1 \mathrm{~mol} \%), \mathrm{NiBr}_{2} \bullet \mathrm{L} 2$ ( $30 \mu \mathrm{mol}, 15 \mathrm{~mol} \%$ ), (TES) ${ }_{3} \mathrm{SiH}$ ( $0.3 \mathrm{mmol}, 1.5$ equiv.), $\mathrm{K}_{3} \mathrm{PO}_{4}$ ( $0.5 \mathrm{mmol}, 2.5$ equiv.) and stirring at room temperature for $24 \mathrm{~h}$ under $30 \mathrm{~W}$ blue LEDs irradiation. The reaction mixture was filtered through a pad of silica gel and concentrated in vacuo, then purified by chromatography (pentane/EtOAc $=96 / 4$ ) to give $47(49.8 \mathrm{mg}$, $0.153 \mathrm{mmol}, 77 \%)$ as a colorless oil. Rf (pentane/EtOAc $=90 / 10) 0.81 .{ }^{1} \mathbf{H} \mathbf{~ N M R}$ (599 MHz, $\mathrm{CDCl}_{3}$ ): $\delta$ ppm ${ }^{1} \mathrm{H}$ NMR (599 MHz, Chloroform- $d$ ) $\delta 8.19-8.11(\mathrm{~m}, 2 \mathrm{H})$, 
$7.63-7.55(\mathrm{~m}, 1 \mathrm{H}), 7.48(\mathrm{dt}, J=15.9,7.6 \mathrm{~Hz}, 3 \mathrm{H}), 6.88(\mathrm{dd}, J=7.2,2.5 \mathrm{~Hz}, 1 \mathrm{H}), 6.63$ - $6.57(\mathrm{~m}, 1 \mathrm{H}), 5.79(\mathrm{~d}, 1 \mathrm{H}), 3.92(\mathrm{~s}, 3 \mathrm{H}), 2.16(\mathrm{~s}, 1 \mathrm{H}), 1.76(\mathrm{~s}, 3 \mathrm{H}), 1.65(\mathrm{t}, J=16.4$ $\mathrm{Hz}, 2 \mathrm{H}), 1.23$ (ddd, $J=45.5,23.1,12.9 \mathrm{~Hz}, 5 \mathrm{H}) .{ }^{13} \mathrm{C} \mathrm{NMR}\left(151 \mathrm{MHz}, \mathrm{CDCl}_{3}\right): \delta \mathrm{ppm}$ 166.0, 163.5, 156.6, 138.6, 132.9, 130.5, 129.7, 128.4, 113.7, 109.2, 80.6, 53.3, 41.9, 29.5, 27.8, 26.4, 26.1, 26.0. HRMS $\left(\mathrm{ESI}^{+}\right)$: calcd. for $\mathrm{C}_{20} \mathrm{H}_{23} \mathrm{NO}_{3} \mathrm{Na}\left(\mathrm{M}+\mathrm{Na}^{+}\right)$: 348.1570. Found: 348.1579.

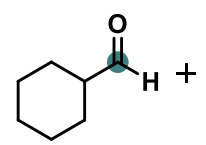

1d<smiles>Brc1ccccn1</smiles>

$2 r$
$\mathrm{ZnBr}_{2}$ (10 mol\%), BzBr (1.5 equiv.)

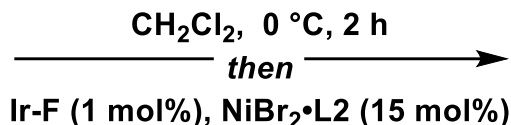

(TES) $)_{3} \mathrm{SiH}(1.5$ equiv.)

$\mathrm{K}_{3} \mathrm{PO}_{4}$ (2.5 equiv.), $\mathrm{CH}_{3} \mathrm{CN}(0.05 \mathrm{M})$

$30 \mathrm{~W} 450 \mathrm{~nm}$ LEDs, RT, $24 \mathrm{~h}$

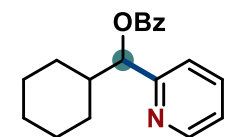

$48,53 \%$

Cyclohexyl(pyridin-2-yl)methyl benzoate (48). According to the GP3, using the aldehyde $1 \mathbf{d}$ ( $0.3 \mathrm{mmol}, 1.5$ equiv.), benzoyl bromide ( $0.3 \mathrm{mmol}, 1.5$ equiv.), aryl bromide $2 q$ ( 0.2 mmol, 1.0 equiv.), Ir-F ( $2 \mu \mathrm{mol}, 1$ mol\%), NiBr2 $\bullet 2$ (30 $\mu \mathrm{mol}, 15$ mol\%), (TES) ${ }_{3} \mathrm{SiH}$ (0.3 mmol, 1.5 equiv.), $\mathrm{K}_{3} \mathrm{PO}_{4}$ ( $0.5 \mathrm{mmol}, 2.5$ equiv.) and stirring at room temperature for $24 \mathrm{~h}$ under $30 \mathrm{~W}$ blue LEDs irradiation. The reaction mixture was filtered through a pad of silica gel and concentrated in vacuo, then purified by chromatography (pentane/EtOAc $=96 / 4)$ to give $48(31.0 \mathrm{mg}, 0.105$ mmol, 53\%) as a colorless oil. Rf (pentane/EtOAc $=90 / 10)$ 0.21. ${ }^{\mathbf{1}} \mathbf{H}$ NMR (599 $\left.\mathrm{MHz}, \mathrm{CDCl}_{3}\right): \delta$ ppm $8.60(\mathrm{~d}, J=4.5 \mathrm{~Hz}, 1 \mathrm{H}), 8.15-8.08(\mathrm{~m}, 2 \mathrm{H}), 7.67-7.62(\mathrm{~m}$, 1H), $7.56(\mathrm{~d}, J=8.1 \mathrm{~Hz}, 1 \mathrm{H}), 7.49-7.42(\mathrm{~m}, 2 \mathrm{H}), 7.33(\mathrm{dd}, J=8.0,3.3 \mathrm{~Hz}, 1 \mathrm{H}), 7.18$ $(\mathrm{dt}, J=7.8,4.2 \mathrm{~Hz}, 1 \mathrm{H}), 5.85(\mathrm{dd}, J=6.4,3.4 \mathrm{~Hz}, 1 \mathrm{H}), 2.25-2.14(\mathrm{~m}, 1 \mathrm{H}), 1.87-1.79$ (m, 1H), $1.78-1.69$ (m, 2H), $1.69-1.61(\mathrm{~m}, 1 \mathrm{H}), 1.60-1.51(\mathrm{~m}, 1 \mathrm{H}), 1.31-1.22$ (m, 3H), 1.20 - $1.12(\mathrm{~m}, 2 \mathrm{H}) .{ }^{13} \mathbf{C}$ NMR (151 MHz, CDCl 3$): \delta$ ppm 165.9, 159.0, 149.2, 136.3, 133.0, 130.3, 129.7, 128.4, 122.5, 121.6, 81.0, 42.1, 29.5, 28.0, 26.3, 26.0, 25.9. HRMS (ESI ${ }^{+}$): calcd. for $\mathrm{C}_{19} \mathrm{H}_{21} \mathrm{NO}_{2} \mathrm{Na}\left(\mathrm{M}+\mathrm{Na}^{+}\right)$: 318.1465. Found: 318.1471.<smiles>O=CC1CCCCC1</smiles>

$1 d$
$\mathrm{ZnBr}_{2}(10 \mathrm{~mol} \%), \mathrm{BzBr}(1.5$ equiv.)

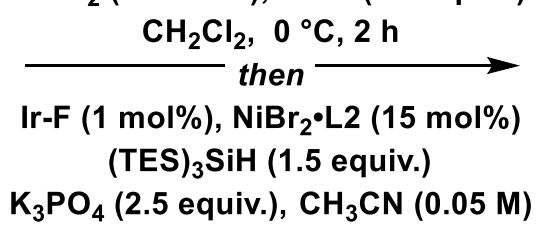

$30 \mathrm{~W} 450 \mathrm{~nm}$ LEDs, RT, $24 \mathrm{~h}$

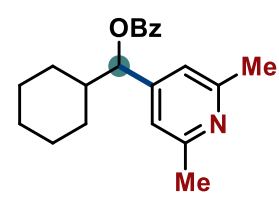

$49,70 \%$

Cyclohexyl(2,6-dimethylpyridin-4-yl)methyl benzoate (49). According to the GP3, using the aldehyde $\mathbf{1 d}(0.3 \mathrm{mmol}, 1.5$ equiv.), benzoyl bromide $(0.3 \mathrm{mmol}$, 
1.5 equiv.), aryl bromide $2 \mathrm{~s}$ ( $0.2 \mathrm{mmol}, 1.0$ equiv.), Ir-F ( $2 \mu \mathrm{mol}, 1 \mathrm{~mol} \%)$, $\mathrm{NiBr}_{2} \bullet \mathrm{L} 2$ (30 $\mu \mathrm{mol}, 15 \mathrm{~mol} \%$ ), (TES) ${ }_{3} \mathrm{SiH}\left(0.3 \mathrm{mmol}, 1.5\right.$ equiv.), $\mathrm{K}_{3} \mathrm{PO}_{4}(0.5 \mathrm{mmol}, 2.5$ equiv.) and stirring at room temperature for $24 \mathrm{~h}$ under $30 \mathrm{~W}$ blue LEDs irradiation. The reaction mixture was filtered through a pad of silica gel and concentrated in vacuo, then purified by chromatography (pentane/EtOAc $=90 / 10)$ to give $49(45.2 \mathrm{mg}$, $0.140 \mathrm{mmol}, 70 \%)$ as a colorless oil. $\mathbf{R}_{\mathbf{f}}($ pentane $/$ EtOAc $=80 / 20) 0.11 .{ }^{1} \mathbf{H}$ NMR (599 MHz, CDCl $): \delta$ ppm 8.09 (dd, $J=7.8,2.9 \mathrm{~Hz}, 2 \mathrm{H}$ ), $7.64-7.55(\mathrm{~m}, 1 \mathrm{H}), 7.47$ (ddd, $J=10.3,6.7,2.5 \mathrm{~Hz}, 2 \mathrm{H}$ ), $6.95-6.87$ (m, 2H), 5.63 (d, J=6.8, 2.6 Hz, 1H), 2.51 (s, 6H), 1.87 (d, J = 12.5 Hz, 2H), $1.79-1.70(\mathrm{~m}, 2 \mathrm{H}), 1.67$ (d, $J=12.2 \mathrm{~Hz}, 1 \mathrm{H}), 1.55$ (d, $J=13.0 \mathrm{~Hz}, 1 \mathrm{H}), 1.18(\mathrm{dt}, J=34.8,13.1 \mathrm{~Hz}, 4 \mathrm{H}), 1.09-1.02(\mathrm{~m}, 1 \mathrm{H}) .{ }^{13} \mathbf{C} \mathbf{~ N M R}$ (151 MHz, $\mathrm{CDCl}_{3}$ ): $\delta$ ppm 165.7, 157.7, 149.1, 133.1, 130.1, 129.6, 128.5, 118.3, 79.4, 42.9, 29.2, 28.4, 26.2, 25.9, 25.8, 24.5. HRMS (ESI+): calcd. for $\mathrm{C}_{21} \mathrm{H}_{25} \mathrm{NO}_{2} \mathrm{Na}$ $\left(\mathrm{M}+\mathrm{Na}^{+}\right): 346.1778$. Found: 346.1777 .

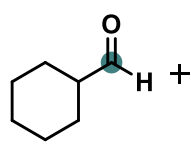

1d

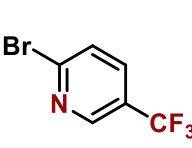

$2 t$

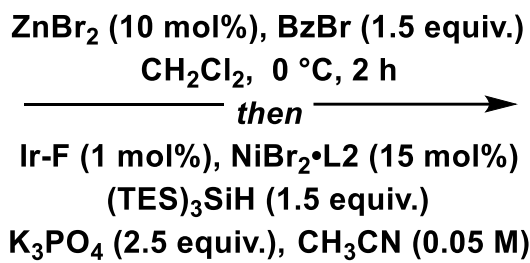

$30 \mathrm{~W} 450 \mathrm{~nm}$ LEDs, RT, $24 \mathrm{~h}$

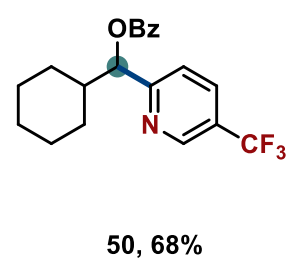

benzoate

According to the GP3, using the aldehyde 1d ( $0.3 \mathrm{mmol}, 1.5$ equiv.), benzoyl bromide ( 0.3 mmol, 1.5 equiv.), aryl bromide $2 \mathrm{t}$ ( $0.2 \mathrm{mmol}, 1.0$ equiv.), Ir-F (2

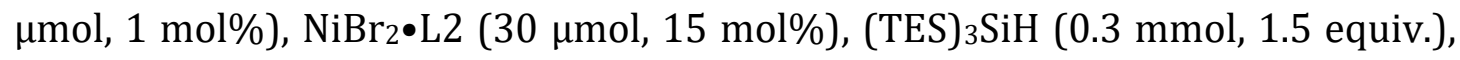
$\mathrm{K}_{3} \mathrm{PO}_{4}$ ( $0.5 \mathrm{mmol}, 2.5$ equiv.) and stirring at room temperature for $24 \mathrm{~h}$ under $30 \mathrm{~W}$ blue LEDs irradiation. The reaction mixture was filtered through a pad of silica gel and concentrated in vacuo, then purified by chromatography $($ pentane $/$ EtOAc $=$ 96/4) to give $\mathbf{5 0}$ (49.2 $\mathrm{mg}, 0.136 \mathrm{mmol}, 68 \%$ ) as a colorless oil. $\mathbf{R} \mathbf{f}$ (pentane/EtOAc = 96/4) 0.35. ${ }^{1} \mathbf{H}$ NMR (599 MHz, $\mathrm{CDCl}_{3}$ ): $\delta$ ppm 8.87 (s, 1H), 8.12 (d, $J=7.6 \mathrm{~Hz}$, 2H), $7.88(\mathrm{~d}, J=8.2 \mathrm{~Hz}, 1 \mathrm{H}), 7.60(\mathrm{t}, J=7.3 \mathrm{~Hz}, 1 \mathrm{H}), 7.51-7.44(\mathrm{~m}, 3 \mathrm{H}), 5.89$ (d, $J=$ $6.2 \mathrm{~Hz}, 1 \mathrm{H}$ ), $2.23-2.15(\mathrm{~m}, 1 \mathrm{H}), 1.77$ (q, J= 13.4, $12.7 \mathrm{~Hz}, 3 \mathrm{H}), 1.68$ (d, $J=12.5 \mathrm{~Hz}$, 1H), $1.58(\mathrm{~d}, J=12.7 \mathrm{~Hz}, 1 \mathrm{H}), 1.31-1.14(\mathrm{~m}, 5 \mathrm{H}) .{ }^{13} \mathrm{C}$ NMR (151 MHz, CDCl 3$): \delta$ ppm 165.9, 163.1 (q, $J=3.0 \mathrm{~Hz}$ ), 146.2 (q, $J=4.5 \mathrm{~Hz}$ ), 133.5 (q, $J=4.5 \mathrm{~Hz}), 133.3$, 129.9, 129.7, 128.5, 125.5 (q, $J=39.0 \mathrm{~Hz}), 123.5$ (q, $J=325.5 \mathrm{~Hz}), 121.1,80.7,42.2$, 
29.5, 27.8, 26.2, 26.0, 25.9. ${ }^{19}$ F NMR (564 MHz, $\mathbf{C D C l}_{3}$ ): $\delta$ ppm -62.39. HRMS (ESI+): calcd. for $\mathrm{C}_{20} \mathrm{H}_{20} \mathrm{NO}_{2} \mathrm{~F}_{3} \mathrm{Na}\left(\mathrm{M}+\mathrm{Na}^{+}\right)$: 386.1338. Found: 386.1336.

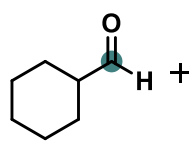

1d

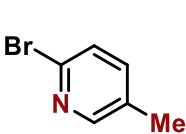

$2 \mathrm{u}$
$\mathrm{ZnBr}_{2}$ (10 mol\%), BzBr (1.5 equiv.)

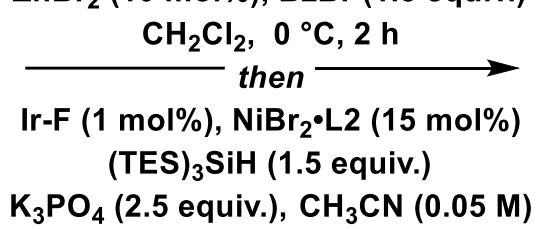

$30 \mathrm{~W} 450 \mathrm{~nm}$ LEDs, RT, $24 \mathrm{~h}$

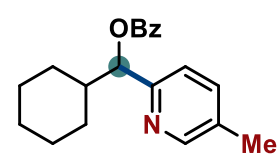

$51,73 \%$

Cyclohexyl(5-methylpyridin-2-yl)methyl benzoate (51). According to the GP3, using the aldehyde $1 \mathbf{d}(0.3 \mathrm{mmol}, 1.5$ equiv.), benzoyl bromide $(0.3 \mathrm{mmol}$, 1.5 equiv.), aryl bromide $2 \mathbf{u}$ ( 0.2 mmol, 1.0 equiv.), Ir-F ( $2 \mu \mathrm{mol}, 1 \mathrm{~mol} \%)$, NiBr2 $\bullet 2$ (30 $\mu \mathrm{mol}, 15 \mathrm{~mol} \%$ ), (TES) ${ }_{3} \mathrm{SiH}\left(0.3 \mathrm{mmol}, 1.5\right.$ equiv.), $\mathrm{K}_{3} \mathrm{PO}_{4}(0.5 \mathrm{mmol}, 2.5$ equiv.) and stirring at room temperature for $24 \mathrm{~h}$ under 30W blue LEDs irradiation. The reaction mixture was filtered through a pad of silica gel and concentrated in vacuo, then purified by chromatography (pentane $/$ EtOAc $=96 / 4)$ to give $\mathbf{5 1}(45.1 \mathrm{mg}$, $0.146 \mathrm{mmol}, 73 \%)$ as a colorless oil. $\mathbf{R}_{\mathbf{f}}($ pentane $/$ EtOAc $=90 / 10) 0.21 .{ }^{1} \mathbf{H}$ NMR (599 MHz, $\left.\mathrm{CDCl}_{3}\right): \delta$ ppm $8.49-8.43(\mathrm{~m}, 1 \mathrm{H}), 8.18$ - $8.09(\mathrm{~m}, 2 \mathrm{H}), 7.60-7.53(\mathrm{~m}$, 1H), $7.49-7.40(\mathrm{~m}, 2 \mathrm{H}), 7.13(\mathrm{~s}, 1 \mathrm{H}), 6.99$ (d, $J=5.0 \mathrm{~Hz}, 1 \mathrm{H}), 5.79$ (d, $J=6.4 \mathrm{~Hz}$, 1H), $2.32(\mathrm{~s}, 3 \mathrm{H}), 1.84(\mathrm{~d}, J=9.5 \mathrm{~Hz}, 1 \mathrm{H}), 1.77-1.62(\mathrm{~m}, 3 \mathrm{H}), 1.53(\mathrm{~d}, J=12.9 \mathrm{~Hz}$, 1H), $1.28-1.08(\mathrm{~m}, 5 \mathrm{H}), 1.02(\mathrm{td}, J=8.2,7.2,3.6 \mathrm{~Hz}, 1 \mathrm{H}) .{ }^{13} \mathrm{C}$ NMR (151 MHz, $\left.\mathrm{CDCl}_{3}\right): \delta$ ppm 166.0, 158.6, 149.0, 147.5, 133.0, 130.3, 129.8, 128.4, 123.5, 122.5, 81.1, 42.0, 29.5, 28.1, 26.3, 26.0, 25.9, 21.2. HRMS (ESI+): calcd. for $\mathrm{C}_{20} \mathrm{H}_{23} \mathrm{NO}_{2} \mathrm{Na}$ $\left(\mathrm{M}+\mathrm{Na}^{+}\right): 332.1621$. Found: 332.1618 .

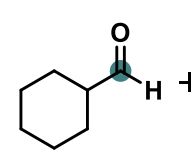

$1 d$<smiles>FC(F)(F)c1cccc(Br)n1</smiles>

$2 \mathbf{v}$

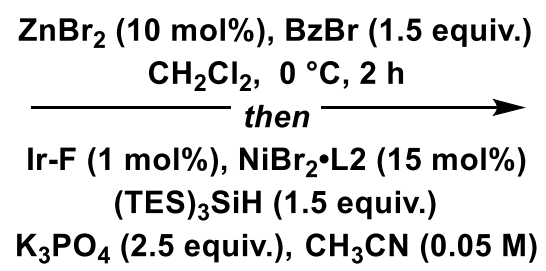

$30 \mathrm{~W} 450 \mathrm{~nm}$ LEDs, RT, $24 \mathrm{~h}$

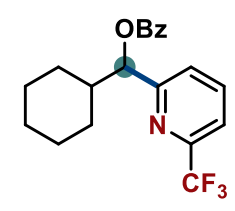

$52,89 \%$

Cyclohexyl(6-(trifluoromethyl)pyridin-2-yl)methyl benzoate

(52).

According to the GP3, using the aldehyde 1d ( $0.3 \mathrm{mmol}, 1.5$ equiv.), benzoyl bromide ( $0.3 \mathrm{mmol}, 1.5$ equiv.), aryl bromide $2 \mathbf{v}$ ( $0.2 \mathrm{mmol}, 1.0$ equiv.), Ir-F (2

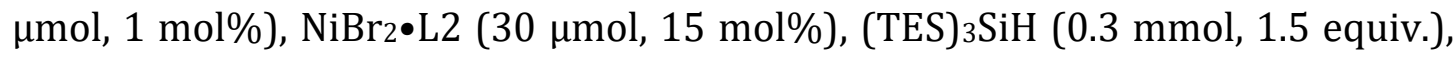
$\mathrm{K}_{3} \mathrm{PO}_{4}(0.5 \mathrm{mmol}, 2.5$ equiv.) and stirring at room temperature for $24 \mathrm{~h}$ under $30 \mathrm{~W}$ 
blue LEDs irradiation. The reaction mixture was filtered through a pad of silica gel and concentrated in vacuo, then purified by chromatography $($ pentane $/$ EtOAc $=$ 96/4) to give 52 (64.3 mg, $0.177 \mathrm{mmol}, 89 \%$ ) as a colorless oil. Rf (pentane/EtOAc = 90/10) 0.51. ${ }^{1} \mathbf{H}$ NMR (599 MHz, $\mathrm{CDCl}_{3}$ ): $\delta \mathrm{ppm} 8.13$ (dd, J = 8.3, $1.4 \mathrm{~Hz}, 2 \mathrm{H}$ ), 7.81 (t, $J=7.8 \mathrm{~Hz}, 1 \mathrm{H}), 7.62-7.54(\mathrm{~m}, 2 \mathrm{H}), 7.53-7.44(\mathrm{~m}, 3 \mathrm{H}), 5.96(\mathrm{~d}, J=5.7 \mathrm{~Hz}, 1 \mathrm{H})$, $2.18(\mathrm{dp}, J=8.3,2.8 \mathrm{~Hz}, 1 \mathrm{H}), 1.81-1.72(\mathrm{~m}, 3 \mathrm{H}), 1.72-1.61(\mathrm{~m}, 2 \mathrm{H}), 1.32-1.12$ (m, 5H). ${ }^{13}$ C NMR (151 MHz, $\left.\mathrm{CDCl}_{3}\right): \delta$ ppm 165.8, 160.1, 147.7 (q, $J=42.2 \mathrm{~Hz}$ ), 137.6, 133.2, 130.0, 129.7, 128.5, 123.7, 121.4 (q, $J=330.7 \mathrm{~Hz}$ ), 119.0 (q, $J=4.5$ $\mathrm{Hz}), 80.4,42.1,29.4,27.6,26.2,26.0,25.9 .{ }^{19} \mathbf{F}$ NMR (564 MHz, $\left.\mathrm{CDCl}_{3}\right): \delta \mathrm{ppm}-$ 67.96. HRMS $\left(\mathrm{ESI}^{+}\right)$: calcd. for $\mathrm{C}_{20} \mathrm{H}_{20} \mathrm{NO}_{2} \mathrm{~F}_{3} \mathrm{Na}\left(\mathrm{M}+\mathrm{Na}^{+}\right)$: 386.1338. Found: 386.1338.

Scale-up reaction. According to the GP3, using the aldehyde $\mathbf{1 d}$ (3.0 mmol, 1.5 equiv.), benzoyl bromide ( $3.0 \mathrm{mmol}, 1.5$ equiv.), aryl bromide $2 \mathbf{v}$ ( $2.0 \mathrm{mmol}, 1.0$ equiv.), Ir-F (20 $\mu \mathrm{mol}, 1 \mathrm{~mol} \%), \mathrm{NiBr}_{2} \bullet \mathrm{L} 2$ (300 $\left.\mu \mathrm{mol}, 15 \mathrm{~mol} \%\right),(\mathrm{TES})_{3} \mathrm{SiH}(3.0$ mmol, 1.5 equiv.), $\mathrm{K}_{3} \mathrm{PO}_{4}$ (5.0 mmol, 2.5 equiv.) and stirring at room temperature for $36 \mathrm{~h}$ under $2 \times 30 \mathrm{~W}$ blue LEDs irradiation. The reaction mixture was filtered through a pad of silica gel and concentrated in vacuo, then purified by chromatography (pentane $/$ EtOAc $=96 / 4)$ to give 52 (567 mg, $1.56 \mathrm{mmol}, 78 \%$ ) as a white waxy solid. The experimental data are in agreement with the $0.2 \mathrm{mmol}$ scale reaction.

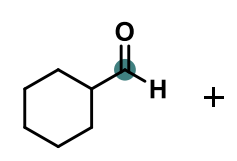

$1 d$<smiles>N#Cc1ccccc1/C=C\Br</smiles>

2w
$\mathrm{ZnBr}_{2}$ (10 mol\%), BzBr (1.5 equiv.)

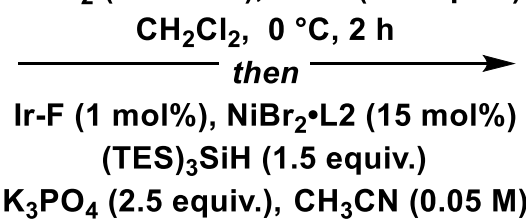

$30 \mathrm{~W} 450 \mathrm{~nm}$ LEDs, RT, $24 \mathrm{~h}$

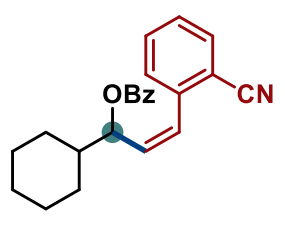

$53,46 \%$ yield $>95: 5$ Z:E

(Z)-3-(2-cyanophenyl)-1-cyclohexylallyl benzoate (53). According to the GP3, using the aldehyde $1 \mathbf{d}$ ( $0.3 \mathrm{mmol}, 1.5$ equiv.), benzoyl bromide ( $0.3 \mathrm{mmol}, 1.5$ equiv.), aryl bromide $2 \mathbf{w}$ (0.2 mmol, 1.0 equiv.), Ir-F ( $2 \mu \mathrm{mol}, 1 \mathrm{~mol} \%), \mathrm{NiBr}_{2} \bullet \mathrm{L} 2$ (30 $\mu \mathrm{mol}, 15 \mathrm{~mol} \%)$, (TES) ${ }_{3} \mathrm{SiH}\left(0.3 \mathrm{mmol}, 1.5\right.$ equiv.), $\mathrm{K}_{3} \mathrm{PO}_{4}(0.5 \mathrm{mmol}, 2.5$ equiv.) and stirring at room temperature for $24 \mathrm{~h}$ under 30W blue LEDs irradiation. The reaction mixture was filtered through a pad of silica gel and concentrated in vacuo, 
then purified by xchromatography (pentane/ $\left.\mathrm{Et}_{2} \mathrm{O}=95 / 5 \rightarrow 93 / 7\right)$ to give $\mathbf{5 3}(31.5$ mg, $0.091 \mathrm{mmol}, 46 \%, Z: E>95: 5$ ) as a colorless solid. Rf (pentane/Et $2 \mathrm{O}=95 / 5$ ) 0.20. ${ }^{1} \mathrm{H}$ NMR (599 MHz, $\left.\mathrm{CDCl}_{3}\right) \delta 8.06-8.02(\mathrm{~m}, 2 \mathrm{H}), 7.87(\mathrm{~d}, J=7.8 \mathrm{~Hz}, 1 \mathrm{H}), 7.66$ (dd, $J=7.7,1.3 \mathrm{~Hz}, 1 \mathrm{H}), 7.62(\mathrm{td}, J=7.7,1.4 \mathrm{~Hz}, 1 \mathrm{H}), 7.56(\mathrm{ddt}, J=8.7,7.3,1.3 \mathrm{~Hz}$, 1H), $7.47-7.43(\mathrm{~m}, 2 \mathrm{H}), 7.36(\mathrm{td}, J=7.7,1.2 \mathrm{~Hz}, 1 \mathrm{H}), 6.84(\mathrm{~d}, J=11.8 \mathrm{~Hz}, 1 \mathrm{H}), 5.94$ (dd, $J=11.8,9.6 \mathrm{~Hz}, 1 \mathrm{H}), 5.64(\mathrm{ddd}, J=9.6,7.1,1.1 \mathrm{~Hz}, 1 \mathrm{H}), 1.80-1.74(\mathrm{~m}, 2 \mathrm{H})$, $1.74-1.67(\mathrm{~m}, 2 \mathrm{H}), 1.66-1.61(\mathrm{~m}, 1 \mathrm{H}), 1.25-1.17(\mathrm{~m}, 2 \mathrm{H}), 1.12(\mathrm{tt}, J=12.7,3.4$ $\mathrm{Hz}, 1 \mathrm{H}), 1.08-1.01(\mathrm{~m}, 1 \mathrm{H}), 0.97-0.88(\mathrm{~m}, 1 \mathrm{H}) .{ }^{13} \mathrm{C}$ NMR $\left(151 \mathrm{MHz}, \mathrm{CDCl}_{3}\right) \delta$ 166.1, 140.4, 133.1, 132.94, 132.93, 132.8, 130.5, 129.8, 129.6, 129.1, 128.5, 127.8, 117.9, 112.4, 75.2, 42.4, 28.6, 28.4, 26.3, 26.0, 25.9. HRMS (ESI ${ }^{+}$): calcd. for $\mathrm{C}_{23} \mathrm{H}_{23} \mathrm{NO}_{2} \mathrm{Na}\left(\mathrm{M}+\mathrm{Na}^{+}\right)$: 368.1626. Found: 368.1621. In addition to NOESY experiment to confirm the $Z$-configuration of the double bond, comparison with close literature precedents for both the $Z^{-36}$ and $E$-isomers ${ }^{37}$ corroborates the assigned stereochemistry.

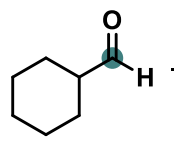

$1 d$

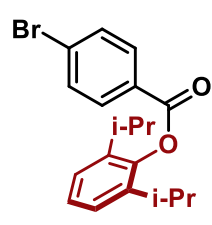

$2 x$

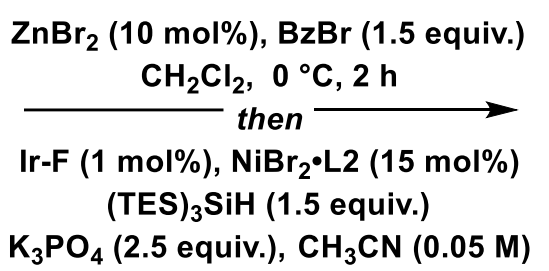

$30 \mathrm{~W} 450 \mathrm{~nm}$ LEDs, RT, $24 \mathrm{~h}$

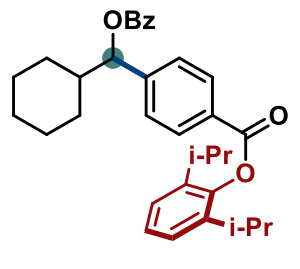

$54,81 \%$

2,6-diisopropylphenyl 4-((benzoyloxy)(cyclohexyl)methyl)benzoate (54).

According to the GP3, using the aldehyde 1d ( $0.3 \mathrm{mmol}, 1.5$ equiv.), benzoyl bromide ( 0.3 mmol, 1.5 equiv.), aryl bromide $2 x$ (0.2 mmol, 1.0 equiv.), Ir-F (2

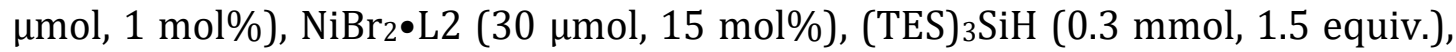
$\mathrm{K}_{3} \mathrm{PO}_{4}$ ( $0.5 \mathrm{mmol}, 2.5$ equiv.) and stirring at room temperature for $24 \mathrm{~h}$ under $30 \mathrm{~W}$ blue LEDs irradiation. The reaction mixture was filtered through a pad of silica gel and concentrated in vacuo, then purified by chromatography $($ pentane $/$ EtOAc $=$ $60 / 1 \rightarrow 45 / 1$ ) to give 54 (80.5 $\mathrm{mg}, 0.161 \mathrm{mmol}, 81 \%$ ) as a colorless gum. $\mathbf{R}_{\mathbf{f}}$ (pentane/EtOAc = 60/1) 0.45. ${ }^{1} \mathbf{H}$ NMR $\left(400 \mathrm{MHz} \mathrm{CDCl}_{3}\right) \delta 8.25(\mathrm{~d}, J=8.3 \mathrm{~Hz}, 2 \mathrm{H})$, $8.15(\mathrm{~d}, J=8.4 \mathrm{~Hz}, 2 \mathrm{H}), 7.64-7.59(\mathrm{~m}, 1 \mathrm{H}), 7.57(\mathrm{~d}, J=8.0 \mathrm{~Hz}, 2 \mathrm{H}), 7.50(\mathrm{t}, J=7.5$ Hz, 2H), $7.31-7.19$ (m, 3H), 5.85 (d, J= $7.1 \mathrm{~Hz}, 1 \mathrm{H}$ ), 2.99 (hept, J = 6.9 Hz, 2H), 2.08 
- $1.94(\mathrm{~m}, 2 \mathrm{H}), 1.87-1.68(\mathrm{~m}, 3 \mathrm{H}), 1.61$ (d, J = $13.2 \mathrm{~Hz}, 1 \mathrm{H}), 1.39-1.08(\mathrm{~m}, 18 \mathrm{H})$. ${ }^{13} \mathrm{C}$ NMR $\left(101 \mathrm{MHz} \mathrm{CDCl}_{3}\right) \delta 166.0,165.0,145.9,145.8,140.7,133.3,130.4,130.3$, 129.8, 128.9, 128.6, 127.3, 126.7, 124.1, 80.5, 43.4, 29.3, 28.9, 27.7, 26.4, 26.1, 26.0, 24.1, 22.8. HRMS $\left(\mathrm{ESI}^{+}\right)$: calcd. for $\mathrm{C}_{33} \mathrm{H}_{38} \mathrm{O}_{4} \mathrm{Na}\left(\mathrm{M}+\mathrm{Na}^{+}\right)$: 521.2662. Found: 521.2661.

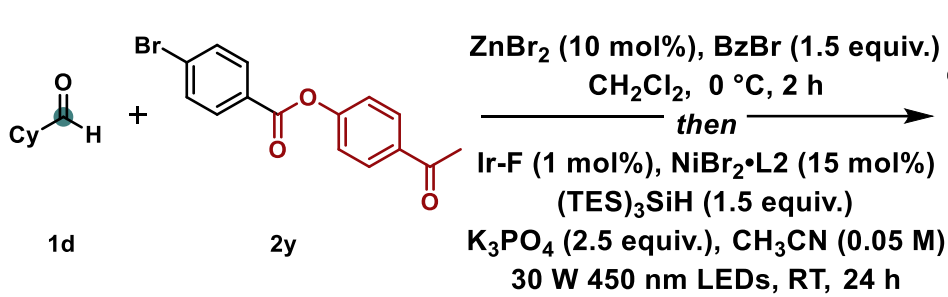

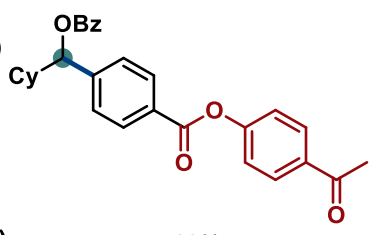

$55,69 \%$

\section{4-acetylphenyl 4-((benzoyloxy)(cyclohexyl)methyl)benzoate}

According to the GP3, using the cyclohexanecarbaldehyde 1d $0.3 \mathrm{mmol}$, 1.5 equiv.), benzoyl bromide (0.3 mmol, 1.5 equiv.), 4-acetylphenyl 4bromobenzoate $2 \mathbf{y}(0.2 \mathrm{mmol}, 1.0$ equiv.), Ir-F (2 $\mu \mathrm{mol}, 1 \mathrm{~mol} \%), \mathrm{NiBr}_{2} \bullet \mathrm{L} 2$ (30 $\mu \mathrm{mol}, 15 \mathrm{~mol} \%$ ), (TES) ${ }_{3} \mathrm{SiH}$ (0.3 mmol, 1.5 equiv.), $\mathrm{K}_{3} \mathrm{PO}_{4}$ (0.5 mmol, 2.5 equiv.) and stirring at room temperature for $24 \mathrm{~h}$ under $30 \mathrm{~W}$ blue LEDs irradiation. The reaction mixture was filtered through a pad of silica gel and concentrated in vacuo, then purified by chromatography (pentane/EtOAc $=16 / 1 \rightarrow 4 / 1)$ to give $55(63.0$ mg, $0.13 \mathrm{mmol}, 69 \%)$ as a colorless solid. $\mathbf{R} \mathbf{f}($ pentane $/$ EtOAc $=9 / 1)=0.50 .{ }^{1} \mathbf{H}$ NMR (400 MHz, $\left.\mathrm{CDCl}_{3}\right) \delta 8.21-8.16(\mathrm{~m}, 2 \mathrm{H}), 8.14-8.08(\mathrm{~m}, 2 \mathrm{H}), 8.08-8.01(\mathrm{~m}$, 2H), $7.62-7.55(\mathrm{~m}, 1 \mathrm{H}), 7.55-7.51(\mathrm{~m}, 2 \mathrm{H}), 7.50-7.44(\mathrm{~m}, 2 \mathrm{H}), 7.34-7.28(\mathrm{~m}$, $2 \mathrm{H}), 5.81(\mathrm{~d}, J=7.0 \mathrm{~Hz}, 1 \mathrm{H}), 2.61(\mathrm{~s}, 3 \mathrm{H}), 2.02-1.88(\mathrm{~m}, 2 \mathrm{H}), 1.84-1.63(\mathrm{~m}, 3 \mathrm{H})$, $1.55(\mathrm{~d}, J=12.5 \mathrm{~Hz}, 1 \mathrm{H}), 1.32-0.93(\mathrm{~m}, 5 \mathrm{H}) .{ }^{13} \mathbf{C ~ N M R}\left(101 \mathrm{MHz}, \mathrm{CDCl}_{3}\right) \delta$ 197.0, 165.9, 164.4, 154.8, 146.2, 134.9, 133.3, 130.4, 130.3, 130.1, 129.7, 128.6, 128.5, 127.3, 122.0, 80.3, 43.4, 29.2, 28.8, 26.7, 26.3, 26.0, 25.9. HRMS (ESI $\left.{ }^{+}\right)$: calcd. for $\mathrm{C}_{29} \mathrm{H}_{28} \mathrm{O}{ }_{5} \mathrm{Na}\left(\mathrm{M}+\mathrm{Na}^{+}\right): 479.1834$. Found: 479.1828 .

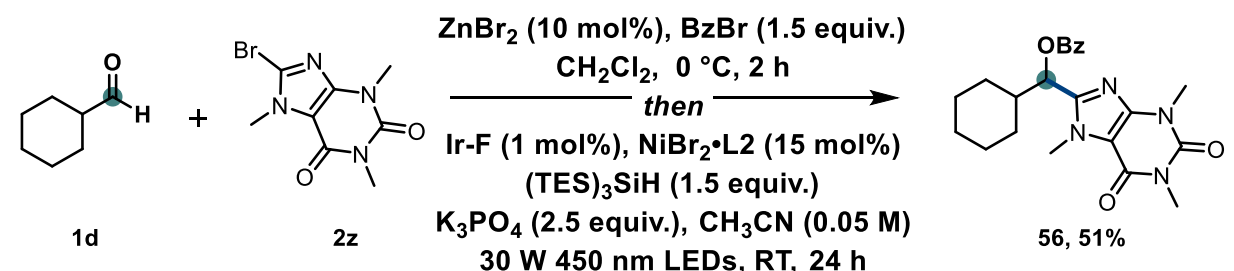


cyclohexyl(1,3,7-trimethyl-2,6-dioxo-2,3,6,7-tetrahydro-1H-purin-8-yl)methyl benzoate (56). According to the GP3, using the cyclohexanecarbaldehyde 1d ( $0.3 \mathrm{mmol}, 1.5$ equiv.), benzoyl bromide ( $0.3 \mathrm{mmol}, 1.5$ equiv.), 8-bromo-1,3,7trimethyl-3,7-dihydro-1H-purine-2,6-dione $2 z$ (0.2 mmol, 1.0 equiv.), Ir-F (2

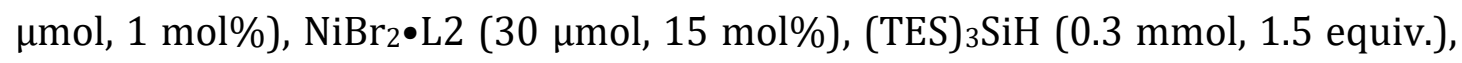
$\mathrm{K}_{3} \mathrm{PO}_{4}$ (0.5 mmol, 2.5 equiv.) and stirring at room temperature for $24 \mathrm{~h}$ under $30 \mathrm{~W}$ blue LEDs irradiation. The reaction mixture was filtered through a pad of silica gel and concentrated in vacuo, then purified by chromatography $($ pentane $/$ EtOAc $=$ $90 / 10 \rightarrow 70 / 30$ ) to give 56 (41.8 $\mathrm{mg}, 0.102 \mathrm{mmol}, 51 \%$ ) as a colorless solid. $\mathbf{R} \mathbf{f}$ $($ pentane $/$ EtOAc $=7 / 3)=0.50 .{ }^{1} \mathbf{H}$ NMR $\left(400 \mathrm{MHz}, \mathrm{CDCl}_{3}\right) \delta 8.09-8.02(\mathrm{~m}, 2 \mathrm{H})$, $7.62-7.53(\mathrm{~m}, 1 \mathrm{H}), 7.44(\mathrm{t}, J=7.7 \mathrm{~Hz}, 2 \mathrm{H}), 5.63(\mathrm{~d}, J=9.7 \mathrm{~Hz}, 1 \mathrm{H}), 4.14(\mathrm{~s}, 3 \mathrm{H})$, 3.55 (s, 3H), 3.38 (s, 3H), $2.43-2.28(\mathrm{~m}, 1 \mathrm{H}), 2.16$ (d, J = $12.8 \mathrm{~Hz}, 1 \mathrm{H}), 1.85-1.68$ (m, 3H), $1.38-0.81(\mathrm{~m}, 6 \mathrm{H}) .{ }^{13} \mathrm{C}$ NMR (101 MHz, $\left.\mathrm{CDCl}_{3}\right) \delta 166.3,155.6,151.8$, 150.9, 148.1, 133.6, 130-0, 129.4, 128.6, 107.6, 71.8, 41.2, 32.3, 30.0, 29.5, 28.6, 28.0, 26.3, 25.6, 25.5. HRMS (ESI+): calcd. for $\mathrm{C}_{22} \mathrm{H}_{26} \mathrm{~N}_{4} \mathrm{O}_{4} \mathrm{Na}\left(\mathrm{M}+\mathrm{Na}^{+}\right)$: 433.1852 . Found: 433.1844 .

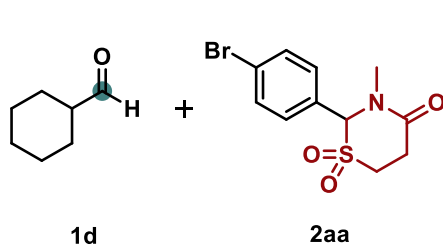

$1 d$

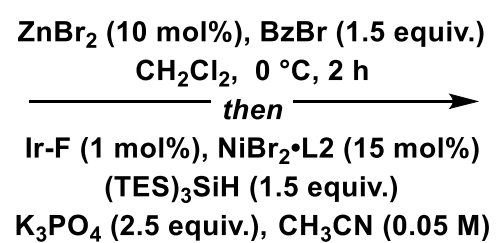

$30 \mathrm{~W} 450 \mathrm{~nm}$ LEDs, RT, $24 \mathrm{~h}$

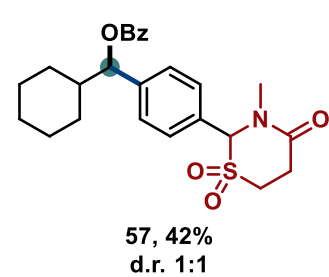

d.r. $1: 1$

cyclohexyl(4-(3-methyl-1,1-dioxido-4-oxo-1,3-thiazinan-2-yl)phenyl)meth-

yl benzoate (57). According to the GP3, using the cyclohexanecarbaldehyde 1d (0.3 mmol, 1.5 equiv.), benzoyl bromide (0.3 mmol, 1.5 equiv.), 2-(4bromophenyl)-3-methyl-1,3-thiazinan-4-one 1,1-dioxide 2 aa (0.2 mmol, 1.0 equiv.), Ir-F ( $2 \mu \mathrm{mol}, 1 \mathrm{~mol} \%), \mathrm{NiBr}_{2} \bullet \mathrm{L} 2$ (30 $\left.\mu \mathrm{mol}, 15 \mathrm{~mol} \%\right)$, (TES) ${ }_{3} \mathrm{SiH}(0.3 \mathrm{mmol}$, 1.5 equiv.), $\mathrm{K}_{3} \mathrm{PO}_{4}$ ( $0.5 \mathrm{mmol}, 2.5$ equiv.) and stirring at room temperature for 24 $\mathrm{h}$ under $30 \mathrm{~W}$ blue LEDs irradiation. The reaction mixture was filtered through a pad of silica gel and concentrated in vacuo, then purified by chromatography (pentane $/$ EtOAc $=50 / 50 \rightarrow 40 / 60)$ to give 57 (38.0 mg, $0.083 \mathrm{mmol}, 42 \%$; d.r. 
1:1) as an off-white solid. $\mathbf{R}_{\mathbf{f}}$ (pentane/EtOAc $\left.=7 / 3\right)=0.50 .{ }^{\mathbf{1}} \mathbf{H} \mathbf{~ N M R}(400 \mathrm{MHz}$, $\left.\mathrm{CDCl}_{3}\right) \delta 8.08(\mathrm{dt}, J=6.6,3.4 \mathrm{~Hz}, 2 \mathrm{H}), 7.57(\mathrm{td}, J=7.4,1.6 \mathrm{~Hz}, 1 \mathrm{H}), 7.51-7.41(\mathrm{~m}$, 4H), 7.39 - $7.27(\mathrm{~m}, 2 \mathrm{H}), 5.77(\mathrm{t}, J=7.8 \mathrm{~Hz}, 1 \mathrm{H}), 5.25$ (s, 1H), 3.32 (dp, J = 17.9, 6.1, $5.3 \mathrm{~Hz}, 1 \mathrm{H}), 3.29$ - 3.03 (m, 3H), 2.95 (s, 3H), 1.92 (tt, J = 8.5, 4.4 Hz, 2H), 1.83 $1.63(\mathrm{~m}, 3 \mathrm{H}), 1.49$ (d, $J=12.6 \mathrm{~Hz}, 1 \mathrm{H}), 1.32-0.98(\mathrm{~m}, 4 \mathrm{H}) .{ }^{13} \mathrm{C}$ NMR $(101 \mathrm{MHz}$, $\left.\mathrm{CDCl}_{3}\right) \delta 166.3,166.3,165.9,165.8,142.5,133.3,130.3,130.3,129.8,129.7,129.6$, 128.6, 128.1, 128.1, 128.1, 128.0, 80.5, 80.4, 80.2, 80.1, 43.5, 43.5, 43.3, 43.3, 36.6, 36.5, 30.6, 29.3, 28.8, 28.7, 26.3, 26.0, 25.9, 25.9. HRMS (ESI+): calcd. for $\mathrm{C}_{25} \mathrm{H}_{29} \mathrm{NO}_{5} \mathrm{SNa}\left(\mathrm{M}+\mathrm{Na}^{+}\right): 478.1664$. Found: 478.1657.
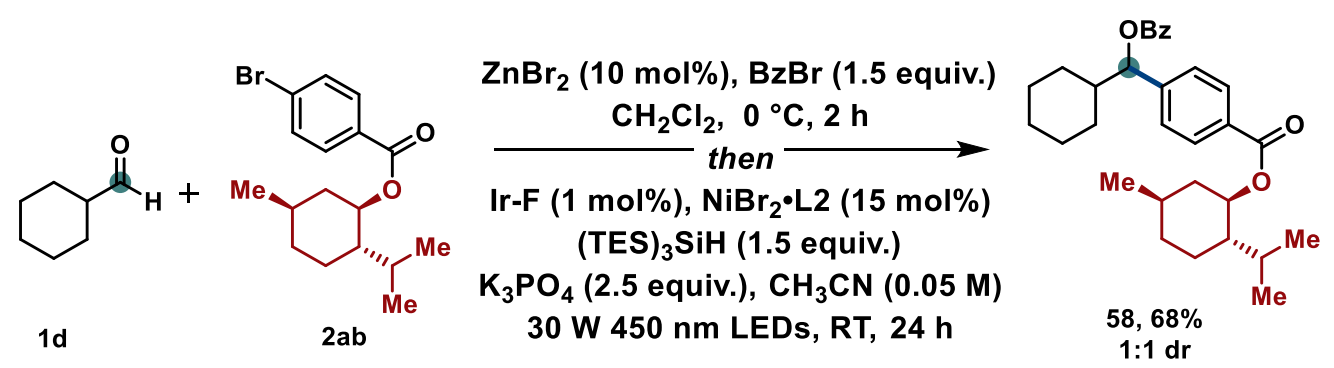

$(1 R, 2 S, 5 R)-2$-isopropyl-5-methylcyclohexyl

((benzoyloxy)(cyclohexyl)methyl)benzoate (58). According to the GP3, using the aldehyde $1 \mathrm{~d}$ ( $0.3 \mathrm{mmol}, 1.5$ equiv.), benzoyl bromide ( $0.3 \mathrm{mmol}, 1.5$ equiv.), aryl bromide $\mathbf{2} \mathbf{a b}$ (0.2 mmol, 1.0 equiv.), Ir-F ( $2 \mu \mathrm{mol}, 1 \mathrm{~mol} \%)$, NiBr $2 \bullet \mathrm{L} 2$ (30 $\mu \mathrm{mol}$, $15 \mathrm{~mol} \%$ ), (TES) $3 \mathrm{SiH}\left(0.3 \mathrm{mmol}, 1.5\right.$ equiv.), $\mathrm{K}_{3} \mathrm{PO}_{4}$ (0.5 mmol, 2.5 equiv.) and stirring at room temperature for $24 \mathrm{~h}$ under $30 \mathrm{~W}$ blue LEDs irradiation. The reaction mixture was filtered through a pad of silica gel and concentrated in vacuo, then purified by chromatography (pentane/EtOAc $=100 / 0 \rightarrow 60 / 1 \rightarrow 50 / 1$ ) to give 58 (73.9 mg, $20 \mathrm{~mol} \%$ of debrominated impurity, $0.136 \mathrm{mmol}, 68 \%, 1: 1 \mathrm{dr}$ ) as a colorless gum. $\mathbf{R} \mathbf{f}($ pentane $/$ EtOAc $=40 / 1)$ 0.65. Note. Integration in the aliphatic region cannot be accurately determined due to the presence of debrominated product signals. ${ }^{13} \mathrm{C}$ NMR signals attributable to a single diastereoisomer are quoted with *. ${ }^{1} \mathrm{H}$ NMR (400 MHz, $\left.\mathrm{CDCl}_{3}\right) \delta 8.12-8.07(\mathrm{~m}, 2 \mathrm{H}), 8.02(\mathrm{~d}, J=8.3 \mathrm{~Hz}, 2 \mathrm{H})$, $7.56(\mathrm{t}, J=7.1 \mathrm{~Hz}, 1 \mathrm{H}), 7.49-7.42(\mathrm{~m}, 4 \mathrm{H}), 5.77$ (apt dd, $J=7.2,3.2 \mathrm{~Hz}, 1 \mathrm{H}), 4.92$ (apt td, $J=10.9,4.3 \mathrm{~Hz}, 1 \mathrm{H}), 2.14-2.07$ (m, 1H), $1.99-1.87$ (m, 3H), $1.80-1.64$ 
(m), $1.58-1.49$ (m), $1.29-1.02$ (m), $0.94-0.86$ (m), 0.78 (apt dd, J = 7.0, 1.4 Hz, 3H). ${ }^{13}$ C NMR (101 MHz, $\left.\mathrm{CDCl}_{3}\right) \delta 166.0$ - $165.8(\mathrm{~m}), 144.8,133.2,130.4,130.1$, 129.7, 129.7, 128.5, $126.99\left(^{*}\right), 126.96\left(^{*}\right), 80.5,80.4,74.90\left(^{*}\right), 74.89\left(^{*}\right), 47.4$, $43.4\left(^{*}\right), 43.3\left(^{*}\right), 41.1,34.4,31.6,29.2,28.85\left(^{*}\right), 28.85\left(^{*}\right), 26.58\left(^{*}\right), 26.55\left(^{*}\right)$, 26.4, $26.02\left(^{*}\right), 25.98\left(^{*}\right), 23.72\left(^{*}\right), 23.70\left(^{*}\right), 22.2,20.9,16.60\left(^{*}\right), 16.58\left(^{*}\right)$. HRMS $\left(\mathrm{ESI}^{+}\right)$: calcd. for $\mathrm{C}_{31} \mathrm{H}_{40} \mathrm{O}_{4} \mathrm{Na}\left(\mathrm{M}+\mathrm{Na}^{+}\right)$: 499.2819. Found: 499.2817.
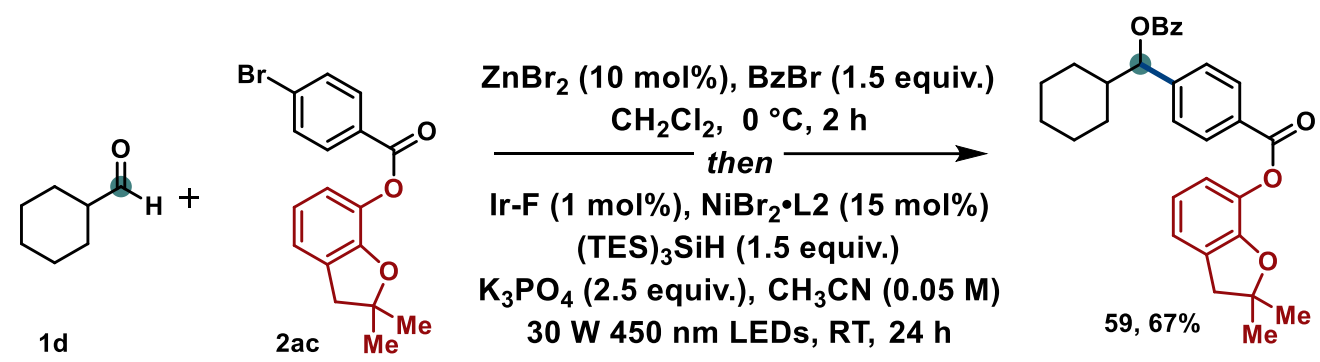

2,2-dimethyl-2,3-dihydrobenzofuran-7-yl

((benzoyloxy)(cyclohexyl)methyl)benzoate (59). According to the GP3, using the aldehyde $1 \mathbf{d}$ ( $0.3 \mathrm{mmol}, 1.5$ equiv.), benzoyl bromide ( $0.3 \mathrm{mmol}, 1.5$ equiv.), aryl bromide 2 ac $(0.2 \mathrm{mmol}, 1.0$ equiv.), Ir-F (2 $\mu \mathrm{mol}, 1 \mathrm{~mol} \%), \mathrm{NiBr}_{2} \bullet \mathrm{L} 2$ (30 $\mu \mathrm{mol}$, 15 mol\%), (TES) ${ }_{3} \mathrm{SiH}$ (0.3 mmol, 1.5 equiv.), $\mathrm{K}_{3} \mathrm{PO}_{4}$ (0.5 mmol, 2.5 equiv.) and stirring at room temperature for $24 \mathrm{~h}$ under $30 \mathrm{~W}$ blue LEDs irradiation. The reaction mixture was filtered through a pad of silica gel and concentrated in vacuo, then purified by chromatography (pentane/EtOAc $=18 / 1)$ to give $59(65.4 \mathrm{mg}$, $0.135 \mathrm{mmol}, 67 \%)$ as a colourless gum. $\mathbf{R}_{\boldsymbol{f}}($ pentane $/$ EtOAc $=18 / 1): 0.45 .{ }^{1} \mathbf{H} \mathbf{~ N M R}$ $\left(400 \mathrm{MHz} \mathrm{CDCl}_{3}\right) \delta 8.21(\mathrm{~d}, J=8.3 \mathrm{~Hz}, 2 \mathrm{H}), 8.15-8.07(\mathrm{~m}, 2 \mathrm{H}), 7.59(\mathrm{tt}, J=7.4,1.1$ $\mathrm{Hz}, 1 \mathrm{H}), 7.54-7.43(\mathrm{~m}, 4 \mathrm{H}), 7.04(\mathrm{dq}, J=7.3,1.2 \mathrm{~Hz}, 1 \mathrm{H}), 6.96(\mathrm{~d}, J=8.1 \mathrm{~Hz}, 1 \mathrm{H})$, $6.83(\mathrm{dd}, J=8.2,7.2 \mathrm{~Hz}, 1 \mathrm{H}), 5.81(\mathrm{~d}, J=7.1 \mathrm{~Hz}, 1 \mathrm{H}), 3.06(\mathrm{~s}, 2 \mathrm{H}), 2.02-1.89(\mathrm{~m}$, 2H), $1.83-1.65(\mathrm{~m}, 3 \mathrm{H}), 1.57$ (d, J = $12.6 \mathrm{~Hz}, 1 \mathrm{H}), 1.49-1.43(\mathrm{~m}, 6 \mathrm{H}), 1.33-1.04$ (m, 5H). ${ }^{13} \mathrm{C}$ NMR $\left(101 \mathrm{MHz}, \mathrm{CDCl}_{3}\right) \delta 165.9,164.1,150.5,145.6,134.6,133.2$, $130.5,130.3,129.9,129.8,129.0,128.6,127.1,122.7,121.6,120.2,88.4,80.4,43.4$, 43.2, 29.2, 28.9, 28.2, 26.3, 26.02, 25.97. HRMS (ESI+): calcd. for $\mathrm{C}_{31} \mathrm{H}_{32} \mathrm{O}_{5} \mathrm{Na}$ $\left(\mathrm{M}+\mathrm{Na}^{+}\right):$507.2142. Found: 507.2142. 

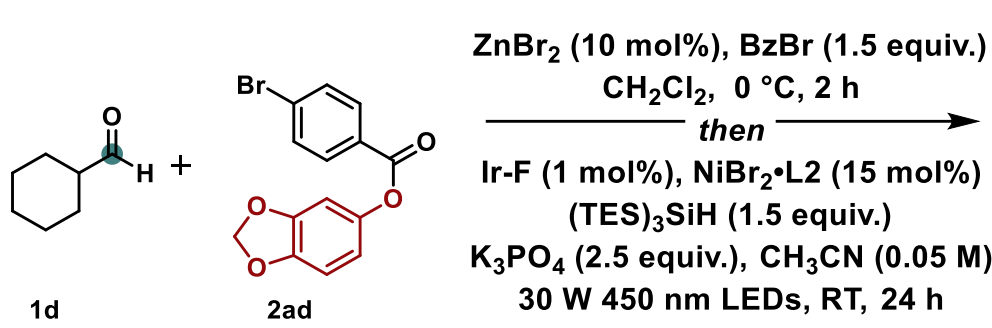

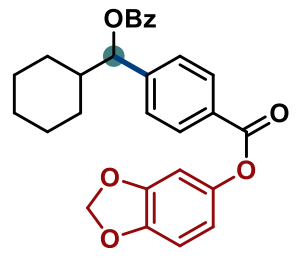

$60,72 \%$

benzo[d][1,3]dioxol-5-yl 4-((benzoyloxy)(cyclohexyl)methyl)benzoate (60). According to the GP3, using the aldehyde 1d (0.3 mmol, 1.5 equiv.), benzoyl bromide ( 0.3 mmol, 1.5 equiv.), aryl bromide 2 ad ( $0.2 \mathrm{mmol}, 1.0$ equiv.), Ir-F (2

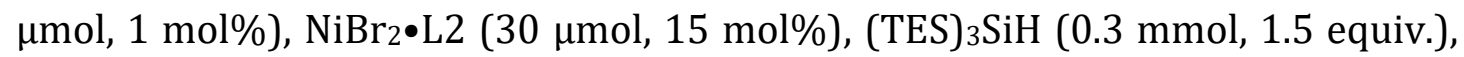
$\mathrm{K}_{3} \mathrm{PO}_{4}$ ( $0.5 \mathrm{mmol}, 2.5$ equiv.) and stirring at room temperature for $24 \mathrm{~h}$ under $30 \mathrm{~W}$ blue LEDs irradiation. The reaction mixture was filtered through a pad of silica gel and concentrated in vacuo, then purified by chromatography (pentane $/$ EtOAc $=$ $15 / 1)$ to give 60 (65.8 $\mathrm{mg}, 0.144 \mathrm{mmol}, 72 \%$ ) as a colourless gum. $\mathbf{R}_{\boldsymbol{f}}$ (pentane/EtOAc = 18/1): 0.40. ${ }^{1} \mathrm{H}$ NMR $\left(400 \mathrm{MHz}, \mathrm{CDCl}_{3}\right) \delta 8.16(\mathrm{~d}, J=8.5 \mathrm{~Hz}, 2 \mathrm{H})$, $8.13-8.07(\mathrm{~m}, 2 \mathrm{H}), 7.59(\mathrm{tt}, J=7.4,1.2 \mathrm{~Hz}, 1 \mathrm{H}), 7.53-7.44(\mathrm{~m}, 4 \mathrm{H}), 6.81(\mathrm{~d}, J=8.4$ $\mathrm{Hz}, 1 \mathrm{H}), 6.71(\mathrm{~d}, J=2.3 \mathrm{~Hz}, 1 \mathrm{H}), 6.63(\mathrm{dd}, J=8.4,2.4 \mathrm{~Hz}, 1 \mathrm{H}), 6.00$ (s, 2H), 5.81 (d, $J=7.0 \mathrm{~Hz}, 1 \mathrm{H}), 2.01-1.90(\mathrm{~m}, 2 \mathrm{H}), 1.85-1.62(\mathrm{~m}, 3 \mathrm{H}), 1.55(\mathrm{~d}, J=12.8 \mathrm{~Hz}, 1 \mathrm{H})$, $1.34-0.94(\mathrm{~m}, 5 \mathrm{H}) .{ }^{13} \mathrm{C}$ NMR $\left(101 \mathrm{MHz}, \mathrm{CDCl}_{3}\right) \delta$ 165.9, 165.3, 148.2, 145.8, 145.5, 145.3, 133.2, 130.3, 129.8, 128.9, 128.6, 127.2, 114.2, 108.1, 104.0, 101.8, 80.3, 43.4, 29.2, 28.8, 26.3, 26.01, 25.96. HRMS (ESI+): calcd. for $\mathrm{C}_{28} \mathrm{H}_{26} \mathrm{O}_{6} \mathrm{Na}\left(\mathrm{M}+\mathrm{Na}^{+}\right)$: 481.1622. Found: 481.1623.

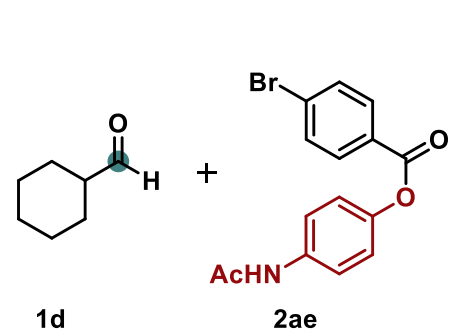

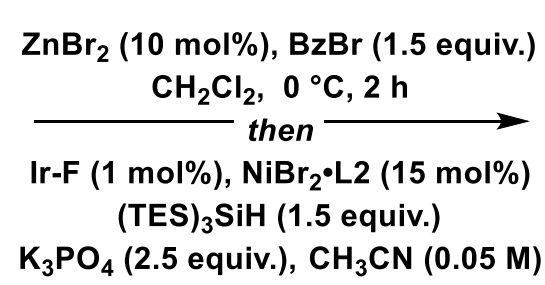

$30 \mathrm{~W} 450 \mathrm{~nm}$ LEDs, RT, $24 \mathrm{~h}$

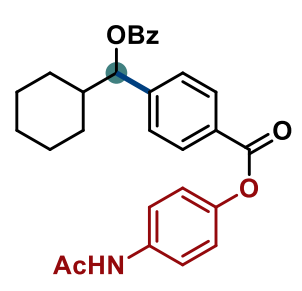

$61,83 \%$

\section{4-acetamidophenyl 4-((benzoyloxy)(cyclohexyl)methyl)benzoate}

(61).

According to the GP3, using the aldehyde 1d ( $0.3 \mathrm{mmol}, 1.5$ equiv.), benzoyl bromide ( 0.3 mmol, 1.5 equiv.), aryl bromide 2ae ( $0.2 \mathrm{mmol}, 1.0$ equiv.), Ir-F (2 


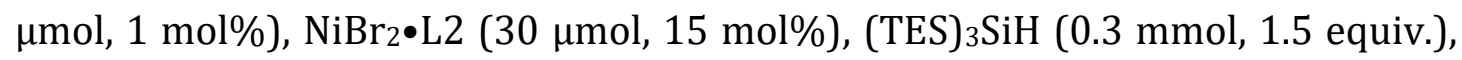
$\mathrm{K}_{3} \mathrm{PO}_{4}$ (0.5 mmol, 2.5 equiv.) and stirring at room temperature for $24 \mathrm{~h}$ under $30 \mathrm{~W}$ blue LEDs irradiation. The reaction mixture was filtered through a pad of silica gel and concentrated in vacuo, then purified by chromatography $($ pentane $/$ EtOAc $=$ 1/1) to give $61(78.0 \mathrm{mg}, 0.165 \mathrm{mmol}, 83 \%)$ as a light-yellow gum. $\mathbf{R}_{\boldsymbol{f}}$ (pentane/EtOAc = 1/1): 0.37. ${ }^{1} \mathrm{H}$ NMR $\left(400 \mathrm{MHz}, \mathrm{CDCl}_{3}\right) \delta 8.16(\mathrm{~d}, J=8.2 \mathrm{~Hz}, 2 \mathrm{H})$, $8.11(\mathrm{~d}, J=7.7 \mathrm{~Hz}, 2 \mathrm{H}), 8.03(\mathrm{~s}, 1 \mathrm{H}), 7.62-7.55(\mathrm{~m}, 1 \mathrm{H}), 7.53-7.44(\mathrm{~m}, 6 \mathrm{H}), 7.08$ $(\mathrm{d}, J=8.5 \mathrm{~Hz}, 2 \mathrm{H}), 5.80(\mathrm{~d}, J=6.9 \mathrm{~Hz}, 1 \mathrm{H}), 2.12(\mathrm{~s}, 3 \mathrm{H}), 2.01-1.89(\mathrm{~m}, 2 \mathrm{H}), 1.83-$ $1.63(\mathrm{~m}, 3 \mathrm{H}), 1.54(\mathrm{~d}, J=12.7 \mathrm{~Hz}, 1 \mathrm{H}), 1.31-1.08(\mathrm{~m}, 5 \mathrm{H}) .{ }^{13} \mathrm{C}$ NMR (101 MHz, $\left.\mathrm{CDCl}_{3}\right) \delta 168.8,166.0,165.4,146.9,145.9,136.1,133.3,130.3,129.7,128.8,128.7$, 128.6, 127.2, 122.0, 121.1, 80.4, 43.4, 29.2, 28.8, 26.3, 26.0, 25.9, 24.4. HRMS (ESI+): calcd. for $\mathrm{C}_{29} \mathrm{H}_{29} \mathrm{NO}_{5} \mathrm{Na}\left(\mathrm{M}+\mathrm{Na}^{+}\right)$: 494.1938. Found: 494.1937.
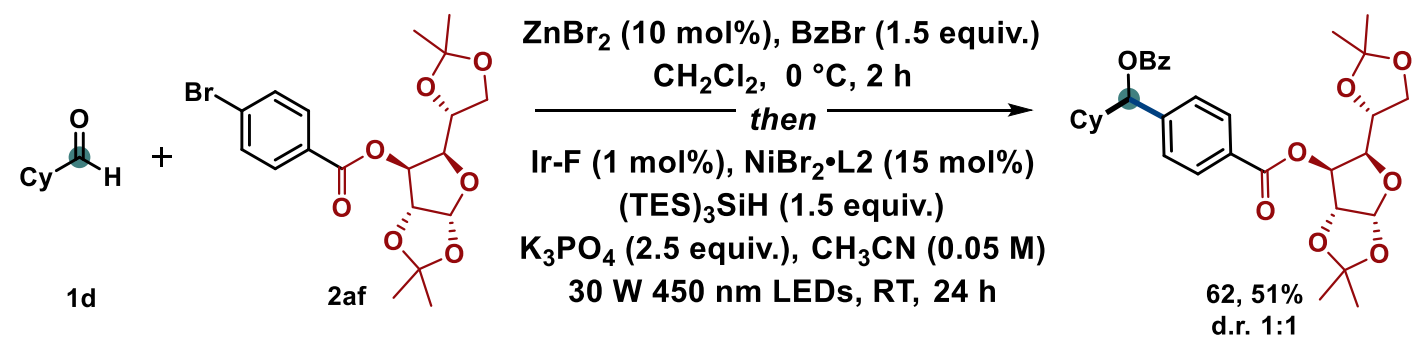

(3aR,6aR)-5-(2,2-dimethyl-1,3-dioxolan-4-yl)-2,2-dimethyltetrahydrofuro [2,3-d][1,3]dioxol-6-yl 4-((benzoyloxy)(cyclohexyl)methyl)benzoate (62). According to the GP3, using the cyclohexanecarbaldehyde $1 \mathbf{d}(0.3 \mathrm{mmol}$, 1.5 equiv.), benzoyl bromide ( $0.3 \mathrm{mmol}, 1.5$ equiv.), (3a $R, 5 R, 6 S, 6 \mathrm{a} R)-5-(2,2-$ dimethyl-1,3-dioxolan-4-yl)-2,2-dimethyltetrahydrofuro[2,3-d][1,3]dioxol-6-yl 4-bromobenzoate 2af (0.2 mmol, 1.0 equiv.), Ir-F ( $2 \mu \mathrm{mol}, 1 \mathrm{~mol} \%)$, NiBr $2 \bullet L 2$ (30 $\mu \mathrm{mol}, 15 \mathrm{~mol} \%$ ), (TES) ${ }_{3} \mathrm{SiH}$ (0.3 mmol, 1.5 equiv.), $\mathrm{K}_{3} \mathrm{PO}_{4}$ (0.5 mmol, 2.5 equiv.) and stirring at room temperature for $24 \mathrm{~h}$ under $30 \mathrm{~W}$ blue LEDs irradiation. The reaction mixture was filtered through a pad of silica gel and concentrated in vacuo, then purified by chromatography (pentane/EtOAc $=90 / 10 \rightarrow 88 / 12$ ) to give 62 (57.2 mg, $0.103 \mathrm{mmol}, \quad 51 \%$; d.r. $1: 1$ ) as a colorless solid. Rf $\left(\right.$ pentane/EtOAc = 9/1): 0.30. ${ }^{1} \mathbf{H}$ NMR $\left(400 \mathrm{MHz} \mathrm{CDCl}_{3}\right) \delta 8.14-8.04(\mathrm{~m}, 2 \mathrm{H}), 7.99$ 
$(\mathrm{d}, J=8.0 \mathrm{~Hz}, 2 \mathrm{H}), 7.62-7.54(\mathrm{~m}, 1 \mathrm{H}), 7.46(\mathrm{td}, J=8.0,1.8 \mathrm{~Hz}, 4 \mathrm{H}), 5.92(\mathrm{dd}, J=6.5$, $3.6 \mathrm{~Hz}, 1 \mathrm{H}), 5.76(\mathrm{dd}, J=7.1,3.5 \mathrm{~Hz}, 1 \mathrm{H}), 5.48(\mathrm{t}, J=2.4 \mathrm{~Hz}, 1 \mathrm{H}), 4.59(\mathrm{~d}, J=3.6 \mathrm{~Hz}$, 1H), $4.38-4.28(\mathrm{~m}, 2 \mathrm{H}), 4.13-4.06(\mathrm{~m}, 2 \mathrm{H}), 1.92(\mathrm{~d}, J=11.5 \mathrm{~Hz}, 2 \mathrm{H}), 1.71$ (ddd, $J$ $=33.1,16.2,7.8 \mathrm{~Hz}, 2 \mathrm{H}), 1.55(\mathrm{~s}, 3 \mathrm{H}), 1.53-1.46(\mathrm{~m}, 1 \mathrm{H}), 1.41(\mathrm{~s}, 3 \mathrm{H}), 1.31(\mathrm{~d}, J=$ $1.6 \mathrm{~Hz}, 3 \mathrm{H}), 1.27(\mathrm{~d}, J=6.2 \mathrm{~Hz}, 4 \mathrm{H}), 1.24-0.81(\mathrm{~m}, 5 \mathrm{H}) .{ }^{13} \mathbf{C ~ N M R}\left(101 \mathrm{MHz}, \mathrm{CDCl}_{3}\right)$ $\delta 165.9,165.9,165.1,165.0,145.8,133.3,130.3,129.9,129.9,129.8,129.8,129.0$, 129.0, 128.6, 127.2, 112.5, 109.6, 109.5, 105.3, 83.5, 80.4, 80.3, 80.1, 80.1, 76.8, 76.7, 72.7, 67.4, 67.3, 43.4, 43.4, 29.3, 28.9, 28.8, 27.0, 26.9, 26.3, 26.0, 26.0, 25.4. HRMS (ESI+): calcd. for $\mathrm{C}_{33} \mathrm{H}_{40}{ }_{9} \mathrm{Na}\left(\mathrm{M}^{+} \mathrm{Na}^{+}\right)$: 603.2570. Found: 603.2567 .

\subsection{Evaluation of the Grignard reaction}

In order to benchmark our newly developed reaction versus the venerable Grignard reaction, we selected four substrates that can potentially experience functional-group compatibility issues under Grignard reagent formation and reaction. In order to maximize tha chances of success, we employed the mild transmetallating conditions reported by Knochel and co-workers ${ }^{38}$ using $i$ PrMgCl-LiCl ("Turbo-Grignard"), which is known to offer greater functional group tolerance, in light of the highly controlled reaction conditions (low temperature, short reaction times).
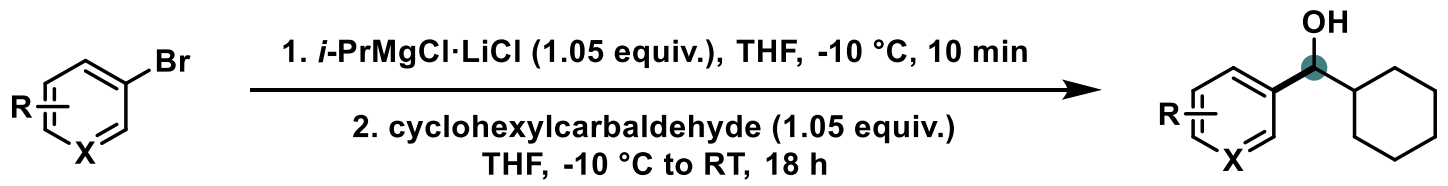

Procedure for the Grignard reagent formation and reaction. In an oven-dried

Schlenk tube equipped with a PTFE-coated stirring bar, $i$-PrMgCl-LiCl $1.3 \mathrm{M}$ in THF ( $250 \mu \mathrm{l}, 0.32 \mathrm{mmol}, 1.05$ equiv.) was charged under argon, then the solution was cooled to $-10{ }^{\circ} \mathrm{C}$. A solution of the appropriate aryl bromide $(0.30 \mathrm{mmol}, 1.00$ equiv.) in anhydrous THF ( $0.6 \mathrm{ml})$ was added dropwise over 5 minutes, then the reaction was stirred for additional 10 minutes. Cyclohexancarbaldehyde (38.8 $\mu \mathrm{l}$, $0.32 \mathrm{mmol}, 1.05$ equiv.) was added dropwise, then the reaction was gently warmed at room temperature and stirred for 18 hours. The reaction was carefully 
quenched with saturated aqueous $\mathrm{NH}_{4} \mathrm{Cl}$, then the reaction was filtered over a short plug of silica, thoroughly rinsing with EtOAc. The volatiles were removed in vacuo and the residue was analyzed by ${ }^{1} \mathrm{H} \mathrm{NMR}$, using mesitylene as internal standard.

\section{Results.}

Supplementary Table 1. Results of the attempted Grignard formation/reaction.

\begin{tabular}{cc}
\hline Bromide substrate & Yield \% ${ }^{\mathbf{a}}$ \\
\hline $\mathbf{4 0}$ & 4 \\
$\mathbf{5 2}$ & 17 \\
$\mathbf{5 4}$ & $<5$ \\
$\mathbf{5 9}$ & 2 \\
a Yield were determined by ${ }^{1}$ H NMR analysis using mesitylene as internal standard.
\end{tabular}

Comment. As shown in Supplementary Table 1, very low yields to complete absence of product was observed, thus testifying the potential utility of our strategy with challenging substrates under more estab lished Grignard reaction conditions. 


\section{Product derivatizations}

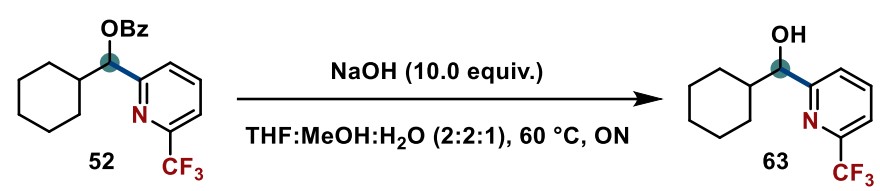

cyclohexyl(6-(trifluoromethyl)pyridin-2-yl)methanol (63). In a Schlenk tube equipped with a PTFE-coated stirring bar, 52 (36.3 mg, $0.100 \mathrm{mmol}, 1.00$ equiv.) and $\mathrm{NaOH}$ (40.0 mg, $1.00 \mathrm{mmol}, 10.0$ equiv.) were charged, followed by THF:MeOH: $\mathrm{H}_{2} \mathrm{O}(2: 2: 1,1 \mathrm{ml})$. The reaction was heathed at $60{ }^{\circ} \mathrm{C}$ overnight, then the reaction was cooled to room temperature and the volatiles were removed in vacuo. The residue was purified by flash column chromatograpgy on silica (pentane/EtOAc = 8/1 $\rightarrow 7 / 1)$, affording $63(22.8 \mathrm{mg}, 0.088 \mathrm{mmol}, 88 \%)$ as a colourless gum. $\mathbf{R}_{\mathbf{f}}($ pentane $/$ EtOAc $=10 / 1)=0.35 .{ }^{\mathbf{1}} \mathbf{H}\left\{{ }^{\mathbf{1}} \mathbf{F}\right\} \mathbf{N M R}\left(599 \mathrm{MHz}, \mathrm{CDCl}_{3}\right)$ $\delta 7.85(\mathrm{t}, J=7.8 \mathrm{~Hz}, 1 \mathrm{H}), 7.58(\mathrm{dd}, J=7.7,1.0 \mathrm{~Hz}, 1 \mathrm{H}), 7.44(\mathrm{~d}, J=7.8 \mathrm{~Hz}, 1 \mathrm{H}), 4.59$ (d, $J=5.0 \mathrm{~Hz}, 1 \mathrm{H}), 4.10-2.88(\mathrm{br}, 1 \mathrm{H},-\mathrm{OH}), 1.78-1.66(\mathrm{~m}, 3 \mathrm{H}), 1.66-1.56(\mathrm{~m}$, 2H), $1.52-1.45(\mathrm{~m}, 1 \mathrm{H}), 1.27-1.18(\mathrm{~m}, 2 \mathrm{H}), 1.17-1.07(\mathrm{~m}, 3 \mathrm{H}) .{ }^{13} \mathbf{C}\left\{{ }^{\mathbf{1}} \mathbf{H},{ }^{19} \mathbf{F}\right\} \mathbf{N M R}$ $\left(151 \mathrm{MHz}, \mathrm{CDCl}_{3}\right) \delta 162.8,147.0,137.7,124.1,121.6,119.0,77.3,45.1,29.7,26.8$, 26.4, 26.1. One ${ }^{13}$ C aliphatic signal missing due to overlap. ${ }^{19} \mathbf{F}\left\{{ }^{1} \mathbf{H}\right\}$ NMR $(564 \mathrm{MHz}$, $\left.\mathrm{CDCl}_{3}\right) \delta$-68.09. HRMS $\left(\mathrm{ESI}^{+}\right)$: calcd. for $\mathrm{C}_{13} \mathrm{H}_{16} \mathrm{NOF}_{3} \mathrm{Na}\left(\mathrm{M}+\mathrm{Na}^{+}\right): 282.1076$. Found: 282.1075.

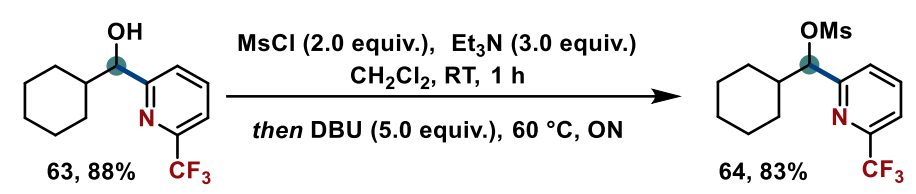

cyclohexyl(6-(trifluoromethyl)pyridin-2-yl)methyl methanesulfonate (64). In an oven-dried Schlenk tube equipped with a PTFE-coated stirring bar, 63 (22.8 mg, $0.088 \mathrm{mmol}, 1.00$ equiv.), Et3 $\mathrm{N}$ (38 $\mu \mathrm{l}, 0.274 \mathrm{mmol}, 3.00$ equiv.) were dissolved in dry $\mathrm{CH}_{2} \mathrm{Cl}_{2}(1 \mathrm{ml})$, then mesyl chloride (13 $\mu \mathrm{l}, 0.166 \mathrm{mmol}, 2.00$ equiv.) was added dropwise and the reaction was stirred at room temperature for 60 minutes, then DBU ( $66 \mu \mathrm{l}, 0.440 \mathrm{mmol}, 5.00$ equiv.) was added and the reaction was heated at $60^{\circ} \mathrm{C}$ overnight. Upon cooling to room temperature, the volatiles were removed in vacuo and the residue was purified by flash column chromatography on silica 
(pentane/EtOAc = 4/1), affording $64(24.6 \mathrm{mg}, 0.073 \mathrm{mmol}, 83 \%)$ as a colourless gum. Rf (pentane $/$ EtOAc $=4 / 1)=0.50 .{ }^{1} \mathbf{H} \mathbf{~ N M R}\left(400 \mathrm{MHz}, \mathrm{CDCl}_{3}\right) \delta 7.92(\mathrm{t}, J=7.9$ Hz, 1H), $7.67-7.59$ (m, 2H), 5.45 (d, J = 6.4 Hz, 1H), 2.92 (s, 3H), $2.10-1.98$ (m, 1H), $1.80-1.61(\mathrm{~m}, 4 \mathrm{H}), 1.54-1.44(\mathrm{~m}, 1 \mathrm{H}), 1.28-1.09$ (m, 5H). ${ }^{13}$ C NMR (101 $\left.\mathrm{MHz}, \mathrm{CDCl}_{3}\right) \delta 158.6,147.9(\mathrm{q}, J=34.9 \mathrm{~Hz}), 138.3,124.5,121.4(\mathrm{q}, J=274.4 \mathrm{~Hz}$ ), $119.9(\mathrm{q}, J=2.8 \mathrm{~Hz}), 87.4,42.5,38.6,29.2,27.6,26.1,25.9,25.7 .{ }^{19}$ F NMR (377 $\left.\mathrm{MHz}, \mathrm{CDCl}_{3}\right) \delta$-68.05. HRMS $\left(\mathrm{ESI}^{+}\right)$: calcd. for $\mathrm{C}_{14} \mathrm{H}_{18} \mathrm{NO}_{3} \mathrm{SF}_{3} \mathrm{Na}\left(\mathrm{M}+\mathrm{Na}^{+}\right): 360.0852$. Found: 360.0851 .
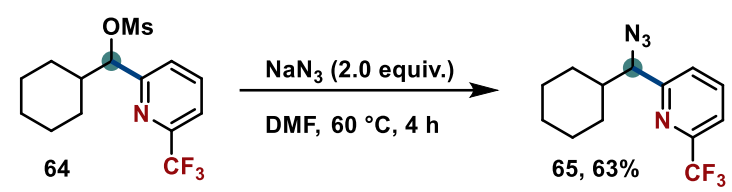

2-(azido(cyclohexyl)methyl)-6-(trifluoromethyl)pyridine (65). In a scintillation vial equipped with a PTFE-coated stirring bar, 64 (24.6 mg, 0.073 mmol, 1.00 equiv.) was dissolved in DMF (1 ml), then $\mathrm{NaN}_{3}(9.5 \mathrm{mg}, 0.146 \mathrm{mmol}$, 2.00 equiv.) was added and the reaction was heated at $60^{\circ} \mathrm{C}$ for 4 hours, then was cooled down to room temperature, partitioned between EtOAc: $\mathrm{H}_{2} \mathrm{O}(1: 1,20 \mathrm{ml})$, then the aqueous layer was extracted twice with EtOAc (10 ml each time). The combined organic layers were washed twice with water (10 ml each time), then dried over $\mathrm{MgSO}_{4}$ and the solvent was removed in vacuo. The residues was purified by flash column chromatography on silica (pentane $/$ EtOAc $=40 / 1$ ), affording 65 (13.0 mg, $0.046 \mathrm{mmol}, 63 \%)$ as a colourless oil. $\mathbf{R}_{\mathbf{f}}$ (pentane $/$ EtOAc $=$ $40 / 1)=0.40 .{ }^{1} \mathbf{H}\left\{{ }^{19} \mathbf{F}\right\}$ NMR $\left(500 \mathrm{MHz}, \mathrm{CDCl}_{3}\right) \delta 7.89(\mathrm{t}, J=7.8 \mathrm{~Hz}, 1 \mathrm{H}), 7.61(\mathrm{dd}, J=$ 7.8, $1.0 \mathrm{~Hz}, 1 \mathrm{H}$ ), 7.50 (dd, $J=8.0,1.0 \mathrm{~Hz}, 1 \mathrm{H}), 4.38$ (d, $J=7.2 \mathrm{~Hz}, 1 \mathrm{H}), 1.95-1.86$ (m, 1H), $1.83-1.73(\mathrm{~m}, 2 \mathrm{H}), 1.72-1.62(\mathrm{~m}, 2 \mathrm{H}), 1.45-1.38(\mathrm{~m}, 1 \mathrm{H}), 1.24-1.03$ $(\mathrm{m}, 5 \mathrm{H}) .{ }^{13} \mathbf{C}\left\{{ }^{1} \mathbf{H},{ }^{19} \mathbf{F}\right\} \mathbf{N M R}\left(126 \mathrm{MHz}, \mathrm{CDCl}_{3}\right) \delta 160.1,147.9,138.1,124.6,121.5$, 119.5, 72.1, 42.8, 30.0, 28.7, 26.3, 26.0, 25.9. ${ }^{19} \mathbf{F}\left\{{ }^{\mathbf{1}} \mathbf{H}\right\} \mathbf{N M R}\left(470 \mathrm{MHz}, \mathrm{CDCl}_{3}\right) \delta-$ 68.09. HRMS $\left(\mathrm{ESI}^{+}\right)$: calcd. for $\mathrm{C}_{13} \mathrm{H}_{15} \mathrm{~N}_{4} \mathrm{~F}_{3} \mathrm{Na}\left(\mathrm{M}+\mathrm{Na}^{+}\right)$: 307.1141. Found: 307.1141. 

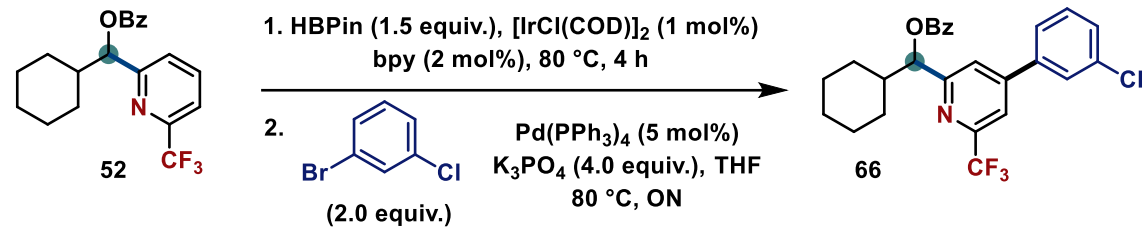

\section{(4-(3-chlorophenyl)-6-(trifluoromethyl)pyridin-2-yl)(cyclohexyl)methyl}

benzoate (66). In an oven-dried Schlenk tube equipped with a PTFE-coated stirring bar, 52 (36.3 mg, 0.100 mmol, 1.00 equiv.), 2,2'-bispyridine (0.3 mg, 0.002, $2 \mathrm{~mol} \%)$ and $\left[\mathrm{IrCl}(\mathrm{COD})_{2}\right](0.7 \mathrm{mg}, 0.001 \mathrm{mmol}, 1 \mathrm{~mol} \%)$ were charged, then the vessel was evacuated and back-filled three times with argon. HBPin (22 $\mu \mathrm{l}, 0.15$ mmol, 1.50 equiv.) was added and the reaction was heated at $80{ }^{\circ} \mathrm{C}$ for 4 hours, then cooled to room temperature and the volatiles were removed in vacuo. The residue was dissolved in THF $(1 \mathrm{ml})$, then $\mathrm{Pd}\left(\mathrm{PPh}_{3}\right) 4(5.8 \mathrm{mg}, 0.005 \mathrm{mmol}, 5 \mathrm{~mol} \%)$, $\mathrm{K}_{3} \mathrm{PO}_{4}$ (85 mg, $0.40 \mathrm{mmol}, 4.0$ equiv.) and 3-chlorobromobenzene (23.4 $\mu \mathrm{l}, 0.20$ mmol, 2.00 equiv.) were consecutively added, then the reaction was heated at $80{ }^{\circ} \mathrm{C}$ overnight. The reaction was cooled to room temperature, the volatiles removed in vacuo and the residue was purified by flash column chromatography on silica (pentane/EtOAc $=50 / 1)$, affording 66 (28.9 mg, $0.061 \mathrm{mmol}, 61 \%$ over two steps) as a colourless gum. $\mathbf{R}_{\mathbf{f}}($ pentane $/$ EtOAc $=50 / 1)=0.40 .{ }^{1} \mathbf{H} \mathbf{~ N M R}(599$ $\left.\mathrm{MHz} \mathrm{CDCl}_{3}\right) \delta 8.16-8.12(\mathrm{~m}, 2 \mathrm{H}), 7.73(\mathrm{~d}, J=1.5 \mathrm{~Hz}, 1 \mathrm{H}), 7.64(\mathrm{~d}, J=1.5 \mathrm{~Hz}, 1 \mathrm{H})$, $7.60(\mathrm{tt}, J=7.5,1.3 \mathrm{~Hz}, 1 \mathrm{H}), 7.58(\mathrm{dt}, J=1.9,0.9 \mathrm{~Hz}, 1 \mathrm{H}), 7.51-7.46(\mathrm{~m}, 3 \mathrm{H}), 7.46$ - $7.40(\mathrm{~m}, 2 \mathrm{H}), 5.99$ (d, J = 6.0 Hz, 1H), $2.27-2.19(\mathrm{~m}, 1 \mathrm{H}), 1.84-1.73(\mathrm{~m}, 3 \mathrm{H})$, $1.72-1.61(\mathrm{~m}, 2 \mathrm{H}), 1.34-1.15(\mathrm{~m}, 5 \mathrm{H}) .{ }^{13} \mathbf{C}\left\{{ }^{1} \mathbf{H}\right\} \mathbf{N M R}\left(151 \mathrm{MHz}, \mathrm{CDCl}_{3}\right) \delta 166.1$, $161.1,149.3,148.7$ (q, J = 34.6 Hz), 139.2, 135.5, 133.4, 130.7, 130.1, 129.9, 129.9, 128.7, 127.5, 125.6, 121.8, 121.6 (q, $J=274.6 \mathrm{~Hz}), 117.4$ (q, $J=2.8 \mathrm{~Hz}), 80.7,42.3$, 29.6, 27.9, 26.4, 26.1, 26.0. ${ }^{13} \mathbf{C}\left\{{ }^{1} \mathbf{H},{ }^{19} \mathbf{F}\right\}$ NMR $\left(151 \mathrm{MHz}, \mathrm{CDCl}_{3}\right) \delta 166.1,161.1$, 149.3, 148.7, 139.2, 135.5, 133.4, 130.7, 130.1, 129.91, 129.89, 128.7, 127.5, 125.6, 121.8, 121.6, 117.4, 80.7, 42.3, 29.6, 27.9, 26.4, 26.1, 26.0. ${ }^{19} \mathbf{F}\left\{{ }^{1} \mathbf{H}\right\}$ NMR (564 MHz, $\left.\mathrm{CDCl}_{3}\right) \delta$-67.83. HRMS (ESI+): calcd. for $\mathrm{C}_{26} \mathrm{H}_{23} \mathrm{NO}^{35} \mathrm{ClF}_{3} \mathrm{Na}\left(\mathrm{M}+\mathrm{Na}^{+}\right): 496.1262$. Found: 496.1260. 
<smiles>O=C(c1cc(-c2cccc(Cl)c2)cc(C(F)(F)F)n1)C1CCCCC1</smiles>

66

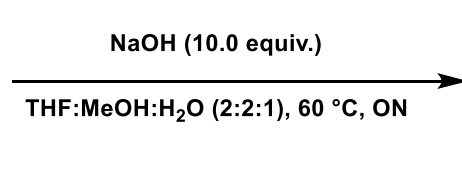

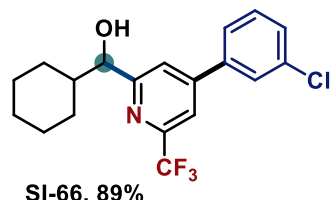

SI-66, 89\%

(4-(3-chlorophenyl)-6-(trifluoromethyl)pyridin-2-yl)(cyclohexyl)methanol

(SI-66). In a scintillation vial equipped with a PTFE-coated stirring bar, 66 (28.9 mg, $0.061 \mathrm{mmol}, 1.00$ equiv.) and $\mathrm{NaOH}$ (24.4 mg, $0.61 \mathrm{mmol}, 10.0$ equiv.) were charged, followed by THF:MeOH: $\mathrm{H}_{2} \mathrm{O}(2: 2: 1,1 \mathrm{ml})$. The reaction was heathed at $60{ }^{\circ} \mathrm{C}$ overnight, then the reaction was cooled to room temperature and the volatiles were removed in vacuo. The residue was purified by flash column chromatograpgy on silica (pentane/EtOAc $=12 / 1 \rightarrow 10 / 1)$, affording SI-66 (20.1 $\mathrm{mg}, 0.053 \mathrm{mmol}, 89 \%)$ as a colourless gum. $\mathbf{R}_{\mathbf{f}}($ pentane $/$ EtOAc $=12 / 1)=0.40 .{ }^{1} \mathbf{H}$ NMR (500 MHz, $\left.\mathrm{CDCl}_{3}\right) \delta 7.75(\mathrm{~d}, J=1.6 \mathrm{~Hz}, 1 \mathrm{H}), 7.65-7.63(\mathrm{~m}, 1 \mathrm{H}), 7.61(\mathrm{~d}, J=$ $1.6 \mathrm{~Hz}, 1 \mathrm{H}), 7.57-7.52(\mathrm{~m}, 1 \mathrm{H}), 7.49-7.44(\mathrm{~m}, 2 \mathrm{H}), 4.66(\mathrm{~d}, J=4.9 \mathrm{~Hz}, 1 \mathrm{H}), 4.10-$ 2.70 (br, 1H, OH), $1.81-1.70(\mathrm{~m}, 3 \mathrm{H}), 1.69-1.60(\mathrm{~m}, 2 \mathrm{H}), 1.58-1.50(\mathrm{~m}, 1 \mathrm{H}), 1.31$ - 1.08 (m, 5H). ${ }^{13} \mathbf{C}\left\{{ }^{1} \mathbf{H},{ }^{19} \mathrm{~F}\right\}$ NMR (126 MHz, $\left.\mathrm{CDCl}_{3}\right) \delta 163.8,149.3,147.9,139.0$, 135.6, 130.8, 130.0, 127.5, 125.5, 121.7, 121.6, 117.2, 77.7, 45.1, 29.8, 26.9, 26.4, 26.1. One aliphatic signal is missing due to overlap. ${ }^{19} \mathbf{F}\left\{{ }^{\mathbf{1}} \mathbf{H}\right\} \mathbf{N M R}\left(470 \mathrm{MHz}, \mathrm{CDCl}_{3}\right)$ $\delta$-67.94. HRMS $\left(\mathrm{ESI}^{+}\right)$: calcd. for $\mathrm{C}_{19} \mathrm{H}_{19} \mathrm{NO}^{35} \mathrm{ClF}_{3} \mathrm{Na}\left(\mathrm{M}+\mathrm{Na}^{+}\right)$: 392.1000. Found: 392.1000.
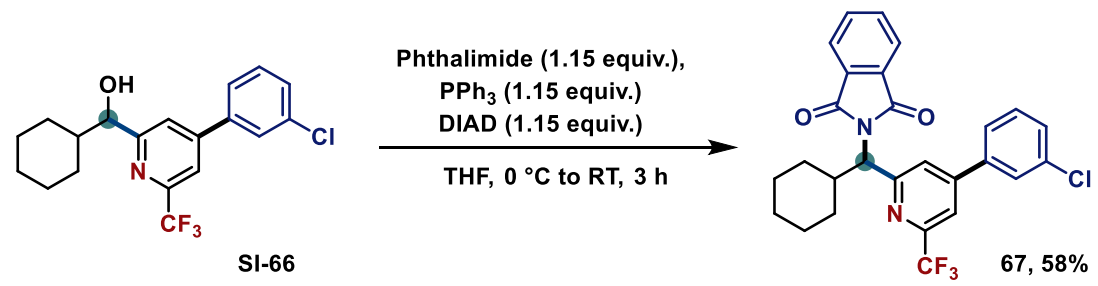

\section{2-((4-(3-chlorophenyl)-6-(trifluoromethyl)pyridin-2-}

yl)(cyclohexyl)methyl)isoindoline-1,3-dione (67). In a Scintillation vial equipped with a PTFE-coated stirring bar and a septum, substrate SI-66 (20.1 mg, $0.053 \mathrm{mmol}, 1.00$ equiv.), $\mathrm{PPh}_{3}(16.0 \mathrm{mg}, 0.061 \mathrm{mmol}, 1.15$ equiv.), phthalimide (9.0 mg, $0.061 \mathrm{mmol}, 1.15$ equiv.) were dissolved in dry THF ( $1 \mathrm{ml}$ ), then the solution was cooled to $0{ }^{\circ} \mathrm{C}$ and DIAD (12 $\mu \mathrm{l}, 0.061 \mathrm{mmol}, 1.15$ equiv.) was added dropwise. The reaction was warmed to room temperature and stirred for 3 hours, 
then the volatiles were removed in vacuo and the reaction was purified by flash column chromatography on silica (pentane/EtOAc $=15 / 1 \rightarrow 13 / 1$ ), affording 67 (15.5 mg, $0.031 \mathrm{mmol}, 58 \%)$ as a colourless gum. $\mathbf{R}_{\mathbf{f}}($ pentane $/$ EtOAc $=12 / 1)=$ 0.50. ${ }^{1} \mathbf{H}\left\{{ }^{19} \mathrm{~F}\right\}$ NMR $\left(500 \mathrm{MHz}, \mathrm{CDCl}_{3}\right) \delta 8.11(\mathrm{~d}, J=1.5 \mathrm{~Hz}, 1 \mathrm{H}), 7.84(\mathrm{dd}, J=5.4,3.0$ $\mathrm{Hz}, 2 \mathrm{H}), 7.73$ (d, $J=1.5 \mathrm{~Hz}, 1 \mathrm{H}), 7.71$ (dd, $J=5.5,3.0 \mathrm{~Hz}, 2 \mathrm{H}), 7.65-7.61(\mathrm{~m}, 1 \mathrm{H})$, $7.57-7.51(\mathrm{~m}, 1 \mathrm{H}), 7.49-7.43(\mathrm{~m}, 2 \mathrm{H}), 5.32(\mathrm{~d}, J=11.3 \mathrm{~Hz}, 1 \mathrm{H}), 2.96(\mathrm{qt}, J=11.2$, $3.3 \mathrm{~Hz}, 1 \mathrm{H}), 1.77-1.55(\mathrm{~m}, 5 \mathrm{H}), 1.38-1.01(\mathrm{~m}, 5 \mathrm{H}) .{ }^{13} \mathbf{C}\left\{{ }^{1} \mathbf{H}\right\}$ NMR (126 MHz, $\left.\mathrm{CDCl}_{3}\right) \delta 168.4,159.9,149.4,148.5(\mathrm{q}, J=34.7 \mathrm{~Hz}), 139.2,135.5,134.2,131.8$, $130.8,129.9,127.5,125.6,124.1,123.6,121.5$ (q, $J=274.7 \mathrm{~Hz}), 117.6$ (q, $J=2.8$ $\mathrm{Hz}), 62.3,37.0,31.0,30.1,26.3,25.7 .{ }^{13} \mathbf{C}\left\{{ }^{1} \mathbf{H},{ }^{19} \mathbf{F}\right\}$ NMR $\left(126 \mathrm{MHz}, \mathrm{CDCl}_{3}\right) \delta 168.4$, 159.9, 149.4, 148.5, 139.1, 135.5, 134.2, 131.8, 130.8, 129.9, 127.5, 125.6, 124.1, 123.6, 121.5, 117.6, 62.3, 37.0, 31.0, 30.1, 26.3, 25.7.19F\{ $\left.{ }^{1} \mathbf{H}\right\}$ NMR (470 MHz, $\left.\mathrm{CDCl}_{3}\right) \delta$-67.82. HRMS $\left(\mathrm{ESI}^{+}\right)$: calcd. for $\mathrm{C}_{27} \mathrm{H}_{22} \mathrm{~N}_{2} \mathrm{O}_{2}{ }^{35} \mathrm{ClF}_{3} \mathrm{Na}\left(\mathrm{M}+\mathrm{Na}^{+}\right): 521.1214$. Found: 521.1212.

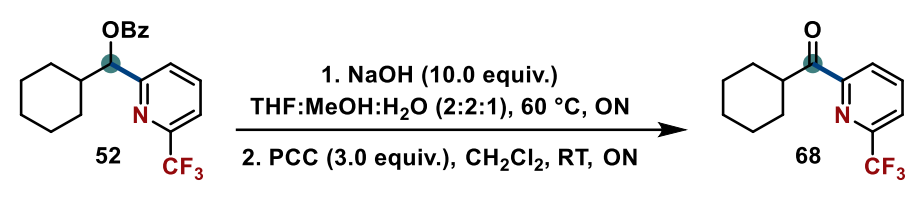

cyclohexyl(6-(trifluoromethyl)pyridin-2-yl)methanone (68). In a Schlenk tube equipped with a PTFE-coated stirring bar, 52 (36.3 mg, $0.100 \mathrm{mmol}, 1.00$ equiv.) and $\mathrm{NaOH}$ (40.0 mg, $1.00 \mathrm{mmol}, 10.0$ equiv.) were charged, followed by THF:MeOH: $\mathrm{H}_{2} \mathrm{O}(2: 2: 1,1 \mathrm{ml})$. The reaction was heathed at $60^{\circ} \mathrm{C}$ overnight, then the reaction was cooled to room temperature, partitioned between $\mathrm{CH}_{2} \mathrm{Cl}_{2}: \mathrm{H}_{2} \mathrm{O}$ $(1: 1,20 \mathrm{ml})$ and the layers were separated. The aqueous layer was extracted twice with $\mathrm{CH}_{2} \mathrm{Cl}_{2}$ (10 ml each time), then the combined organic layers were dried over $\mathrm{MgSO}_{4}$ and the solvent was removed in vacuo. The residue was dissolved in $\mathrm{CH}_{2} \mathrm{Cl}_{2}$ (1 ml), then pyridinium chlorochromate (64 $\mathrm{mg}, 0.30 \mathrm{mmol}, 3.00$ equiv.) was added and the reaction was stirred at room temperature for four hours, then filtered over a short pad of silica, rinsing with $\mathrm{CH}_{2} \mathrm{Cl}_{2}$. The volatiles were removed in vacuo and the residue was purified by flash column chromatography on silica 
(pentane/EtOAc $=20 / 1)$, affording 68 (20.5 $\mathrm{mg}, 0.080 \mathrm{mmol}, 80 \%$ over two steps) as a colourless gum. $\mathbf{R f}($ pentane $/$ EtOAc $=20 / 1)=0.60 .{ }^{1} \mathbf{H}\left\{{ }^{19} \mathbf{F}\right\} \mathbf{N M R}(500 \mathrm{MHz}$, $\left.\mathrm{CDCl}_{3}\right) \delta 8.19(\mathrm{dd}, J=7.9,1.0 \mathrm{~Hz}, 1 \mathrm{H}), 8.02(\mathrm{t}, J=7.8 \mathrm{~Hz}, 1 \mathrm{H}), 7.83(\mathrm{dd}, J=7.8,1.0$ Hz, 1H), $3.91-3.81$ (m, 1H), $1.97-1.88$ (m, 2H), $1.88-1.78$ (m, 2H), $1.78-1.70$ (m, 1H), $1.52-1.39(\mathrm{~m}, 4 \mathrm{H}), 1.34-1.19(\mathrm{~m}, 1 \mathrm{H}) .{ }^{13} \mathbf{C}$ NMR $\left(126 \mathrm{MHz}, \mathrm{CDCl}_{3}\right) \delta$ 203.9, $153.1,147.7(\mathrm{q}, J=35.1 \mathrm{~Hz}), 138.7,124.9(\mathrm{q}, J=1.0 \mathrm{~Hz}), 123.4(\mathrm{q}, J=2.7 \mathrm{~Hz})$, $121.4(\mathrm{q}, J=274.3 \mathrm{~Hz}), 44.3,28.9,26.2,25.8 .{ }^{13} \mathrm{C}\left\{{ }^{1} \mathrm{H},{ }^{19} \mathrm{~F}\right\} \mathrm{NMR}\left(126 \mathrm{MHz}, \mathrm{CDCl}_{3}\right) \delta$ 203.9, 153.1, 147.7, 138.7, 124.9, 123.4, 121.4, 44.3, 28.9, 26.2, 25.8. ${ }^{19} \mathbf{F}\left\{{ }^{1} \mathbf{H}\right\}$ NMR (470 MHz, $\left.\mathrm{CDCl}_{3}\right) \delta-68.06$. HRMS $\left(\mathrm{ESI}^{+}\right)$: calcd. for $\mathrm{C}_{13} \mathrm{H}_{14} \mathrm{NOF}_{3} \mathrm{Na}\left(\mathrm{M}+\mathrm{Na}^{+}\right)$: 280.0920. Found: 280.0920 .

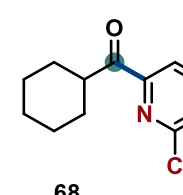

68

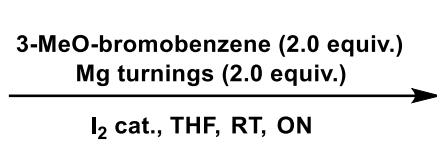

$\mathrm{I}_{2}$ cat., THF, RT, ON

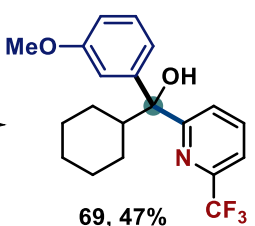

$69,47 \% \quad \mathrm{CF}_{3}$

\section{cyclohexyl(3-methoxyphenyl)(6-(trifluoromethyl)pyridin-2-yl)methanol}

(69). In an oven-dried Schlenk tube equipped with a PTFE-coated stirring bar, magnesium turnings ( $3.7 \mathrm{mg}, 0.146 \mathrm{mmol}, 2.00$ equiv.) were charged, then a small crystal of iodine was added, followed by dry THF $(1.0 \mathrm{ml})$. The suspension was stirred for 10 minutes, then 3-methoxybromobenzene (18.5 $\mu \mathrm{l}, 0.146 \mathrm{mmol}, 2.00$ equiv.) was added in one portion and the suspension was briefly heated at reflux to initiate the Grignard reagent formation, then the reaction was stirred at room temperature for 30 minutes. In a scintillation vial, ketone $68(24.0 \mathrm{mg}, 0.073 \mathrm{mmol}$, 1.00 equiv.) was dissolved in dry THF (0.5 ml), then the Grignard solution was added dropwise at room temperature, and the reaction was stirred overnight. The reaction was quenched with water (two drops), then the volatiles were removed in vacuo and the residue was purified by flash column chromatography on silica (pentane/EtOAc = 20/1), affording $69(12.3 \mathrm{mg}, 0.034 \mathrm{mmol}, 47 \%)$ as a colourless solid. $\mathbf{R}_{\mathbf{f}}($ pentane $/$ EtOAc $=20 / 1)=0.40 .{ }^{1} \mathbf{H}\left\{{ }^{19} \mathbf{F}\right\} \mathbf{~ N M R}\left(599 \mathrm{MHz}, \mathrm{CDCl}_{3}\right) \delta 7.81$ (dd, $J=8.1,7.6 \mathrm{~Hz}, 1 \mathrm{H}), 7.68(\mathrm{dd}, J=8.1,0.9 \mathrm{~Hz}, 1 \mathrm{H}), 7.51(\mathrm{dd}, J=7.6,0.9 \mathrm{~Hz}, 1 \mathrm{H}$ ), $7.26-7.22$ (m, 2H), 7.20 (ddd, $J=7.8,1.7,1.1 \mathrm{~Hz}, 1 \mathrm{H}$ ), 6.74 (ddd, $J=8.0,2.7,1.0$ 
$\mathrm{Hz}, 1 \mathrm{H}), 5.28(\mathrm{~s}, 1 \mathrm{H}), 3.79(\mathrm{~s}, 3 \mathrm{H}), 2.48-2.38(\mathrm{~m}, 1 \mathrm{H}), 1.78-1.62(\mathrm{~m}, 3 \mathrm{H}), 1.61-$ $1.55(\mathrm{~m}, 1 \mathrm{H}), 1.34-1.13(\mathrm{~m}, 6 \mathrm{H}) .{ }^{13} \mathbf{C}\left\{{ }^{19} \mathrm{~F}\right\}$ NMR $\left(151 \mathrm{MHz}, \mathrm{CDCl}_{3}\right) \delta 164.7,159.8$, 146.9, 145.9, 138.4, 129.4, 123.6, 121.4, 118.6, 118.3, 112.4, 112.1, 80.0, 55.3, 46.6, 26.9, 26.8, 26.6, 26.5. ${ }^{19} \mathbf{F}\left\{{ }^{1} \mathbf{H}\right\}$ NMR (564 MHz, $\left.\mathrm{CDCl}_{3}\right) \delta$-68.04. HRMS (ESI $)$ : calcd. for $\mathrm{C}_{20} \mathrm{H}_{22} \mathrm{NO}_{2} \mathrm{~F}_{3} \mathrm{Na}\left(\mathrm{M}+\mathrm{Na}^{+}\right): 388.1495$. Found: 388.1493.

\section{Troubleshooting: Frequently Asked Questions}

1. Is the reaction air and moisture sensitive? Which precautions should be taken to ensure reproducible yields?

The reaction proved sensitive towards the presente of oxygen, to the extent that sparging with inert gas is required. Further freeze-pump-thaw is convenient to avoid any need of degassing liquid reagents by any other mean. Moisture should be avoided, as commonly encountered in nickel catalysis. We used commercially available dry solvents, stored under molecular sieves and inert atmosphere.

\section{Is using excess silane needed to ensure optimal yields?}

Yes, we observed that a small excess of silane ensures complete conversion of the bromide to the ketyl radical through halogen atom transfer step.

\section{Are pre-formed $\mathrm{Ni}(\mathrm{II})$ compleses required?}

While we observed slightly improved yields when using pre formed Ni(II) complex rather than Ni precursor-ligand mixtures, the in situ formation can be employed. For the interested reader, we show some preliminary optimization results with different $\mathrm{Ni}(\mathrm{II})$ sources:

\begin{tabular}{ccc}
\hline Ni(II) source & Ligand & Yield \% \\
\hline $\mathrm{NiCl}_{2}(10 \mathrm{~mol} \%)$ & L2 $(12 \mathrm{~mol} \%)$ & $<5$ \\
$\mathrm{NiBr}_{2} \cdot$ glyme $(10 \mathrm{~mol} \%)$ & $\mathrm{L} 2(12 \mathrm{~mol} \%)$ & 40 \\
$\mathrm{Ni}$ (acac) 2 & L2 $(12 \mathrm{~mol} \%)$ & 8
\end{tabular}




\section{Can I lower the catalyst's loadings?}

Using $5 \mathrm{~mol} \%$ of nickel complex has also been performed and we provided comparatively lower yield. As nickel is comparatively cheap, we aimed at maintaining the iridium loading as low as possible, while increasing the nickel loading to maintain good conversion towards the desired product.

\section{Is filtration of the reaction required?}

In general, the reaction mixture could be directly evaporated and purified by column chromatography without the need of additional filtration, which proved useful only in case of NMR analysis of the crude mixture.

\section{Can the reaction be performed in a single flask?}

The reaction can be performed by adding-after the aldehyde activation-the other reaction components in the same flask with the solvent (one pot). We opted for the transfer of the aldehyde activation solution in order to simplify the set-up when multiple reactions featuring the same aldehyde were performed.

\section{Can benzaldehydes and related carbonyls react in the process?}

We observed lack of activation by formation of the $\alpha$-oxy bromides when benzaldehyde was attempted as coupling partner. Interestingly, benzaldehydesin particular bearing significant steric bulk-were excellent reactive partner under Weix's conditions. ${ }^{39}$

\section{Can ketones be used as coupling partners?}

Under the optimized reaction conditions, ketones did not afford synthetically useful yields of the corresponding arylated product. We attempted-without success-both cyclic and acyclic symmetric ketones, as well as methylphenethylketone. 


\section{Mechanistic investigation}

\subsection{Synthesis of oxidative addition complex Ni-I}
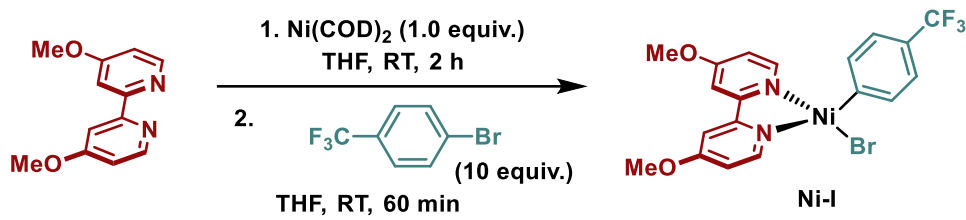

The compound was synthesized in accordance with the procedure reported by Martin and co-workers. ${ }^{40}$

An oven-dried Schlenk tube equipped with a PTFE-coated stirring bar was charged with $\mathrm{Ni}(\mathrm{COD})_{2}$ (138 mg, $0.50 \mathrm{mmol}, 1.00$ equiv.) in an argon filled glovebox, then the vessel was brought out and connected to the argon-vacuum manifold. 4,4'-dimethoxy-2,2'-bispyridine (118 mg, $0.50 \mathrm{mmol}, 1.00$ equiv.) was charged under argon, followed by the addition of dry and degassed THF ( $5 \mathrm{ml}$ ) under vigorous stirring. The stirring was continued at room temperature for 2 hours (the solution turns almost immediately to dark purple), then 4bromotrifluorotoluene $(0.70 \mathrm{ml}, 5.0 \mathrm{mmol}, 10.0$ equiv.) was added dropwise (the reaction turns to yellow-orange and becomes cloudy) and the reaction was stirred at room temperature for an additional hour. The reaction was diluted with dry hexane $(20 \mathrm{ml})$, causing the precipitation of a fine precipitate, which was collected by filtration over a frit (porosity 4), rinsed three times with dry hexane $(10 \mathrm{ml}$ each time) and thoroughly dried in vacuo to afford the Ni(II) complex Ni-I as a bright yellow solid (202 mg, $0.40 \mathrm{mmol}, 81 \%$ ). The analytical data were consistent with the previously reported ones. ${ }^{40}$

\subsection{Use of different catalytic systems}
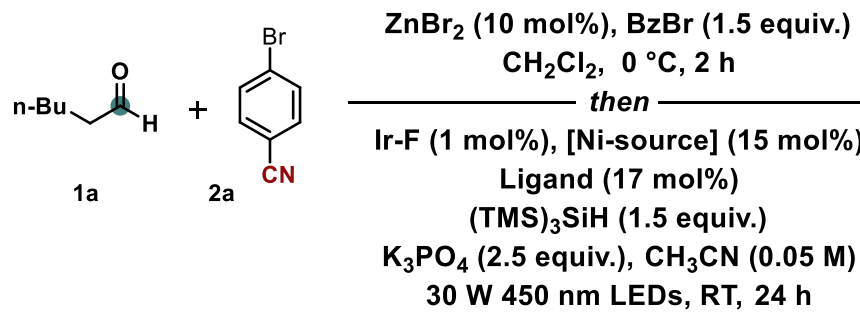
According to the GP3, using aldehyde $1 \mathrm{a}(0.15 \mathrm{mmol}$, 1.5 equiv.), benzoyl bromide ( 0.15 mmol, 1.5 equiv.), aryl bromide $2 a(0.1 \mathrm{mmol}, 1.0$ equiv.), Ir-F ( $1 \mu \mathrm{mol}, 1$ mol\%), the appropriate nickel source (0.015 mmol, $15 \mathrm{~mol} \%)$, the appropriate ligand (if needed, $0.017 \mathrm{mmol}, 17 \mathrm{~mol} \%$ ), (TES) 3 SiH (0.15 mmol, 1.5 equiv.), $\mathrm{K}_{3} \mathrm{PO}_{4}$ ( $0.25 \mathrm{mmol}, 2.5$ equiv.) and stirring at room temperature for $24 \mathrm{~h}$ under $30 \mathrm{~W}$ blue LEDs irradiation. The reaction mixture was filtered through a pad of silica gel and concentrated in vacuo, then was analyzed by means of ${ }^{1} \mathrm{H}$ NMR (using dibromomethane as internal standard).

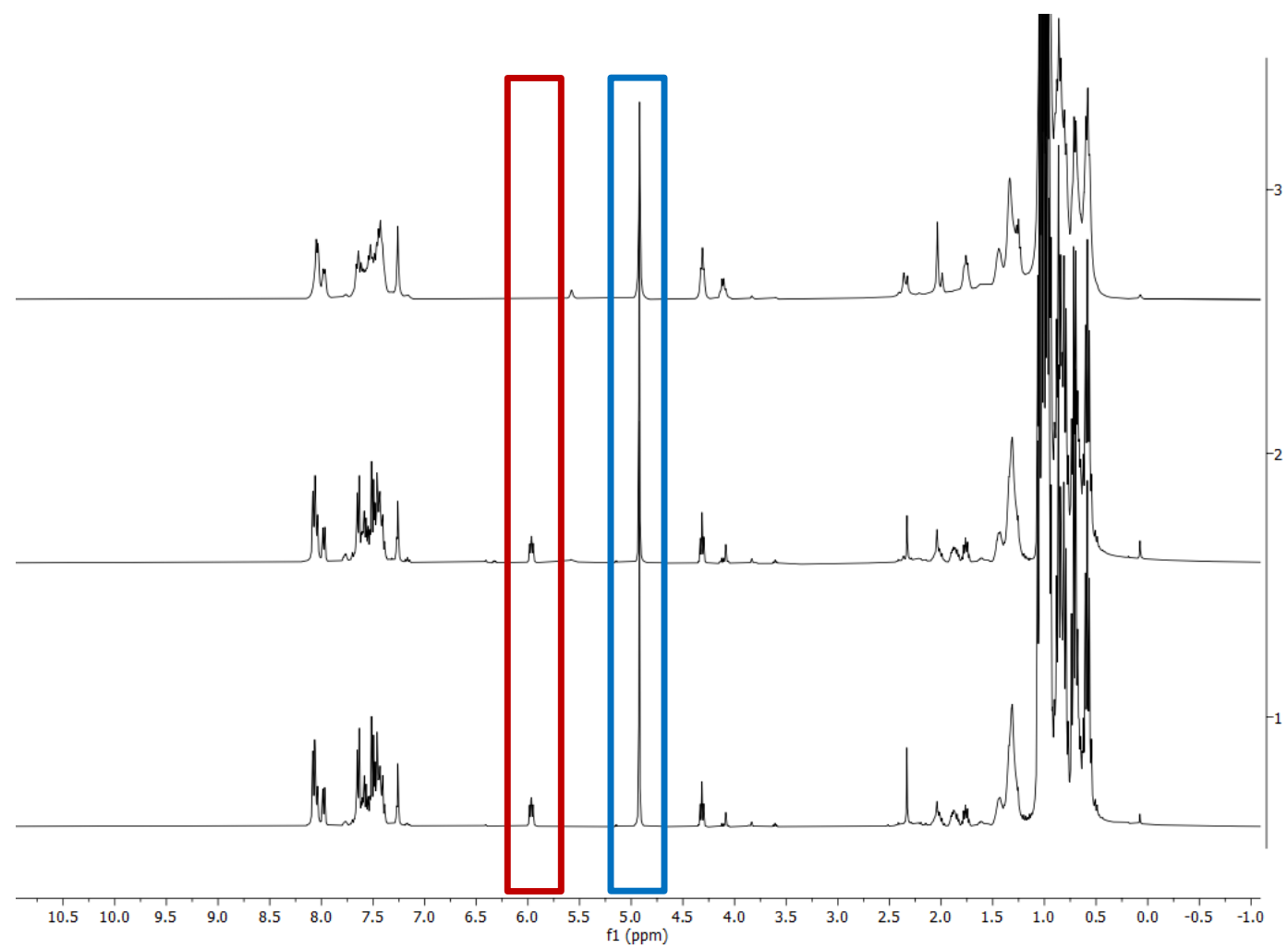

Supplementary Figure 3. ${ }^{1} \mathrm{H}$ NMR $\left(400 \mathrm{MHz}, \mathrm{CDCl}_{3}\right)$ of (1) entry 1, (2) entry 2; (3) entry 3. Red box: product 3; blue box: dibromomethane.

Supplementary Table 2. Results upon use of different catalytic systems

\begin{tabular}{|c|c|c|c|}
\hline Entry & [Ni source] & ligand & yield \\
\hline 1 & $\mathrm{Ni}(\mathrm{COD})_{2}(15 \mathrm{~mol} \%)$ & - & $<5 \%$ \\
\hline 2 & $\mathrm{Ni}(\mathrm{COD})_{2}(15 \mathrm{~mol} \%)$ & $\mathbf{L 2}(17 \mathrm{~mol} \%)$ & $48 \%$ \\
\hline 3 & $\mathrm{Ni}-\mathrm{I}(15 \mathrm{~mol} \%)$ & - & $57 \%$ \\
\hline
\end{tabular}




\subsection{Stoichiometric reaction of $\mathrm{Ni}(\mathrm{II})$-oxidative addition complex}

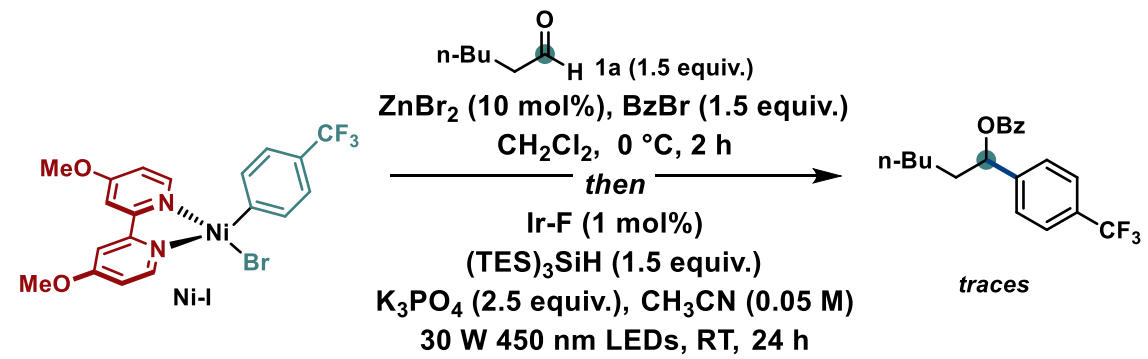

In an oven-dried Schlenk tube equipped with a PTFE-coated stirring bar, $\mathrm{ZnBr}_{2}$ ( $0.015 \mathrm{mmol}, 10 \mathrm{~mol} \%$ ) was charged under air, then the vessel was evacuated and back-filled three times with argon. Dry $\mathrm{CH}_{2} \mathrm{Cl}_{2}(0.1 \mathrm{ml})$ was added, then the solution was cooled to $0{ }^{\circ} \mathrm{C}$ and benzoyl bromide ( $0.15 \mathrm{mmol}, 1.50$ equiv.) was added dropwise, followed by the appropriate aldehyde $\mathbf{1 a}(0.15 \mathrm{mmol}, 1.50$ equiv.). The reaction was stirred at $0{ }^{\circ} \mathrm{C}$ for two hours. In another oven-dried Schlenk tube equipped with a PTFE-coated stirring bar, Ir-F (1 $\mu \mathrm{mol}, 1 \mathrm{~mol} \%)$, NiI ( 0.1 mmol, 1.0 equiv.), $\mathrm{K}_{3} \mathrm{PO}_{4}$ ( $0.25 \mathrm{mmol}, 2.50$ equiv.) were charged under air, then the vessel was evacuated and back-filled three times with argon. Dry MeCN ( $1 \mathrm{ml}, 0.075 \mathrm{M}$ ) was added, then the bromide solution was transferred via syringe, rinsing with $\mathrm{MeCN}\left(1 \mathrm{ml}, 0.075 \mathrm{M}\right.$ ), followed by (TES) ${ }_{3} \mathrm{SiH}$ ( $0.15 \mathrm{mmol}, 1.50$ equiv.). The reaction was further degassed by freeze-pump-thaw (three times), then sealed and irradiated with 30W $450 \mathrm{~nm}$ LEDs using the standard set-up. After 24 hours, the irradiation was interrupted, then the reaction was filtered through a short pad of silica, rinsing with EtOAc. The volatiles were removed in vacuo, then the crude residue was analyzed by ${ }^{1} \mathrm{H}$ NMR. 


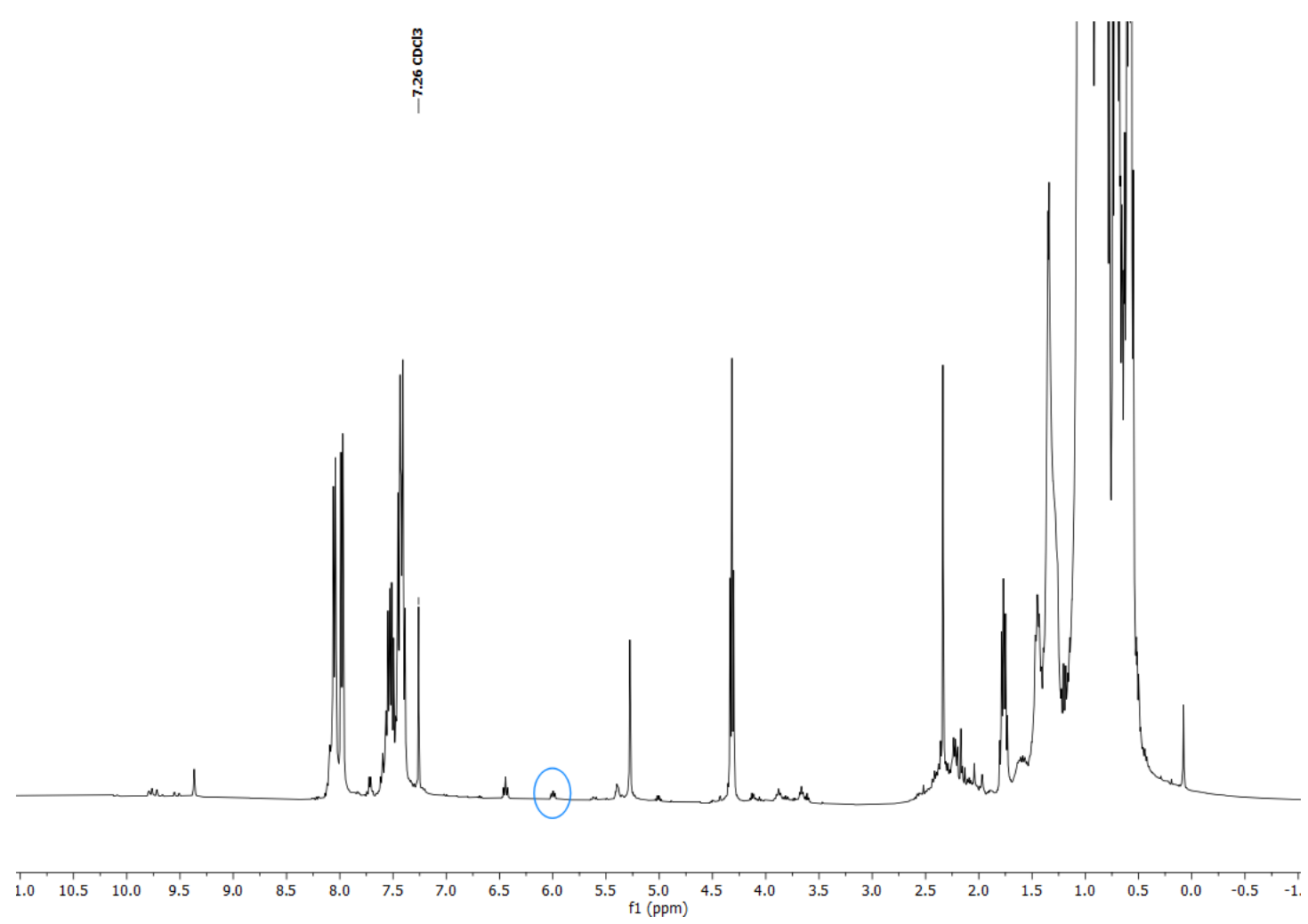

Supplementary Figure 4. ${ }^{1} \mathrm{H}$ NMR spectrum $\left(\mathrm{CDCl}_{3}, 400 \mathrm{MHz}\right)$ of the stoichiometric reaction of $\mathrm{Ni}(\mathrm{II})$-oxidative addition complex.

Comment. As visible in Supplementary Figure 4, only trace amounts of coupling product could be detected. We can hypothesize that the catalytic turnover that generates $\mathrm{H}$-atom abstracting species cannot be operative under the reaction conditions. Is is known that nickel species can generate radicals from alkyl halides, ${ }^{41}$ thus potentially explaining the observation of trace product formation.

\subsection{Radical probe experiment}

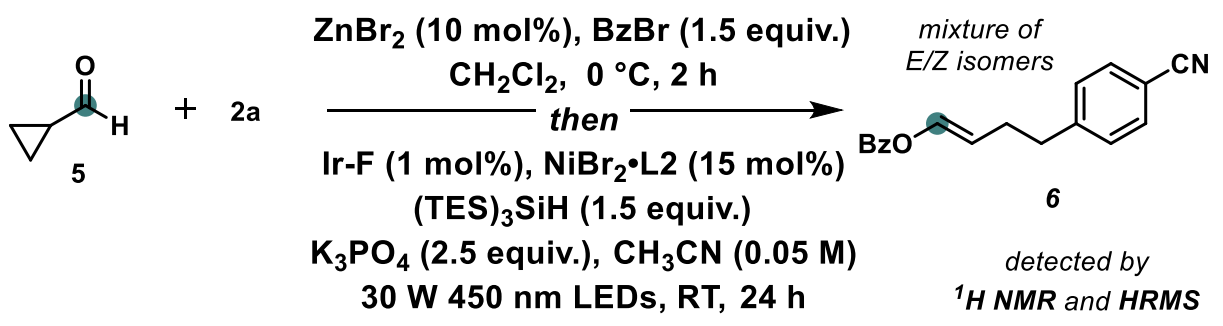

(Z)-4-(4-cyanophenyl)but-1-en-1-yl benzoate (6). According to the GP3, using the aldehyde 5 ( $0.3 \mathrm{mmol}, 1.5$ equiv.), benzoyl bromide ( $0.3 \mathrm{mmol}, 1.5$ equiv.), aryl bromide 2 a (0.2 mmol, 1.0 equiv.), Ir-F ( $2 \mu \mathrm{mol}, 1 \mathrm{~mol} \%)$, NiBr $2 \bullet L 2(30 \mu \mathrm{mol}, 15$ mol\%), (TES) ${ }_{3} \mathrm{SiH}$ (0.3 mmol, 1.5 equiv.), $\mathrm{K}_{3} \mathrm{PO}_{4}$ ( $0.3 \mathrm{mmol}, 1.5$ equiv.) and stirring 
at room temperature for $24 \mathrm{~h}$ under $30 \mathrm{~W}$ blue LEDs irradiation. The reaction mixture was filtered through a pad of silica gel and concentrated in vacuo, then partially purified by chromatography (pentane/EtOAc $=94 / 6)$ to give $6(11 \mathrm{mg}$, as a mixture of $E / Z$ isomers) as a colorless oil. $\mathbf{R}_{\mathbf{f}}$ (pentane $/$ EtOAc $\left.=94 / 6\right) 0.22$. HRMS (ESI ${ }^{+}$): calcd. for $\mathrm{C}_{18} \mathrm{H}_{15} \mathrm{NO}_{2} \mathrm{Na}\left(\mathrm{M}+\mathrm{Na}^{+}\right)$: 300.0995. Found: 300.0994 . The ${ }^{1} \mathrm{H}$ NMR spectra clearly show the alkene peaks and the main correlations are established by means of 2D NMR.

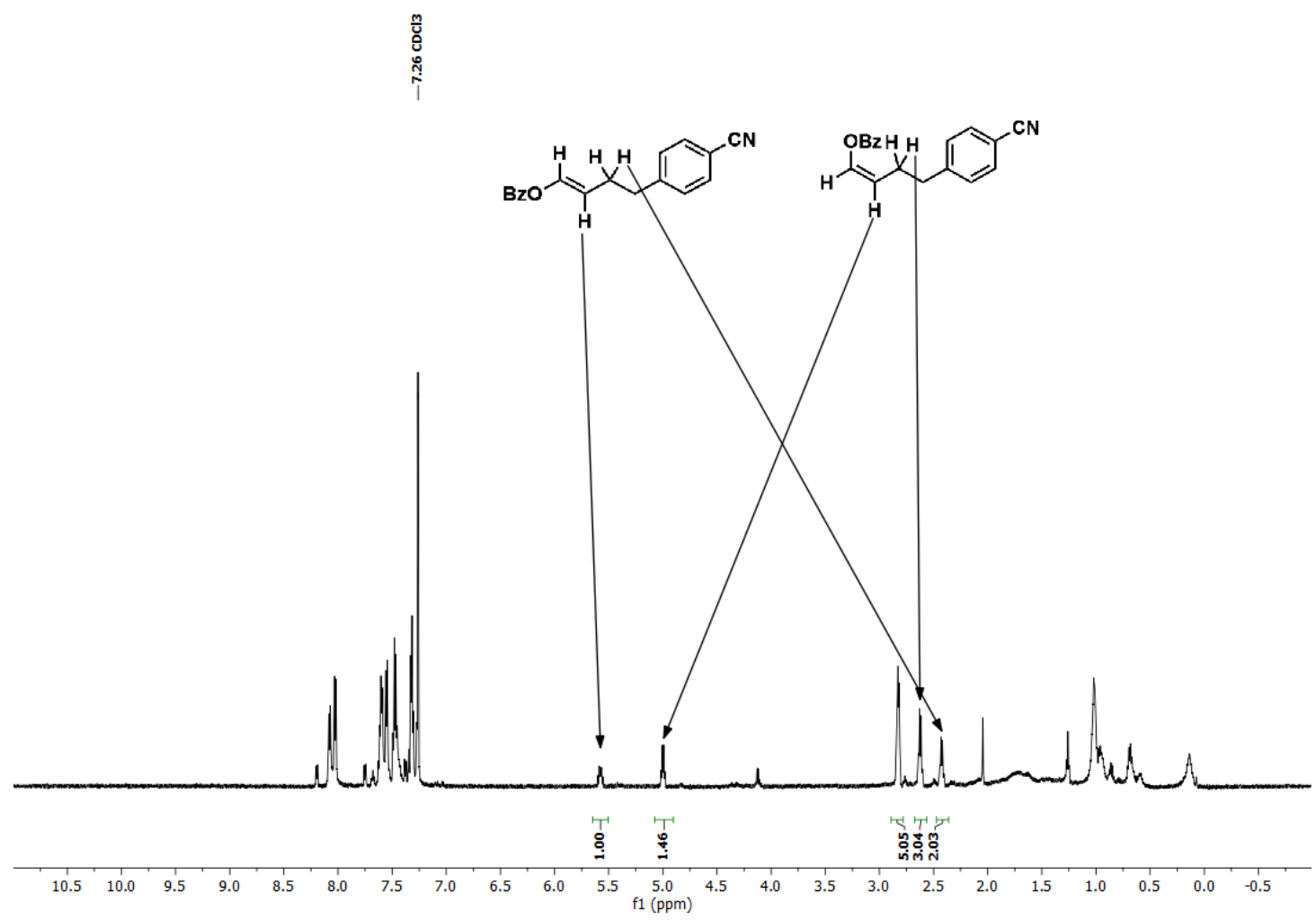

Supplementary Figure $5 .{ }^{1} \mathrm{H}$ NMR $\left(600 \mathrm{MHz}, \mathrm{CDCl}_{3}\right)$ of the alkene product. 


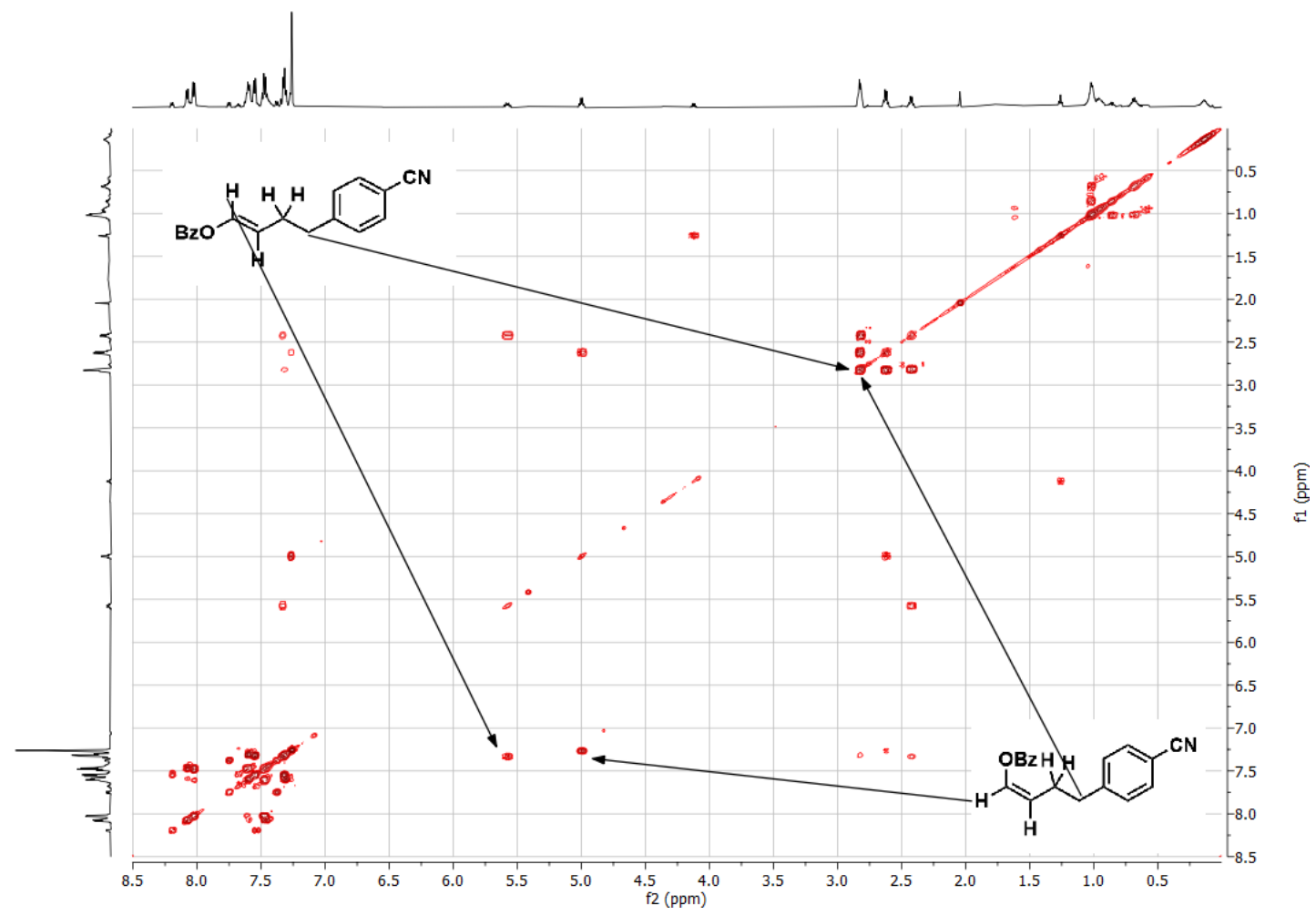

Supplementary Figure $6 .{ }^{1} \mathrm{H}^{-1} \mathrm{H} \operatorname{COSY}\left(600 \mathrm{MHz}, \mathrm{CDCl}_{3}\right)$ of the alkene product.

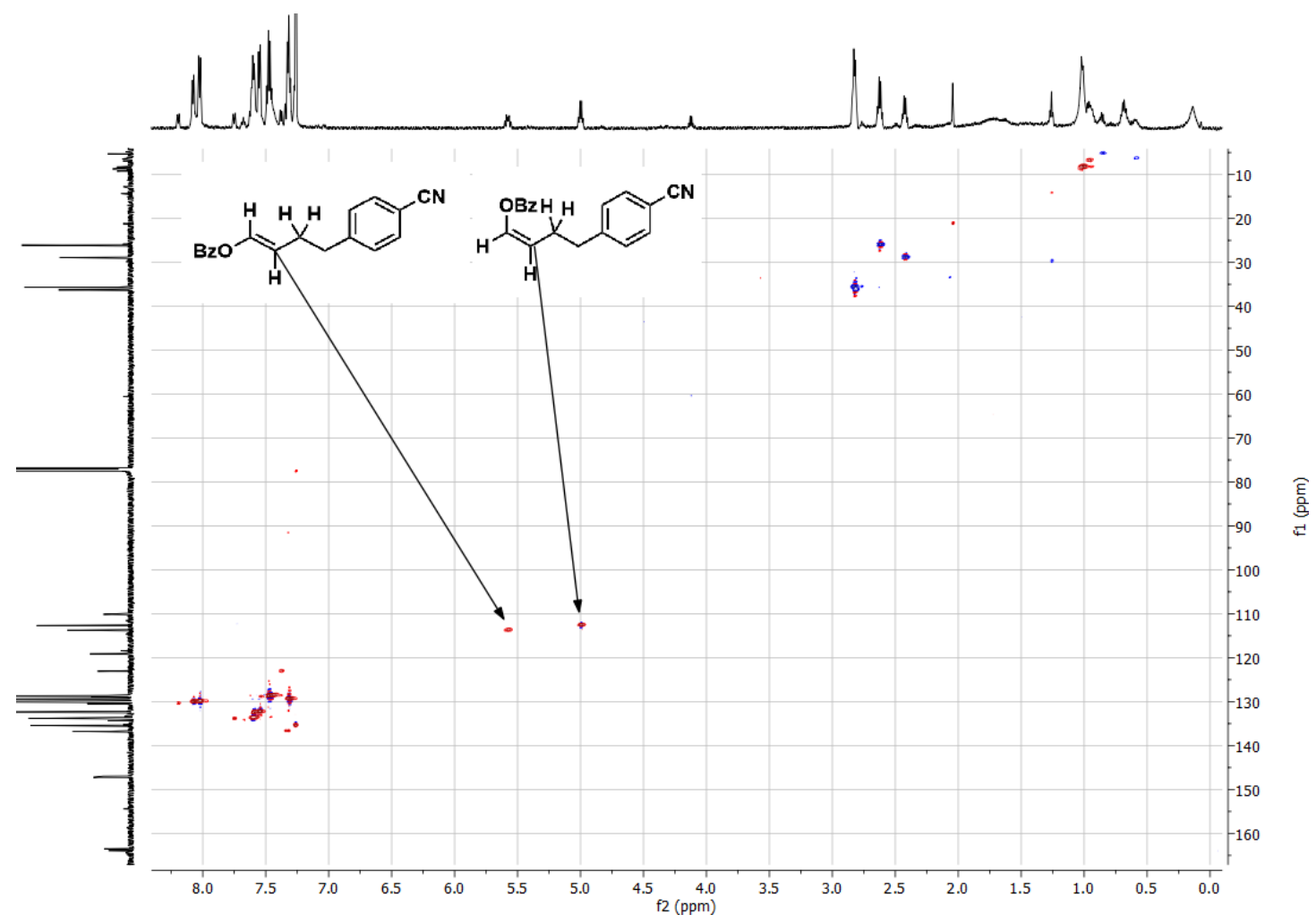

Supplementary Figure $7 .{ }^{1} \mathrm{H}^{-13} \mathrm{C} \mathrm{HSQC}\left(600 \mathrm{MHz}, \mathrm{CDCl}_{3}\right)$ of the alkene product. 


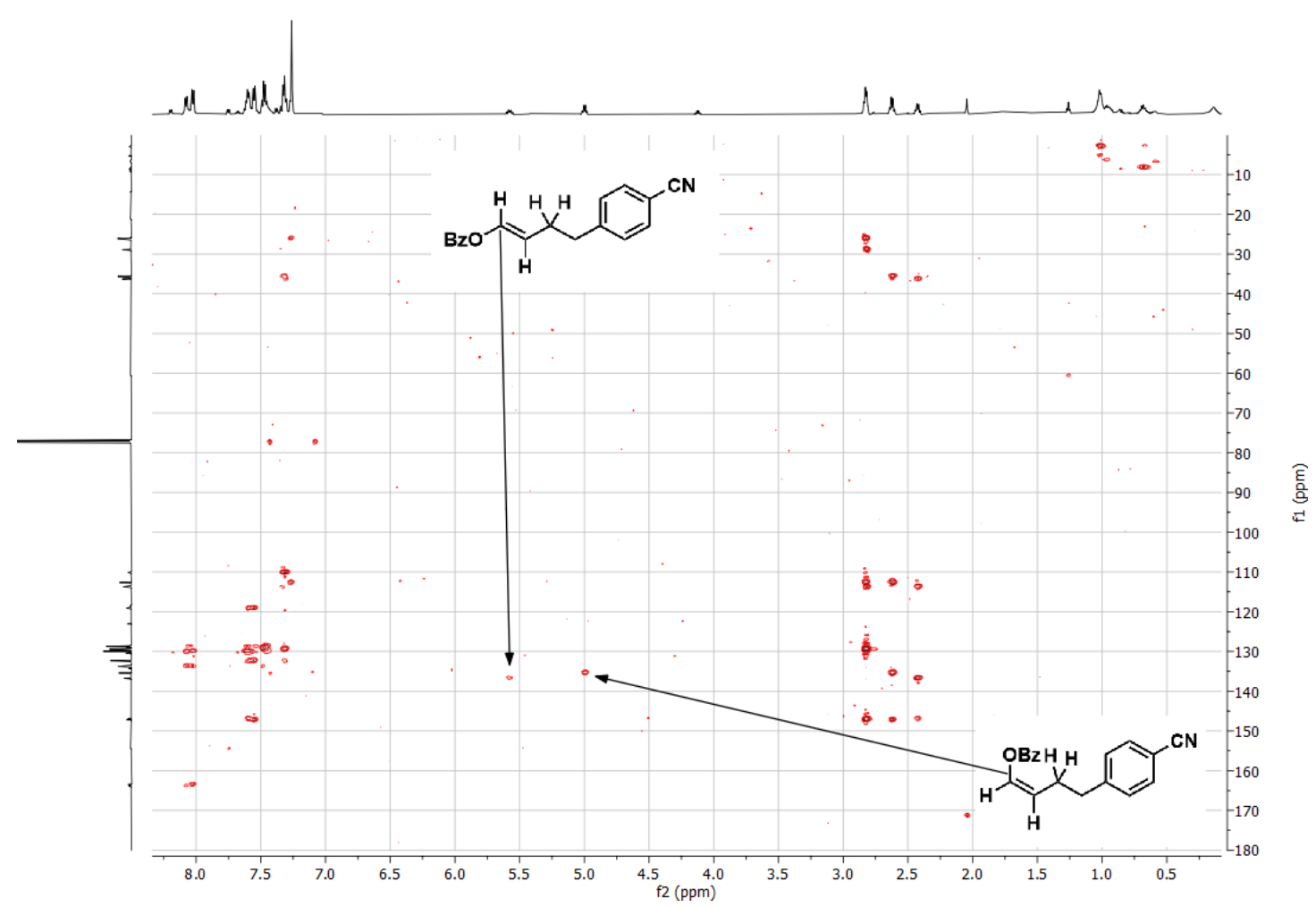

Supplementary Figure 8. ${ }^{1} \mathrm{H}^{-13} \mathrm{C} \mathrm{HMBC}\left(600 \mathrm{MHz}, \mathrm{CDCl}_{3}\right)$ of the alkene product.

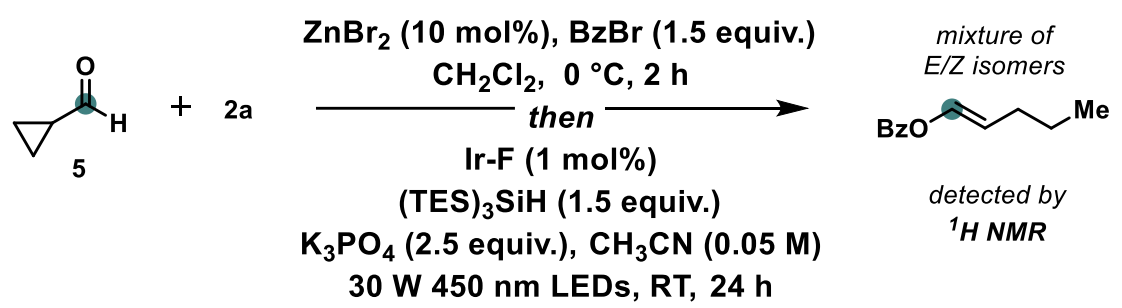

Reaction in the absence of Ni(II)-complex. According to the GP3, using the aldehyde 5 ( 0.3 mmol, 1.5 equiv.), benzoyl bromide ( $0.3 \mathrm{mmol}, 1.5$ equiv.), aryl bromide $2 a(0.2 \mathrm{mmol}, 1.0$ equiv.), Ir-F ( $2 \mu \mathrm{mol}, 1 \mathrm{~mol} \%)$, (TES) $3 \mathrm{SiH}(0.3 \mathrm{mmol}$, 1.5 equiv.), $\mathrm{K}_{3} \mathrm{PO}_{4}(0.3 \mathrm{mmol}, 1.5$ equiv.) and stirring at room temperature for 24 $\mathrm{h}$ under $30 \mathrm{~W}$ blue LEDs irradiation. The reaction mixture was filtered through a pad of silica gel and concentrated in vacuo, then analyzed by ${ }^{1} \mathrm{H}$ NMR. 


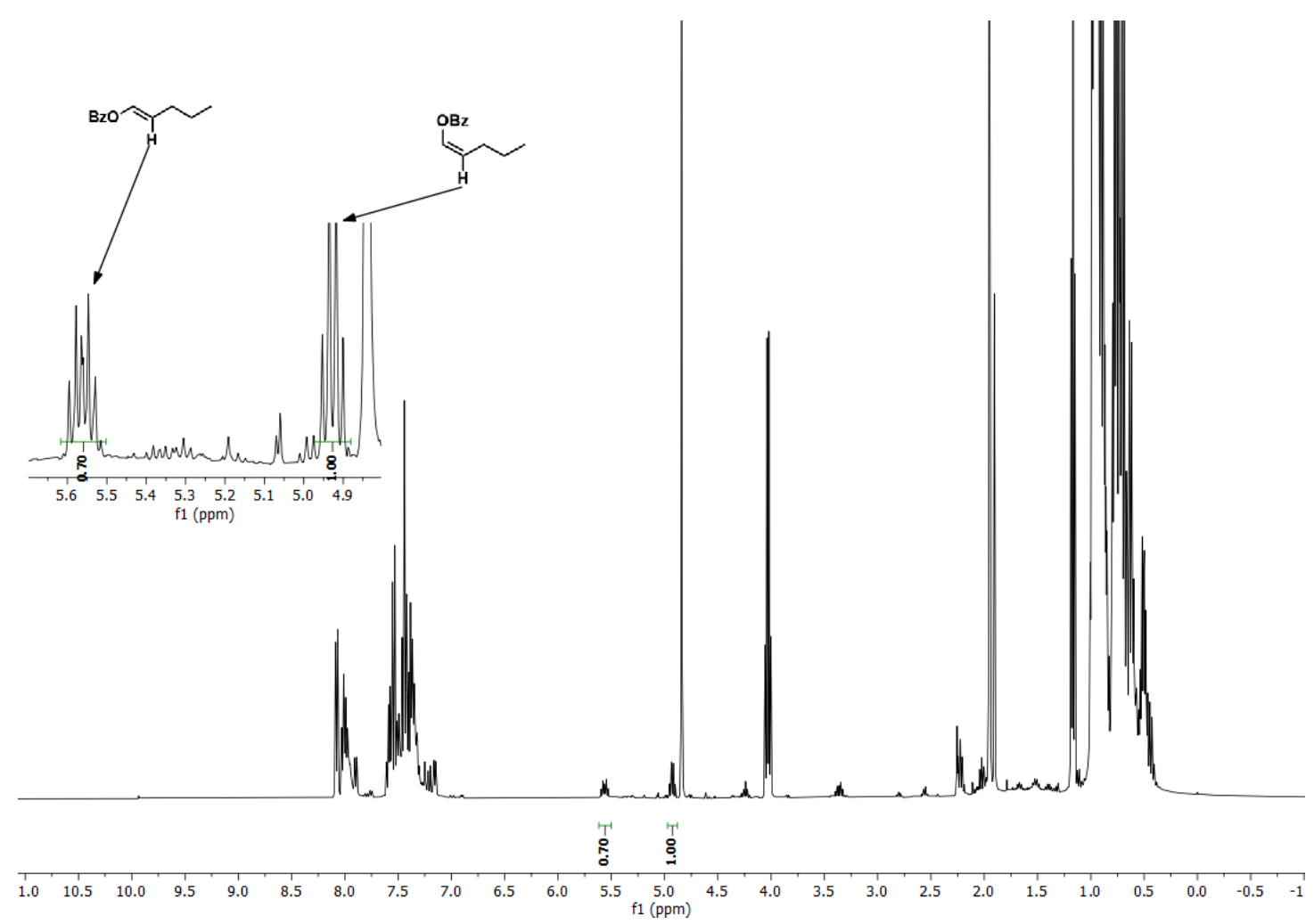

Supplementary Figure 9. ${ }^{1} \mathrm{H}$ NMR $\left(400 \mathrm{MHz}^{\mathrm{CDCl}} 3\right)$ of the alkene product without $\mathrm{Ni}(\mathrm{II})$ species.

Comment. Differently from Weix ${ }^{42}$ and Montgomery ${ }^{43}$ reports, whereby the $\mathrm{Ni}(\mathrm{II})$-coordination assists and drive the ring-opening to the Z-enol ester, we observed a Z:E mixture of ring-opening coupling products (see Supplementary Figures 5-8) Furthermore, be performing the reaction in the absence of Ni(II)precatalytic species, a Z:E mixture of ring-opening/HAT product was detected, thus suggesting that the ring-opening process (but obviously not the coupling) can also operate in the absence of the metal species (Supplementary Figure 9). As the ring-opening process of methylcyclopropyl proceeds without stereochemical control with regards to the forming alkene, ${ }^{44}$ the observed reaction outcome is compatible with a process operating via a radical ring-opening.

\subsection{Radical cyclization experiment}

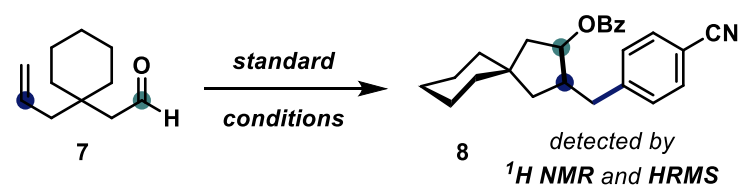


According to the GP3, using aldehyde 7 ( $0.3 \mathrm{mmol}, 1.5$ equiv.), benzoyl bromide ( $0.3 \mathrm{mmol}, 1.5$ equiv.), aryl bromide $2 \mathrm{a}(0.2 \mathrm{mmol}, 1.0$ equiv.), Ir-F ( $2 \mu \mathrm{mol}, 1$ mol\%), NiBr2•L2 (30 $\mu \mathrm{mol}, 15 \mathrm{~mol} \%$ ), (TES) $3 \mathrm{SiH}$ (0.3 mmol, 1.5 equiv.), $\mathrm{K}_{3} \mathrm{PO}_{4}(0.5$ mmol, 2.5 equiv.) and stirring at room temperature for $24 \mathrm{~h}$ under $30 \mathrm{~W}$ blue LEDs irradiation. The reaction mixture was filtered through a pad of silica gel and concentrated in vacuo, then purified by chromatography (pentane/EtOAc $=20 / 1$ $\rightarrow 10 / 1 \rightarrow 5 / 1$ ) a set of three fractions, which were analyzed by means of ${ }^{1} \mathrm{H}$ NMR and HRMS.

Fraction 1 mainly consists of $\mathrm{TES}_{3} \mathrm{SiH}$ derived side products (aliphatic region) and monosubstituted arene (likely benzoyl fragment).

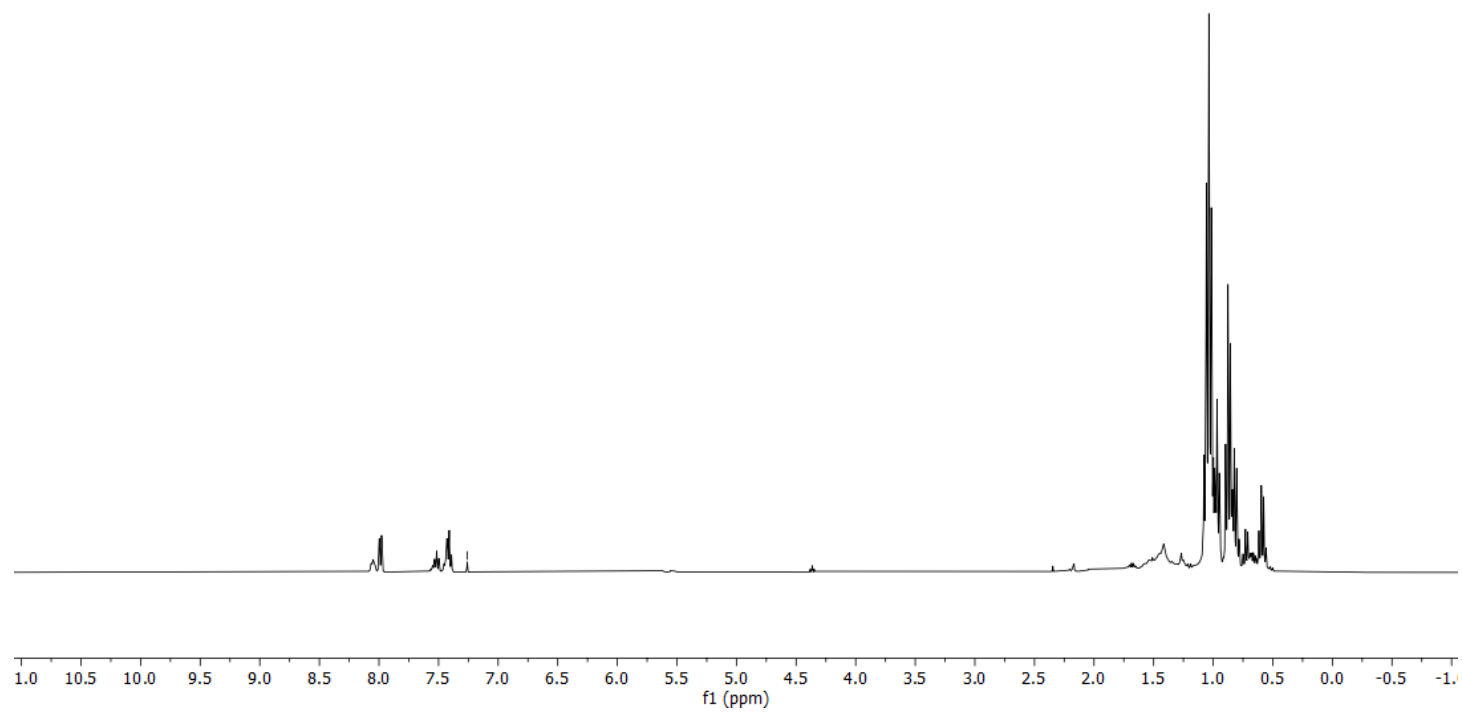

Supplementary Figure 10. ${ }^{1} \mathbf{H}$ NMR $\left(400 \mathrm{MHz}^{\mathrm{CDCl}} 3\right)$ of fraction 1.

Fraction 2. Cyclization product 8 could be detected by ${ }^{1} \mathrm{H}$ NMR and HRMS analysis. 


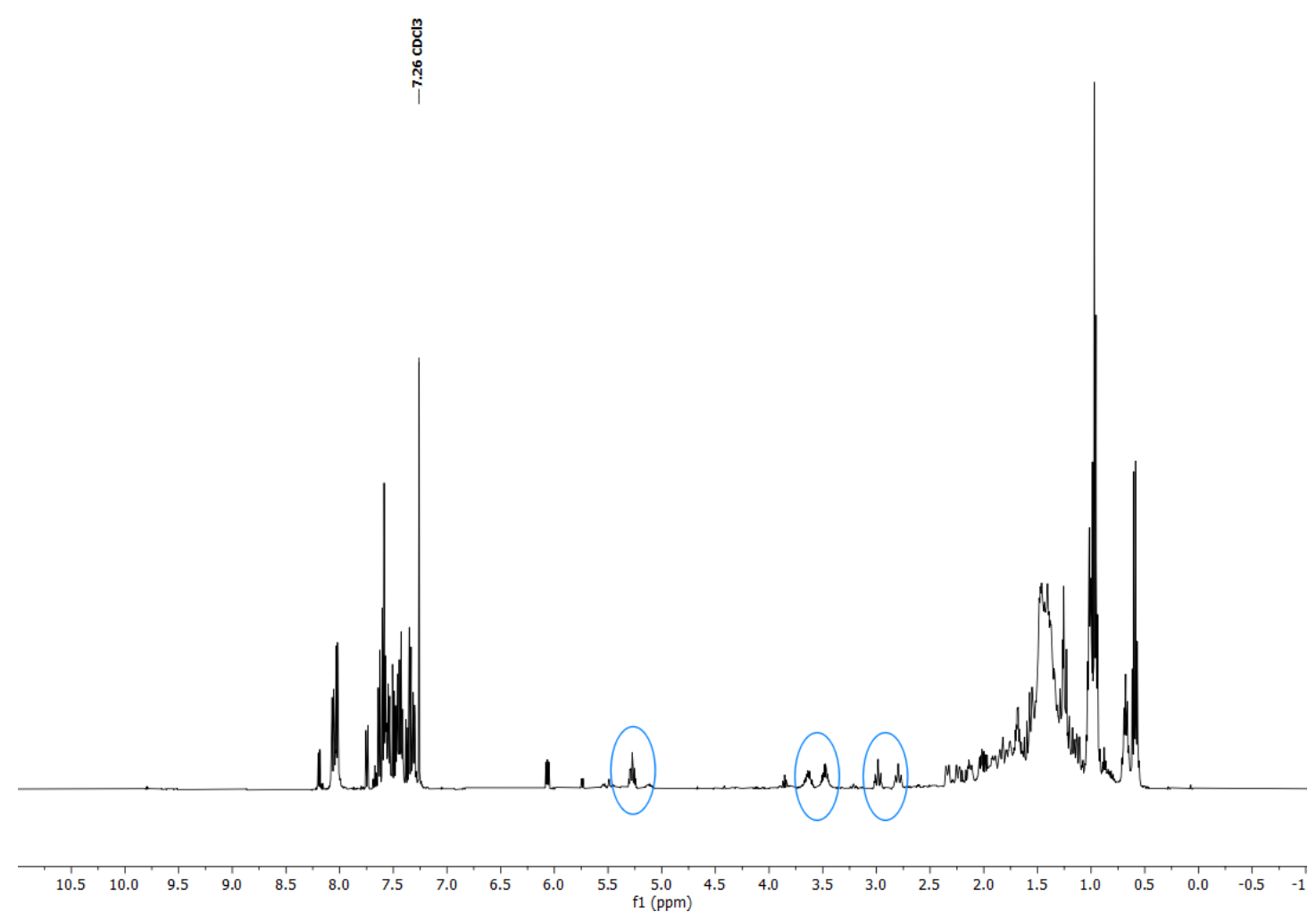

Supplementary Figure 11. ${ }^{1} \mathrm{H}$ NMR $\left(500 \mathrm{MHz}, \mathrm{CDCl}_{3}\right)$ of fraction 2.

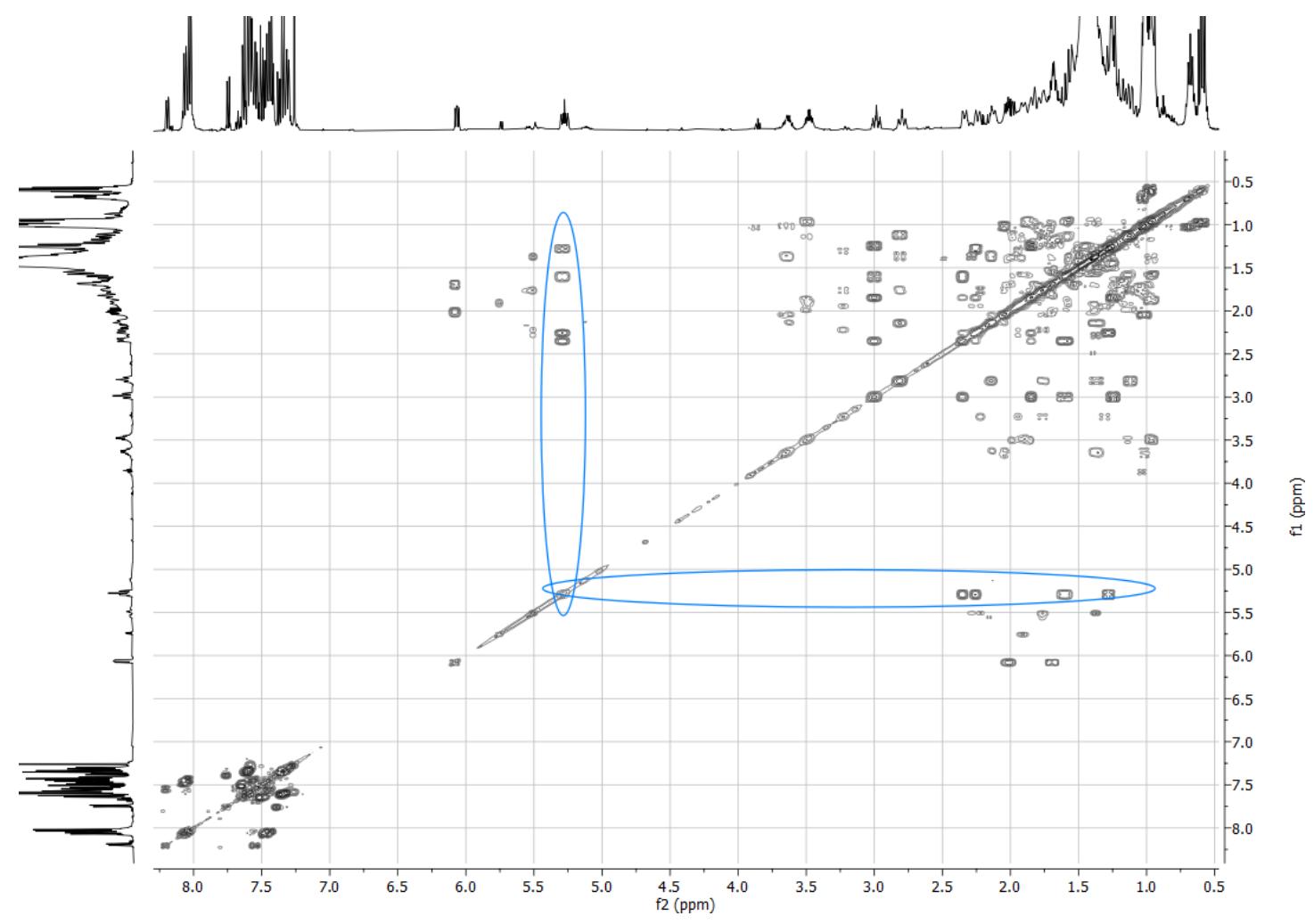

Supplementary Figure 12. ${ }^{1} \mathrm{H}-{ }^{1} \mathrm{H}$ COSY NMR $\left(500 \mathrm{MHz}, \mathrm{CDCl}_{3}\right)$ of fraction 2. 


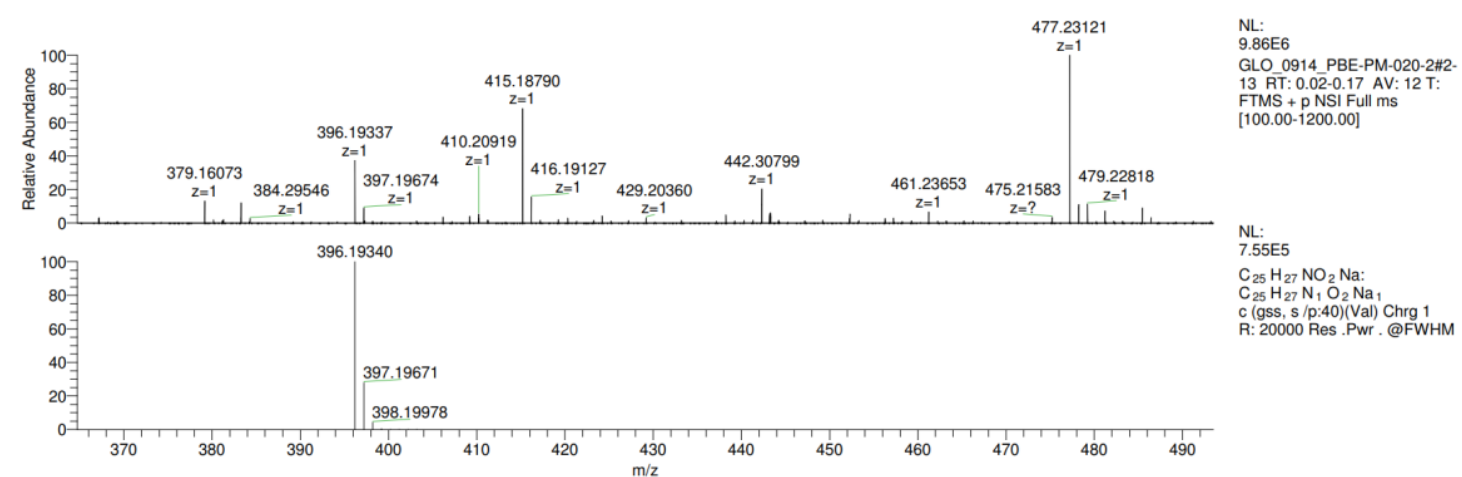

Supplementary Figure 13. HRMS analysis of fraction 2, showing the molecular ion of the coupled product 8 .

Fraction 3 consisted of 4,4'-dicyanodiphenylmethane (CAS 10466-37-2), as confirmed by ${ }^{1} \mathrm{H}$ NMR analysis. ${ }^{45}$ The product likely arises from twofold formal substitution of benzonitrile with dichloromethane (reaction co-solvent).
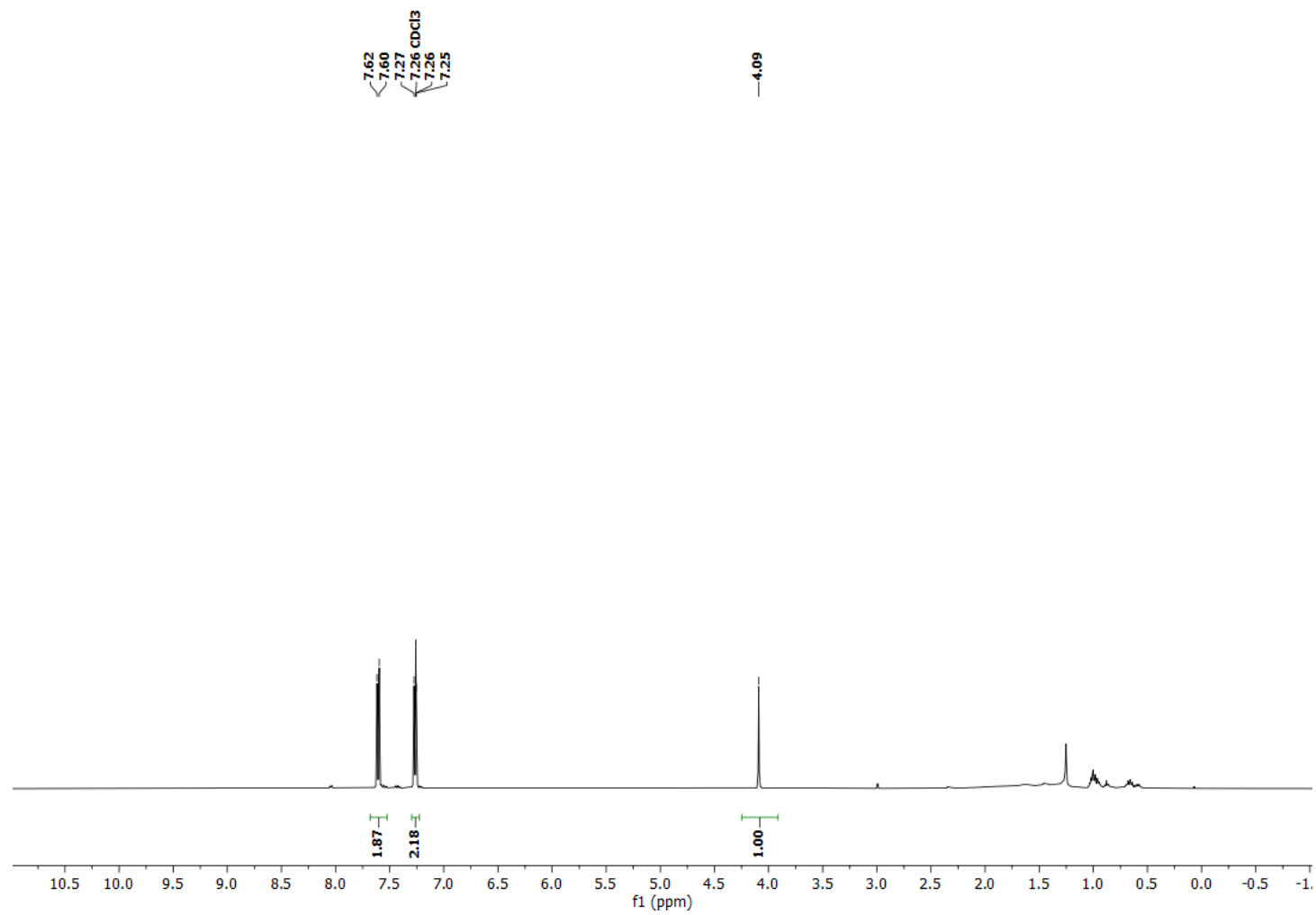

Supplementary Figure 14. ${ }^{\mathbf{1}} \mathrm{H}$ NMR $\left(400 \mathrm{MHz}, \mathrm{CDCl}_{3}\right)$ of fraction 3.

\subsection{UV-Visible spectroscopy}

UV-visible absorption spectra were recorded on a Jasco V-650 spectrophotometer, equipped with a temperature control unit at $25^{\circ} \mathrm{C}$. The samples were measured in 
Starna Fluorometer Microquartz cuvettes (volume: $1.4 \mathrm{ml}$, path length: $10 \mathrm{~mm}$ ) equipped with a PTFE-stopper. The spectra were aquired from 400 to $700 \mathrm{~nm}$ using $0.5 \mathrm{~nm}$ steps. All measurements were performed in dry MeCN at concentrations used for the standard reaction (for the in-situ formed alkyl bromide, conversion was estimated to be quantitative). Note. Due to substantial insolubility of the Ni(II) complex, in some instances the baseline appears deviating from the zero value, due to scattering effects.

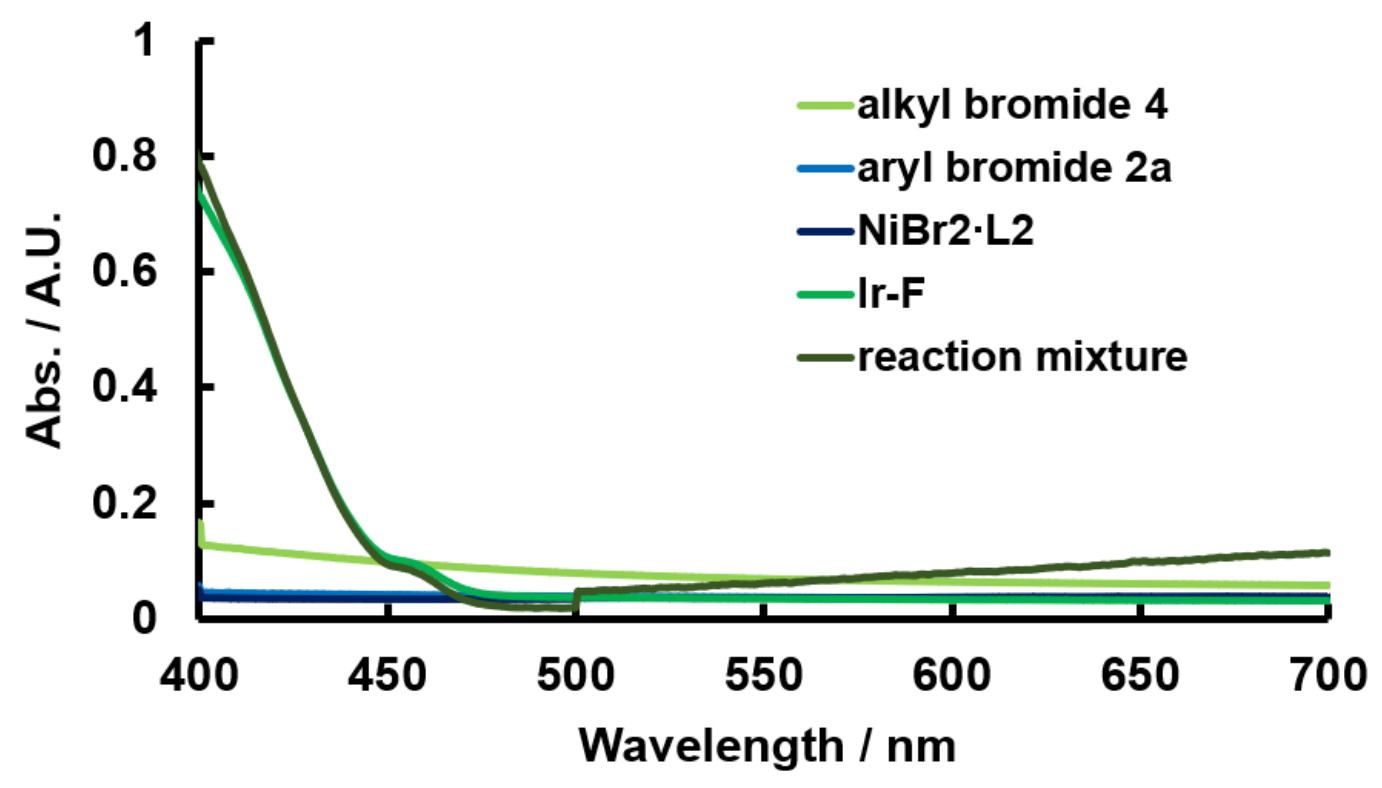

Supplementary Figure 15. UV-visible spectra.

Comment on the experimental data. As visible in Supplementary Figure 15, the only species that are substantially absorbing in the visible range

\subsection{Stern-Volmer quenching analysis}

Stern-Volmer luminescence quenching analysis was conducted using a Jasco FP8300 spectrofluorometer at $25{ }^{\circ} \mathrm{C}$. The following parameters were employed: Excitation bandwidth $=5 \mathrm{~nm}$, data interval $=0.2 \mathrm{~nm}$, scan speed $=500 \mathrm{~nm} / \mathrm{min}$, response time $=0.2 \mathrm{sec}$. The samples were measured in Hellma fluorescence QS quartz cuvettes (chamber volume $=1.4 \mathrm{~mL}, \mathrm{H} \times \mathrm{W} \times \mathrm{D}=46 \mathrm{~mm} \times 12.5 \mathrm{~mm}, 12.5$ $\mathrm{mm}$ ) fitted with a PTFE stopper.

Samples were prepared in $1.4 \mathrm{~mL}$ quartz cuvettes equipped with PTFE stoppers inside an argon filled glovebag. The solutions of $\operatorname{Ir}\left[d F\left(\mathrm{CF}_{3}\right) \mathrm{ppy}\right]_{2}(\mathrm{dtbbpy}) \mathrm{PF}_{6}(2.5$ 
$\mu \mathrm{M}$ in $\mathrm{MeCN}$ ) were excited at $\lambda_{\mathrm{ex}}=400 \mathrm{~nm}$ and the emission was collected at 474 $\mathrm{nm}$. A saturated LiBr solution in MeCN was prepared by stirring excess anhydrous $\mathrm{LiBr}$ in dry and degassed MeCN under argon atmosphere. For each quenching experiment, $100 \mu \mathrm{l}$ of the saturated $\mathrm{LiBr}$ were titrated to a solution (1 mL) of $\operatorname{Ir}\left[d F\left(\mathrm{CF}_{3}\right) \text { ppy }\right]_{2}(\mathrm{dtbbpy}) \mathrm{PF}_{6}$ in a screw-top $10.0 \mathrm{~mm}$ quartz cuvette $\mathrm{I}_{0}$ is the luminescence intensity without the quencher, I is the intensity in the presence of the quencher. The results are listed below:

Supplementary Table 3. Stern-Volmer quenching data.

\begin{tabular}{cccc}
\hline Entry & Quencher addition $/ \mu \mathrm{l}$ & I / A.U. & I0/I \\
1 & 0 & 39.1 & 1.000 \\
2 & 100 & 33.3 & 1.174 \\
3 & 200 & 29.3 & 1.334 \\
4 & 300 & 27.5 & 1.422 \\
5 & 400 & 25.9 & 1.510 \\
\hline
\end{tabular}

By linear fitting of the datapoints and standard linear regression:

Slope $=0.0013 \pm 0.0001$

$$
\text { Intercept }=1.03 \pm 0.03
$$

$R^{2}=0.972$

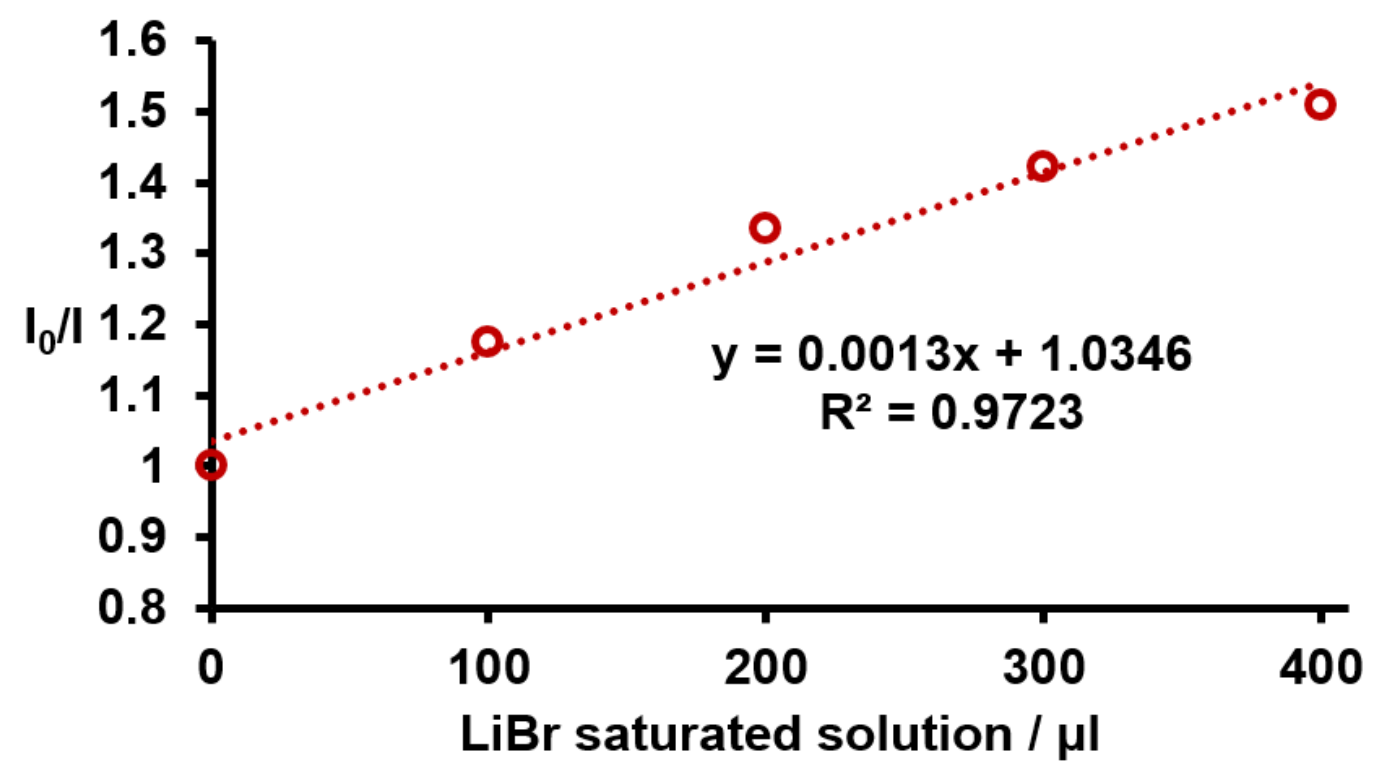

Supplementary Figure 10. Stern-Volmer quenching analysis with respect to LiBr. 
Comment. As visible from Supplementary Figure 10, LiBr salts can quench the fluorescence of the photocatalyst in accordance to the observation by MacMillan and co-workers. ${ }^{46}$ This suggests that bromine radicals can be formed upon photoirradiation.

\section{Condition-based sensitivity screening}

A condition-based sensitivity screening approach was undertaken according to the report of Glorius and co-workers, ${ }^{47}$ as detailed below.

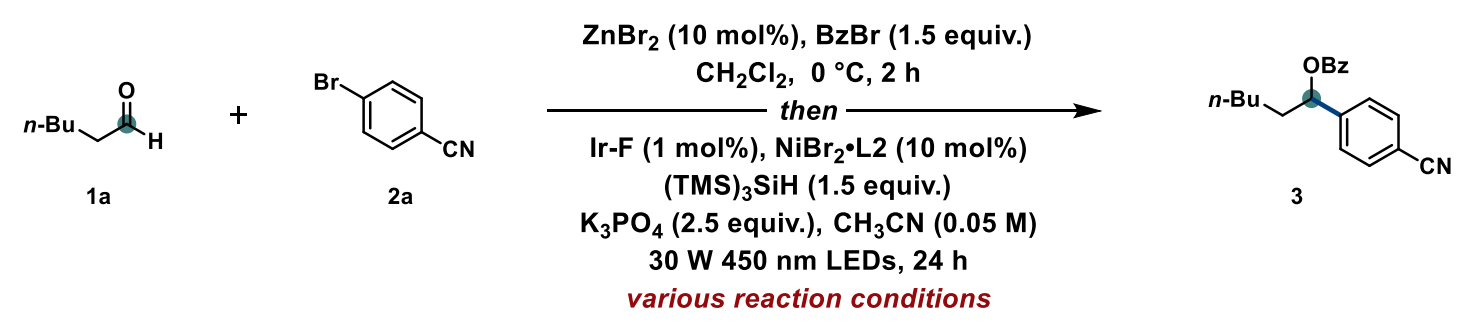

Preparation of stock solution 1. In an oven-dried Schlenk tube equipped with a PTFE-coated stirring bar, $\mathrm{ZnBr} 2$ (40.5 mg, $0.015 \mathrm{mmol}$ for each reaction, $10 \mathrm{~mol} \%$ ) was suspended in dry $\mathrm{CH}_{2} \mathrm{Cl}_{2}(1 \mathrm{ml})$, then the suspension was cooled to $0{ }^{\circ} \mathrm{C}$ and benzoyl bromide ( $212 \mu \mathrm{l}, 0.15 \mathrm{mmol}$ for each reaction, 1.50 equiv.) was added, followed hexanal (222 $\mu \mathrm{l}, 0.15 \mathrm{mmol}$ for each reaction, 1.50 equiv.). The reaction was stirred at $0{ }^{\circ} \mathrm{C}$ for two hours

Preparation of stock solution 2. In an oven-dried Schlenk tube equipped with a PTFE-coated stirring bar, 4-bromobenzonitrile $(282 \mathrm{mg}, 0.10 \mathrm{mmol}$ for each reaction), Ir-F (13.5 $\mathrm{mg}, 0.001 \mathrm{mmol}$ for each reaction, $1 \mathrm{~mol} \%)$ and (TES) ${ }_{3} \mathrm{SiH}$ (760 $\mu \mathrm{l}, 0.15 \mathrm{mmol}$ for each reaction, 1.50 equiv.) were charged, then the vessel was evacuated and back-filled with argon (three times). Dry MeCN (18 ml) was added, then the solution was virogoursly stirred for 30 minutes.

Reaction set-up. In the individual oven-dried Schlenk tube equipped with a PTFEcoated stirring bar, $\mathrm{NiBr}_{2} \cdot \mathrm{L} 2$ (6.2 mg, $0.015 \mathrm{mmol}, 15 \mathrm{~mol} \%$ ), $\mathrm{K}_{3} \mathrm{PO}_{4}$ (53 mg, 0.25 mmol, 2.50 equiv.) were charged under air, then the vessel was evacuated and back-filled three times with argon. The appropriate amount of dry MeCN 
(indicated in table) was added, then additives (indicated in table). Stock solution 1 $(1.5 \mathrm{ml})$ and stock solution $2(100 \mu \mathrm{l})$ were transferred via syringe, then the reaction was degassed as detailed in the individual entries, then sealed and irradiated with 30W $450 \mathrm{~nm}$ LEDs using the standard set-up or the variation detailed in ${ }^{47}$. After 24 hours, the irradiation was interrupted, then the reaction was filtered through a short pad of silica, rinsing with EtOAc. The volatiles were removed in vacuo, then the crude residue was analyzed by $1 \mathrm{H}$ NMR using dibromomethane as internal standard (relaxation delay $=30 \mathrm{~s}$ ).

Supplementary Table 4. Reactions of the condition-based sensitivity screening

\begin{tabular}{|c|c|c|c|}
\hline Entry & Reaction & Added MeCN & Other \\
\hline 1 & Standard & $500 \mu \mathrm{l}$ & - \\
\hline 2 & High C (+10\%) & $300 \mu \mathrm{l}$ & - \\
\hline 3 & Low C (-10\%) & $700 \mu \mathrm{l}$ & - \\
\hline 4 & High $\mathrm{H}_{2} \mathrm{O}$ & $500 \mu \mathrm{l}$ & $20 \mu \mathrm{l}$ of $\mathrm{H}_{2} \mathrm{O}$ added \\
\hline 5 & Medium $\mathrm{O}_{2}$ & $500 \mu \mathrm{l}$ & Argon sparging \\
\hline 6 & High $\mathrm{O}_{2}$ & $500 \mu \mathrm{l}$ & $20 \mathrm{ml}$ of air injected \\
\hline 7 & High T & $500 \mu \mathrm{l}$ & Aluminum foil wrapping \\
\hline 8 & High I (16 x) & $500 \mu \mathrm{l}$ & $\mathrm{d}=0.8 \mathrm{~cm}$ \\
\hline 9 & Low I (1/16 x) & $500 \mu \mathrm{l}$ & $\mathrm{d}=8.8 \mathrm{~cm}$ \\
\hline 10 & Standard low T & $500 \mu \mathrm{l}$ & Water jacket, no flow \\
\hline 11 & Low $\mathrm{T}$ & $500 \mu \mathrm{l}$ & Flowing water jacket \\
\hline 12 & Large scale reaction & - & $\begin{array}{c}\text { Isolated reaction, see details in the scope } \\
\text { entry }\end{array}$ \\
\hline
\end{tabular}

Supplementary Table 5. Results the condition-based sensitivity screening

\begin{tabular}{|c|c|c|c|}
\hline Entry & Reaction & 80 & Variation \% \\
\hline 1 & Standard & 78 & -2.5 \\
\hline 2 & High C $(+10 \%)$ & 81 & 1.3 \\
\hline 3 & Low C $(-10 \%)$ & 18 & -77.5 \\
\hline 4 & High $\mathrm{H}_{2} \mathrm{O}$ & 81 & 1.3 \\
\hline 5 & Medium $\mathrm{O}_{2}$ & 20 & -75 \\
\hline 6 & High $\mathrm{O}_{2}$ & 0 & -100 \\
\hline 7 & High T & 78 & -2.5 \\
\hline 8 & High I $(16 \mathrm{x})$ & 40 & -50 \\
\hline 9 & Low I $(1 / 16 \mathrm{x})$ & 30 & - \\
\hline 10 & Standard low T & 31 & 3.3 \\
\hline 11 & Low T & 78 & -12 \\
\hline 12 & Large scale reaction & & \\
\hline
\end{tabular}




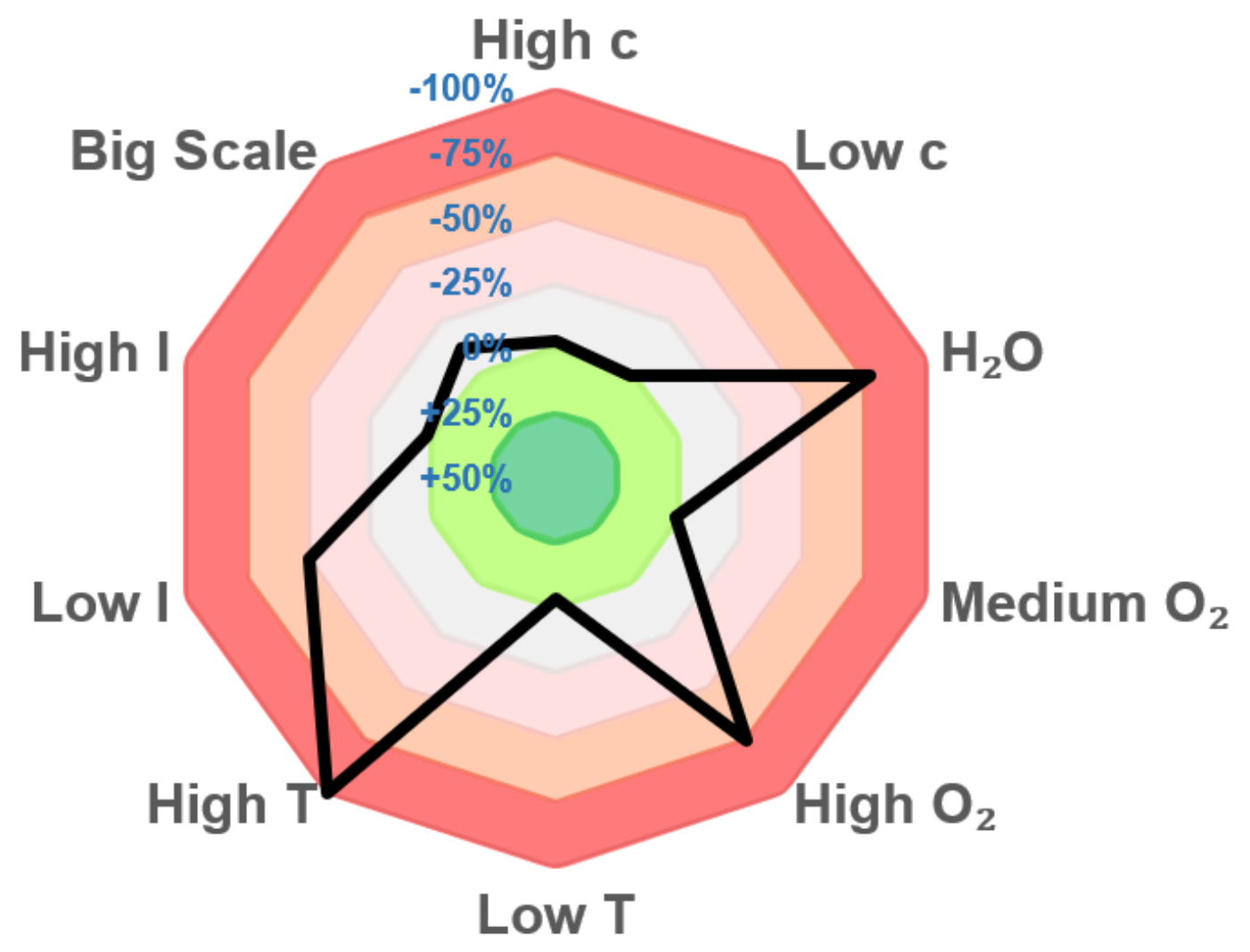

Supplementary Figure 11. Radar diagram for the condition-based sensitivity screening.

Comment on the data. As reported in Supplementary Table 5 and Supplementary Figure 17, the reaction proved sensitive towads high oxygen and water content, as well as towards increased reaction temperature and moderately sensitive towards low light intensity. On the other hand, high light intensities, concentration fluctuations, scale-up and reduced temperatures had a negligible impact. Interestingly, simple argon sparging proved sufficient to ensure comparable reaction efficiency to freeze-pump-thaw methodology. 
8. ${ }^{1} \mathrm{H},{ }^{13} \mathrm{C}$ and ${ }^{19} \mathrm{~F}$ NMR Spectra of Compounds

${ }^{1} \mathrm{H}$ NMR (400 MHz, $\left.\mathrm{CDCl}_{3}\right)$

啇

然<smiles>O=C(OCCCCO)C1CCC(F)(F)C1</smiles>

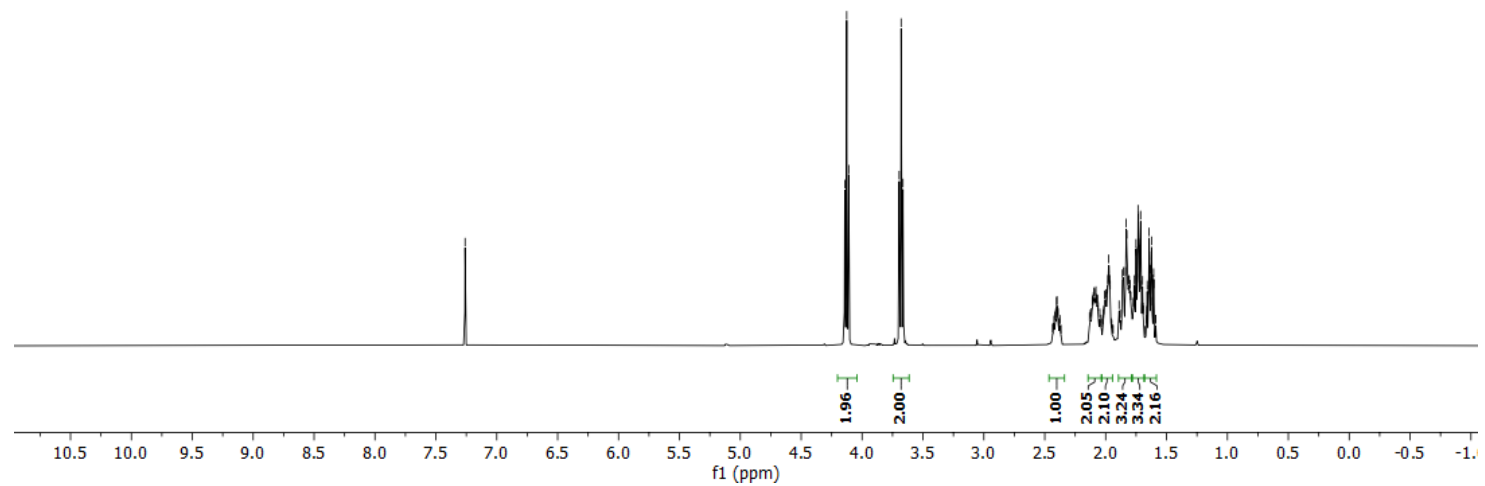

${ }^{13} \mathrm{C}$ NMR (101 MHz, $\left.\mathrm{CDCl}_{3}\right)$
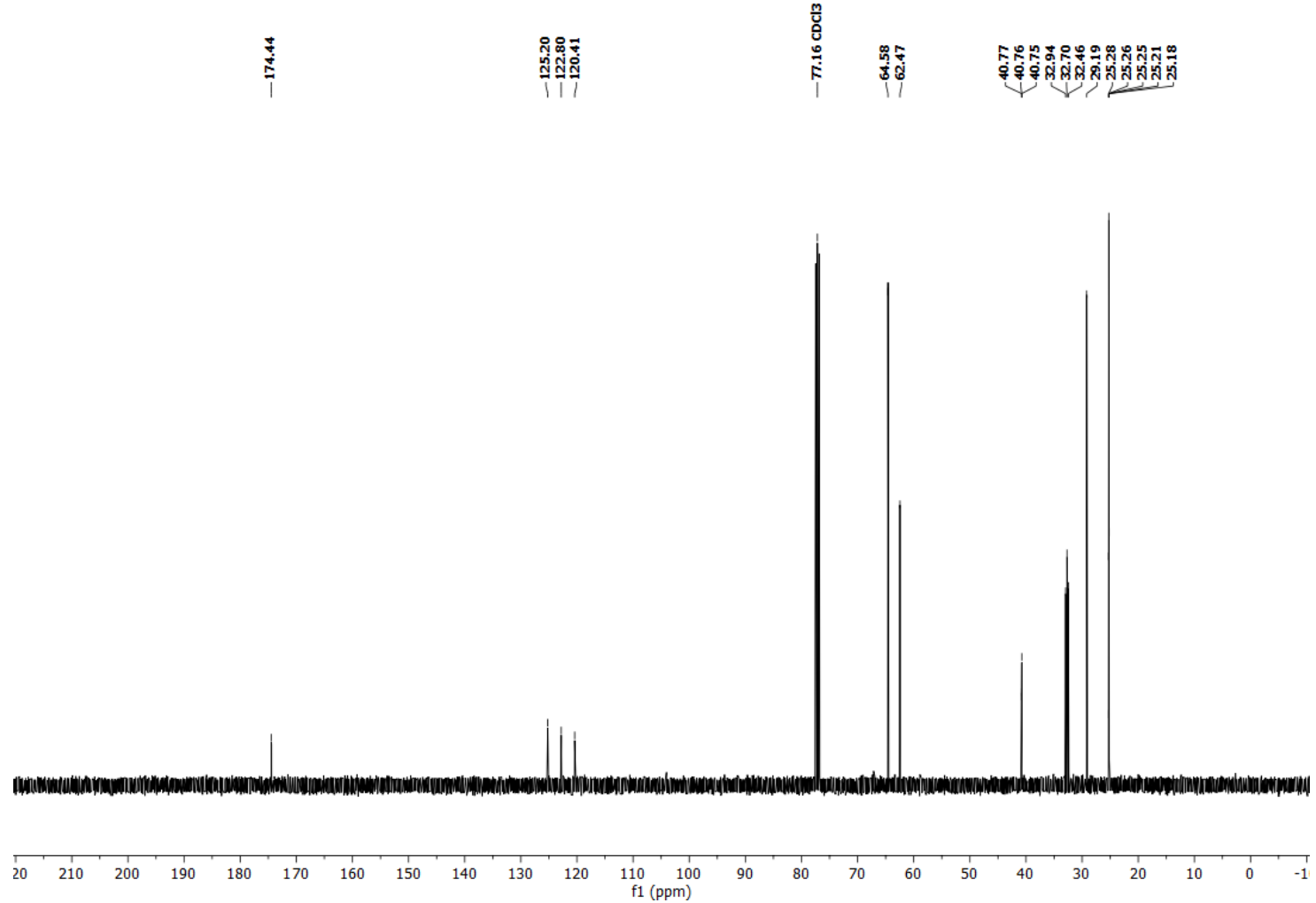
19F NMR (377 MHz, $\mathrm{CDCl}_{3}$ )

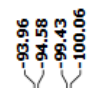

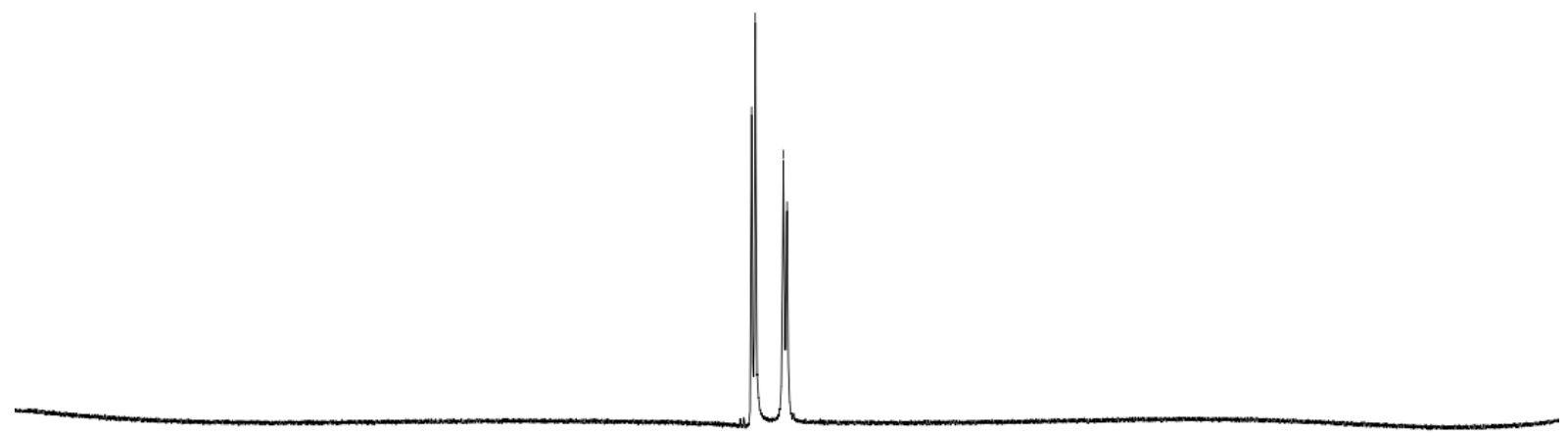

\begin{tabular}{llllllllllllllllllllllllllllllllllll}
\hline 30 & 20 & 10 & 0 & -10 & -20 & -10 & -40 & -50 & -60 & -70 & -80 & -90 & -100 & -110 & -120 & -130 & -140 & -150 & -160 & -170 & -180 & -190 & -200 & -210 & -220 & -230
\end{tabular}

${ }^{1} \mathrm{H}$ NMR (400 $\left.\mathrm{MHz} \mathrm{CDCl}_{3}\right)$
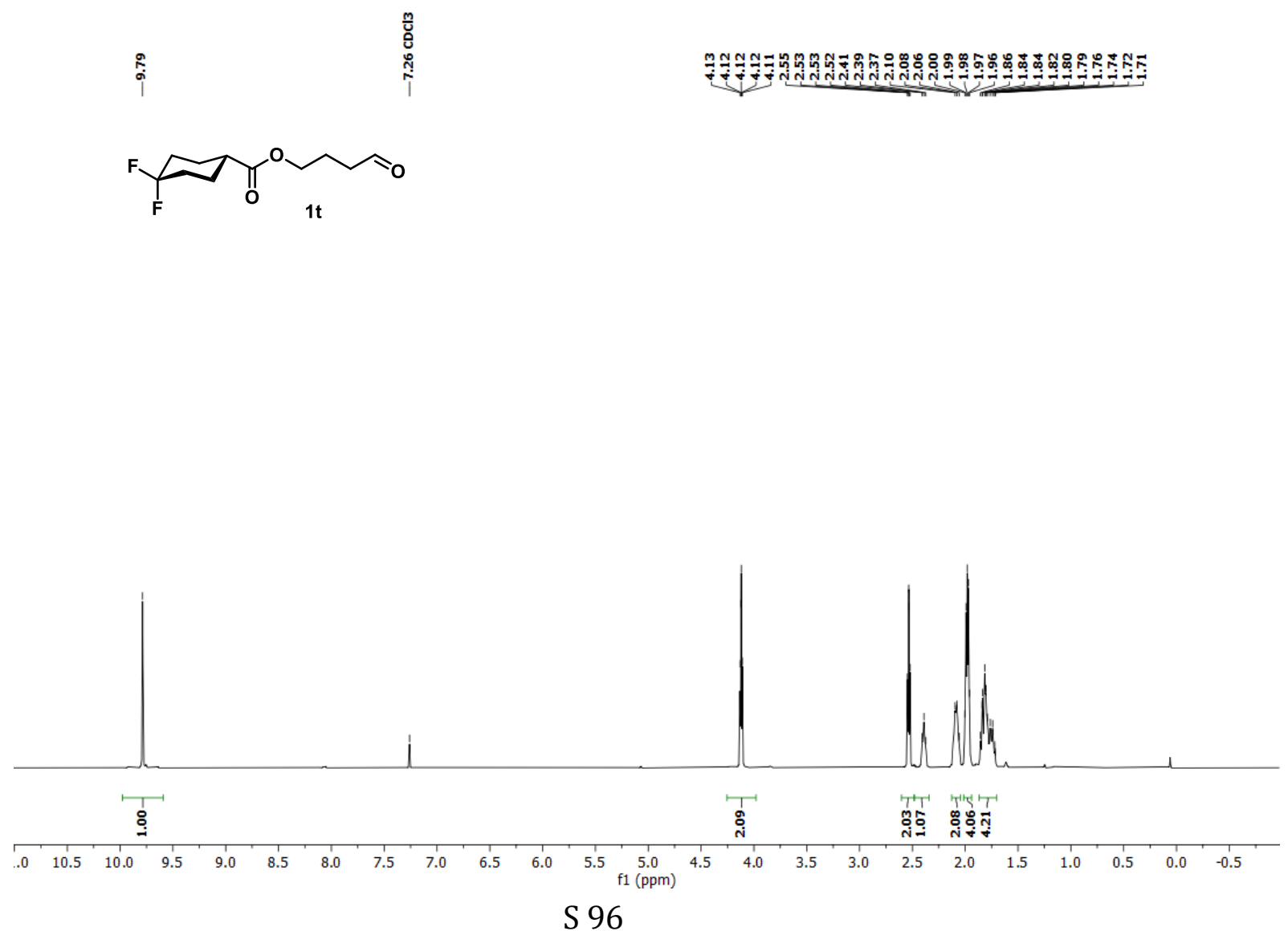
${ }^{13} \mathrm{C}$ NMR (101 $\mathrm{MHz}, \mathrm{CDCl}_{3}$ )

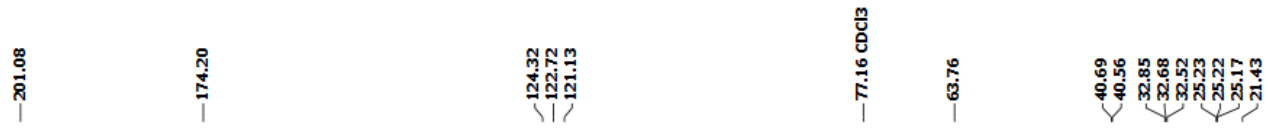
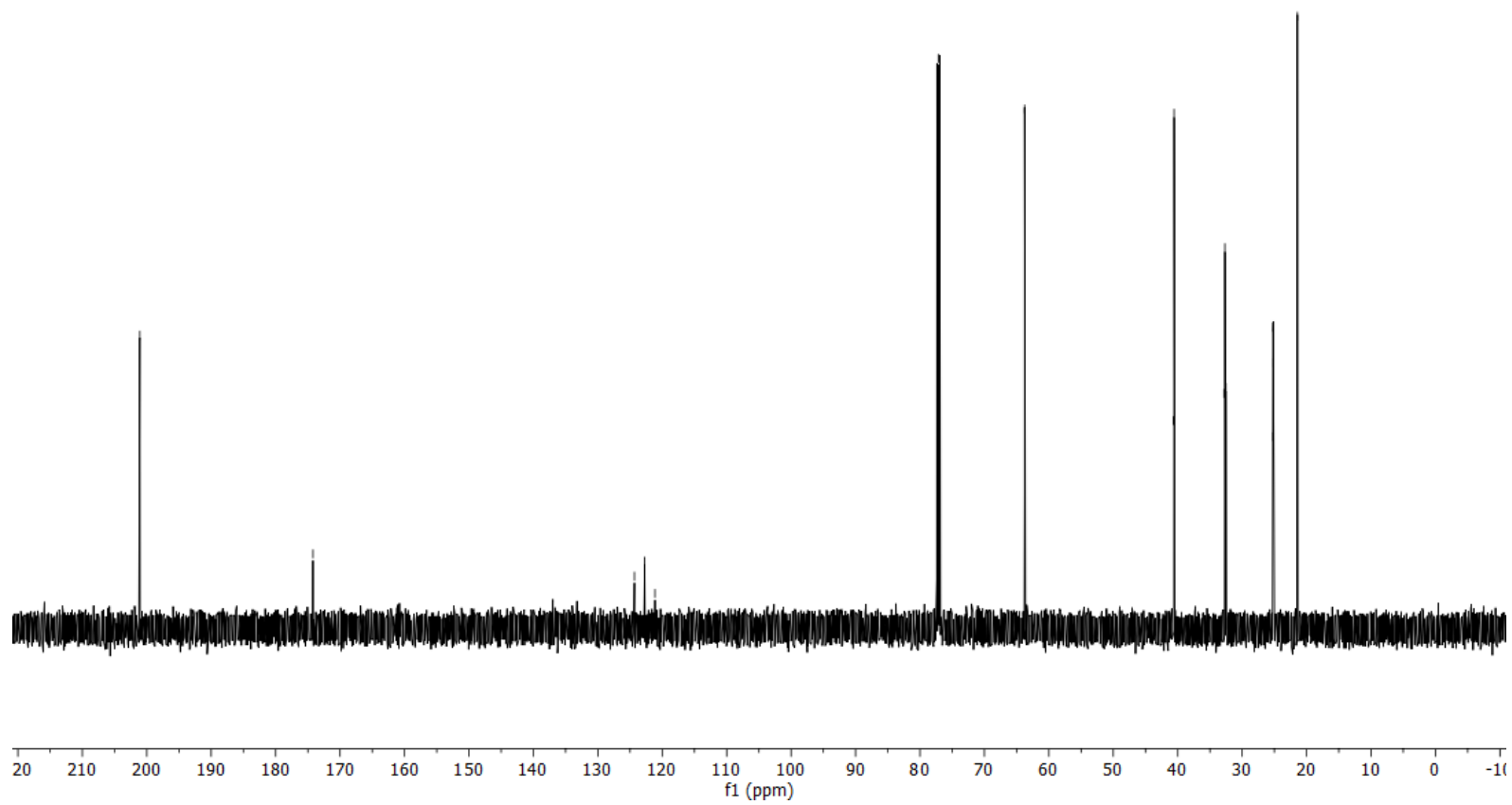

${ }^{19}$ F NMR (377 MHz, $\mathrm{CDCl}_{3}$ )

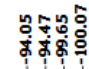

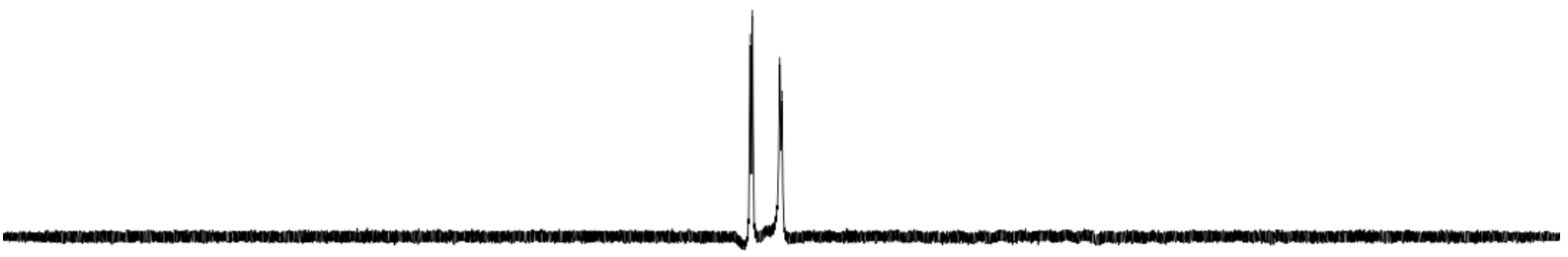

$\begin{array}{llllllllllllllllllllllllllllllllllll}40 & 30 & 20 & 10 & 0 & -10 & -20 & -30 & -40 & -50 & -60 & -70 & -80 & -90 & -100 & -110 & -120 & -130 & -140 & -150 & -160 & -170 & -180 & -190 & -200 & -210 & -220 & -230 & -240\end{array}$ S 97 
1H NMR (400 MHz, $\mathrm{CDCl}_{3}$ )

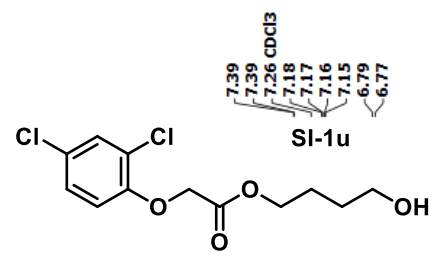

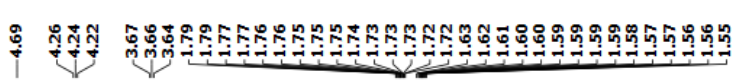

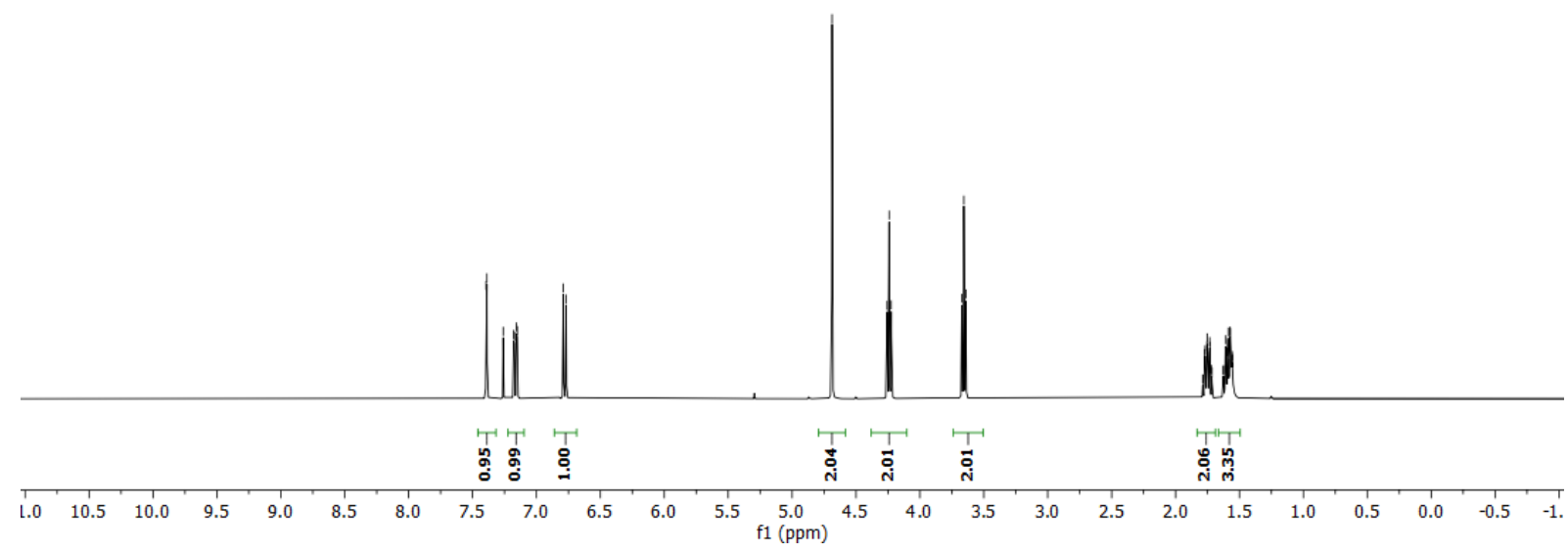

${ }^{13} \mathrm{C}$ NMR (101 MHz, $\mathrm{CDCl}_{3}$ )

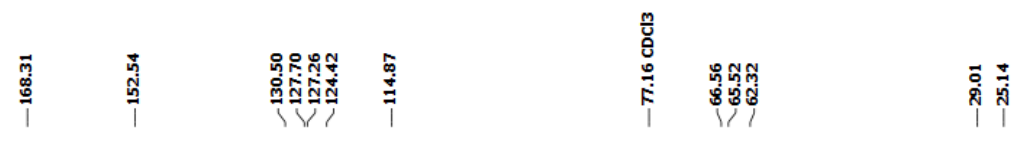

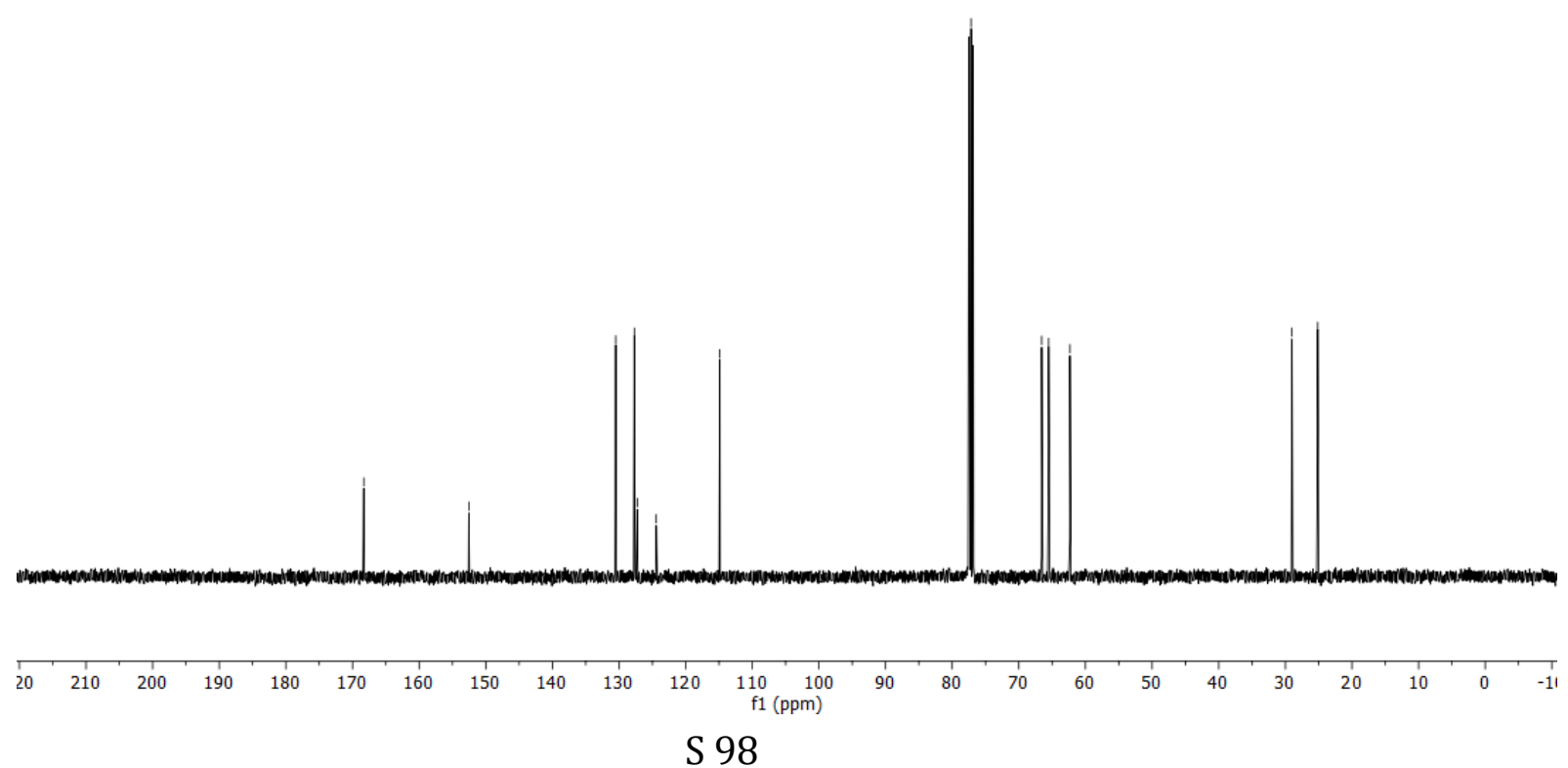


1H NMR (599 MHz, $\mathrm{CDCl}_{3}$ )
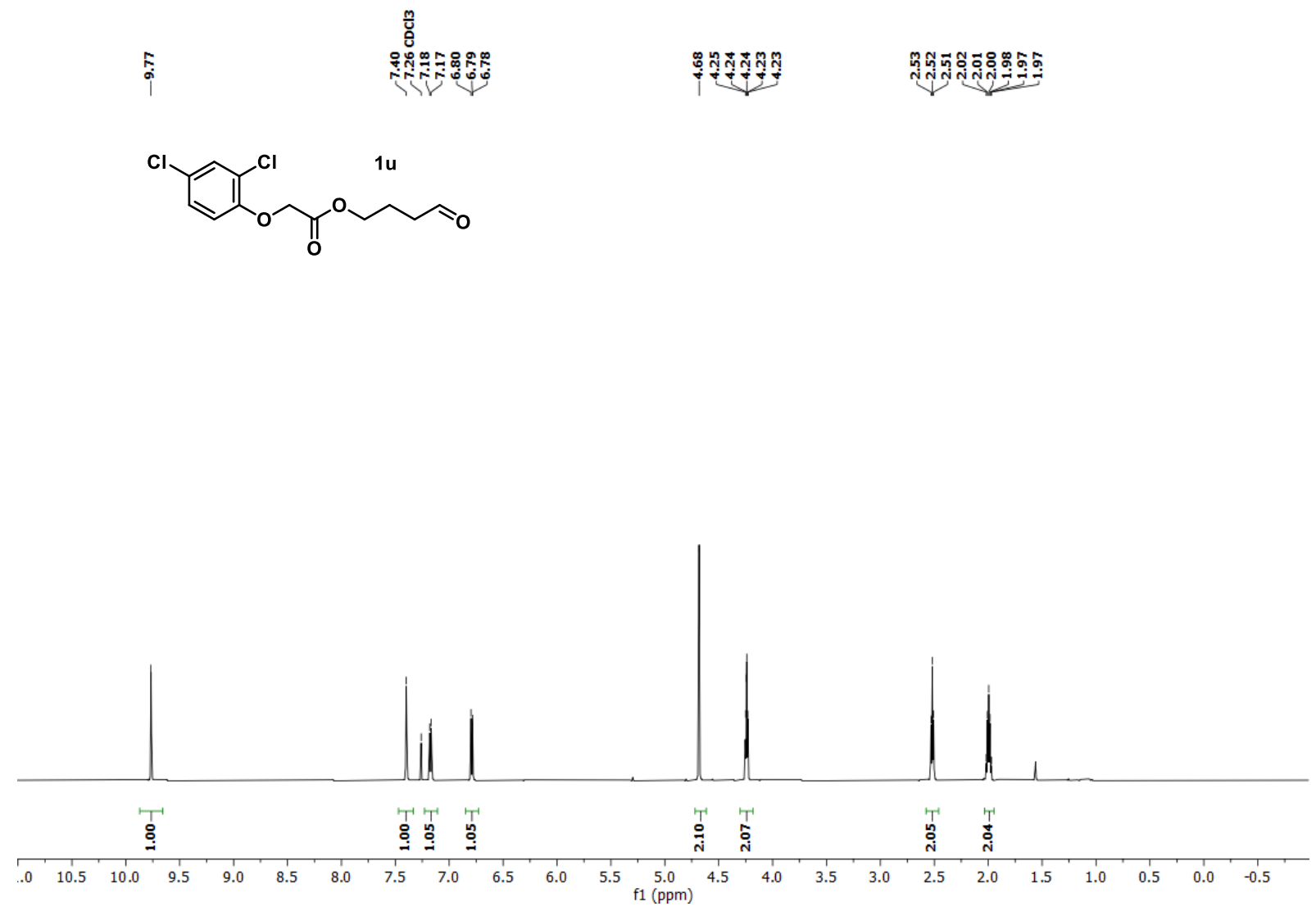

${ }^{13} \mathrm{C}$ NMR (151 MHz, $\mathrm{CDCl}_{3}$ )

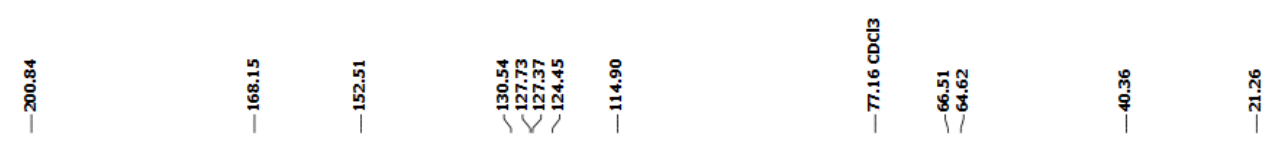

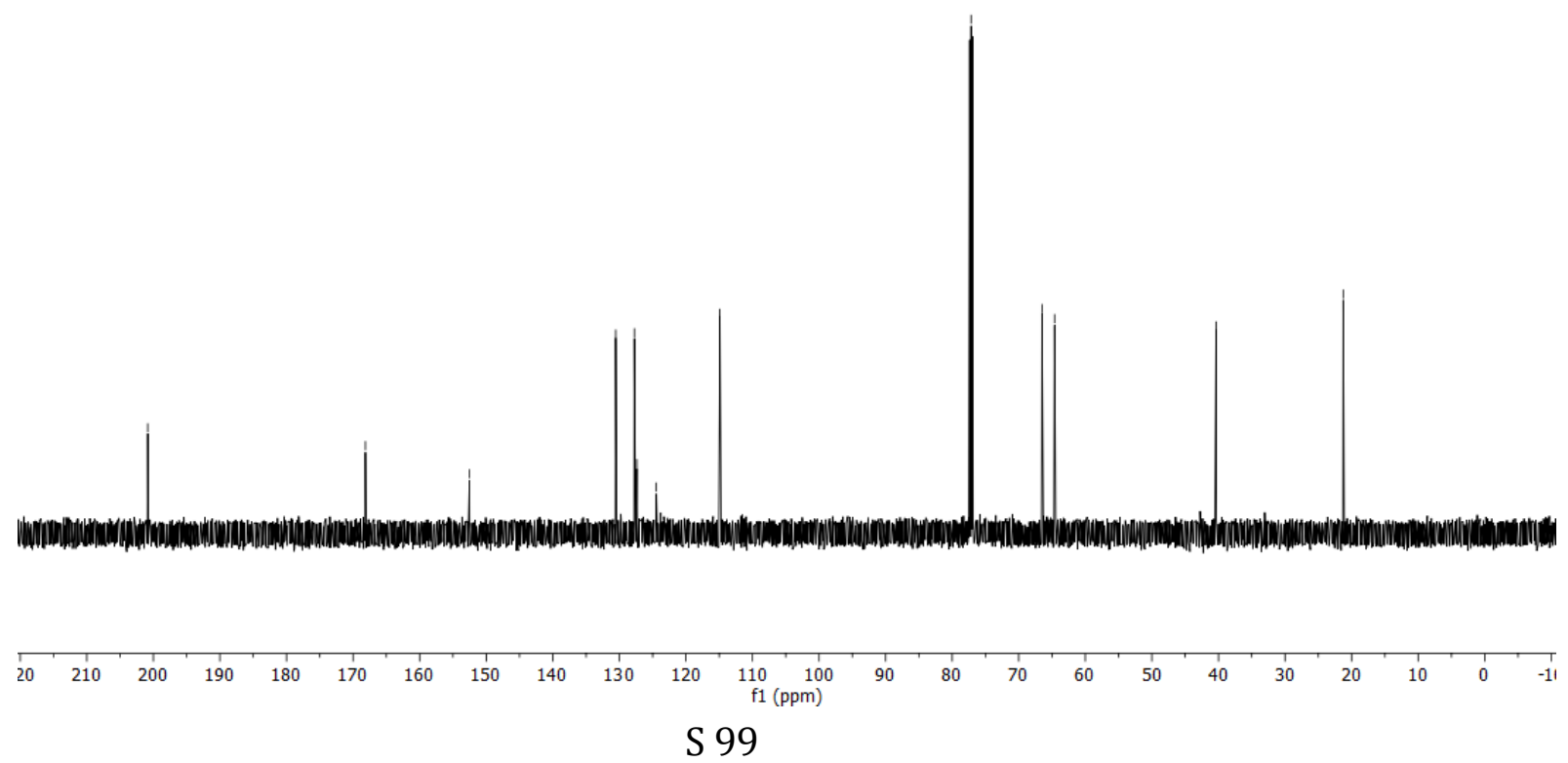


1H NMR (599 MHz, $\mathrm{CDCl}_{3}$ )

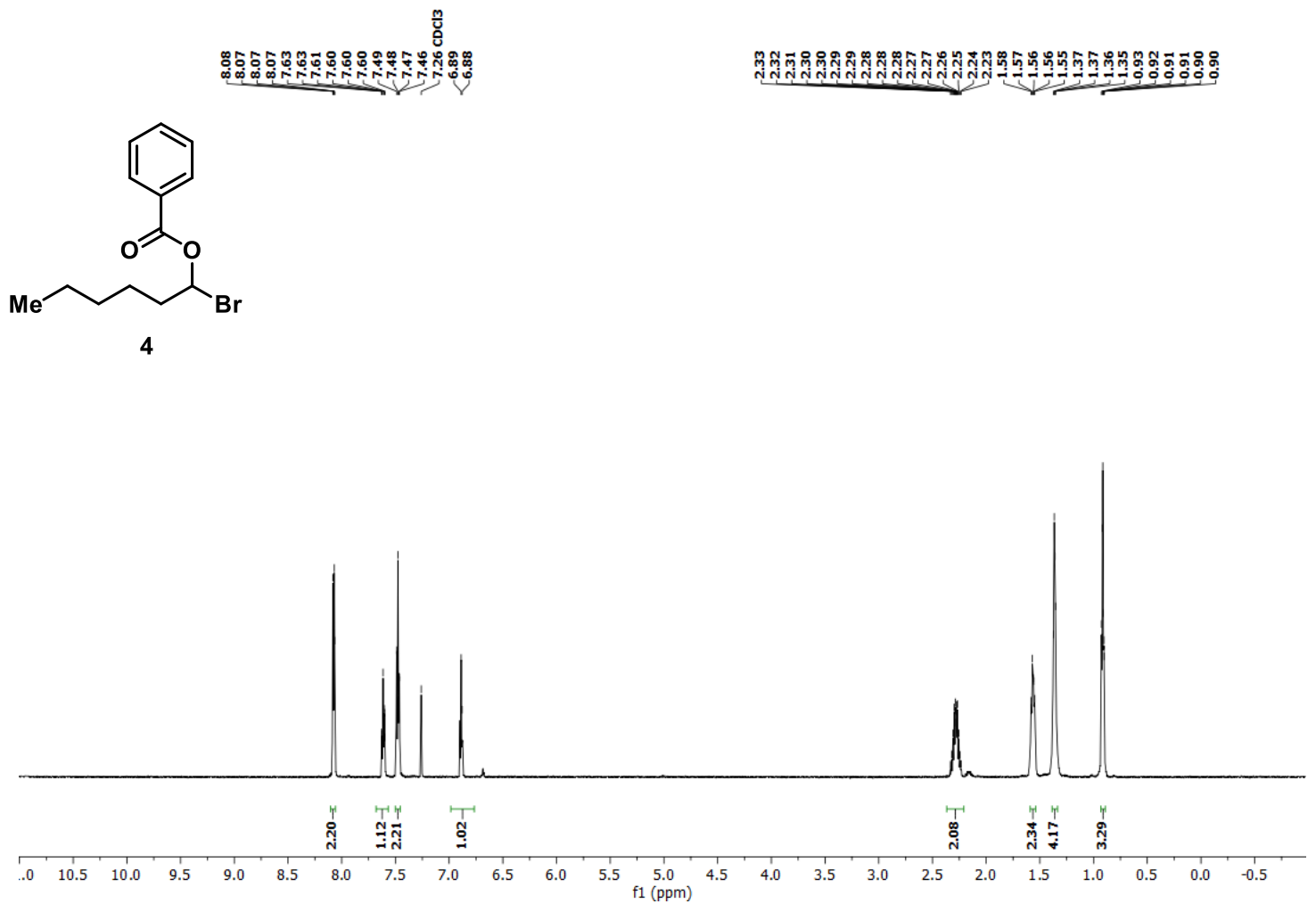

${ }^{13} \mathrm{C}$ NMR (151 MHz, $\mathrm{CDCl}_{3}$ )

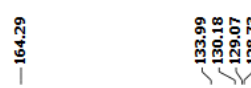

1ִ

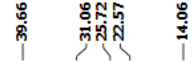
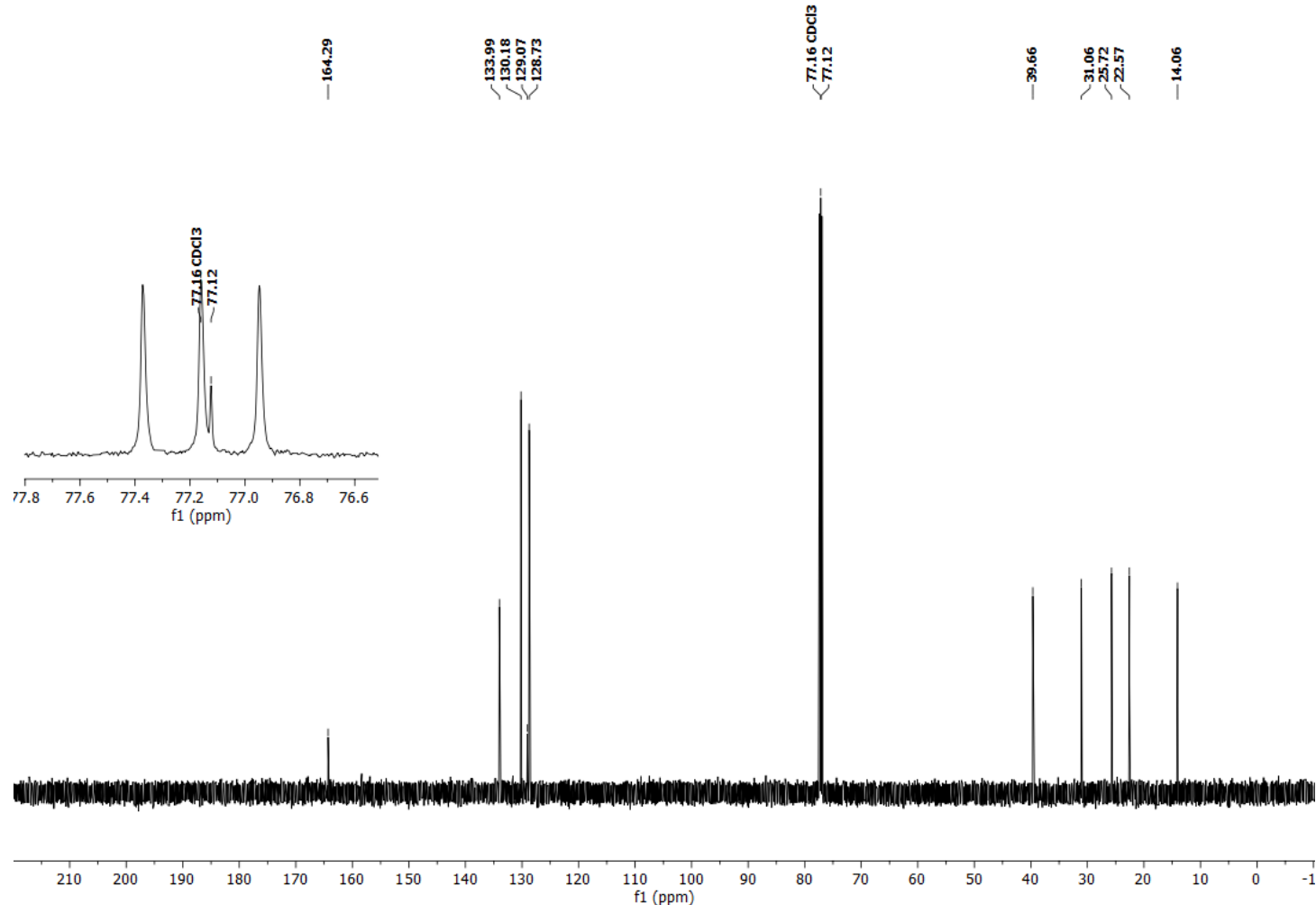
${ }^{1} \mathrm{H}$ NMR (599 MHz, $\mathrm{CDCl}_{3}$ )
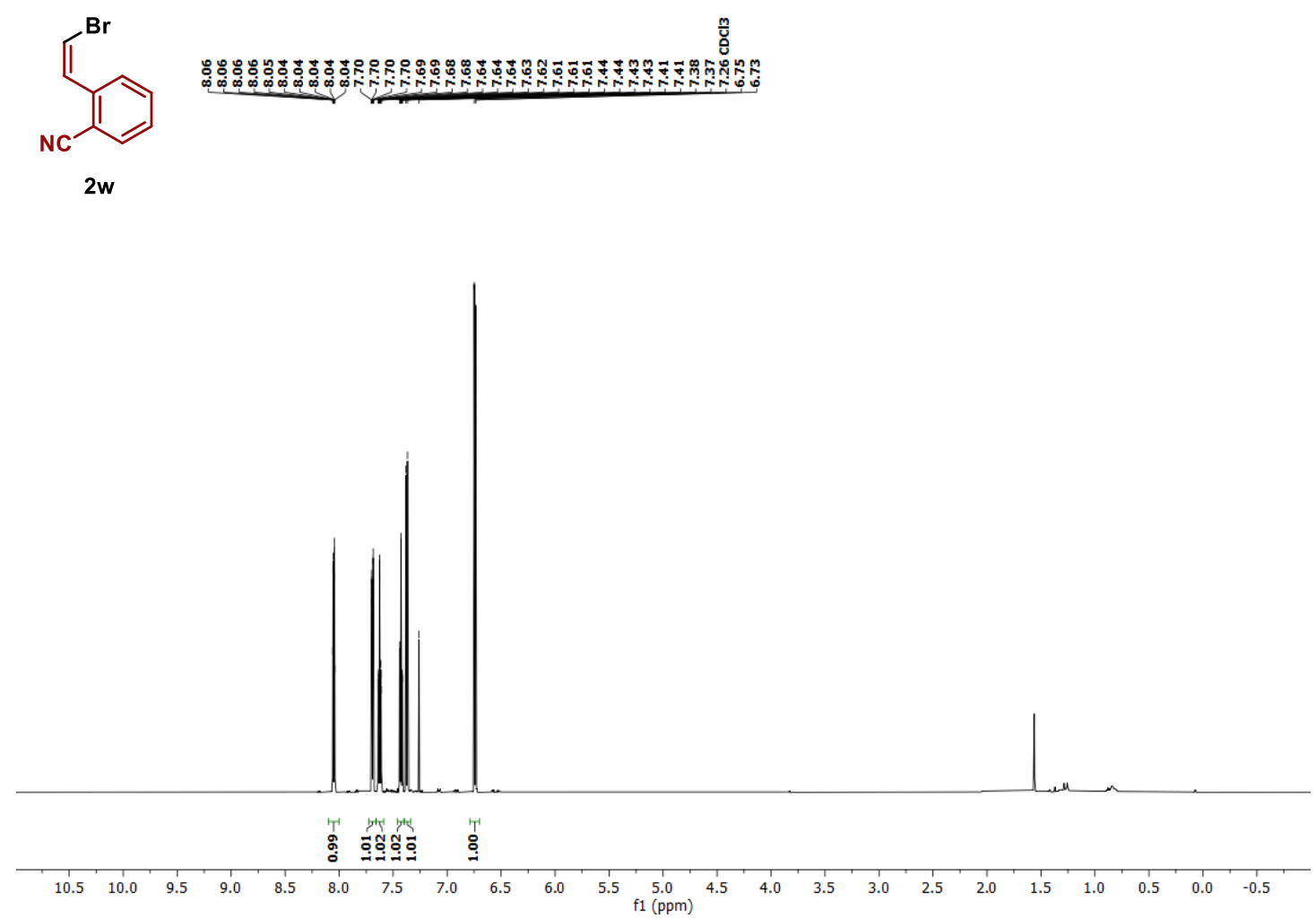

${ }^{13} \mathrm{C}$ NMR (151 MHz, $\mathrm{CDCl}_{3}$ )

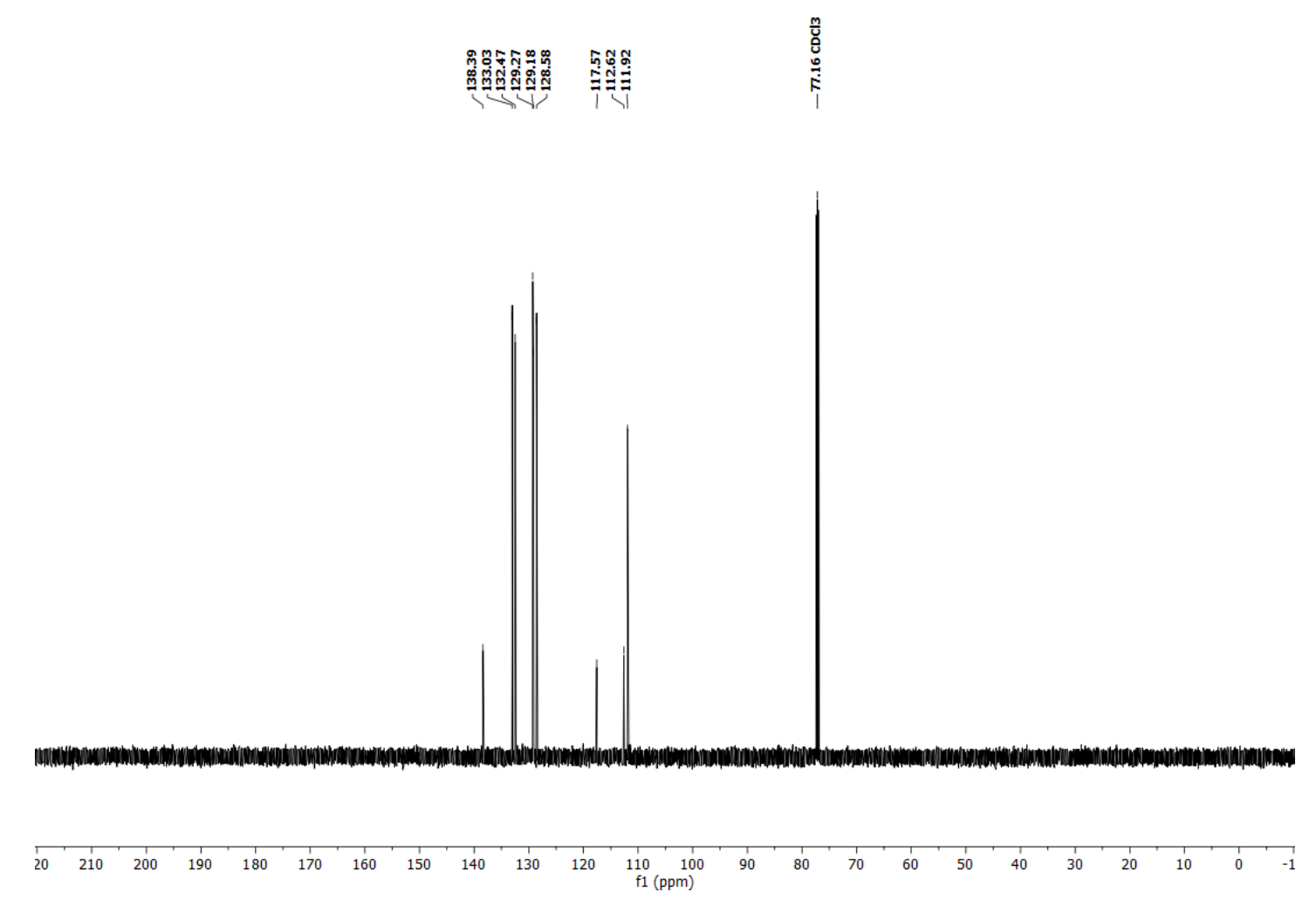


${ }_{1}^{1} \mathrm{H}$ NMR (400 MHz, $\mathrm{CDCl}_{3}$ )
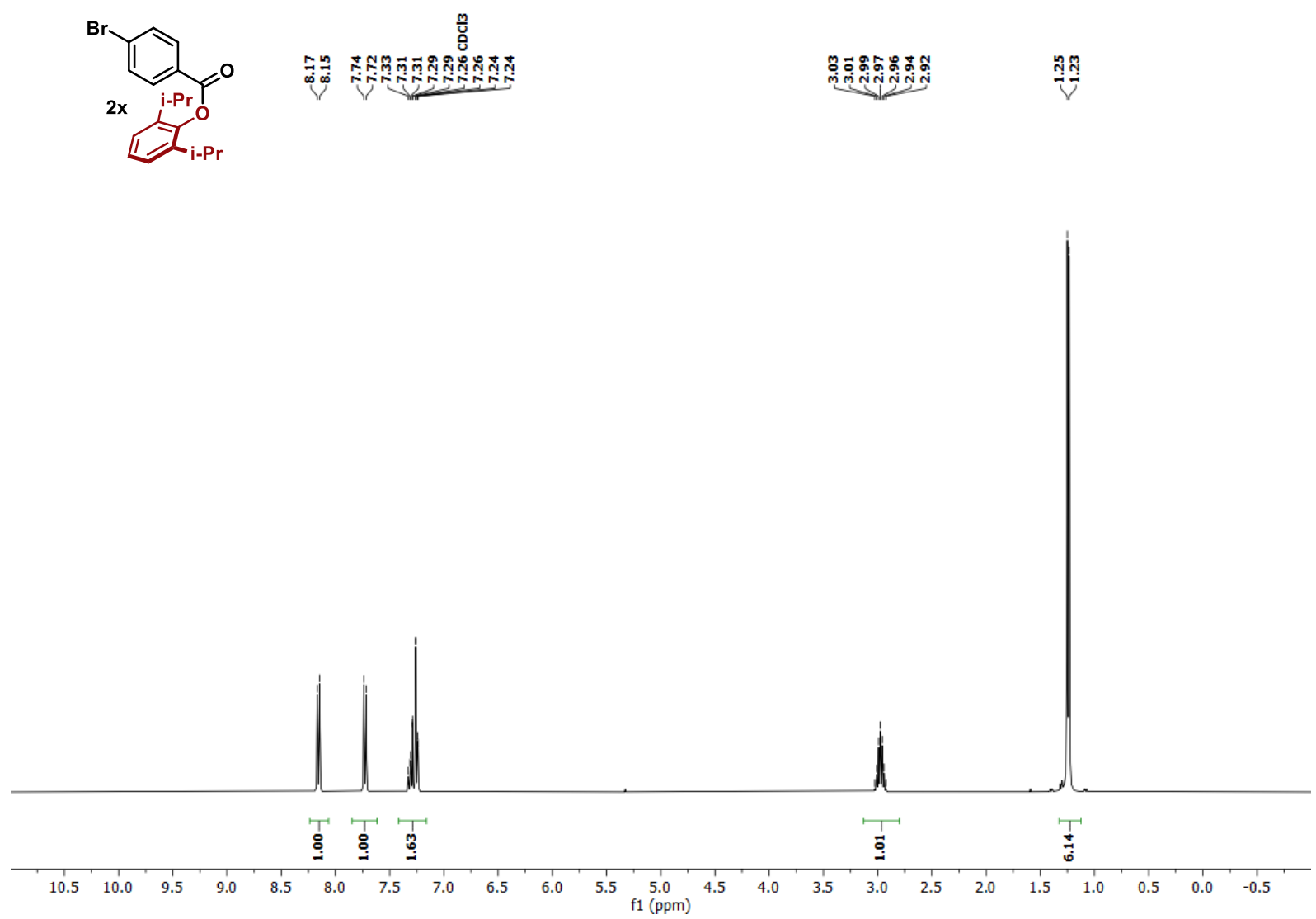

${ }^{13} \mathrm{C}$ NMR (101 MHz, $\mathrm{CDCl}_{3}$ )
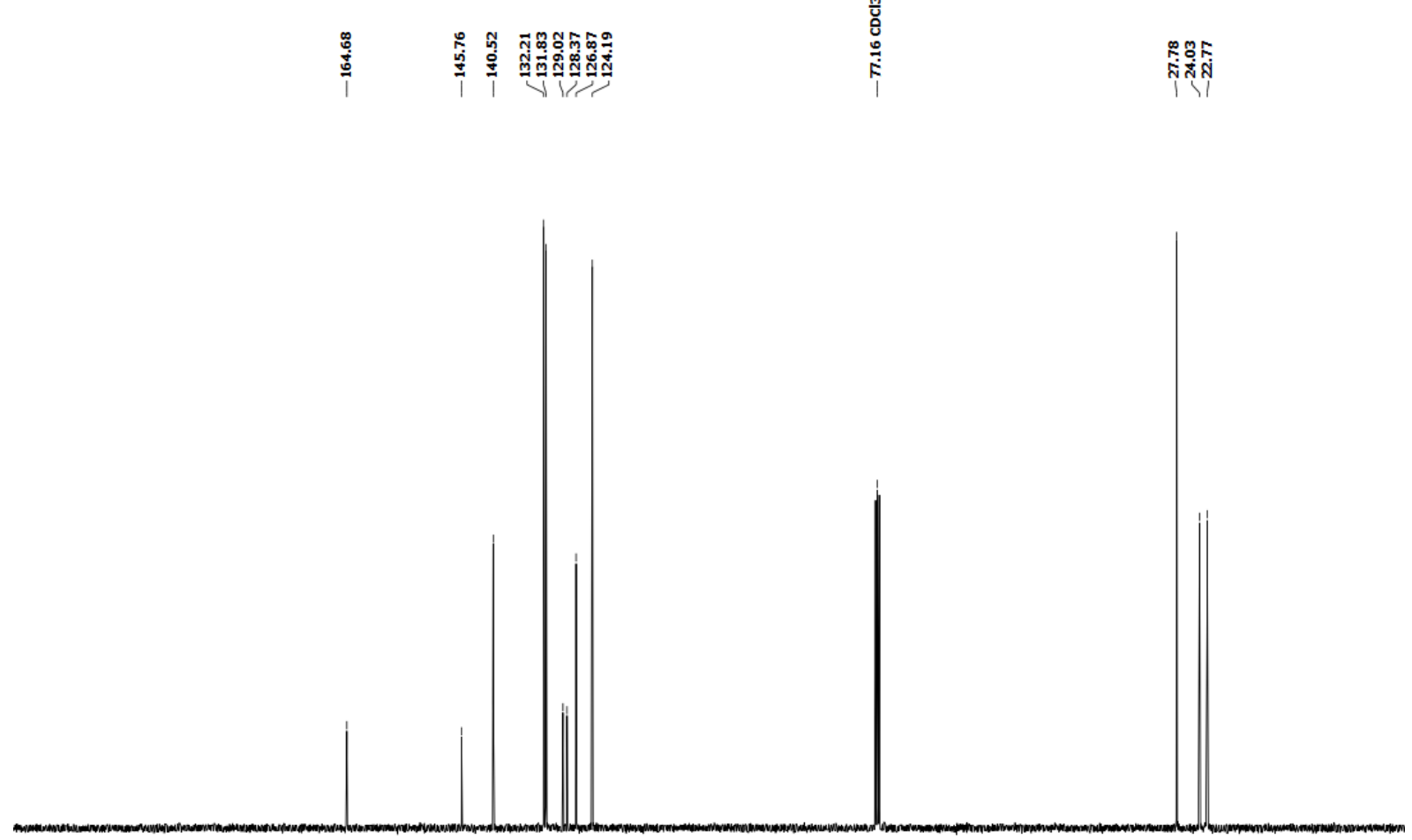

210
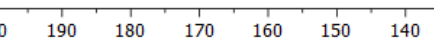


\section{${ }^{1} \mathrm{H}$ NMR (400 MHz, $\left.\mathrm{CDCl}_{3}\right)$}
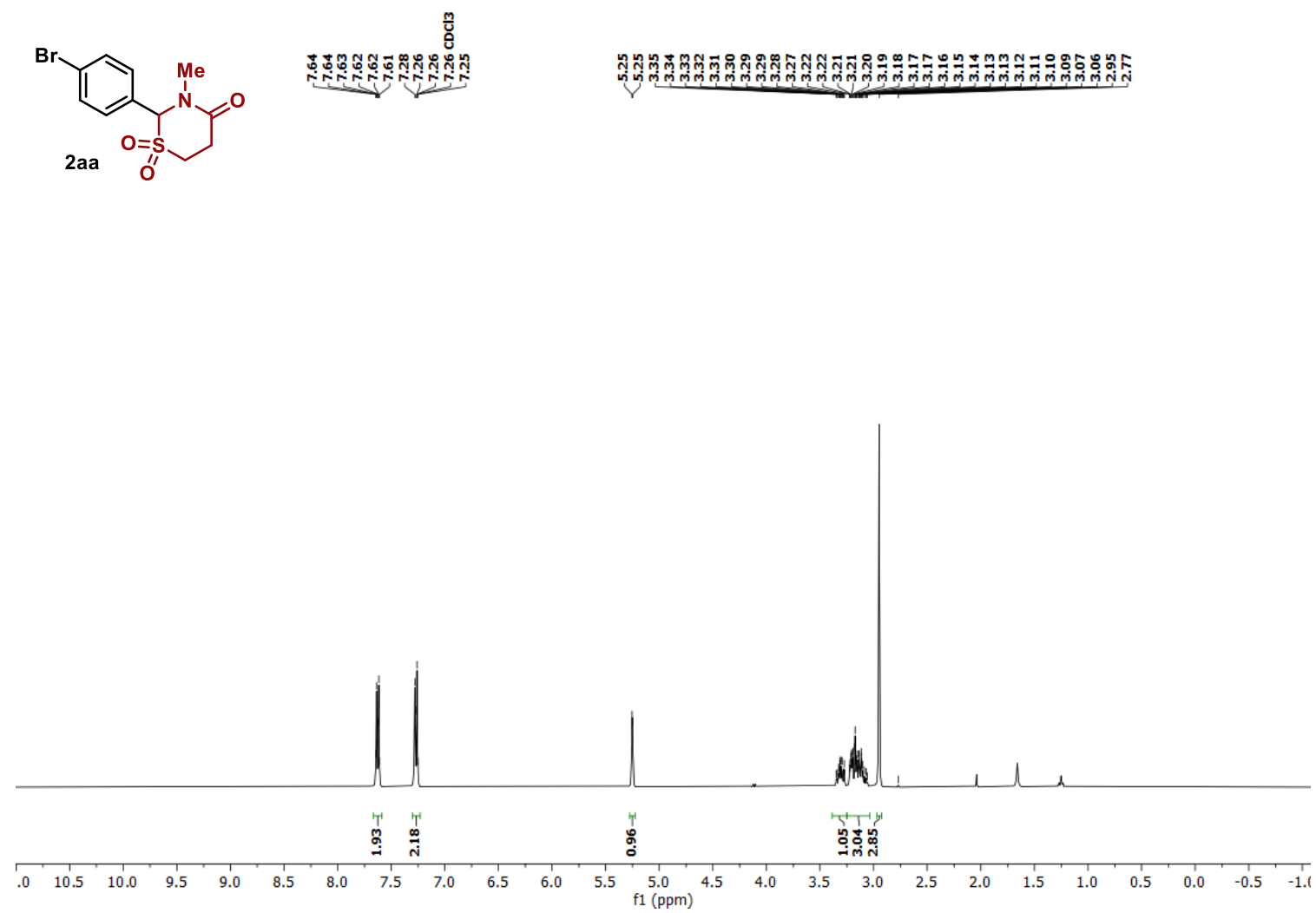

${ }^{13} \mathrm{C}$ NMR (101 $\mathrm{MHz}, \mathrm{CDCl}_{3}$ )

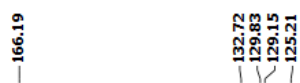

㖞

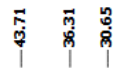

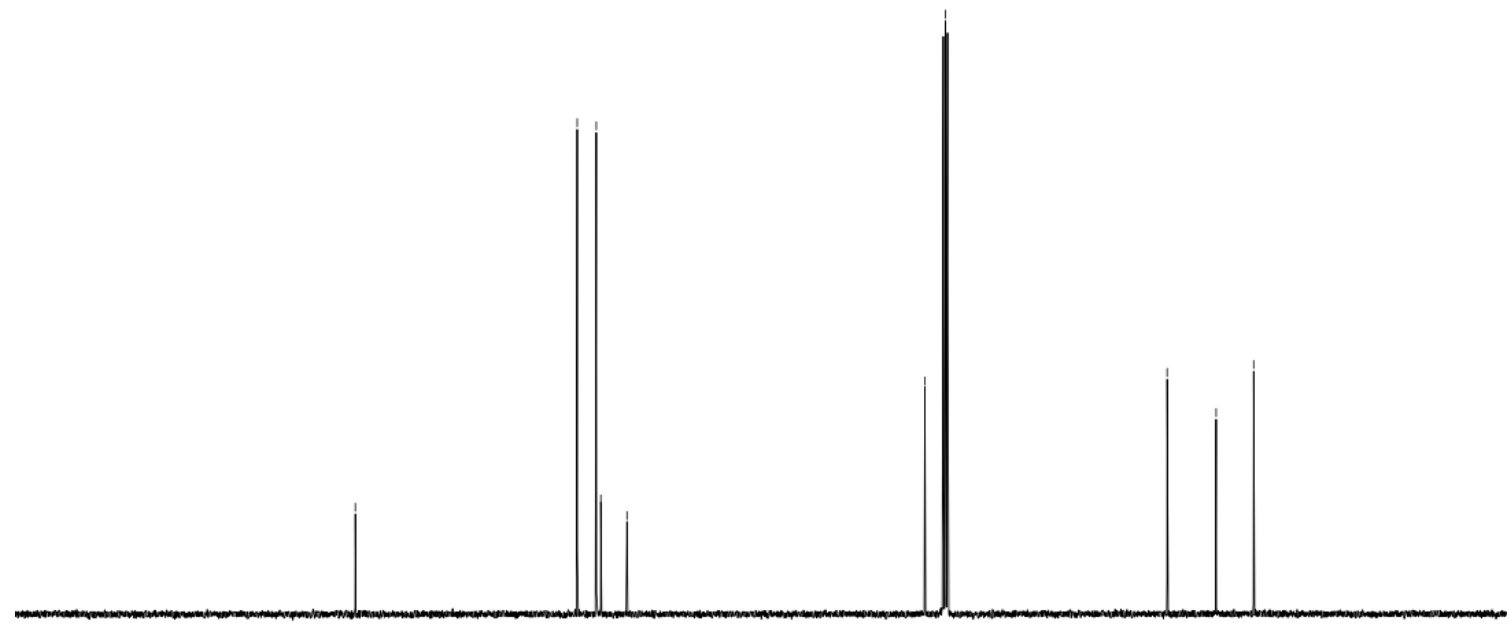

$\begin{array}{rrrrrrrrrrr}210 & 200 & 190 & 180 & 170 & 160 & 150 & 140 & 130 & 120 & \begin{array}{c}110 \\ \mathrm{f} 1(\mathrm{ppm})\end{array}\end{array}$ 
1H NMR (400 MHz, $\mathrm{CDCl}_{3}$ )

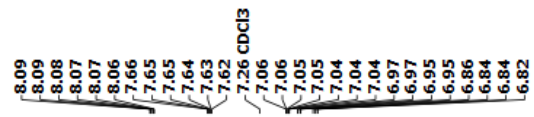

ตัตุตั ฺ
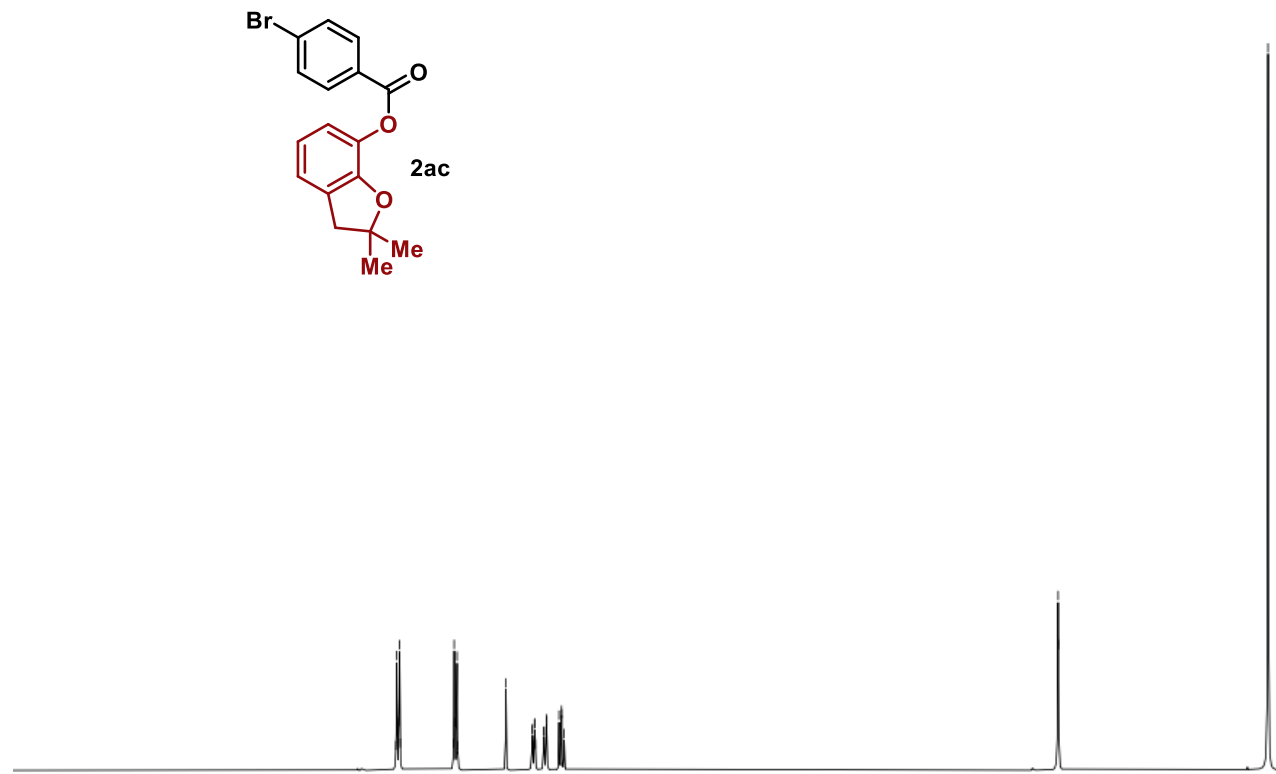

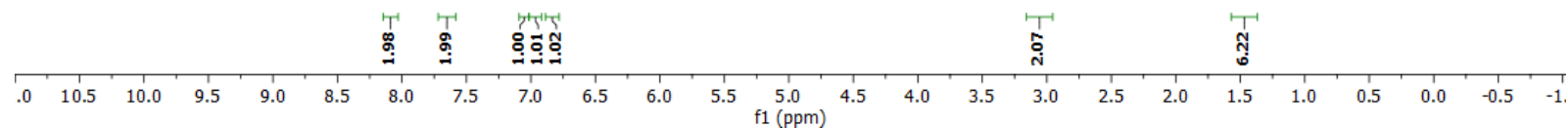

${ }^{13} \mathrm{C}$ NMR (101 MHz, $\mathrm{CDCl}_{3}$ )
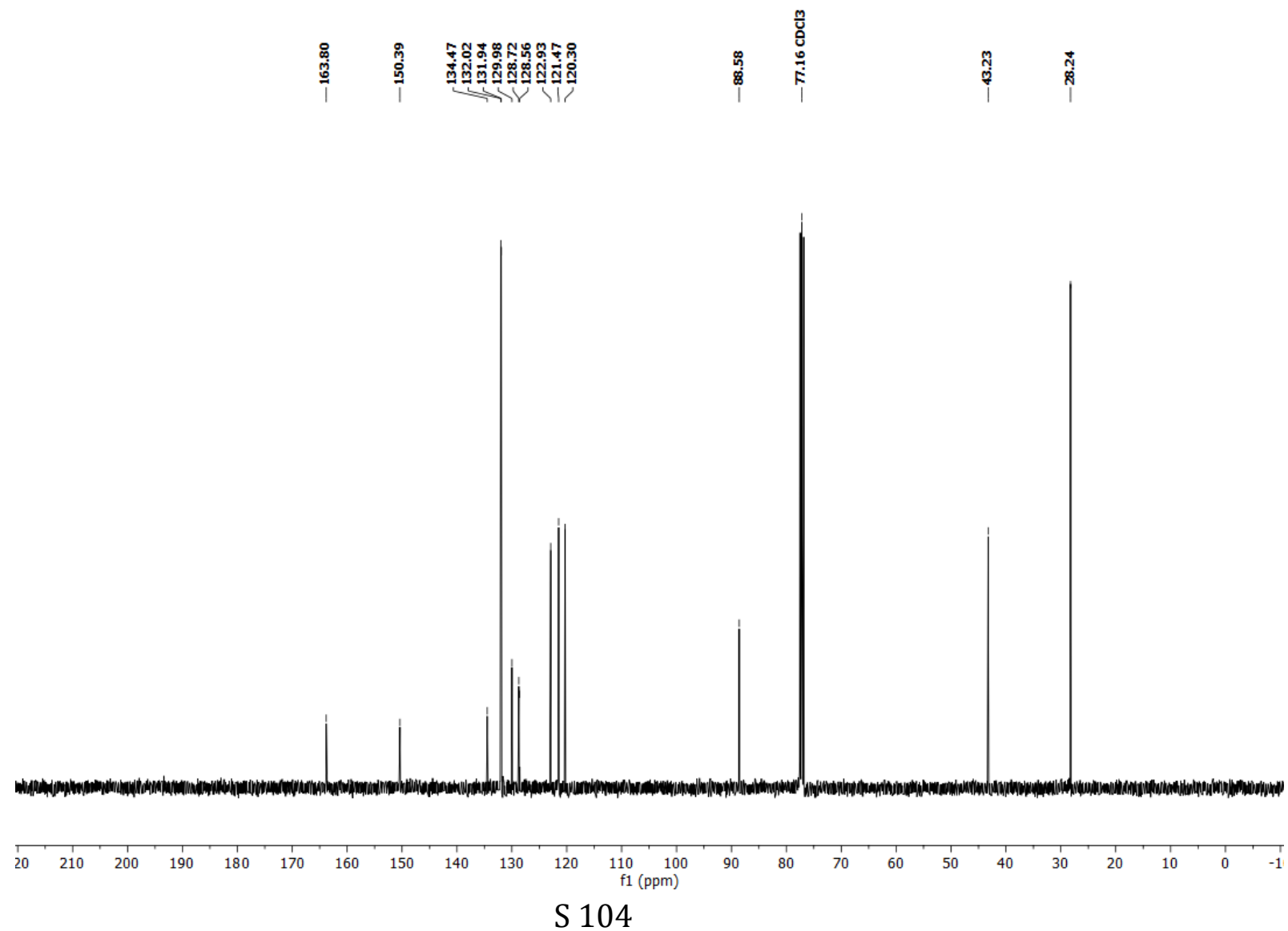


\section{${ }^{1} \mathrm{H}$ NMR (400 MHz, $\mathrm{CDCl}_{3}$ )}
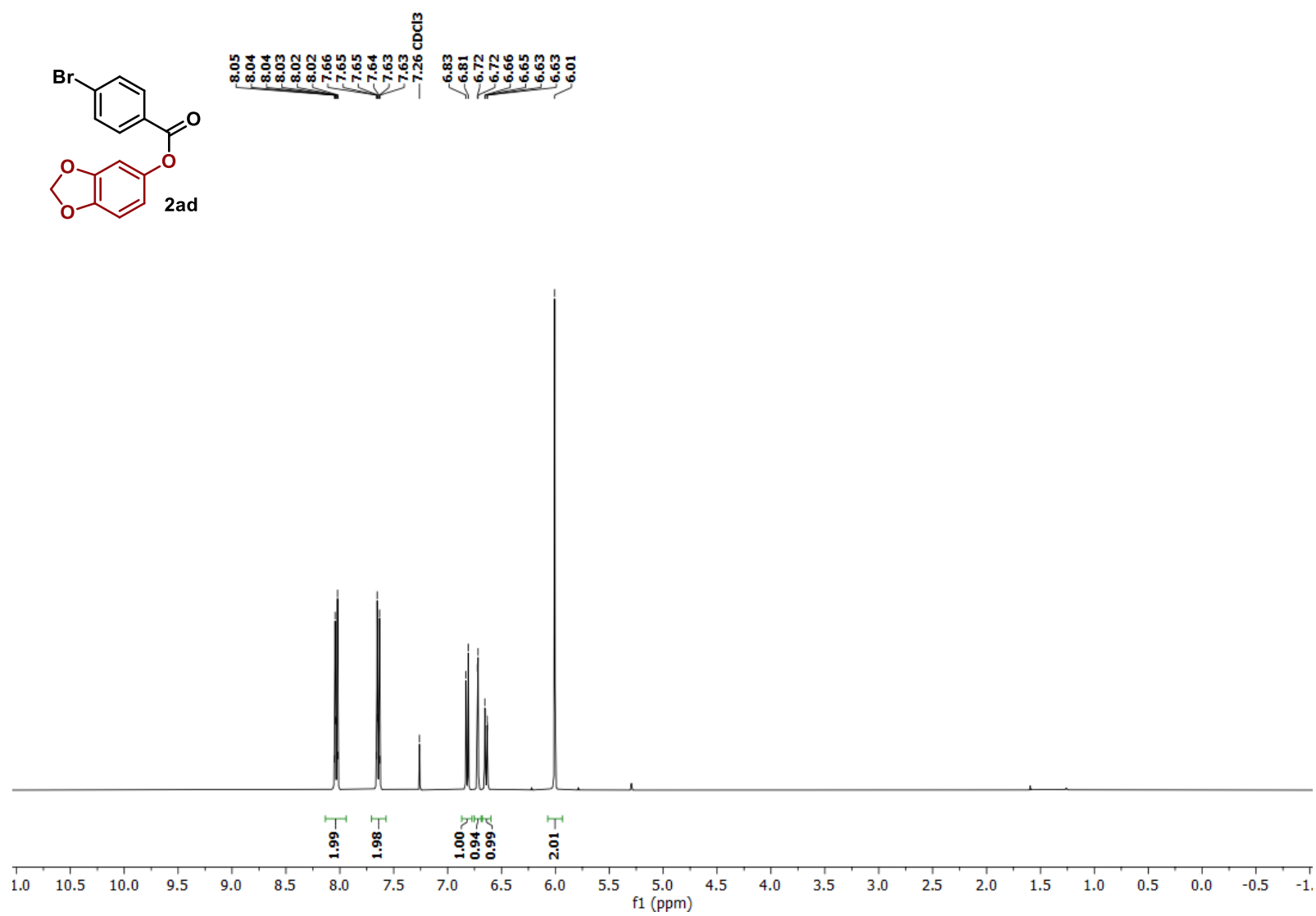

${ }^{13} \mathrm{C}$ NMR (101 MHz, $\mathrm{CDCl}_{3}$ )

\begin{tabular}{|c|c|c|}
\hline 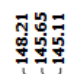 & 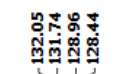 & 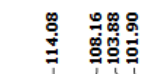 \\
\hline
\end{tabular}

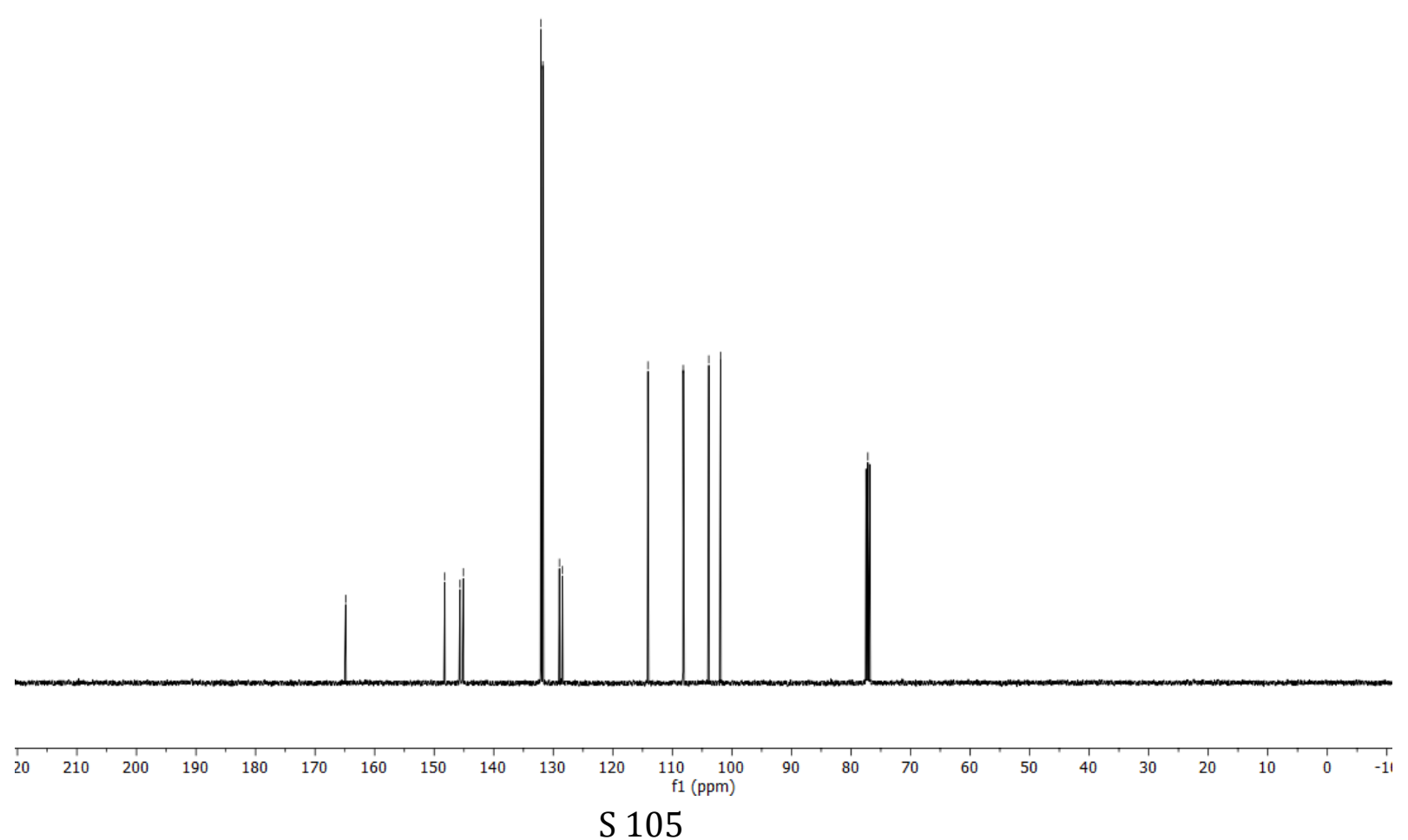


1H NMR (400 MHz, DMSO-d6)

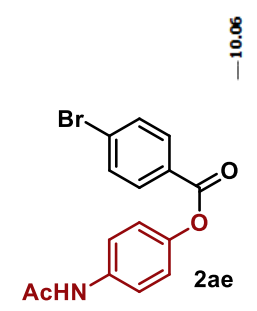

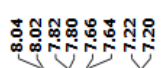

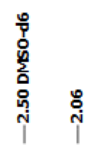

$2 \mathrm{ae}$

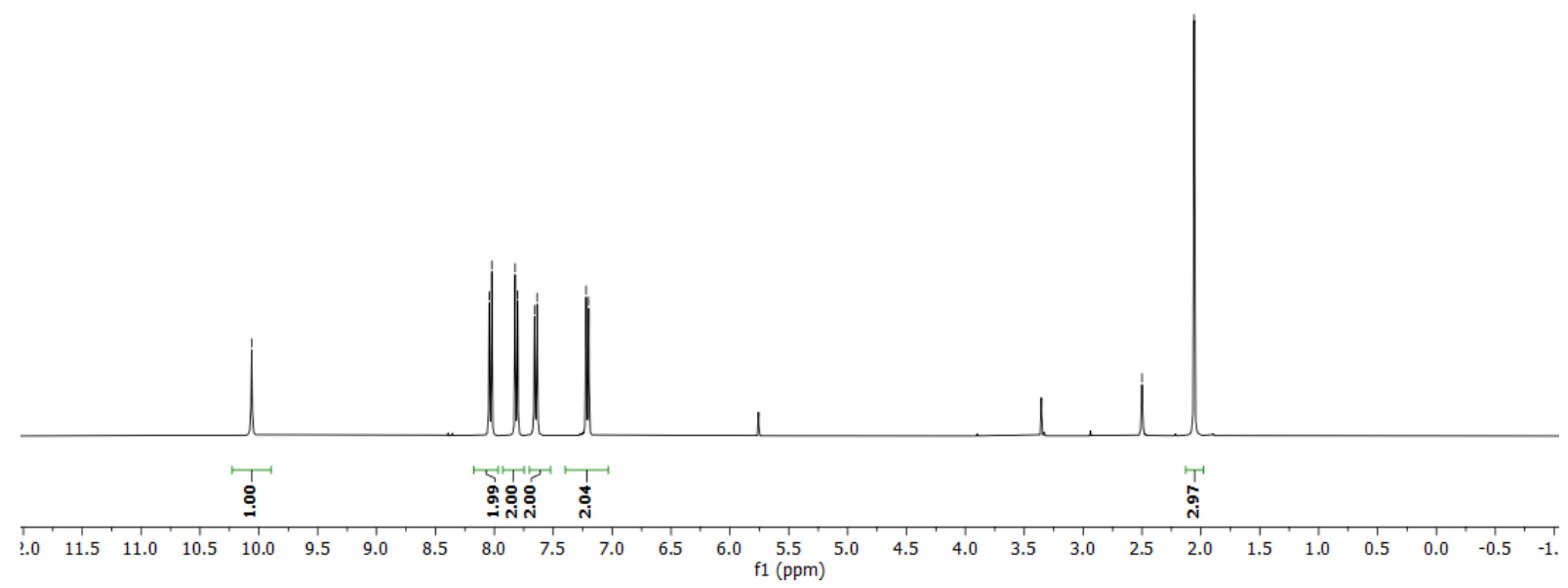

${ }^{13}$ C NMR (101 MHz, DMSO-d6)

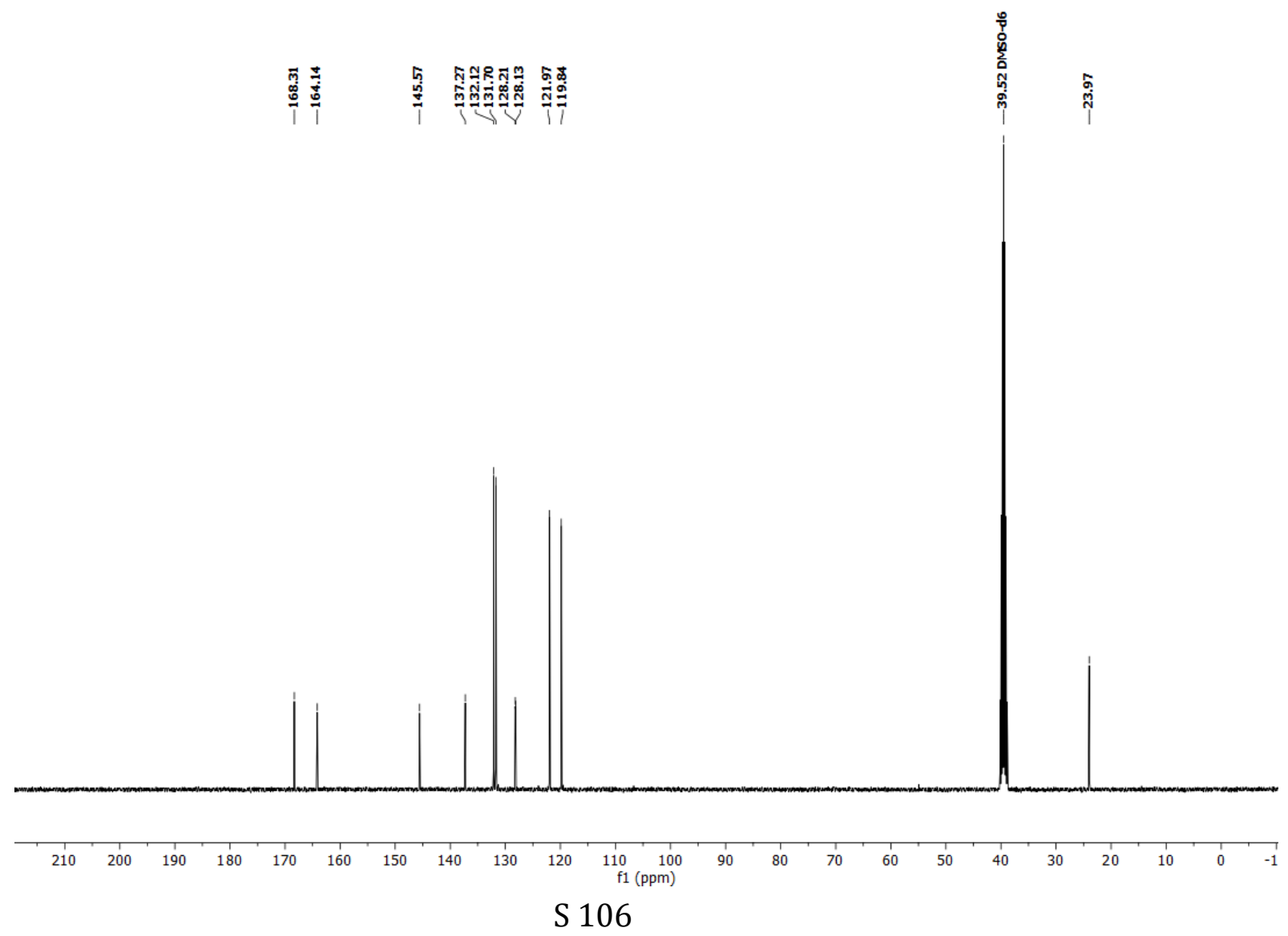


1H NMR (599 MHz, $\mathrm{CDCl}_{3}$ )
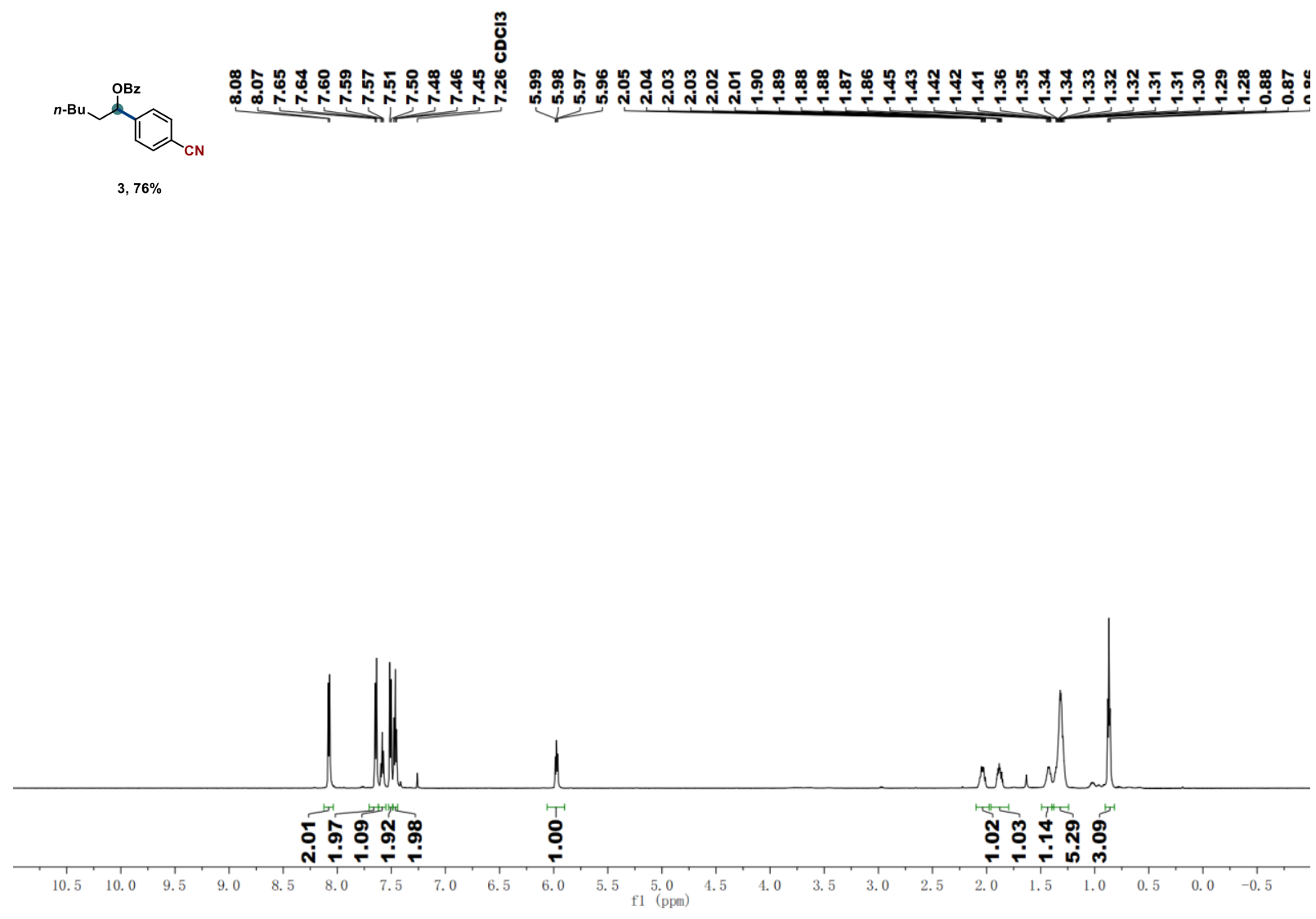

${ }^{13} \mathrm{C}$ NMR (151 MHz, $\left.\mathrm{CDCl}_{3}\right)$

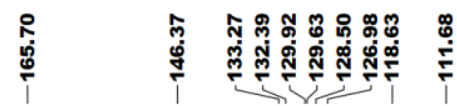

คูก

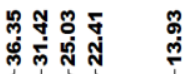

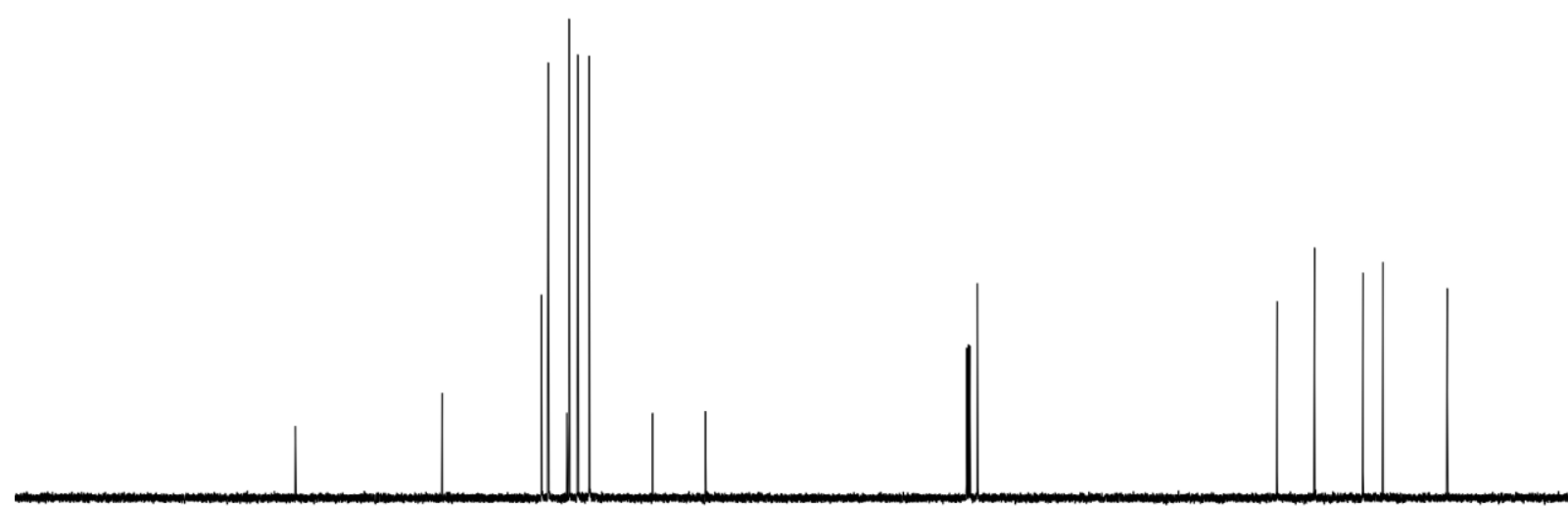


1H NMR (400 MHz, $\mathrm{CDCl}_{3}$ )
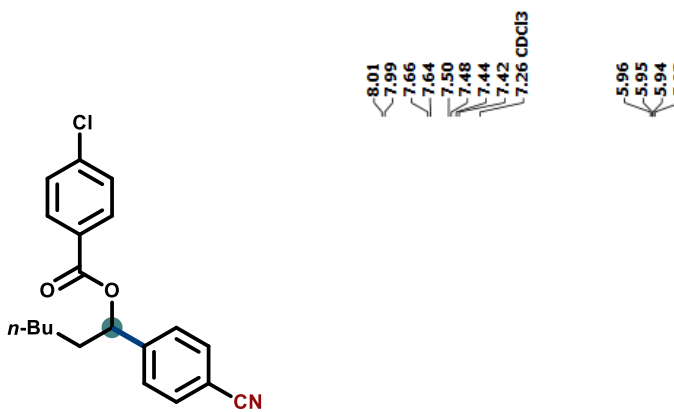

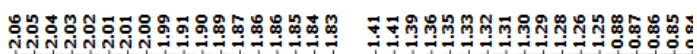

$9,49 \%$

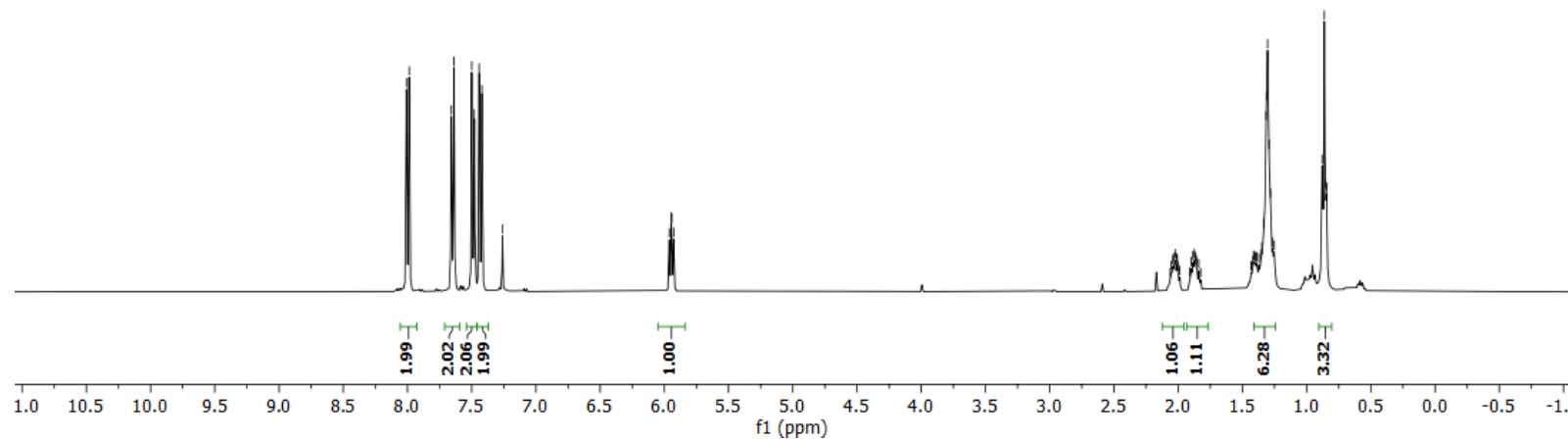

${ }^{13} \mathrm{C}$ NMR (101 MHz, $\mathrm{CDCl}_{3}$ )
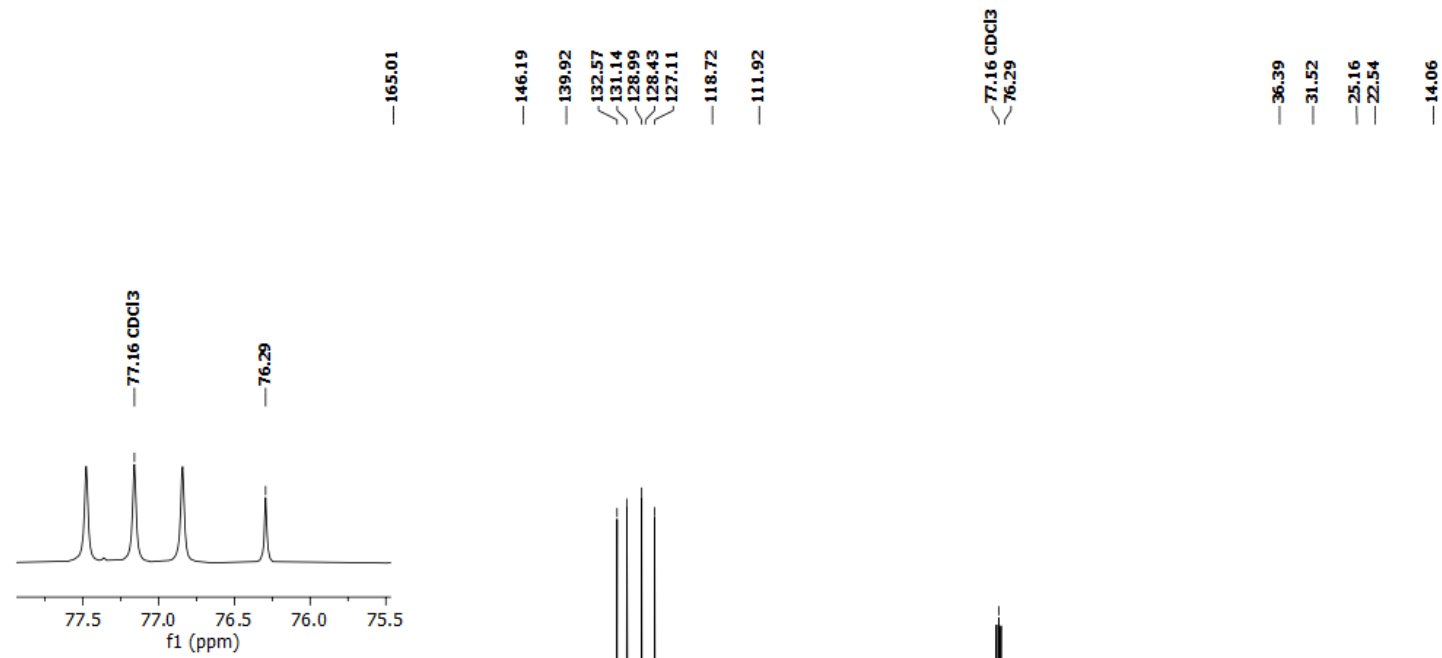

$\begin{array}{llllllllllll}210 & 200 & 190 & 180 & 170 & 160 & 150 & 140 & 130 & 120 & 110 & 100\end{array}$

S 108 
1H NMR (599 MHz, $\left.\mathrm{CDCl}_{3}\right)$

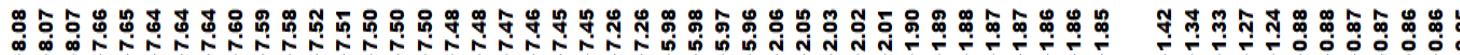<smiles>CCC(O)c1ccc(N)cc1</smiles>

$10,53 \%$

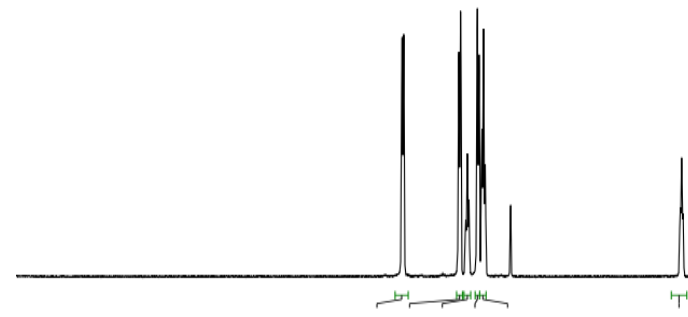

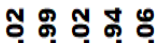
8

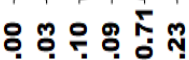

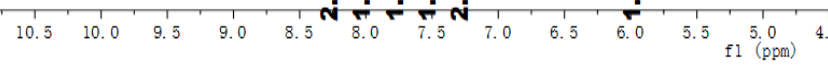

${ }^{13} \mathrm{C}$ NMR (151 MHz, $\left.\mathrm{CDCl}_{3}\right)$
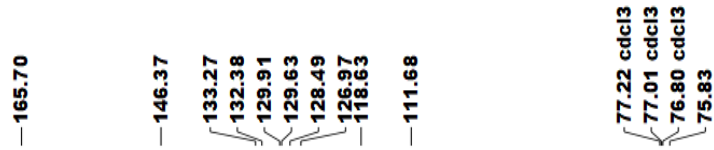

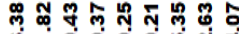

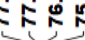

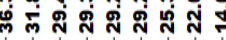

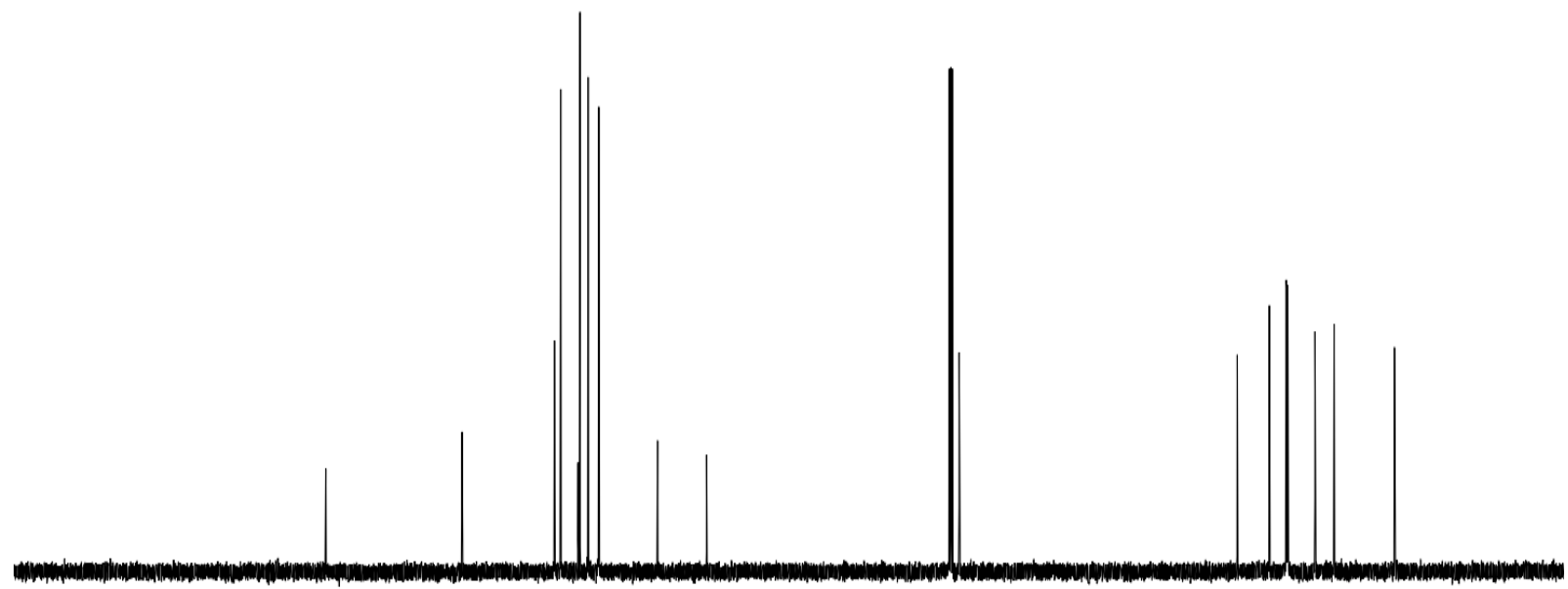

$\begin{array}{lllllllllll}200 & 190 & 180 & 170 & 160 & 150 & 140 & 130 & 120 & 110 & 100\end{array}$ 
1H NMR (599 MHz, $\mathrm{CDCl}_{3}$ )

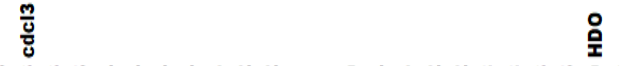

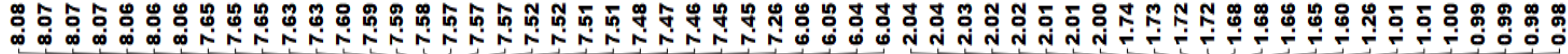<smiles>CC(C)CC(=O)c1ccc(C#N)cc1</smiles>

$11,54 \%$

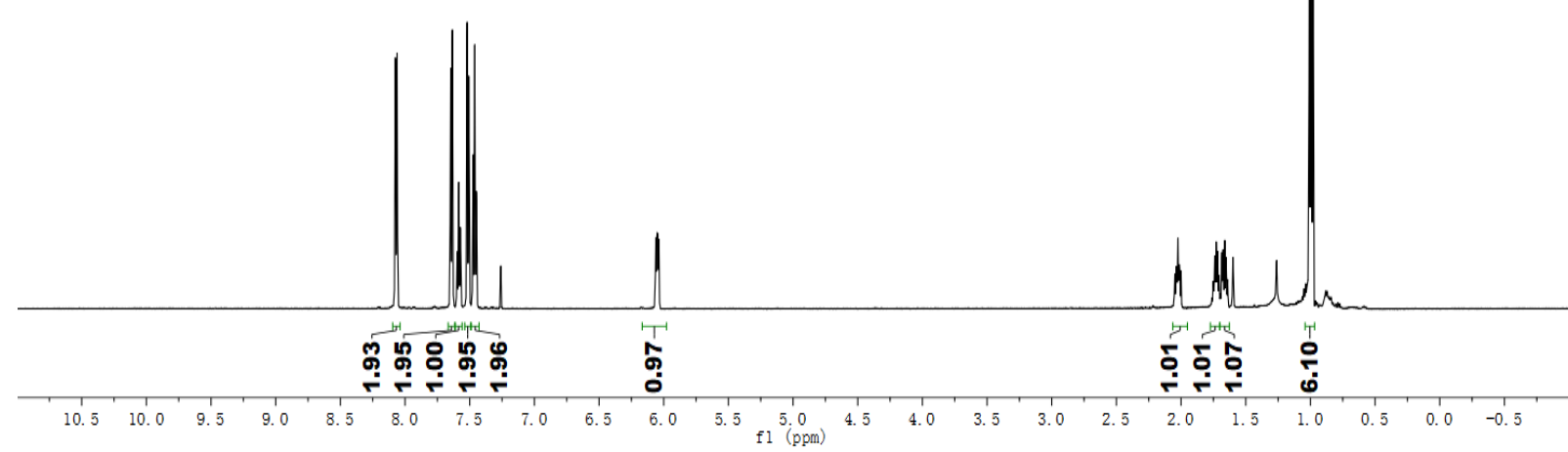

${ }^{13} \mathrm{C}$ NMR (151 MHz, $\left.\mathrm{CDCl}_{3}\right)$

告
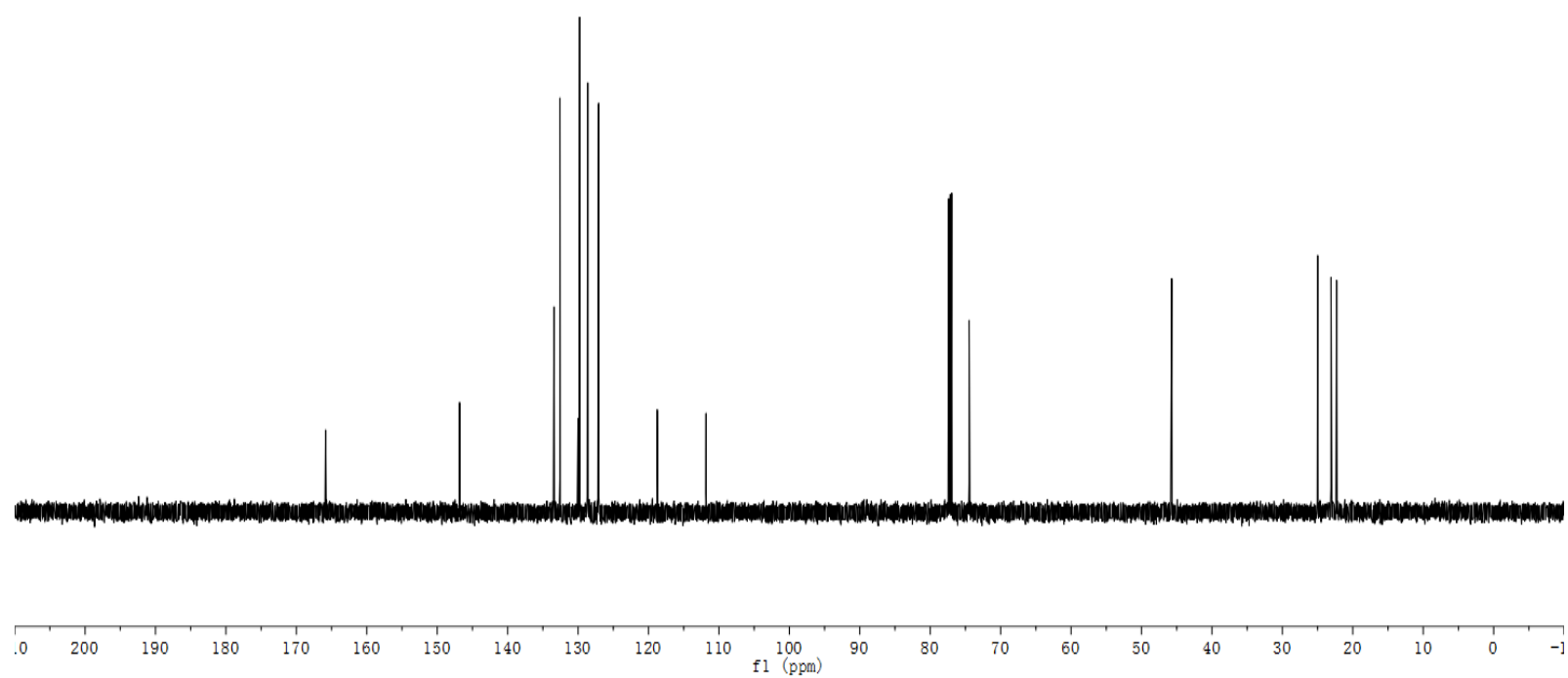

S 110 
1H NMR (599 MHz, $\mathrm{CDCl}_{3}$ )
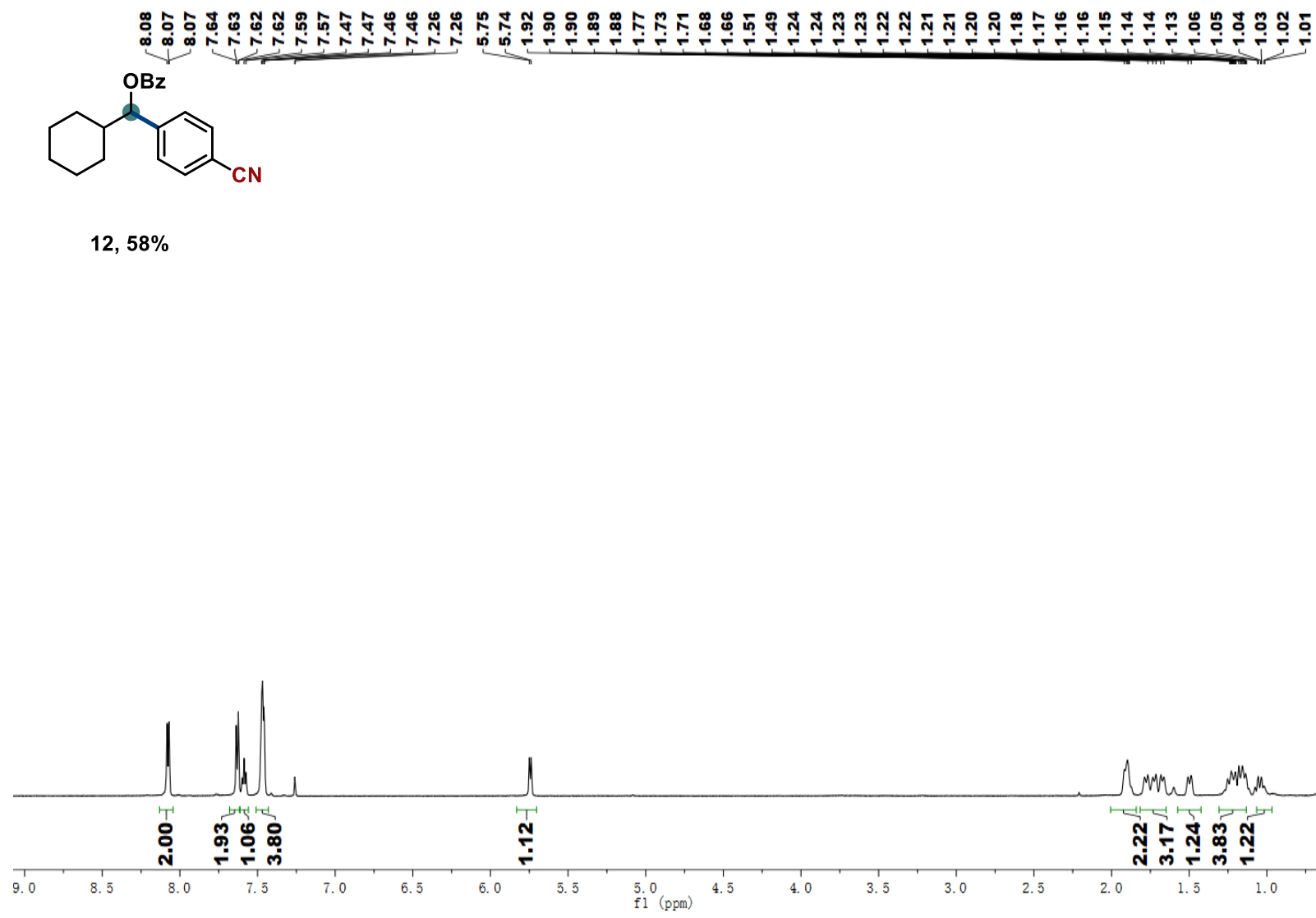

${ }^{13} \mathrm{C}$ NMR (151 $\mathrm{MHz}, \mathrm{CDCl}_{3}$ )

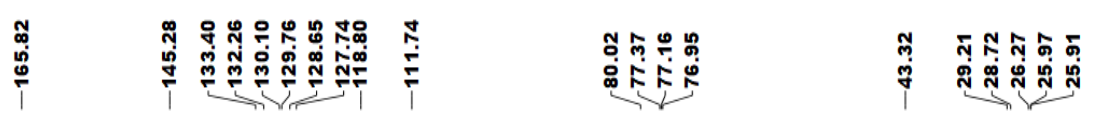
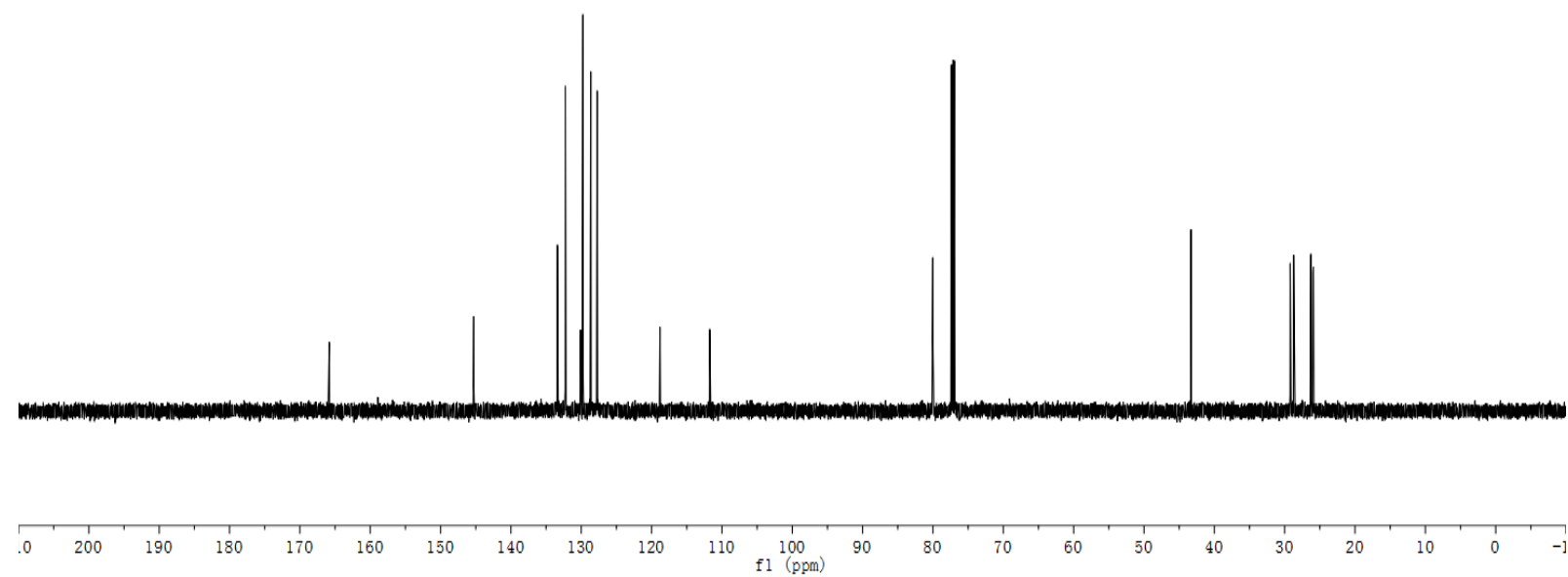


\section{H NMR (400 MHz, $\mathrm{CDCl}_{3}$ )}<smiles>N#Cc1ccc([C@@H](O)C2CCC3(CCCCC3)CC2)cc1</smiles>

$\frac{0}{8}$

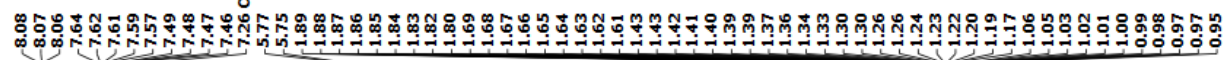
$13,64 \%$

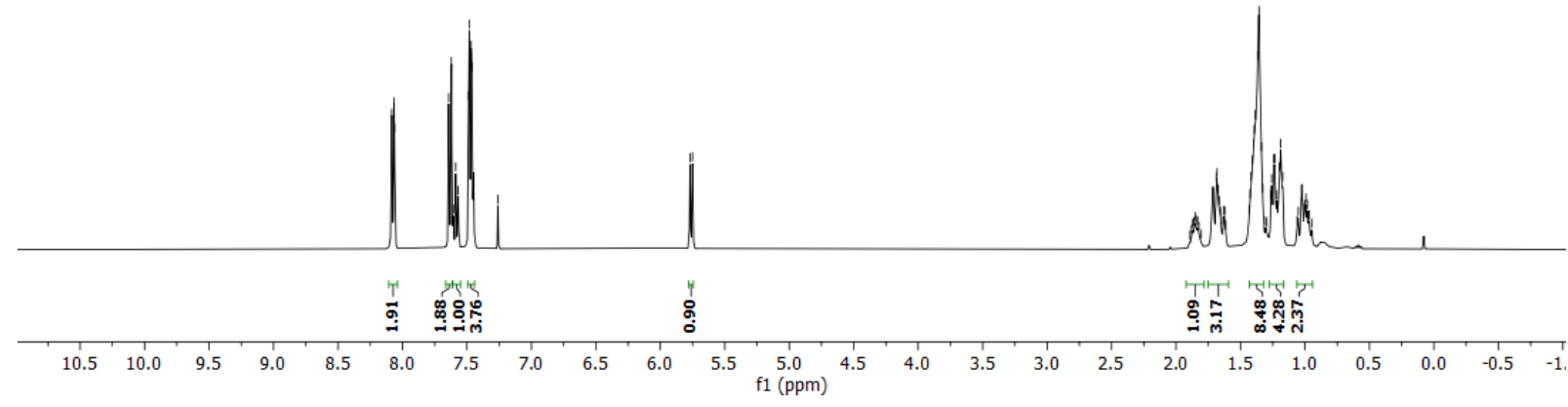

${ }^{13} \mathrm{C}$ NMR (101 MHz, $\mathrm{CDCl}_{3}$ )
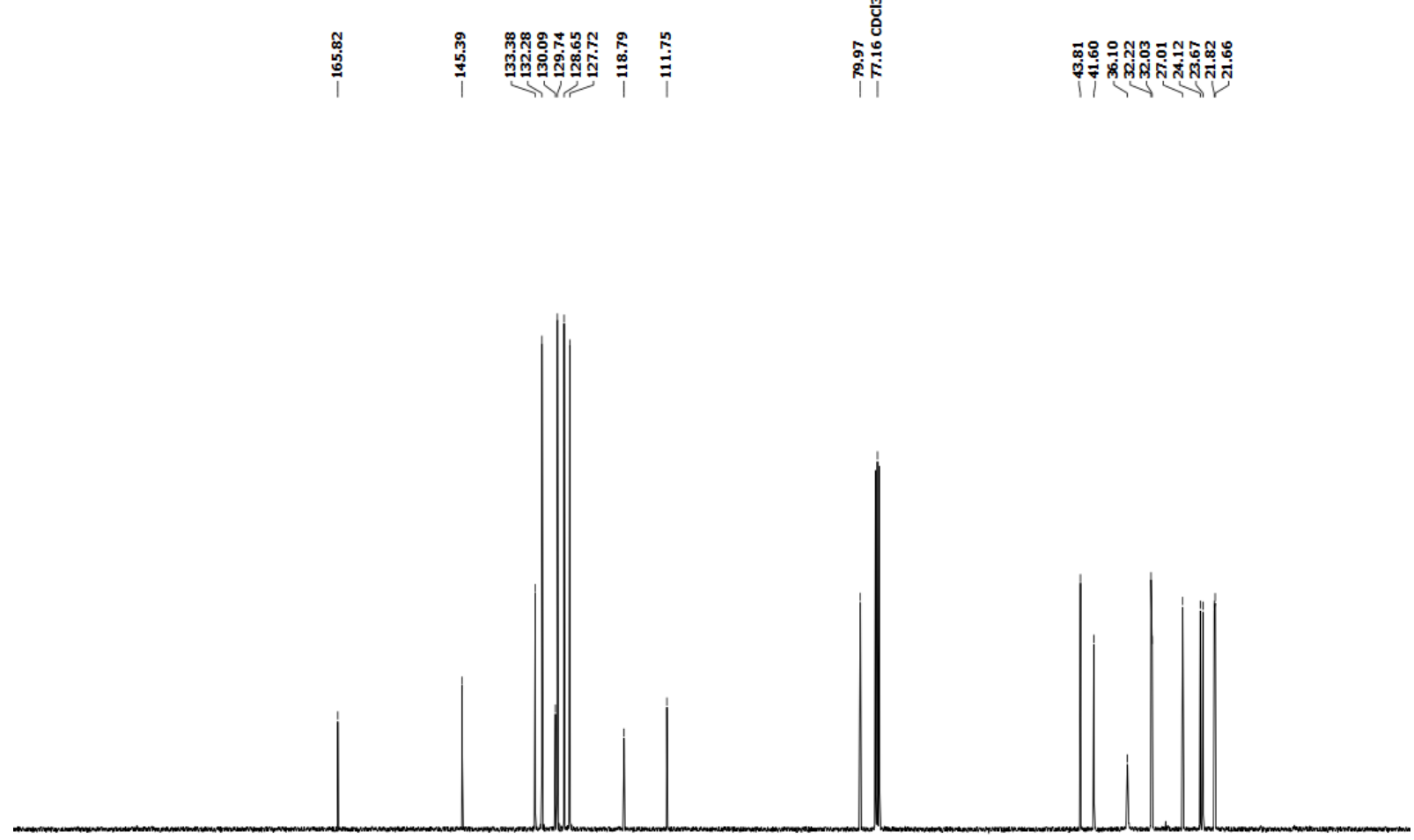
${ }^{1} \mathrm{H}$ NMR (599 MHz, $\mathrm{CDCl}_{3}$ )

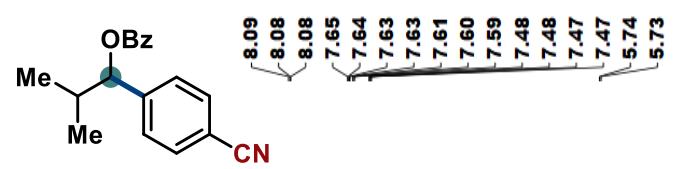

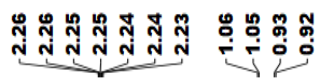

$14,54 \%$

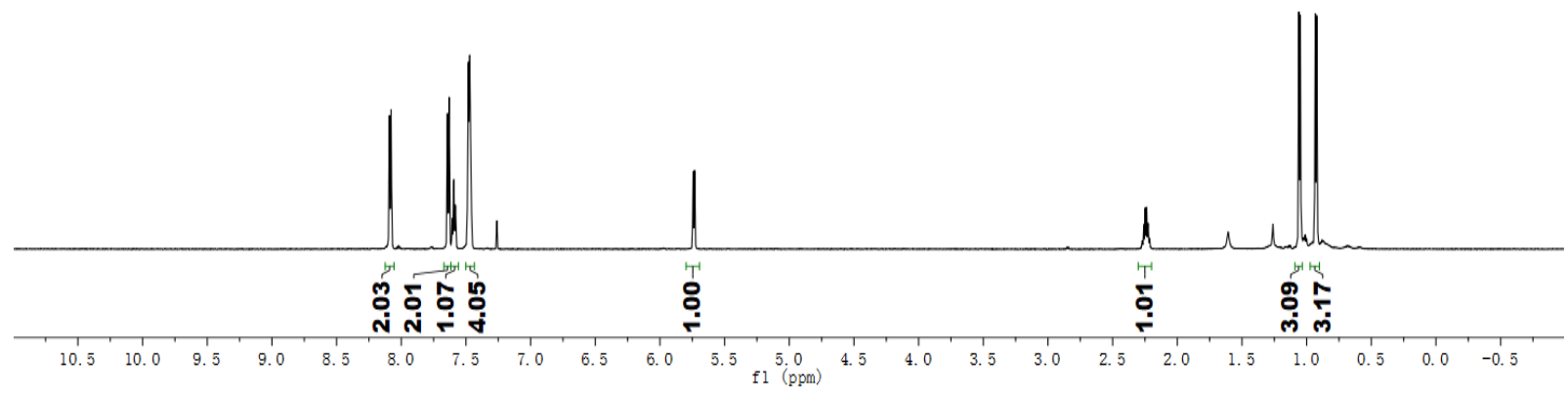

${ }^{13} \mathrm{C}$ NMR (151 $\left.\mathrm{MHz}, \mathrm{CDCl}_{3}\right)$
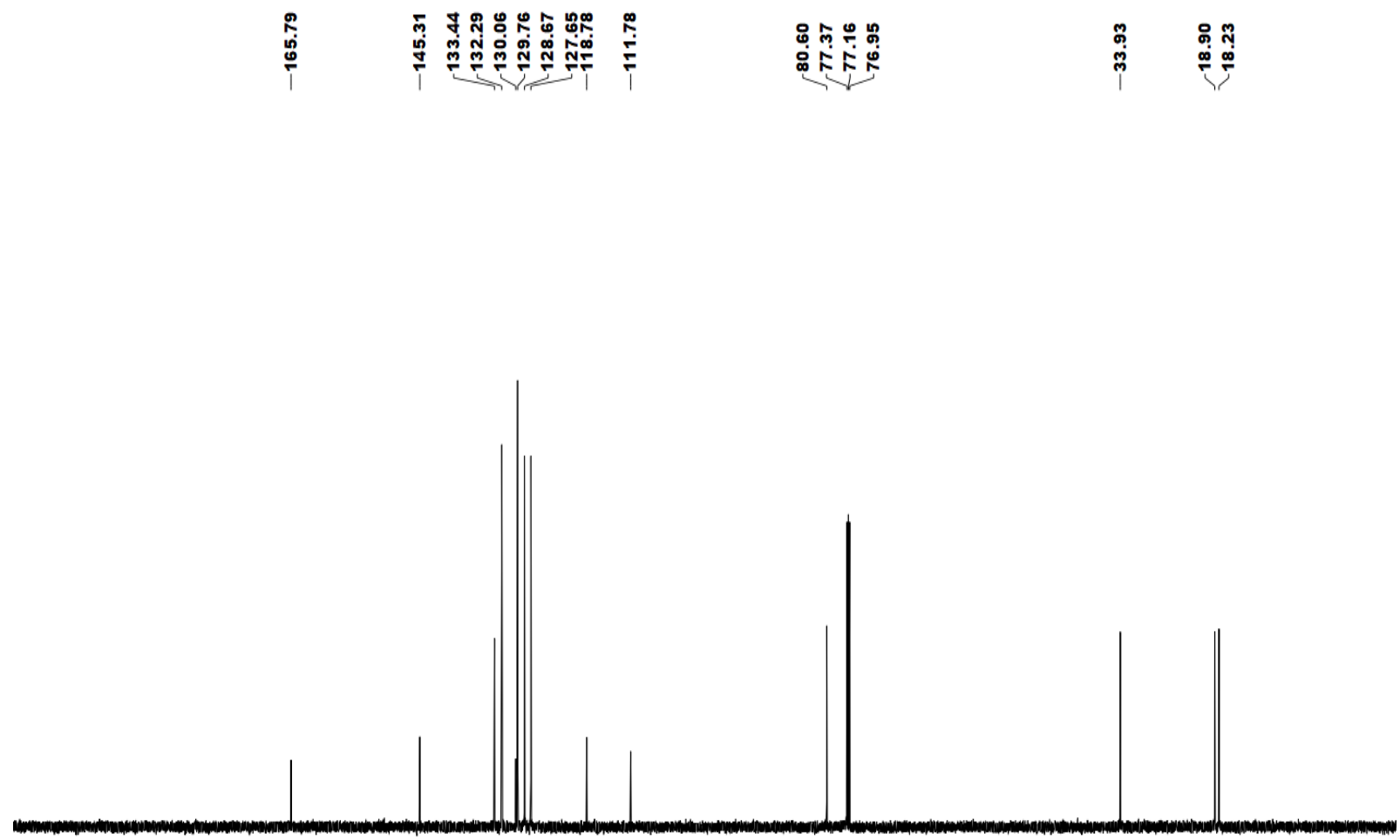

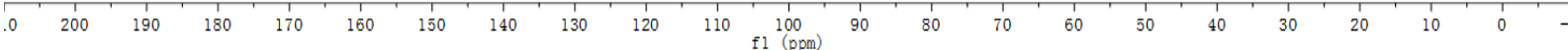
S 113 
1H NMR (599 MHz, $\mathrm{CDCl}_{3}$ )

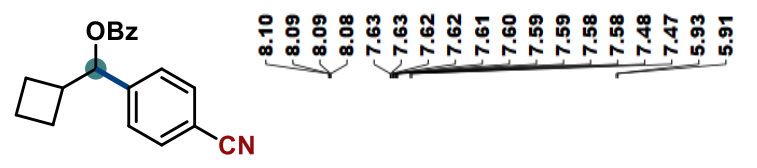

暨

$15,56 \%$

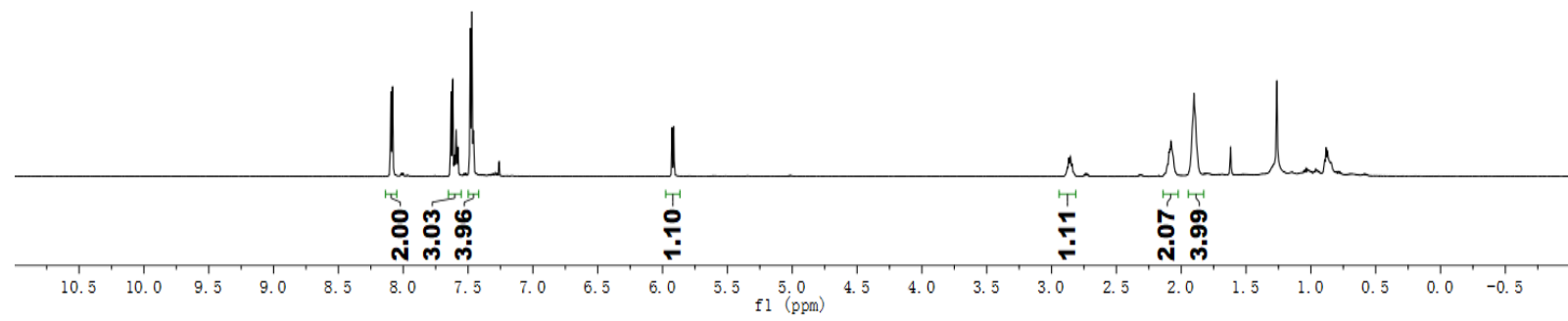

${ }^{13} \mathrm{C}$ NMR (151 MHz, $\mathrm{CDCl}_{3}$ )

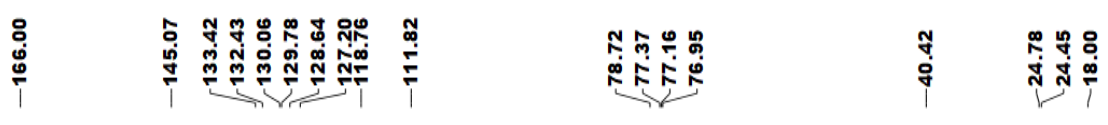
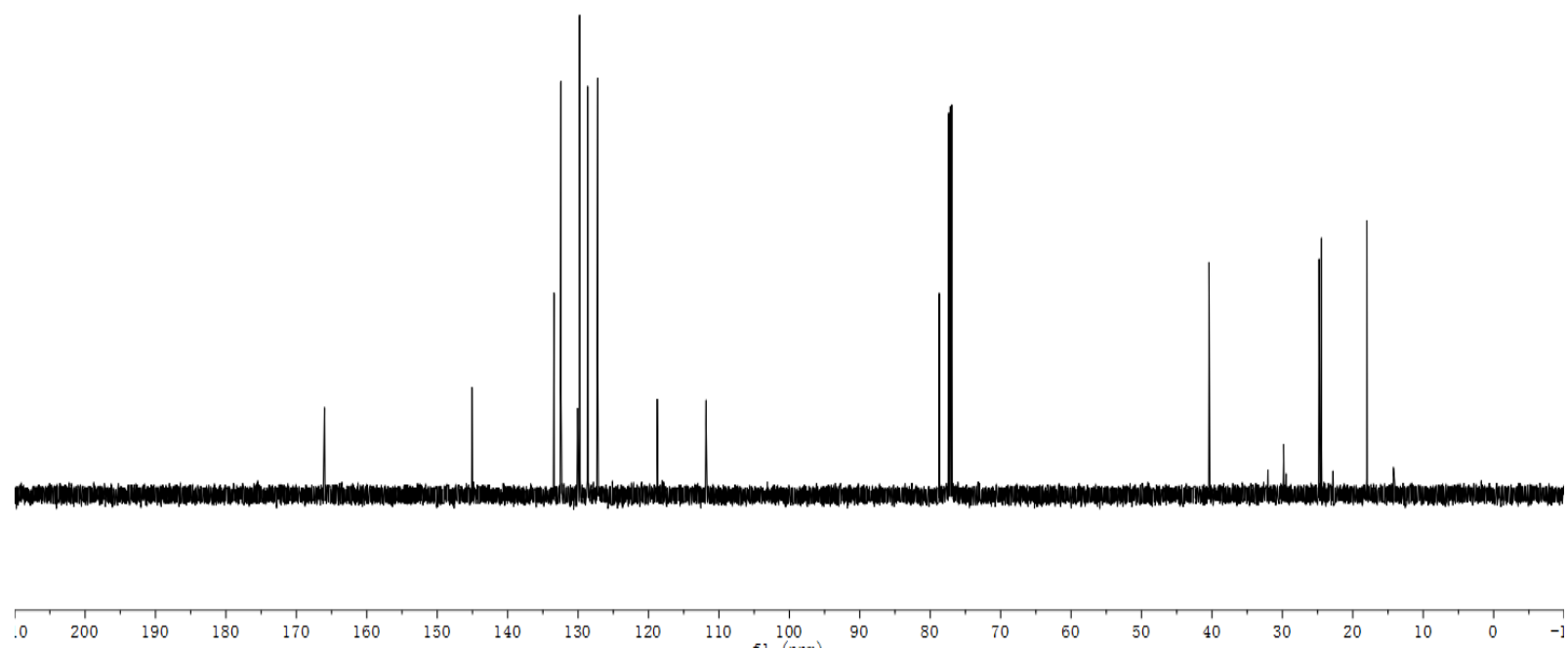

S 114 
${ }^{1} \mathrm{H}$ NMR (400 MHz, $\mathrm{CDCl}_{3}$ )

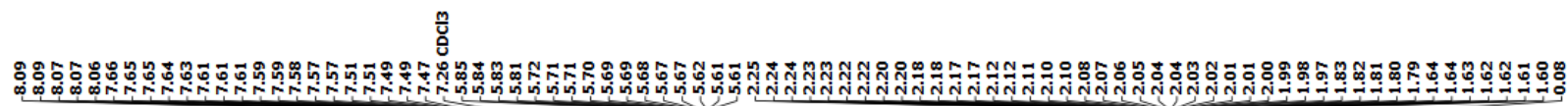<smiles>N#Cc1ccc(C(O)C2CC=CCC2)cc1</smiles>

$16,52 \%$ yield

$1: 1 \mathrm{dr}$

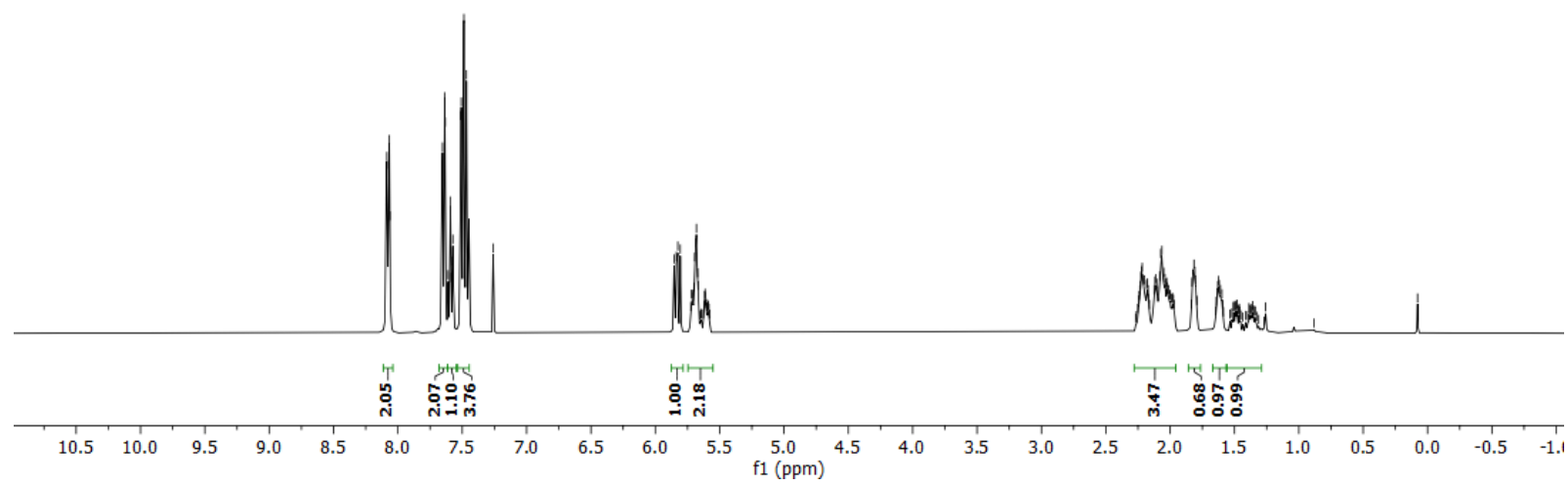

${ }^{13} \mathrm{C}$ NMR (101 MHz, $\left.\mathrm{CDCl}_{3}\right)$

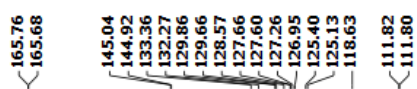

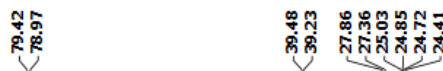

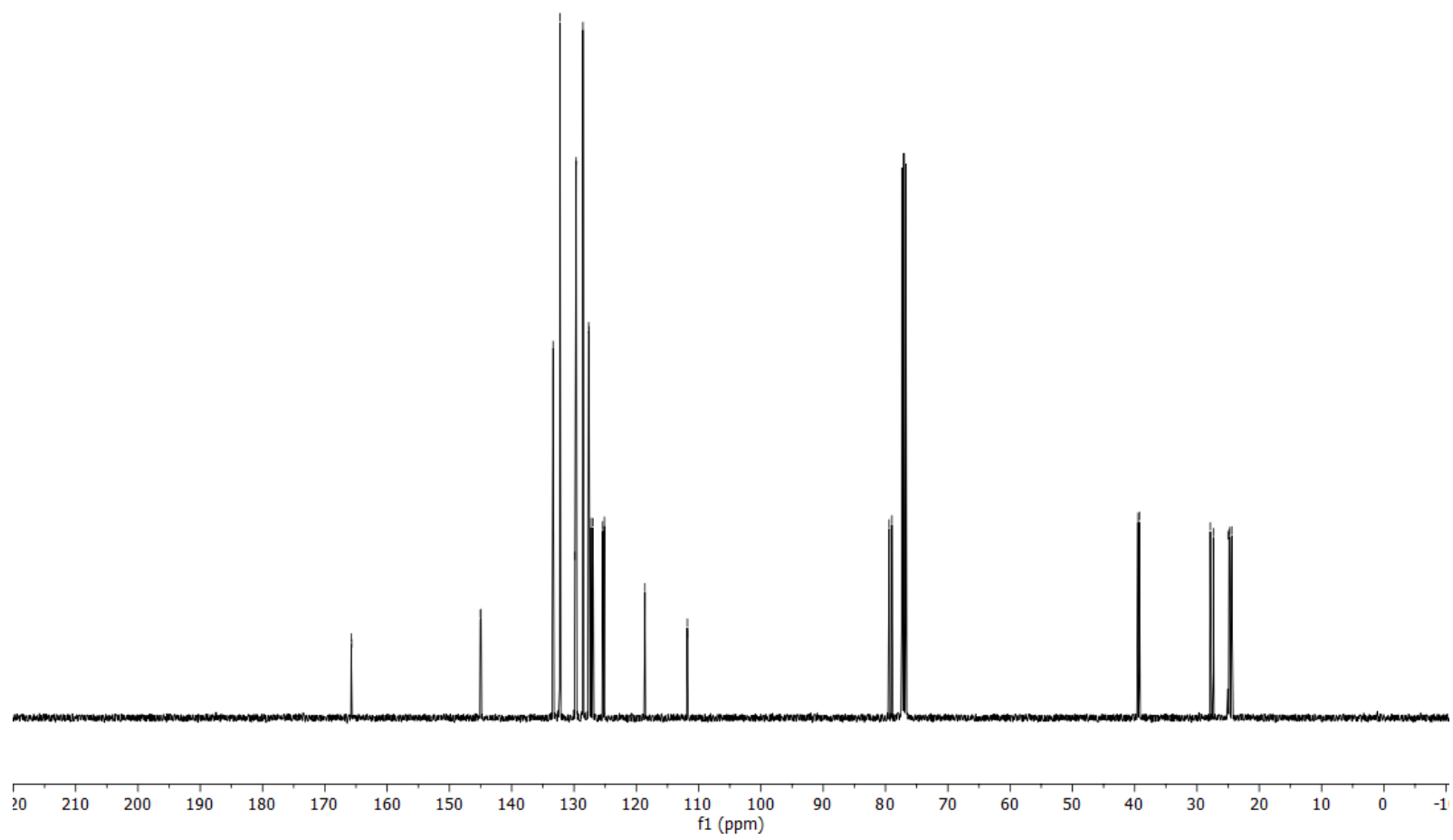

S 115 
${ }^{1} \mathrm{H}$ NMR (400 MHz, $\mathrm{CDCl}_{3}$ )

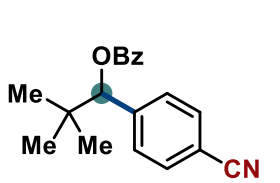

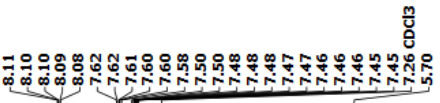

$17,60 \%$

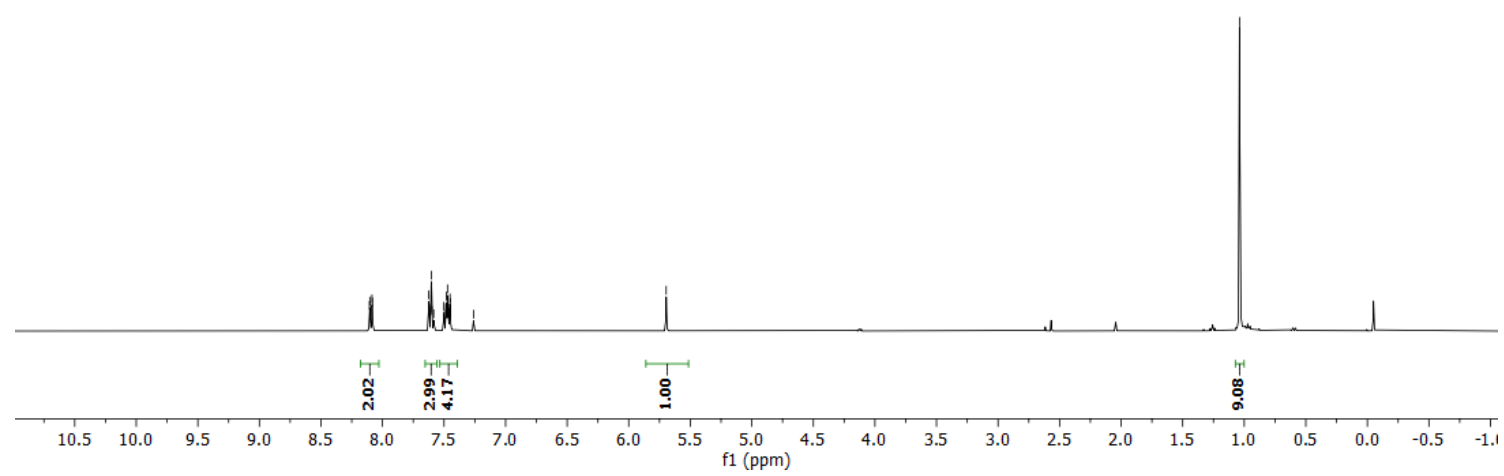

${ }^{13} \mathrm{C}$ NMR (101 MHz, $\left.\mathrm{CDCl}_{3}\right)$

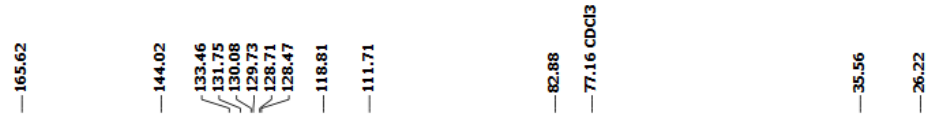

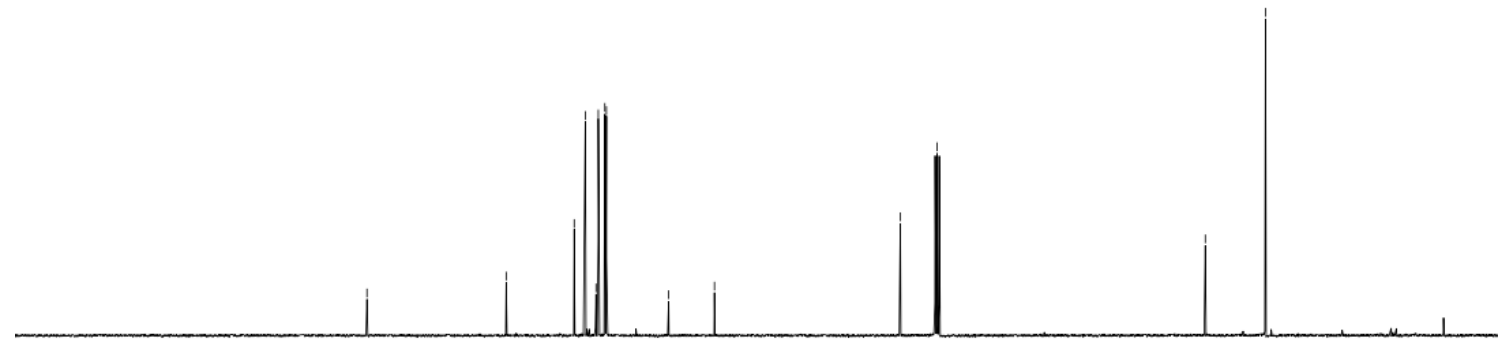

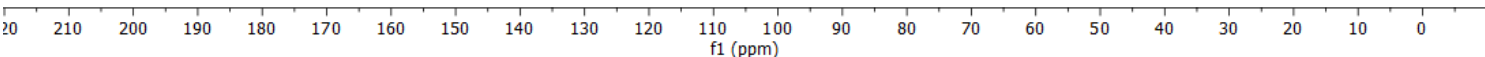


${ }^{1} \mathrm{H}$ NMR (400 MHz, $\mathrm{CDCl}_{3}$ )

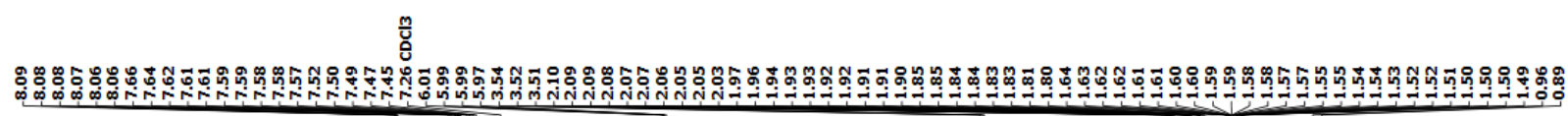<smiles>N#Cc1ccc(C(=O)CCCCCl)cc1</smiles>

$18,67 \%$ yield

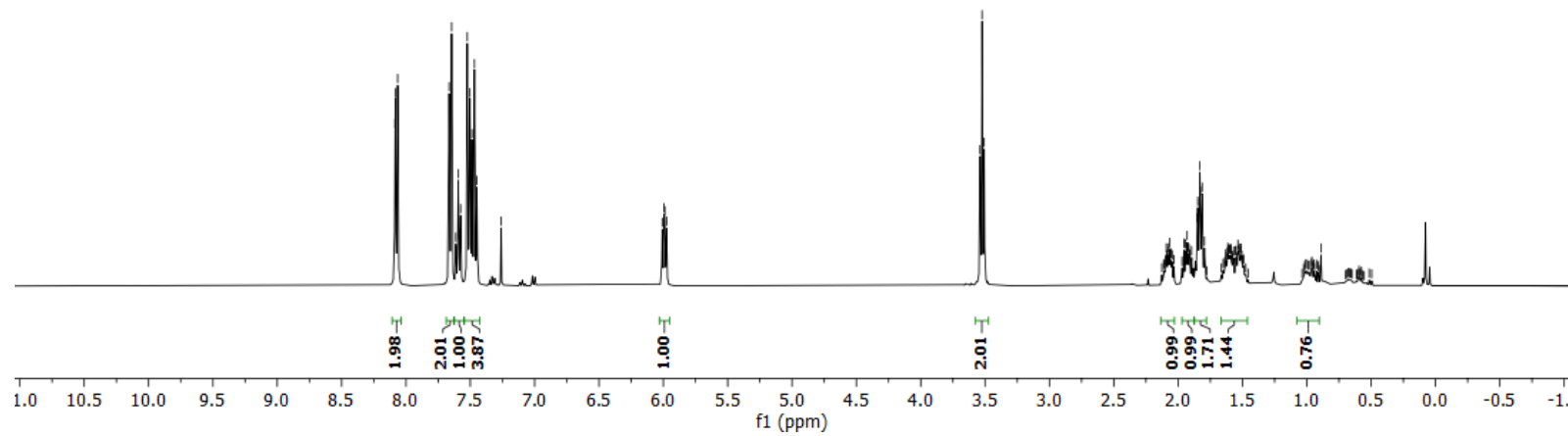

${ }^{13} \mathrm{C}$ NMR (101 MHz, $\left.\mathrm{CDCl}_{3}\right)$

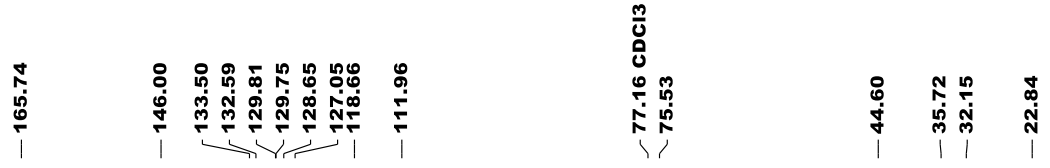

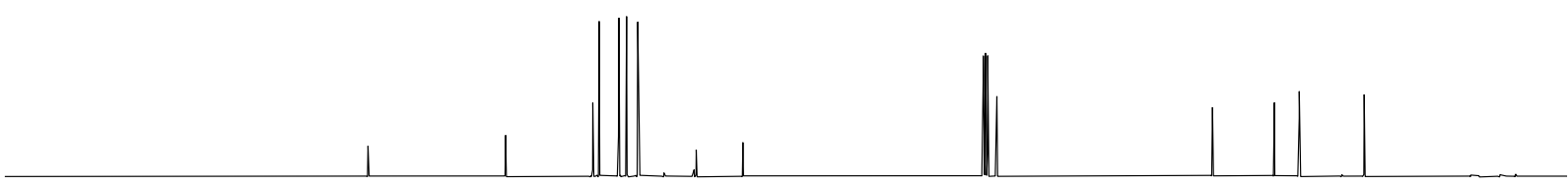

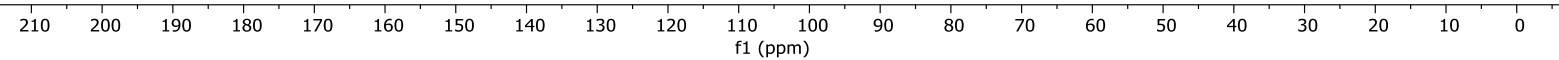


${ }^{1} \mathrm{H}$ NMR (400 MHz, $\mathrm{CDCl}_{3}$ )

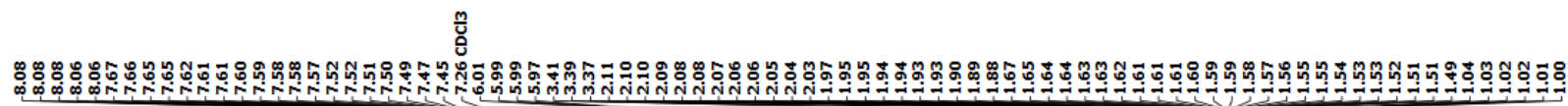<smiles>N#Cc1ccc(C(O)CCCCBr)cc1</smiles>

$19,52 \%$ yield

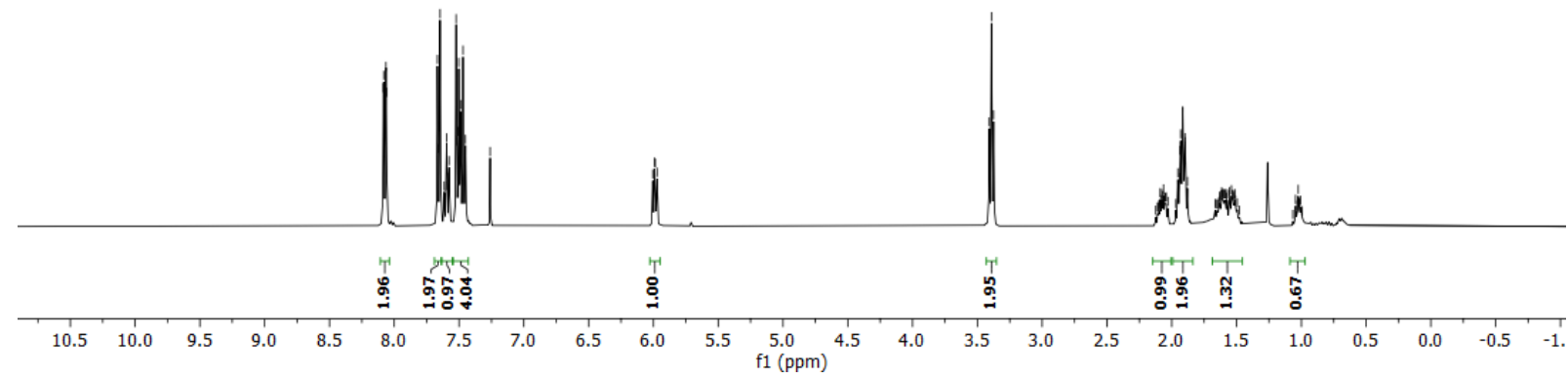

${ }^{13} \mathrm{C}$ NMR (101 MHz, $\left.\mathrm{CDCl}_{3}\right)$
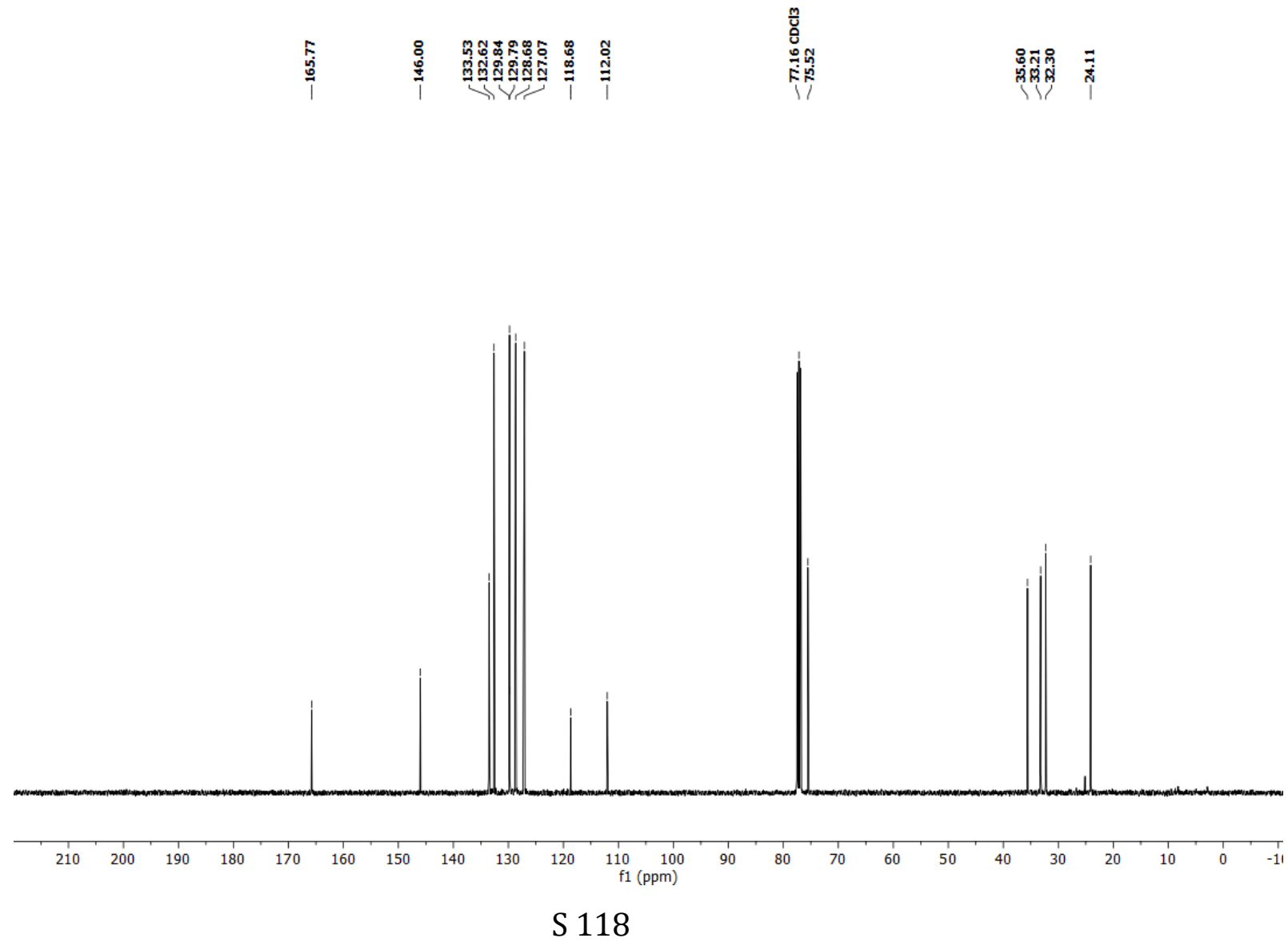
1H NMR (599 MHz, $\mathrm{CDCl}_{3}$ )

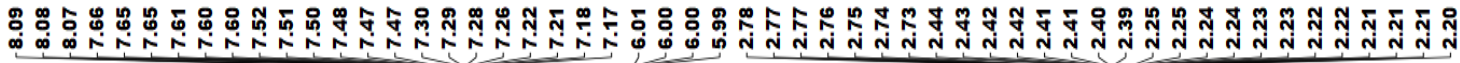

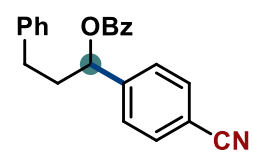

$20,63 \%$

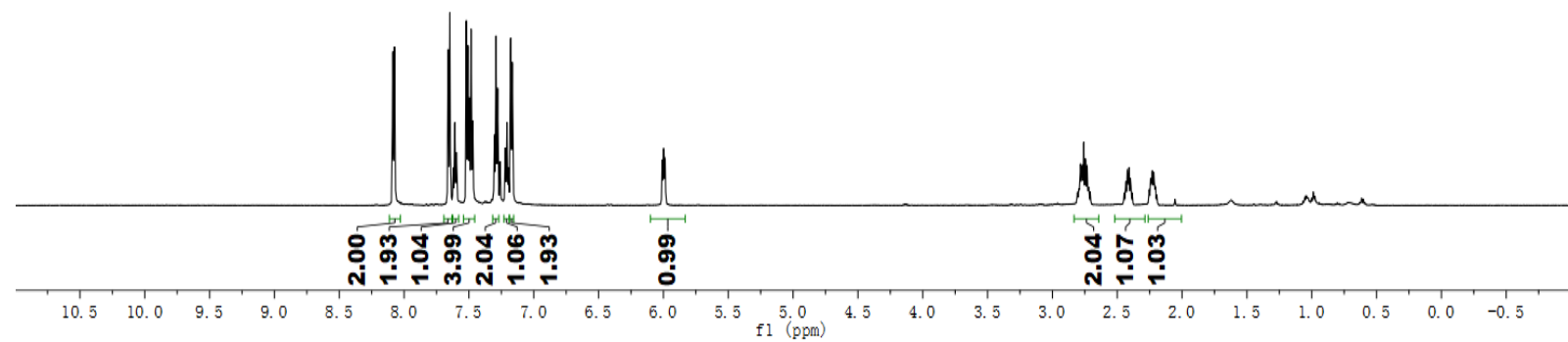

${ }^{13} \mathrm{C}$ NMR (151 MHz, $\mathrm{CDCl}_{3}$ )

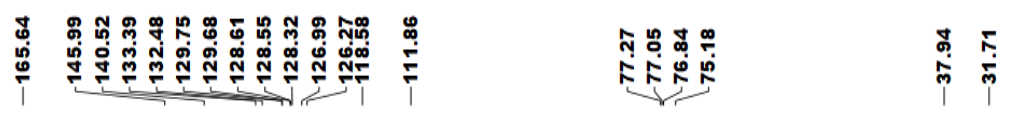
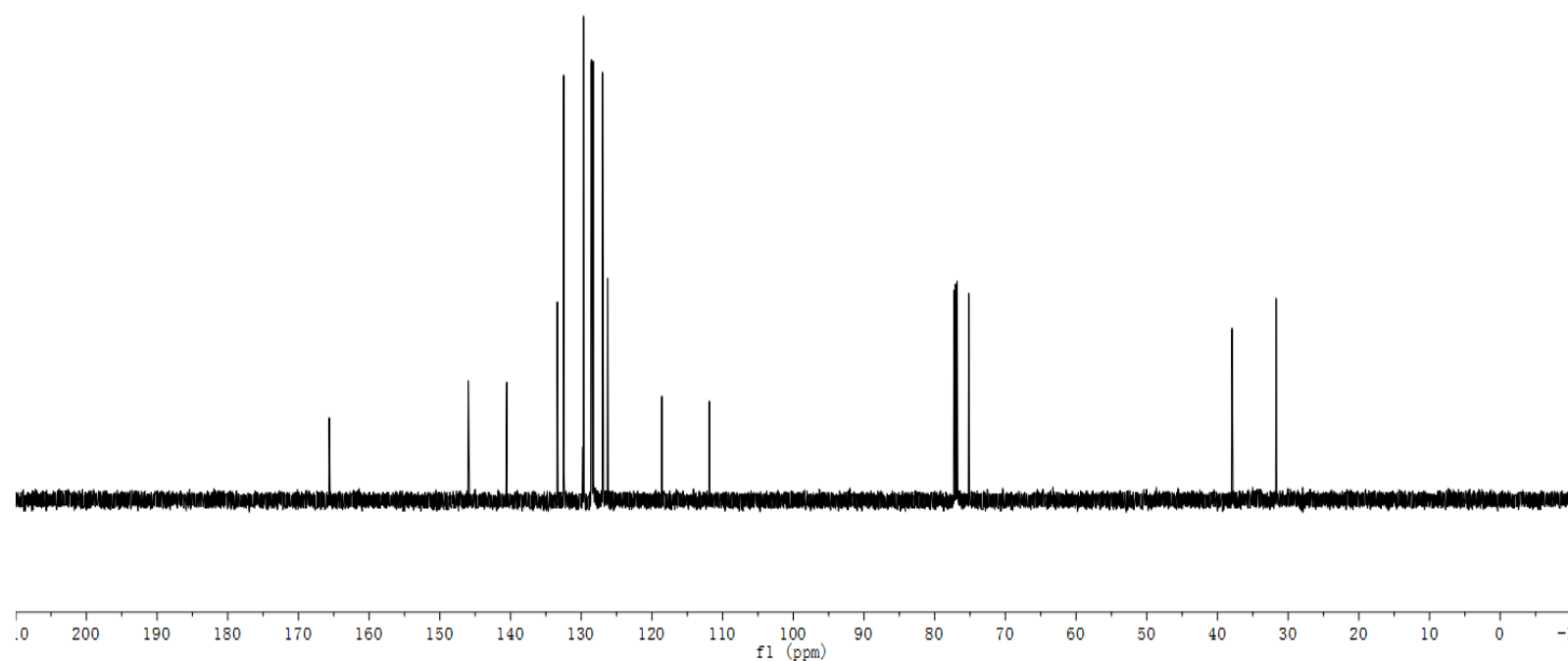

S 119 
1H NMR (599 MHz, $\mathrm{CDCl}_{3}$ )

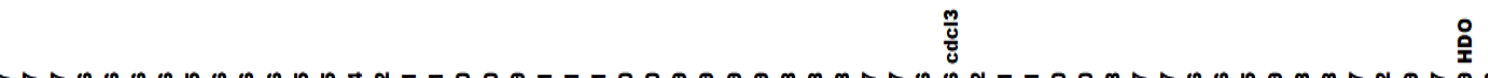

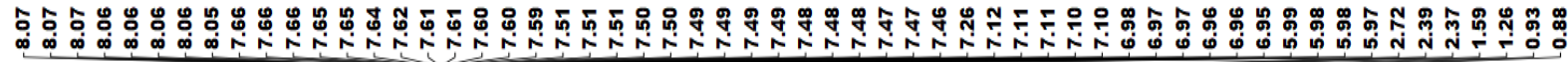<smiles>N#Cc1ccc(C(=O)CCc2ccc(F)cc2)cc1</smiles>

$21,56 \%$

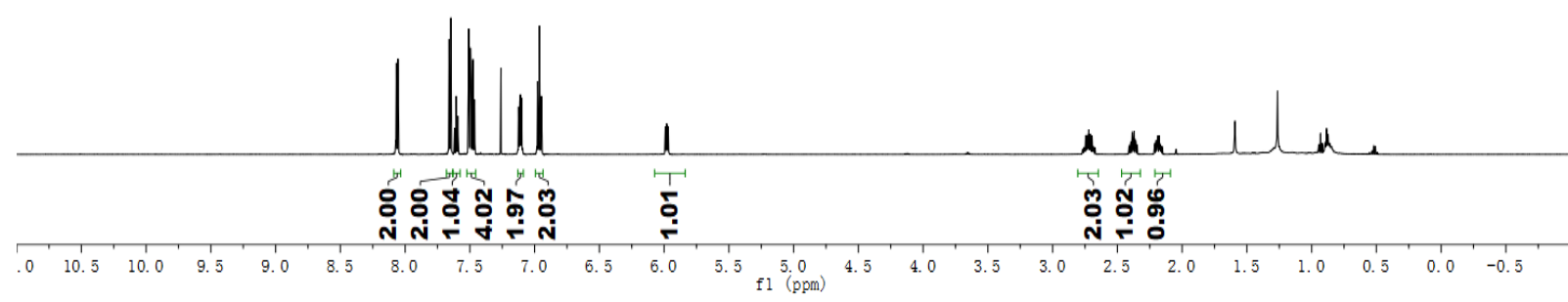

${ }^{13} \mathrm{C}$ NMR (151 $\mathrm{MHz}, \mathrm{CDCl}_{3}$ )

\begin{tabular}{|c|c|c|}
\hline 至 & 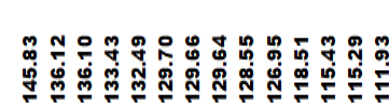 & 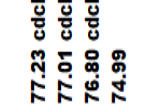 \\
\hline
\end{tabular}
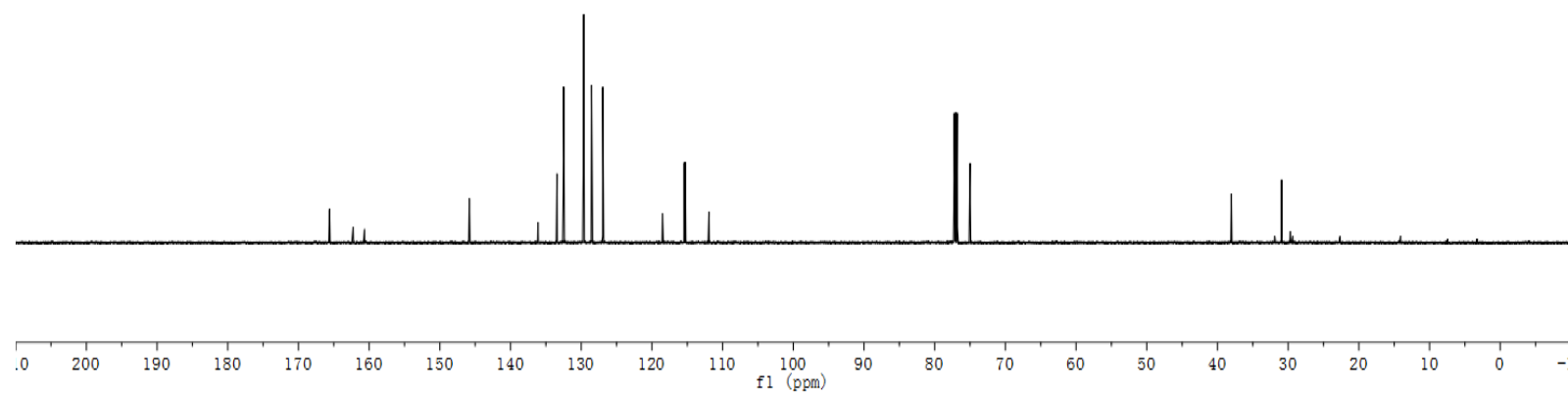

S 120 
${ }^{19}$ F NMR (564 MHz, $\left.\mathrm{CDCl}_{3}\right)$

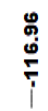

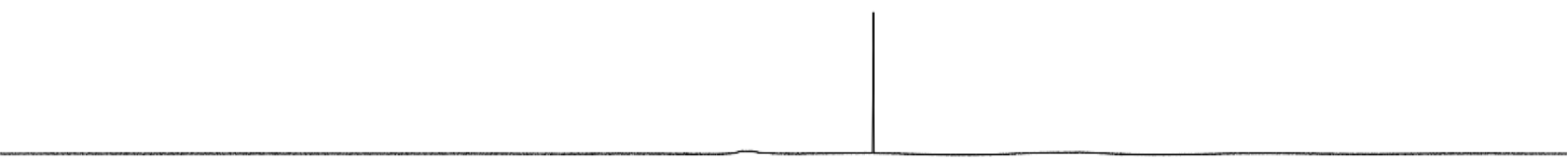

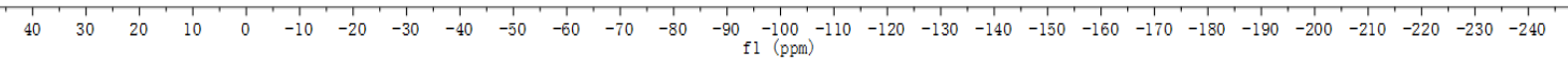


1H NMR (599 MHz, $\mathrm{CDCl}_{3}$ )

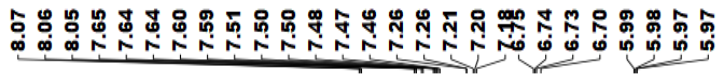

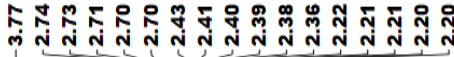

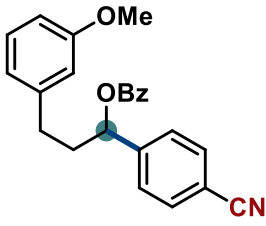

$22,47 \%$

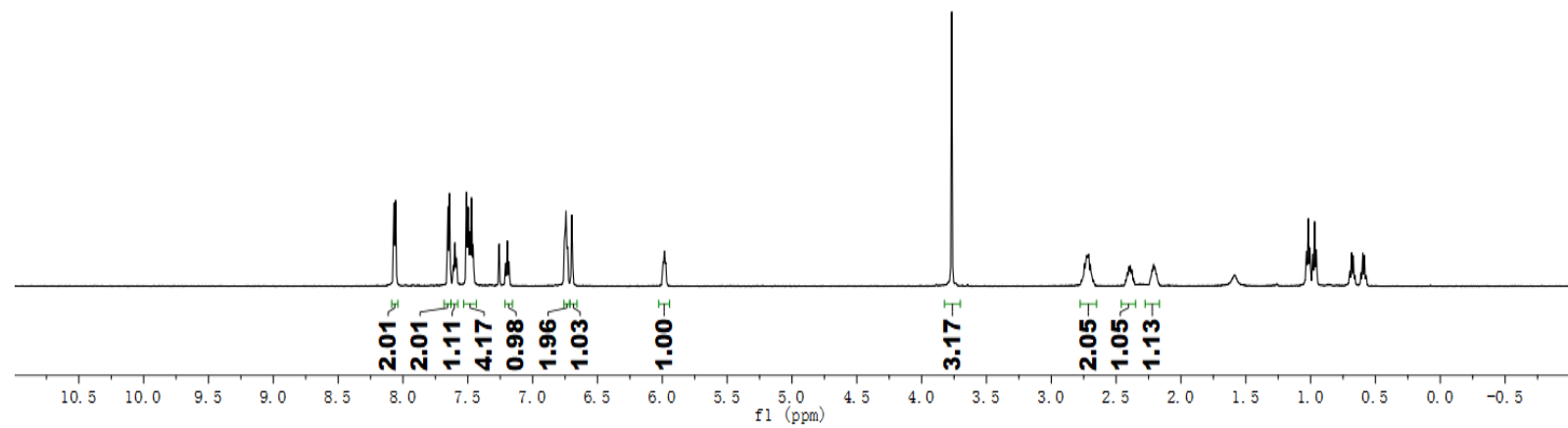

${ }^{13} \mathrm{C}$ NMR (151 MHz, $\mathrm{CDCl}_{3}$ )

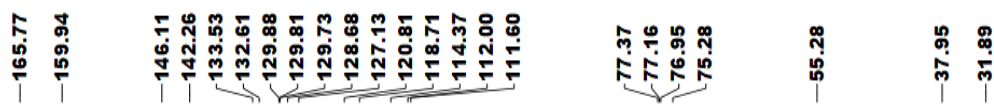

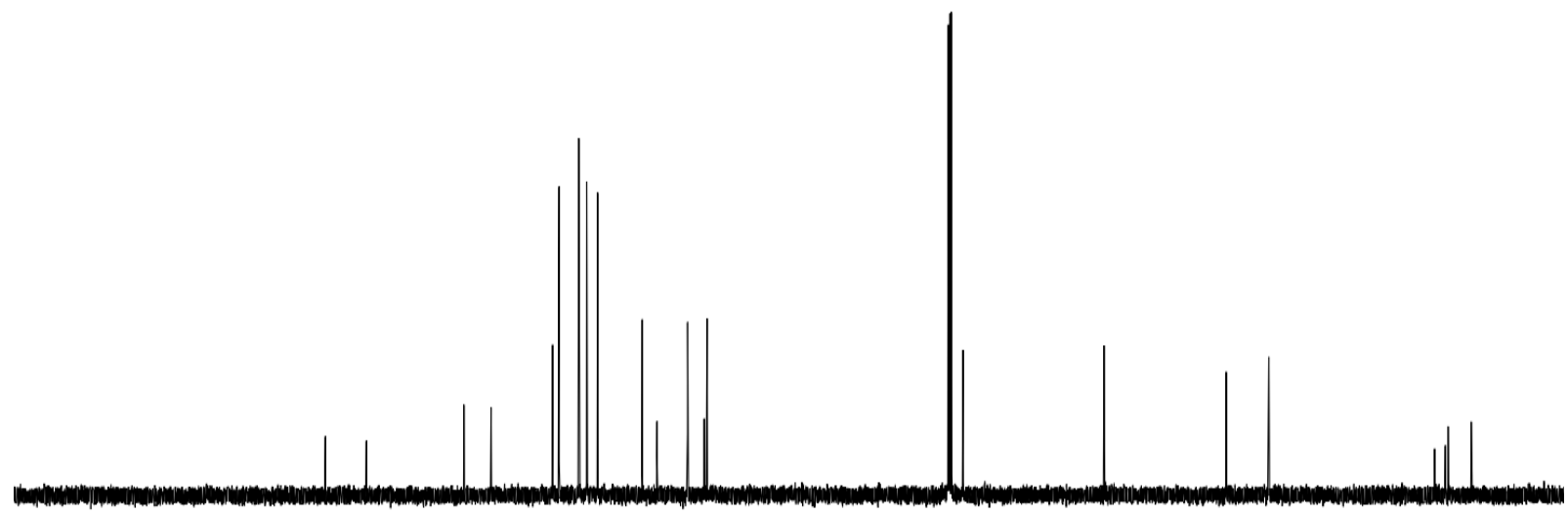

$\begin{array}{llllllllllllllllllllllll} & 0 & 200 & 190 & 180 & 170 & 160 & 150 & 140 & 130 & 120 & 110 & 100 & 90 & 80 & 70 & 60 & 50 & 40 & 30 & 20 & 10 & 0 & -1\end{array}$

S 122 
1H NMR (599 MHz, $\mathrm{CDCl}_{3}$ )

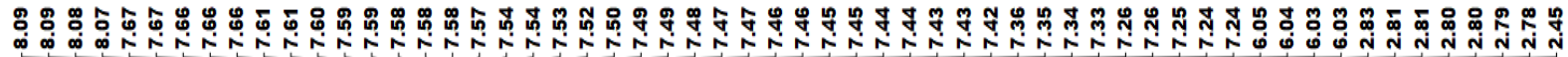<smiles>N#Cc1ccc(C(=O)CCc2ccc(-c3ccccc3)cc2)cc1</smiles>

$23,54 \%$

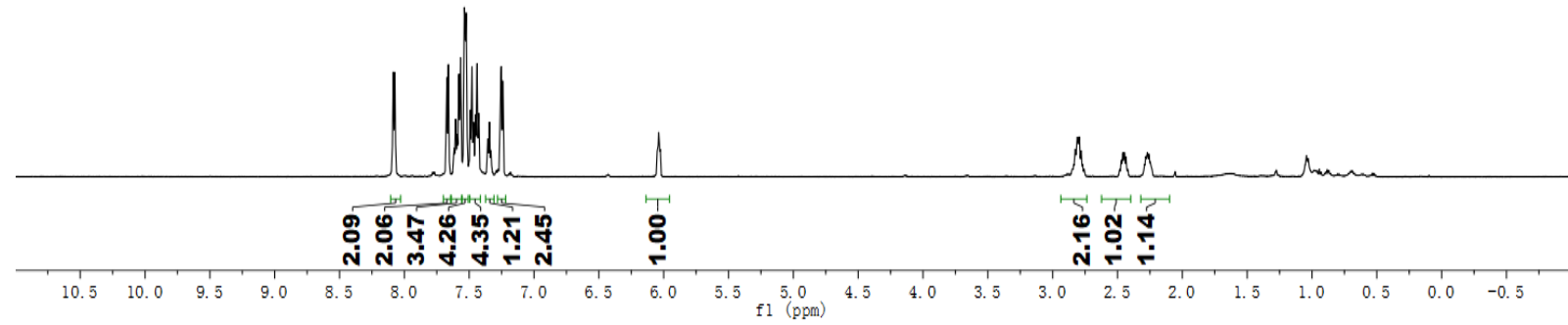

${ }^{13} \mathrm{C}$ NMR (151 MHz, $\mathrm{CDCl}_{3}$ )
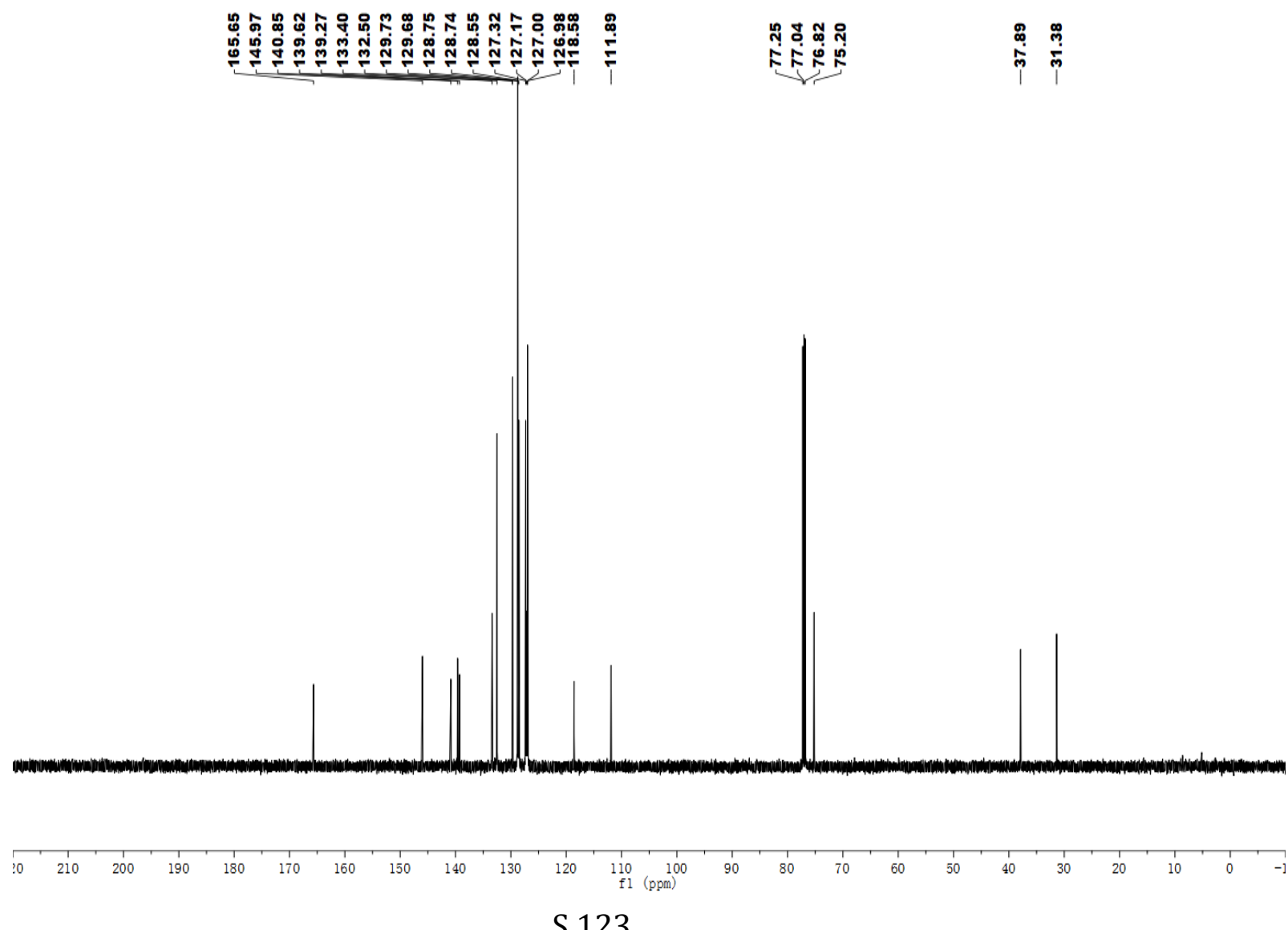

S 123 
${ }^{1} \mathrm{H}$ NMR (599 $\mathrm{MHz}^{\mathrm{CDCl}_{3}}$ )

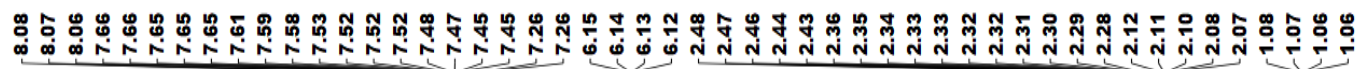

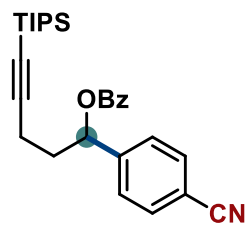

$24,43 \%$

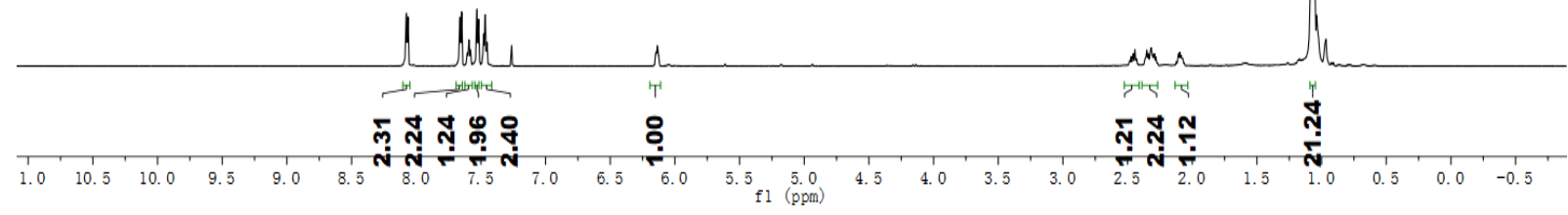

${ }^{13} \mathrm{C}$ NMR (151 MHz, $\mathrm{CDCl}_{3}$ )

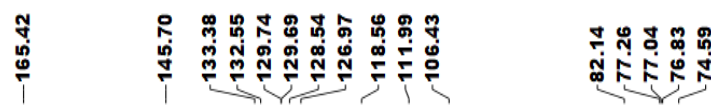

管

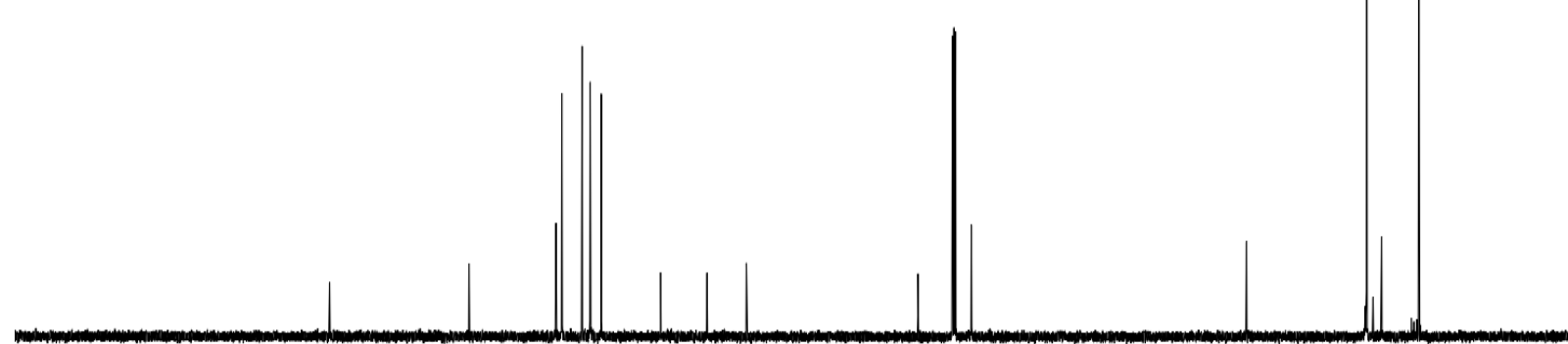

$\begin{array}{lllllllllll}200 & 190 & 180 & 170 & 160 & 150 & 140 & 130 & 120 & 110 & 100 \\ \mathrm{fl} 1 & (\mathrm{ppm})\end{array}$

S 124 
1H NMR (599 MHz, $\mathrm{CDCl}_{3}$ )
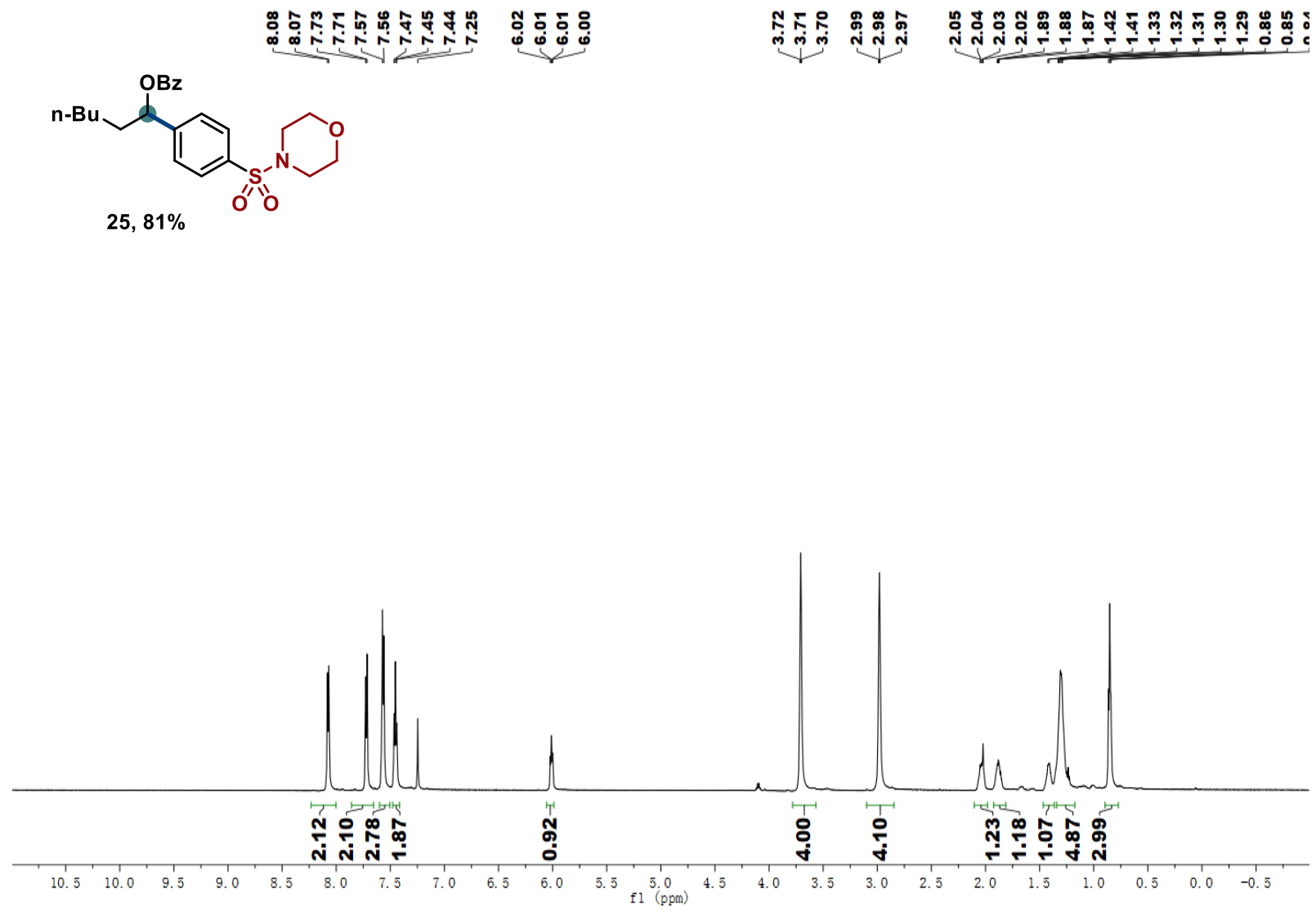

${ }^{13} \mathrm{C}$ NMR (151 MHz, $\mathrm{CDCl}_{3}$ )
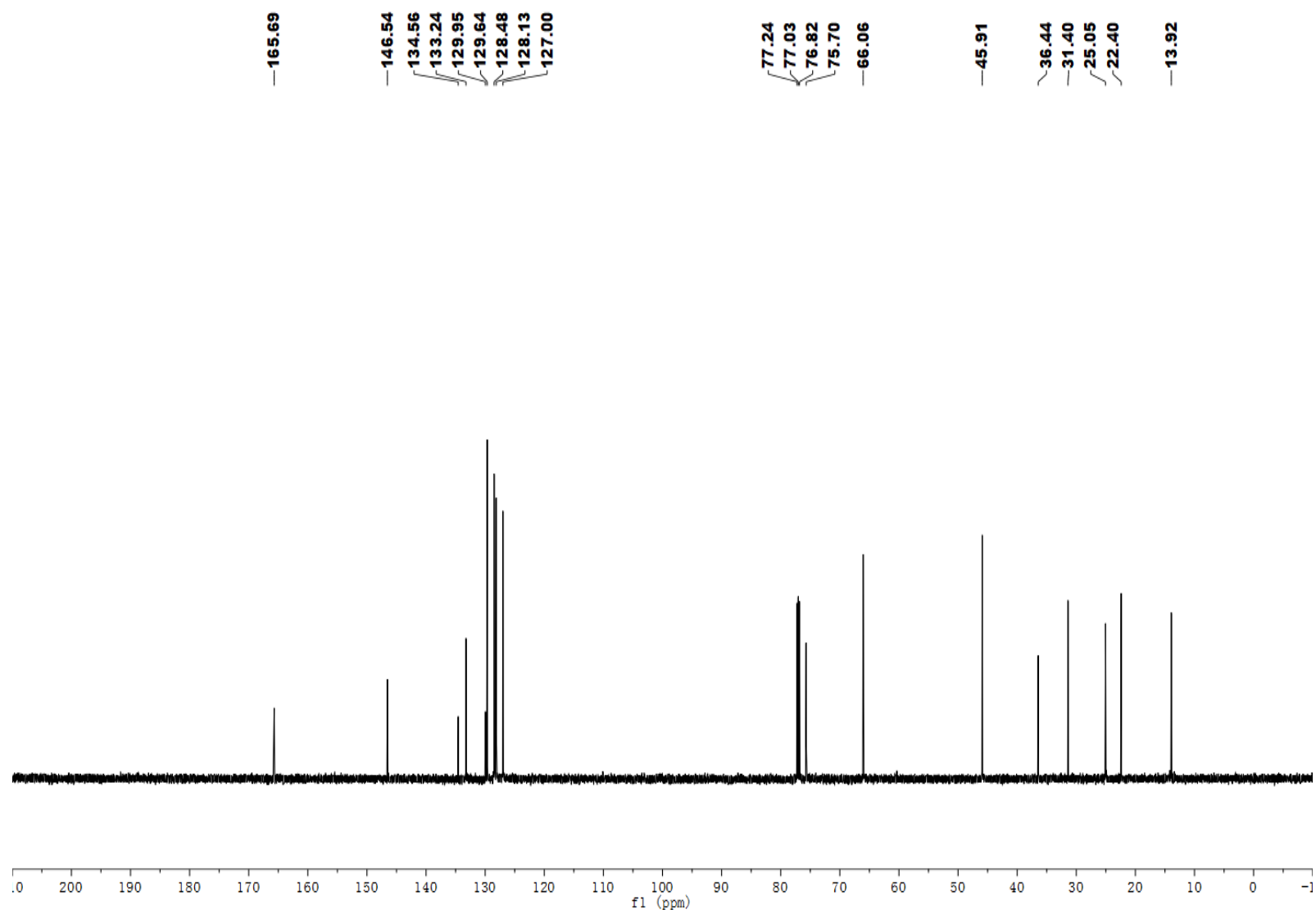
1H NMR (599 MHz, $\left.\mathrm{CDCl}_{3}\right)$

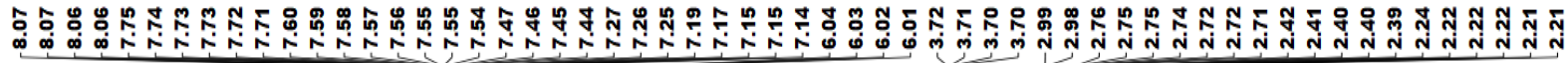<smiles></smiles>

$6,51 \%$

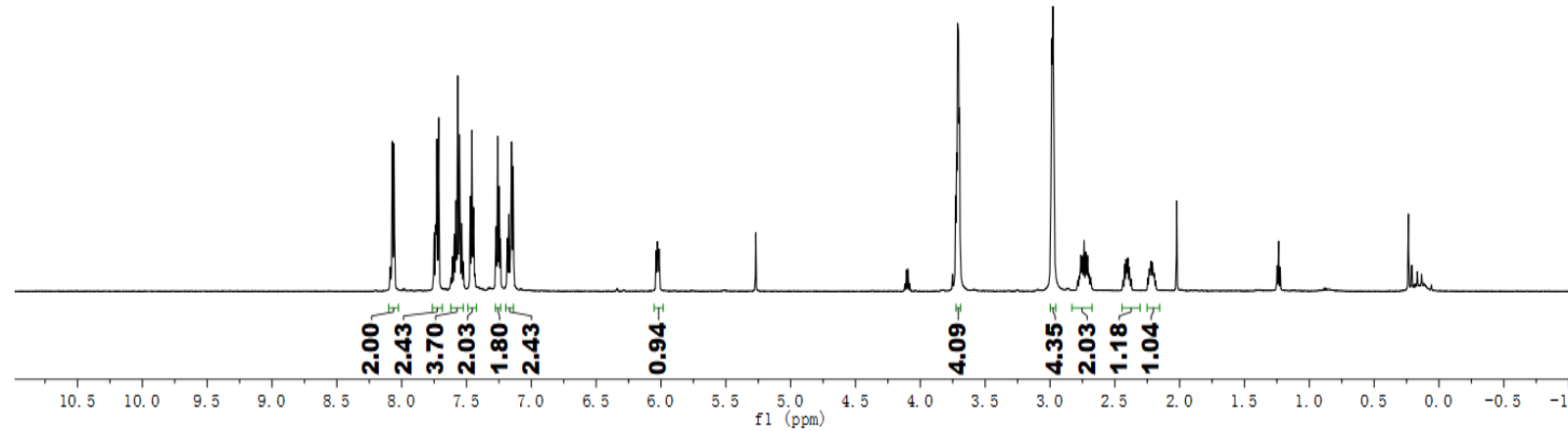

${ }^{13} \mathrm{C}$ NMR (151 MHz, $\mathrm{CDCl}_{3}$ )

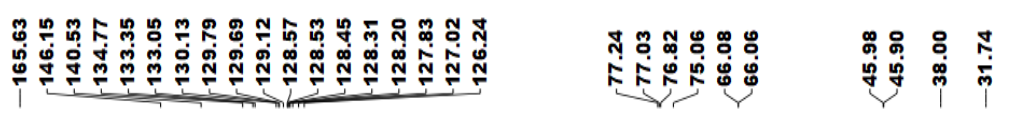
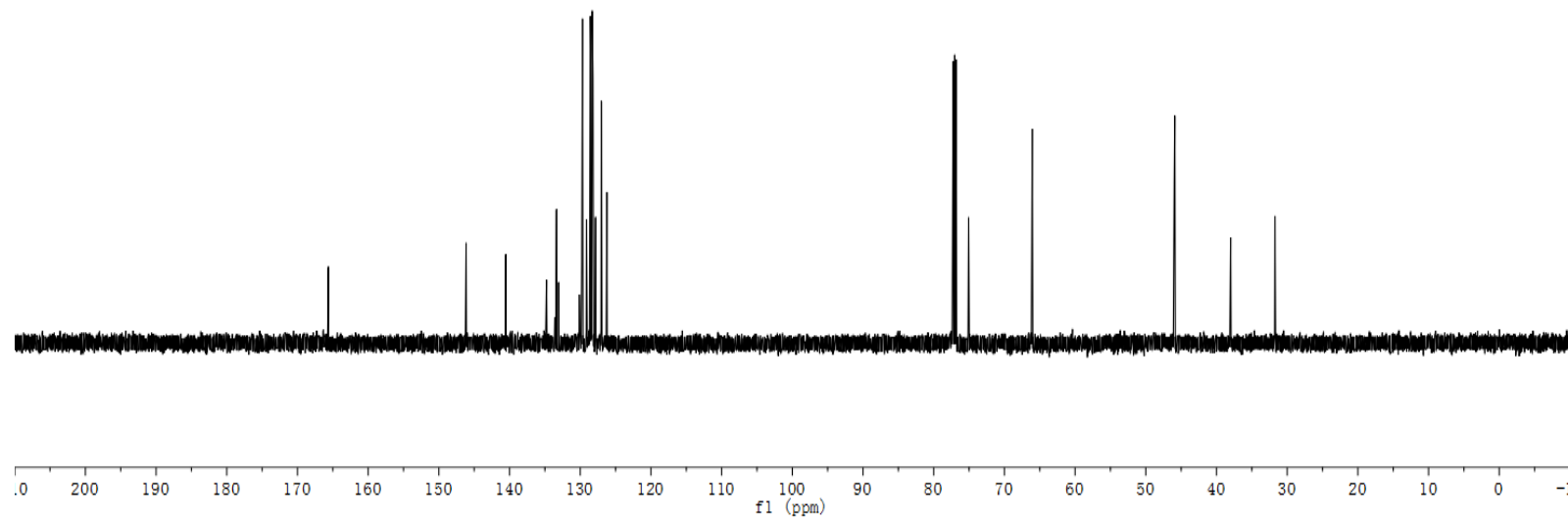

S 126 
1H NMR (599 MHz, $\mathrm{CDCl}_{3}$ )

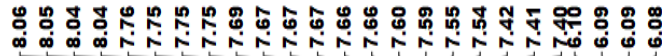

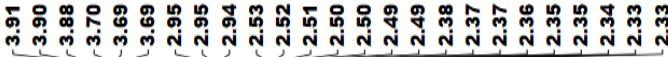
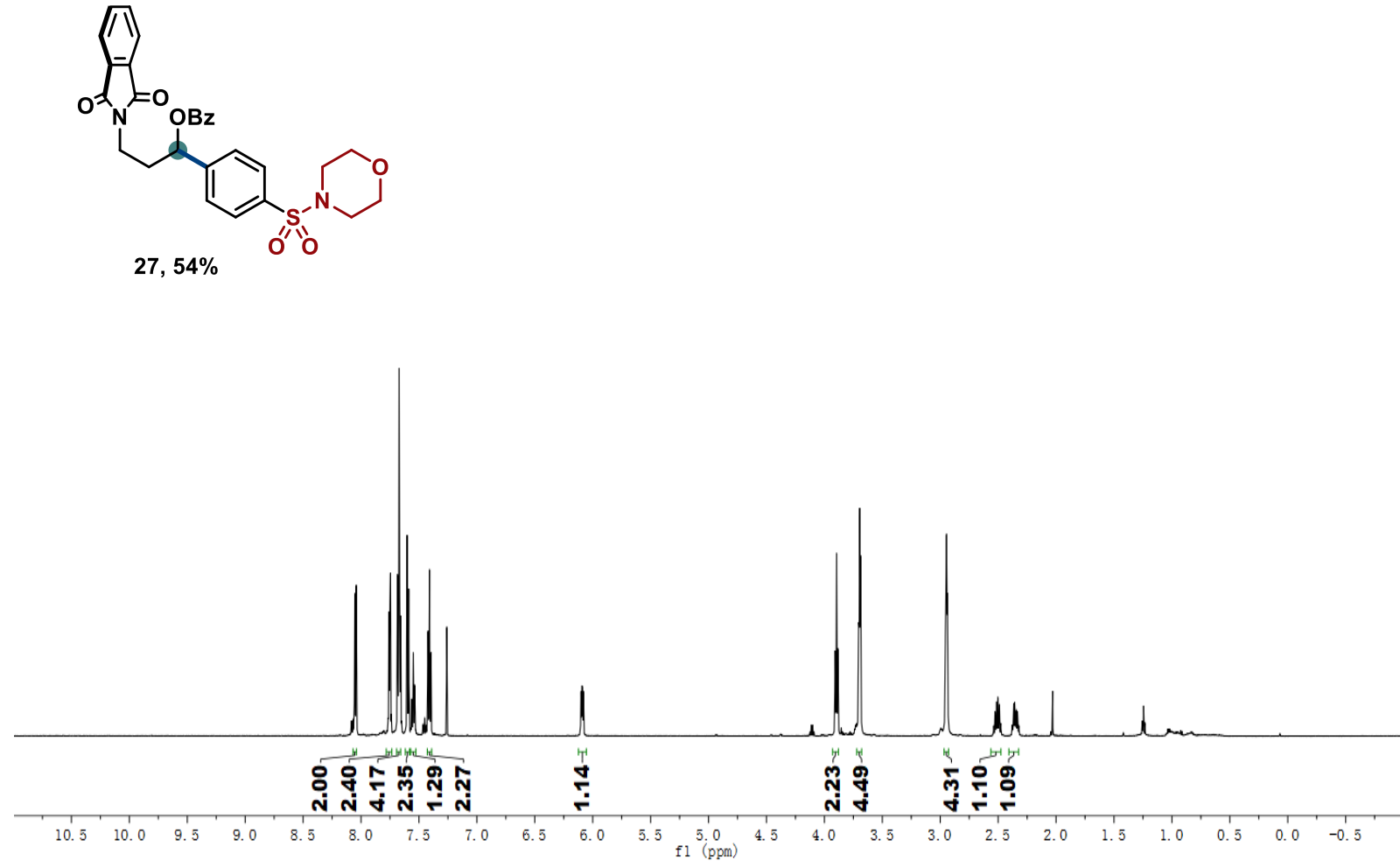

${ }^{13} \mathrm{C}$ NMR (151 MHz, $\mathrm{CDCl}_{3}$ )

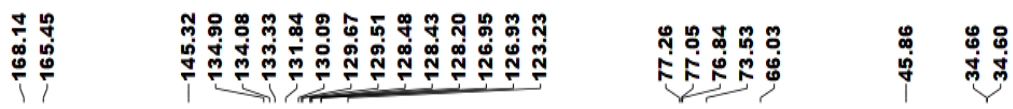

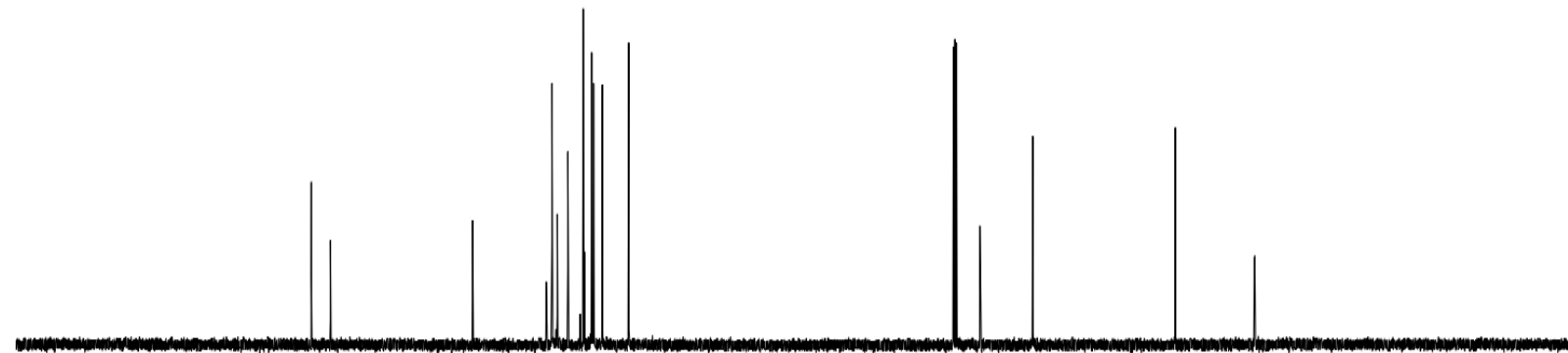

$\begin{array}{lllllllllllllllllllllll}0 & 200 & 190 & 180 & 170 & 160 & 150 & 140 & 130 & 120 & 110 & \begin{array}{l}100 \\ \mathrm{f} 1\end{array}(\mathrm{ppm}) & 90 & 80 & 70 & 60 & 50 & 40 & 30 & 20 & 10 & 0 & -10\end{array}$ 
1H NMR (400 MHz, $\mathrm{CDCl}_{3}$ )

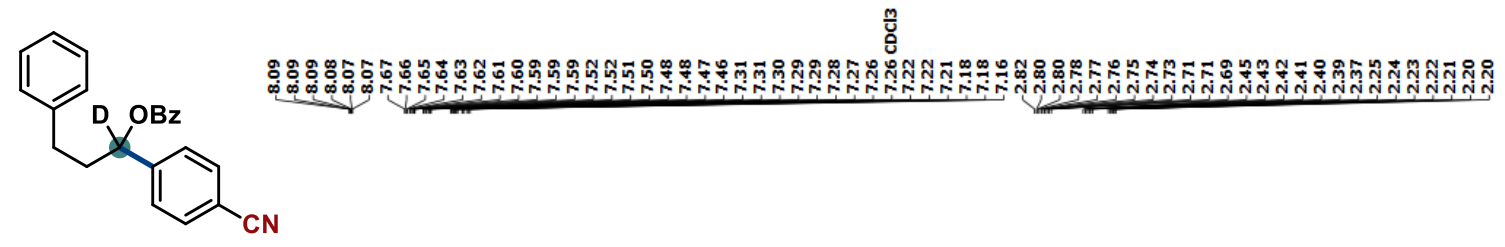

$28,72 \%$

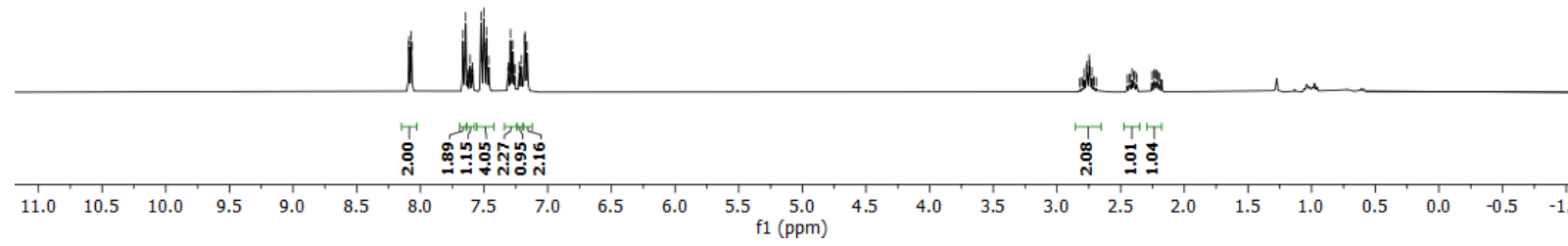

${ }^{13} \mathrm{C}$ NMR (101 MHz, $\mathrm{CDCl}_{3}$ )
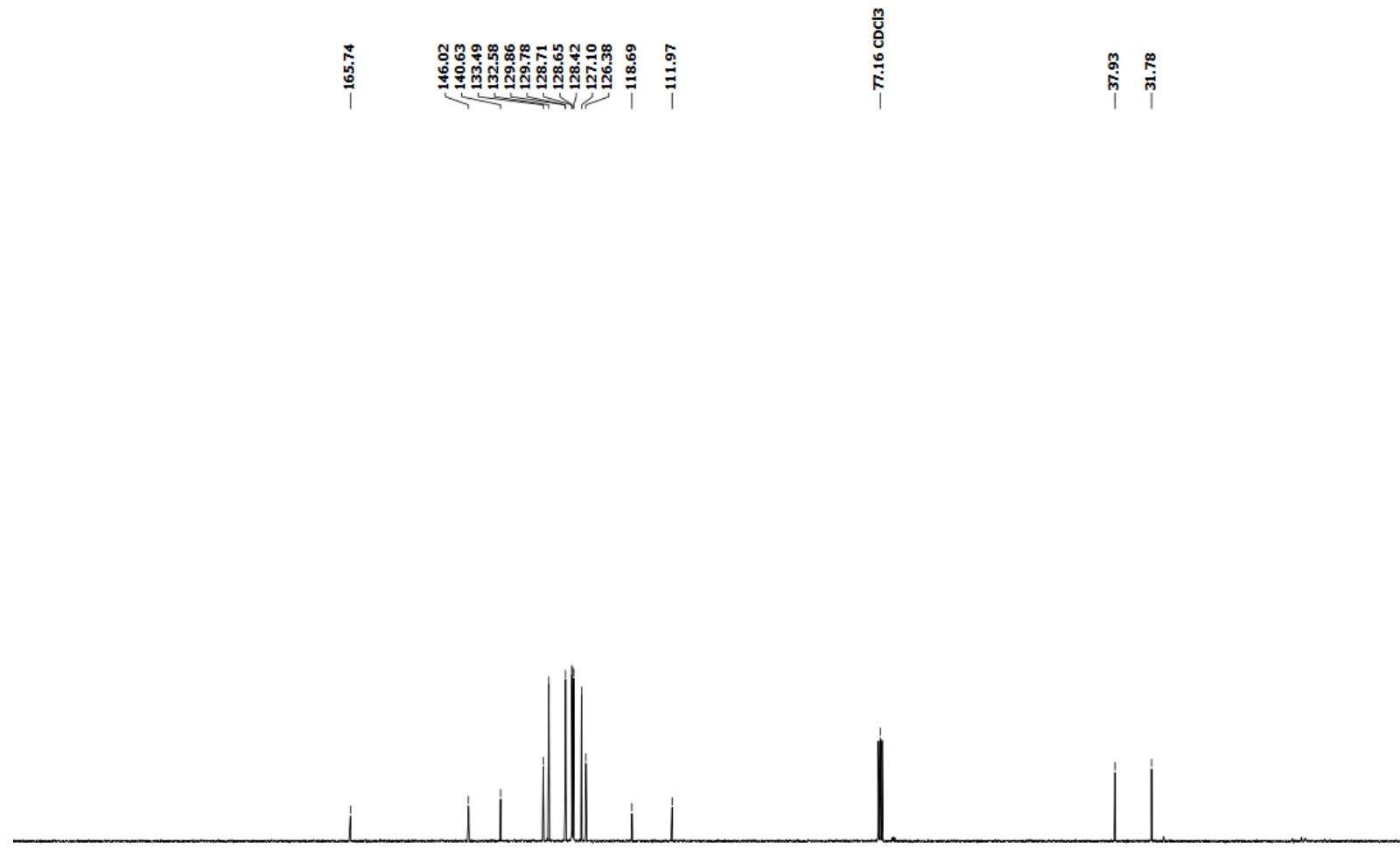

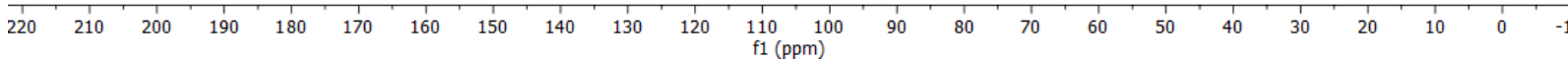
S 128 
1H NMR (500 MHz, $\mathrm{CDCl}_{3}$ )

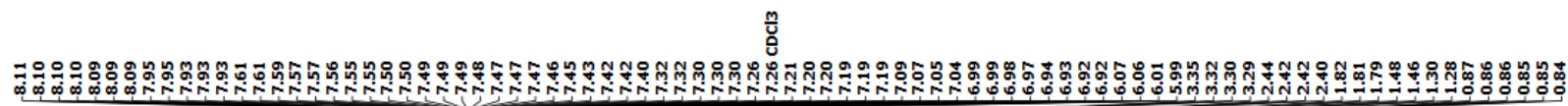<smiles>CCCCOC(=C(c1ccc(C#N)cc1)C(C)N)c1ccc(CC(C)C)cc1</smiles>

$29,40 \%$ yield, $63: 37 \mathrm{dr}$.

from ibuprofen (painkiller)

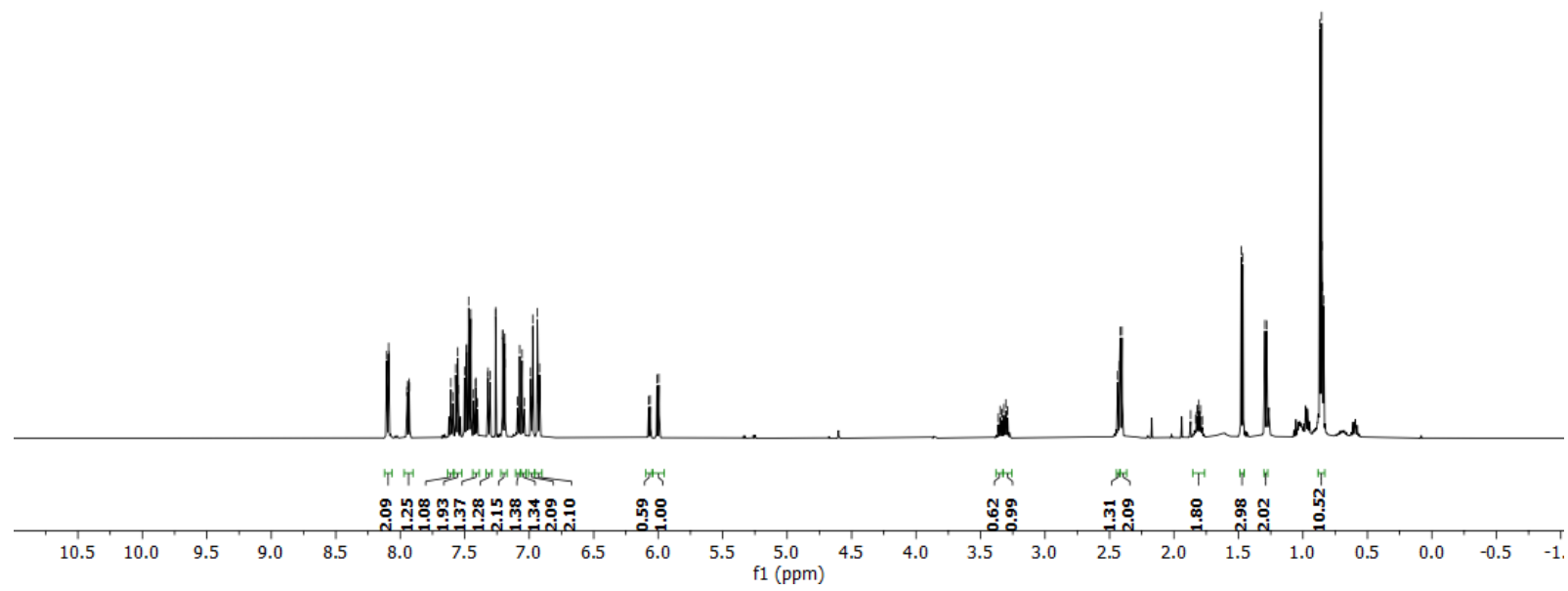

${ }^{13} \mathrm{C}$ NMR (126 $\left.\mathrm{MHz}, \mathrm{CDCl}_{3}\right)$

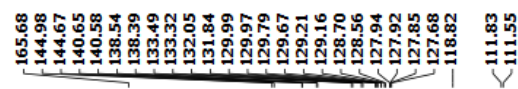

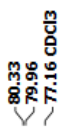

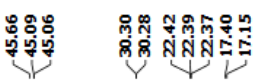

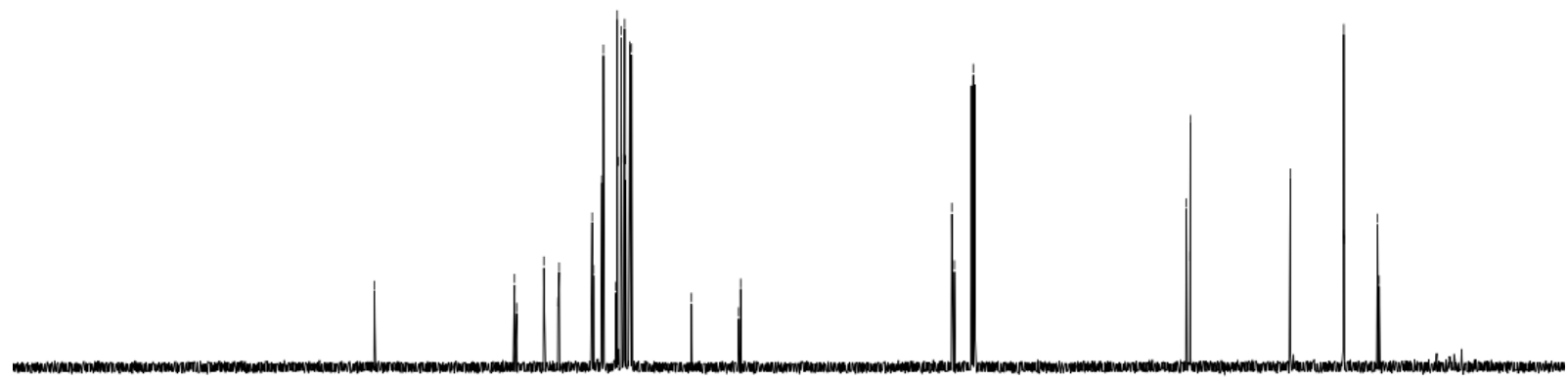

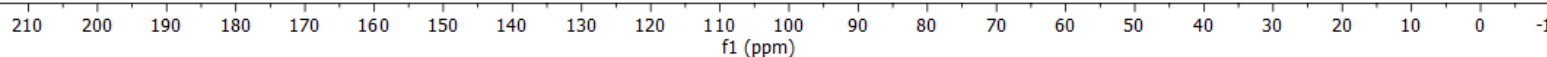


${ }^{1} \mathrm{H}^{-1} \mathrm{H}$ COSY NMR (500 $\left.\mathrm{MHz}, \mathrm{CDCl}_{3}\right)$

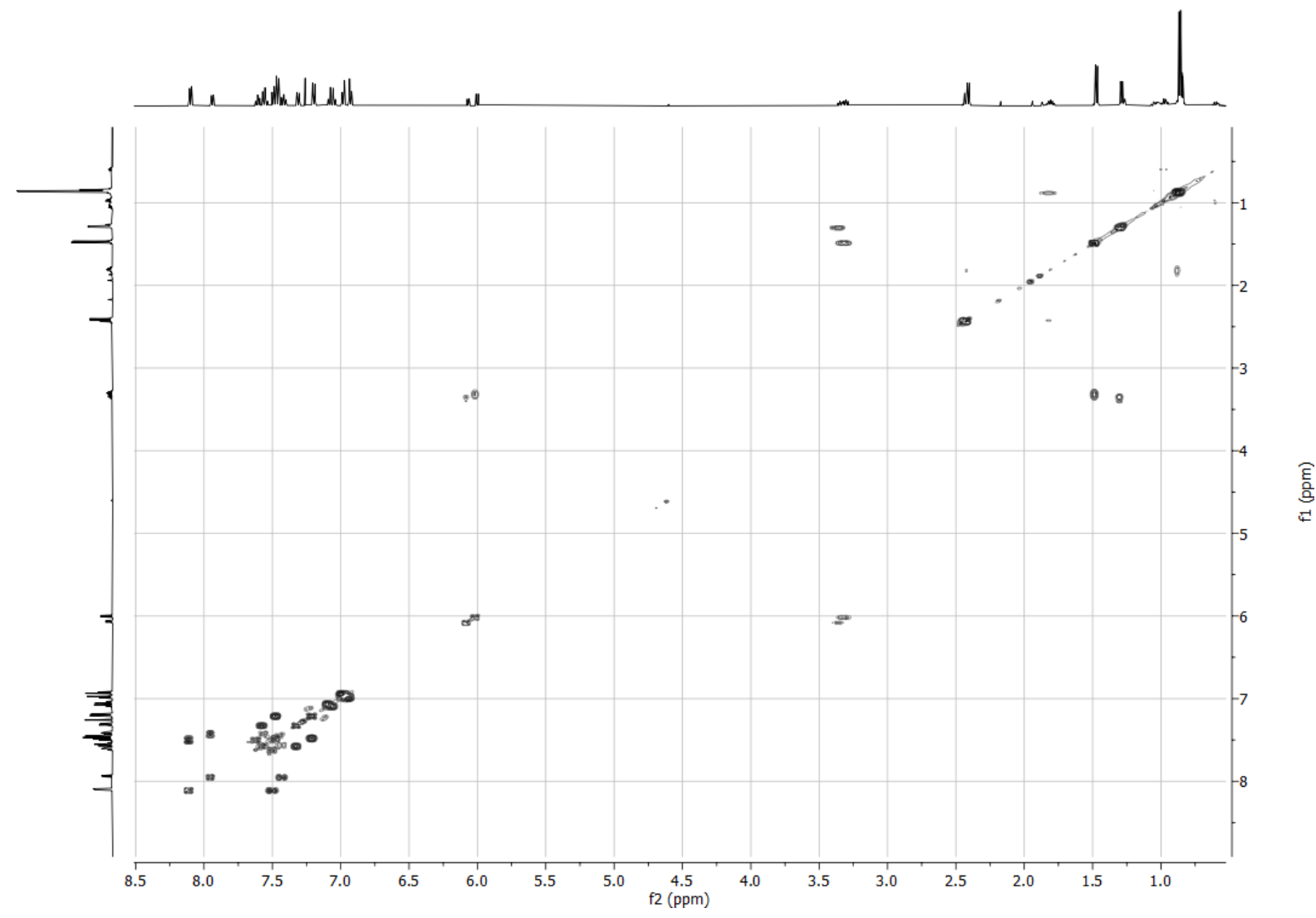

${ }^{1} \mathrm{H}^{-13} \mathrm{C}$ HSQC NMR (500 MHz, $\mathrm{CDCl}_{3}$ )

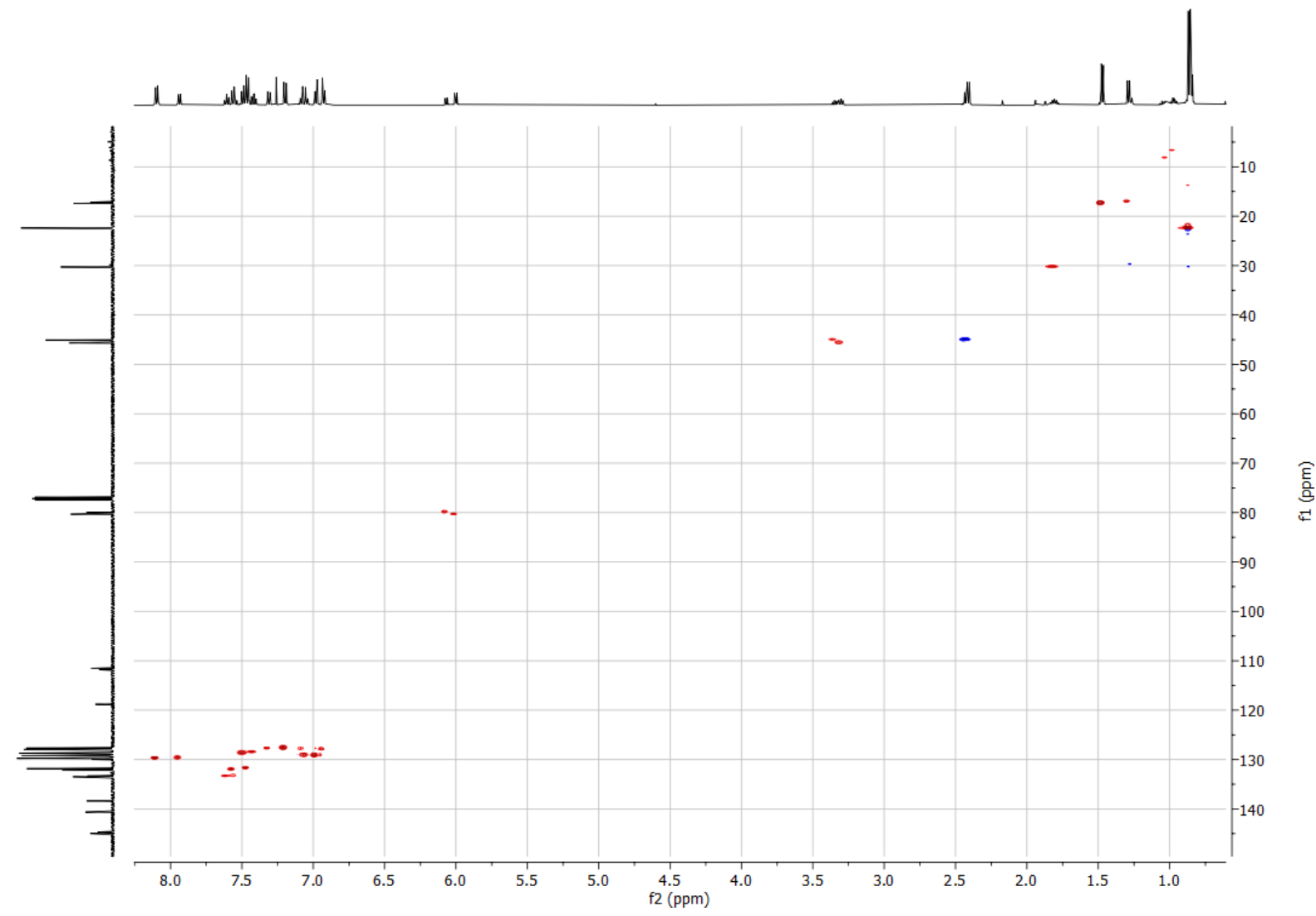

S 130 
${ }^{1}{ }^{13-13} \mathrm{C}$ HMBC NMR (500 MHz, $\mathrm{CDCl}_{3}$ )

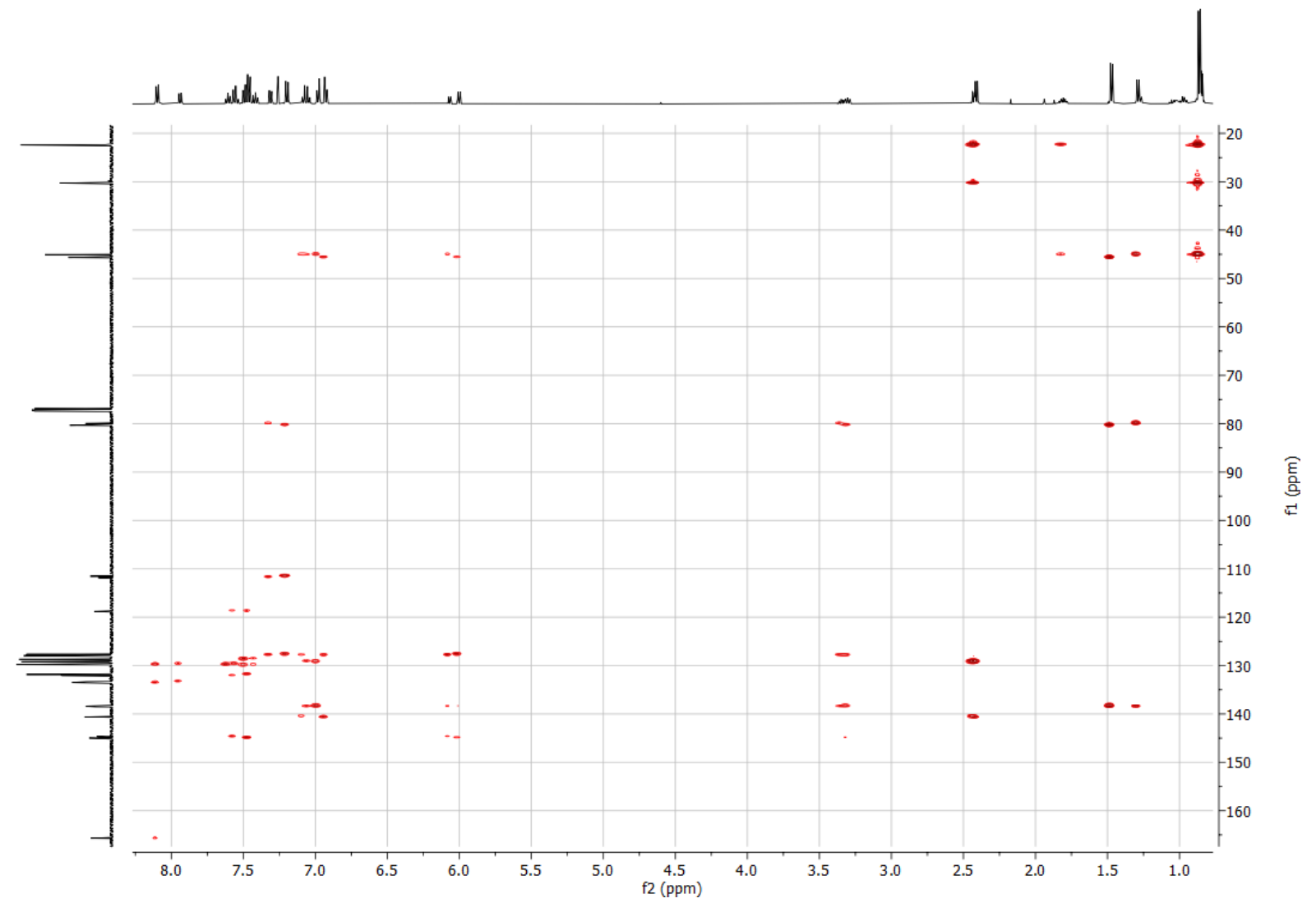


${ }^{1} \mathrm{H}$ NMR (400 MHz, $\left.\mathrm{CDCl}_{3}\right)$

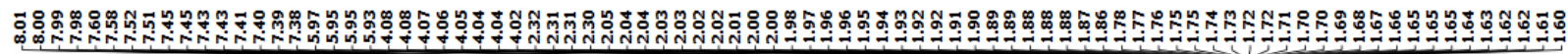<smiles>N#Cc1ccc(C(=O)CCCOC(=O)CC=CC(F)(F)F)cc1</smiles>

$30,71 \%$

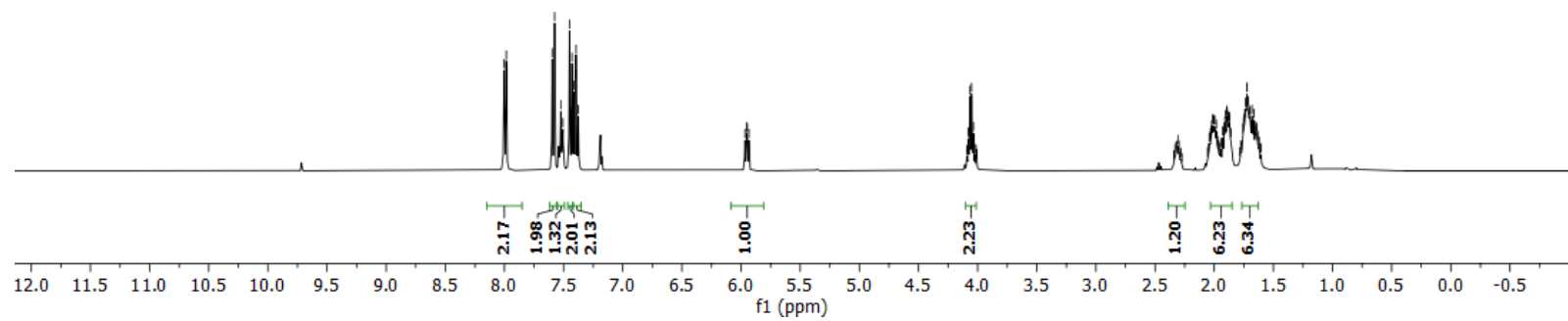

${ }^{13} \mathrm{C}$ NMR (101 MHz, $\left.\mathrm{CDCl}_{3}\right)$
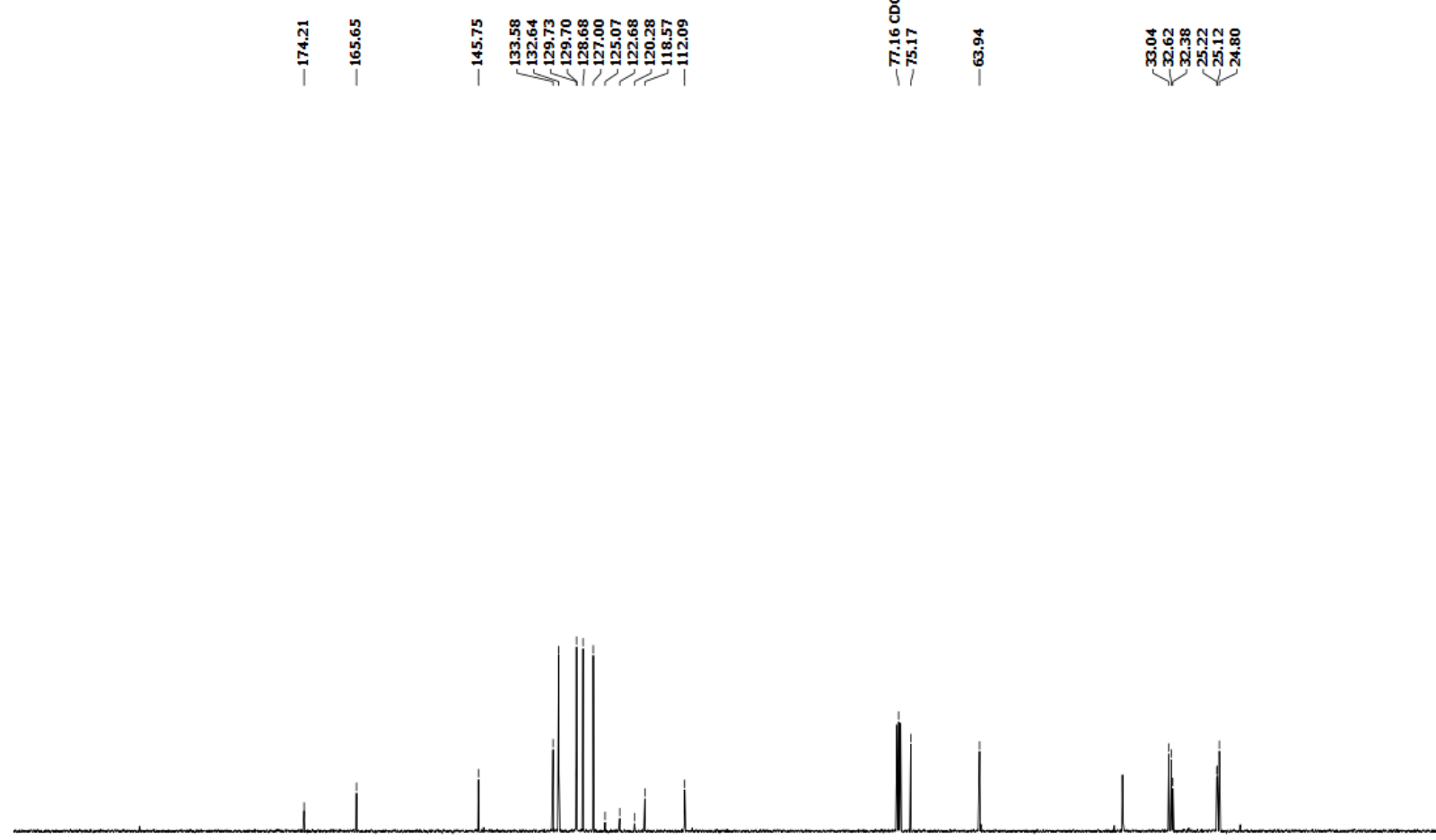

$\begin{array}{llllllllll}220 & 210 & 200 & 190 & 180 & 170 & 160 & 150 & 140 & 130\end{array}$ $110 \stackrel{100}{f}(\mathrm{ppm})$
$\mathrm{f}$ 
${ }^{19}$ F NMR (377 MHz, $\left.\mathrm{CDCl}_{3}\right)$

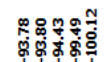

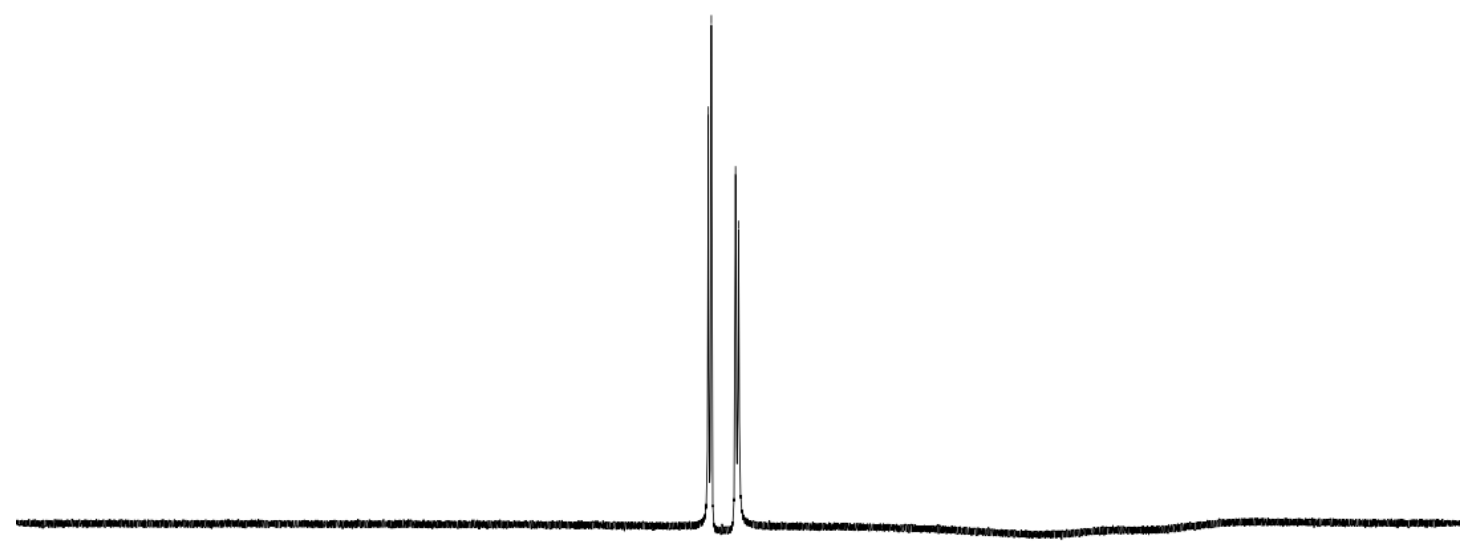

\begin{tabular}{lllllllllllllllllllllllllllllllllllllll}
\hline 0 & 40 & 30 & 20 & 10 & 0 & -10 & -20 & -30 & -40 & -50 & -60 & -70 & -80 & -90 & -100 & -110 & -120 & -130 & -140 & -150 & -160 & -170 & -180 & -190 & -200 & -210 & -220 & -230 & -240 & -25
\end{tabular} 
1H NMR (400 MHz, $\mathrm{CDCl}_{3}$ )

产

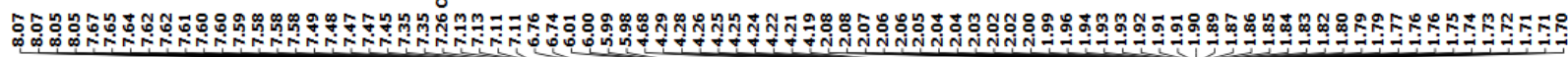<smiles>N#Cc1ccc(C(=O)OCCCOC(=O)COc2ccc(Cl)cc2Cl)cc1</smiles>

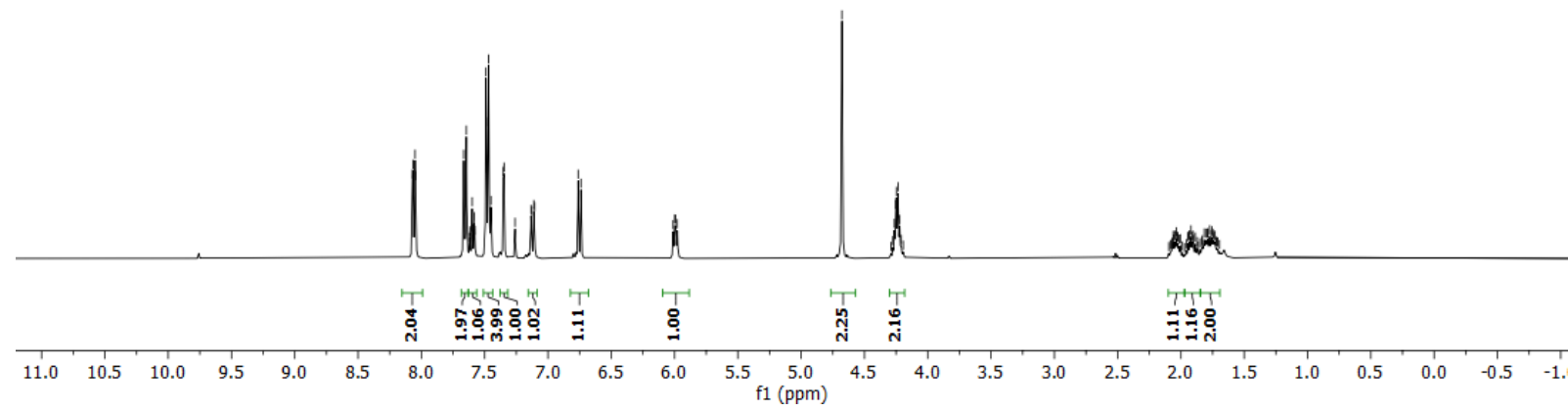

${ }^{13} \mathrm{C}$ NMR (101 MHz, $\mathrm{CDCl}_{3}$ )
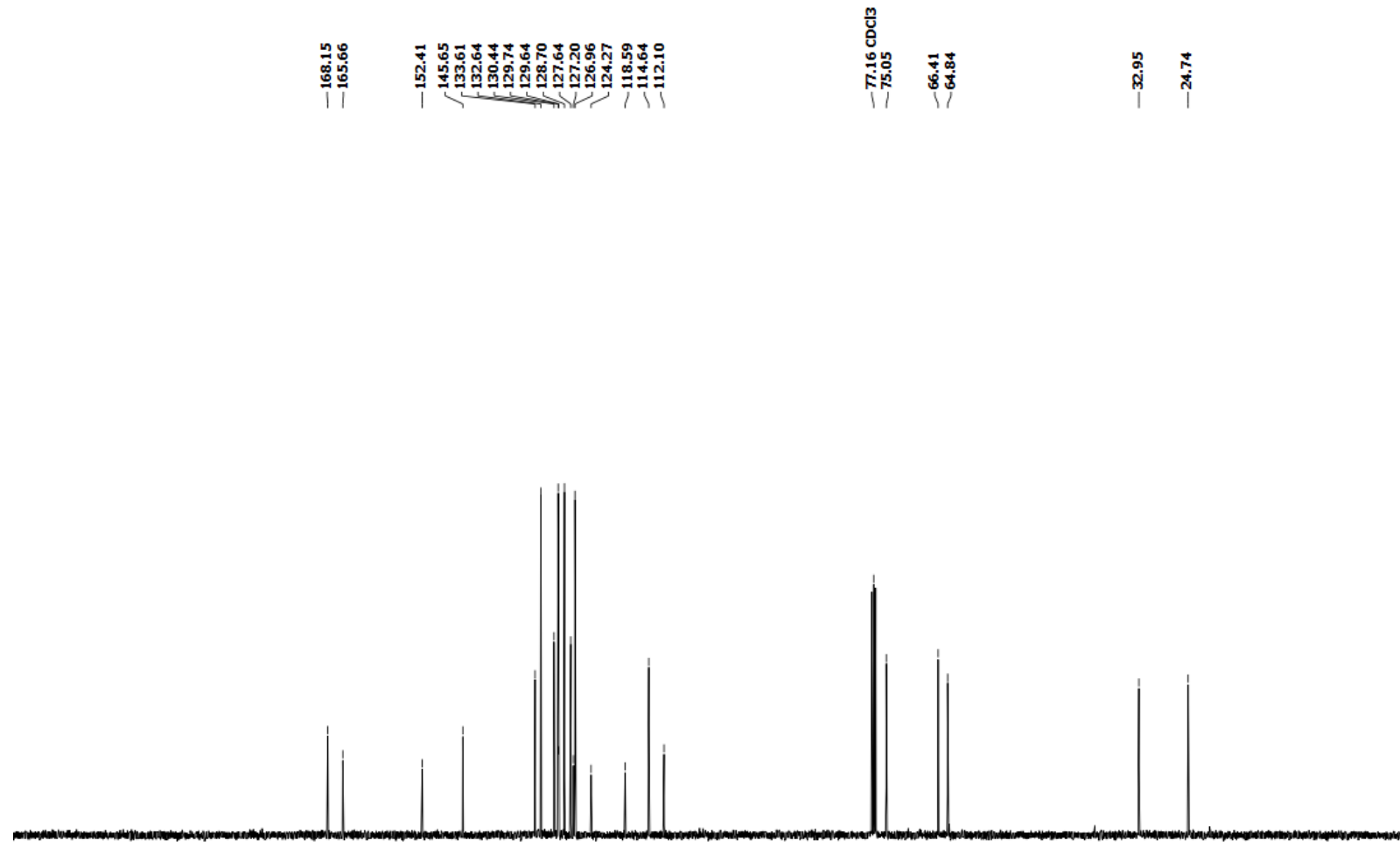

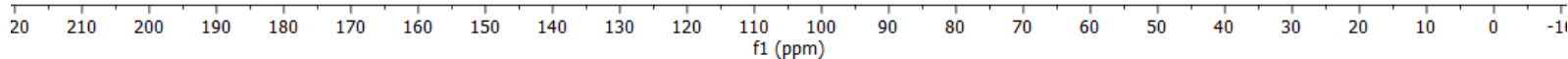
S 134 
1H NMR (599 MHz, $\mathrm{CDCl}_{3}$ )
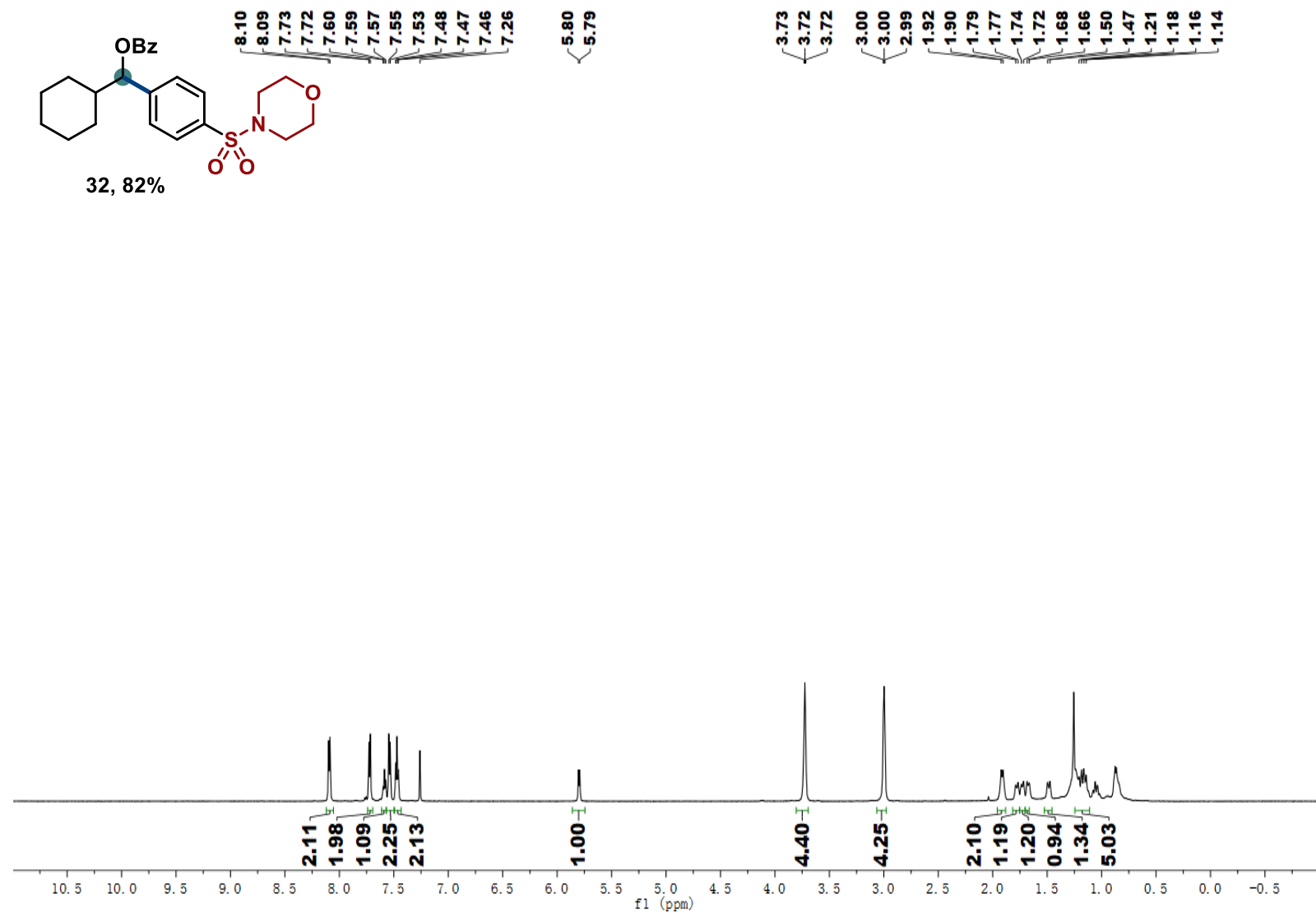

${ }^{13} \mathrm{C}$ NMR (151 MHz, $\mathrm{CDCl}_{3}$ )
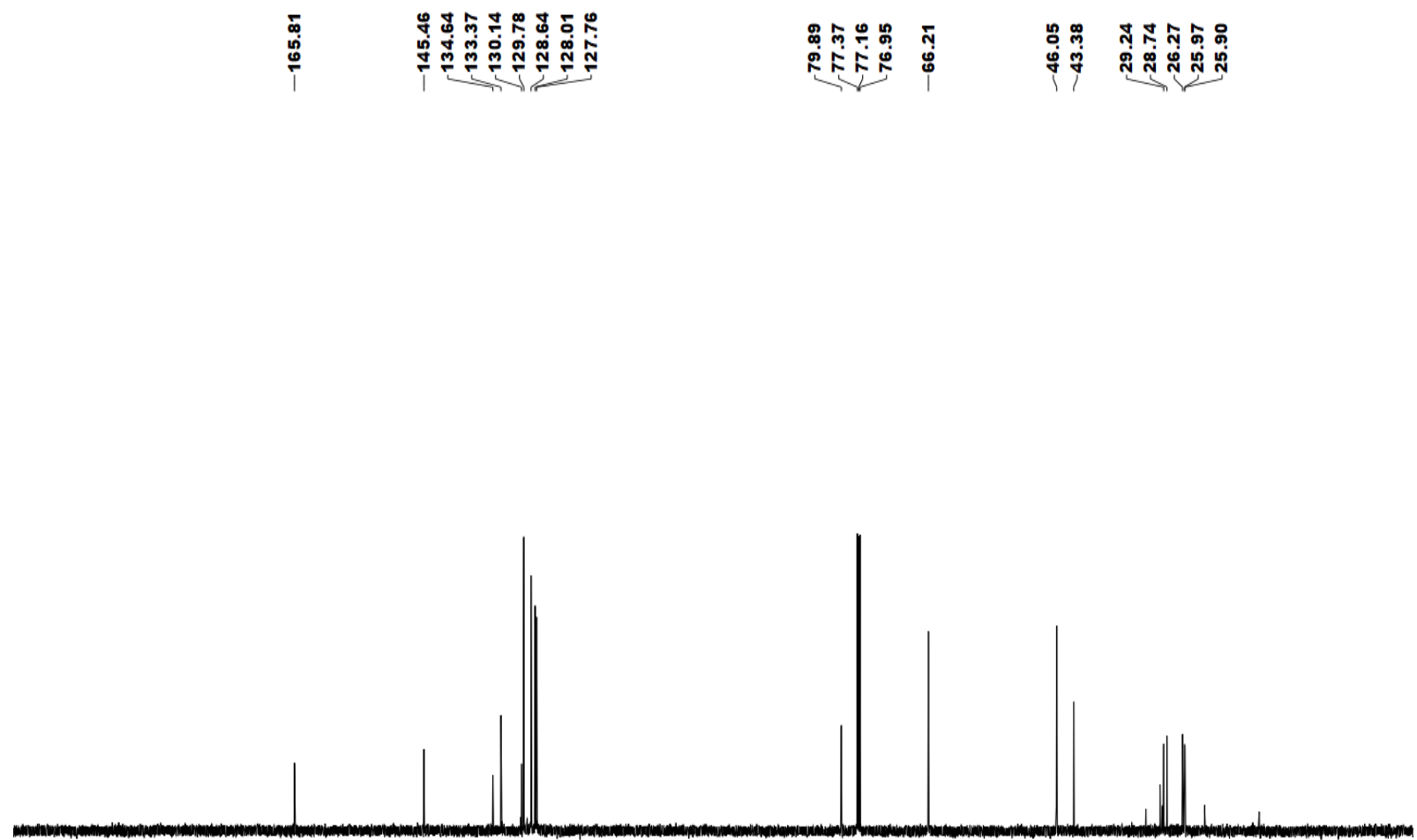

$\begin{array}{llllllllll}200 & 190 & 180 & 170 & 160 & 150 & 140 & 130 & 120 & 110 \\ \mathrm{fl} & 100 \\ (\mathrm{ppm})\end{array}$ 
1H NMR (599 MHz, $\mathrm{CDCl}_{3}$ )

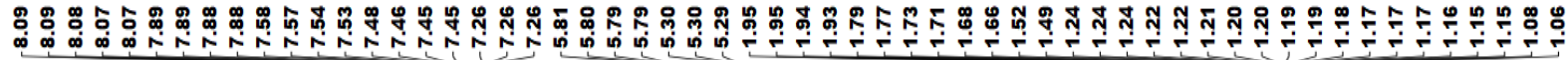<smiles>O=C1OCc2cc([C@@H](O)C3CCCCC3)ccc21</smiles>

$33,86 \%$

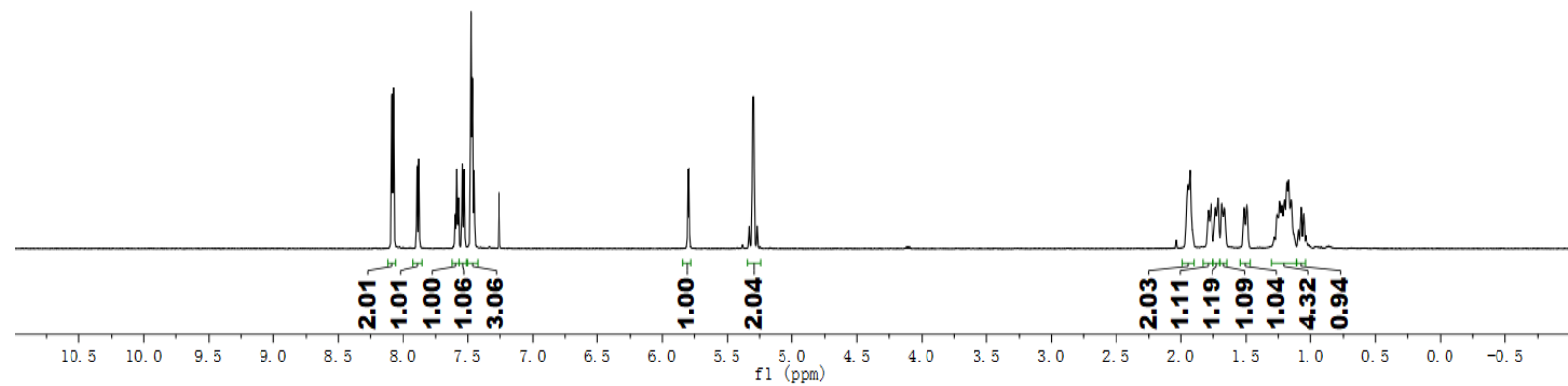

${ }^{13} \mathrm{C}$ NMR (151 MHz, $\mathrm{CDCl}_{3}$ )

草

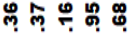

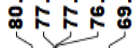

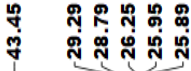

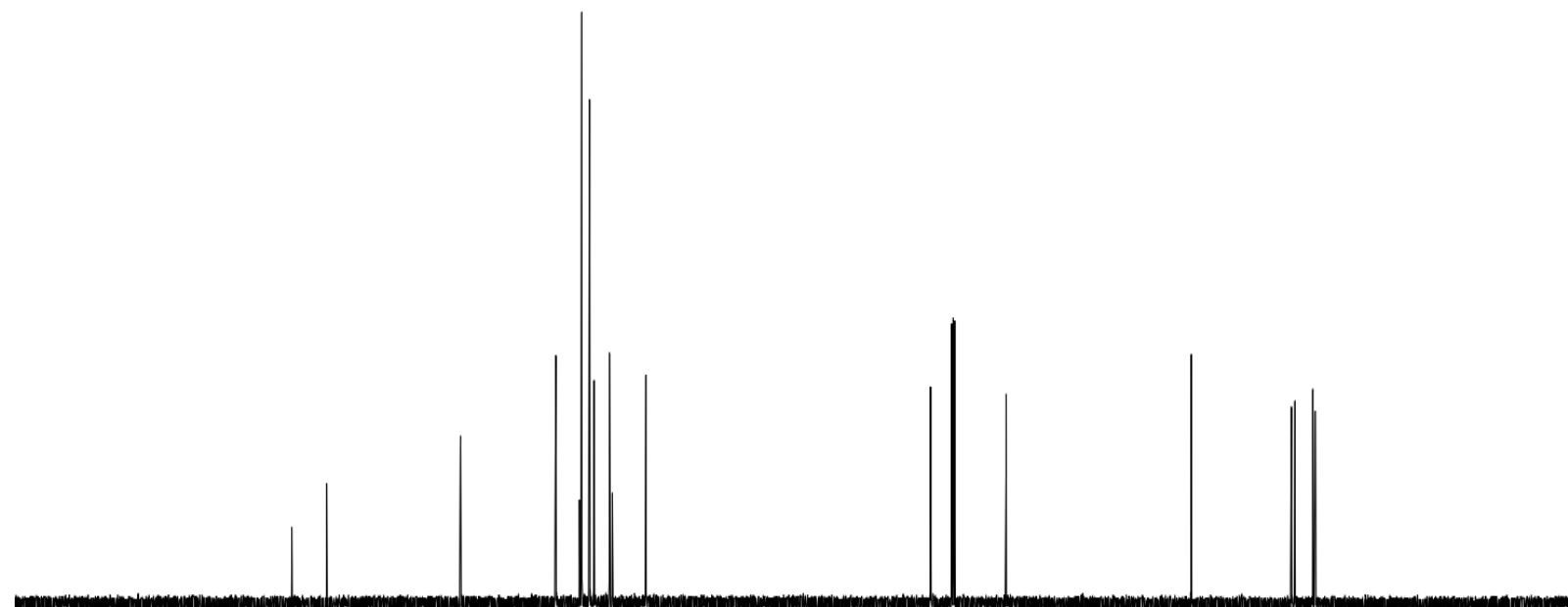

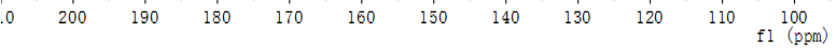

S 136 
1H NMR (599 MHz, $\mathrm{CDCl}_{3}$ )

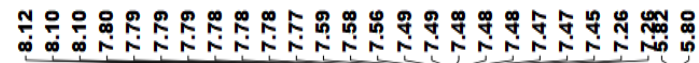<smiles>CC(C)(C)OC(=O)c1ccc(C(=O)c2ccccc2)cc1</smiles>

$34,79 \%$

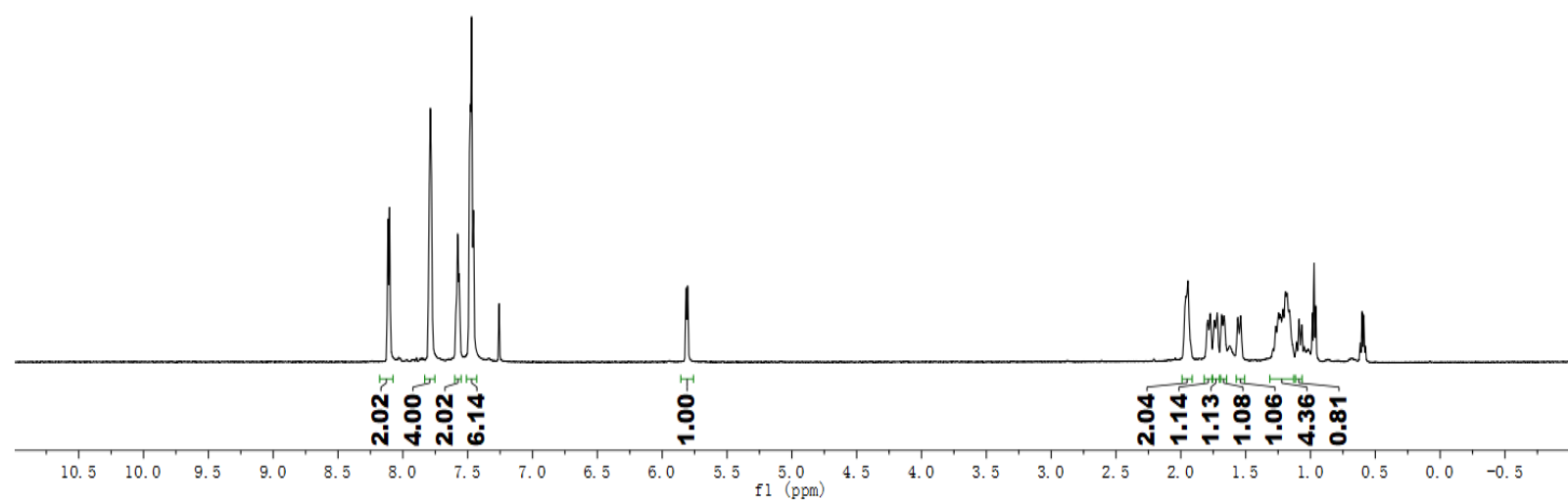

${ }^{13} \mathrm{C}$ NMR (151 MHz, $\mathrm{CDCl}_{3}$ )

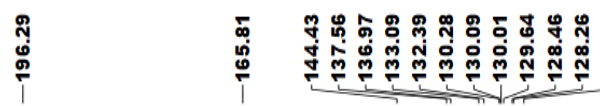

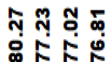

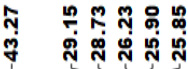

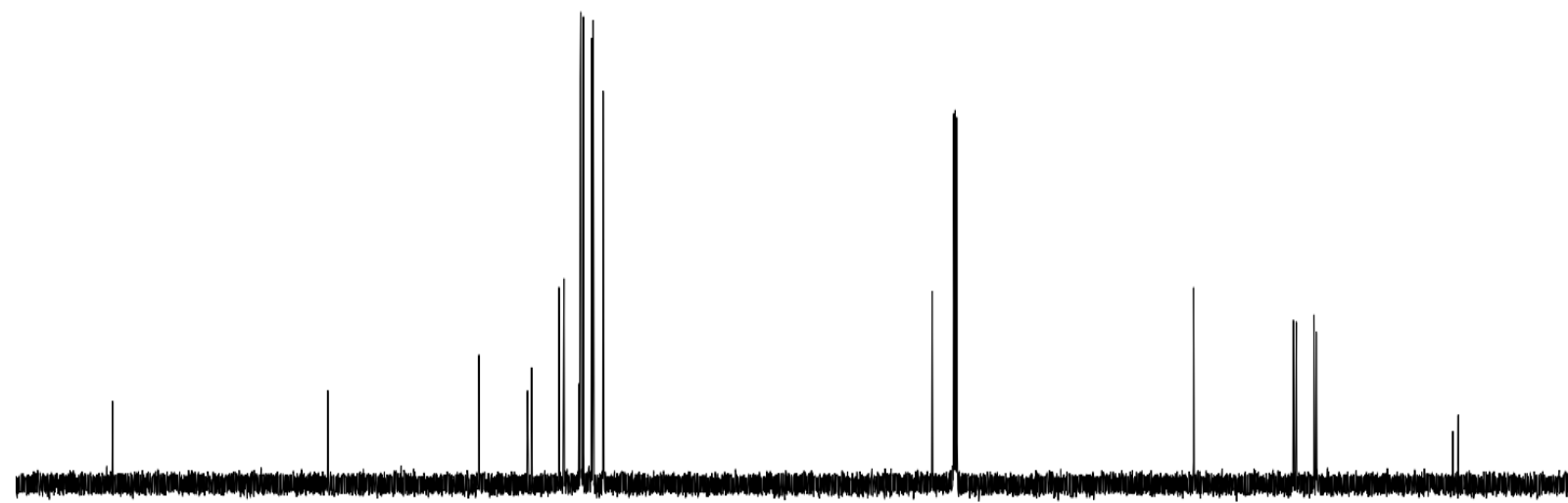


1H NMR (400 MHz, $\mathrm{CDCl}_{3}$ )

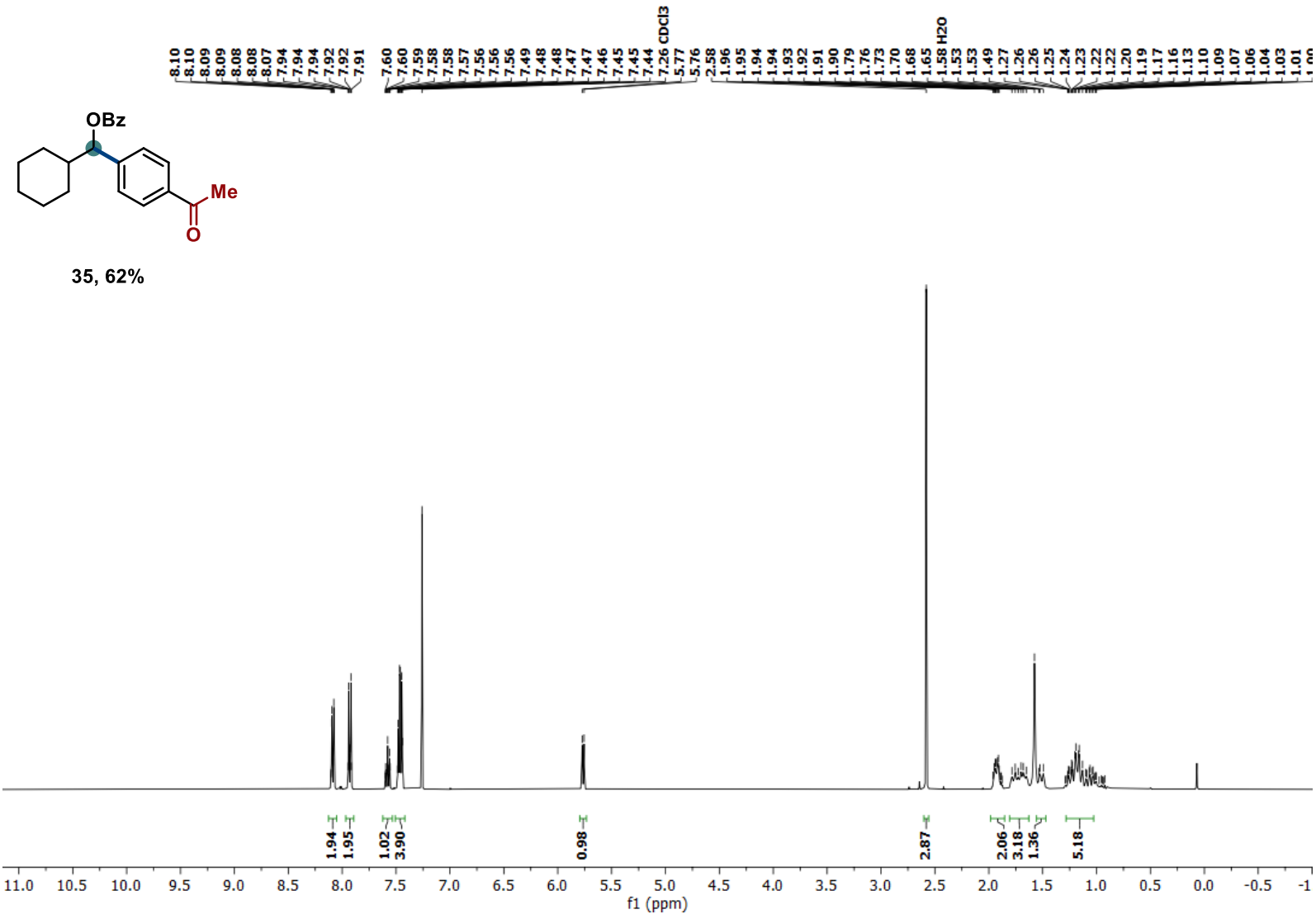

${ }^{13} \mathrm{C}$ NMR (101 MHz, $\left.\mathrm{CDCl}_{3}\right)$

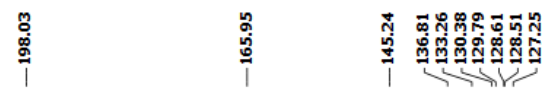

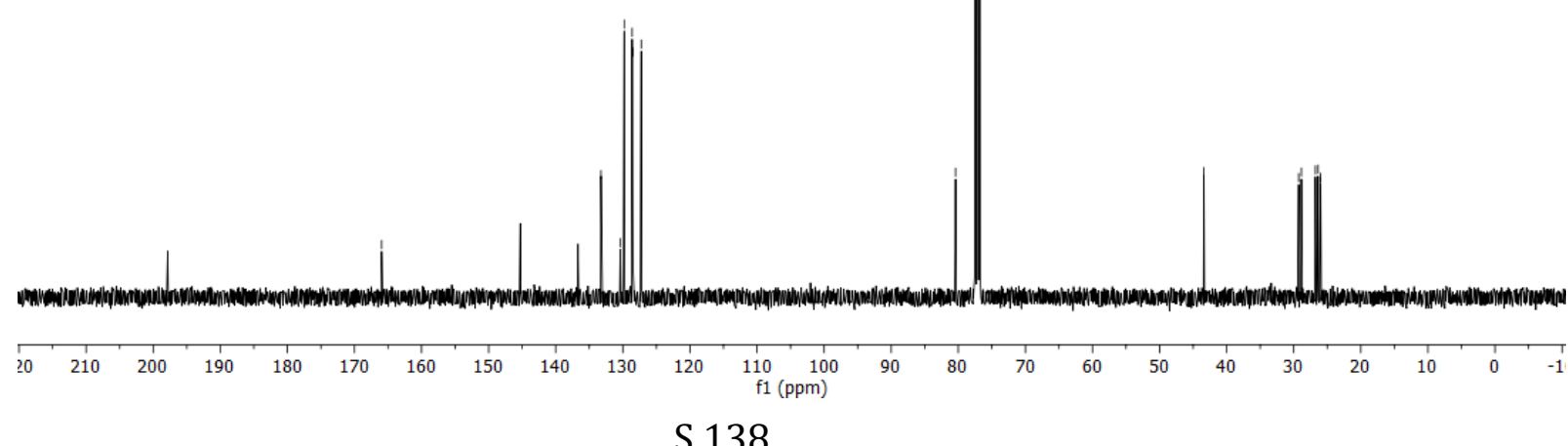


1H NMR (400 MHz, $\mathrm{CDCl}_{3}$ )
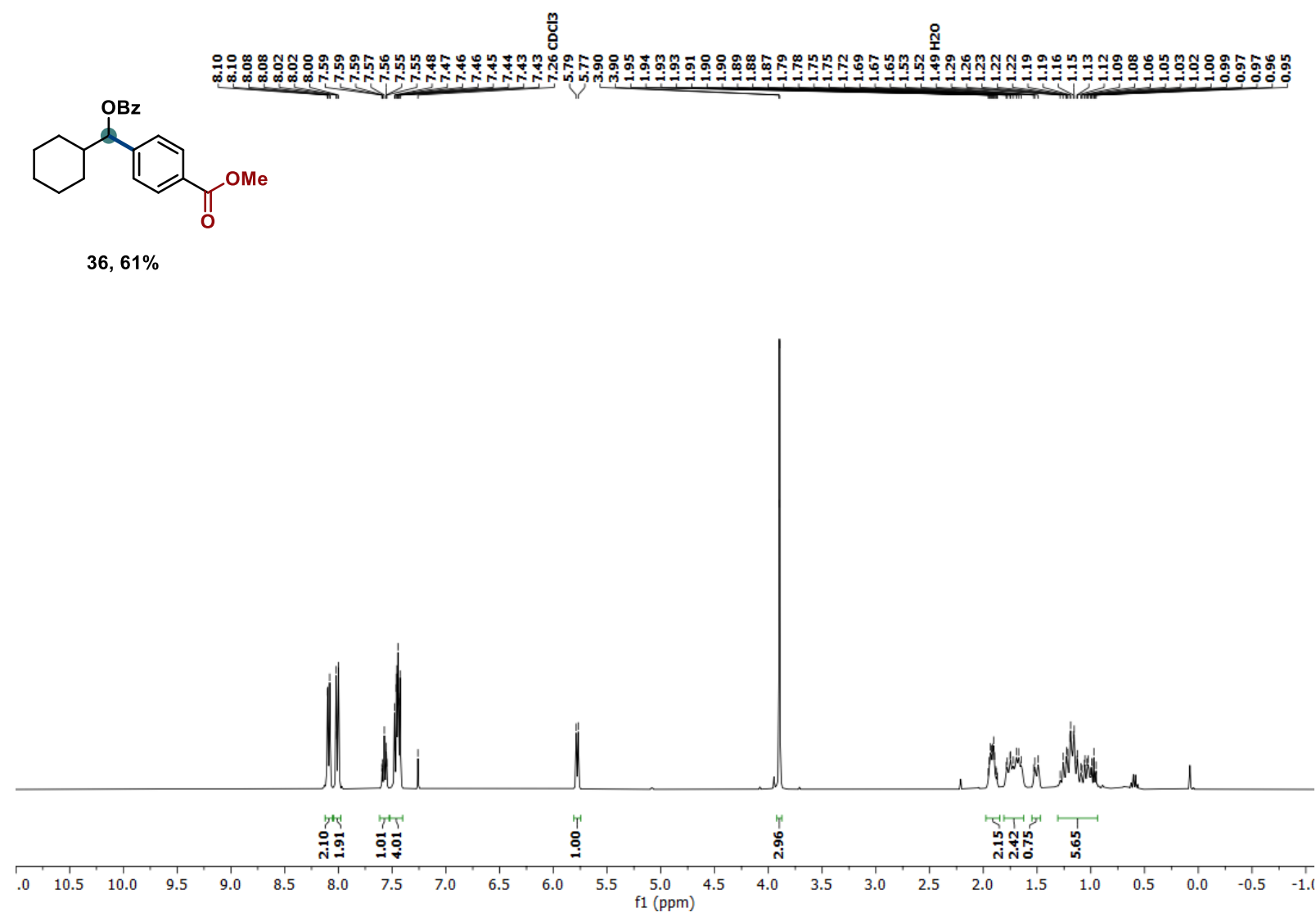

${ }^{13} \mathrm{C}$ NMR (101 MHz, $\mathrm{CDCl}_{3}$ )
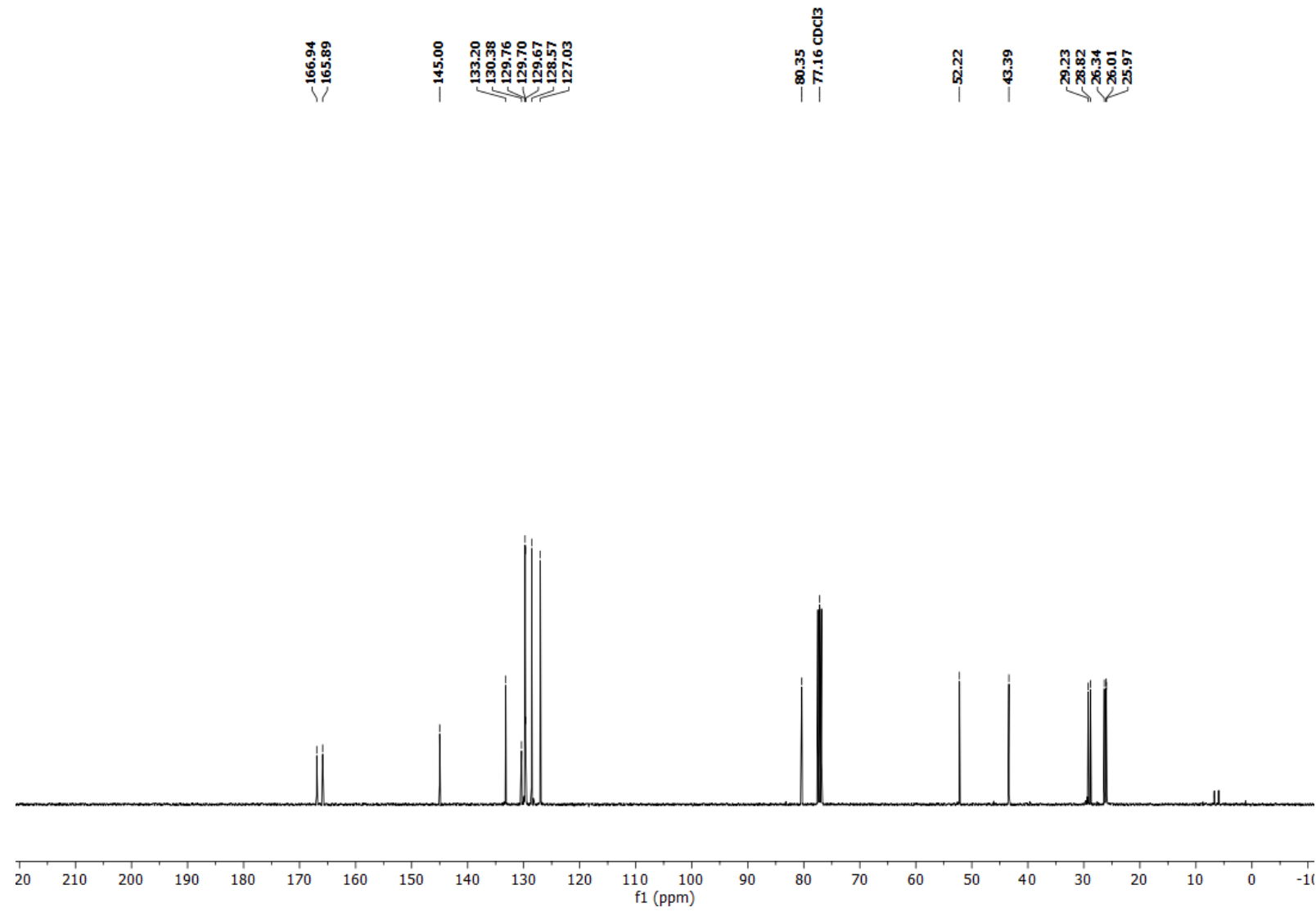
1H NMR (599 MHz, $\mathrm{CDCl}_{3}$ )

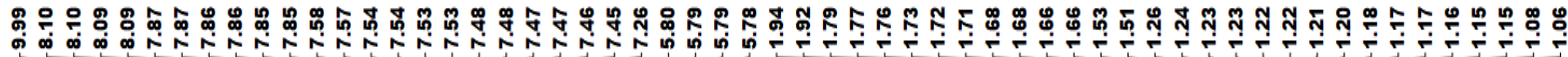<smiles>O=Cc1ccc(C(=O)C2CCCCC2)cc1</smiles>

$37,77 \%$

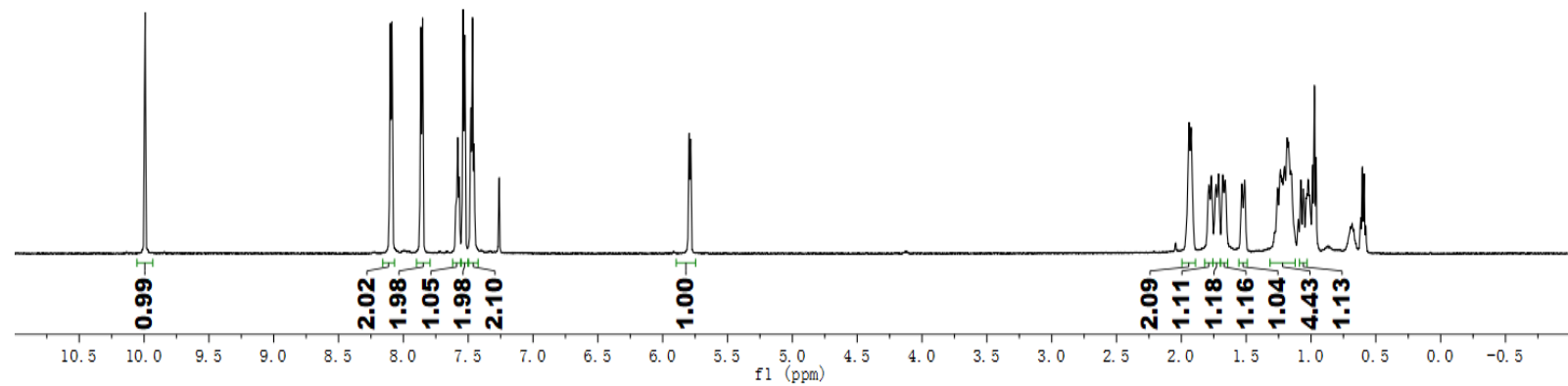

${ }^{13} \mathrm{C}$ NMR (151 MHz, $\mathrm{CDCl}_{3}$ )

商

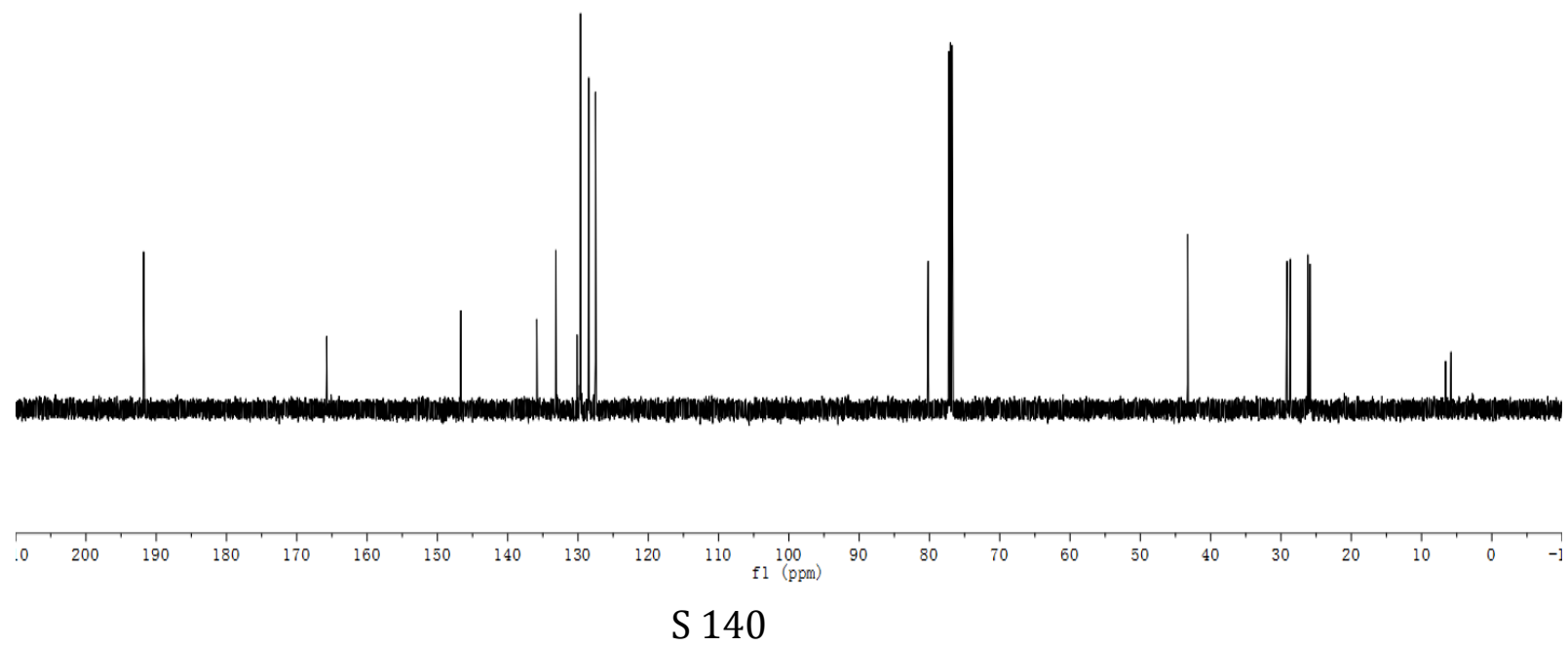


1H NMR (599 MHz, $\mathrm{CDCl}_{3}$ )

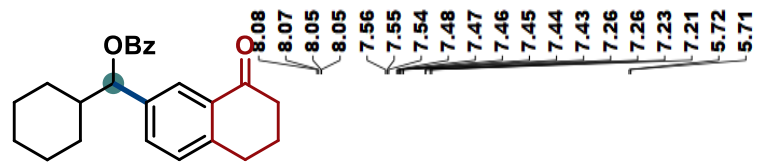

ผू.

$38,30 \%$

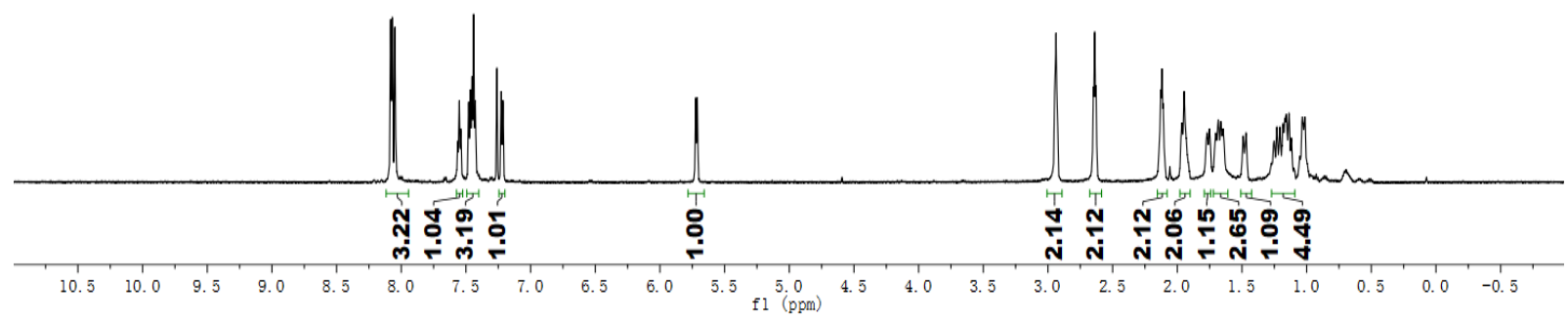

${ }^{13} \mathrm{C}$ NMR (151 MHz, $\mathrm{CDCl}_{3}$ )
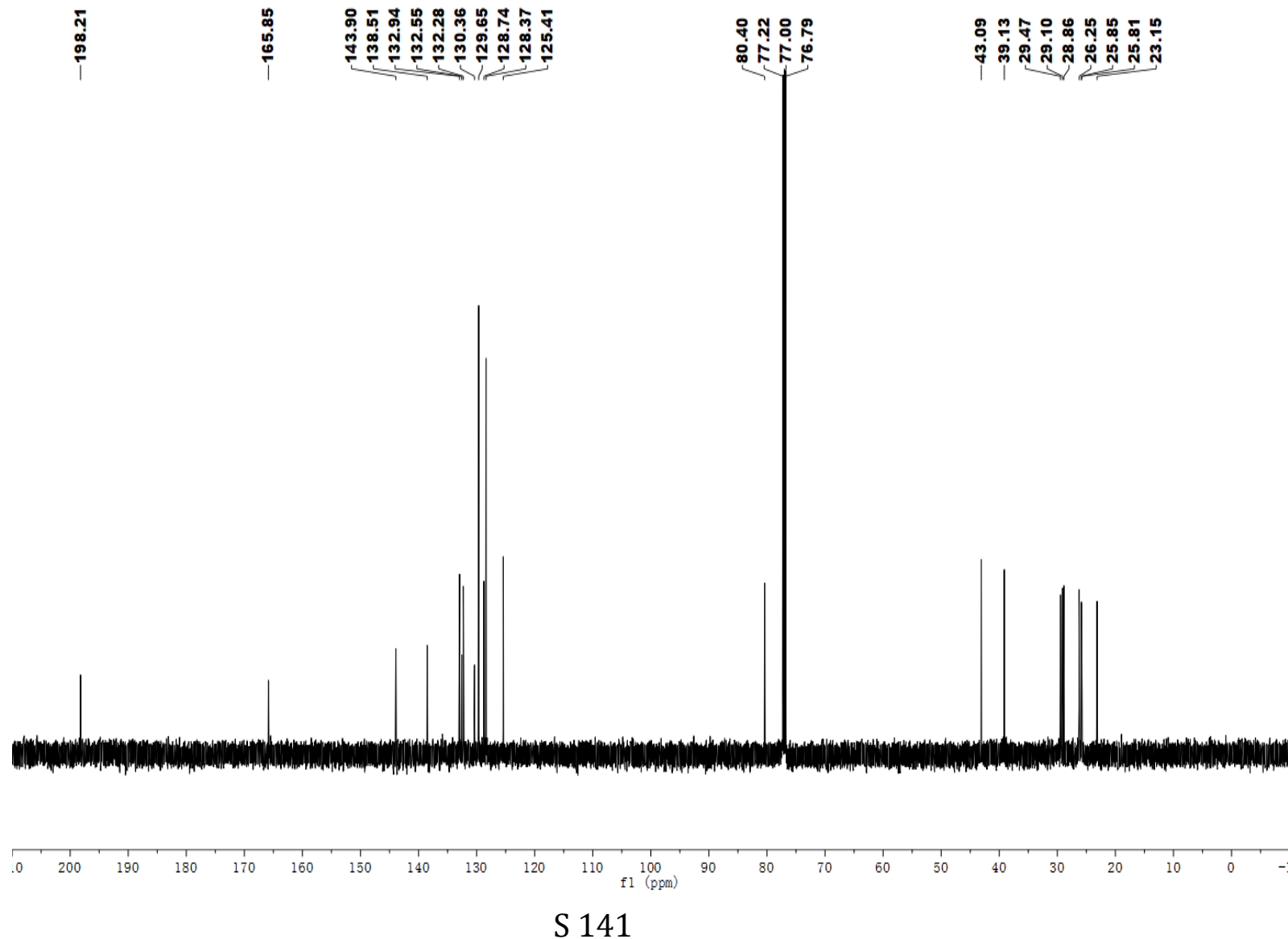
1H NMR (599 MHz, $\mathrm{CDCl}_{3}$ )

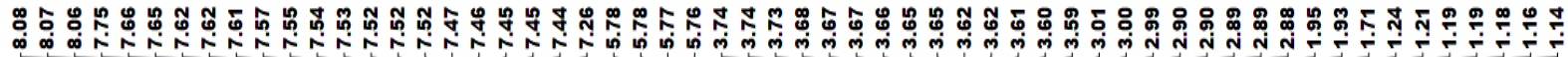<smiles>O=C(c1cccc(S(=O)(=O)N2CCOCC2)c1)C1CCCCC1</smiles>

$39,69 \%$

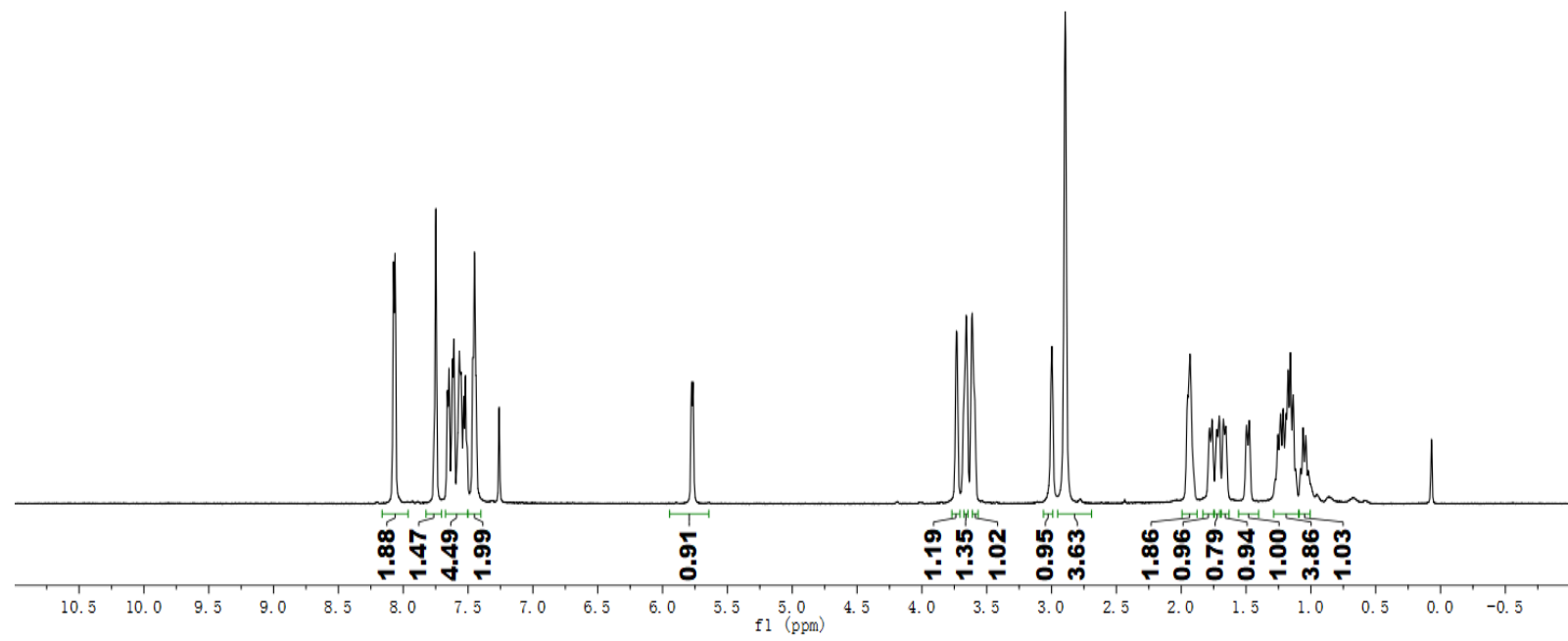

${ }^{13} \mathrm{C}$ NMR (151 MHz, $\mathrm{CDCl}_{3}$ )

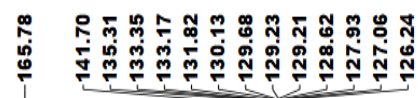

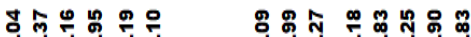

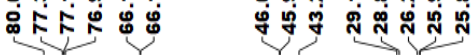

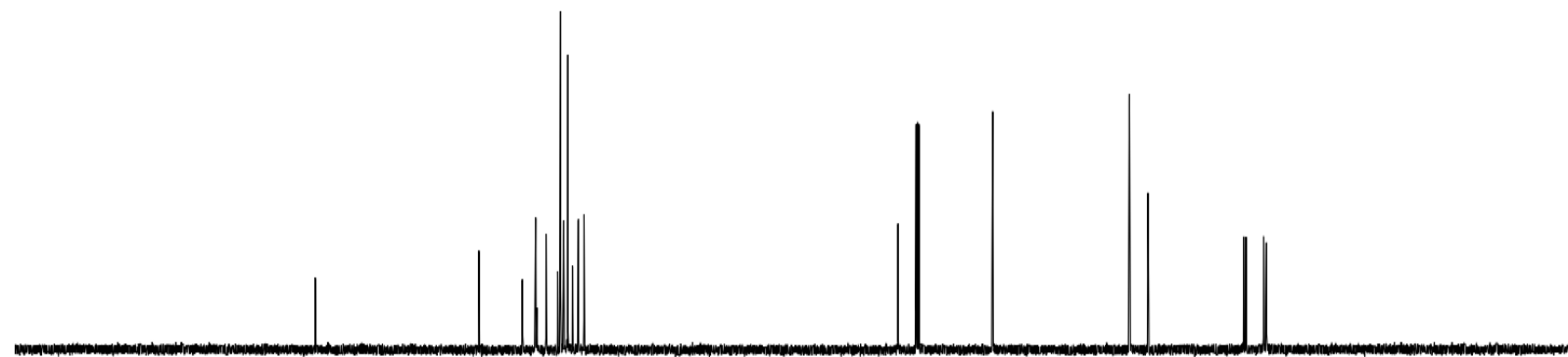

$\begin{array}{lllllllll}200 & 190 & 180 & 170 & 160 & 150 & 140 & 130 & 120\end{array}$

${ }_{\mathrm{f}}^{100}(\mathrm{ppm})^{90}$

S 142 
1H NMR (599 MHz, $\mathrm{CDCl}_{3}$ )

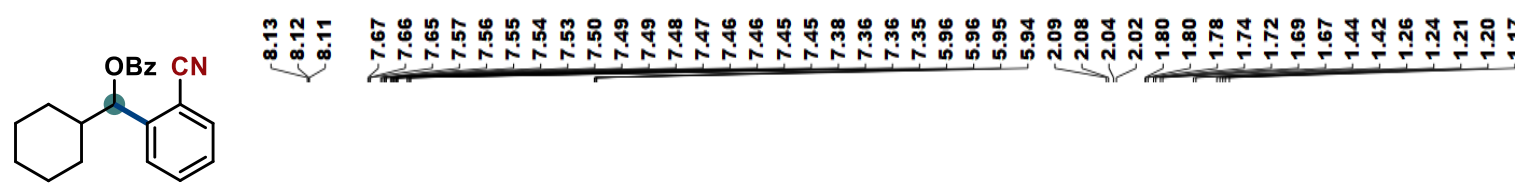

$40,55 \%$

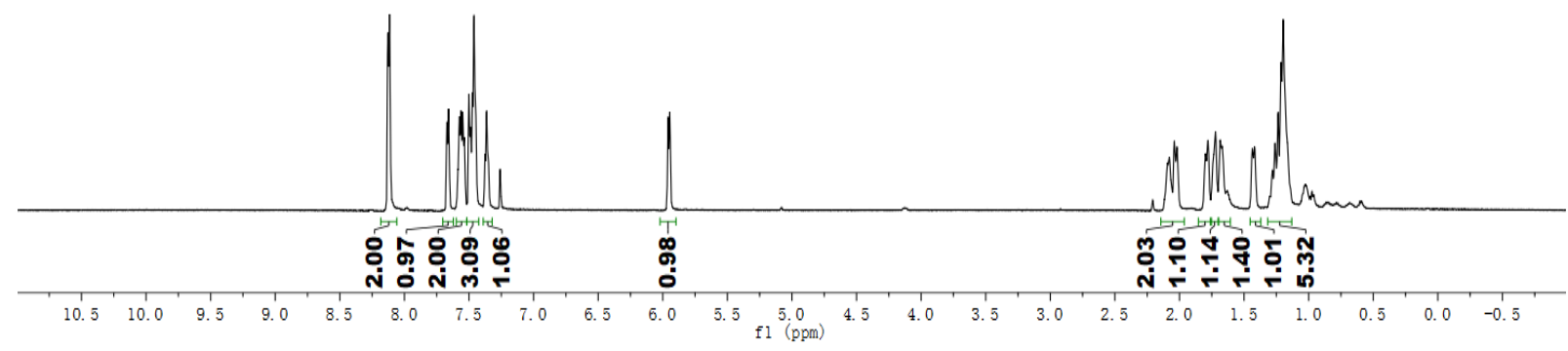

${ }^{13} \mathrm{C}$ NMR (151 $\mathrm{MHz}, \mathrm{CDCl}_{3}$ )

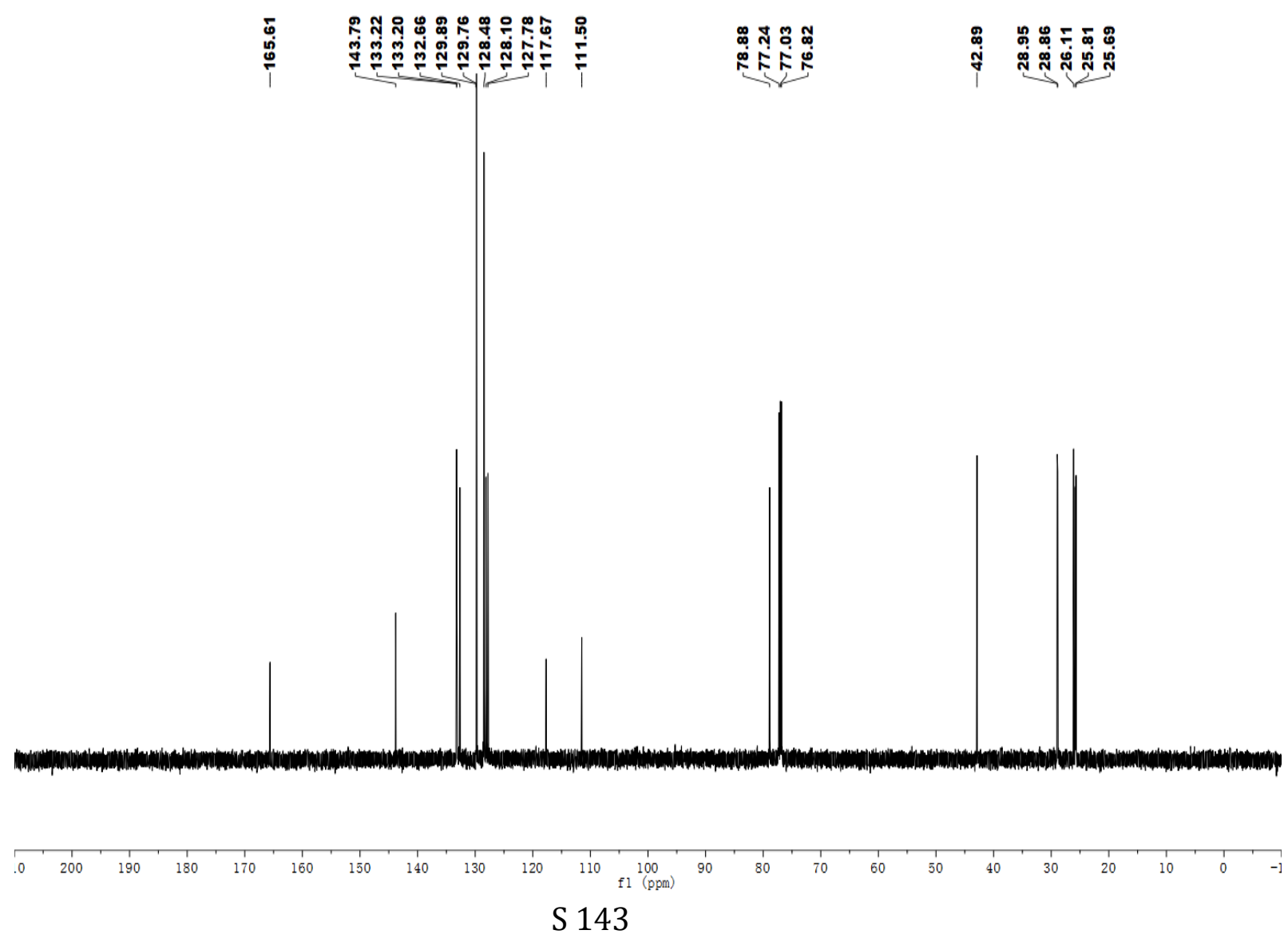


${ }^{1} \mathrm{H}$ NMR (599 MHz, $\mathrm{CDCl}_{3}$ )

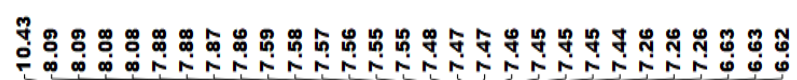

策

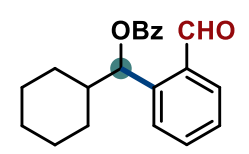

$41,71 \%$

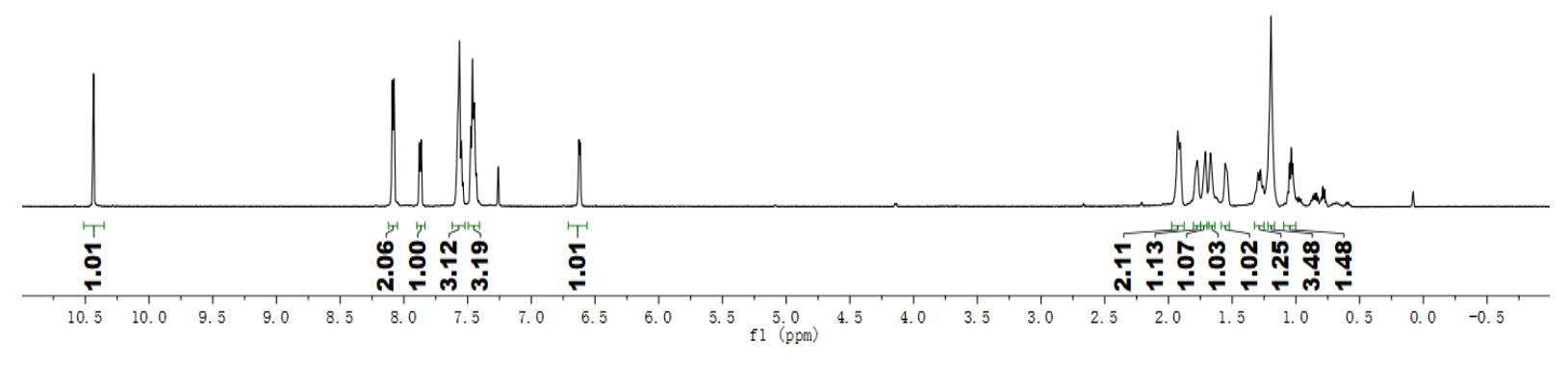

${ }^{13} \mathrm{C}$ NMR (151 MHz, $\left.\mathrm{CDCl}_{3}\right)$

至

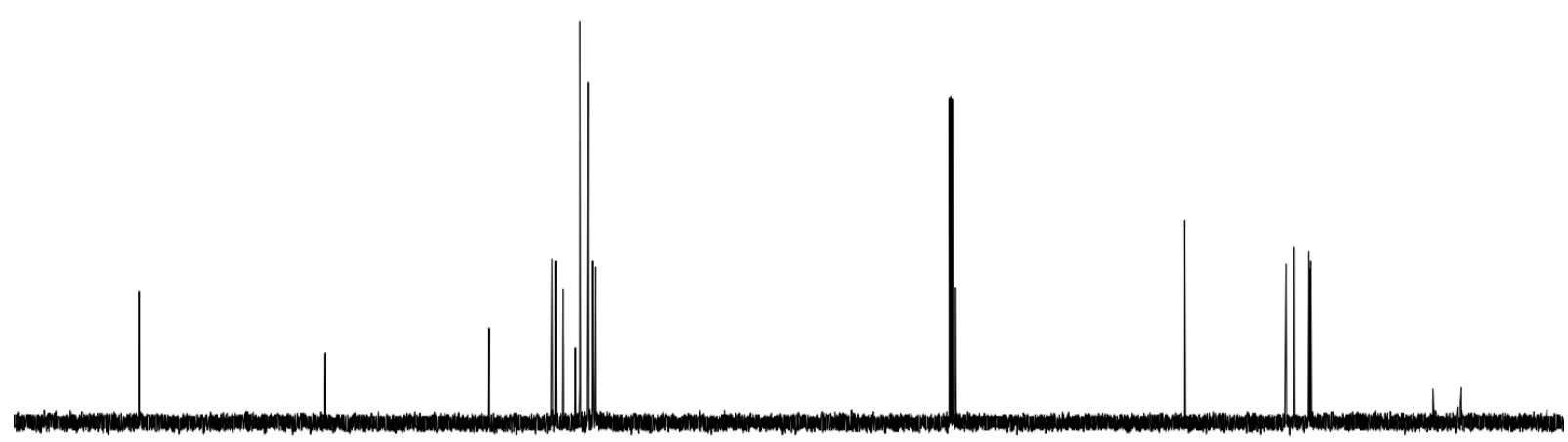

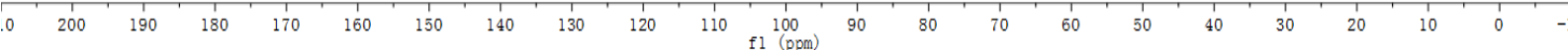
S 144 
1H NMR (599 MHz, $\mathrm{CDCl}_{3}$ )

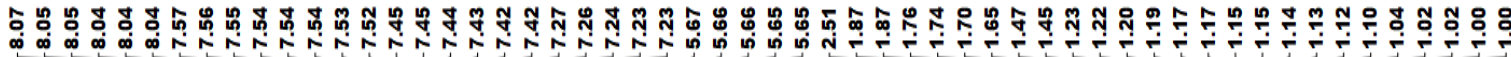<smiles>N#Cc1ccc(C(=O)C2CCCCC2)cc1N</smiles>

$43,69 \%$

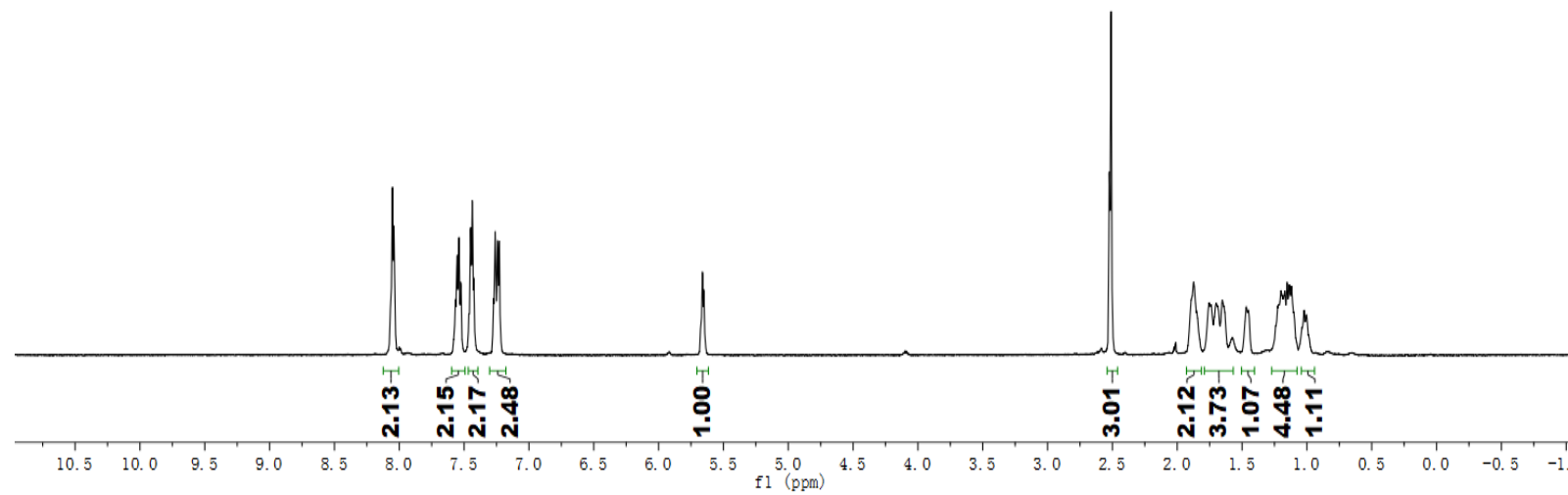

${ }^{13} \mathrm{C}$ NMR (151 MHz, $\mathrm{CDCl}_{3}$ )
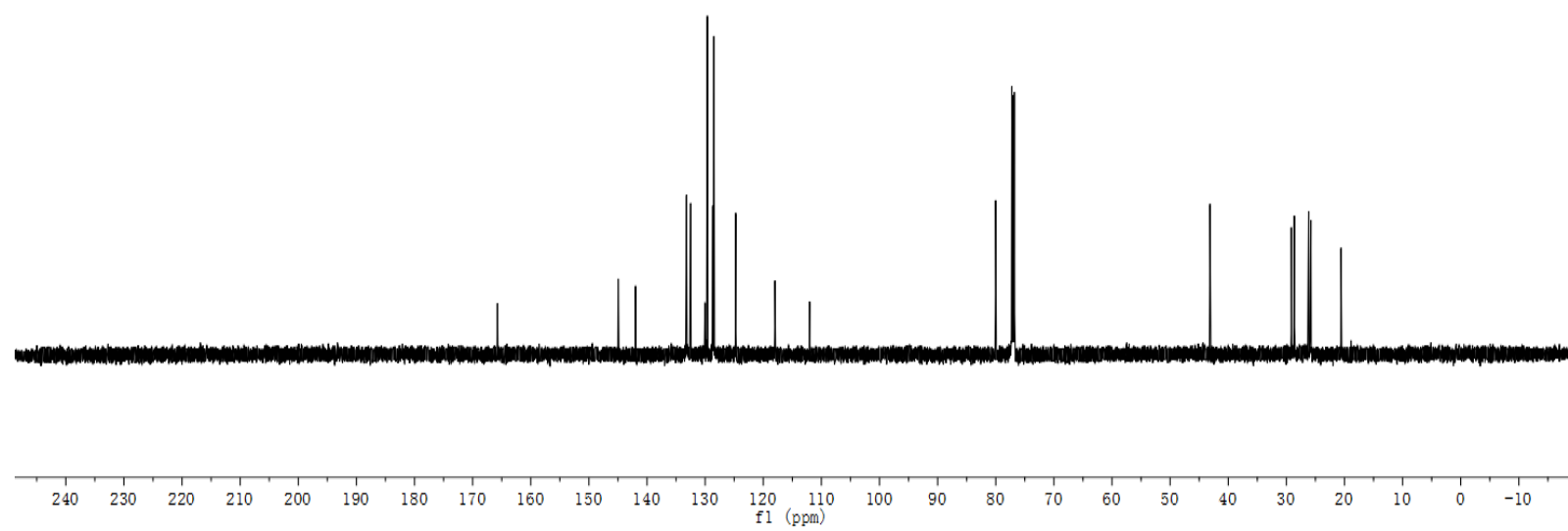
1H NMR (599 MHz, $\mathrm{CDCl}_{3}$ )<smiles>O=C(c1cc(C(F)(F)F)cc(C(F)(F)F)c1)C1CCCCC1</smiles>

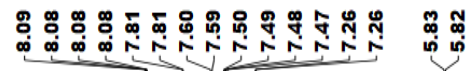

$44,87 \%$

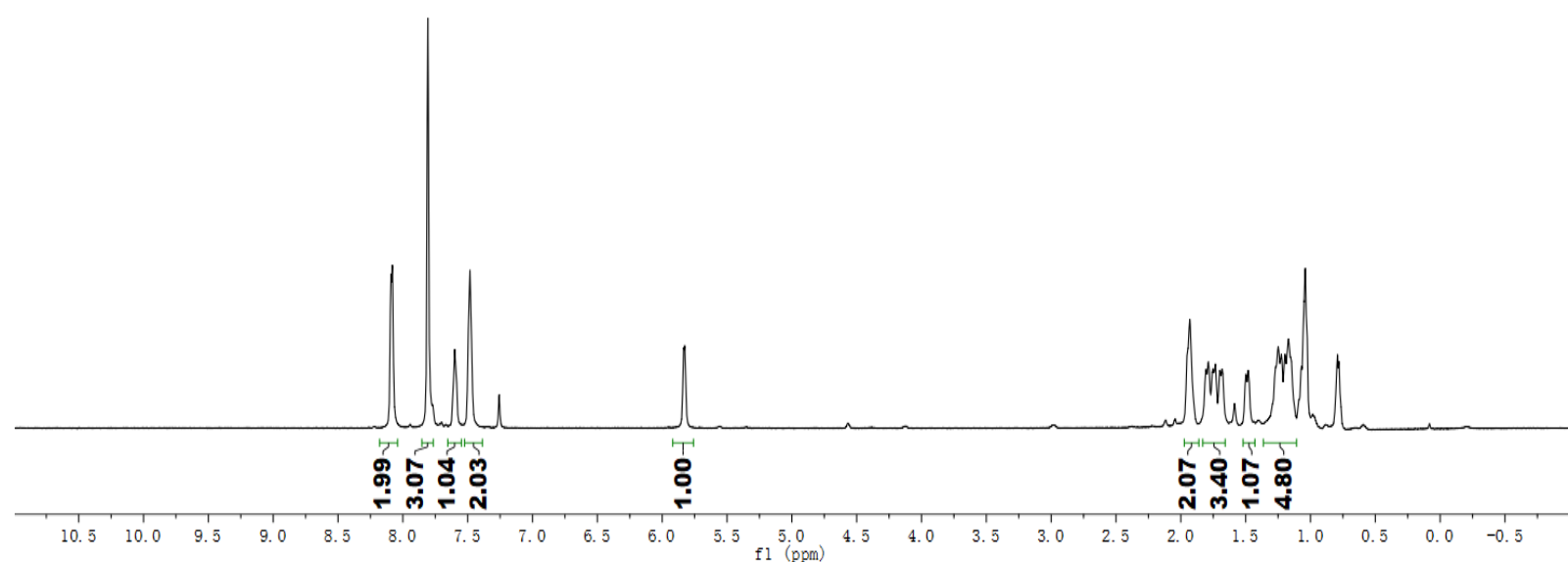

${ }^{13} \mathrm{C}$ NMR (151 MHz, $\mathrm{CDCl}_{3}$ )

告
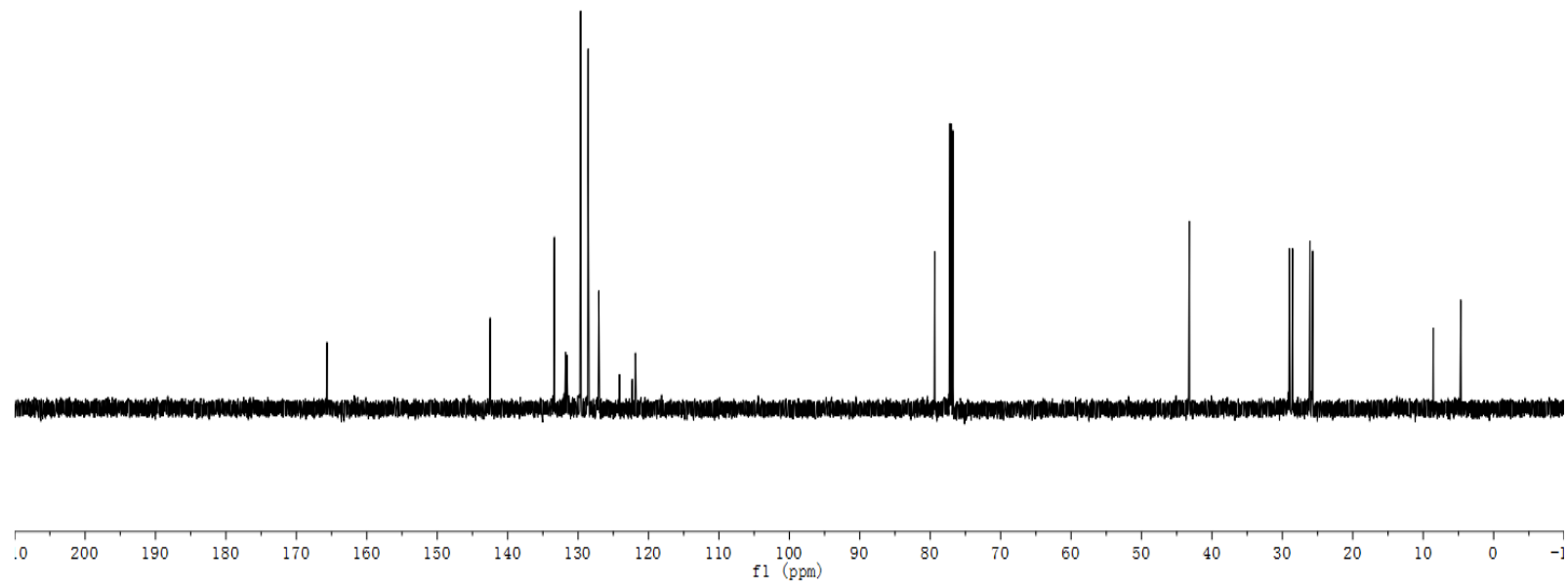
19F NMR (470 MHz, $\left.\mathrm{CDCl}_{3}\right)$

ஸَ.

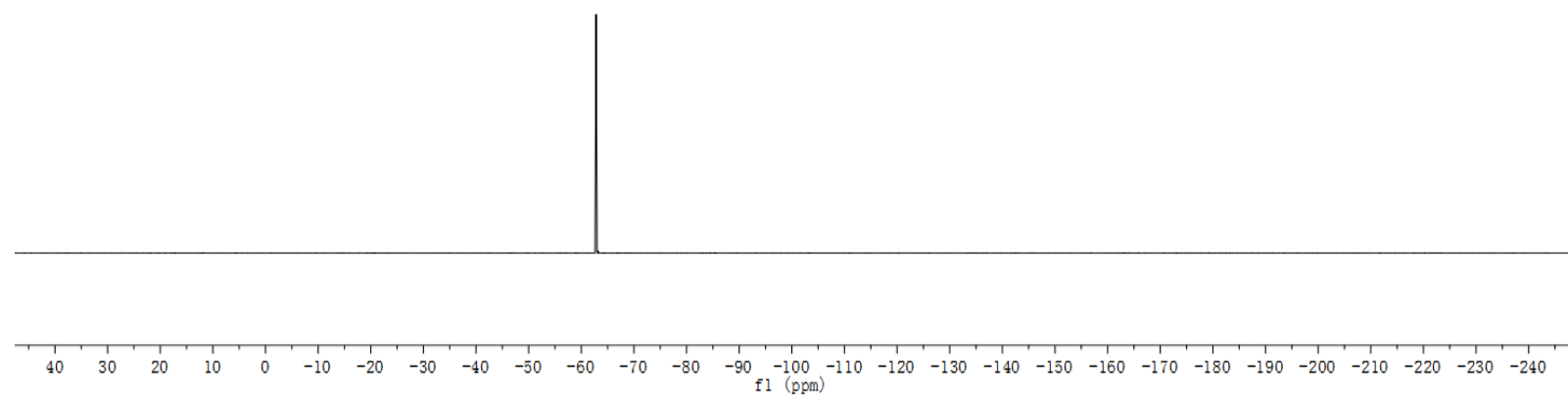


1H NMR (599 MHz, $\mathrm{CDCl}_{3}$ )

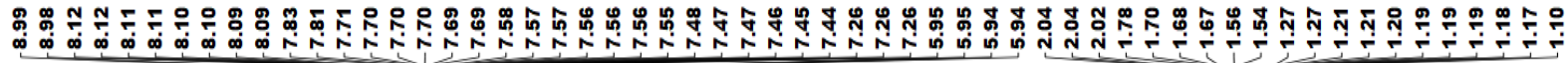<smiles>CCCCC(=O)c1cnc2ccccc2c1</smiles>

$45,45 \%$

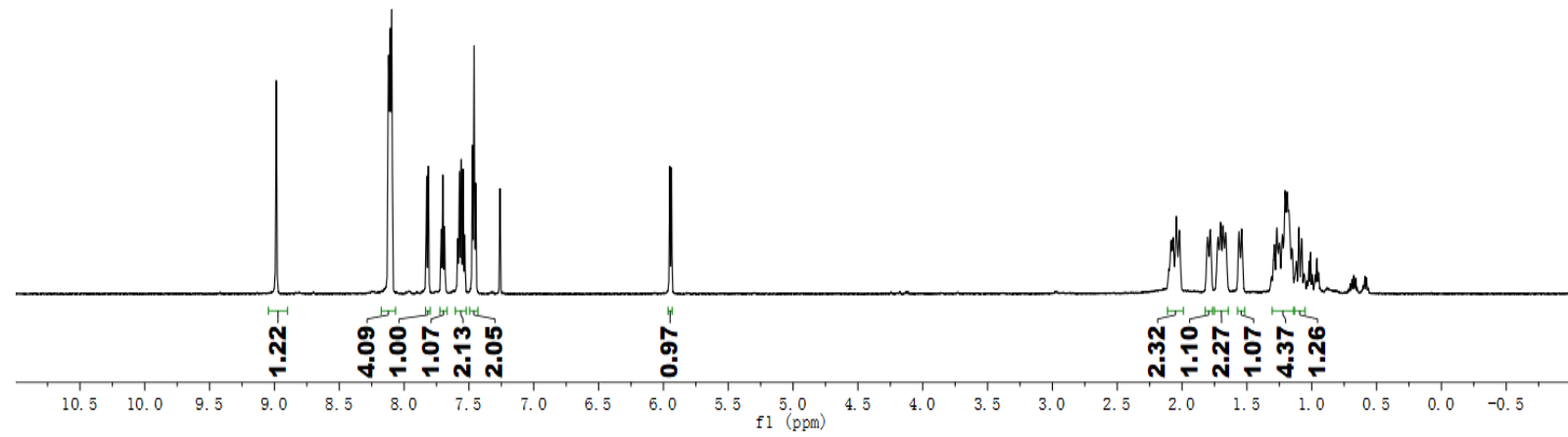

${ }^{13} \mathrm{C}$ NMR (151 MHz, $\mathrm{CDCl}_{3}$ )

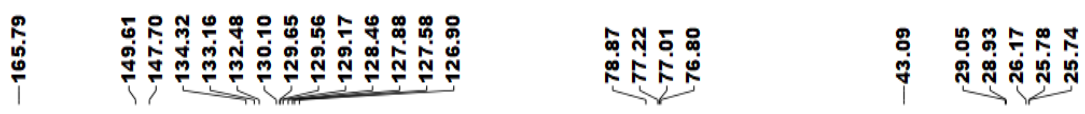

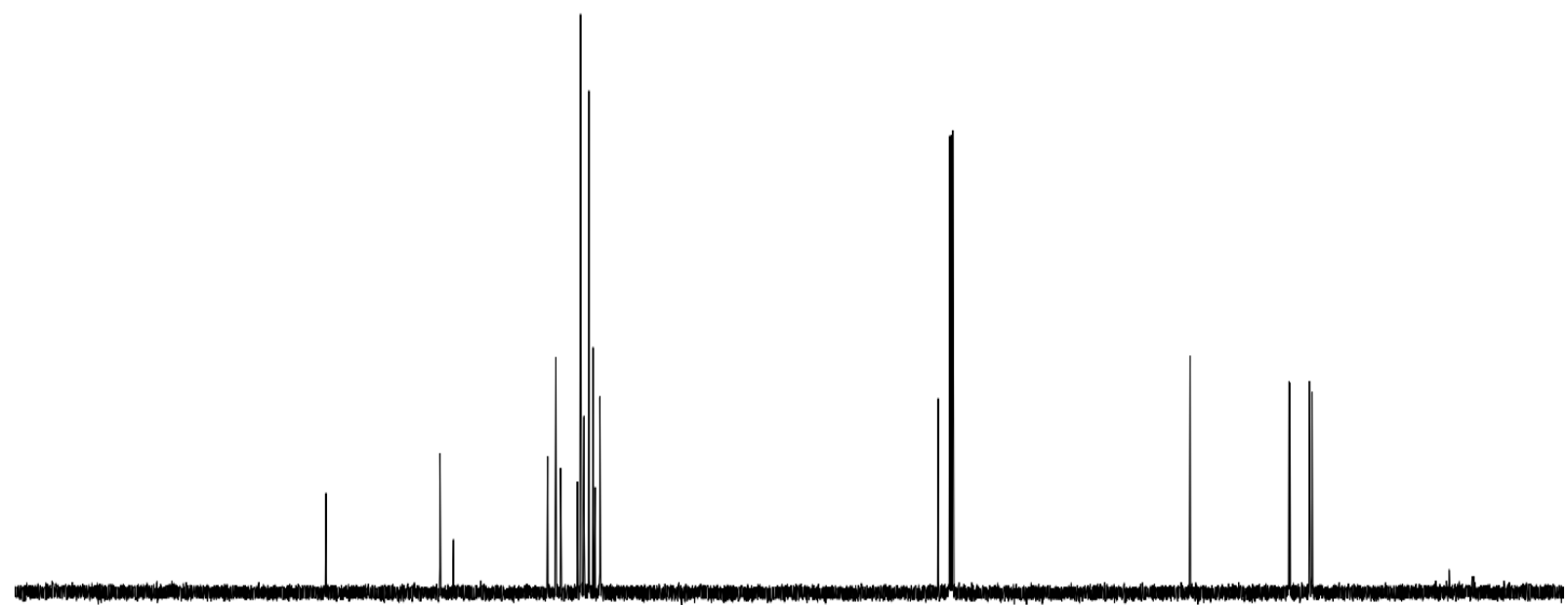

$\begin{array}{lllllllllllllllllllllllll}0 & 200 & 190 & 180 & 170 & 160 & 150 & 140 & 130 & 120 & 110 & \mathrm{f} 1(\mathrm{ppm}) & 90 & 80 & 70 & 60 & 50 & 40 & 30 & 20 & 10 & 0 & -1\end{array}$ S 149 
1H NMR (599 MHz, $\mathrm{CDCl}_{3}$ )

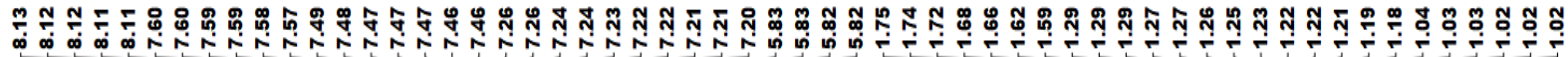<smiles>CC(C)(C)OC(=Nc1cccc(Cl)n1)C1CCCCC1</smiles>

$46,64 \%$

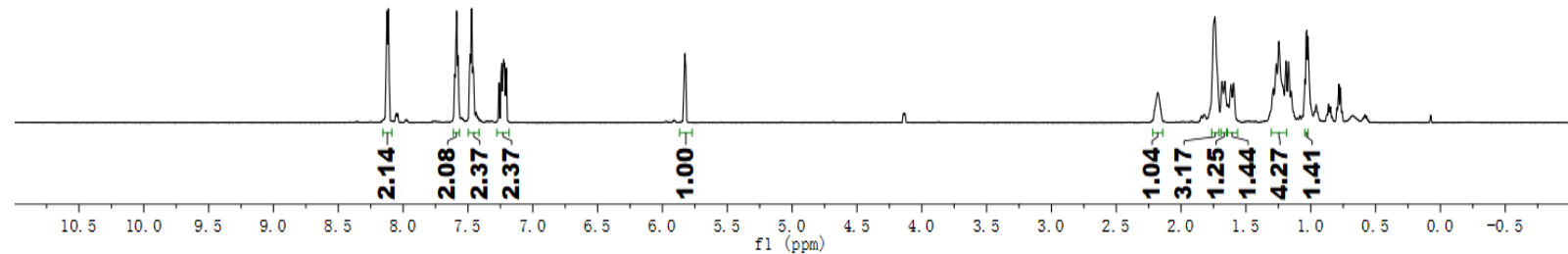

${ }^{13} \mathrm{C}$ NMR (151 MHz, $\mathrm{CDCl}_{3}$ )

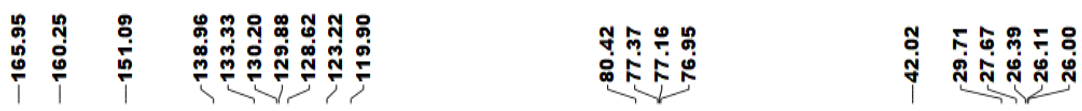

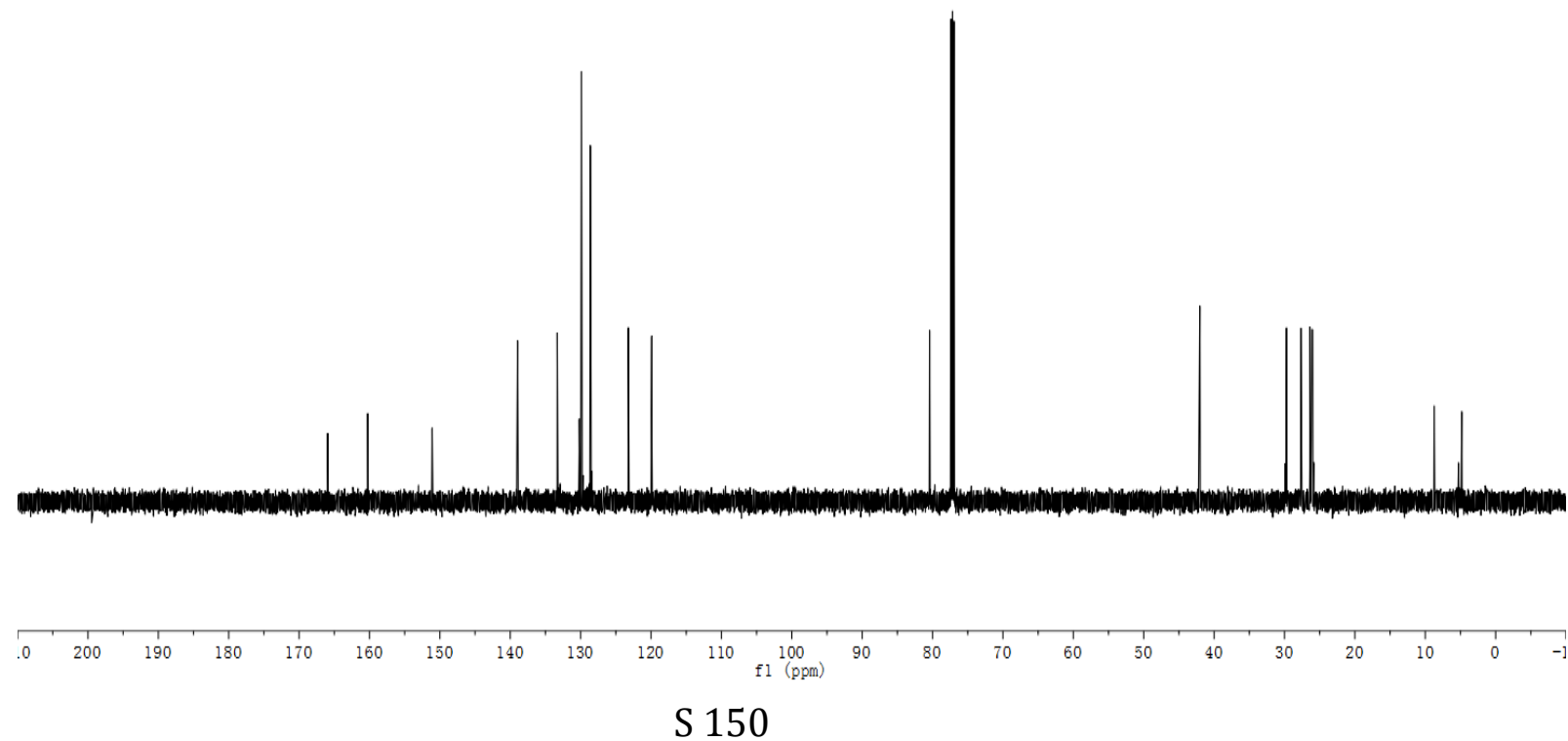


${ }^{1} \mathrm{H}$ NMR (599 $\mathrm{MHz}^{\mathrm{CDCl}_{3}}$ )

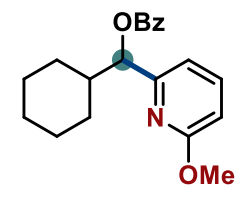

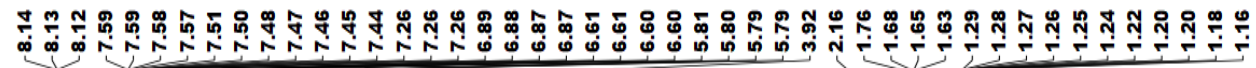

$47,77 \%$

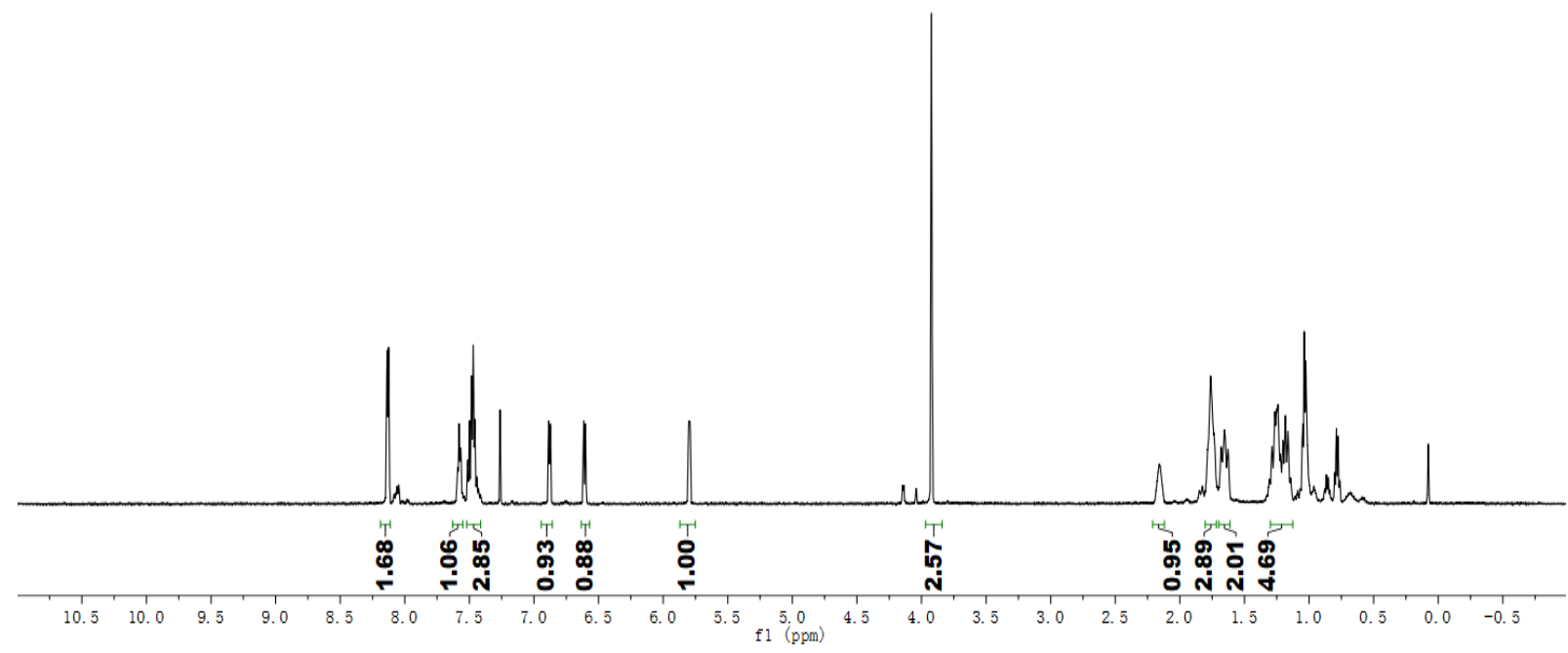

${ }^{13} \mathrm{C}$ NMR (151 MHz, $\left.\mathrm{CDCl}_{3}\right)$

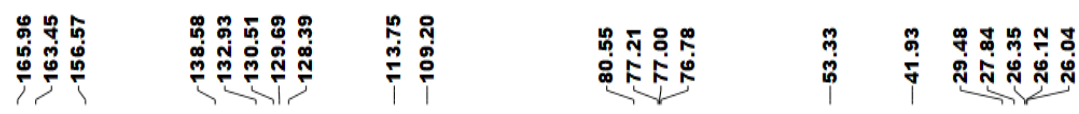

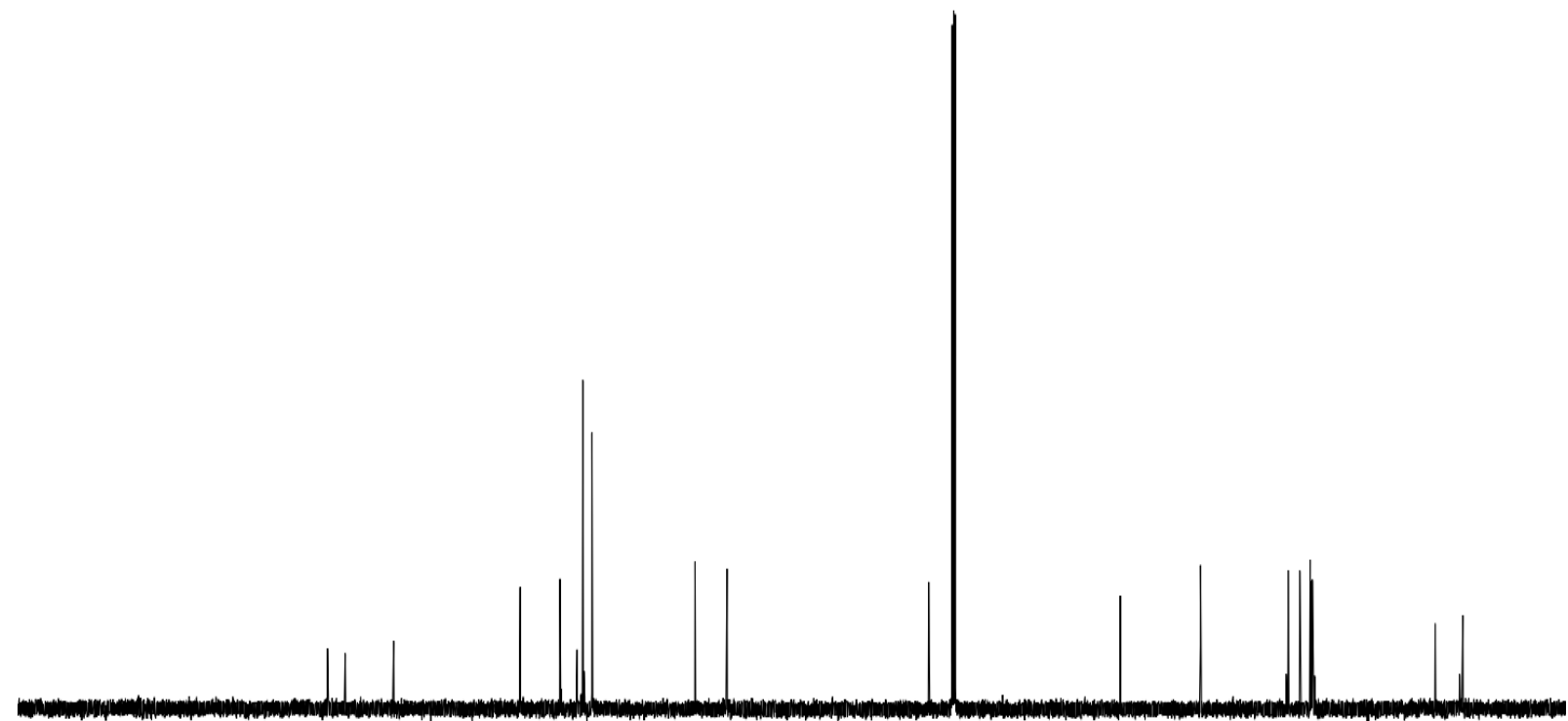

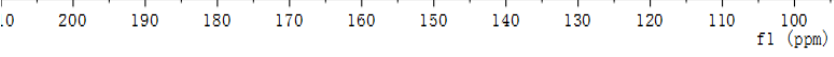

S 151 
${ }^{1} \mathrm{H}$ NMR (599 MHz, $\mathrm{CDCl}_{3}$ )

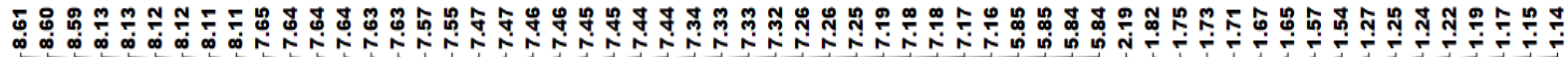<smiles>O=C(c1ccccn1)C1CCCCC1</smiles>

$48,53 \%$

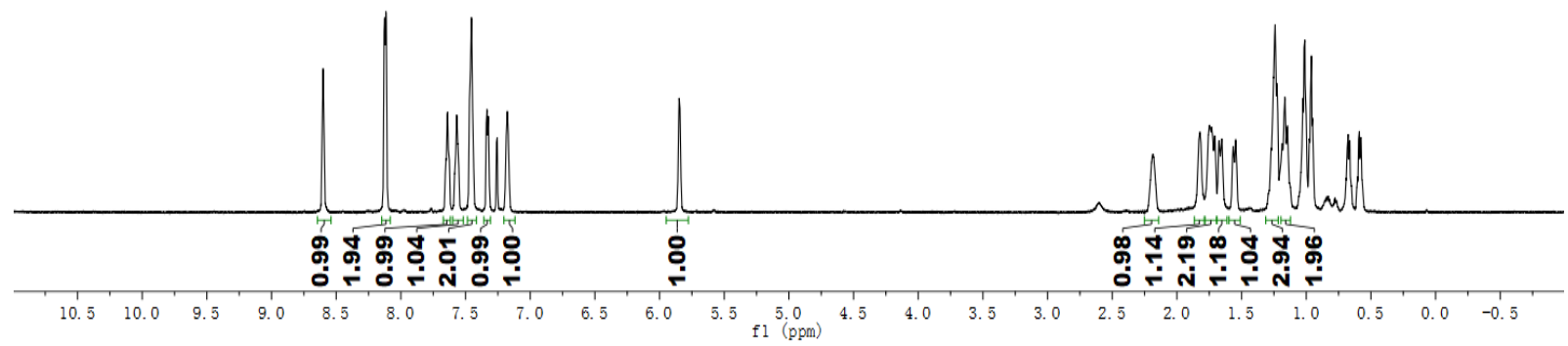

${ }^{13} \mathrm{C}$ NMR (151 MHz, $\mathrm{CDCl}_{3}$ )

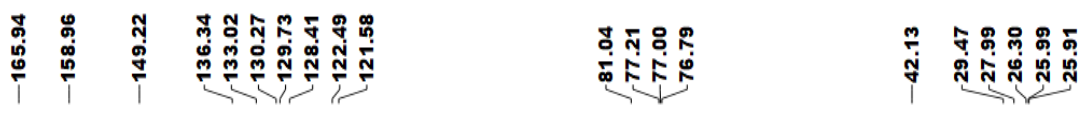
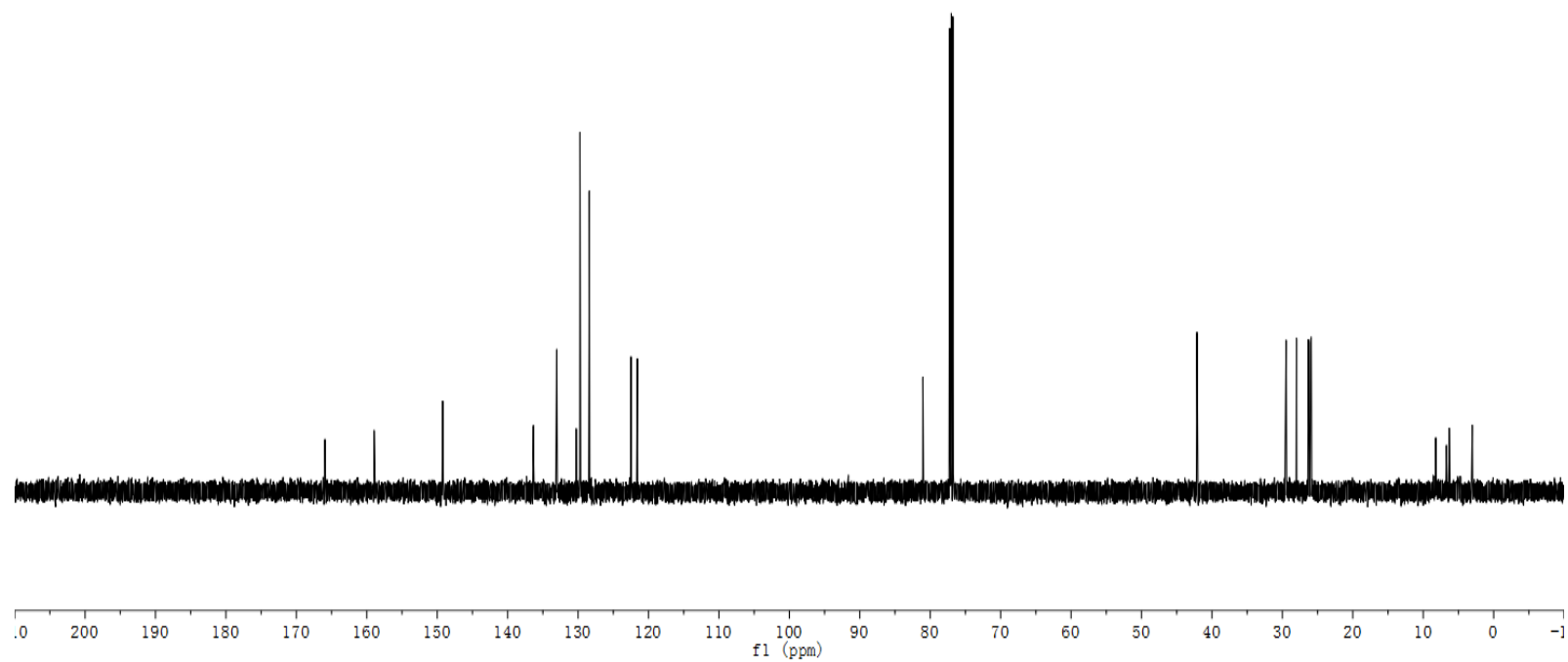

S 152 
${ }^{1} \mathrm{H}$ NMR (599 $\mathrm{MHz}, \mathrm{CDCl}_{3}$ )

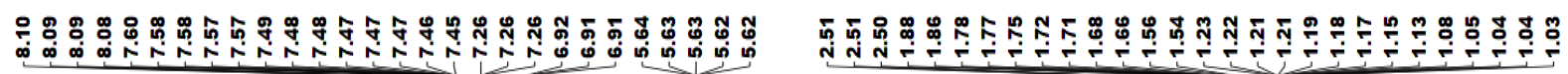<smiles>Cc1cc(C(=O)C2CCCCC2)cc(C)n1</smiles>

$49,70 \%$

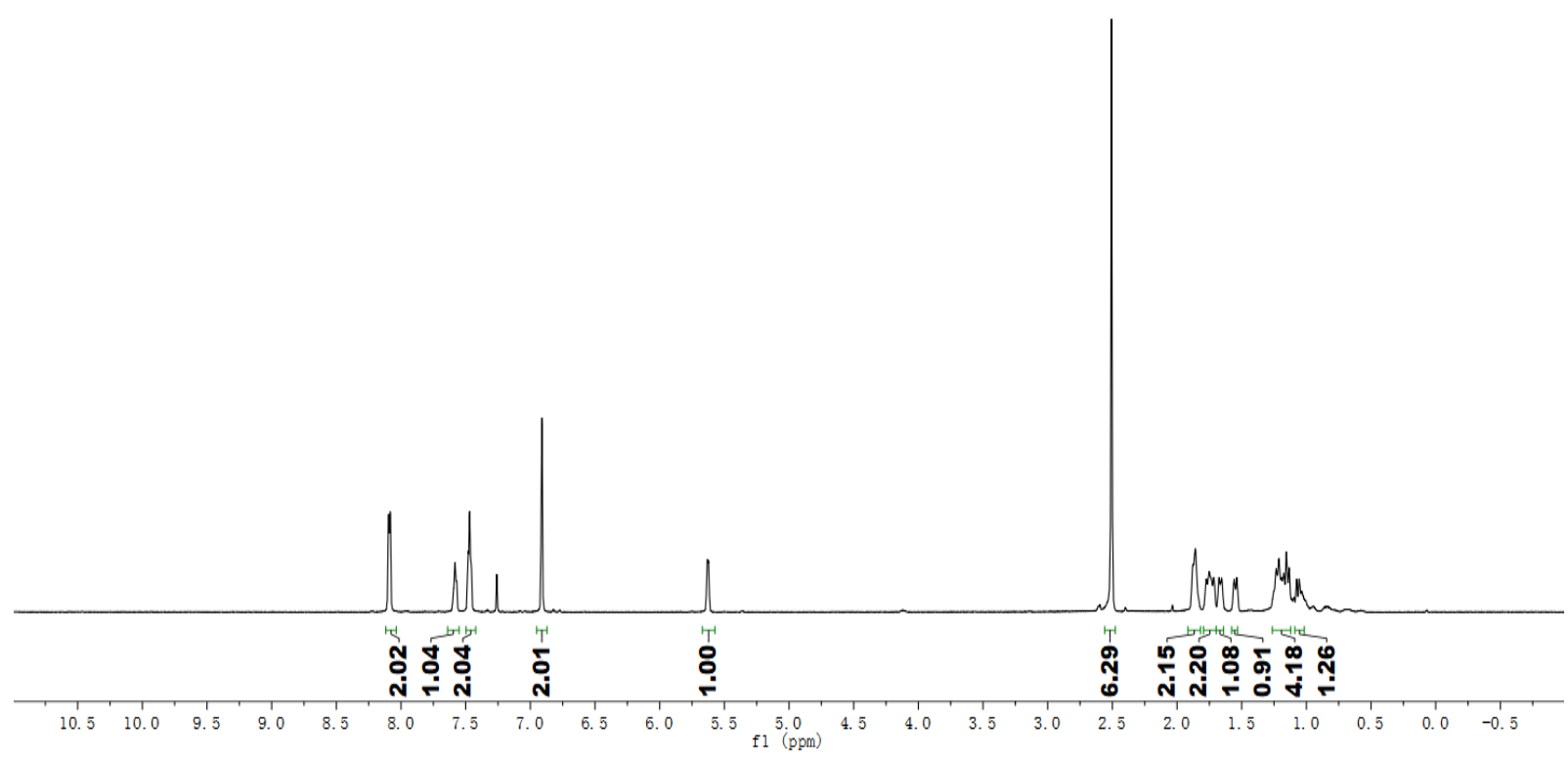

${ }^{13} \mathrm{C}$ NMR (151 $\left.\mathrm{MHz}, \mathrm{CDCl}_{3}\right)$

疍

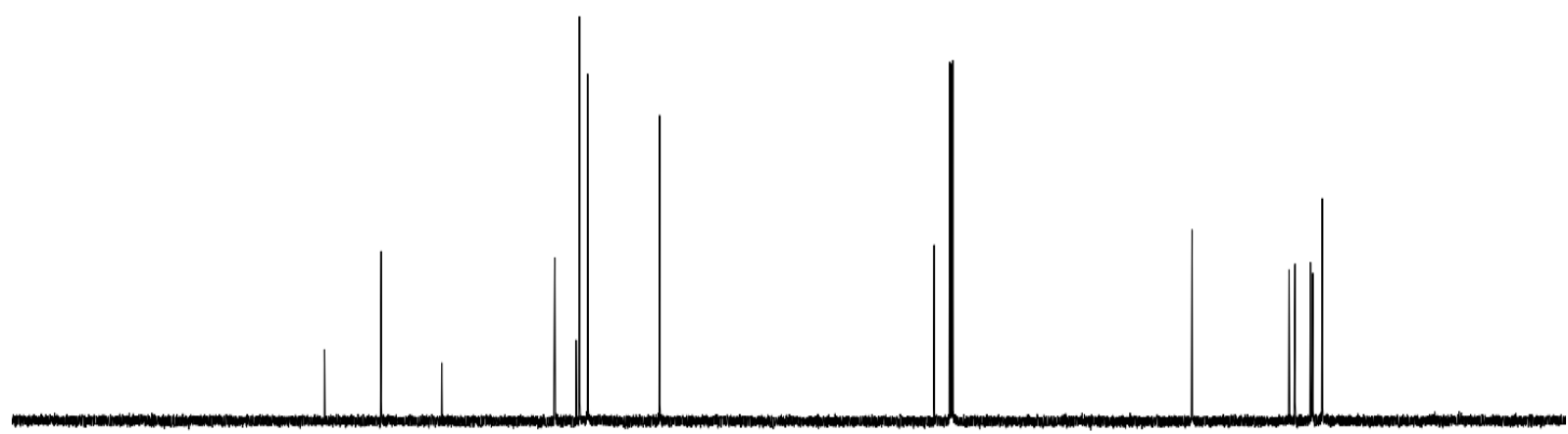


${ }^{1} \mathrm{H}$ NMR (599 $\mathrm{MHz}, \mathrm{CDCl}_{3}$ )<smiles>CCCCOC(=O)C1CCCCC1</smiles>

$50,68 \%$

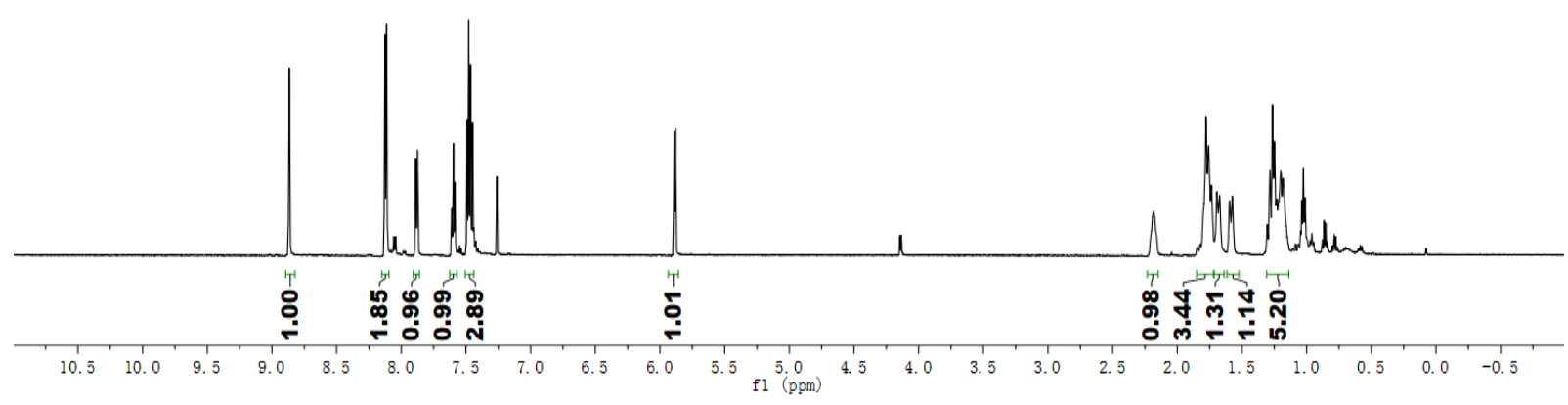

${ }^{13} \mathrm{C}$ NMR (151 MHz, $\left.\mathrm{CDCl}_{3}\right)$

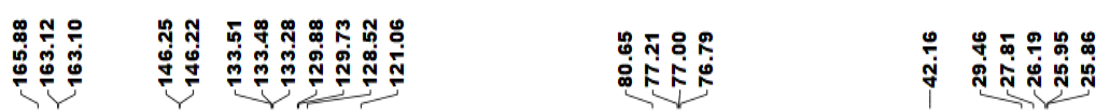

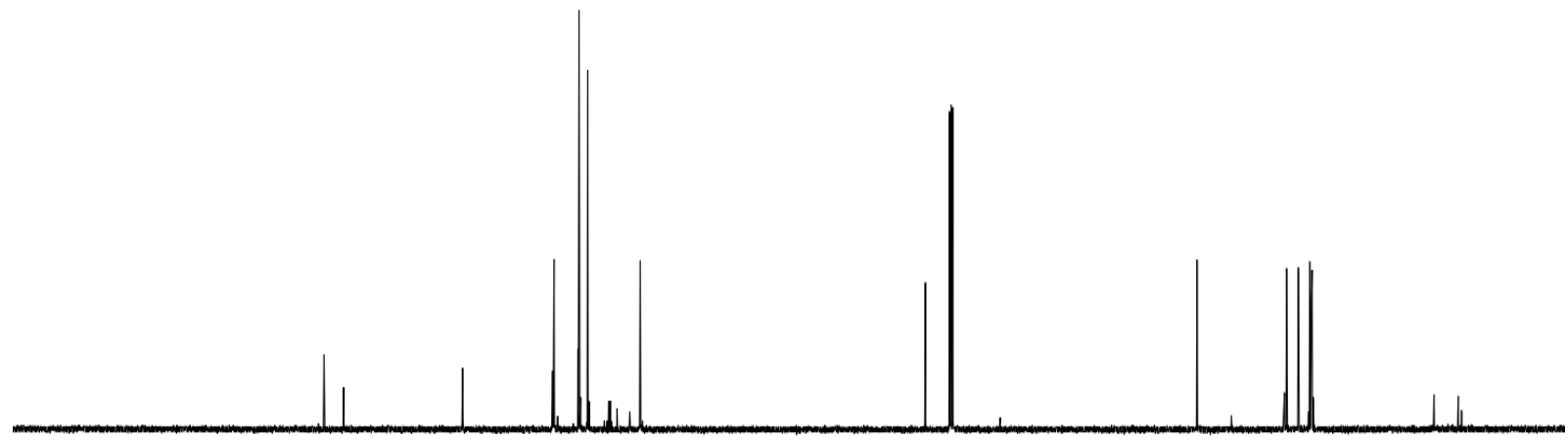


${ }^{19}$ F NMR (564 MHz, $\mathrm{CDCl}_{3}$ )

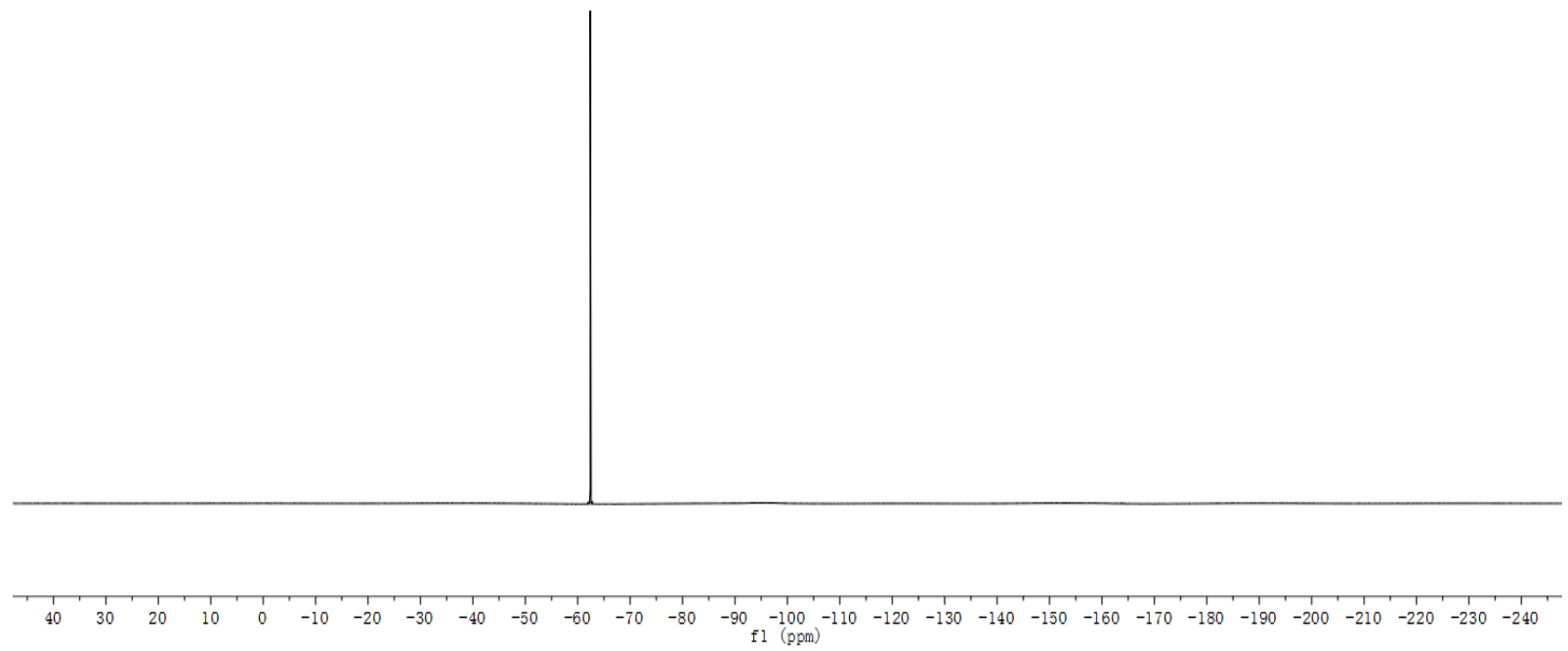


${ }^{1} \mathrm{H}$ NMR (599 $\mathrm{MHz}^{\mathrm{CDCl}_{3}}$ )

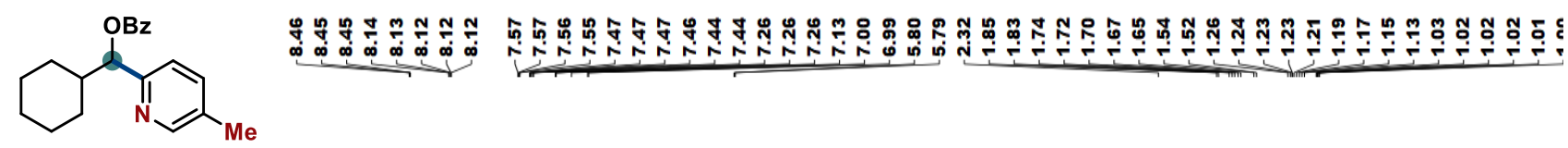

$51,73 \%$

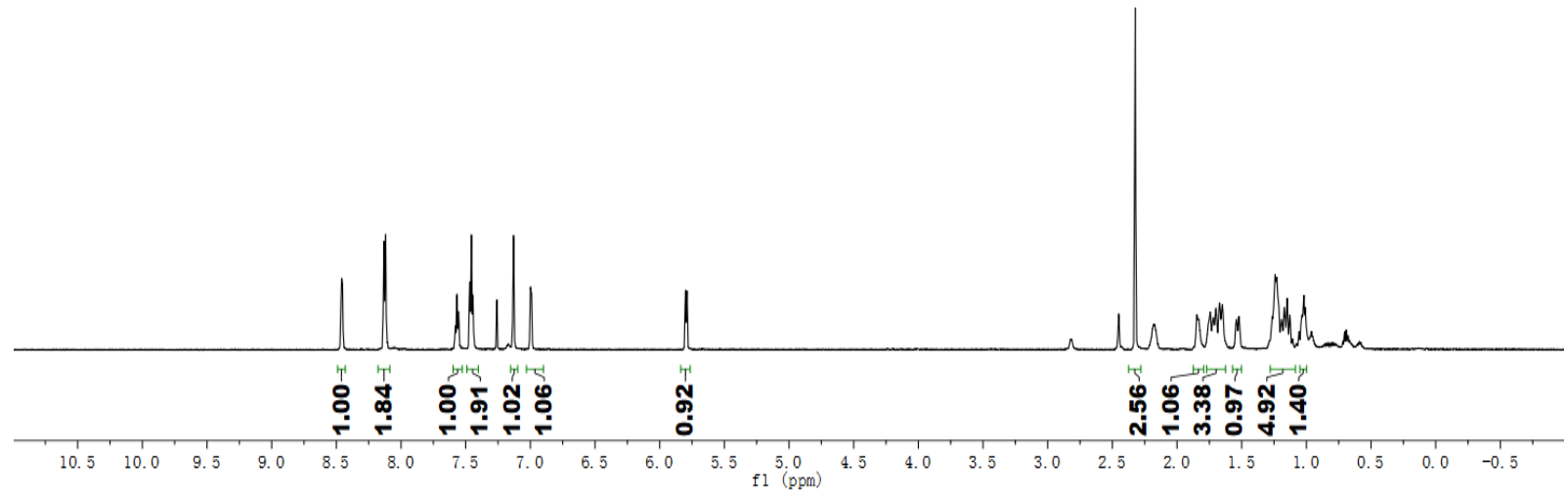

${ }^{13} \mathrm{C}$ NMR (151 MHz, $\left.\mathrm{CDCl}_{3}\right)$

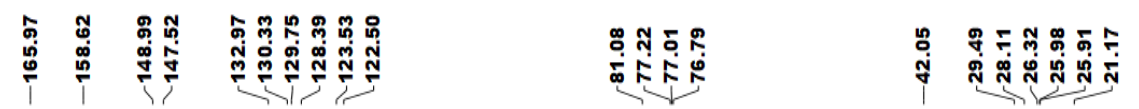

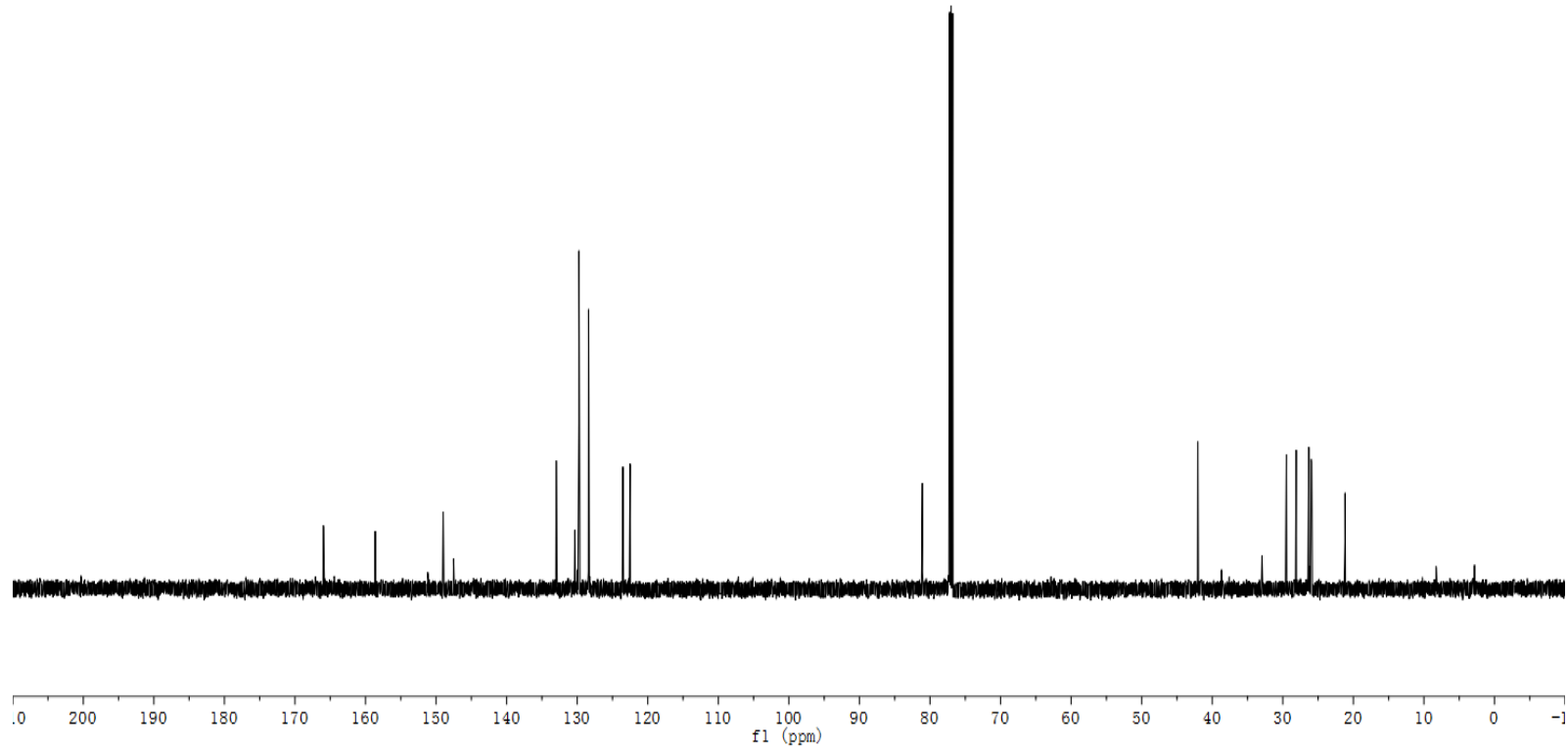


${ }^{1} \mathrm{H}$ NMR (599 $\mathrm{MHz}^{\mathrm{CDCl}_{3}}$ )

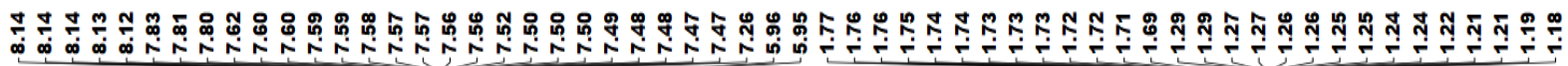<smiles>O=C(c1cccc(C(F)(F)F)n1)C1CCCCC1</smiles>

$52,89 \%$

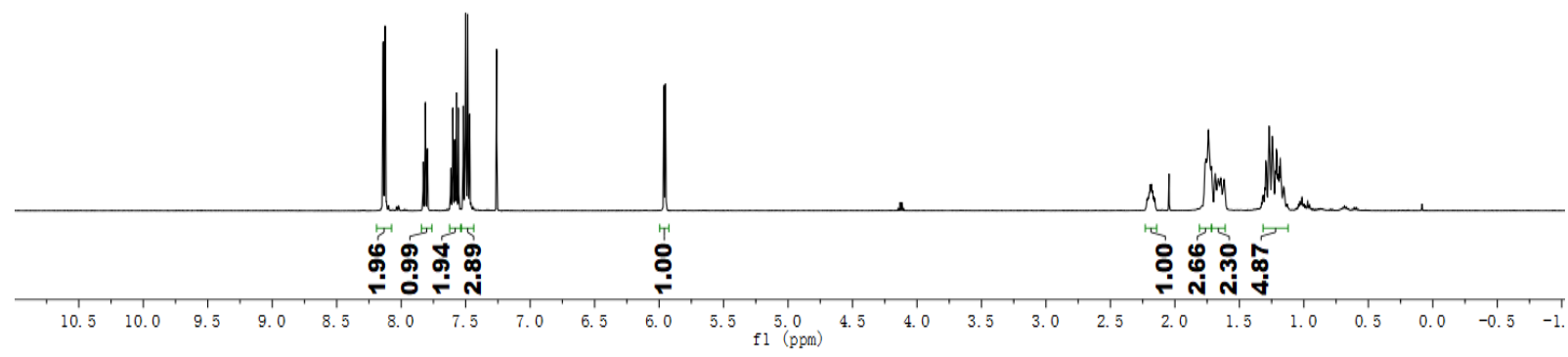

${ }^{13} \mathrm{C}$ NMR (151 MHz, $\left.\mathrm{CDCl}_{3}\right)$

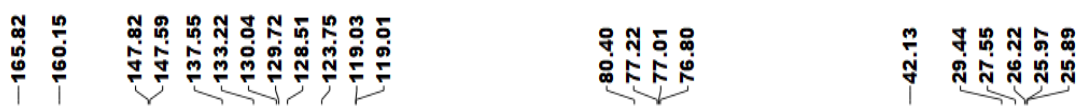

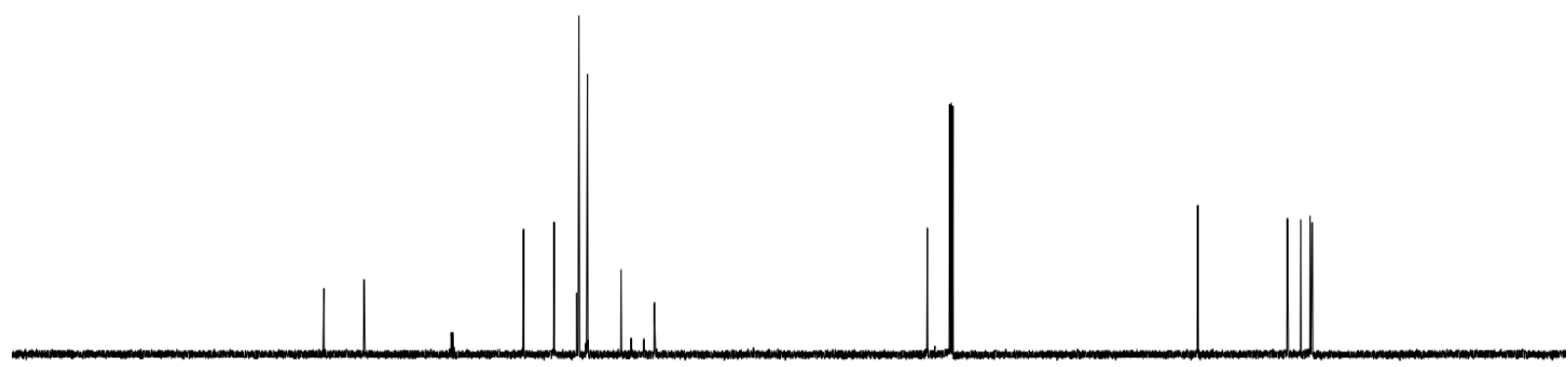

$\begin{array}{lllllllllllllllllllllllll}1 & 200 & 190 & 180 & 170 & 160 & 150 & 140 & 130 & 120 & 110 & 100 & 90 & 80 & 70 & 60 & 50 & 40 & 30 & 20 & 10 & 0 & -1\end{array}$ 
${ }^{19}$ F NMR (564 MHz, CDCl 3 )

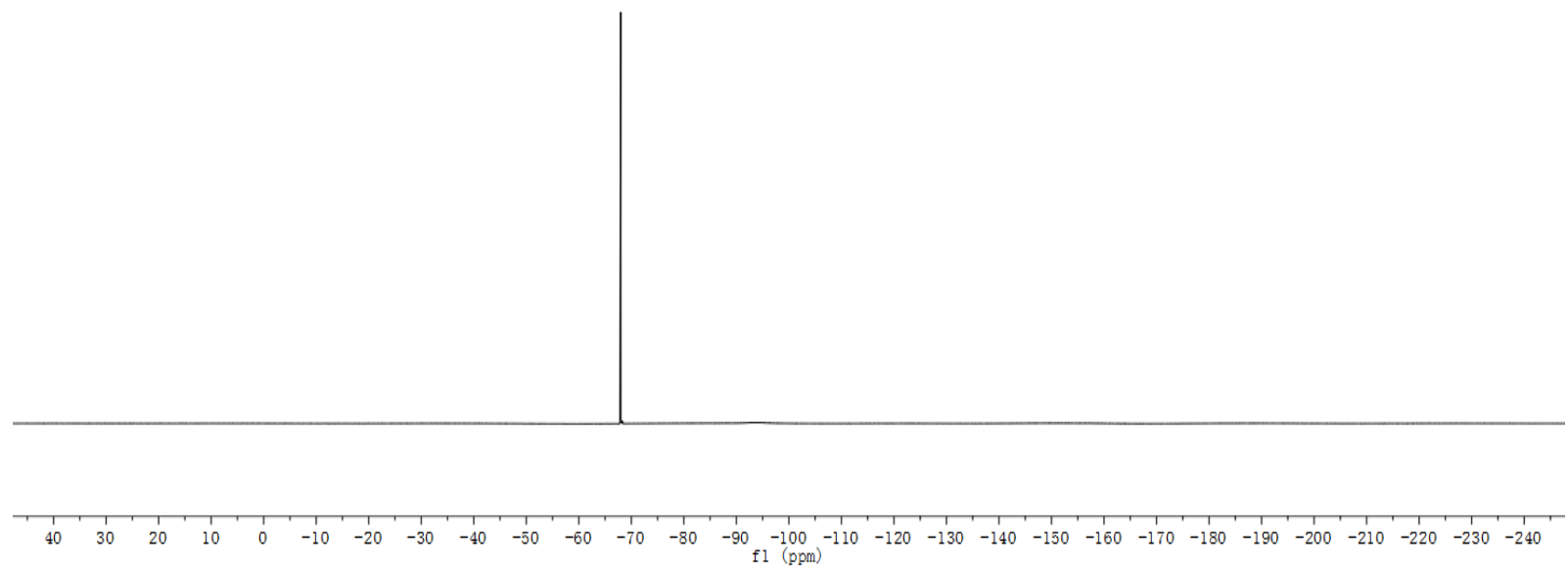


${ }^{1} \mathrm{H}$ NMR (599 $\left.\mathrm{MHz}, \mathrm{CDCl}_{3}\right)$

뭉

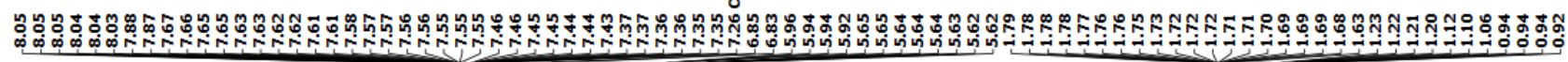

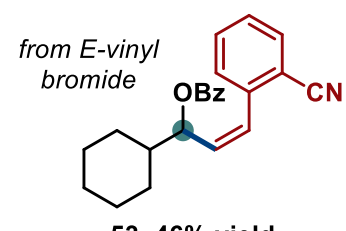

$53,46 \%$ yield

$>95: 5$ Z:E

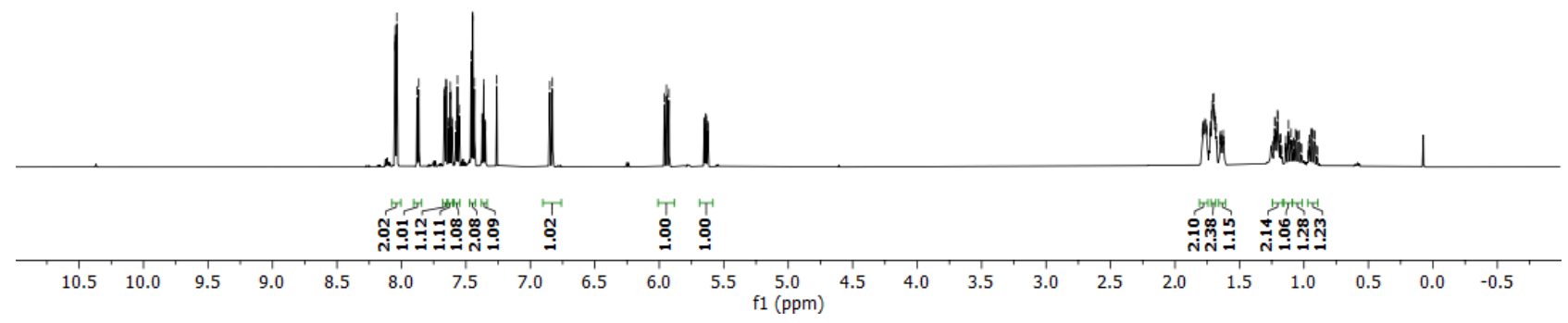

${ }^{13} \mathrm{C}$ NMR (151 MHz, $\left.\mathrm{CDCl}_{3}\right)$
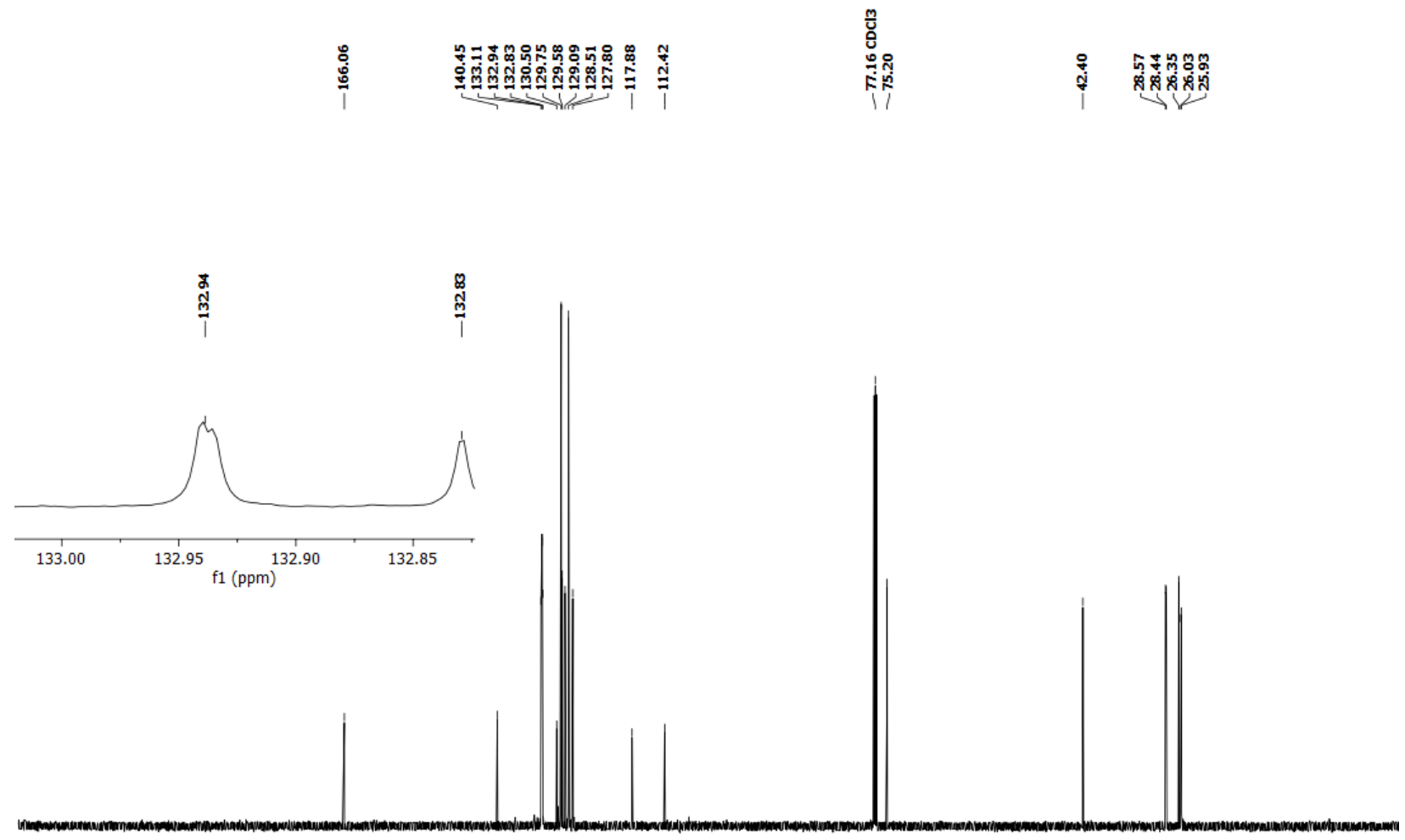

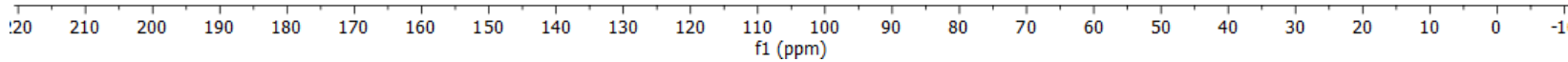


${ }^{1} \mathrm{H}-\mathrm{NOESY}$ NMR (599 MHz, $\mathrm{CDCl}_{3}$ ) - selected resonances

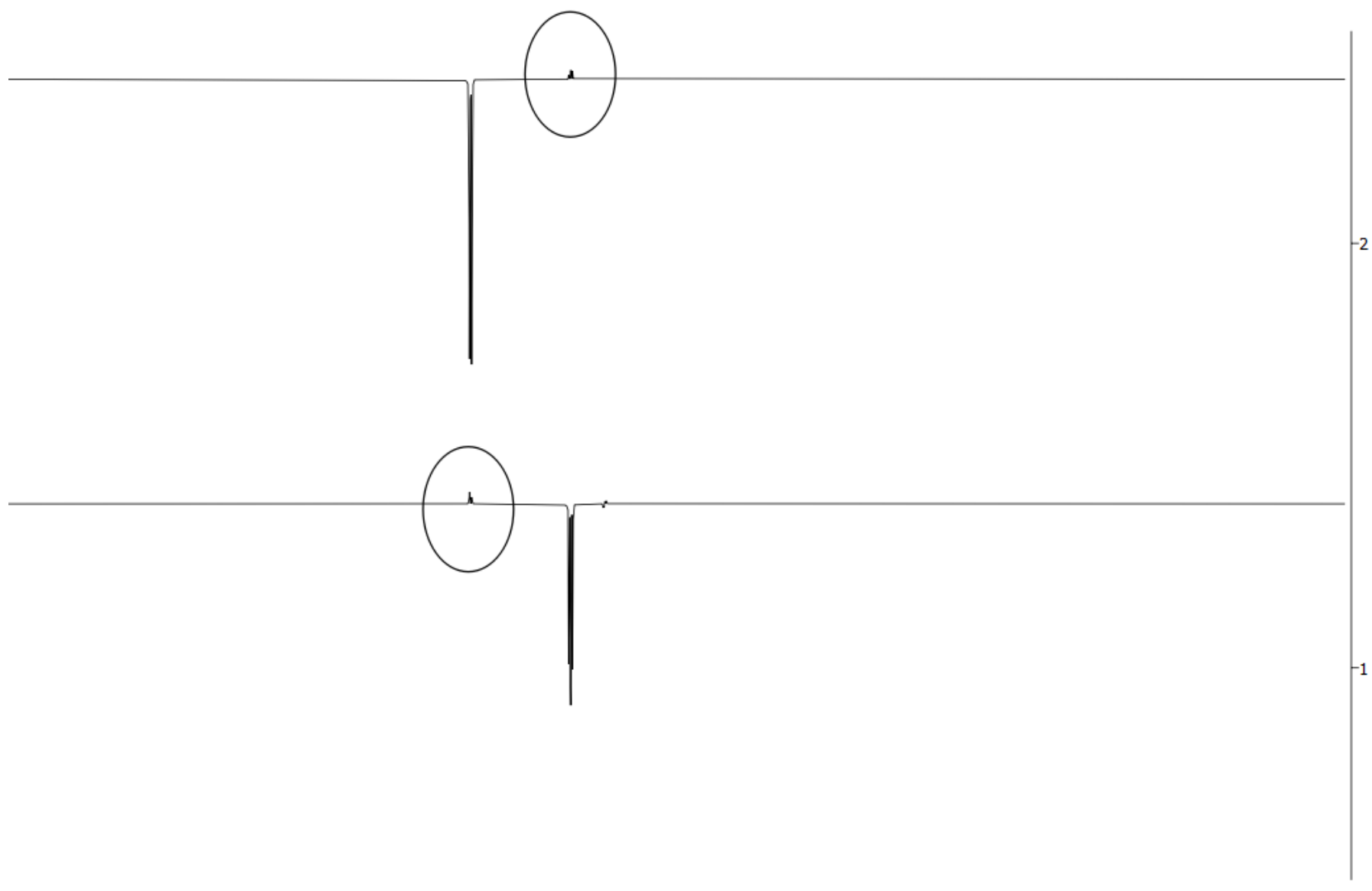

$\begin{array}{llllllllllllllllllllllllll}10.5 & 10.0 & 9.5 & 9.0 & 8.5 & 8.0 & 7.5 & 7.0 & 6.5 & 6.0 & 5.5 & \begin{array}{c}1.0 \\ \mathrm{f} 1(\mathrm{ppm})\end{array} & 4.5 & 4.0 & 3.5 & 3.0 & 2.5 & 2.0 & 1.5 & 1.0 & 0.5 & 0.0 & -0.5\end{array}$ 
${ }^{1} \mathrm{H}$ NMR (400 MHz, $\mathrm{CDCl}_{3}$ )

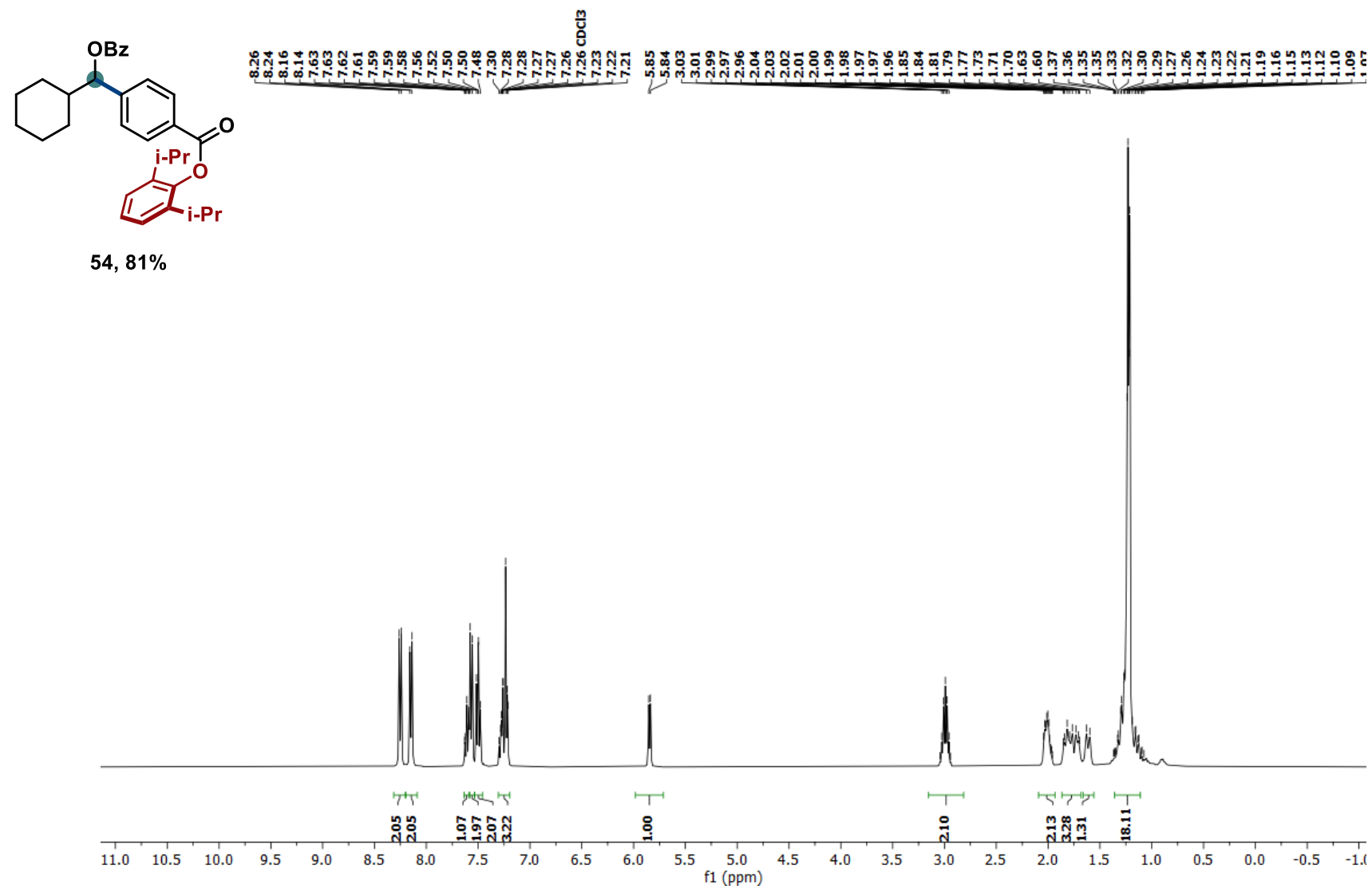

${ }^{13} \mathrm{C}$ NMR (101 MHz, $\mathrm{CDCl}_{3}$ )

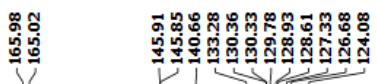

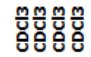

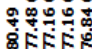

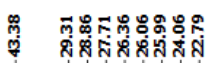

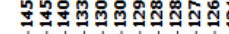

等

-

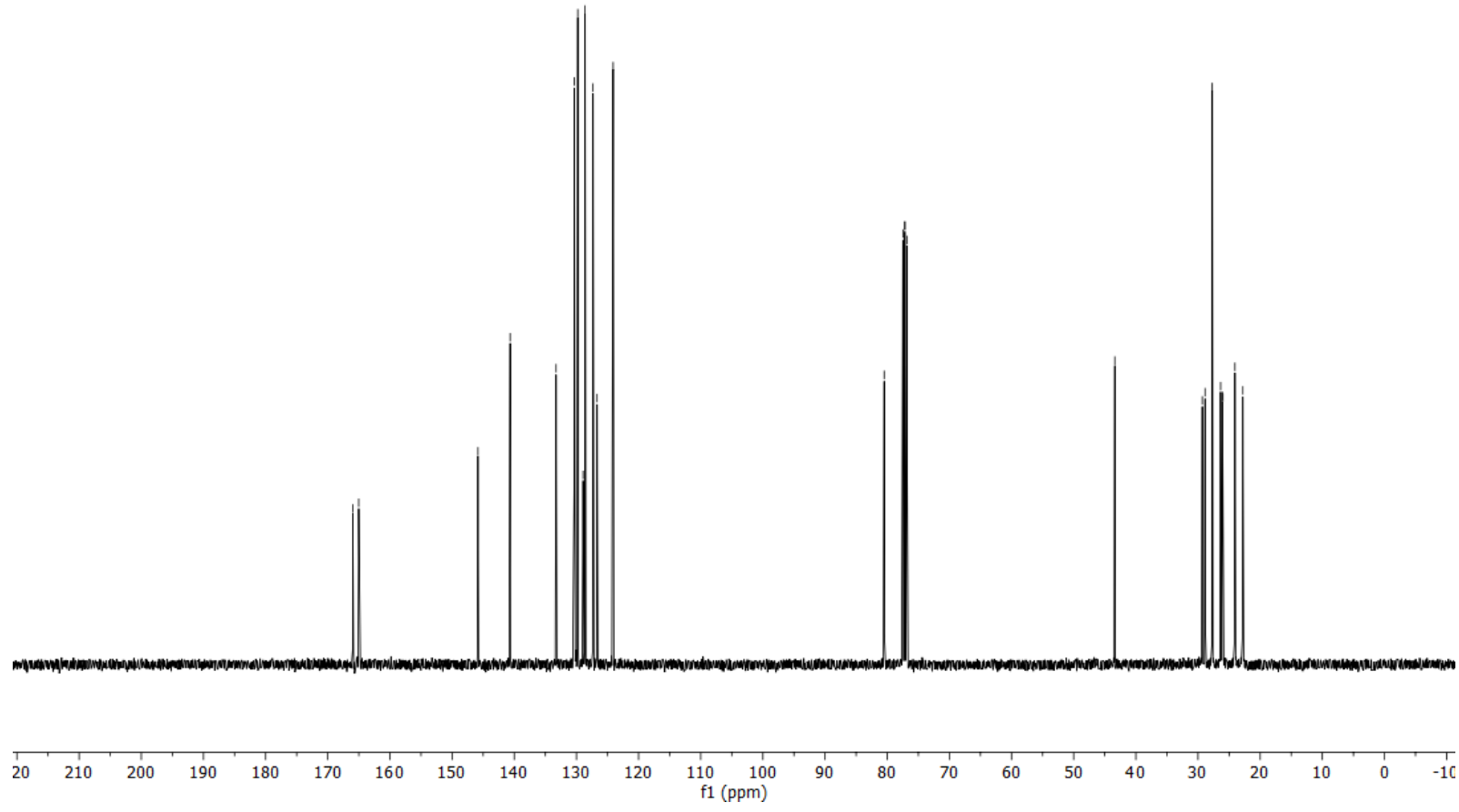


${ }^{1} \mathrm{H}$ NMR (400 MHz, $\left.\mathrm{CDCl}_{3}\right)$

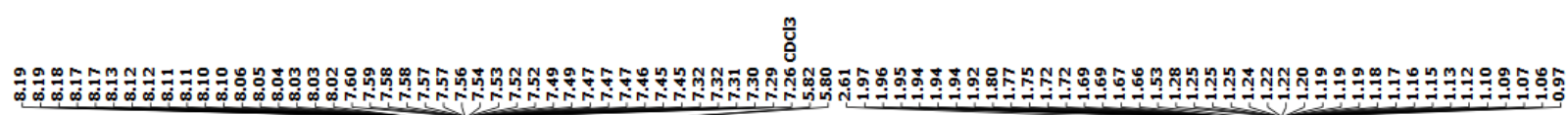<smiles>CC(=O)c1ccc(OC(=O)c2ccc([C@@H](O)C3CCCCC3)cc2)cc1</smiles>
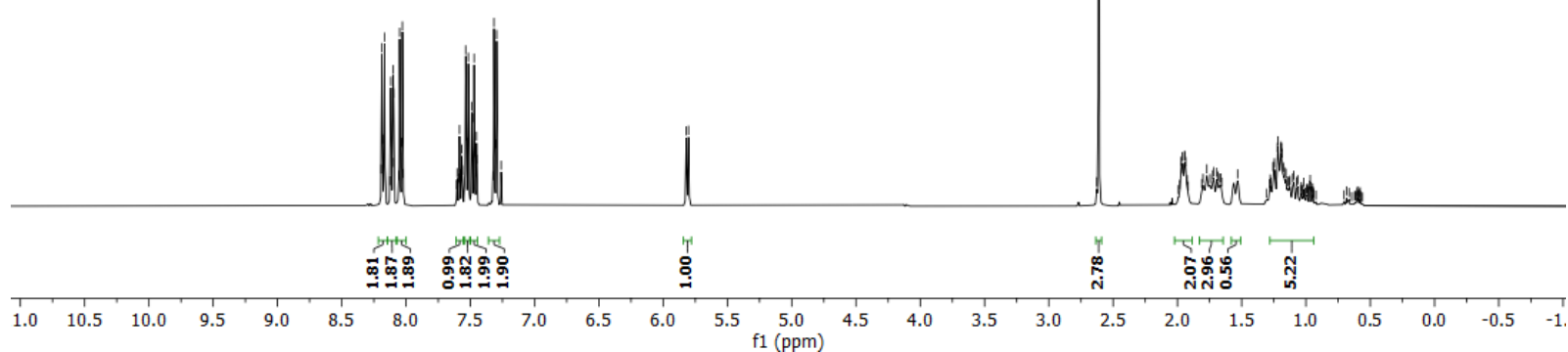

${ }^{13} \mathrm{C}$ NMR (101 MHz, $\left.\mathrm{CDCl}_{3}\right)$
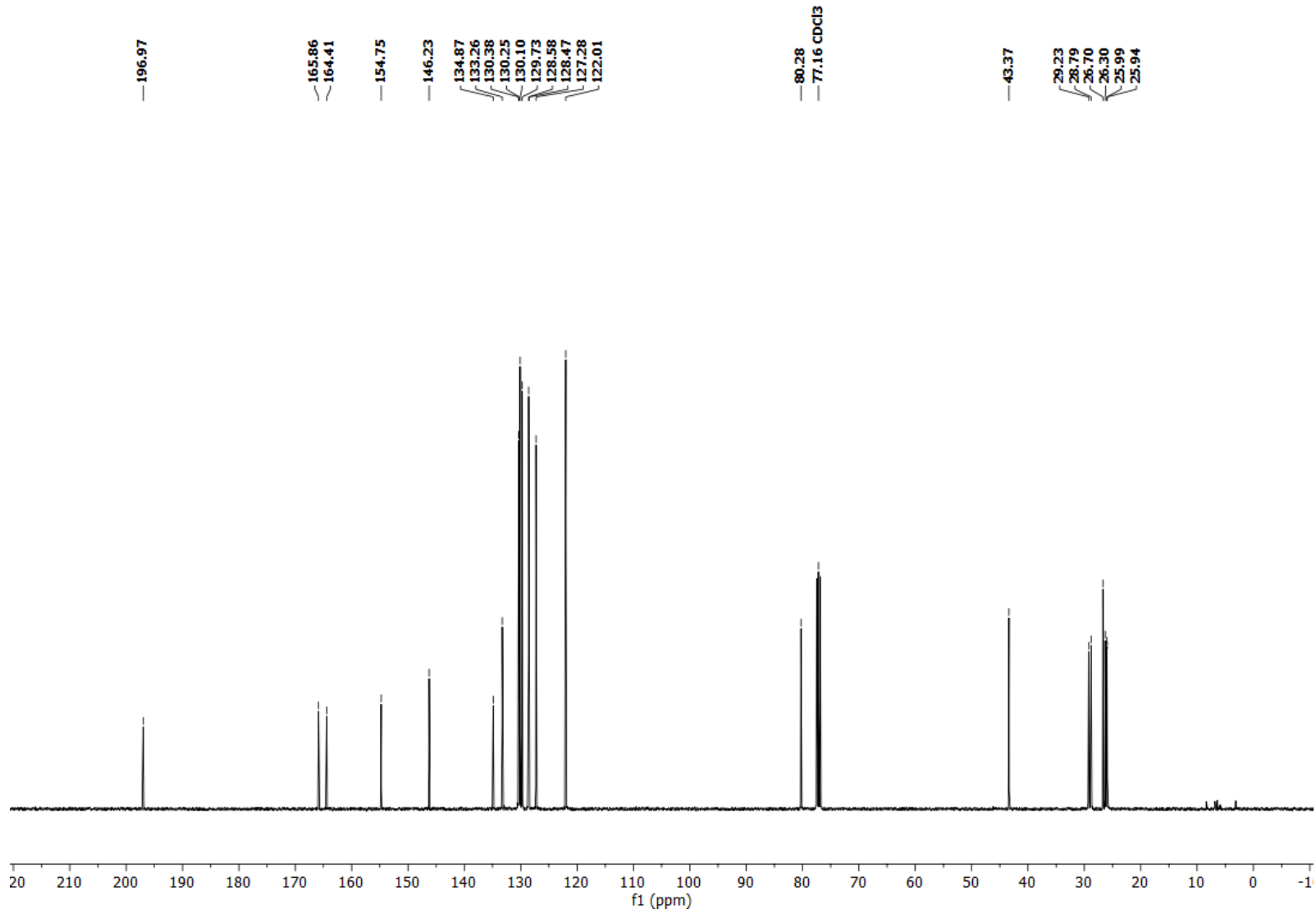
${ }^{1} \mathrm{H}$ NMR (400 $\left.\mathrm{MHz}, \mathrm{CDCl}_{3}\right)$

高

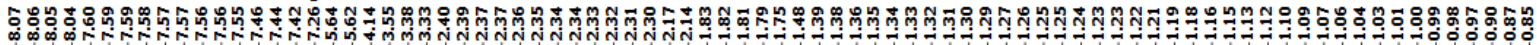<smiles>CCCCC(=O)c1nc2c(c(=O)n(C)c(=O)n2C)n1C</smiles>

$56,51 \%$ yield from caffeine

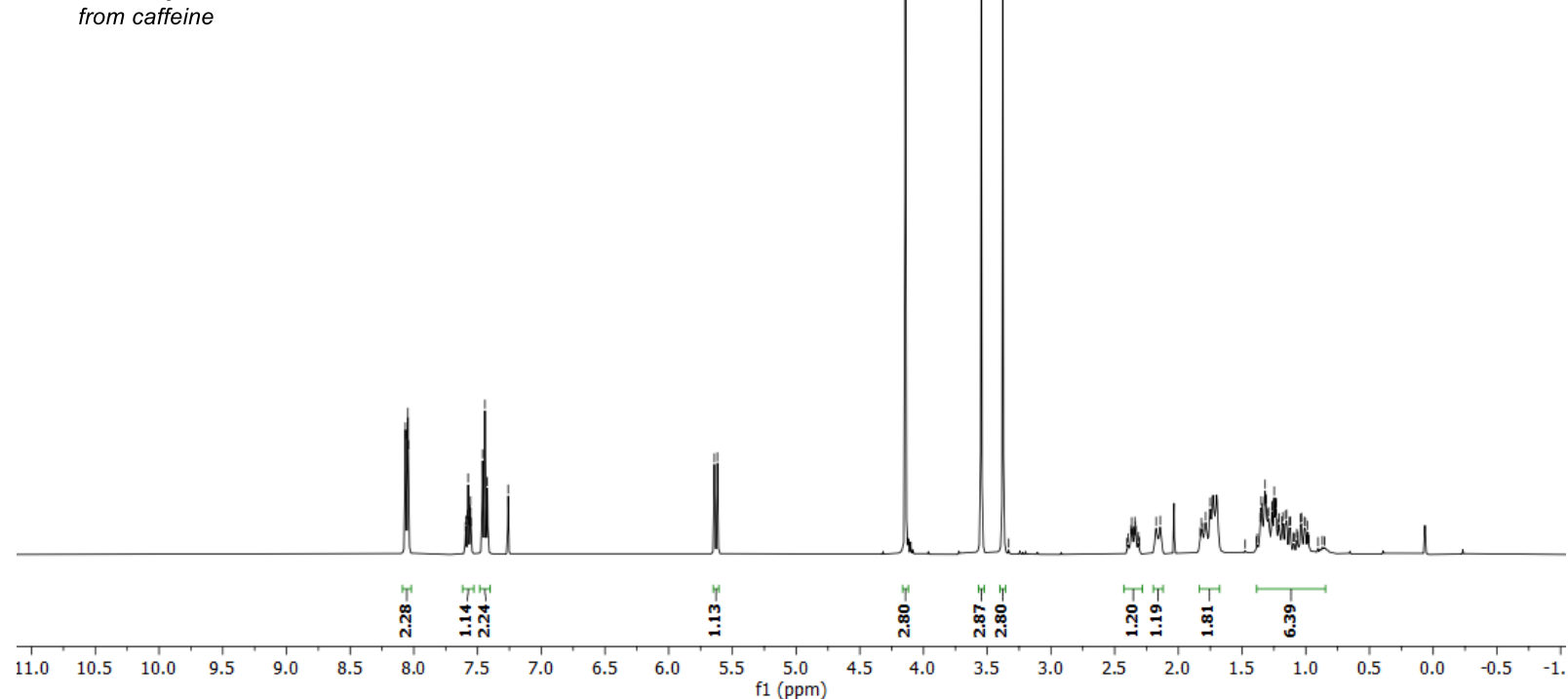

${ }^{13} \mathrm{C}$ NMR (101 MHz, $\left.\mathrm{CDCl}_{3}\right)$

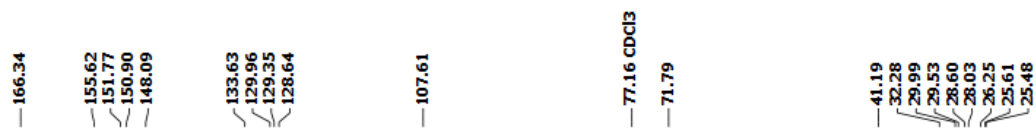

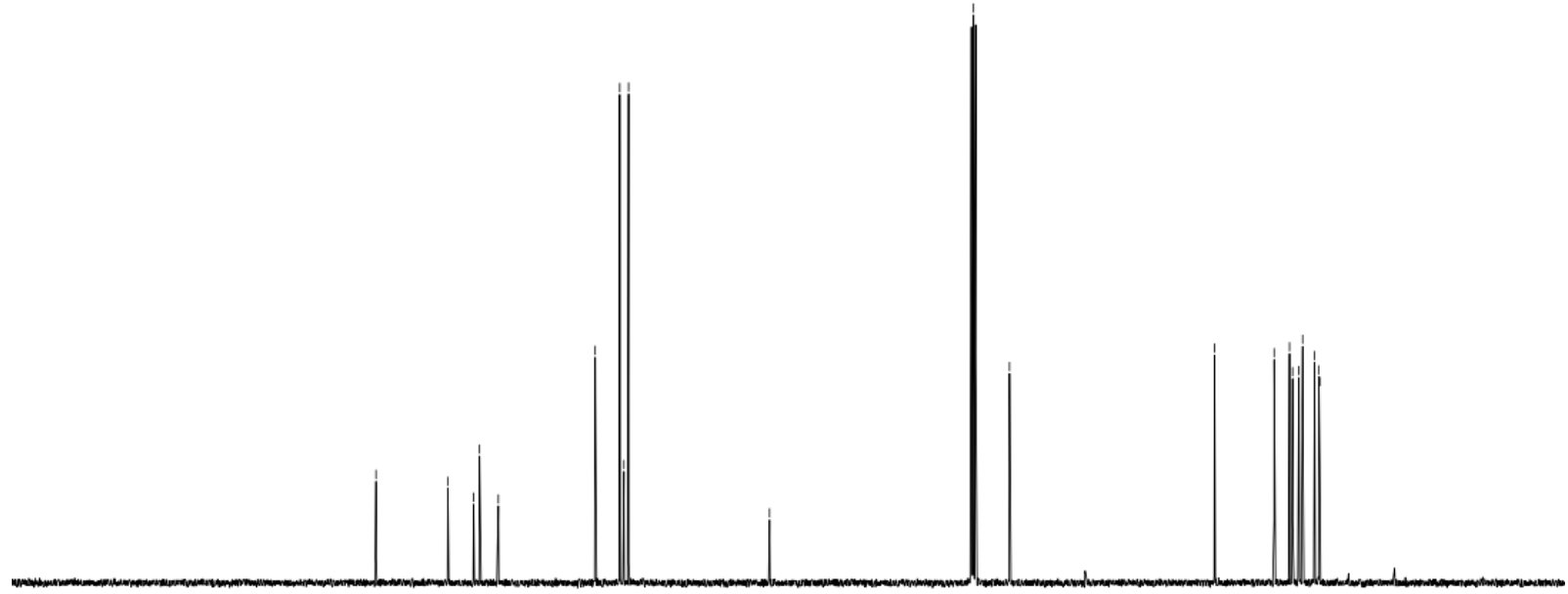

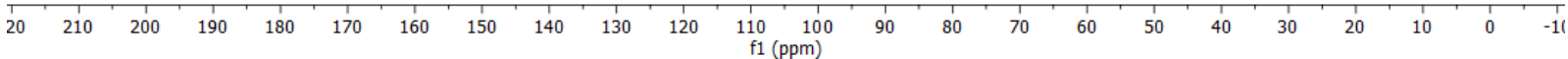


${ }^{1} \mathrm{H}$ NMR (400 $\mathrm{MHz}, \mathrm{CDCl}_{3}$ )
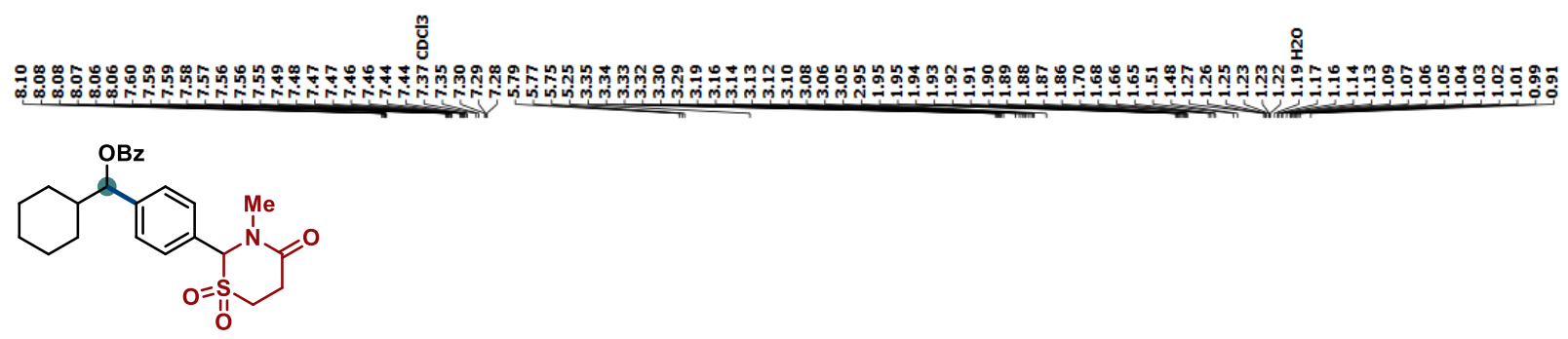

$57,42 \%$ yield

$1: 1 \mathrm{dr}$

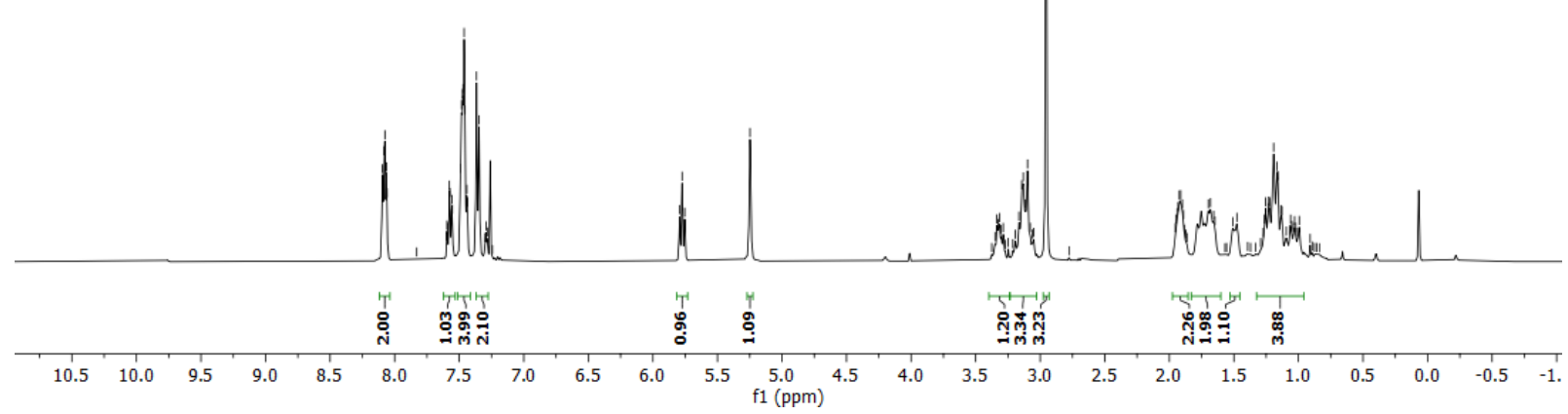

${ }^{13} \mathrm{C}$ NMR (101 MHz, $\mathrm{CDCl}_{3}$ )

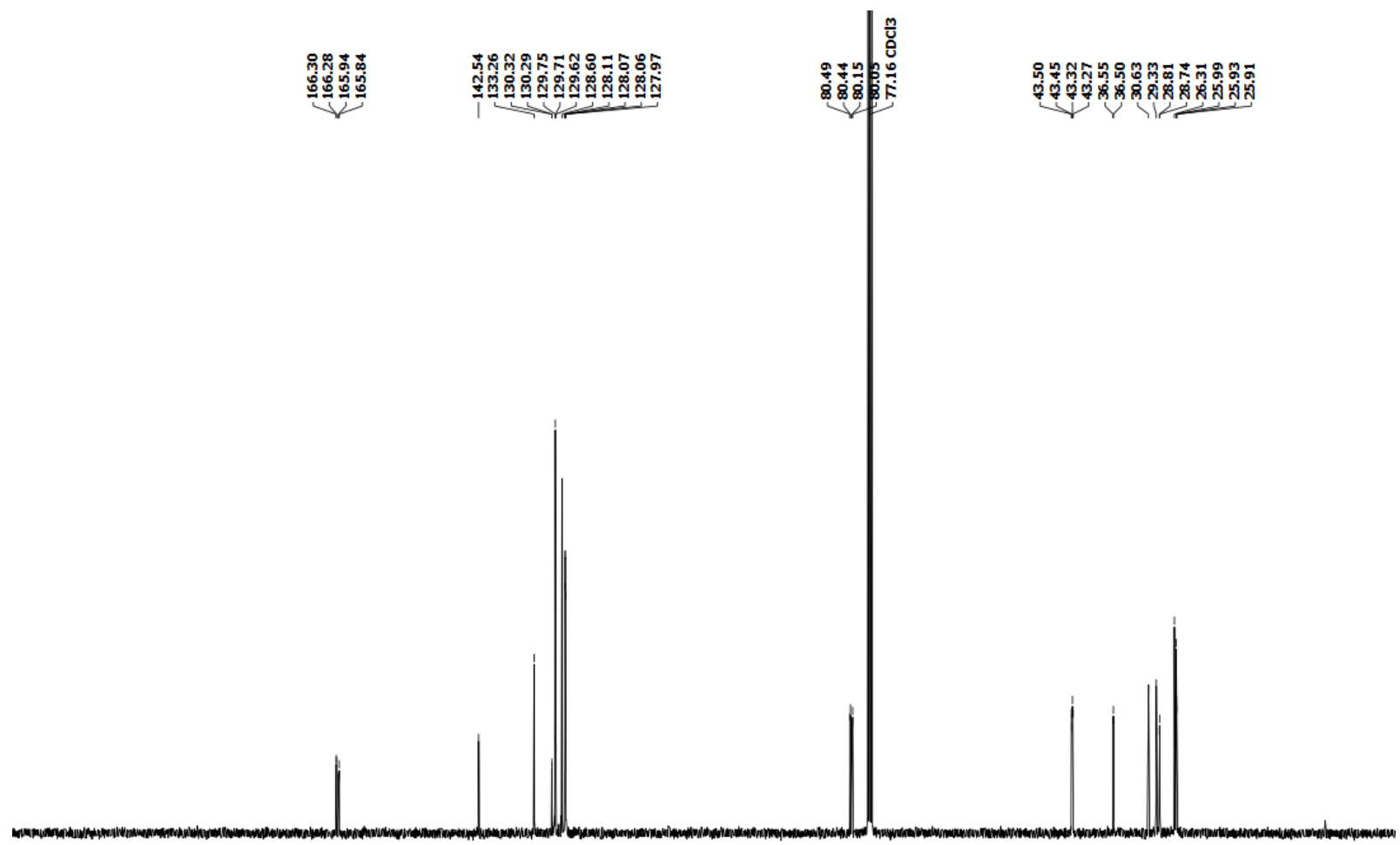


${ }^{1} \mathrm{H}$ NMR (400 $\left.\mathrm{MHz}, \mathrm{CDCl}_{3}\right)$

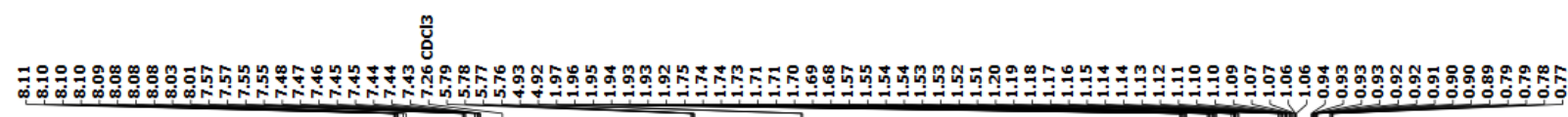

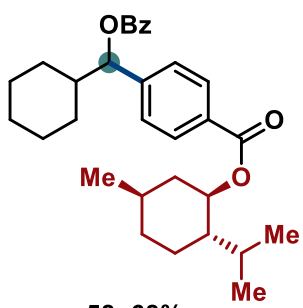

$58,68 \%$

$1: 1 \mathrm{dr}$

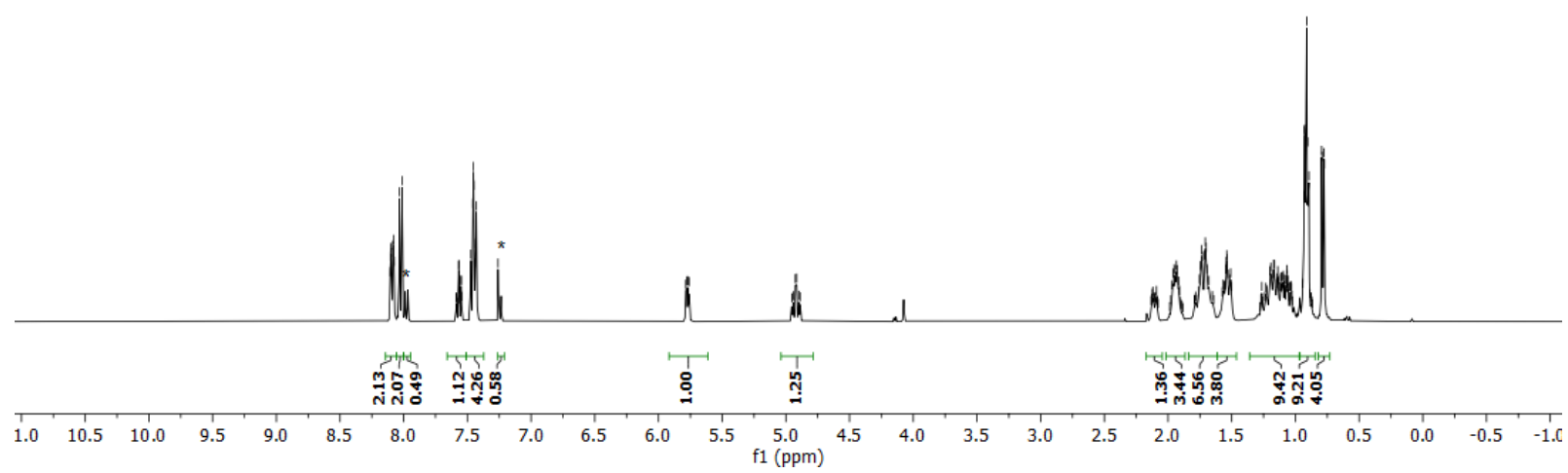

${ }^{13} \mathrm{C}$ NMR (101 MHz, $\left.\mathrm{CDCl}_{3}\right)$
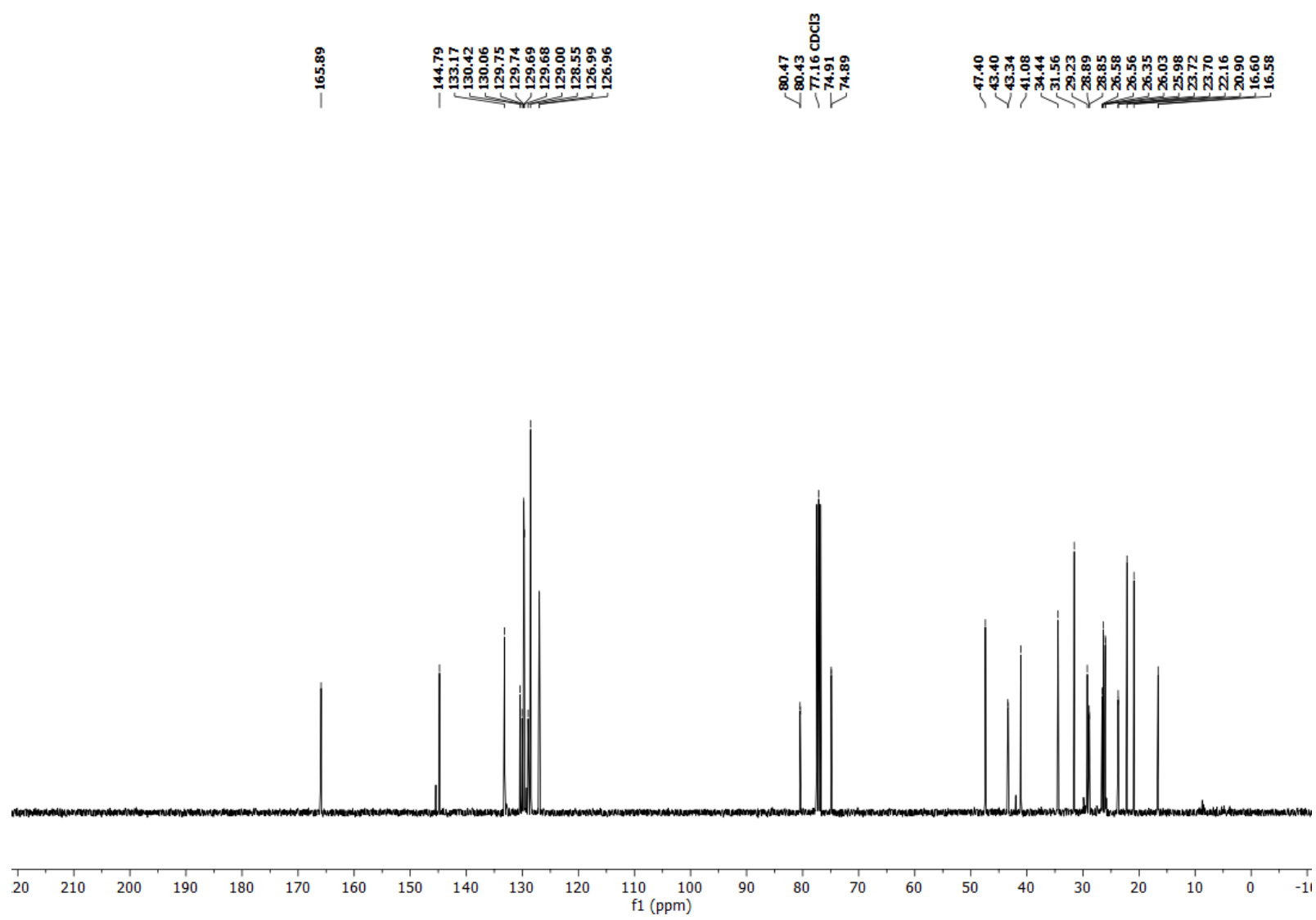
${ }^{1} \mathrm{H}$ NMR (400 $\mathrm{MHz}, \mathrm{CDCl}_{3}$ )

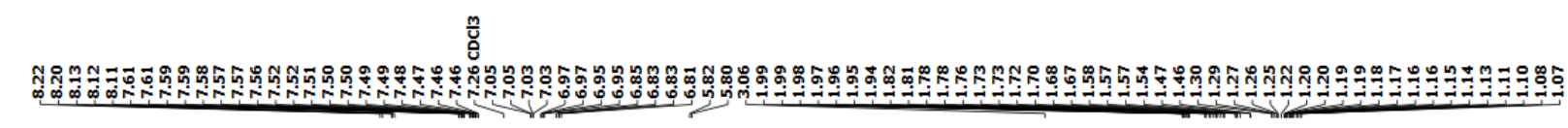<smiles>CC1(C)Cc2cccc(OC(=O)c3ccc(C(=O)C4CCCCC4)cc3)c2O1</smiles>

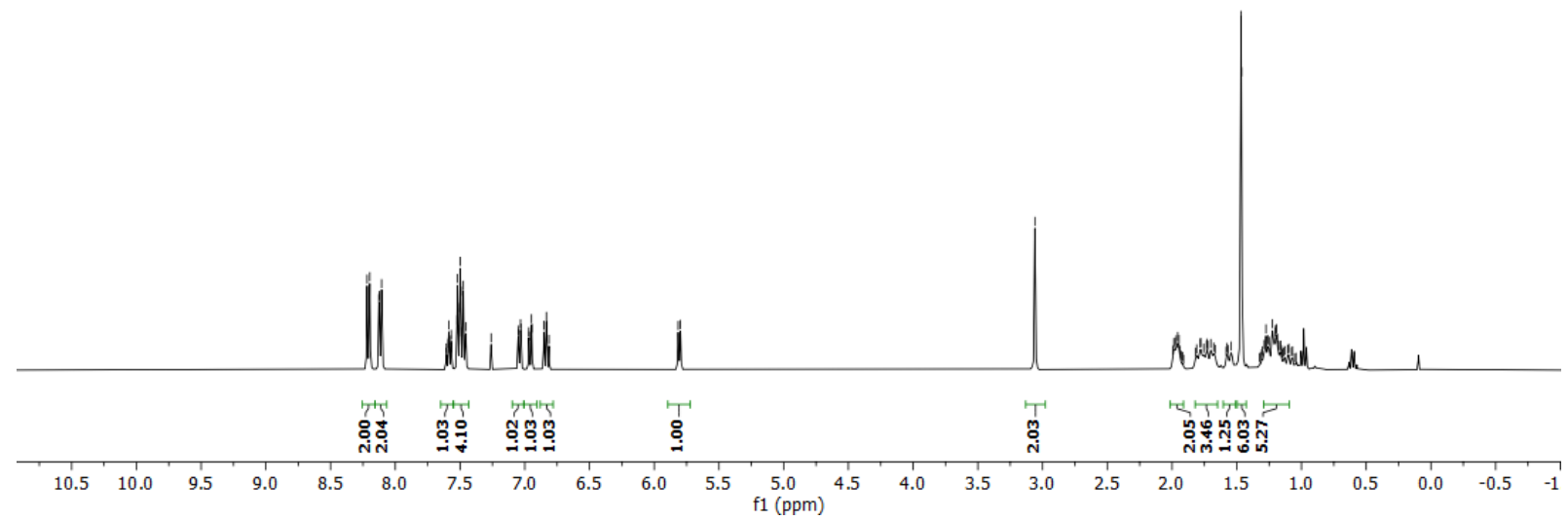

${ }^{13} \mathrm{C}$ NMR (101 MHz, $\mathrm{CDCl}_{3}$ )
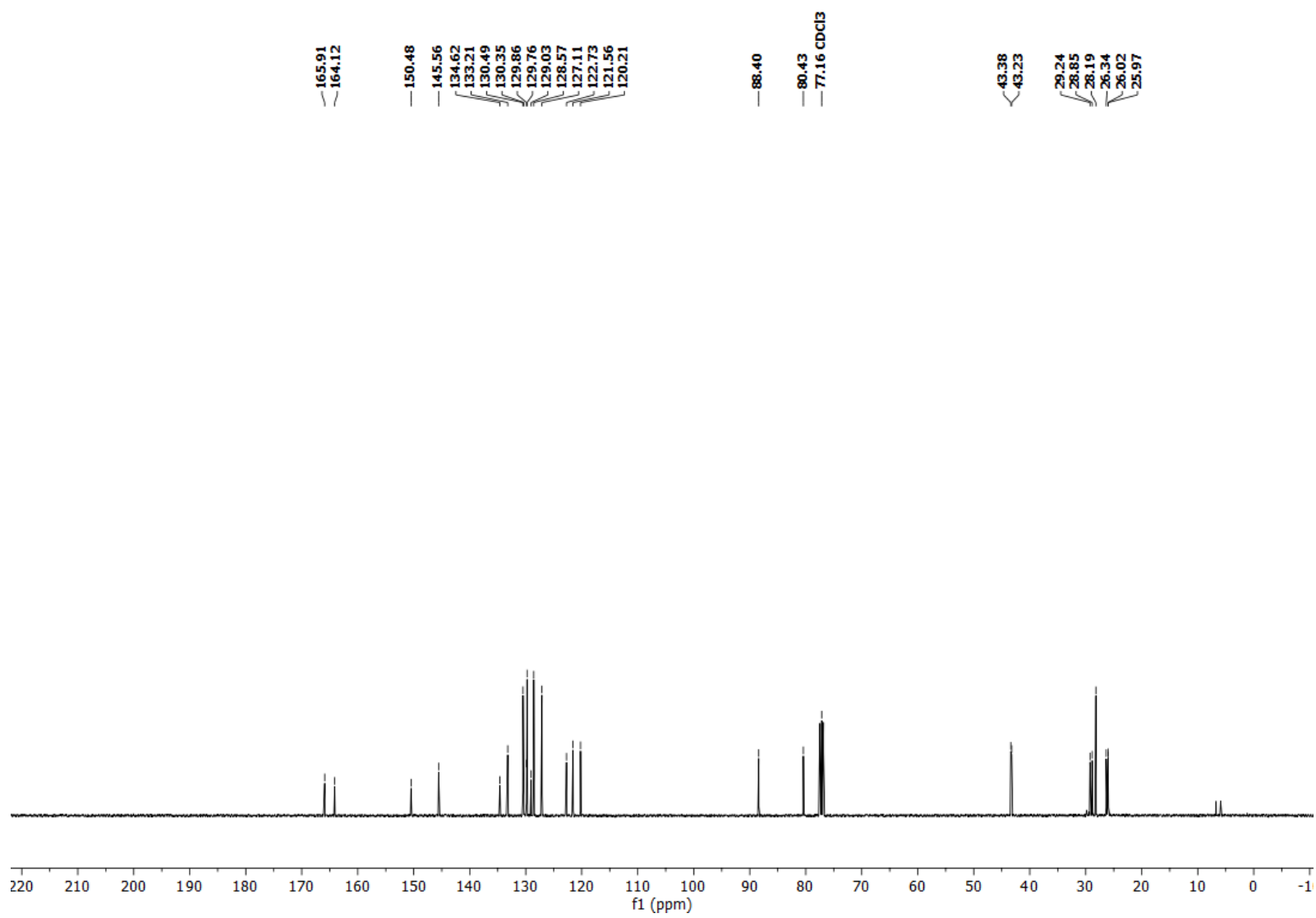
${ }^{1} \mathrm{H}$ NMR (400 MHz, $\left.\mathrm{CDCl}_{3}\right)$

$$
\text { 范 }
$$

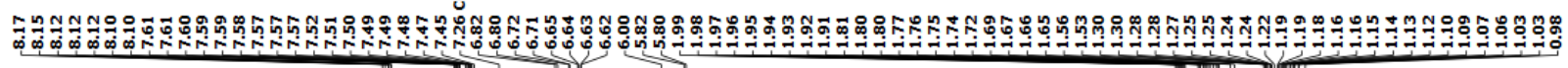<smiles>O=C(OC(=O)C1CCCCC1)c1ccc(C(=O)Oc2ccc3c(c2)OCO3)cc1</smiles>

$60,72 \%$

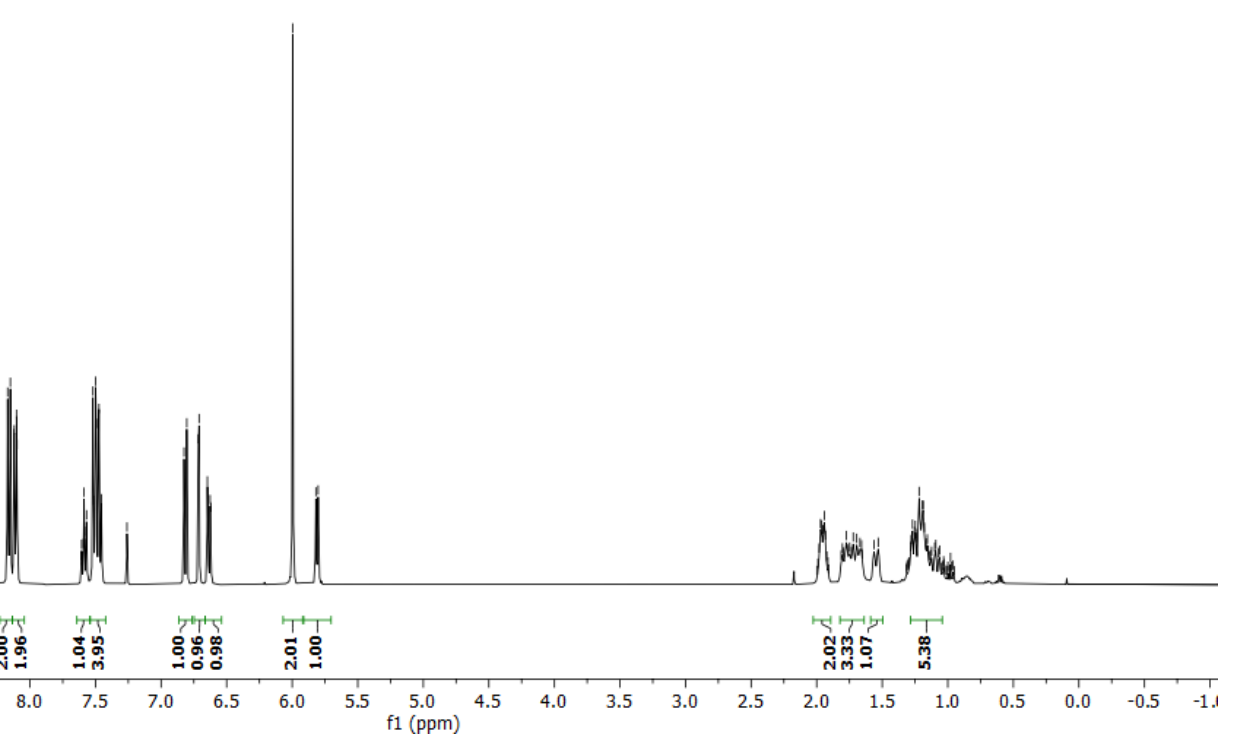

${ }^{13} \mathrm{C}$ NMR (101 $\left.\mathrm{MHz}, \mathrm{CDCl}_{3}\right)$

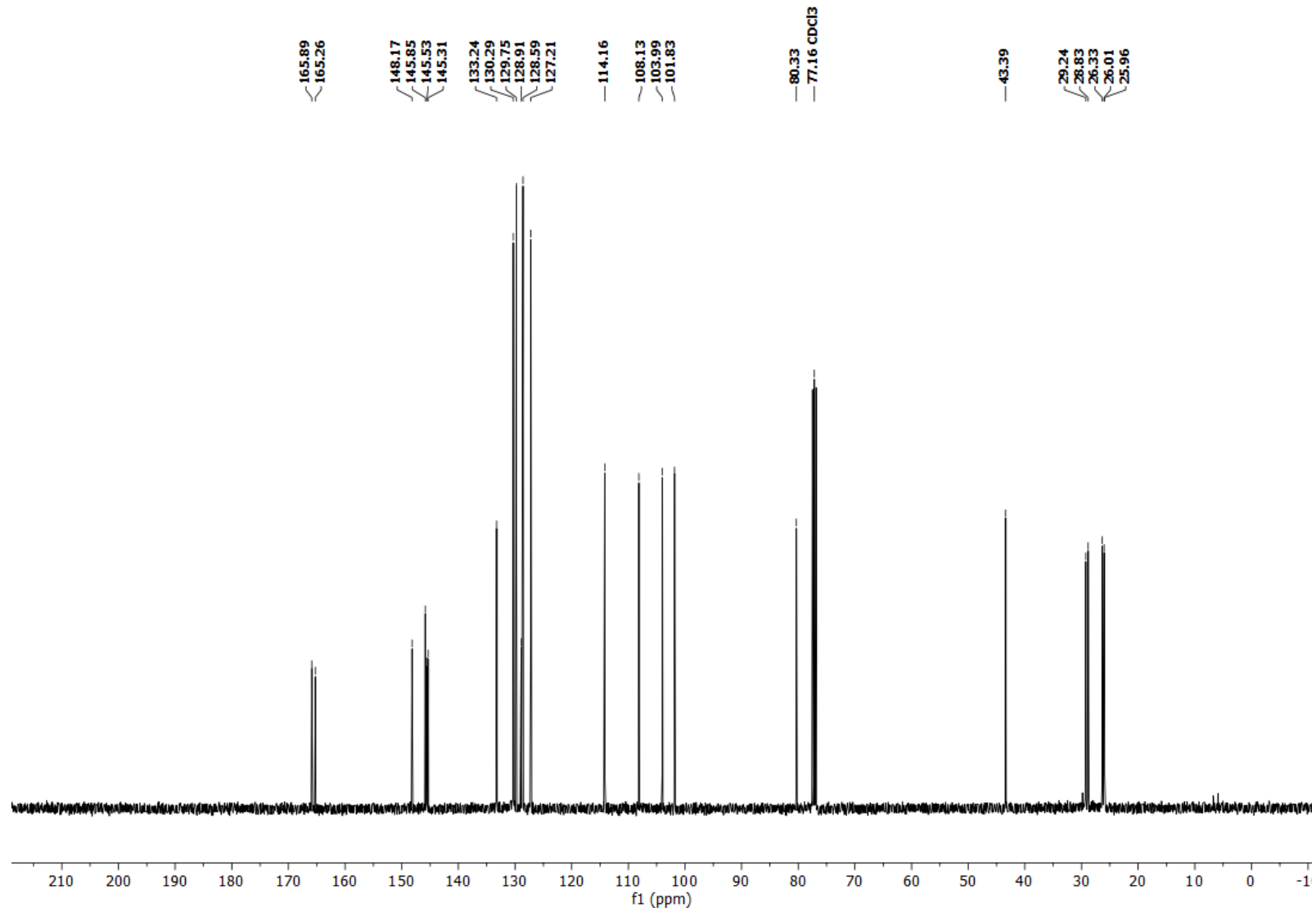


${ }^{1} \mathrm{H}$ NMR (400 MHz, $\mathrm{CDCl}_{3}$ )

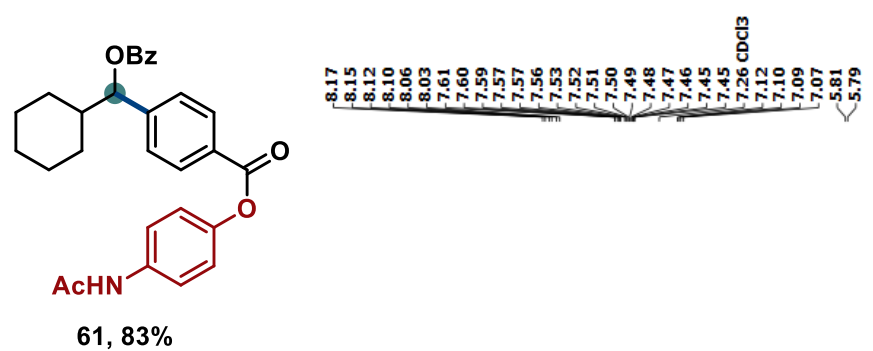

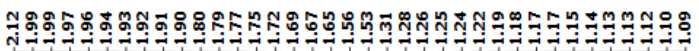

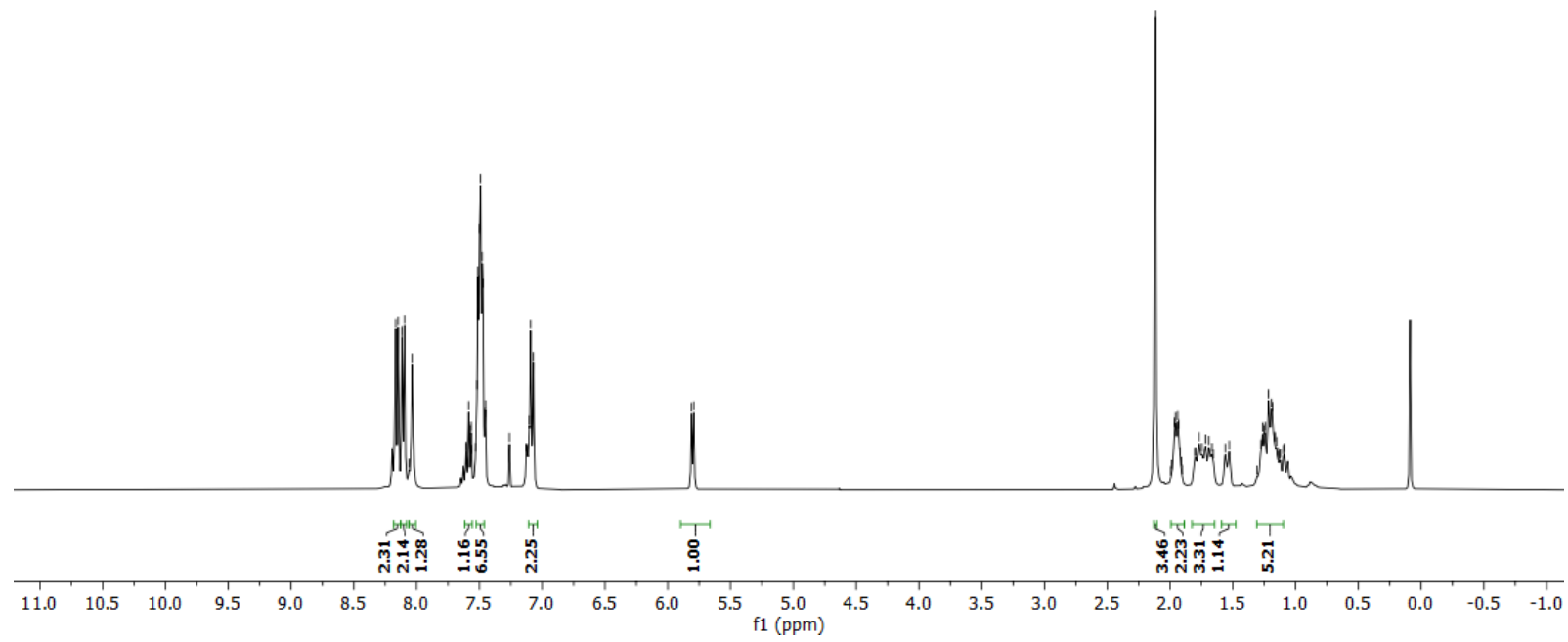

${ }^{13} \mathrm{C}$ NMR (101 MHz, $\left.\mathrm{CDCl}_{3}\right)$

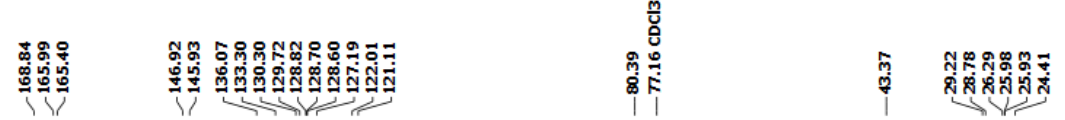

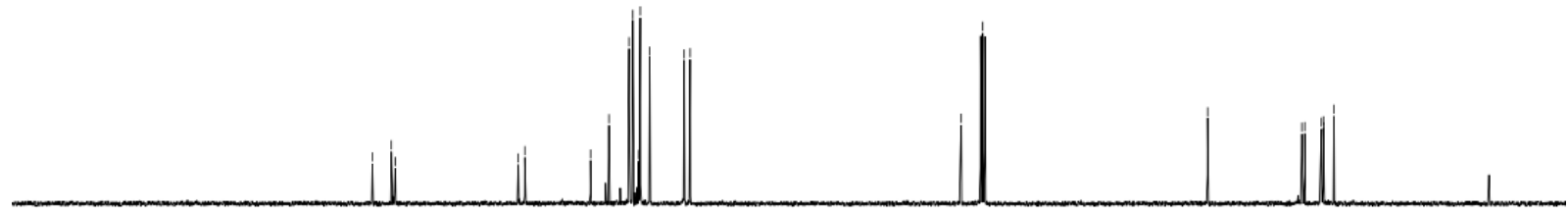

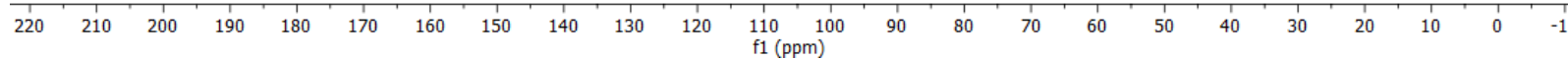


${ }^{1} \mathrm{H}$ NMR (400 $\mathrm{MHz}, \mathrm{CDCl}_{3}$ )

范

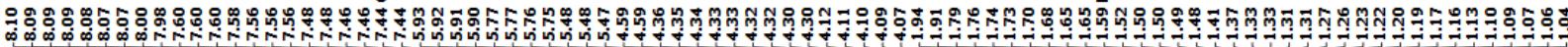

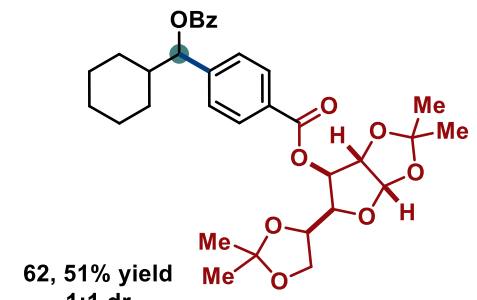

$$
1: 1 \mathrm{dr}
$$

from $D$-glucose

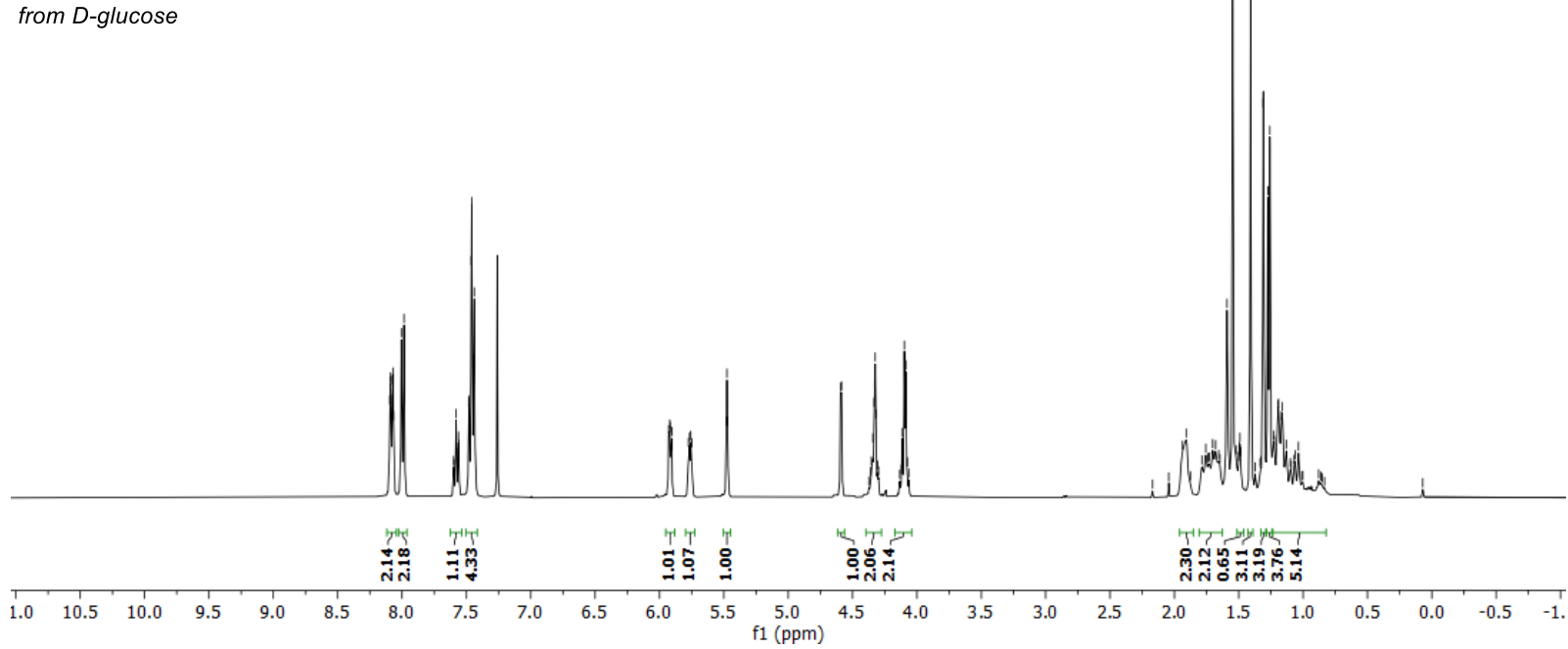

${ }^{13} \mathrm{C}$ NMR (101 MHz, $\left.\mathrm{CDCl}_{3}\right)$

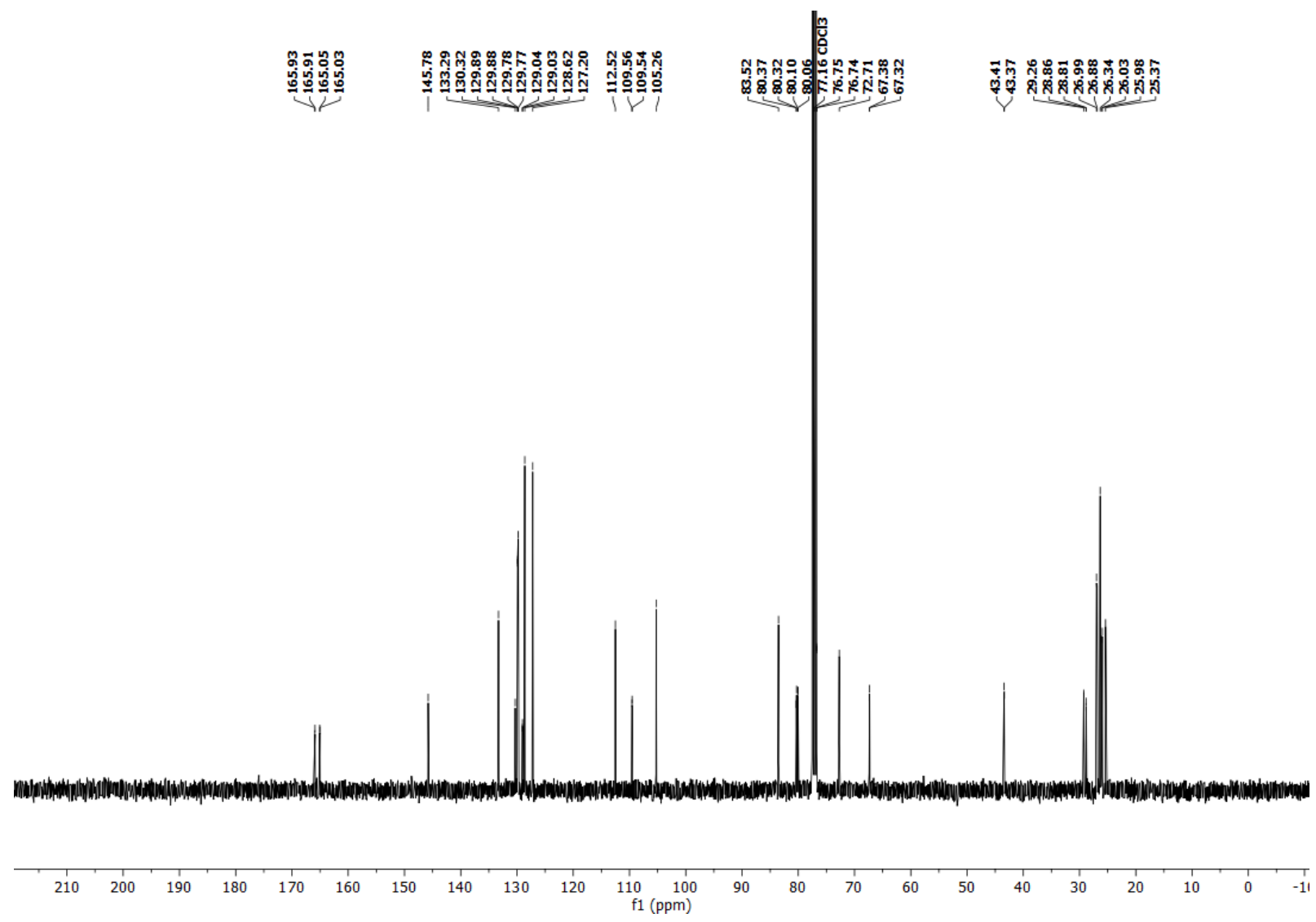


${ }^{1} \mathrm{H}$ NMR (599 $\left.\mathrm{MHz} \mathrm{CDCl}_{3}\right)$

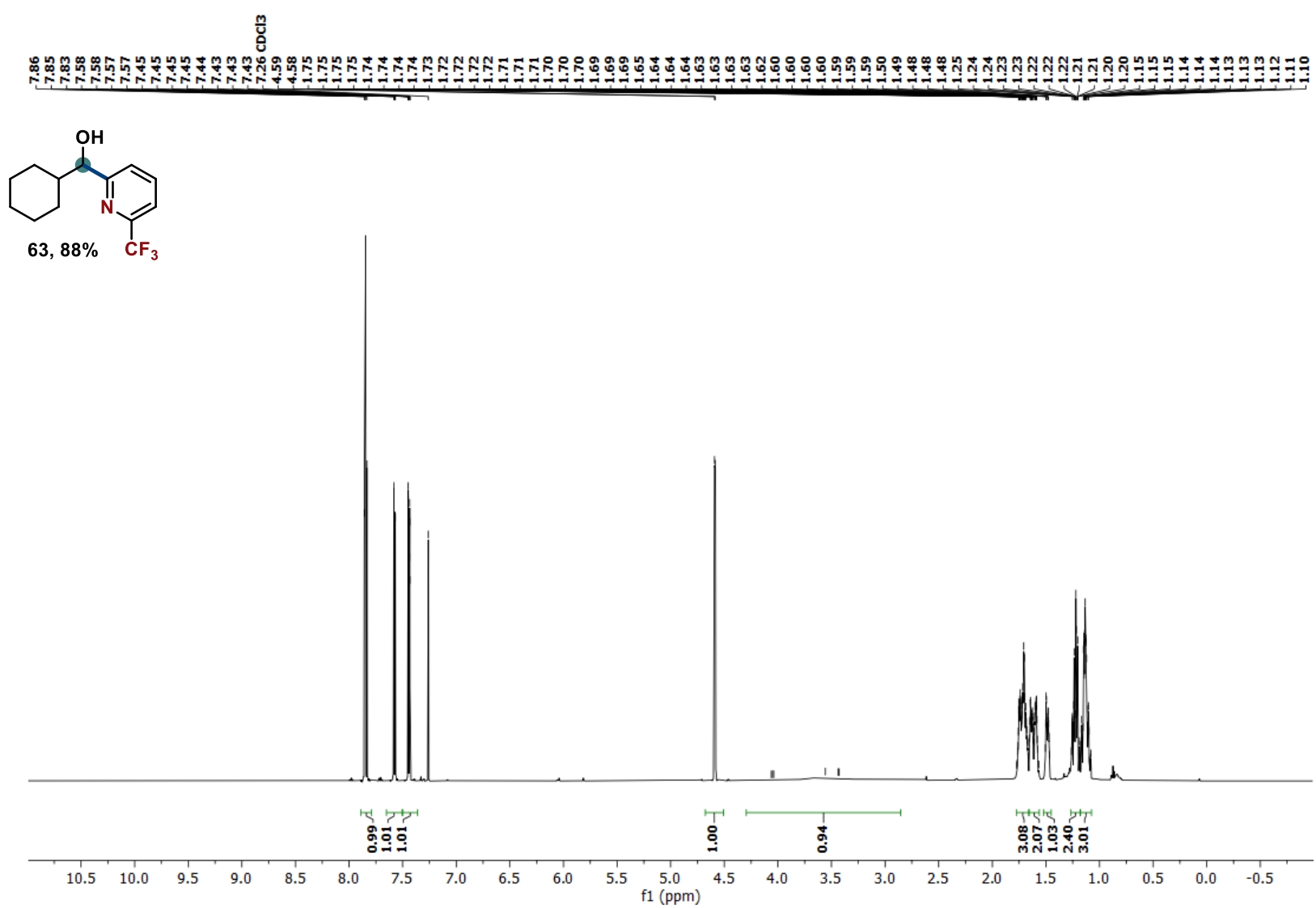

${ }^{13} \mathrm{C}$ NMR (151 MHz, $\mathrm{CDCl}_{3}$ )
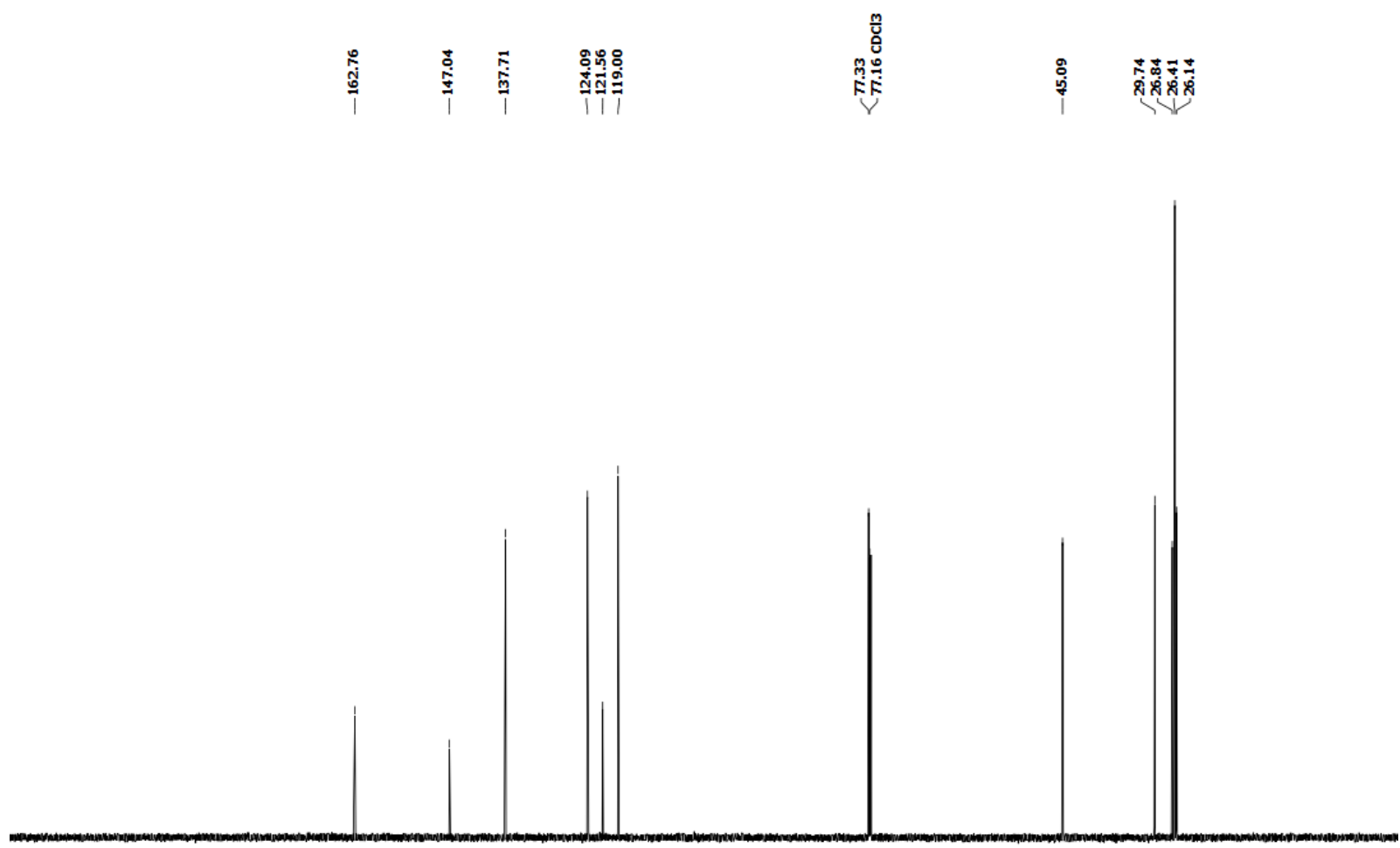

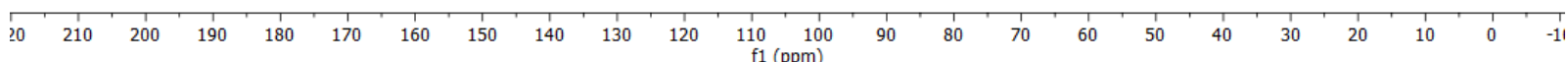


${ }^{19}$ F NMR (564 MHz, $\mathrm{CDCl}_{3}$ )

o̊

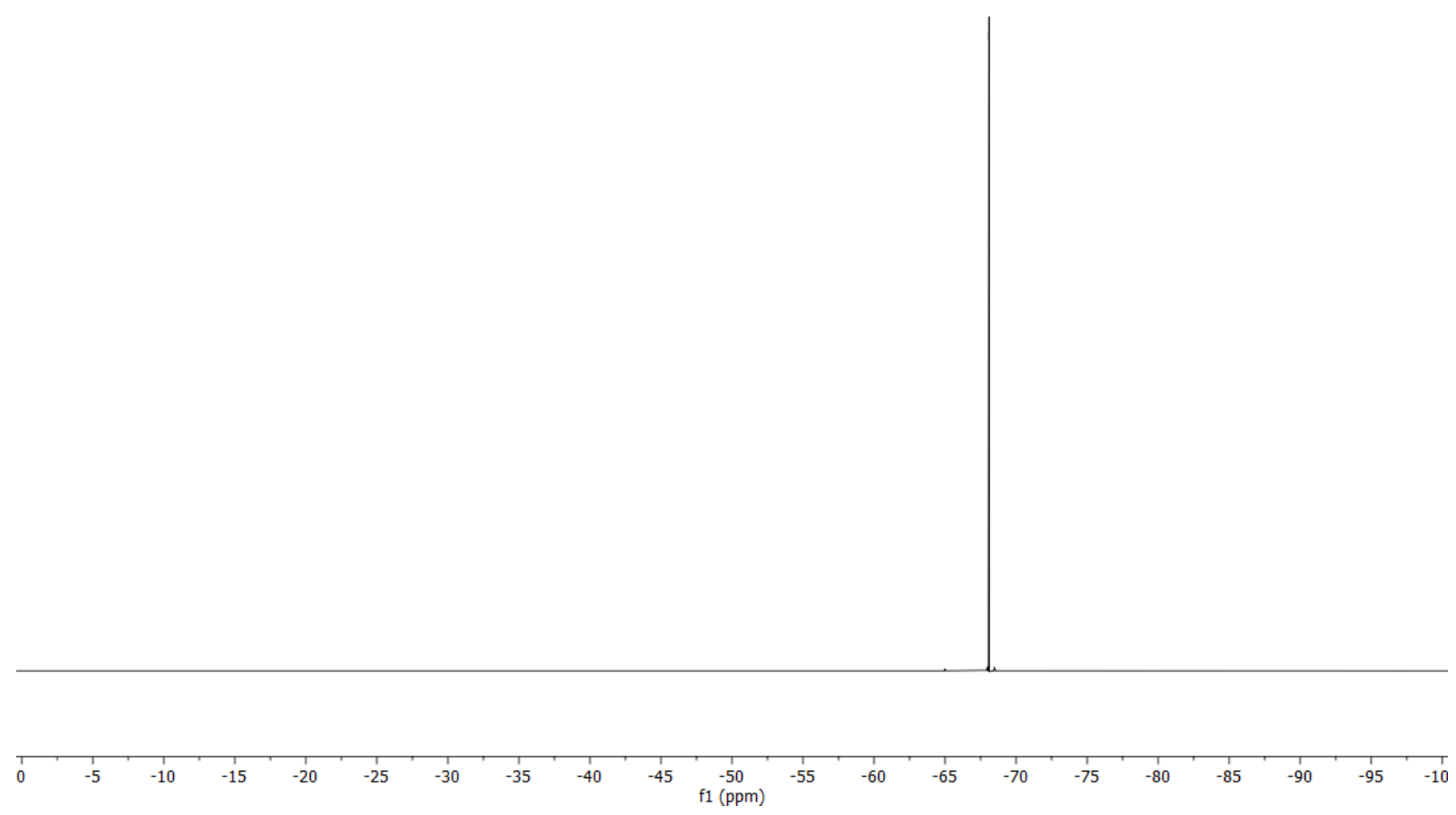


${ }^{1} \mathrm{H}$ NMR (400 $\mathrm{MHz}, \mathrm{CDCl}_{3}$ )

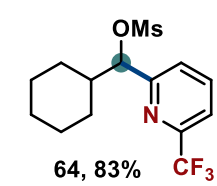

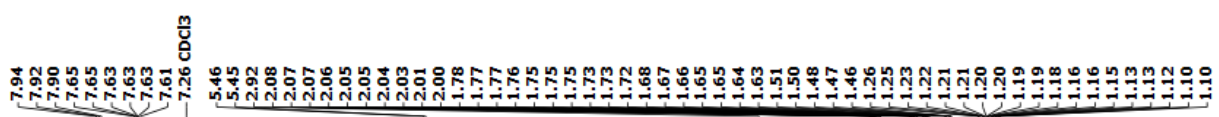

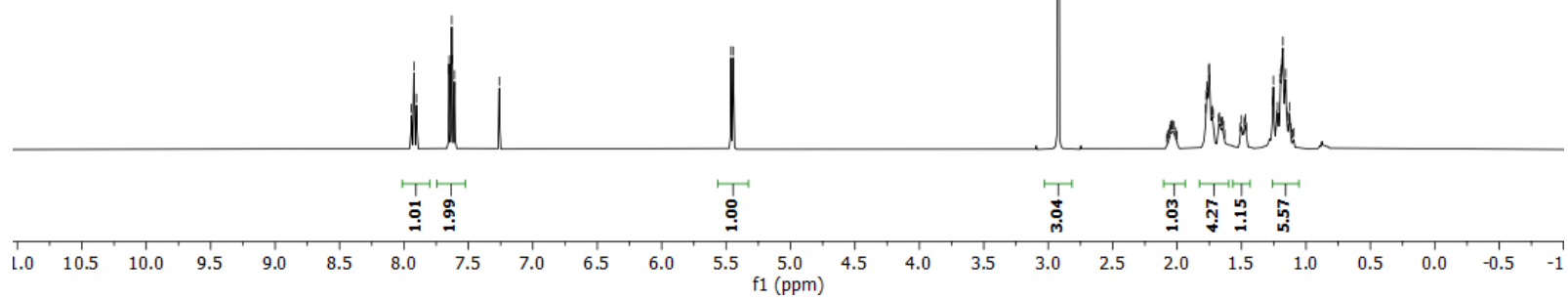

${ }^{13} \mathrm{C}$ NMR (101 MHz, $\mathrm{CDCl}_{3}$ )

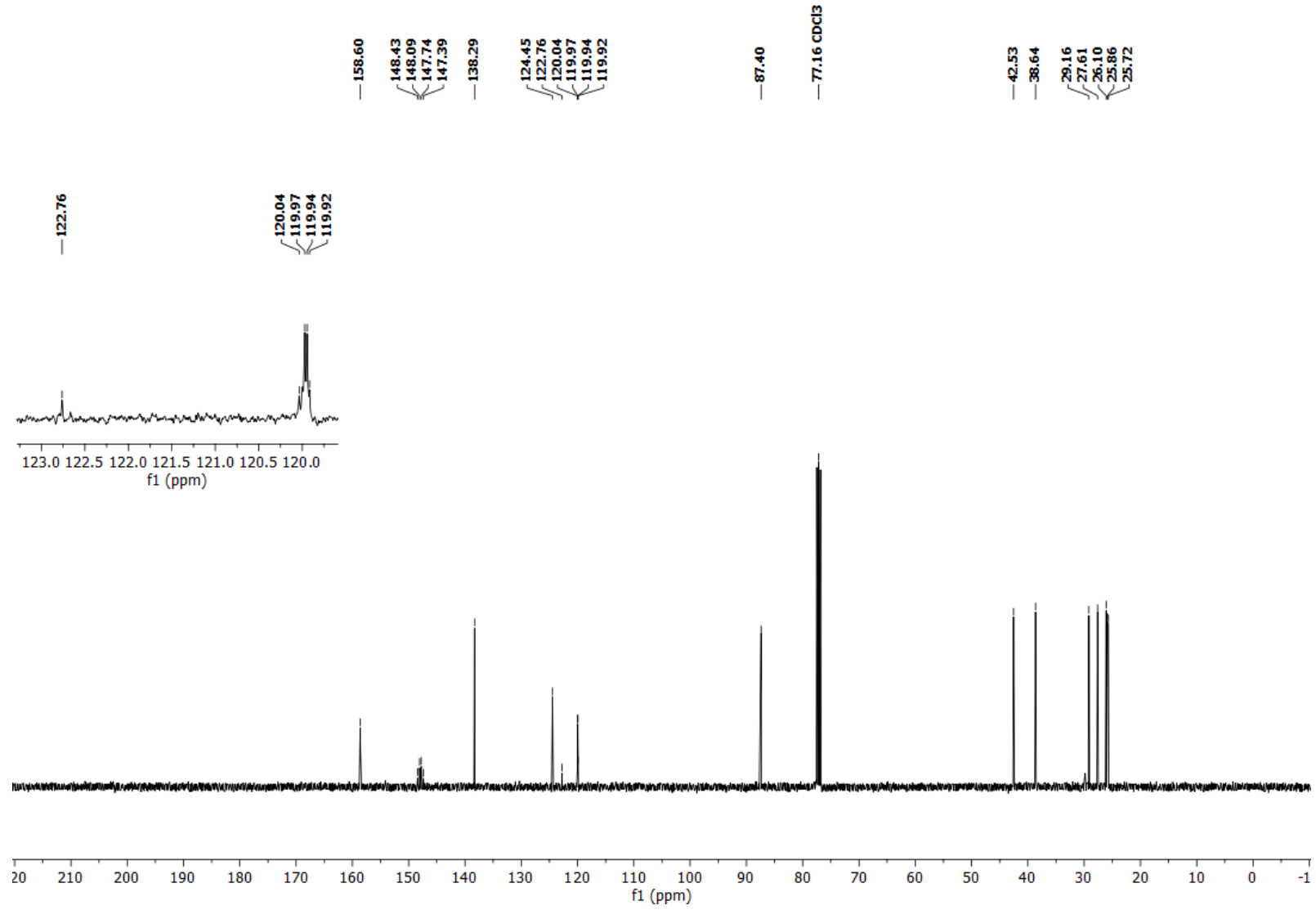


19F NMR (377 MHz, $\mathrm{CDCl}_{3}$ )

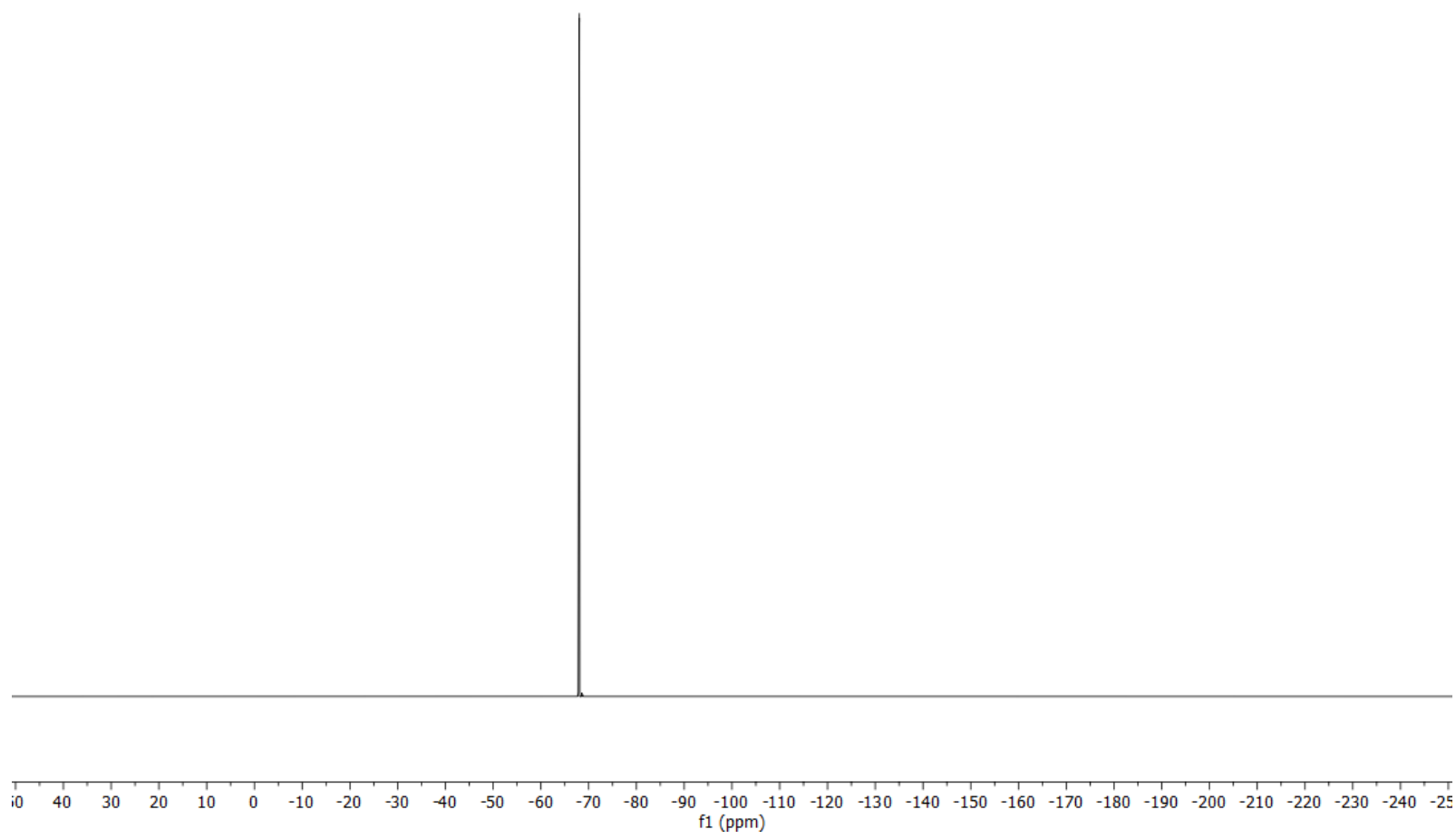


${ }^{1} \mathrm{H}\left\{{ }^{19} \mathrm{~F}\right\}$ NMR (500 $\left.\mathrm{MHz}, \mathrm{CDCl}_{3}\right)$

$\frac{m}{4}$

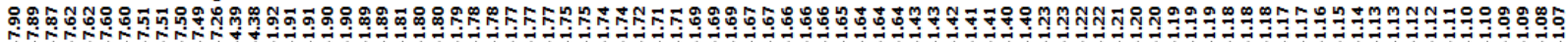
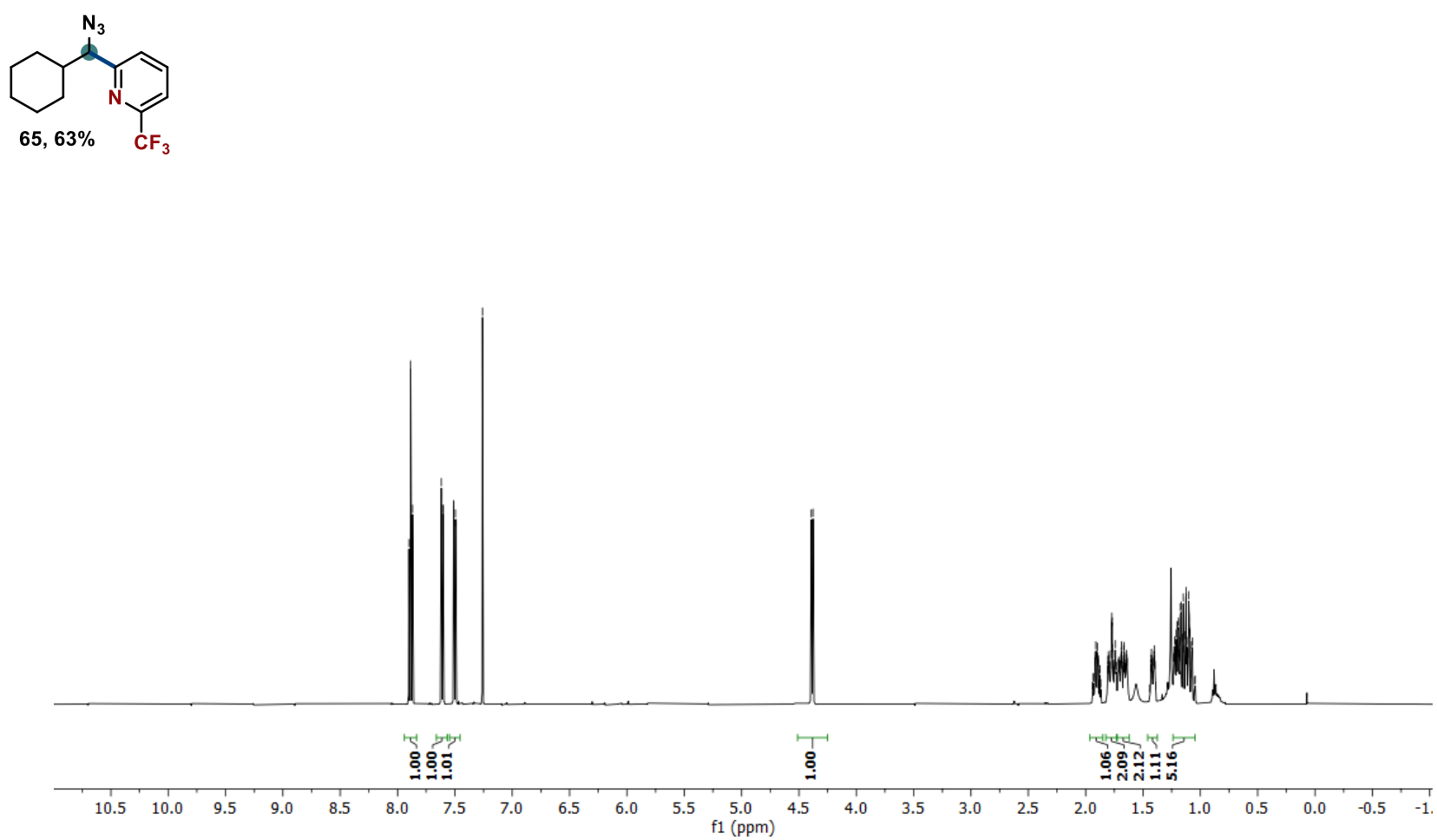

${ }^{13} \mathrm{C}\left\{{ }^{1} \mathrm{H},{ }^{19} \mathrm{~F}\right\}$ NMR (126 $\left.\mathrm{MHz}, \mathrm{CDCl}_{3}\right)$

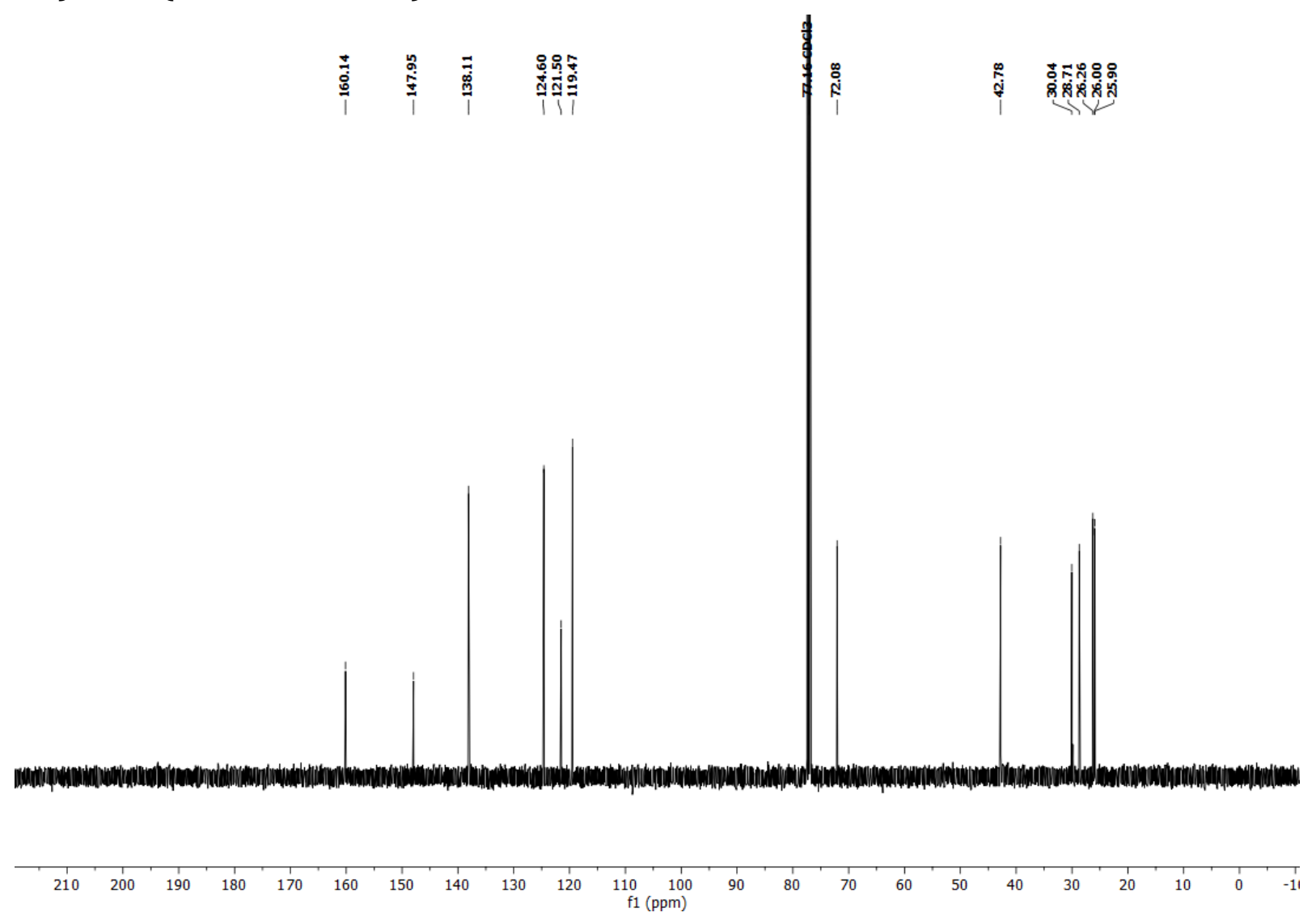


${ }^{19} \mathrm{~F}\left\{{ }^{1} \mathrm{H}\right\}$ NMR (470 $\left.\mathrm{MHz}, \mathrm{CDCl}_{3}\right)$

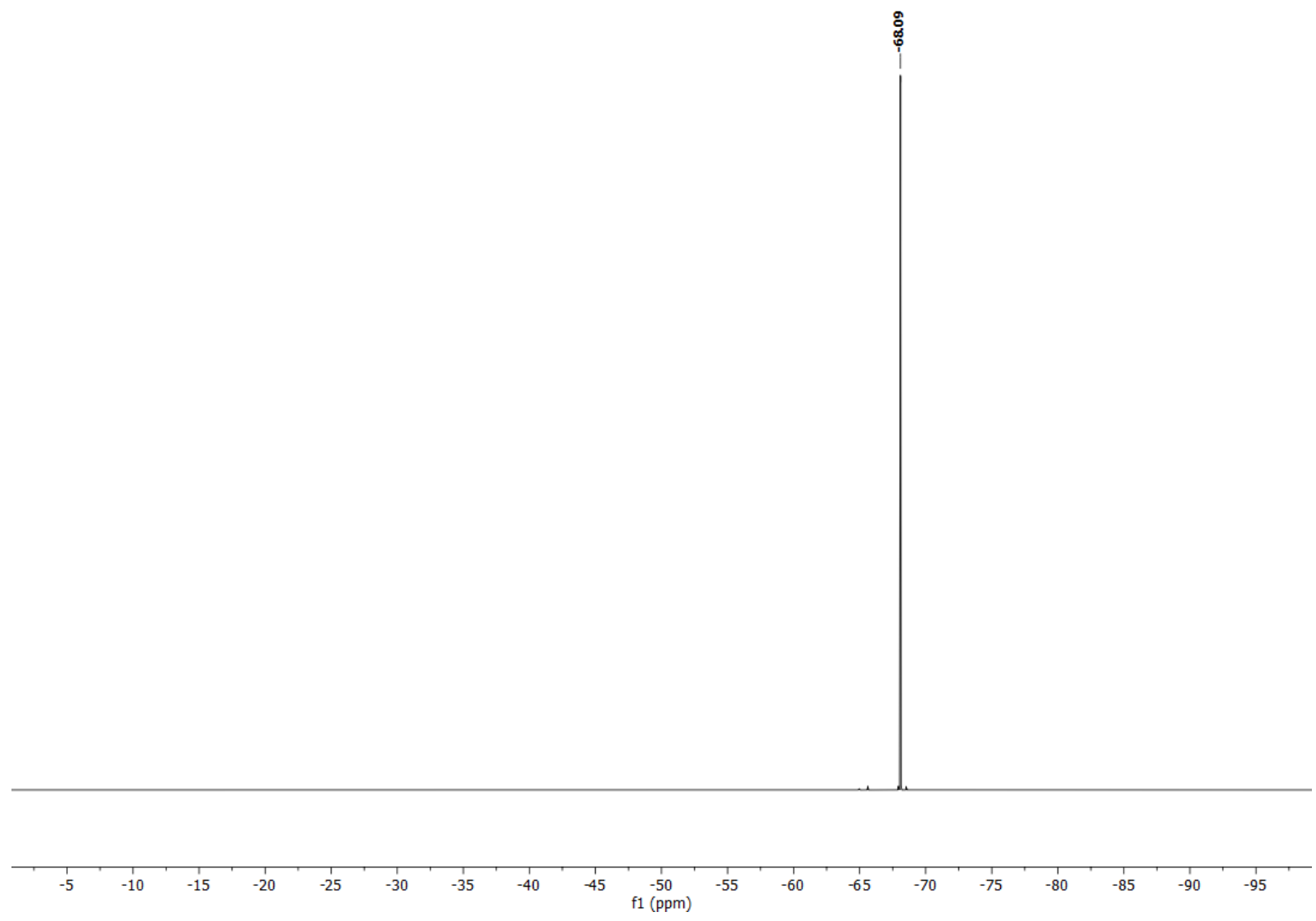


${ }^{1} \mathrm{H}$ NMR (599 $\mathrm{MHz} \mathrm{CDCl}_{3}$ )<smiles>OC(c1cc(-c2cccc(Cl)c2)cc(C(F)(F)F)n1)C1CCCCC1</smiles>

$66,60 \%$ over two steps

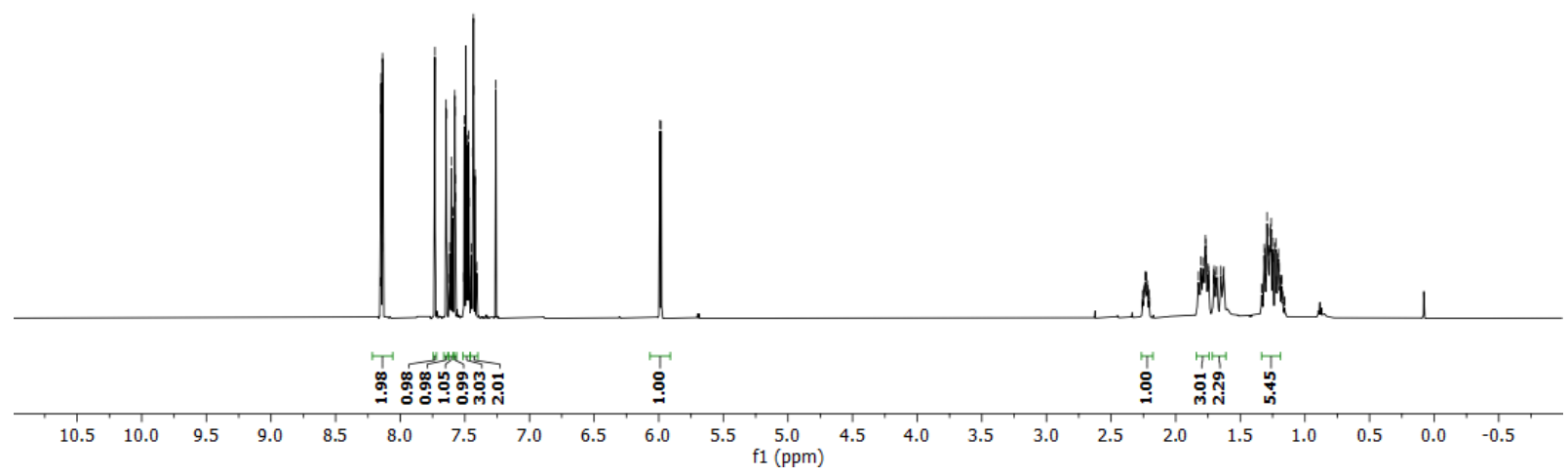

${ }^{13} \mathrm{C}$ NMR (151 MHz, $\mathrm{CDCl}_{3}$ )
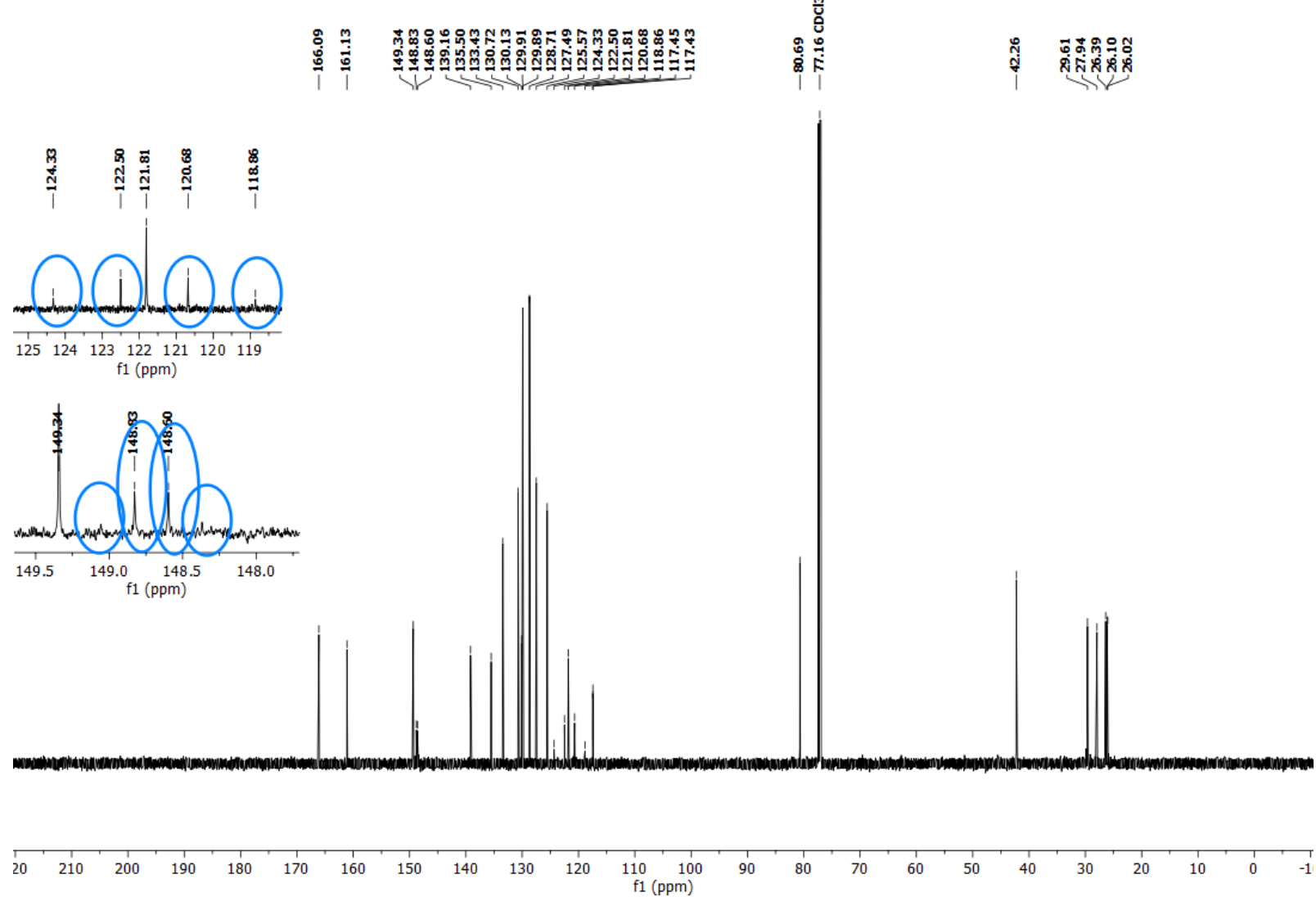
${ }^{13} \mathrm{C}\left\{{ }^{1} \mathrm{H},{ }^{19} \mathrm{~F}\right\}$ NMR (151 $\left.\mathrm{MHz}, \mathrm{CDCl}_{3}\right)$

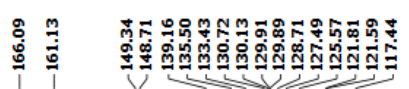

i

สู่

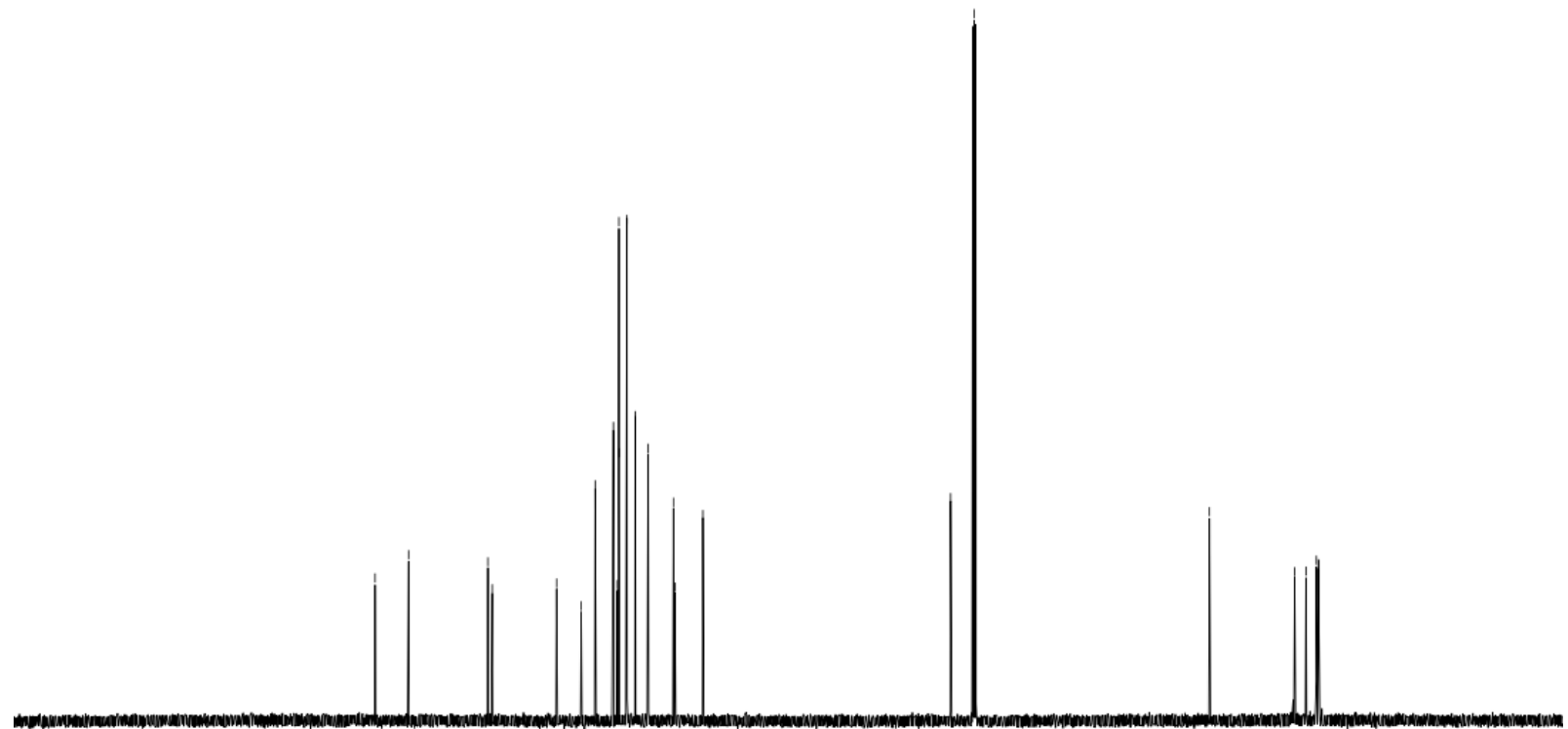

19F NMR (564 MHz, $\mathrm{CDCl}_{3}$ )

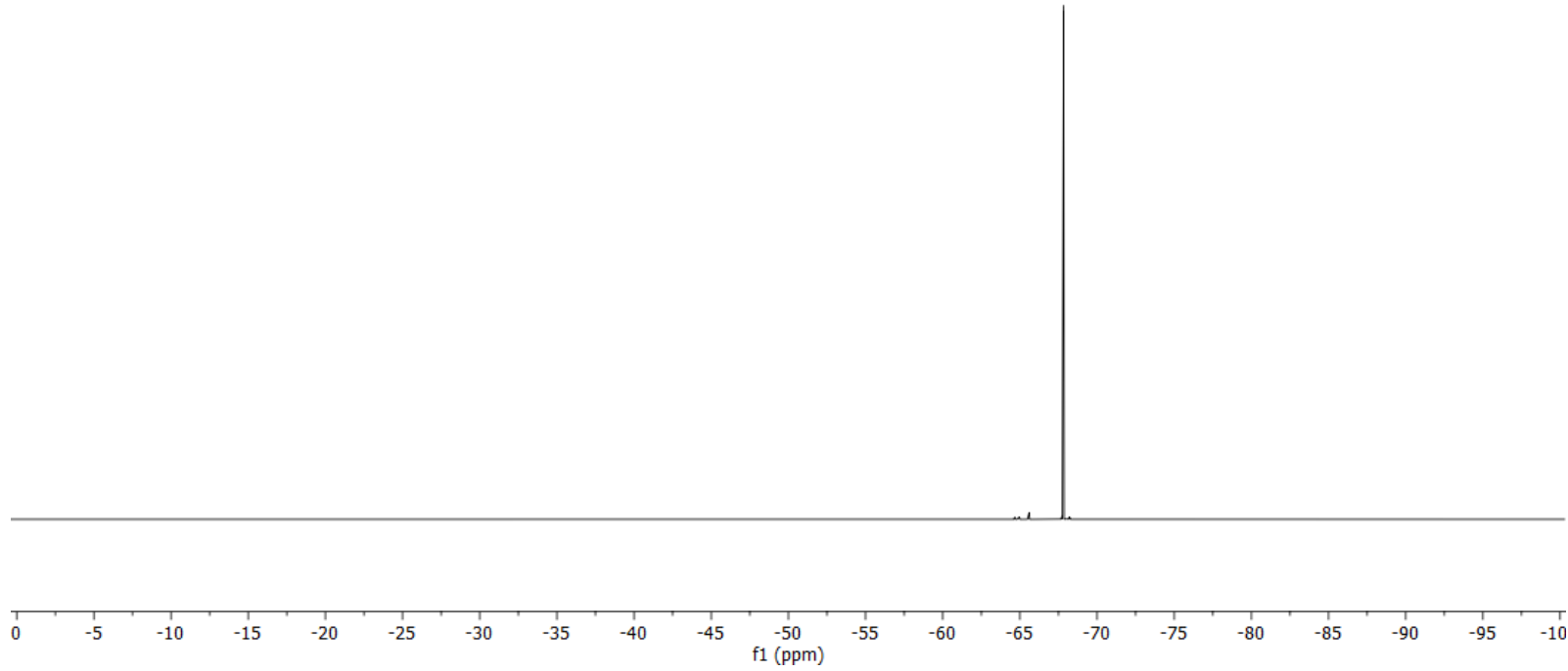


${ }^{1} \mathrm{H}\left\{{ }^{19} \mathrm{~F}\right\}$ NMR (500 $\left.\mathrm{MHz}, \mathrm{CDCl}_{3}\right)$

学

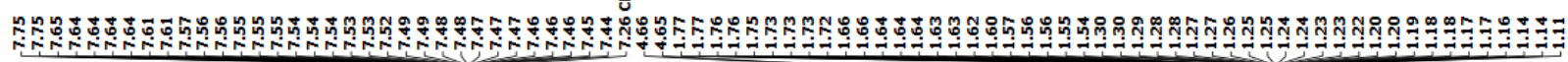<smiles>OC(c1cc(-c2cccc(Cl)c2)cc(C(F)(F)F)n1)C1CCCCC1</smiles>

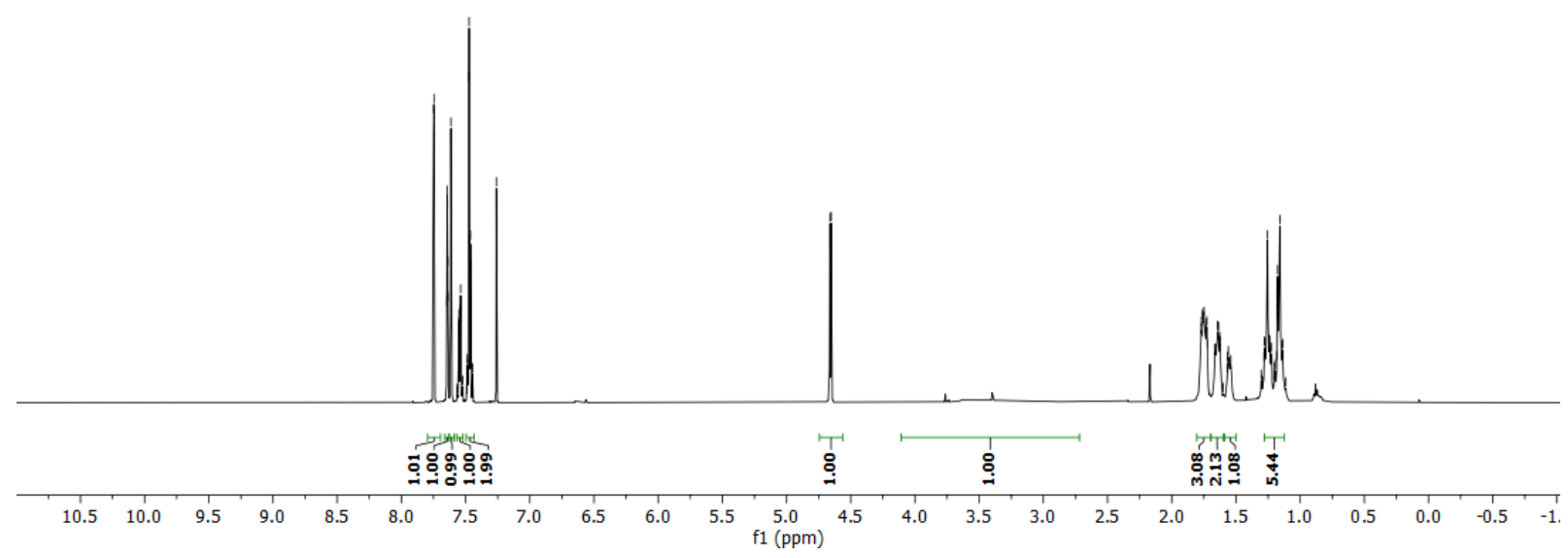

${ }^{13} \mathrm{C}\left\{{ }^{1} \mathrm{H},{ }^{19} \mathrm{~F}\right\}$ NMR (126 $\left.\mathrm{MHz}, \mathrm{CDCl}_{3}\right)$

क

啇

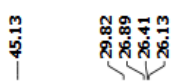

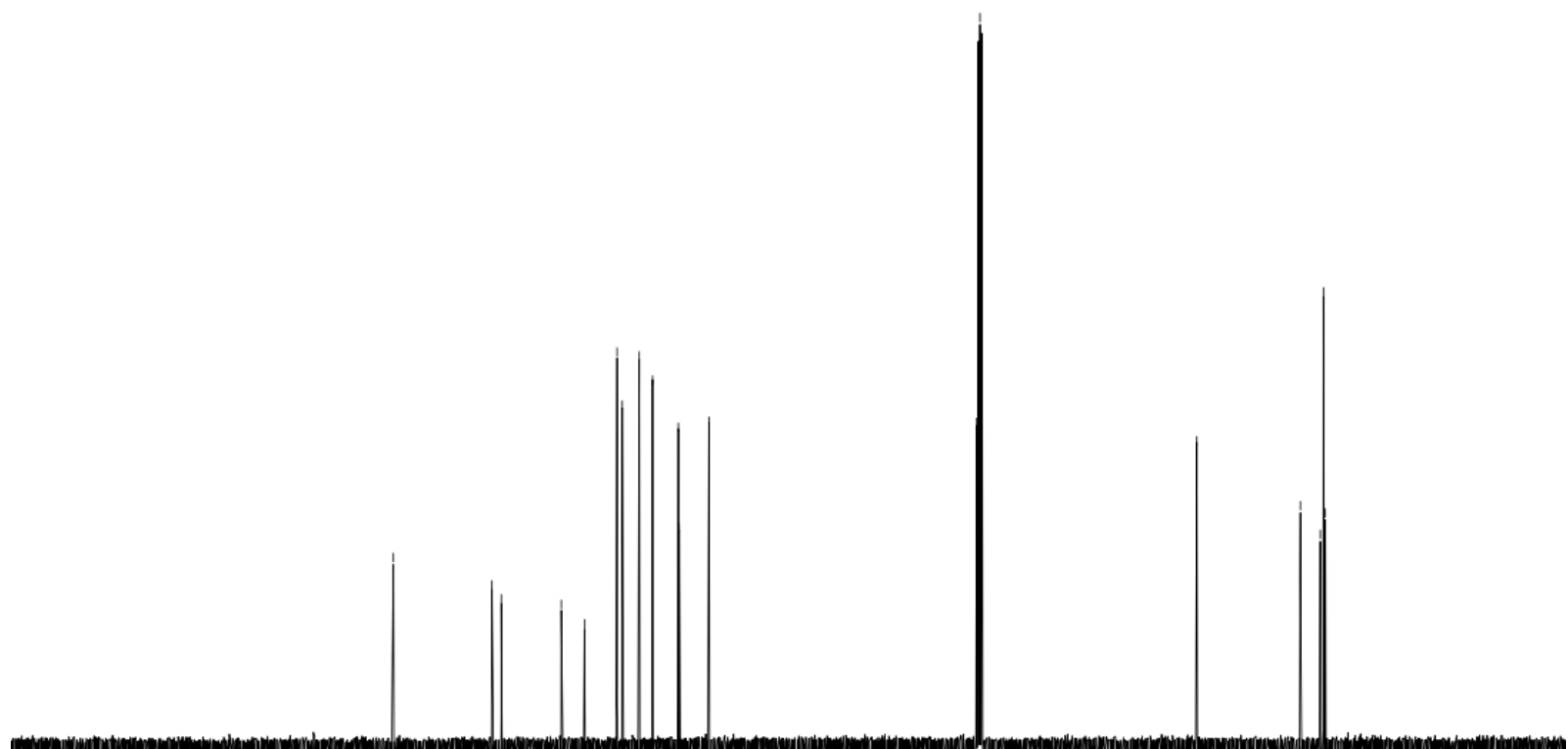

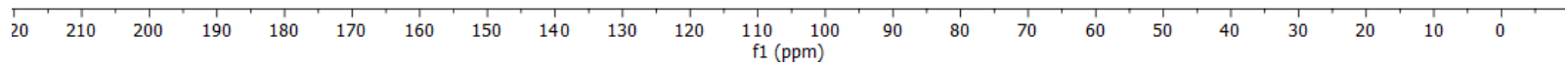


${ }^{19} \mathrm{~F}\left\{{ }^{1} \mathrm{H}\right\}$ NMR (470 $\left.\mathrm{MHz}, \mathrm{CDCl}_{3}\right)$

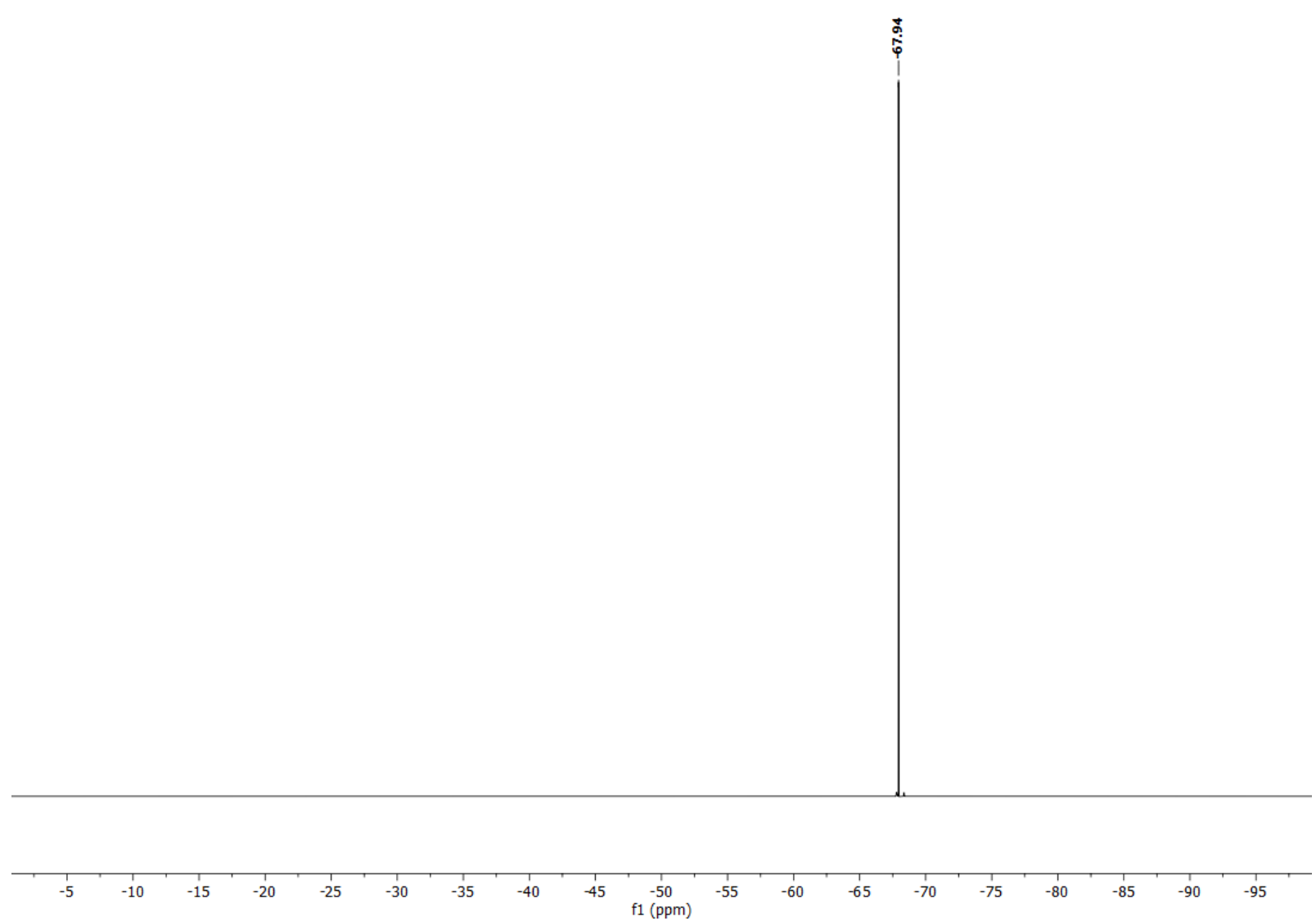


${ }^{1} \mathrm{H}\left\{{ }^{19} \mathrm{~F}\right\}$ NMR (500 $\left.\mathrm{MHz}, \mathrm{CDCl}_{3}\right)$

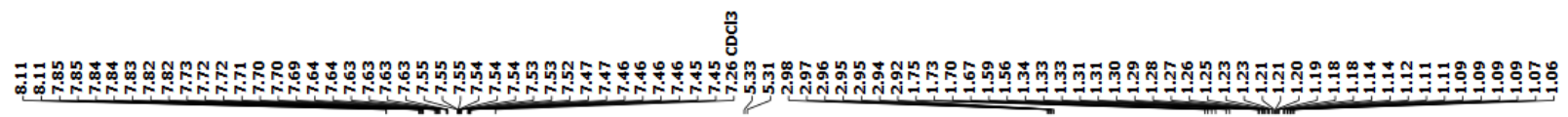<smiles>C[13CH2]c1cc(-c2cccc(Cl)c2)cc([C@H](N)C2CCCCC2)n1</smiles>

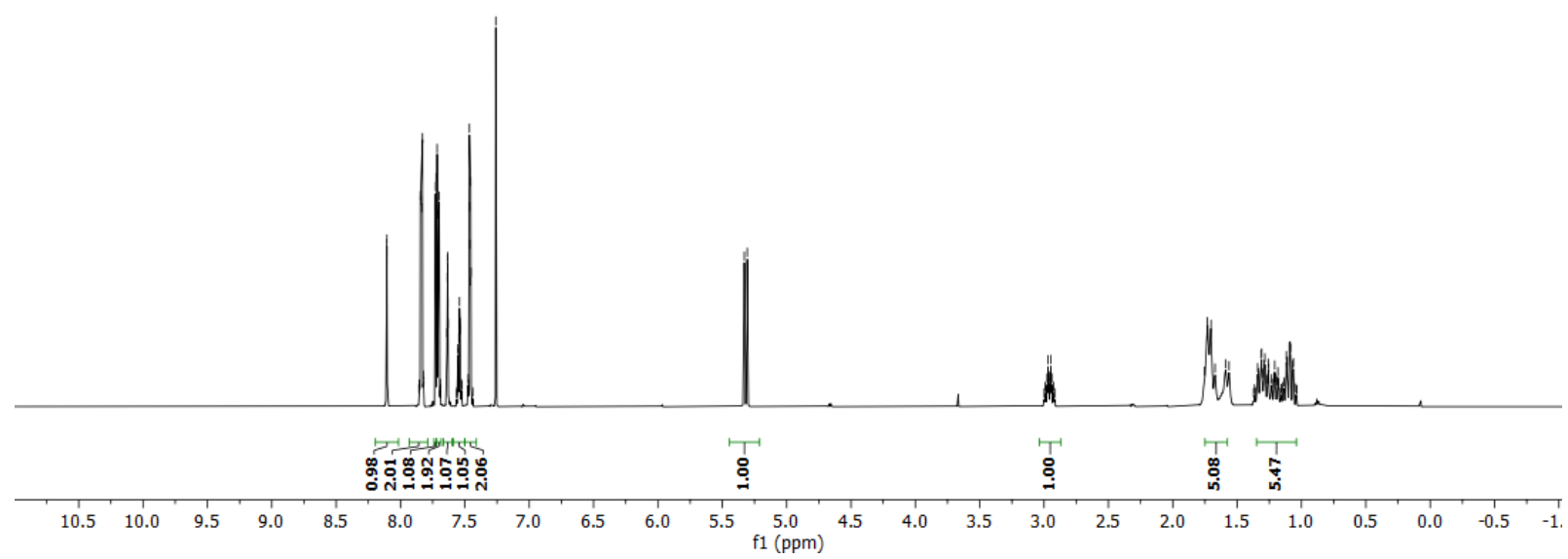

${ }^{13} \mathrm{C}\left\{{ }^{1} \mathrm{H}\right\}$ NMR (126 $\left.\mathrm{MHz}^{\mathrm{CDCl}} 3\right)$

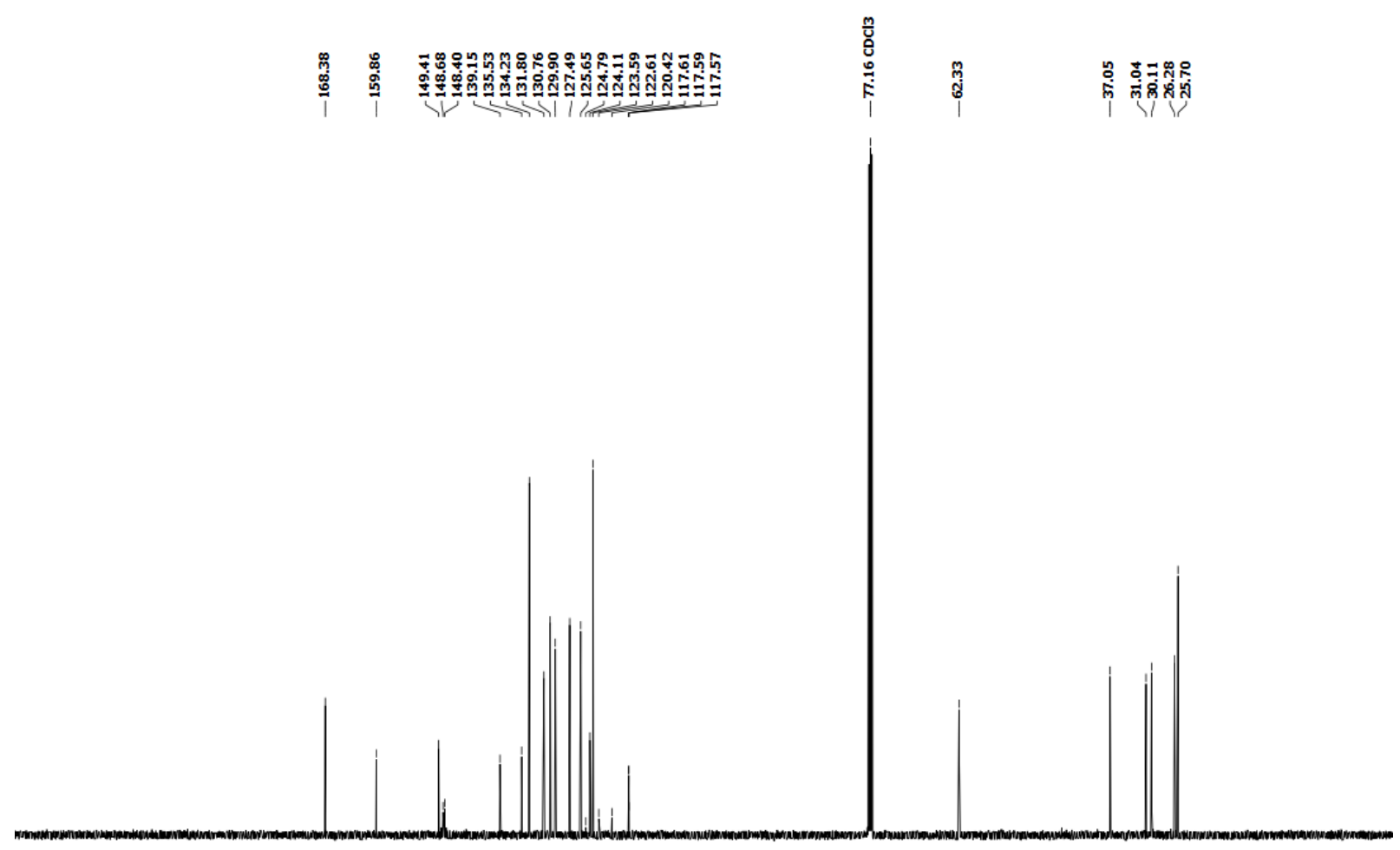

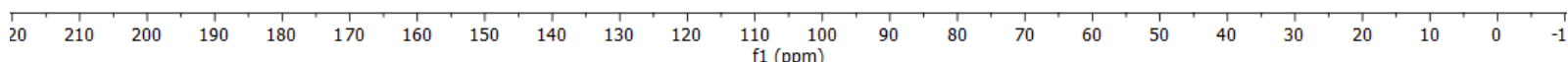


${ }^{13} \mathrm{C}\left\{{ }^{1} \mathrm{H},{ }^{19} \mathrm{~F}\right\}$ NMR (126 $\left.\mathrm{MHz}, \mathrm{CDCl}_{3}\right)$

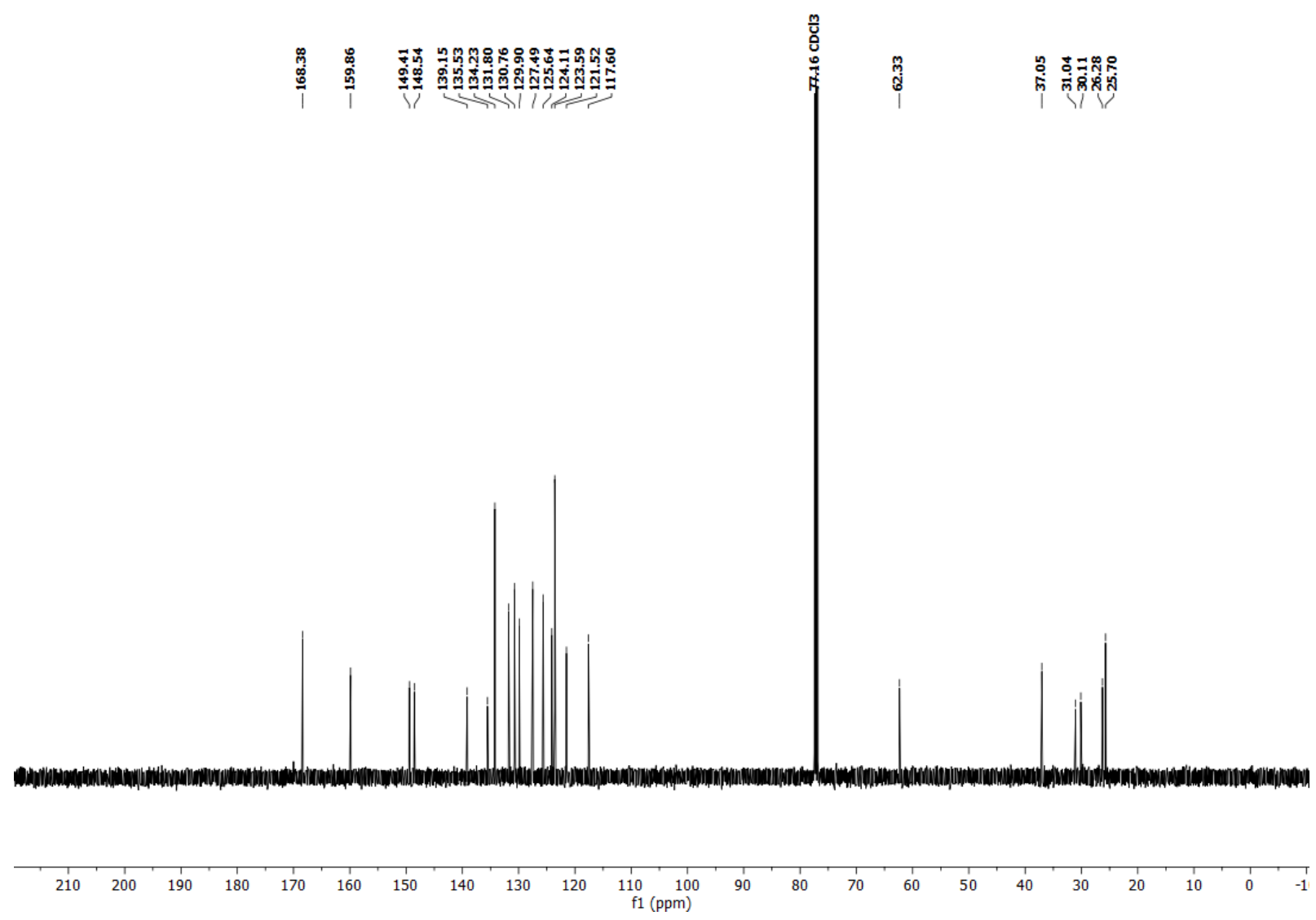

${ }^{19} \mathrm{~F}\left\{{ }^{1} \mathrm{H}\right\}$ NMR (470 $\left.\mathrm{MHz}, \mathrm{CDCl}_{3}\right)$

$$
\stackrel{0}{\circ}
$$

$\begin{array}{lllllllllllllllllllllllllllllllll}40 & 30 & 20 & 10 & 0 & -10 & -20 & -30 & -40 & -50 & -60 & -70 & -80 & -90 & -100 & -110 & -120 & -130 & -140 & -150 & -160 & -170 & -180 & -190 & -200 & -210 & -220 & -230 & -240\end{array}$ 
${ }^{1} \mathrm{H}\left\{{ }^{19} \mathrm{~F}\right\}$ NMR (500 $\left.\mathrm{MHz}, \mathrm{CDCl}_{3}\right)$

范

\%을<smiles>CC(C)(C)c1cccc(C(=O)C2CCCCC2)n1</smiles>

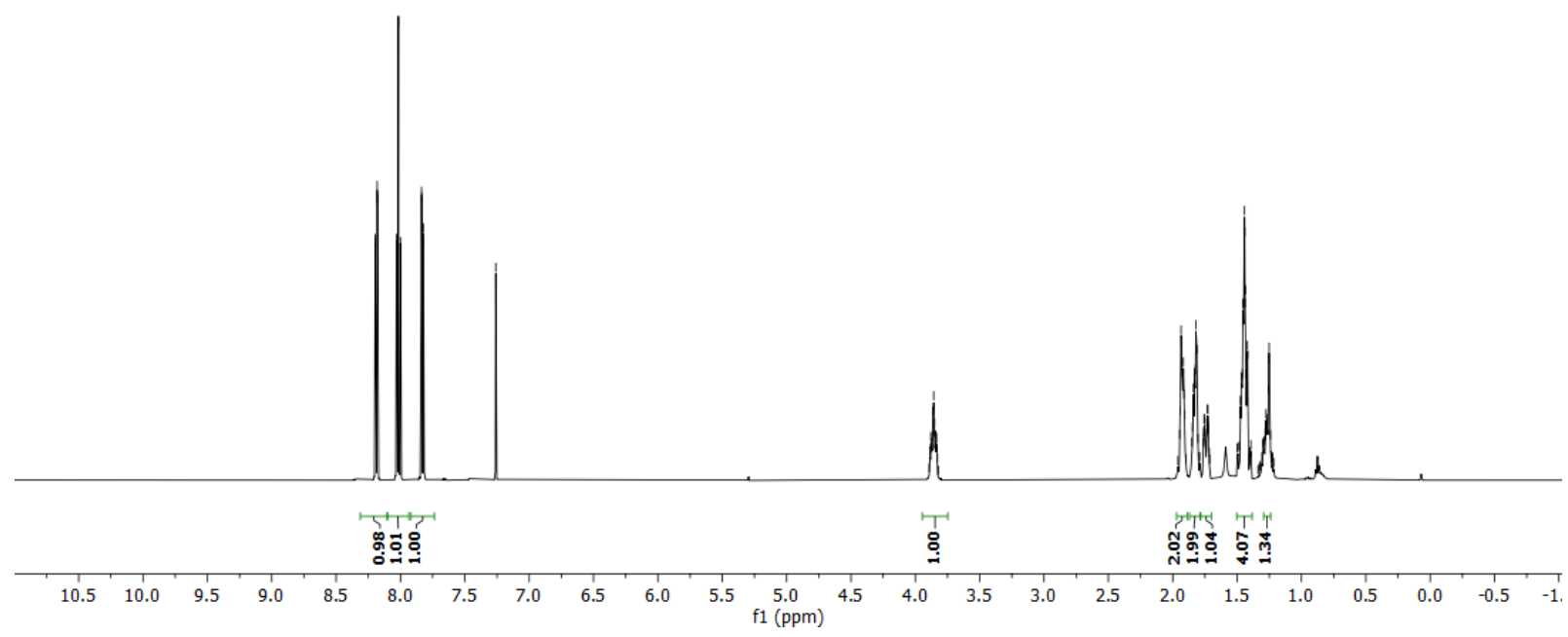

${ }^{13} \mathrm{C}\left\{{ }^{1} \mathrm{H}\right\}$ NMR (126 $\left.\mathrm{MHz}, \mathrm{CDCl}_{3}\right)$

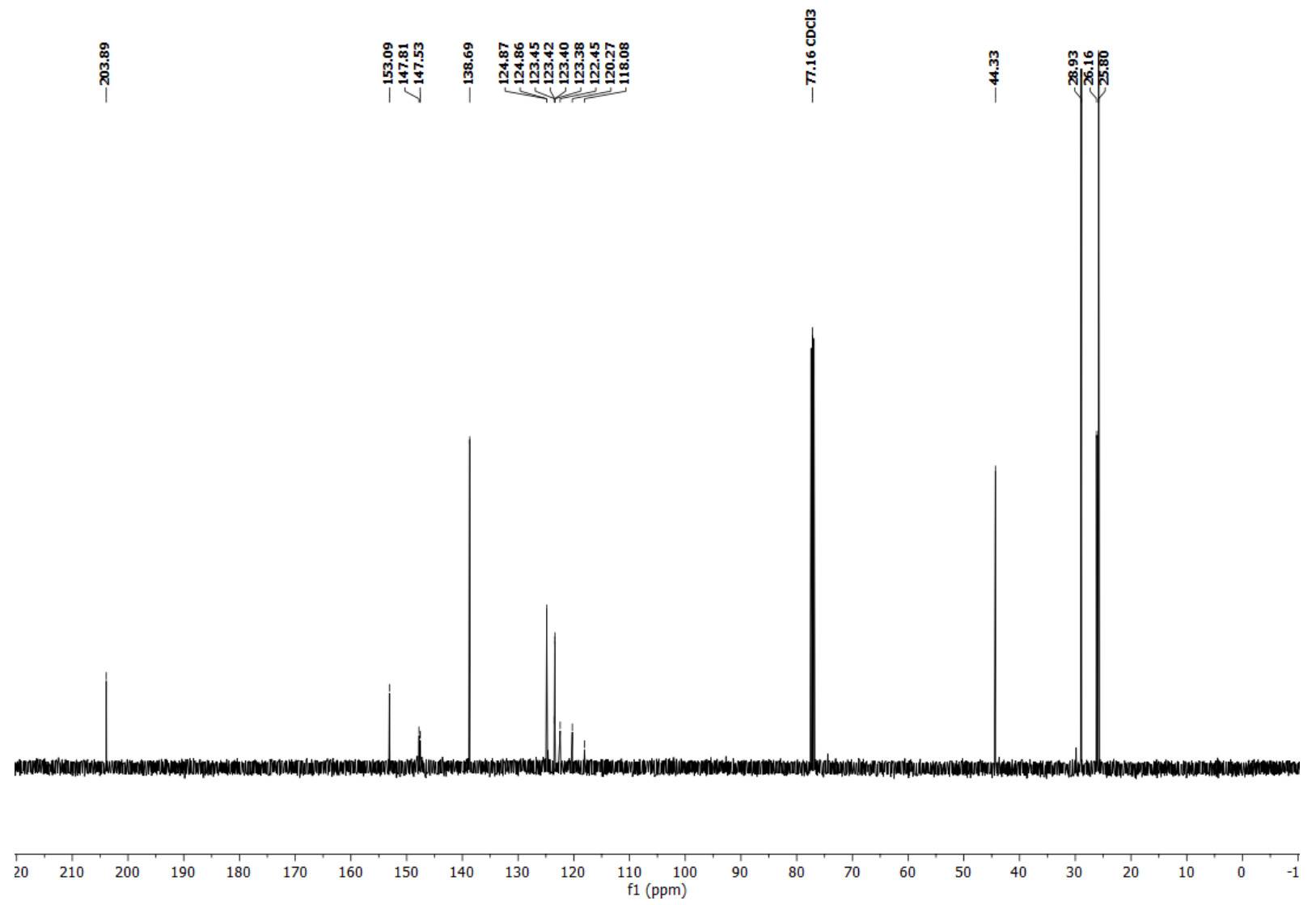


${ }^{13} \mathrm{C}\left\{{ }^{1} \mathrm{H},{ }^{19} \mathrm{~F}\right\}$ NMR (126 $\left.\mathrm{MHz}, \mathrm{CDCl}_{3}\right)$

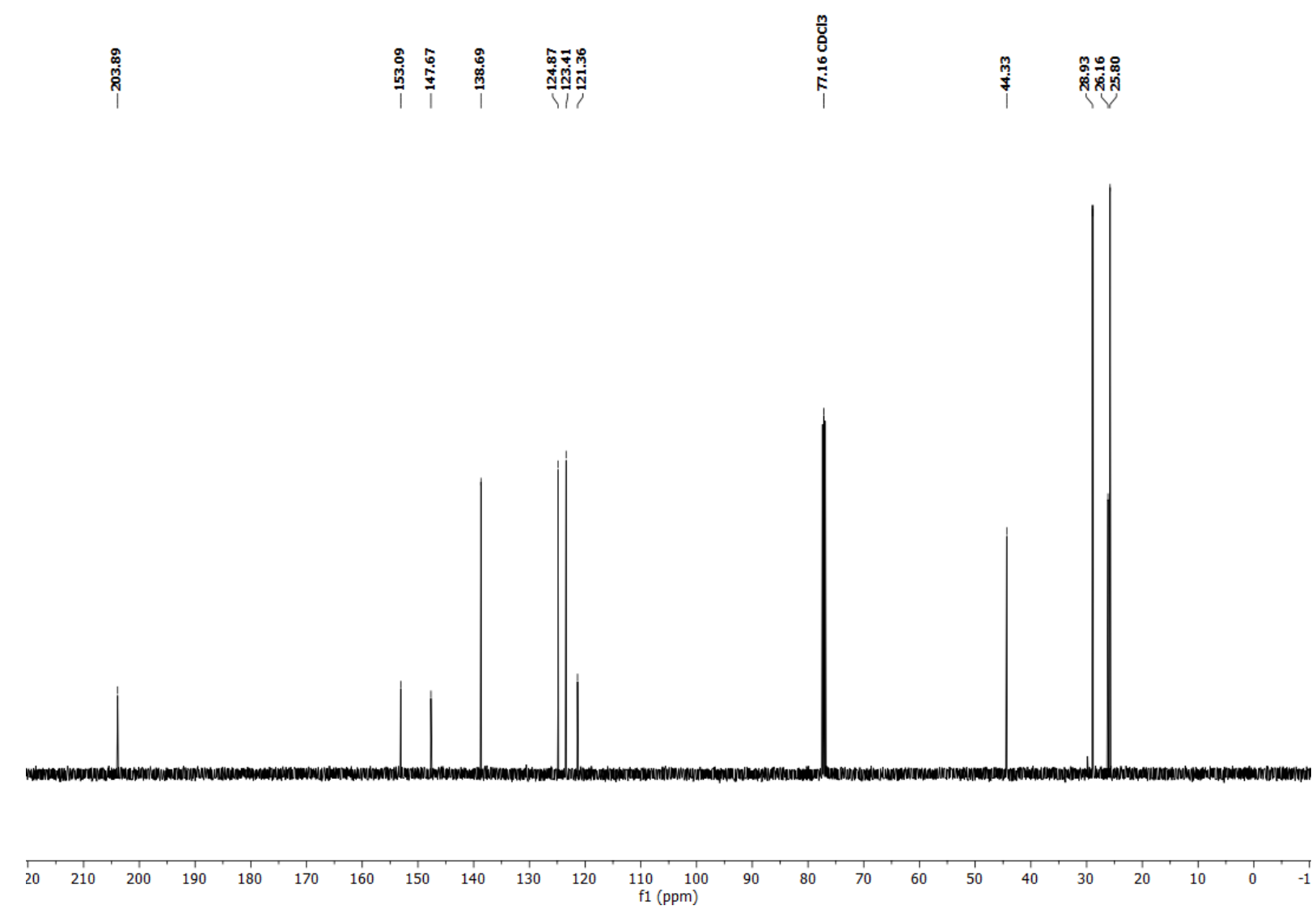

${ }^{19} \mathrm{~F}\left\{{ }^{1} \mathrm{H}\right\}$ NMR (470 $\left.\mathrm{MHz}, \mathrm{CDCl}_{3}\right)$

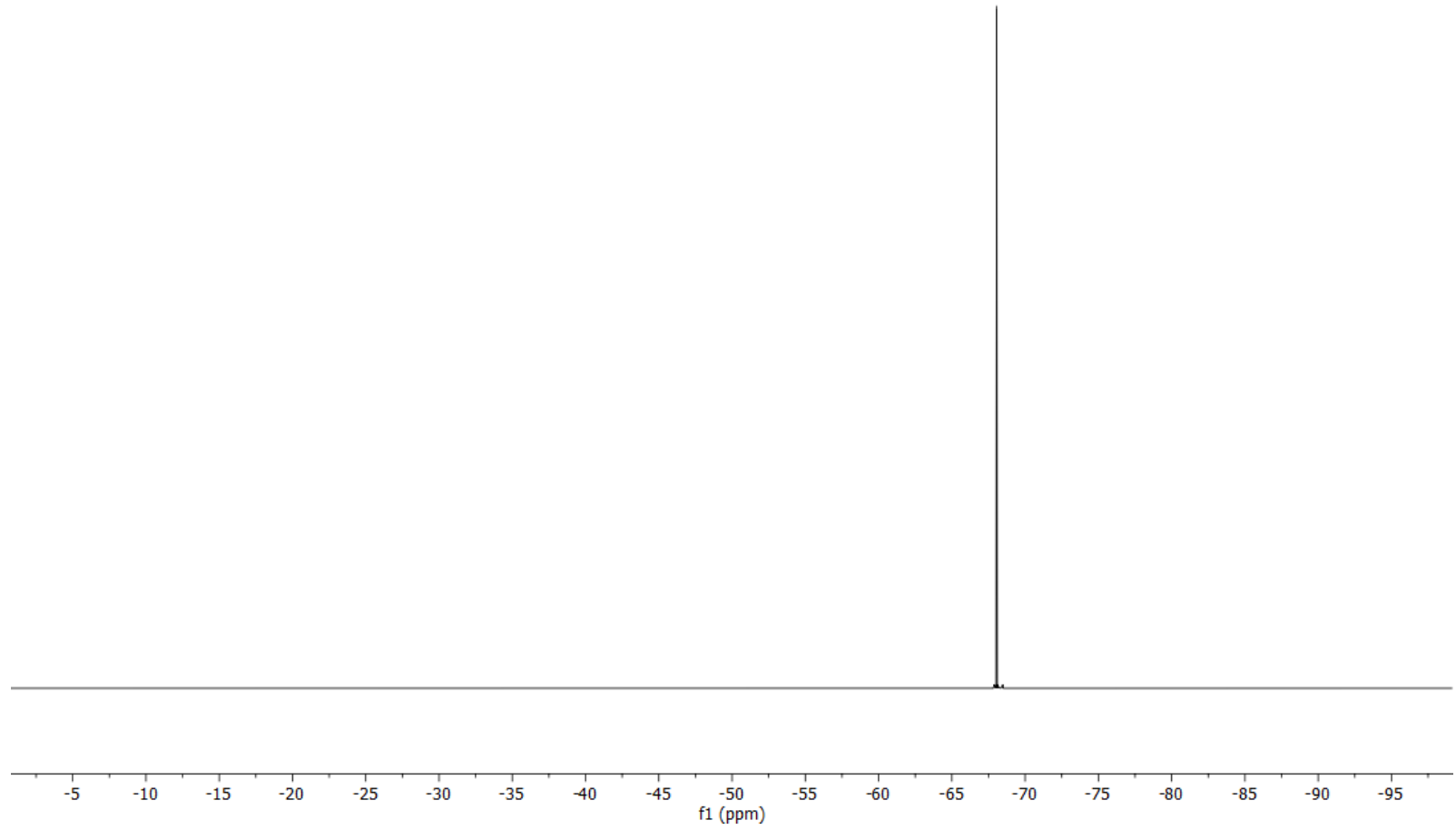


${ }^{1} \mathrm{H}\left\{{ }^{19} \mathrm{~F}\right\}$ NMR (599 $\mathrm{MHz}, \mathrm{CDCl}_{3}$ )

范

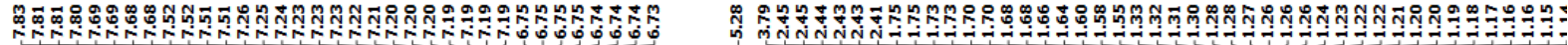<smiles>COc1cccc(C(O)(c2cccc(C(F)(F)F)n2)C2CCCCC2)c1</smiles>

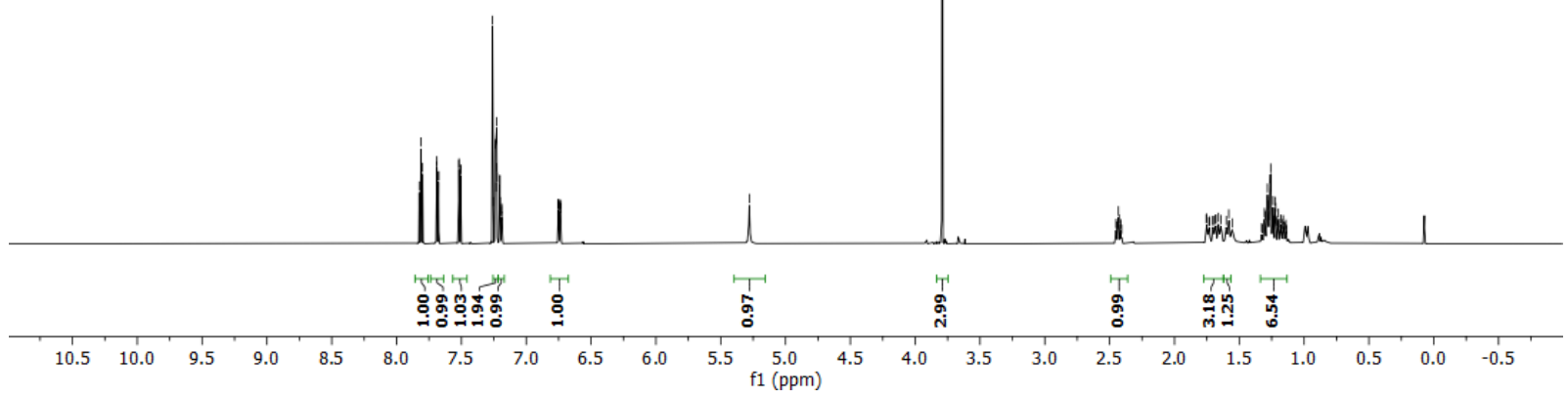

${ }^{13} \mathrm{C}\left\{{ }^{1} \mathrm{H},{ }^{19} \mathrm{~F}\right\}$ NMR (151 $\left.\mathrm{MHz}, \mathrm{CDCl}_{3}\right)$

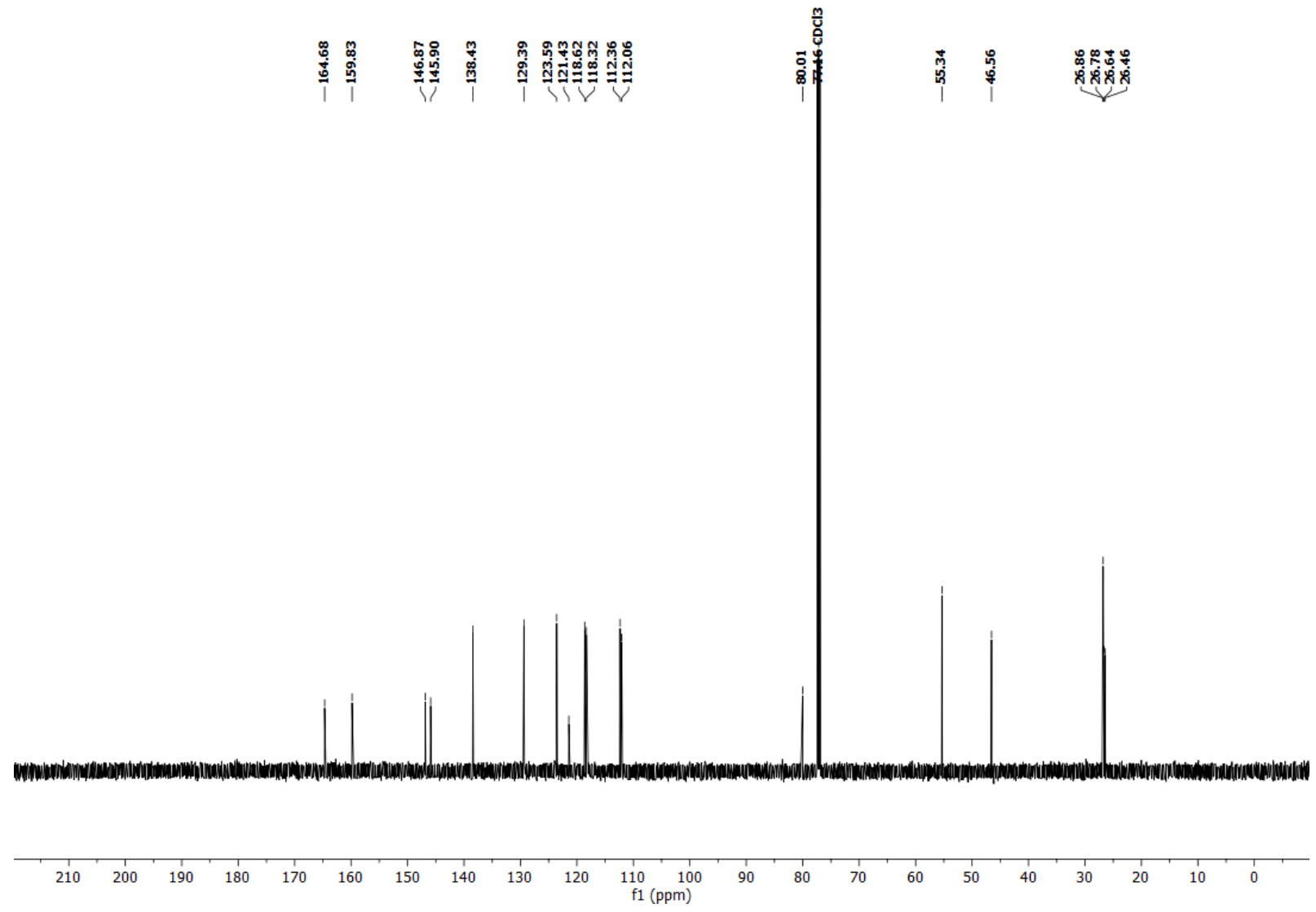


${ }^{19}$ F $\left\{{ }^{1} \mathrm{H}\right\}$ NMR (564 MHz, $\mathrm{CDCl}_{3}$ )

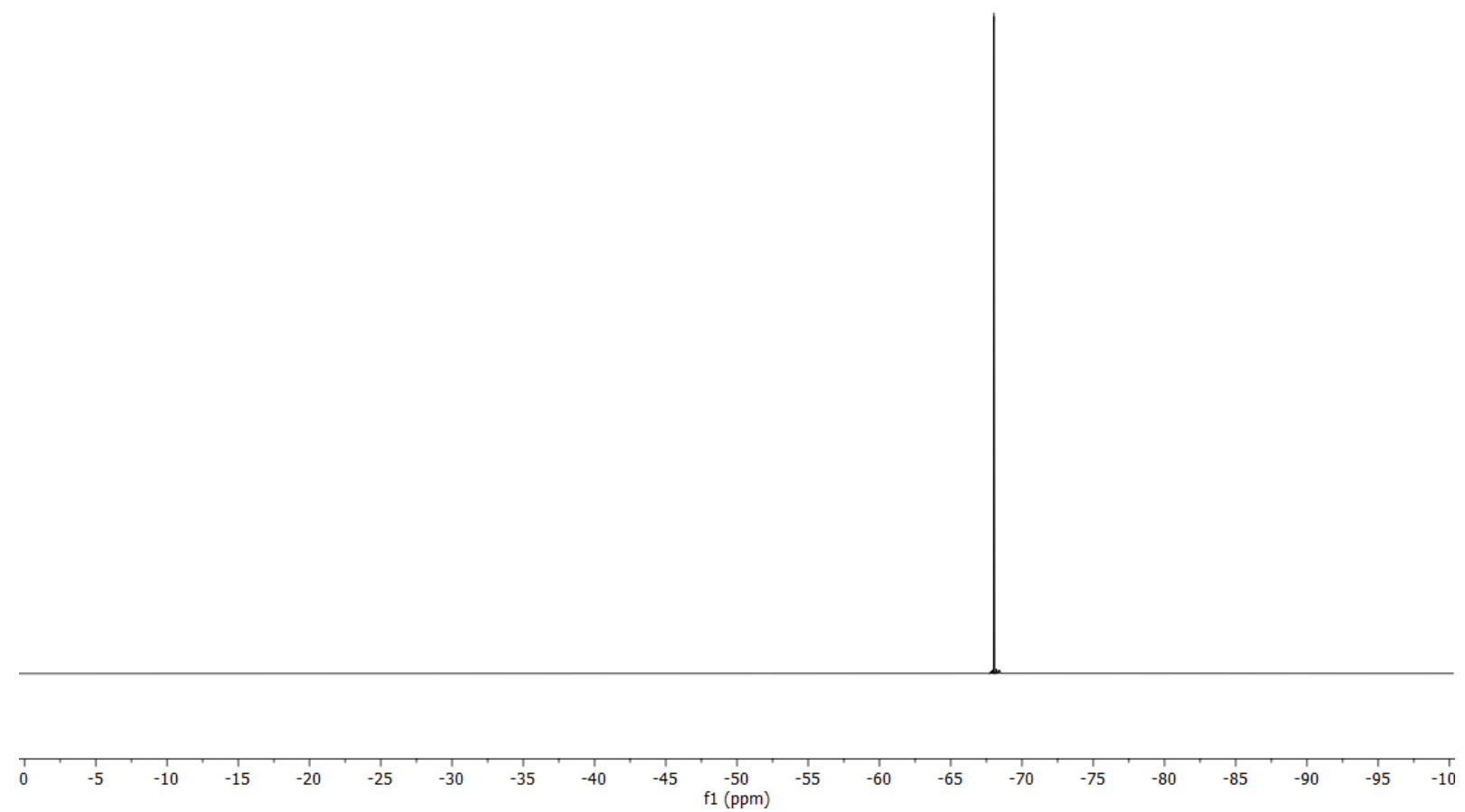




\section{References}

(1) Harris, R. K.; Becker, E. D.; Cabral de Menezes, S. M.; Goodfellow, R.; Granger, P. NMR Nomenclature. Nuclear Spin Properties and Conventions for Chemical Shifts (IUPAC Recommendations 2001). Pure Appl. Chem. 2001, 73, 1795-1818.

(2) Still, W. C.; Kahn, M.; Mitra, A. Rapid Chromatographic Technique for Preparative Separations with Moderate Resolution. J. Org. Chem. 1978, 14, 2923-2925.

(3) Pangborn, A. B.; Giardello, M. A.; Grubbs, R. H.; Rosen, R. K.; Timmers, F. J. Safe and Convenient Procedure for Solvent Purification. Organometallics 1996, 15, 15181520 .

(4) Huang, H.-M.; Bellotti, P.; Daniliuc, C. G.; Glorius, F. Radical Carbonyl Propargylation by Dual Catalysis. Angew. Chem. Int. Ed. 2021, 60, 2464-2471.

(5) Aizpurua, J. M.; Palomo, C. Reagents and Synthetic Methods; 14. A Facile Synthesis of Carboxylic Acid Bromides and Esters under Neutral Conditions via Reaction of the Trimethylsilyl Esters with Triphenylphophine Dibromide. Synthesis (Stuttg). 1982, 1982, 684-687.

(6) Yamaoka, Y.; Yamamoto, H. Super Silyl Stereo-Directing Groups for Complete 1,5Syn and -Anti Stereoselectivities in the Aldol Reactions of $\beta$-Siloxy Methyl Ketones with Aldehydes. J. Am. Chem. Soc. 2010, 132, 5354-5356.

(7) Sahoo, B.; Bellotti, P.; Juliá-Hernández, F.; Meng, Q.-Y.; Crespi, S.; König, B.; Martin, R. Site-Selective, Remote $\mathrm{Sp}^{3} \mathrm{C}-\mathrm{H}$ Carboxylation Enabled by the Merger of Photoredox and Nickel Catalysis. Chem. - A Eur. J. 2019, 25.

(8) Kelly, C. B.; Patel, N. R.; Primer, D. N.; Jouffroy, M.; Tellis, J. C.; Molander, G. A. Preparation of Visible-Light-Activated Metal Complexes and Their Use in Photoredox/Nickel Dual Catalysis. Nat. Protoc. 2017, 12, 472-492.

(9) Shiner, V. J.; Ensinger, M. W.; Rutkowske, R. D. ..Gamma.-Silicon Stabilization of Carbonium Ions in Solvolysis. 2. Solvolysis of 4-(Trimethylsilyl)-2-Butyl pBromobenzenesulfonates. J. Am. Chem. Soc. 1987, 109, 804-809.

(10) Wang, J.; Ma, C.; Fiorin, G.; Carnevale, V.; Wang, T.; Hu, F.; Lamb, R. A.; Pinto, L. H.; Hong, M.; Klein, M. L.; DeGrado, W. F. Molecular Dynamics Simulation Directed Rational Design of Inhibitors Targeting Drug-Resistant Mutants of Influenza A Virus M2. J. Am. Chem. Soc. 2011, 133, 12834-12841.

(11) Snider, B. B.; Vo, N. H.; Foxman, B. M. Manganese(III)-Based Oxidative Fragmentation-Cyclization Reactions of Unsaturated Cyclobutanols. J. Org. Chem. 1993, 58, 7228-7237.

(12) Matsuda, D.; Kawamura, M.; Kobashi, Y.; Shiozawa, F.; Suga, Y.; Fusegi, K.; Nishimoto, S.; Kimura, K.; Miyoshi, M.; Takayama, N.; Kakinuma, H.; Ohtake, N. Design, Synthesis and Biological Evaluation of Novel 7-Azaspiro[3.5]Nonane Derivatives as GPR119 Agonists. Bioorg. Med. Chem. 2018, 26, 1832-1847.

(13) Balannik, V.; Wang, J.; Ohigashi, Y.; Jing, X.; Magavern, E.; Lamb, R. A.; DeGrado, W. F.; Pinto, L. H. Design and Pharmacological Characterization of Inhibitors of 
Amantadine-Resistant Mutants of the M2 Ion Channel of Influenza A Virus. Biochemistry 2009, 48, 11872-11882.

(14) CASON, J.; WALLCAVE, L.; WHITESIDE, C. N. A CONVENIENT PREPARATION OF SUBERIC ACID. CONCERNING THE HOMOGENEITY AND USE IN SYNTHESIS OF POLYMETHYLENE CHLOROBROMIDE PREPARATIONS. J. Org. Chem. 1949, 14, 3744.

(15) Hanack, M.; Fuchs, K. A.; Collins, C. J. Vinyl Cations. 40. .Pi.- and .Sigma.-Routes to Vinyl Cations. Solvolyses of 2-Methylcyclohexenyl, Cyclopentylideneethyl, Hex-5Yn-1-Yl, and Related Triflates. J. Am. Chem. Soc. 1983, 105, 4008-4017.

(16) Ausín, C.; Kauffman, J. S.; Duff, R. J.; Shivaprasad, S.; Beaucage, S. L. Assessment of Heat-Sensitive Thiophosphate Protecting Groups in the Development of Thermolytic DNA Oligonucleotide Prodrugs. Tetrahedron 2010, 66, 68-79.

(17) Furnival, R. C.; Saruengkhanphasit, R.; Holberry, H. E.; Shewring, J. R.; Guerrand, H. D. S.; Adams, H.; Coldham, I. Cascade Oxime Formation, Cyclization to a Nitrone, and Intermolecular Dipolar Cycloaddition. Org. Biomol. Chem. 2016, 14, 10953-10962.

(18) Chong, J. M.; Heuft, M. A.; Rabbat, P. Solvent Effects on the Monobromination of $\alpha, \omega-$ Diols: A Convenient Preparation of $\omega$-Bromoalkanols. J. Org. Chem. 2000, 65, 5837-5838.

(19) Pearson, W. H.; Hutta, D. A.; Fang, W. Azidomercurations of Alkenes: MercuryPromoted Schmidt Reactions. J. Org. Chem. 2000, 65, 8326-8332.

(20) Layton, M. E.; Morales, C. A.; Shair, M. D. Biomimetic Synthesis of (-)-Longithorone A. J. Am. Chem. Soc. 2002, 124, 773-775.

(21) Zhang, Y.; Torker, S.; Sigrist, M.; Bregović, N.; Dydio, P. Binuclear Pd(I)-Pd(I) Catalysis Assisted by Iodide Ligands for Selective Hydroformylation of Alkenes and Alkynes. J. Am. Chem. Soc. 2020, 142, 18251-18265.

(22) Yang, P.-F.; Shu, W. Direct Synthesis of Mono- $\alpha$-Arylated Ketones from Alcohols and Olefins via Ni-Catalyzed Oxidative Cross-Coupling. Org. Lett. 2020, 22, 6203-6208.

(23) Li, H.; Hou, Y.; Liu, C.; Lai, Z.; Ning, L.; Szostak, R.; Szostak, M.; An, J. Pentafluorophenyl Esters: Highly Chemoselective Ketyl Precursors for the Synthesis of $\alpha, \alpha$-Dideuterio Alcohols Using SmI2 and D20 as a Deuterium Source. Org. Lett. 2020, 22, 1249-1253.

(24) Friest, J. A.; Maezato, Y.; Broussy, S.; Blum, P.; Berkowitz, D. B. Use of a Robust Dehydrogenase from an Archael Hyperthermophile in Asymmetric Catalysis-Dynamic Reductive Kinetic Resolution Entry into (S)-Profens. J. Am. Chem. Soc. 2010, 132, 5930-5931.

(25) Catti, L.; Tiefenbacher, K. Brønsted Acid-Catalyzed Carbonyl-Olefin Metathesis inside a Self-Assembled Supramolecular Host. Angew. Chem. Int. Ed. 2018, 57, 14589-14592. 
(26) Vulovic, B.; Trmcic, M.; Matovic, R.; Saicic, R. N. Cyclization Reactions of Oxyallyl Cation. A Method for Cyclopentane Ring Formation. Org. Lett. 2019, 21, 96189621.

(27) Polites, V. C.; Badir, S. O.; Keess, S.; Jolit, A.; Molander, G. A. Nickel-Catalyzed Decarboxylative Cross-Coupling of Bicyclo[1.1.1]Pentyl Radicals Enabled by Electron Donor-Acceptor Complex Photoactivation. Org. Lett. 2021, 23, 48284833.

(28) Flegeau, E. F.; Harrison, J. M.; Willis, M. C. One-Pot Sulfonamide Synthesis Exploiting the Palladium-Catalyzed Sulfination of Aryl Iodides. Synlett 2016, 27, 101-105.

(29) Vassilikogiannakis, G.; Hatzimarinaki, M.; Orfanopoulos, M. Mechanism of the [2+ 2] Photocycloaddition of Fullerene C60 with Styrenes. J. Org. Chem. 2000, 65, 81808187.

(30) Bao, Y.-S.; Chen, C.-Y.; Huang, Z.-Z. Transesterification for Synthesis of Carboxylates Using Aldehydes as Acyl Donors via $\mathrm{C}-\mathrm{H}$ and $\mathrm{C}-\mathrm{O}$ Bond Activations. J. Org. Chem. 2012, 77, 8344-8349.

(31) Arsenyan, P.; Vasiljeva, J.; Domracheva, I.; Kanepe-Lapsa, I. 8-Ethynylxanthines as Promising Antiproliferative Agents, Angiogenesis Inhibitors, and Calcium Channel Activity Modulators. Chem. Heterocycl. Compd. 2020, 56, 776-785.

(32) Alandini, N.; Buzzetti, L.; Favi, G.; Schulte, T.; Candish, L.; Collins, K. D.; Melchiorre, P. Amide Synthesis by Nickel/Photoredox-Catalyzed Direct Carbamoylation of (Hetero)Aryl Bromides. Angew. Chem. Int. Ed. 2020, 59, 5248-5253.

(33) Guo, J.; Frost, J. W. Kanosamine Biosynthesis: A Likely Source of the Aminoshikimate Pathway's Nitrogen Atom. J. Am. Chem. Soc. 2002, 124, 1064210643.

(34) An, L.; Xiao, Y.-L.; Min, Q.-Q.; Zhang, X. Facile Access to Fluoromethylated Arenes by Nickel-Catalyzed Cross-Coupling between Arylboronic Acids and Fluoromethyl Bromide. Angew. Chem. Int. Ed. 2015, 54, 9079-9083.

(35) Bandaev, S. G.; Šabarov, Y. S.; Hantschmann, A.; Weissenfels, M. Zur Stereochemie Der Ringöffnung von Trans-1,2-Diphenylcyclopropanen Mit Quecksilber(II)Salzen. J. für Prakt. Chemie 1980, 322, 643-648.

(36) Fleming, I.; Higgins, D.; Lawrence, N. J.; Thomas, A. P. A Regioselective and Stereospecific Synthesis of Allylsilanes from Secondary Allylic Alcohol Derivatives. J. Chem. Soc. Perkin Trans. 1 1992, No. 24, 3331-3349.

(37) Huang, H.; Kang, J. Y. Mitsunobu Reaction Using Basic Amines as Pronucleophiles. J. Org. Chem. 2017, 82, 6604-6614.

(38) Krasovskiy, A.; Knochel, P. A LiCl-Mediated Br/Mg Exchange Reaction for the Preparation of Functionalized Aryl- and Heteroarylmagnesium Compounds from Organic Bromides. Angew. Chem. Int. Ed. 2004, 43, 3333-3336. 
(39) Garcia, K. J.; Gilbert, M. M.; Weix, D. J. Nickel-Catalyzed Addition of Aryl Bromides to Aldehydes to Form Hindered Secondary Alcohols. J. Am. Chem. Soc. 2019, 141, 1823-1827.

(40) Sun, S.-Z.; Martin, R. Nickel-Catalyzed Umpolung Arylation of Ambiphilic $\alpha$ Bromoalkyl Boronic Esters. Angew. Chem. Int. Ed. 2018, 57, 3622-3625.

(41) Biswas, S.; Weix, D. J. Mechanism and Selectivity in Nickel-Catalyzed CrossElectrophile Coupling of Aryl Halides with Alkyl Halides. J. Am. Chem. Soc. 2013, $135,16192-16197$.

(42) Gilbert, M. M.; Trenerry, M. J.; Longley, V. R.; Berry, J. F.; Weix, D. J. Ligand-Metal Cooperation Enables C-C Activation Cross-Coupling Reactivity of Cyclopropyl Ketones. ChemRxiv 2021.

(43) Xiao, J.; Li, Z.; Montgomery, J. Nickel-Catalyzed Decarboxylative Coupling of RedoxActive Esters with Aliphatic Aldehydes. J. Am. Chem. Soc. 2021.

(44) Nonhebel, D. C. The Chemistry of Cyclopropylmethyl and Related Radicals. Chem. Soc. Rev. 1993, 22, 347-359.

(45) Zhao, C.; Zha, G.-F.; Fang, W.-Y.; Rakesh, K. P.; Qin, H.-L. Construction of Di(Hetero)Arylmethanes Through Pd-Catalyzed Direct Dehydroxylative CrossCoupling of Benzylic Alcohols and Aryl Boronic Acids Mediated by Sulfuryl Fluoride (SO2F2). European J. Org. Chem. 2019, 2019, 1801-1807.

(46) Zhang, P.; Le, C. “Chip”; MacMillan, D. W. C. Silyl Radical Activation of Alkyl Halides in Metallaphotoredox Catalysis: A Unique Pathway for Cross-Electrophile Coupling. J. Am. Chem. Soc. 2016, 138, 8084-8087.

(47) Pitzer, L.; Schäfers, F.; Glorius, F. Rapid Assessment of the Reaction-ConditionBased Sensitivity of Chemical Transformations. Angew. Chem. Int. Ed. 2019, 58, 8572-8576. 